\title{
An Integrated Approach to Naval Ship Survivability in Preliminary Ship Design
}

Alexander Spyro Piperakis

Thesis submitted for the degree of Doctor of Philosophy

Department of Mechanical Engineering, UCL 


\section{$\underline{\text { Declaration }}$}

I, Alexander Spyro Piperakis, confirm that the work presented in this thesis is my own. Where information has been derived from other sources, I confirm that this has been indicated in the thesis. 


\section{$\underline{\text { Abstract }}$}

Alongside deploying weapons and sensors what makes a warship distinct is survivability, being the measure that enables a warship to survive in a militarily hostile environment. The rising cost of warship procurement, coupled with declining defence budgets, has led to a reduction in the number of ships in most western navies. Moreover, cost cutting is often aimed at aspects which may be difficult to quantify, such as survivability, and can lead to potentially inadequate designs. Innovation in both the design process and the design of individual ships is, therefore, necessary, especially at the crucial early design stages. Computer technology can be utilised to exploit architecturally orientated preliminary design approaches which can address innovation early in the ship design process and in issues such as survivability. A number of survivability assessment tools currently exist; however, most fail to integrate all survivability constituents (i.e. susceptibility, vulnerability and recoverability), in that they are unable to balance between the component aspects of survivability. Some are qualitative, therefore less than ideal for requirement specification, others are aimed towards the detailed design stages where implementing changes is heavily constrained or even impractical.

Since a ship's survivability is dependent on layout, the approach adopted in this research takes advantage of an architecturally orientated ship design approach applicable to early stage design. Such a method is proposed and demonstrated on five combatant (including a trimaran configuration) and two auxiliary ship design studies. The proposed method combines various tools used by UCL and the UK Ministry of Defence, as well as a new approach for recoverability assessment and, therefore, tackles difficulties currently associated with the latter (e.g.: lack of data, human performance and time dependence) by using weighted performance measures.

An overall approach for survivability assessment has been applied across the range of designs produced and conclusions drawn on their relative merits for overall survivability. The approach and implications of the integration of survivability assessment in the preliminary ship design stages, as well as the identification of major survivability design drivers, are discussed. Through the identification of problematic topics, areas for further research are suggested. It is envisaged that this research will assist in developing the design process of what are, according to Captain C. Graham, USN, "the most complex, diverse and highly integrated of any engineering systems" produced today on a regular basis. 


\section{Acknowledgements}

First and foremost, I would like to express my gratitude to my supervisor, Prof D.J. Andrews, for giving me the opportunity to work in his research group. His constant support and guidance throughout my $\mathrm{PhD}$ were admirable.

I would also like to acknowledge the generous assistance provided to me by the staff of the Department of Mechanical Engineering of UCL, and in particular by Dr R.G. Pawling.

Funding for this project was provided by EPSRC (CASE studentship) and sponsorship by Dstl, both institutes to which I am grateful. Special thanks go to the Dstl sponsoring officer, Dr J.S. Thornton, as well as to Lt. Cdr. (rtd.) T. Day, RN, Dr K. White and Mr S.J. Parry, all of whom belong to the Maritime Integrated Survivability team (part of the Maritime Systems Engineering Group in Naval Systems Department) of Dstl.

I am also thankful for the assistance provided by 1st Lt. P. Fonseca, Portuguese Navy, and by Cdr. J. Sutcliffe, Lt. Cdr. (rtd.) J. Mant, Lt. Cdr. J. Boughton, Lt. Cdr. K. Hood, Lt. Cdr. B. Kadinopoulos, Lt. Cdr. T. O'Brien and Lt. M. Koheeallee, RN.

Finally, I am especially grateful to my family for their continued support and encouragement. 
Chapter 1: Introduction $\quad 25$

\begin{tabular}{ll}
1.1 Preamble & 25 \\
\hline
\end{tabular}

\begin{tabular}{lr}
1.2 Research Scope and Aim & 26 \\
\hline
\end{tabular}

\begin{tabular}{lr}
\hline .3 Structure of the Thesis & 27 \\
\hline
\end{tabular}

Chapter 2: Ship Design Issues $\quad 30$

2.1 Ship Design $\quad 30$

2.1.1 Background to Naval Ship Design and Procurement 30

\begin{tabular}{ll}
2.1 .2 Introduction to Ship Design & 31 \\
\hline
\end{tabular}

2.1.3 Traditional Preliminary Ship Design 33

2.1.4 Architecturally Orientated Preliminary Ship Design 37

2.1.5 The Design Building Block Approach to Preliminary Ship Design 43

2.2 Ship Costing $\quad 48$

\begin{tabular}{lr}
\hline .3 Conclusions on Ship Design Issues & 57 \\
\hline
\end{tabular}

Chapter 3: Background to Survivability $\quad \mathbf{5 8}$

\begin{tabular}{lr}
3.1 Introduction to Survivability & 58 \\
\hline 3.2 Threats
\end{tabular}

\begin{tabular}{ll}
3.2 Threats & 63 \\
\hline 3.3 Surviability Assessment
\end{tabular}

\begin{tabular}{lr}
3.3 Survivability Assessment & 65 \\
\hline
\end{tabular}

3.3.1 Introduction $\quad 65$

\begin{tabular}{ll}
3.3 .2 Susceptibility Assessment & 67 \\
\hline 3.3 .3 tulnerabs
\end{tabular}

\begin{tabular}{ll}
3.3 .3 Vulnerability Assessment & 69 \\
\hline 3.3 .4 Recoraty
\end{tabular}

\begin{tabular}{lr} 
3.3.4 Recoverability Assessment & 75 \\
\hline 3.5 Total Survabilty Assessment
\end{tabular}

\begin{tabular}{lr}
3.3 .5 Total Survivability Assessment & 83 \\
\hline 3.3 .6 Survivabilty Requirement Specification
\end{tabular}

\begin{tabular}{ll}
3.3 .6 Survivability Requirement Specification & 87 \\
\hline
\end{tabular}

3.4 Conclusions on Background to Survivability 90

Chapter 4: Survivability Assessment Method Development $r$

\begin{tabular}{ll}
4.1 Susceptibility Assessment & 91 \\
\hline 4.2 Vulnerability Assessment
\end{tabular}

\begin{tabular}{lr}
4.2 Vulnerability Assessment & 94 \\
\hline 4.3 Recoverability Assessment
\end{tabular}

\begin{tabular}{lr}
4.3 Recoverability Assessment & 98 \\
\hline
\end{tabular}

4.3.1 Category 1 Performance Measures $\quad 99$

4.3.2 Category 2 Performance Measures 103

\begin{tabular}{ll}
4.3 .3 Category 3 Performance Measures & 105 \\
\hline 4.3.4 Recoverabity Performance Measures Matix
\end{tabular}

\begin{tabular}{lr}
4.3 .4 Recoverability Performance Measures Matrix & 109 \\
\hline 4.3 .5 Equipment Cate
\end{tabular}

4.3.5 Equipment Categorisation and Repair Data 115

4.4 Total Survivability Assessment 118

\begin{tabular}{lr} 
Chapter 5: Ship Design Studies & 120 \\
\hline
\end{tabular}

\begin{tabular}{lr}
5.1 Frigate Design Studies & 121 \\
\hline
\end{tabular}

\begin{tabular}{lr}
5.1 .1 Baseline Frigate & 123 \\
\hline 5.1 .2 Frgate & 131
\end{tabular}

\begin{tabular}{ll}
5.1 .2 Frigate Variants & 131 \\
\hline
\end{tabular}

5.2 Corvette and Destroyer Design Studies 150

\begin{tabular}{ll}
5.3 Replenishment Ship Design Studies & 170 \\
\hline
\end{tabular}

Chapter 6: Results of Applying Proposed Survivability Assessment Approach 190

\begin{tabular}{lr} 
6.1 Susceptibility Assessment Results & 190 \\
\hline
\end{tabular}

6.1.1 Frigate Variants (Including Baseline) 190 
6.1.2 Corvette, Baseline Frigate and Destroyer $\quad 194$

\begin{tabular}{ll} 
6.1.3 AOR Variants & 198 \\
\hline
\end{tabular}

6.2 Susceptibility Assessment Sensitivity Studies 201

\begin{tabular}{ll}
6.2 .1 External Features & 202 \\
\hline
\end{tabular}

6.2.2 Defensive Missile Targeting Pattern 205

\begin{tabular}{ll}
\hline 6.2 .3 Defensive Missile Type & 206 \\
\hline 6.2 .4
\end{tabular}

\begin{tabular}{lr} 
6.2.4 Lengthwise Probability Hit Distribution & 209 \\
\hline
\end{tabular}

6.3 Vulnerability Assessment Results $\quad 209$

\begin{tabular}{ll} 
6.3.1 Frigate Variants (Including Baseline) & 211 \\
\hline
\end{tabular}

\begin{tabular}{ll} 
6.3.2 Corvette, Baseline Frigate and Destroyer & 212 \\
\hline
\end{tabular}

\begin{tabular}{ll} 
6.3.3 AOR Variants & 214 \\
\hline
\end{tabular}

6.4 Vulnerability Assessment Sensitivity Studies $\quad 216$

6.4.1 System Weighting Schemes 216

6.4.2 Lengthwise Probability Hit Distribution 221

\begin{tabular}{ll} 
6.4.3 ASM Attack Angle & 224 \\
\hline
\end{tabular}

\begin{tabular}{lr}
6.5 Recoverability Assessment Results & 225 \\
\hline 6.5 .1 Frigat Varants (Including Baseine)
\end{tabular}

6.5.1 Frigate Variants (Including Baseline) $\quad 225$

\begin{tabular}{lr}
6.5 .2 Corvette, Baseline Frigate and Destroyer & 229 \\
\hline 6.5 .32
\end{tabular}

\begin{tabular}{ll}
6.5 .3 AOR Variants & 233 \\
\hline
\end{tabular}

6.6 Recoverability Assessment Sensitivity Studies $\quad 237$

\begin{tabular}{ll} 
6.6.1 Weighting Schemes & 237 \\
\hline
\end{tabular}

6.6.2 Lengthwise Probability Hit Distribution 248

\begin{tabular}{ll} 
6.6.3 SURVIVE Lite Decoupling 249 \\
\hline
\end{tabular}

6.7 Total Survivability Assessment Results 253

6.8 Ship Costing Results 256

6.8.1 Unit Procurement Cost $\quad 256$

\begin{tabular}{lr}
\hline 6.8 .2 Through Life Cost & 257 \\
\hline
\end{tabular}

\begin{tabular}{ll}
6.8 .3 Whole Life Cost & 261 \\
\hline
\end{tabular}

\begin{tabular}{lr} 
Chapter 7: Discussion & $\mathbf{2 6 4}$ \\
\hline
\end{tabular}

7.1 Susceptibility Assessment Results Analysis $\quad 264$

7.1.1 Frigate Variants (Including Baseline) 264

7.1.2 Corvette, Baseline Frigate and Destroyer 265

\begin{tabular}{ll}
7.1 .3 AOR Variants & 266 \\
\hline 7.4 Gental
\end{tabular}

7.1.4 General Observations on Susceptibility Assessment $\quad 267$

\begin{tabular}{ll}
7.2 Vulnerability Assessment Results Analysis & 270 \\
\hline
\end{tabular}

\begin{tabular}{ll}
7.2 .1 Frigate Variants (Including Baseline) & 271 \\
\hline
\end{tabular}

\begin{tabular}{ll}
7.2 .2 Corvette, Baseline Frigate and Destroyer & 273 \\
\hline $7.2 .320 R$
\end{tabular}

\begin{tabular}{ll}
7.2 .3 AOR Variants & 275 \\
\hline 7.2 .4 General & 277 \\
\hline
\end{tabular}

7.2.4 General Observations on Vulnerability Assessment $\quad 277$

7.3 Recoverability Assessment Results Analysis 280

7.3.1 Frigate Variants (Including Baseline) 280

\begin{tabular}{ll} 
7.3.2 Corvette, Baseline Frigate and Destroyer & 287 \\
\hline
\end{tabular}

\begin{tabular}{ll}
7.3 .3 AOR Variants & 291 \\
\hline 7.3 .4 General
\end{tabular}

7.3.4 General Observations on Recoverability Assessment 294

7.4 Total Survivability Assessment Results Analysis 297

\begin{tabular}{ll} 
7.4.1 Total Survivability Star (Triangle) Plots & 297 \\
\hline 7.4 .297
\end{tabular}

\begin{tabular}{lr}
\hline 7.4 .2 Total Survivability Bar Charts & 299 \\
\hline
\end{tabular}

7.5 Ship Costing Results Analysis $\quad 300$

\begin{tabular}{lr}
7.5 .1 Unit Procurement Cost & 300 \\
\hline 7.5 .2 Through
\end{tabular}

\begin{tabular}{ll}
7.5 .2 Through Life Cost & 301 \\
\hline 7.5 .3 Whole Life Cost
\end{tabular}

\begin{tabular}{ll}
7.5 .3 Whole Life Cost & 302 \\
\hline
\end{tabular} 
7.5.4 General Observations on Ship Costing and Survivability Assessment 303 7.6 Research Review 303

7.6.1 The Need for Survivability Quantification 304

7.6.2 The Importance of Survivability Assessment in Preliminary Ship Design

7.6.3 Survivability Assessment and Architecturally Orientated Design 306

\begin{tabular}{ll} 
7.6.4 Susceptibility Assessment & 307 \\
\hline 7.6 .5 Vulnerabilty Assessment & 307 \\
\hline
\end{tabular}

\begin{tabular}{ll}
7.6 .5 Vulnerability Assessment & 307 \\
\hline 7.6 .6
\end{tabular}

\begin{tabular}{ll} 
7.6.6 Recoverability Assessment & 308 \\
\hline
\end{tabular}

\begin{tabular}{l} 
7.6.7 Comparison between MISSION and Proposed Approach $\quad 310$ \\
\hline 7.6 .8
\end{tabular}

\begin{tabular}{ll}
7.6 .8 Advantages of the Proposed Approach & 312 \\
\hline
\end{tabular}

\begin{tabular}{ll}
7.6 .9 Future Work & 313 \\
\hline
\end{tabular}

Chapter 8: Conclusions and Future Work

$\underline{\text { References }}$

Appendices 340

Appendix 1: Margins in Ship Design 340

Appendix 2: Ship Type Design Drivers 341

A2.1 Combatants 341

\begin{tabular}{ll} 
A2.2 Trimaran Combatants & 343 \\
\hline
\end{tabular}

\begin{tabular}{ll} 
A2.3 Replenishment Ships & 344 \\
\hline
\end{tabular}

Appendix 3: Survivability Definitions $\quad 346$

Appendix 4: Survivability Constituents $\quad 352$

\begin{tabular}{lr} 
A4.1 Susceptibility Features & 352 \\
\hline A4.2 Vun
\end{tabular}

\begin{tabular}{lr} 
A4.2 Vulnerability Features & 358 \\
\hline
\end{tabular}

\begin{tabular}{lr} 
A4.3 Recoverability Features & 369 \\
\hline
\end{tabular}

Appendix 5: An Integrated Approach to Naval Ship Survivability in Preliminary

\begin{tabular}{ll} 
Ship Design (RINA Warship 2012) & 382 \\
\hline
\end{tabular}

Appendix 6: Vulnerability and Recoverability Equipment and Compartment

\begin{tabular}{ll} 
Categorisation & 401 \\
\hline
\end{tabular}

Appendix 7: Ship System Tree Diagrams and Architecture 403

\begin{tabular}{ll} 
A7.1 Combatant System Tree Diagrams & 403 \\
\hline
\end{tabular}

\begin{tabular}{ll}
\hline A7.2 Combatant System Architecture & 408 \\
\hline
\end{tabular}

\begin{tabular}{lr}
\hline A7.3 AOR System Tree Diagrams & 413 \\
\hline
\end{tabular}

A7.4 AOR System Architecture 419

Appendix 8: General Arrangements - Provided in attached CD ROM (inside

cover)_ 421

\begin{tabular}{ll} 
A8.1 Frigate Variant 1 & 421 \\
\hline
\end{tabular}

\begin{tabular}{ll} 
A8.2 Frigate Variant 2 & 421 \\
\hline
\end{tabular}

\begin{tabular}{ll} 
A8.3 Frigate Variant 3 & 421 \\
\hline
\end{tabular}

\begin{tabular}{ll} 
A8.4 Corvette & 421 \\
\hline
\end{tabular}

\begin{tabular}{ll} 
A8.5 Destroyer & 421 \\
\hline A8.6
\end{tabular}

\begin{tabular}{lr}
\hline A8.6 AOR Variant 1 & 421 \\
\hline A8.7
\end{tabular}

\begin{tabular}{ll}
\hline A8.7 AOR Variant 2 & 421 \\
\hline
\end{tabular}

Appendix 9: Ship Design Studies Data 4422

A9.1 Complement and Accommodation Requirements 422

\begin{tabular}{ll}
\hline A9.2 Weight Breakdown & 423 \\
\hline
\end{tabular}

A9.3 Margin Philosophy 426 
A9.4 Powering Requirements

A9.5 Hydrostatics Analysis

Appendix 10: CSEE Data and Results

A10.1 Frigate Variants (Including Baseline)

A10.2 Corvette, Baseline Frigate and Destroyer

A10.3 AOR Variants

A10.4 Destroyer Sensitivity Studies

Appendix 11: SURVIVE Lite Results 457

A11.1 Frigate Variants (Including Baseline) 457

\begin{tabular}{ll}
\hline A11.2 Corvette and Destroyer & 458 \\
\hline
\end{tabular}

A11.3 AOR Variants 459

A11.4 ASM Attack Angle Variation 459

Appendix 12: Weighting Scheme Form 460

Appendix 13: Cost Analysis Data and Results 462

A13.1 UPC Analysis Data and Results 462

A13.2 TLC Analysis Data and Results 468

A13.3 WLC Analysis Results 471

Appendix 14: Detailed Vulnerability Assessment Results Analysis 472

A14.1 Frigate Variants (Including Baseline) 472

A14.2 Corvette, Baseline Frigate and Destroyer 476 A14.3 AOR Variants 479

CD ROM (Appendix 8) - inside cover 


\section{List of Figures}

Figure 2.1: A Summary Representation of Current Sequential Synthesis (Andrews 1986)

Figure 2.2: A Simplified Model of Initial Ship Sizing (Andrews 1986)

Figure 2.3: A Schematic Modification of the above Initial Ship Synthesis with Spatial Totals which are Laid Out Graphically before Reiterating (Andrews 1986)......40

Figure 2.4: A Summary Representation of a more 'Holistic' Approach to a Fully Integrated Ship Synthesis (Andrews 1986)....

Figure 2.5: Circles of Influence leading to Full Synthesis (Andrews 1986).

Figure 2.6: Building Block Design approach applied to Surface Ships (Andrews and Dicks 1997)

Figure 2.7: Building Block Design Steps (Andrews and Dicks 1997) ........................45

Figure 2.8: SURFCON Representation Showing the Three Panes for Tree Structure, Graphics (Design Building Blocks) and Tabular Interfaces, with the Results of Stability and Resistance Analyses Also Visible (Andrews and Pawling 2008).. 47

Figure 2.9: Unit Procurement Cost, Through Life Cost and Whole Life Cost Breakdown (UCL 2010b).

Figure 2.10: Breakdown of Warship Cost and Typical Example of the Breakdown in Life Cycle Cost for a Warship (Brown and Andrews 1980).

Figure 2.11: Typical Warship UPC Breakdowns (Dirksen 1996) ..................................51

Figure 2.12: Life Costs of a 3,000 Tonne frigate (Rawson 1973) .................................52

Figure 2.13: The Greatest Opportunity to Reduce Life Cycle Costs Usually Occurs During the Early Stages of a Programme (NATO 2009) ......................................53

Figure 2.14: Cost Estimating Methods (NATO 2009)................................................53

Figure 2.15: Example of a Baseline Life Cycle Cost Spend Profile (left) and a Life Cycle Cost Allocation (right) (NATO 2009)

Figure 2.16: The Relationship Between Data Maturity and Level of Assumptions to be Applied (NATO 2009)

Figure 3.1: Survivability is a Subject, Which Depends on the Interrelation of Many Subjects. Not Only Design but Also Operational Effects Can play an Important Role in Ship Survivability (NATO 2003a)

Figure 3.2: Anti-shipping Threat Weapons and Effects (Said 1995).

Figure 3.3: Traditional Design Spiral and the New Approach With Fully Integrated Survivability Assessment (Holmberg and Kotiranta 2012)

Figure 3.4: The Process of Placing the Hazards Within the Matrix Converts Them Into Risks (Barnett 1998)

Figure 3.5: Vulnerability Assessment Models by Navy (Ashe et al 2006)...................69

Figure 3.6: REVS Vulnerability Assessment Code (Tozer 1993) ................................ 70

Figure 3.7: SURVIVE Equipment Layout (Turner et al 2006)................................... 71

Figure 3.8: SURVIVE Type 23 Frigate Model in External and Transparent Views

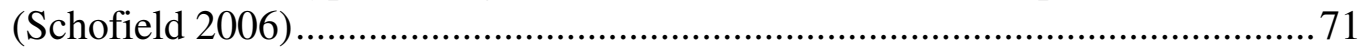

Figure 3.9: Systems Onboard a Frigate Concept (QinetiQ 2005a) ............................. 72

Figure 3.10: Example of the PREVENT Tool (Heywood and Lear 2006) ....................73

Figure 3.11: Sample Concept Attack Configuration (Pugh 2006) ...............................73

Figure 3.12: SURVIVE Lite Rapid Analysis Output (Pugh 2006) ...............................74

Figure 3.13: Proposed EB-FSRS for Old High-rise Buildings (Chow 2002) ................. 76

Figure 3.14: Example List of Performance Measures (Deere et al 2009)......................78

Figure 3.15: Flooding Analysis Model for Project Genesis (Vassalos 2012)............... 79

Figure 3.16: Generic Drawings for Fire Analysis (Left) and Preparation for Fire and Evacuation Simulation (Right) (Mermiris et al 2012). 
Figure 3.17: Node Network Within SURVIVE (Bain 2006) ...................................... 81

Figure 3.18: SURVIVE view showing locations of DCFF equipment (Bain 2006)......81

Figure 3.19: Equipment Systems and Functions Pre-damage (Bain 2006) ....................82

Figure 3.20: Equipment Systems and Functions Post-damage (Bain 2006) .................. 82

Figure 3.21: The Relationship of Various Measures of Probability (Ball and Calvano 1994)

Figure 3.22: Survivability Performance Analysis Flowchart (Papanikolaou and

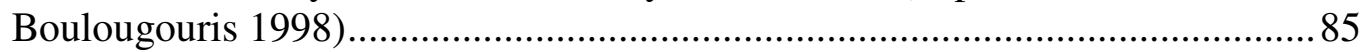

Figure 3.23: Baseline Set of Survivability Design Principles (Richards et al 2008) .....86

Figure 3.24: Top-level View of Survivability Analysis (including feedback loops as green arrows) (Thornton et al 2006) ........................................................... 87

Figure 3.25: The Scenario Expressed in Tasks and Threat (NATO 2003a) .................. 88

Figure 3.26: Probabilistic Approach to Specifying OOVRs for a Surface Ship (Reese et al 1998)

Figure 3.27: Example Threat / Function Table (MOD 2001) ...................................... 90

Figure 4.1: Threat Scenario (McDonald 2010).... 91

Figure 4.2: Relationship Between the Probability That the Missile Locks on the Ship,

P(1), Against the Ratio of (Ship RCS)/(Chaff RCS) ......................................... 92

Figure 4.3: Typical Missile Velocity V. Time Profile (Adams 1988) ............................94

Figure 4.4: SURVIVE Lite Hit Grid (Frigate Variant 1, port side attack) ....................96

Figure 4.5: Simplified Major Ship System Tree Diagram ......................................... 107

Figure 4.6: Example Architecture of Simplified Major Ship System 1......................108

Figure 4.7: A Sample of Star Plots From (Vasudevan and Rusling 2007) .................. 118

Figure 5.1: Frigate Variant 1 Ship Design Study........................................................ 123

Figure 5.2: Frigate Variant 1 Top Level Functional Breakdown................................. 124

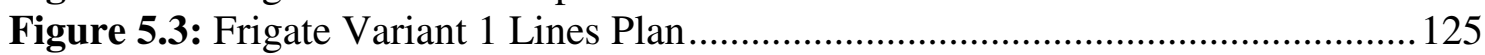

Figure 5.4: Frigate Variant 1 Power and Propulsion Arrangement ............................. 125

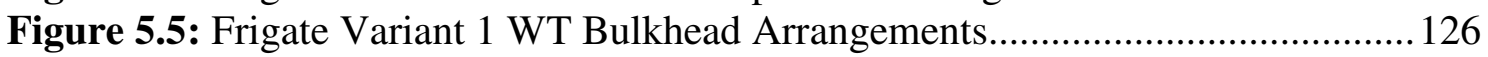

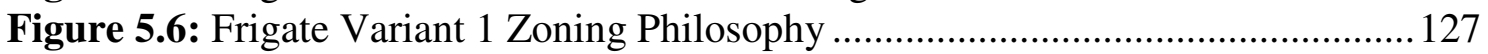

Figure 5.7: Frigate Variant 1 Major Fight System Arrangement................................. 128

Figure 5.8: Frigate Variant 1 Access Philosophy ................................................... 129

Figure 5.9: Frigate Variant 1 Category 1 PM Elements .................................................129

Figure 5.10: Frigate Variant 1 Category 2 PM Elements ............................................ 130

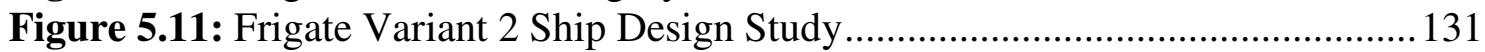

Figure 5.12: Frigate Variant 3 Ship Design Study..................................................... 132

Figure 5.13: Frigate Variant 2 Top Level Functional Breakdown............................. 133

Figure 5.14: Frigate Variant 3 Top Level Functional Breakdown.............................. 133

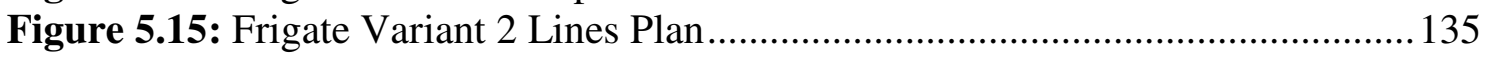

Figure 5.16: Frigate Variant 2 Power and Propulsion Arrangement .......................... 135

Figure 5.17: Frigate Variant 3 Lines Plan................................................................. 136

Figure 5.18: Frigate Variant 3 Power and Propulsion Arrangement ........................... 137

Figure 5.19: Frigate Variant 2 WT Bulkhead Arrangements.......................................139

Figure 5.20: Frigate Variant 3 WT Bulkhead Arrangements..................................... 140

Figure 5.21: Frigate Variant 2 Zoning Philosophy .................................................. 141

Figure 5.22: Frigate Variant 3 Zoning Philosophy .................................................... 142

Figure 5.23: Frigate Variant 2 Major Fight System Arrangement............................... 144

Figure 5.24: Frigate Variant 3 Major Fight System Arrangement................................ 145

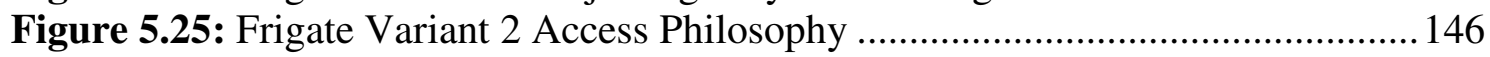

Figure 5.26: Frigate Variant 3 Access Philosophy .................................................. 147

Figure 5.27: Frigate Variant 2 Category 1 PM Elements ......................................... 148 
Figure 5.28: Frigate Variant 3 Category 1 PM Elements ........................................... 148

Figure 5.29: Frigate Variant 2 Category 2 PM Elements ......................................... 149

Figure 5.30: Frigate Variant 3 Category 2 PM Elements ............................................ 150

Figure 5.31: Principal Particulars of the Corvette, Frigate Variant 1 and Destroyer

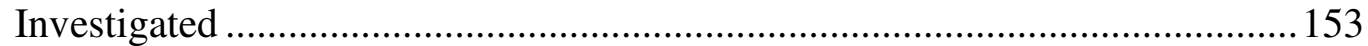

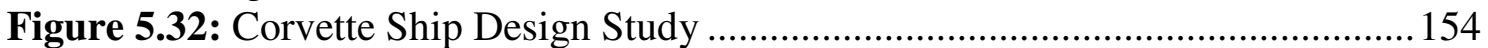

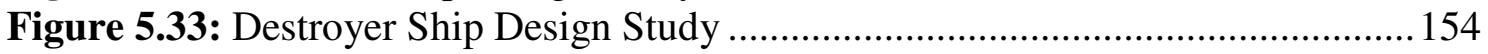

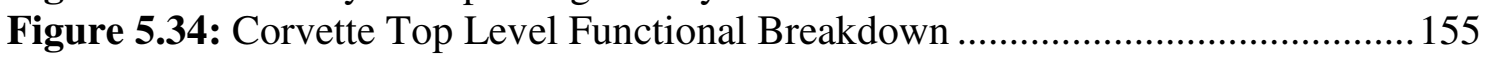

Figure 5.35: Destroyer Top Level Functional Breakdown ...................................... 155

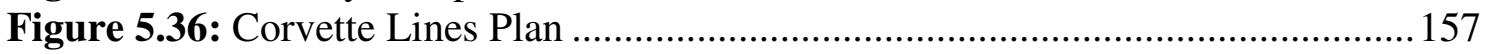

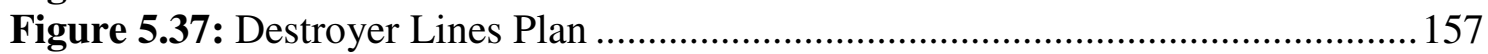

Figure 5.38: Corvette Power and Propulsion Arrangement...................................... 158

Figure 5.39: Destroyer Power and Propulsion Arrangement.......................................159

Figure 5.40: Corvette WT Bulkhead Arrangements .................................................. 161

Figure 5.41: Destroyer WT Bulkhead Arrangements ................................................... 161

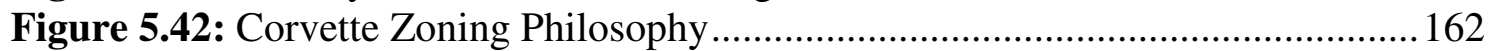

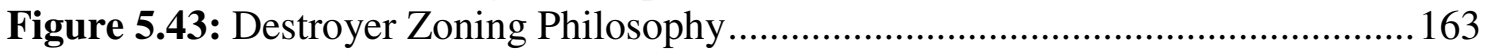

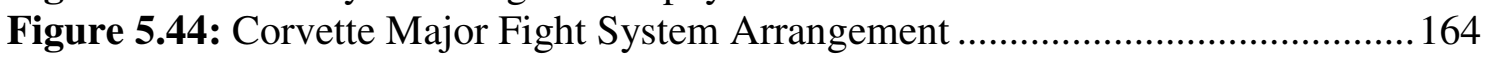

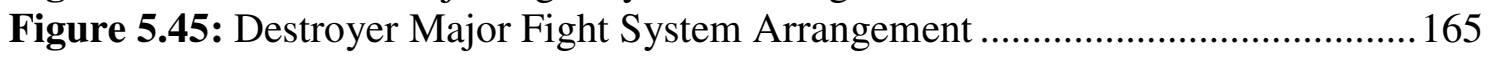

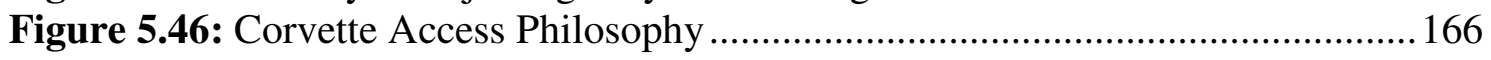

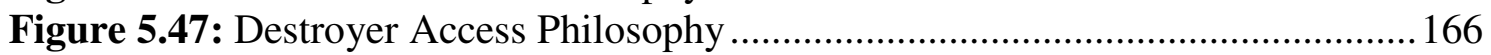

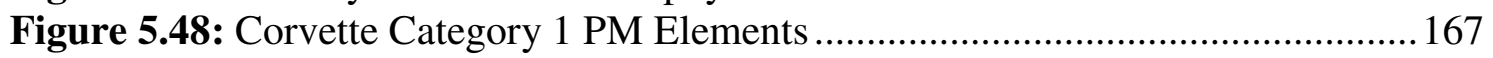

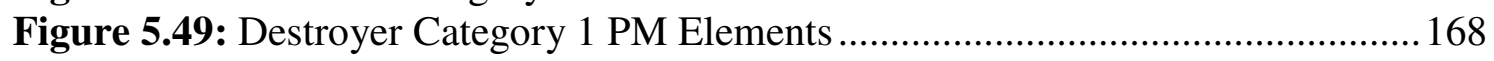

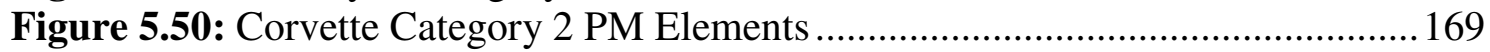

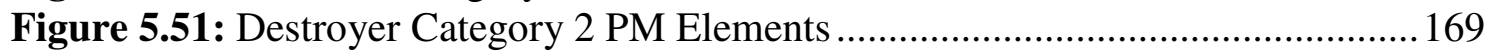

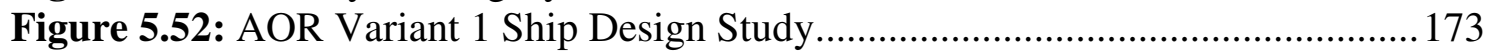

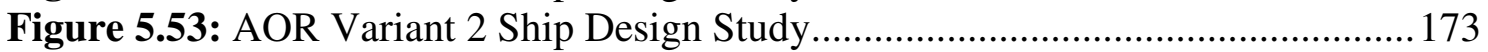

Figure 5.54: AOR Variant 1 Top Level Functional Breakdown ................................ 174

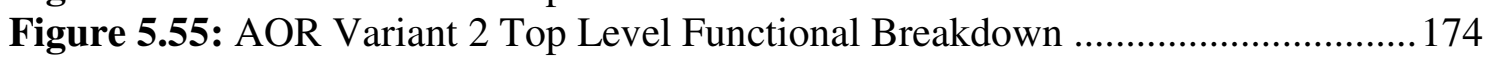

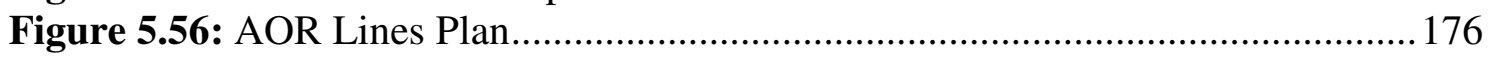

Figure 5.57: AOR Variant 1 Power and Propulsion Arrangement ............................... 177

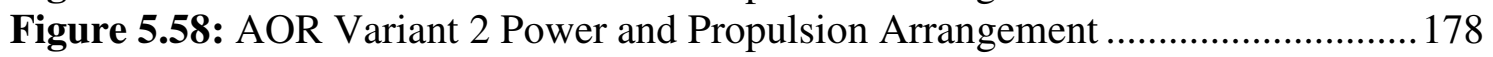

Figure 5.59: AOR Variant 1 WT Bulkhead Arrangements ........................................ 179

Figure 5.60: AOR Variant 2 WT Bulkhead Arrangements ....................................... 179

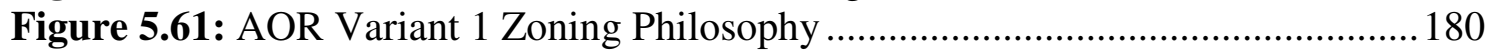

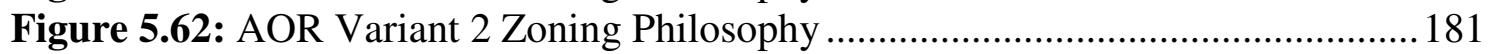

Figure 5.63: AOR Variant 1 Major Fight System Arrangement ................................ 182

Figure 5.64: AOR Variant 2 Major Fight System Arrangement ............................... 183

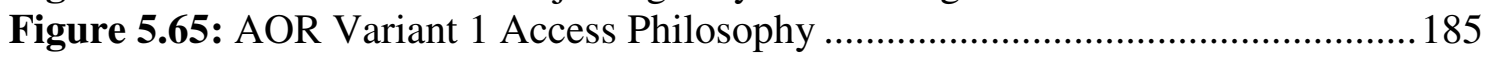

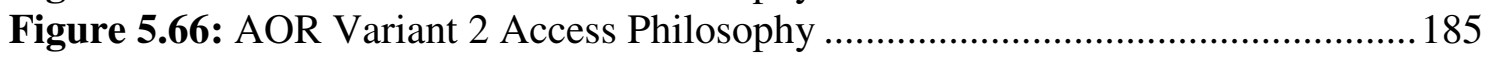

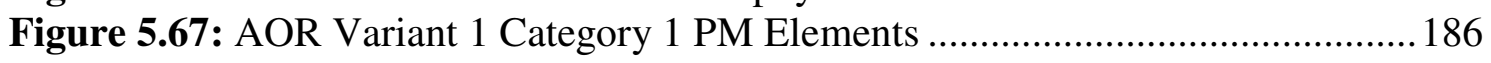

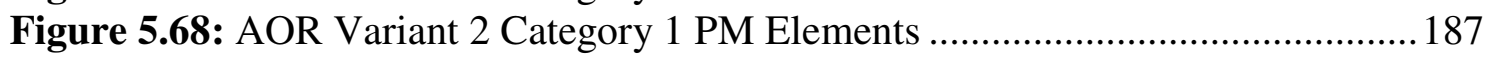

Figure 5.69: AOR Variant 1 Category 2 PM Elements ............................................ 188

Figure 5.70: AOR Variant 2 Category 2 PM Elements ........................................... 188

Figure 6.1: Azimuth Plots of RCS (dBsm) Showing Peak Returns for the Three Frigate

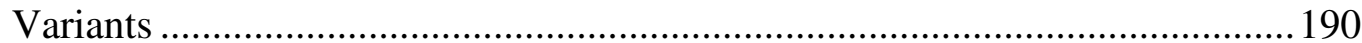

Figure 6.2: Combat System Reaction Timeline for the Three Frigate Variants .......... 191

Figure 6.3: Normalised Plot of Susceptibility for the Three Frigate Variants ............. 193

Figure 6.4: Lengthwise Probability Hit Distribution for the Three Frigate Variants .. 194

Figure 6.5: Azimuth Plots of RCS (dBsm) Showing Peak Returns for the Corvette,

Baseline Frigate and Destroyer ................................................................... 195 
Figure 6.6: Combat System Reaction Timeline for the Destroyer.

Figure 6.7: Normalised Plot of Susceptibility for the Corvette, Frigate Variants and Destroyer

Figure 6.8: Lengthwise Probability Hit Distribution for the Corvette, Baseline Frigate and Destroyer

Figure 6.9: Azimuth Plots of RCS (dBsm) Showing Peak Returns for the Two AOR Variants

Figure 6.10: Combat System Reaction Timeline for the Two AOR Variants ............. 199

Figure 6.11: Normalised Plot of Susceptibility for the Two AOR Variants................201

Figure 6.12: Lengthwise Probability Hit Distribution for the Two AOR Variants .....201

Figure 6.13: Baseline Frigate Microgeometry Signature Reduction Feature .............. 202

Figure 6.14: Before and After Azimuth Plots of RCS (dBsm) Showing Peak Returns for the Baseline Frigate ..............................................................................202

Figure 6.15: Cross-connecting Structure in Trimaran Frigate Variant .......................203

Figure 6.16: Trimaran Frigate Variant Shaping Signature Reduction Feature ............203

Figure 6.17: Before and After Azimuth Plots of RCS (dBsm) Showing Peak Returns for the Trimaran Frigate Variant.

Figure 6.18: Before and After $\mathrm{P}(\mathrm{l})$ Values for Frigate Variants 1 and 3.....................204

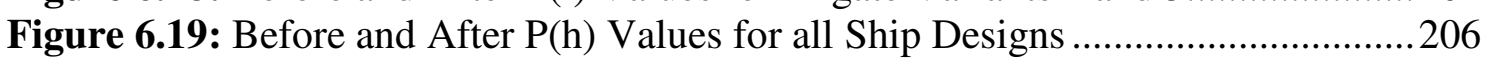

Figure 6.20: Combat System Reaction Timeline for the Destroyer (Aster-30, Maximum Range limited).

Figure 6.21: Combat System Reaction Timeline for the Destroyer (Aster-30, MFR

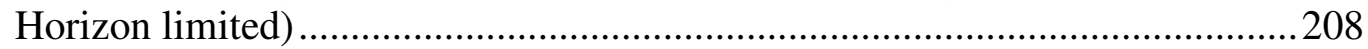

Figure 6.22: Destroyer $\mathrm{P}(\mathrm{h})$ Comparison for Aster-15 and Aster-30 .........................208

Figure 6.23: Linear Lengthwise Probability Hit Distributions for the Frigate Design Studies

Figure 6.24: Total Major Ship System Vulnerability for the Frigate Design Studies . 211

Figure 6.25: Normalised Plot of Vulnerability for the Three Frigate Variants...........212

Figure 6.26: Total Major Ship System Vulnerability for the Corvette, Baseline Frigate and Destroyer Design Studies ....

Figure 6.27: Normalised Plot of Vulnerability for the Corvette, Frigate Variants and Destroyer

Figure 6.28: Total Major Ship System Vulnerability for the AOR Design Studies ....214

Figure 6.29: Normalised Plot of Vulnerability for the Two AOR Variants ................2215

Figure 6.30: System Weighting Scheme Philosophies for the Sensitivity Tests on Frigate Vulnerability...

Figure 6.31: Normalised Plot of Vulnerability for the Three Frigate Variants (Sensitivity Test, Scenario 1)

Figure 6.32: Normalised Plot of Vulnerability for the Three Frigate Variants (Sensitivity Test, Scenario 2)

Figure 6.33: Normalised Plot of Vulnerability for the Three Frigate Variants (Sensitivity Test, Scenario 3)

Figure 6.34: Total Major Ship System Vulnerability for Frigate Variant 1 (Varying Lengthwise Hit Distribution)

Figure 6.35: Total Major Ship System Vulnerability for Frigate Variant 2 (Varying Lengthwise Hit Distribution)

Figure 6.36: Total Major Ship System Vulnerability for Frigate Variant 3 (Varying Lengthwise Hit Distribution)

Figure 6.37: Normalised Plot of Vulnerability for the Three Frigate Variants (Varying Lengthwise Hit Distribution)

Figure 6.38: Total Major Ship System Vulnerability for the Destroyer (Varying Missile Attack Angle) 
Figure 6.39: Normalised Plot of Difficulty of Recoverability for the Three Frigate Variants

Figure 6.40: Normalised Plot of Difficulty of Recoverability for the Corvette, Frigate Variants and Destroyer.

Figure 6.41: Normalised Plot of Difficulty of Recoverability for the Two AOR Variants

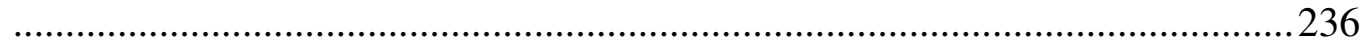

Figure 6.42: Performance Measures Weighting Scheme Philosophies for the Sensitivity Tests

Figure 6.43: Normalised Plot of Difficulty of Recoverability for the Three Frigate

Variants (Sensitivity Test, Scenario 1).

Figure 6.44: Normalised Plot of Difficulty of Recoverability for the Three Frigate Variants (Sensitivity Test, Scenario 2).

Figure 6.45: Normalised Plot of Difficulty of Recoverability for the Three Frigate Variants (Sensitivity Test, Scenario 3).

Figure 6.46: Normalised Plot of Difficulty of Recoverability for the Three Frigate Variants (Varying Lengthwise Hit Distribution)

Figure 6.47: Normalised Plot of Difficulty of Recoverability for the Three Frigate Variants (Assuming All Items Affected By Every Hit).

Figure 6.48: Normalised Star Plot of Survivability for the Three Frigate Design Variants

Figure 6.49: Normalised Star Plot of Survivability for the Corvette, Baseline Frigate and Destroyer

Figure 6.50: Normalised Star Plot of Survivability for the Two AOR Variants ........255

Figure 6.51: Normalised Plot of Difficulty of Survivability for the Three Frigate Design

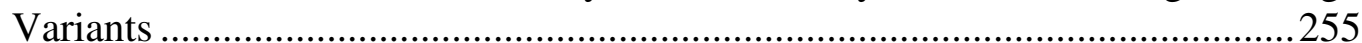

Figure 6.52: UPC Proportions for the Three Frigate Variants ....................................2257

Figure 6.53: Annual Costs for the Three Frigate Variants.........................................260

Figure 6.54: TLC Proportions for the Three Frigate Variants including UPC .............261

Figure 6.55: WLC Proportions for the Three Frigate Variants (for a Class of Twelve)

Figure A1: Frigate Layout Consideration (Andrews 2003) ........................................341

Figure A2: A Qualitative Assessment of Different Hull Forms (Andrews 2004) ........344

Figure A3: Fleet Tanker Size Drivers (Cooper et al 2007) ..........................................345

Figure A4: The Three Elements of Survivability (Ashe et al 2006) ............................346

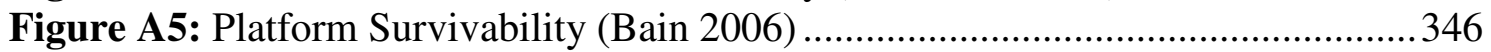

Figure A6: Visualisation of Vulnerability and Recoverability (Boulougouris and Papanikolaou 2012)..................................................................................... 348

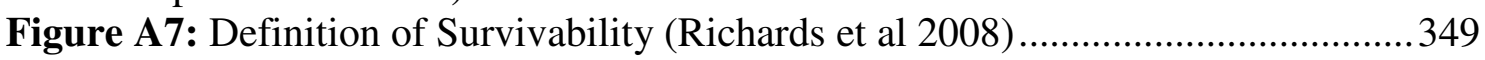

Figure A8: The Three Components of Survivability (Sajdak and Karni 2006).............350

Figure A9: Capability Versus Time (Turner et al 2006)........................................... 351

Figure A10: Nice Slopes - Shame About the Microgeometry (Martin 2007)..............353

Figure A11: Main (propulsion plant) Noise Sources on a Ship (Gallin and Lemenkuhler

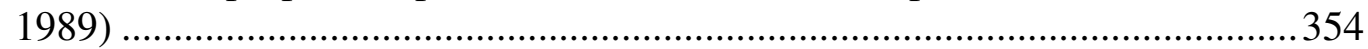

Figure A12: A Noise Reduction Flow Diagram for a Surface Ship With Primary and Secondary Measures for Structurebourne Noise (Foxwell 1991a) ................... 355

Figure A13: Naval Electronic Warfare Tree (Pakenham 1989) ..................................356

Figure A14: Coverage Provided By Supporting Defence Missile System (SDMS) (Adams 1988)....

Figure A15: Point Defence Missile - Performance Against Subsonic Missile (left) and Supersonic Missile (right). 
Figure A16: Medium Range Missile (Left) and Long Range Missile Performance Data

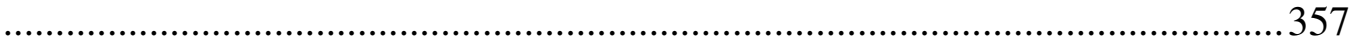

Figure A17: Available Conventional Weapons (Brown 1990)...................................359

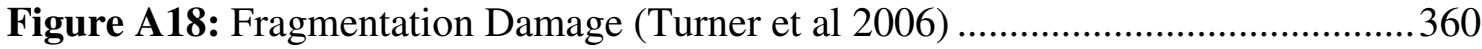

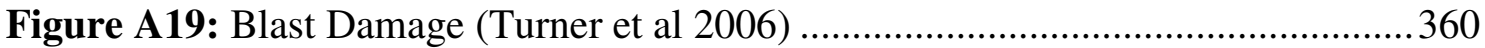

Figure A20: Concentrating and Duplicating Equipment for the Gun System (Tozer 1993)

Figure A21: Improving System Survival by Concentrating, Duplicating and Separating

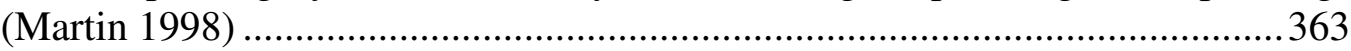

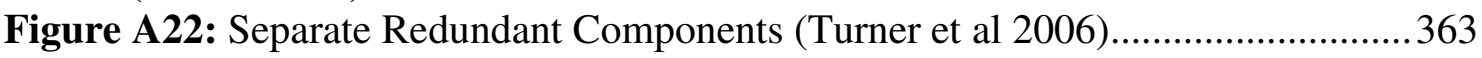

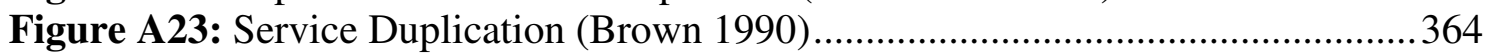

Figure A24: Normal vs. Blast Hardened Watertight Doors (Martin 2007) .................. 364

Figure A25: Possible Box-Girder Configurations (Chalmers 1993b) ...........................366

Figure A26: Example for Girders and Stringers Retaining Residual Strength (Petersen 2006) 366

Figure A27: Vertical Watertight Boundaries (Boulougouris and Papanikolaou 2004)

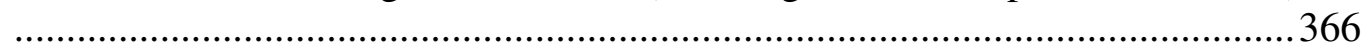

Figure A28: Comparison of Length-of-Damage Criteria (Surko 1994) ......................367

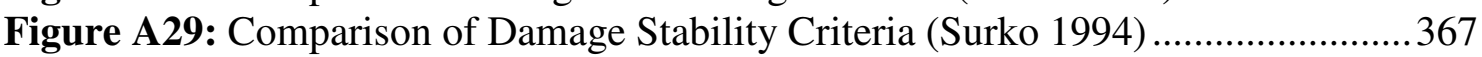

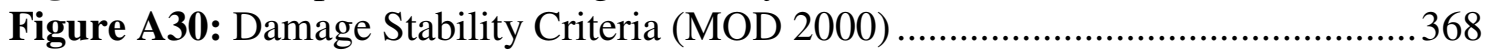

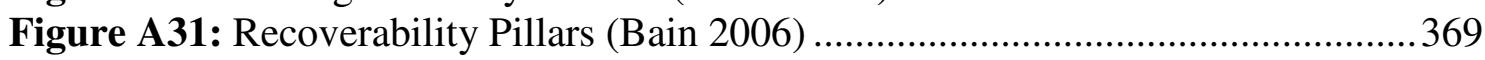

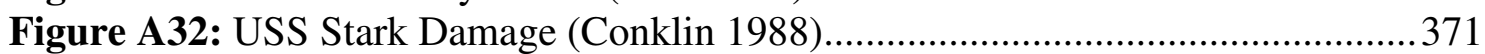

Figure A33: Underwater Explosion Damage to the Samuel B. Roberts (FFG-58) (Glass

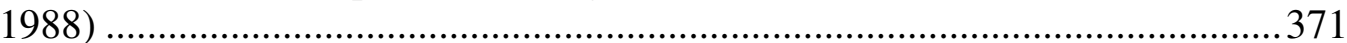

Figure A34: Current SCC Layout in a New Design Ship With Proposed Manning (see Figure A37) (Clements and Kneebone 1985) ....................................................371

Figure A35: Damage Control Features (Herman and Loeser 1992) ............................372

Figure A36: Firefighting Organization (State 1) - Smaller Ships (MOD 1988) .........372

Figure A37: Proposed SCC Manning For a New Design (Clements and Kneebone 1985)

Figure A38: Watertight Bulkhead and Deck Locations (Herman and Loeser 1992)... 373

Figure A39: Full-Time Collective Protection System (CPS) (Herman and Loeser 1992)

Figure A40: Full Firefighting Rig (MOD 1988)

Figure A41: Personnel Decontamination Station (Herman and Loeser 1992) ............. 377

Figure A42: Chemical Protective Overgarment (Herman and Loeser 1992) ............... 377

Figure A43: SINGRAR Decision Support Screen (Sarmento and Duarte 2005) .........379

Figure A44: Modular Weapon and Electronic Systems and Palletized Operator and Display Consoles, such as those Featured in the West German MEKO Frigates, Facilitate Rapid, Inexpensive Damage Repair both in Forward Areas and Domestic Shipyards (Rogers 1988) ................................................................380

Figure A45: Vessels With Known Sinking Times in WWII (Barton 2009)................. 381

Figure A46: Frigate Variants 1 and 2 and Destroyer Move System Tree Diagram.....403

Figure A47: Frigate Variant 3 and Corvette Move System Tree Diagram Tree Diagram

Figure A48: Frigate Variants 1, 2 and 3 Medium Calibre Naval Gun System Tree

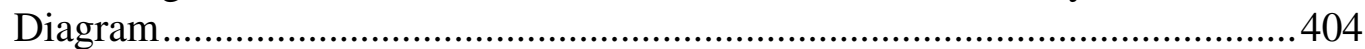

Figure A49: Corvette Medium Calibre Naval Gun System Tree Diagram...................404

Figure A50: Destroyer Medium Calibre Naval Gun System Tree Diagram.................405

Figure A51: Frigate Variants 1, 2 and 3 and Corvette ASM System Tree Diagram ... 405

Figure A52: Destroyer ASM System Tree Diagram...............................................406 
Figure A53: Frigate Variants 1, 2 and 3 and Corvettes fwd/aft SAM Systems Tree

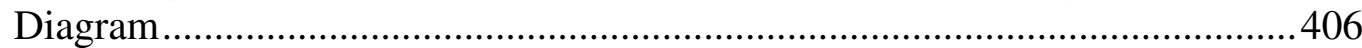

Figure A54: Destroyer fwd/aft SAM Systems Tree Diagram....................................407

Figure A55: Frigate Variants 1, 2 and 3, Corvette and Destroyer Helicopter Systems Tree Diagram

Figure A56: Frigate Variant 1 System Architecture ...................................................408

Figure A57: Frigate Variant 2 System Architecture .....................................................409

Figure A58: Frigate Variant 3 System Architecture ...................................................410

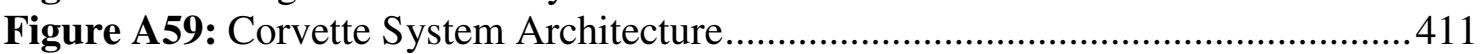

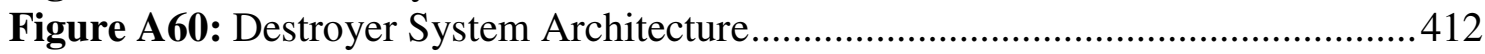

Figure A61: AOR Variant 1 Move System Tree Diagram .........................................413

Figure A62: AOR Variant 2 Move System Tree Diagram ........................................413

Figure A63: AOR Variant 1 Ability to RAS AVCAT Tree Diagram ..........................414

Figure A64: AOR Variant 2 Ability to RAS AVCAT Tree Diagram ..........................414

Figure A65: AOR Variant 1 Ability to RAS Dieso Tree Diagram ..............................415

Figure A66: AOR Variant 2 Ability to RAS Dieso Tree Diagram ..............................415

Figure A67: AOR Variant 1 Ability to RAS Dry Stores Tree Diagram .......................416

Figure A68: AOR Variant 2 Ability to RAS Dry Stores Tree Diagram ......................416

Figure A69: AOR Variant 1 Ability to RAS Ordnance Tree Diagram.........................417

Figure A70: AOR Variant 2 Ability to RAS Ordnance Tree Diagram........................417

Figure A71: AOR Variants 1 and 2 Aviation Support System Tree Diagram..............418

Figure A72: AOR Variants 1 and 2 CIWS Tree Diagram .......................................418

Figure A73: AOR Variant 1 System Architecture ....................................................419

Figure A74: AOR Variant 2 System Architecture ..................................................... 420

Figure A75: Frigate Variant 1 Power Speed Curve ..................................................428

Figure A76: Frigate Variant 2 Power Speed Curve ......................................................428

Figure A77: Frigate Variant 3 Power Speed Curve ....................................................429

Figure A78: Corvette Power Speed Curve (Low Speeds) .......................................430

Figure A79: Corvette Power Speed Curve (High Speeds) ...........................................430

Figure A80: Destroyer Power Speed Curve............................................................ 431

Figure A81: AOR Variant 1 Power Speed Curve ..................................................... 432

Figure A82: AOR Variant 2 Power Speed Curve ..................................................... 432

Figure A83: Frigate Variant 1 Deep Condition GZ Curve ........................................433

Figure A84: Frigate Variant 1 Light Condition GZ Curve .........................................434

Figure A85: Frigate Variant 2 Deep Condition GZ Curve .........................................435

Figure A86: Frigate Variant 2 Light Condition GZ Curve ........................................435

Figure A87: Frigate Variant 3 Deep Condition GZ Curve .......................................436

Figure A88: Frigate Variant 3 Light Condition GZ Curve ......................................437

Figure A89: Frigate Variant 3 Fuel and Ballast tank Location..................................438

Figure A90: Frigate Variant 1 Damage Stability Assessment Cases..........................438

Figure A91: Frigate Variant 2 Damage Stability Assessment Cases..........................438

Figure A92: Frigate Variant 3 Damage Stability Assessment Cases..........................439

Figure A93: Corvette Deep Condition GZ Curve ....................................................439

Figure A94: Corvette Light Condition GZ Curve .....................................................440

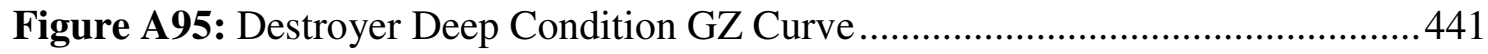

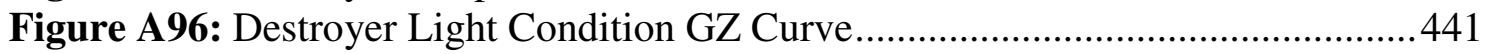

Figure A97: Destroyer Fuel Tank Arrangement ......................................................442

Figure A98: Corvette Damage Stability Assessment Cases ...................................... 443

Figure A99: Destroyer Damage Stability Assessment Cases ......................................443

Figure A100: AOR Variant 1 Deep Condition GZ Curve ...........................................44

Figure A101: AOR Variant 1 Light Condition GZ Curve .......................................... 444

Figure A102: AOR Variant 2 Deep Condition GZ Curve ........................................445 
Figure A103: AOR Variant 2 Light Condition GZ Curve 446

Figure A104: AOR Ballast and Trim Tank Arrangement.........................................44

Figure A105: AOR Variant 1 Damage Stability Assessment Cases.............................447

Figure A106: AOR Variant 2 Damage Stability Assessment Cases.............................448

Figure A107: Operational Profile for the Three Frigate Variants..............................468

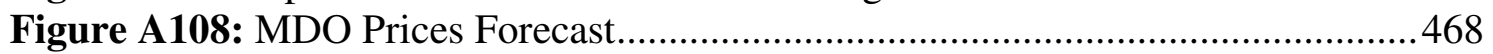




\section{List of Tables}

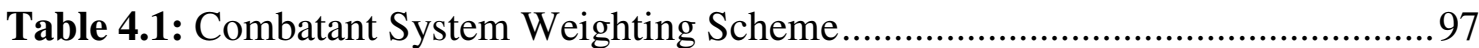

Table 4.2: Replenishment Ship System Weighting Scheme ......................................97

Table 4.3: Immediate DCFF Performance Measures (Category 1) .............................100

Table 4.4: Major System Recovery Performance Measures (Category 2)................... 104

Table 4.5: Individual Major System Recovery Performance Measures (Category 3) . 106

Table 4.6: Scheme for Compiling the Performance Measures Matrix of a Combatant

Table 4.7: Recoverability Weighting Scheme for a Combatant..................................112

Table 4.8: Scheme for Compiling the Performance Measures Matrix of a Replenishment Ship .............................................................................. 114

Table 4.9: Recoverability Weighting Scheme for a Replenishment Ship................... 115

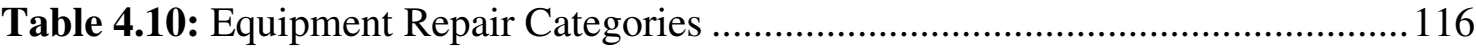

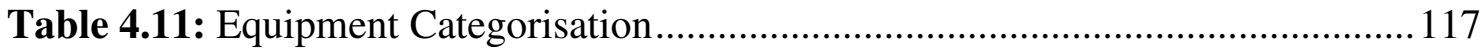

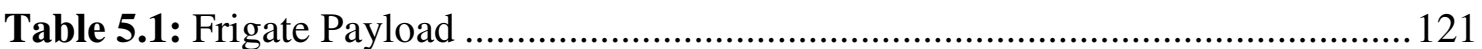

Table 5.2: Frigate Performance Requirements Adopted for Design Studies ............... 122

Table 5.3: Principal Particulars of the Frigate Variants Investigated .......................... 122

Table 5.4: Corvette, Frigate Variant 1 and Destroyer Payload ................................... 151

Table 5.5: Corvette, Frigate Variant 1 and Destroyer Performance Requirements

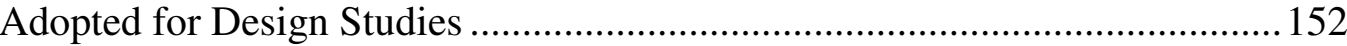

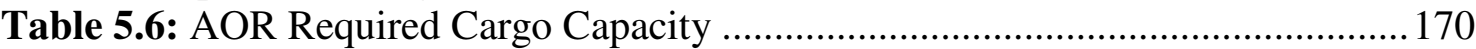

Table 5.7: AOR Performance Requirements Adopted for Design Studies .................. 171

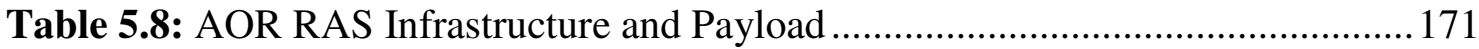

Table 5.9: Principal Particulars of the AOR Variants Investigated ..............................172

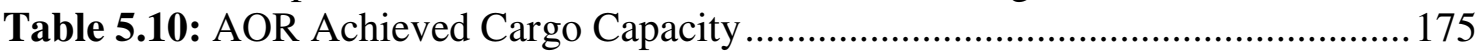

Table 6.1: Probabilities of Kill of Attacking ASMs for the Three Frigate Variants.... 192

Table 6.2: Susceptibility Results for the Three Frigate Variants ................................. 192

Table 6.3: Probabilities of Kill of Attacking ASMs for the Destroyer .........................196

Table 6.4: Susceptibility Results for the Corvette, Baseline Frigate and Destroyer.... 197

Table 6.5: Probabilities of Kill of Attacking ASMs for the Two AOR Variants.........200

Table 6.6: Susceptibility Results for the Two AOR Variants ...................................2200

Table 6.7: Probabilities of Kill of Attacking ASMs for the Three Frigate Variants and Corvette (Sensitivity Test) ...........................................................................205

Table 6.8: Probabilities of Kill of Attacking ASMs for the Destroyer (Sensitivity Test)

Table 6.9: Probabilities of Kill of Attacking ASMs for the Two AOR Variants (Sensitivity Test)

Table 6.10: Scenarios for System Weighting Schemes of the Frigate Design Studies 216

Table 6.11: System Weighting Schemes for the Frigate Design Study Sensitivity Tests

Table 6.12: Performance Measure Matrix for Frigate Variant 1

Table 6.13: Performance Measure Matrix for Frigate Variant 2.

Table 6.14: Performance Measure Matrix for Frigate Variant 3 ...............................228

Table 6.15: Performance Measure Matrix for the Corvette ........................................2230

Table 6.16: Performance Measure Matrix for the Destroyer .....................................2231

Table 6.17: NBCD Store Weighting Scheme for the Corvette, Baseline Frigate and

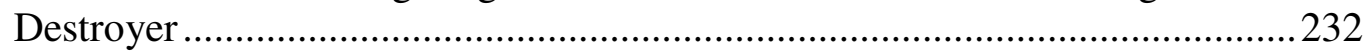

Table 6.18: Performance Measure Matrix for AOR Variant 1 ..................................2234

Table 6.19: Performance Measure Matrix for AOR Variant 2 ...................................2235 
Table 6.20: Category 1 Performance Measures Weighting Schemes for the Frigate Design Study Sensitivity Tests

Table 6.21: Category 2 Performance Measures Weighting Schemes for the Frigate Design Study Sensitivity Tests

Table 6.22: Category 3 Performance Measures Weighting Schemes for the Frigate Design Study Sensitivity Tests ....

Table 6.23: Float and Recovery Support System Weighting Schemes for the Frigate Design Study Sensitivity Tests

Table 6.24: Performance Measure Matrix for Frigate Variant 1 (Assuming All Items Affected By Every Hit)

Table 6.25: Performance Measure Matrix for Frigate Variant 2 (Assuming All Items Affected By Every Hit)

Table 6.26: Performance Measure Matrix for Frigate Variant 3 (Assuming All Items Affected By Every Hit)

Table A1: Vulnerability and Recoverability Categorisation of Equipment Items and Compartments Comprising the Major Ship Systems.....

Table A2: Frigate Variant 1 Accommodation Breakdown 422

Table A3: Frigate Variant 2 Accommodation Breakdown 422

Table A4: Frigate Variant 3 Accommodation Breakdown ........................................ 422

Table A5: Corvette Accommodation Breakdown. 422

Table A6: Destroyer Accommodation Breakdown ...................................................423

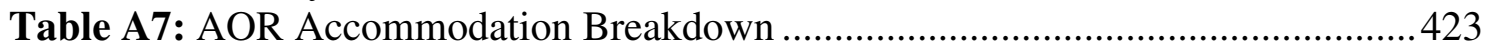

Table A8: Frigate Variant 1 Top Level Weight Breakdown......................................423

Table A9: Frigate Variant 2 Top Level Weight Breakdown......................................423

Table A10: Frigate Variant 3 Top Level Weight Breakdown......................................424

Table A11: Corvette Top Level Weight Breakdown ..................................................424

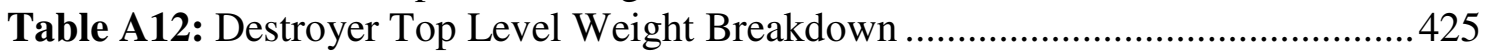

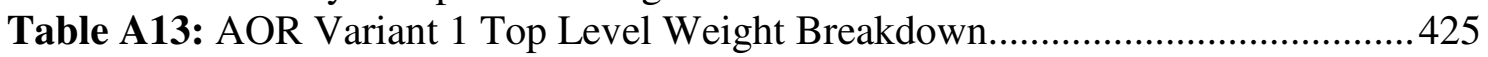

Table A14: AOR Variant 2 Top Level Weight Breakdown.........................................425

Table A15: Design and Construction Margins for Frigate Variant 1..........................426

Table A16: Design and Construction Margins for AOR Designs...............................427

Table A17: Frigate Variant 1 Deep Condition Hydrostatics...................................... 434

Table A18: Frigate Variant 1 Light Condition Hydrostatics........................................434

Table A19: Frigate Variant 2 Deep Condition Hydrostatics ........................................435

Table A20: Frigate Variant 2 Light Condition Hydrostatics.......................................436

Table A21: Frigate Variant 3 Deep Condition Hydrostatics.......................................436

Table A22: Frigate Variant 3 Light Condition Hydrostatics.......................................437

Table A23: Corvette Deep Condition Hydrostatics .....................................................440

Table A24: Corvette Light Condition Hydrostatics ................................................440

Table A25: Destroyer Deep Condition Hydrostatics ............................................... 441

Table A26: Destroyer Light Condition Hydrostatics ..................................................442

Table A27: AOR Variant 1 Deep Condition Hydrostatics..........................................44

Table A28: AOR Variant 1 Light Condition Hydrostatics ..........................................445

Table A29: AOR Variant 2 Deep Condition Hydrostatics..........................................445

Table A30: AOR Variant 2 Light Condition Hydrostatics ..........................................446

Table A31: Weapon System Data for the Three Frigate Variants ..............................449

Table A32: Radar Horizon for the Three Frigate Variants ......................................449

Table A33: Sequence of Events for the Three Frigate Variants ................................450

Table A34: Radar Horizon for the Corvette, Baseline Frigate and Destroyer .............450

Table A35: Weapon System Data for the Destroyer................................................ 451

Table A36: Sequence of Events for the Destroyer..................................................452 
Table A37: Weapon System Data for the Two AOR Variants

Table A38: Radar Horizon for the Two AOR Variants

Table A39: Sequence of Events for the Two AOR Variants

Table A40: Sequence of Events for the Destroyer (Aster-30, MFR horizon limited) . 454

Table A41: Sequence of Events for the Destroyer (Aster-30, Maximum Range limited)

Table A42: Vulnerability Results Given a Hit at Each WT Section of Frigate Variant 1

Table A43: Vulnerability Results Given a Hit at Each WT Section of Frigate Variant 2

Table A44: Vulnerability Results Given a Hit at Each WT Section of Frigate Variant 3

Table A45: Vulnerability Results Given a Hit at Each WT Section of the Corvette...458

Table A46: Vulnerability Results Given a Hit at Each WT Section of the Destroyer.458

Table A47: Vulnerability Results Given a Hit at Each WT Section of AOR Variant 1

Table A48: Vulnerability Results Given a Hit at Each WT Section of AOR Variant 2

Table A49: Effect of ASM Attack Angle on System Vulnerability for the Destroyer 459

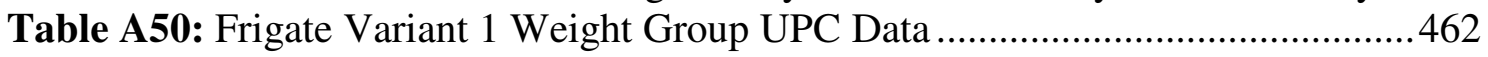

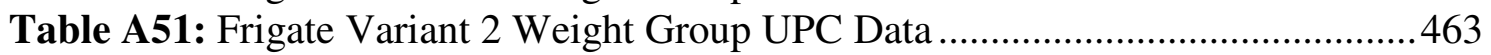

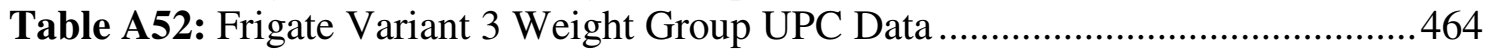

Table A53: Frigate Variant 1, Group 4 Specific Item Cost Data .................................465

Table A54: Frigate Variant 2, Group 4 Specific Item Cost Data ...............................465

Table A55: Frigate Variant 3, Group 4 Specific Item Cost Data .................................465

Table A56: Frigate Variant 1, 2 and 3, Group 6 Specific Item Cost Data ....................465

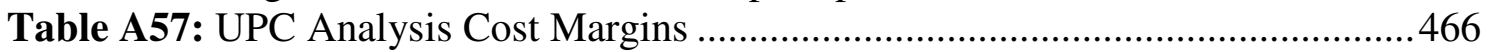

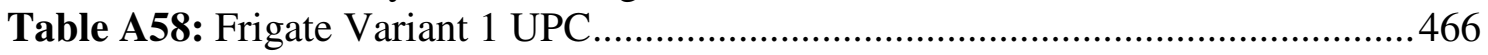

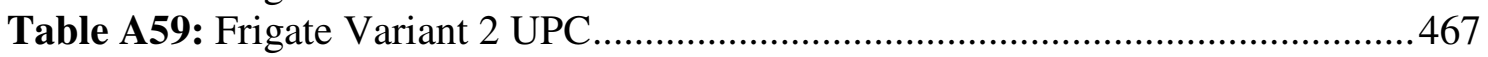

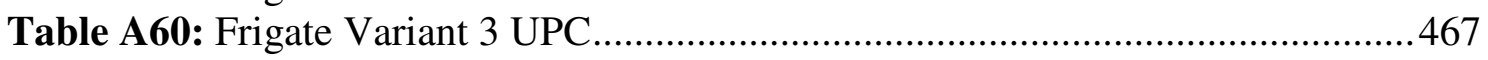

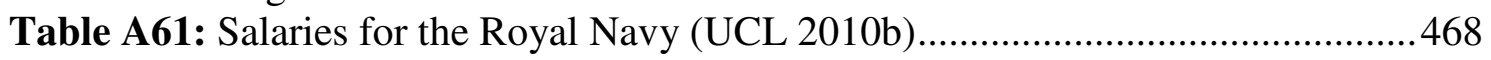

Table A62: Consumables Costs (UCL 2010b)...........................................................469

Table A63: Annual Maintenance Cost for Selected Items of Equipment (UCL 2010b)

Table A64: Costs Incurring During Refit Periods (UCL 2010b) ...............................469

Table A65: Frigate Variant 1, 2 and 3 TLC Results ..................................................470

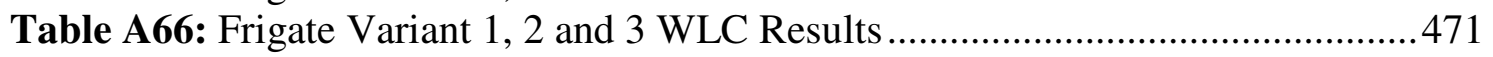




\section{$\underline{\text { Nomenclature }}$}

3D: $\quad$ Three-dimensional.

AAW: $\quad$ Anti-air Warfare.

ADMS: $\quad$ Area-defence Missile System.

AE: $\quad$ Air Engineer.

AMR: $\quad$ Auxiliary Machinery Room.

AMV: $\quad$ Advanced Marine Vehicle.

ANEP: $\quad$ Allied Naval Engineering Publication.

ANSWER: UK MOD ASW simulation software.

AOR: $\quad$ Auxiliary Oiler Replenishment.

ASM: $\quad$ Anti-ship Missile.

ASuW: $\quad$ Anti-surface Warfare.

ASW: $\quad$ Anti-submarine Warfare.

ASyW: Asymmetric warfare.

ATU: $\quad$ Air Treatment Unit.

AVCAT: Aviation Carrier Turbine fuel.

B: $\quad$ Ship's maximum beam on design waterline.

BDCS: Battle Damage Control System.

BGFRS: Board of Governors of the Federal Reserve System, Washington, D.C., USA.

BMT: $\quad$ British Maritime Technology Defence Services Ltd., Bath, UK.

BN: $\quad$ Bayesian Network.

CAD: $\quad$ Computer Aided Design.

CADMID: Concept, Assessment, Demonstration, Manufacture, In-service and Disposal.

CASD: $\quad$ Computer Aided Ship Design.

$\mathbf{C}_{\mathbf{B}}$ : $\quad$ Block Coefficient. A measure of the fullness of the underwater volume of a vessel.

CBR: $\quad$ Chemical, Biological and Radiological.

CCTV: Closed-circuit Television.

Cdr:: Commander.

CER: $\quad$ Cost Estimating Relationship.

CFD: $\quad$ Computational Fluid Dynamics.

CHENG: Chief Engineer, USN.

CIWS: $\quad$ Close-in Weapon System.

$\mathbf{C}_{\mathbf{M}}$ : $\quad$ Midship Coefficient. A measure of the fullness of midships of a vessel.

CNGF: Common New Generation Frigate. Anglo-French-Italian programme of the 1990s.

CO: Commanding Officer.

Comms.: Communications.

$\mathbf{C}_{\mathbf{P}}$ : $\quad$ Prismatic Coefficient. A measure of the distribution of the underwater volume of a vessel.

CPO: $\quad$ Chief Petty Officer.

CPS: $\quad$ Collective Protection System.

CSEE: Combat Systems Effectiveness Exercise. Defensive systems (hard-kill) effectiveness estimation method used by UCL as part of its MSc in Naval Architecture course.

CV: $\quad$ Aircraft Carrier.

CVF: $\quad$ Aircraft Carrier, Future (Queen Elizabeth Class).

CVS: $\quad$ Aircraft Carrier, ASW (Invincible Class).

$\mathbf{C}_{\mathbf{W}}$ : Waterplane Coefficient. A measure of the fullness of the waterplane of a 
vessel.

CWP: $\quad$ Chilled Water Plant.

DBB: $\quad$ Design Building Block. Designation used in the UCL DRC DBB design approach.

DC: $\quad$ Damage Control.

DCA: Damage Control Assistant.

DCFF: Damage Control and Firefighting.

DD 21: Destroyer for the 21st century (Zumwalt Class Destroyer).

Dieso: Diesel fuel, general purpose.

DINCS: Distributed Intelligent Networked Control System.

DRC: $\quad$ Design Research Centre, Marine Research Group, Department of

Mechanical Engineering, UCL, London, UK.

Dstl: $\quad$ Defence Science and Technology Laboratory, MOD, Salisbury, UK.

EB-FSRS: Fire Safety Ranking System.

ECCM: $\quad$ Electronic Counter-counter Measures.

ECM: $\quad$ Electronic Countermeasure.

EMCON: Electronic Emission Control.

EMP: $\quad$ Electro-magnetic Pulse.

Eqpt: Equipment.

ESM: $\quad$ Electronic Support Measures.

EW: $\quad$ Electronic Warfare.

EVI: $\quad$ Evacuability Index. Passenger evacuation model.

FFDC: $\quad$ Firefighting and Damage Control.

FOC: $\quad$ First of Class. Term used in UK naval ship procurement.

FP: $\quad$ Fire Pump.

FPP: $\quad$ Fixed Pitch Propeller.

FRP: $\quad$ Fire and Repair Party.

FRPP: $\quad$ Fire and Repair Party Post.

GA: General Arrangement.

Genset: $\quad$ Engine-generator.

GMt: $\quad$ Transverse Metacentric Height. A measure of stability.

GNP: $\quad$ Gross National Product. The value of goods and services produced

GPEOD: $\quad$ within a country during one year.

GPS: Global Positioning System.

GRC: $\quad$ QinetiQ Graphics Research Corporation Ltd., Gosport, UK.

GT: $\quad$ Gas Turbine.

GZ curve: Righting lever curve of a vessel experiencing a heeling force. A measure of stability.

h: $\quad$ Height of SR/MFR above sea level.

Helo: Helicopter.

HF: Human Factor.

HMS: $\quad$ Her/His Majesty's Ship.

HPAC: $\quad$ High Pressure Air Compressor.

HPM: $\quad$ Human Performance Matrix.

HQ: Headquarters.

HTS: High-temperature Superconducting.

HVAC: $\quad$ Heating, Ventilation, and Air Conditioning.

HVME: UK MOD software monitoring platforms self-noise.

IFEP: Integrated Full Electric Propulsion.

IMO: International Maritime Organization.

IPMS: $\quad$ Integrated Platform Management System. 


\begin{tabular}{|c|c|}
\hline IR: & Infra-red. \\
\hline IRST: & Infra-red Search and Track. \\
\hline JR: & Junior Rate. \\
\hline JSS: & Joint Support Ship. \\
\hline L: & Ship's length between perpendiculars. \\
\hline LCC: & Life Cycle Cost. \\
\hline LCR: & Local Control Room. \\
\hline LCS: & Littoral Combat Ship. \\
\hline LCU: & Local Control Unit. \\
\hline LPCR: & Local Power and Control Room. \\
\hline LPD: & Landing Platform Dock. \\
\hline LPH: & Landing Platform Helicopter. \\
\hline Lt.: & Lieutenant. \\
\hline Lt. Cdr.: & Lieutenant Commander. \\
\hline MARS: & $\begin{array}{l}\text { Military Afloat Reach and Sustainability ships. Designation of UK } \\
\text { auxiliary programme in procurement since } 2010 \text {. }\end{array}$ \\
\hline MASS: & Multi Ammunition Soft-kill System. \\
\hline MBB: & $\begin{array}{l}\text { Master Building Block. Designation used in the UCL DRC DBB design } \\
\text { approach. }\end{array}$ \\
\hline MCR: & Machinery Control Room. \\
\hline MDO: & Marine Diesel Oil. \\
\hline MEO: & Marine Engineering Officer. \\
\hline MFR: & Multi-function Radar. \\
\hline MESM: & Marine Engineer, Submarines. \\
\hline MICA: & $\begin{array}{l}\text { Missile d'Interception et de Combat Aérien (Interception and Aerial } \\
\text { Combat Missile). }\end{array}$ \\
\hline MISSION: & $\begin{array}{l}\text { Maritime Integrated Survivability Simulation. An event simulation tool } \\
\text { designed to simulate a maritime mission used by the UK MOD. }\end{array}$ \\
\hline MMR: & Main Machinery Room. \\
\hline MOD: & Ministry of Defence, UK. \\
\hline MOT: & Ministry of Transport, NZ. \\
\hline MOTISS: & Measure of Total Integrated System Survivability. \\
\hline MSc: & Master of Science. \\
\hline NAAFI: & Navy, Army and Air Force Institutes. \\
\hline NATO: & North Atlantic Treaty Organisation. \\
\hline NBC: & Nuclear, Biological, and Chemical. \\
\hline NBCD: & Nuclear, Biological, and Chemical Defence. \\
\hline NBCDO: & Nuclear, Biological, and Chemical Defence Officer. \\
\hline NES: & Naval Engineering Standards. \\
\hline ODIN: & UK MOD torpedo activation simulation software. \\
\hline ONS: & Office for National Statistics, UK Statistics Authority, Cardiff, UK. \\
\hline OOVR: & Operationally Oriented Vulnerability Requirement. \\
\hline PAAMS: & Principal Anti Air Missile System. \\
\hline $\mathbf{P}(\mathbf{d i}):$ & Probability of the ship being detected and identified. \\
\hline $\mathbf{P}(\mathbf{h}):$ & Probability of the ship being hit by at least one ASM. \\
\hline $\mathbf{P}(\mathbf{k}):$ & Probability of kill. \\
\hline P(l): & Probability that the ASM locks on the ship. \\
\hline P(susc.): & $\mathrm{P}(\mathrm{di}) \times \mathrm{P}(\mathrm{l}) \times \mathrm{P}(\mathrm{h})$ \\
\hline PDMS: & Point-defence Missile System. \\
\hline PM: & Performance Measure. \\
\hline PMS: & Platform Management System. \\
\hline PN: & Portuguese Navy. \\
\hline
\end{tabular}


PO: $\quad$ Petty Officer.

PREVENT: Preliminary Vulnerability Evaluation of Enemy Threats. A vulnerability assessment software for concept stage ship designs developed by BMT Defence Services.

RAM: $\quad$ Radar-absorbent Material.

RAS: $\quad$ Replenishment at Sea.

RASco: Replenishment at Sea control room.

RCN: Royal Canadian Navy.

RCS: Radar Cross Section.

REVS: $\quad$ Rapid Evaluation of Vulnerability of Ships. A vulnerability assessment

RFA: $\quad$ Royal Fleet Auxiliary.

RN: $\quad$ Royal Navy.

RNN: $\quad$ Royal Netherlands Navy.

RPI: $\quad$ Retail Price Index. A measure of inflation published by the ONS.

Rtd.: $\quad$ Retired.

$\mathbf{S}^{\mathbf{5}}$ : $\quad$ Speed, Seakeeping, Stability, Strength and Style. Descriptive ship design term due to Brown and Andrews (1980).

SAM: $\quad$ Surface-to-air Missile.

SCC: $\quad$ Ship Control Centre.

SeaRAM: Rolling Airframe Missile.

SINGRAR: Sistema Integrado para a Gestão de Prioridades de Reparação e Afectação de Recursos (Integrated System for Repair Priority Management and Resource Assignment).

SIREX: UK MOD IR signature prediction software.

SOLAS: $\quad$ Safety of Life at Sea. An IMO international maritime safety treaty.

SPECTRE: UK MOD RCS prediction software.

SRD: $\quad$ System Requirement Document. Generic document used in UK defence procurement.

SR: $\quad$ Senior Rate.

SR: $\quad$ Surveillance Radar.

SSTDS: $\quad$ Surface Ship Torpedo Defence System.

SSVUL: $\quad$ Surface Ship Vulnerability Assessment. A vulnerability assessment code for underwater threats used by the UK MOD during the 1990s.

SUBCON: Submarine Concept. An implementation of the architecturally orientated DBB approach to preliminary submarine design.

SURFCON: Surface Ship Concept. An implementation of the architecturally orientated DBB approach to preliminary ship design.

SURVIVE: Surface and Underwater Ship Visual Vulnerability Evaluation. A Vulnerability (and recoverability) assessment code developed by QinetiQ and used by the UK MOD.

SWATH: Small Waterplane Area Twin Hull.

SYLVER: Système de Lancement Vertical (Vertical Launching Systems).

TEU: $\quad$ Twenty-foot Equivalent Unit. A unit of cargo capacity.

ThreeDim: UK MOD AAW and ASuW simulation software.

TLC: $\quad$ Through Life Cost.

TMSS: $\quad$ Total Mine Simulation System. An UK MOD mine activation simulation Software.

UCL: $\quad$ University College London, London, UK.

UPC: $\quad$ Unit Procurement/Production Cost.

URD: User Requirement Document. Generic document used in UK defence procurement. 


$\begin{array}{ll}\text { USN: } & \text { United States Navy. } \\ \text { USS: } & \text { United States Ship. } \\ \text { VCG: } & \text { Vertical Centre of Gravity. } \\ \text { VLS: } & \text { Vertical Launching Systems. } \\ \text { VL: } & \text { Vertical Launch. } \\ \text { VR: } & \text { Virtual Reality. } \\ \text { WA: } & \text { Warfare Aviation. } \\ \text { WEO: } & \text { Weapon Engineering Officer. } \\ \text { WE: } & \text { Weapon Engineer. } \\ \text { WESM: } & \text { Weapon Engineer, Submarines. } \\ \text { WLC: } & \text { Whole Life Cost. } \\ \text { wrt: } & \text { With respect to. } \\ \text { WT: } & \text { Watertight. } \\ \text { WTD: } & \text { Watertight Door. } \\ \text { WWII: } & \text { World War 2. } \\ \text { XO: } & \text { Executive Officer. }\end{array}$




\section{Chapter 1: Introduction}

\subsection{Preamble}

Survivability is defined by NATO as "the capability of a weapon system to continue to carry out its designated mission(s) in a combat environment" (NATO 2003a), where in the current case the 'weapon system' signifies a naval ship. Survivability is generally considered to encompass three constituents, susceptibility, vulnerability and recoverability (Said 1995). Susceptibility is the measure of a ship being detected, identified, targeted and hit; vulnerability addresses the damage caused by the incident; while recoverability the extent to which capability can be recovered and the time needed to recover it. Each of the three constituents is highly dependent (amongst other factors) on the ship configuration which in turn is produced during the crucial early ship design stages. However, the driving issues in preliminary ship design have traditionally been powering, stability, strength and seakeeping (Brown and Andrews 1980). Therefore, survivability related issues have been investigated in detail during the later design stages but have been heavily constrained by the major design features fixed beforehand. In addition, the lack of an integrated survivability assessment and quantification method which can be utilised during the early design stages, combined with rising warship procurement costs can make attractive the option of cost cutting in this complex and highly critical topic, despite resulting in inadequate ship designs due to inappropriate levels of survivability.

This thesis investigates and describes an approach to ship design which produces an integrated survivability assessment. It focuses on integrating survivability assessment (with emphasis on the least mature aspect of recoverability) in preliminary ship design and exploits the architecturally orientated Design Building Block approach to preliminary ship design. This approach, developed by Andrews (1984; 1986) and demonstrated in (Andrews and Dicks 1997) integrates architectural description with the traditional numerical synthesis. It was initially implemented for submarines through the SUBCON implementation (Andrews et al 1996) and subsequently for surface ships through GRC's SURFCON implementation in Paramarine (Munoz and Forrest 2002), described in (Andrews and Pawling 2003). The use of architecturally orientated design approaches enables the assessment of survivability at the earliest design stages, which are characterised by the expenditure of minimum design resources while having a large impact on the final solution in terms of vessel configuration and cost. 
The feasibility of such an approach to ship survivability is illustrated through the development of various ship designs, including combatants and auxiliary ships adopting both conventional and unconventional hullform configurations. These designs have been analysed using tools such as Paramarine CASD and various survivability assessment techniques.

\subsection{Research Scope and Aim}

The research on survivability assessment outlined in this thesis focuses on the divergent and creative preliminary stages of the ship design process where a large variety of alternative ship designs should be investigated by the designers and the designs are still able to be modified relatively easily. This research does not address aspects which are addressed in the contract design and detailed design stages, where high levels of design definition are necessary.

The thesis focuses on the application of survivability assessment methods on surface warships (including both combatants and auxiliaries) in order to investigate battle damage. This research has applicability on all types of surface warships of both conventional and novel configurations and could also be of relevance to subsurface naval vessels. It is envisaged that the research will encourage innovative ship design and increase the confidence of the designer by proposing an assessment and quantification method of what is an important aspect of naval ship design.

The thesis initially investigates the importance and advantages of preliminary ship design and the full integration of architectural considerations in the preliminary ship design process. This is done in order to justify the application of survivability assessment techniques in the early ship design stages. Current survivability assessment techniques and their applicability to integrate total survivability assessment in the preliminary design process have been examined. This is followed by the proposition of an integrated survivability assessment approach in preliminary ship design, which is then demonstrated on a variety of concept ship design studies. This also allows the investigation of principal survivability drivers in a given design.

The approach adopted for this research was primarily characterised by the combination of various bespoke computer tools for survivability assessment and the development of numerous design studies. Due to the information sensitivity characterising the area of naval ship survivability the developed designs draw on the UCL Naval Architecture database (UCL 2010a; UCL 2010b) which is unclassified but 
essentially representative of UK MOD design practice. In addition, classified tools, as well as classified data for weapon systems and ship designs, were not utilised. This research is primarily concentrated at seagoing naval combatants but variations in size, configuration novelty and role have been investigated to test out the robustness of the proposed method of survivability assessment in preliminary ship design.

The overall aim of the thesis is summarised as:

To propose an integrated survivability assessment approach and demonstrate it on a range of ship types and hullform configurations. The ship designs used throughout the research need to have a level of definition appropriate to preliminary stage design. The principal drivers for survivability and their cost-effective incorporation in ship design need to be explored. In addition, the advantages of using an architecturally orientated preliminary ship design approach, in conjunction with the proposed survivability assessment method, should be investigated. It is argued that such preliminary design approaches can bring survivability performance issues into appropriate consideration in the earliest design stages.

\subsection{Structure of the Thesis}

The thesis is composed of eight main chapters accompanied by separate appendices providing additional material relevant to the main chapters.

This chapter provides a brief introduction to the research topic as well as the scope and aim.

The second and third chapters outline the background for the research work. Chapter 2, focusing on ship design issues, is split into three main sections. The first gives an introduction to ship design, mainly focusing on preliminary warship design. It describes the evolution of the preliminary warship design process through the latter part of the twentieth century to date and how it has been affected and enhanced by the extraordinary developments in computer based tools and methods. The second section focuses on the importance of ship cost estimating during the early design stages as a measure against which survivability measures can be assessed. The final section outlines the main conclusions of this chapter.

The third chapter focuses on naval ship survivability and is split into four parts. In the first two parts, survivability and its relevance are defined in detail and current threats faced by navies are examined. The third part gives a detailed account of current survivability assessment techniques and their implications in ship design. The final part 
provides the main conclusions of this chapter while identifying the gaps in current survivability assessment approaches. Thus an indication as to the way forward is given.

The fourth chapter addresses the gaps identified in survivability assessment essentially through a detailed description of the proposed survivability assessment method. This chapter consists of four main sections. The first two sections focus on the susceptibility and vulnerability assessment parts of the method, each of which are met by current established techniques the applicability of which has been proven at preliminary stage ship design. The third and largest section describes the development, reasoning behind and applicability of the new recoverability assessment approach. The development of a new recoverability assessment approach was necessary due to the limitations of current approaches, which are mentioned in Chapter 3. Finally, the fourth section describes how the three elements of survivability assessment can be combined into the proposed total survivability assessment approach.

The fifth chapter describes the application of the proposed method to seven ship design studies. This chapter is separated into three main parts. The first part gives a detailed account of the three frigate variant design studies conducted, the second describes the corvette and destroyer design studies developed and the final part explores the two replenishment ships which were designed. The choice of ship design is justified and the design procedure, as well as the main characteristics and features of the ship designs are explained, focusing mainly in features relevant to survivability performance.

The sixth chapter presents the results from the application of the method described in Chapter 4 to the selected ship design studies detailed in Chapter 5. Results of all survivability constituents for all ship designs are presented, including various sensitivity studies. In addition, cost estimations are presented from a ship costing study undertaken in order to explore the cost-effective incorporation of the principal features of survivability in the ship designs undertaken.

The discussion in the seventh chapter analyses the results (Chapter 6) of applying the proposed survivability assessment approach in Chapter 4 to the ship design studies in Chapter 5. It is argued that the major survivability drivers for the various ship designs have been identified and validate the proposed method. In addition the discussion considers whether the proposed approach in Chapter 4 addresses the gaps identified in the research background outlined in Chapters 2 and 3. Finally, consideration is given as to whether the research aims in Chapter 1 were met and suitable ways forward for this on-going research are suggested. 
The eighth and final chapter summarises the major conclusions that have resulted from the research work. 


\section{Chapter 2: Ship Design Issues}

This chapter, consisting of three main sections, provides the ship design related background and context for the research. The first section outlines the nature of ship design and developments in preliminary ship design approaches through the latter part of the twentieth century to date. It specifically focuses on and gives an adequate explanation of the importance of preliminary ship design and the utility of architecturally orientated processes, such as the Design Building Block approach to preliminary ship design. Such design approaches are crucial with regards to the proposed survivability assessment approach. The second section focuses on ship costing during the preliminary ship design process which could assist in the cost effective incorporation of the principal survivability drivers. The final section provides the main conclusions of the research review related to issues concerning ship design by highlighting the importance of architecturally orientated preliminary ship design approaches.

\subsection{Ship Design}

\subsubsection{Background to Naval Ship Design and Procurement}

A warship design project is initiated to meet a need (operational, new major equipment) (Andrews 1987; Brown 1993) which is set by the customer either in the form of a high-level URD or a lower-level SRD (MOD 2001). After a baseline design is developed, the initial requirement is produced based on operational research and dialogue with designers on what is achievable (Andrews 1992). Achieving a realistic and affordable requirement is of vital importance (Heather 1990; Crow 2001) as the design process and the end result are largely based on the designer's interpretation of the often crude requirements (Andrews 1986; Brown 1986a). Correct interpretation of the requirements can also lead to cost saving (Andrews and Brown 1982; Crow 2001).

Naval ships have moved from aiming to design the technically best to the cost effective and finally to the current cash limited ship (Brown 1986a) as a result of the growth in Unit Procurement Cost (UPC) (approximately 9\% per annum (Gates 2005)), although it should be recognised that this is only about $20 \%$ of the Through Life Cost (TLC) (Andrews and Brown 1982; Brown and Tupper 1989). This real cost increase is attributed to increased capability (which has to be balanced with safety, supportability 
and sustainability (Knight 2012a; Knight 2012b)), greater complexity and higher standards (Brown and Andrews 1980; Brown and Tupper 1989). Coupled with declining defence budgets post-cold war (Andrews and Hall 1995) this has led to a decrease in the number of warships for most navies (Andrews and Brown 1982; Brown and Tupper 1989) despite no reduction in commitments (Collins and Ward 2012), leading to the aforementioned requirement of greater capability per ship (Jones and Kimber 2012) and greater reliability (Manley 2012). A number of ways to reduce cost, which have all affected the design process, have been suggested, such as adoption of commercial standards for naval ships (Brown and Andrews 1980; Rattenbury 2004) (especially for non-combatants such as auxiliary ships (Cooper et al 2007)), open system architecture (Vasilakos et al 2000), flexibility through modularity/cellularity (Brown and Andrews 1980; Jones and Kimber 2012) (which, however could lead to a heavier ship (Gates and Rusling 1982)), restricting the number of roles intended for a new ship design (Brown and Andrews 1980; Collins and Ward 2012), technical innovation (Andrews and Brown 1982) and complement reduction (Brown and Andrews 1980; Collins and Ward 2012). It should be noted that cost cutting is usually focused on UPC rather than TLC (Brown and Andrews 1980) and contentious areas such as resistance to damage which can be difficult to quantify (Brown 1986a).

\subsubsection{Introduction to Ship Design}

Many attempts to describe ship design have been made. Gale (2003) defined it as "the activity involved in producing the drawings, specifications and other data needed to construct an object (ship)", emphasising both the scientific and artistic nature of design (Gale 2003; Gates 2005). This approach is in agreement with Andrews (1986) remark that ship design cannot be described as a purely scientific/engineering field as it involves the arrangement of spaces and equipment, giving it an architectural element. Andrews (1981) also states that what differentiates design from other human activities is synthesis ("putting the pieces together in a new way" (Jones 1970)), therefore again stressing the fundamental creative aspect of design (Gale 2003) which is growing in importance given the increased information currently available to naval architects due to technology advances (Jasionowski 2012). Archer (1979) took a further step and classified design as a separate culture to humanities and sciences, (Figure 1 of (Archer 1979)). It is therefore understandable that the personal characteristics of the designer largely influence the ship design process (Andrews 1986; Gale 2003). 
According to Rawson and Tupper (2001) ship design is the raison d'être of naval architecture. Ship design is an iterative process, where evolving the requirement and designer judgments are part of the process to arrive to a solution (Gale 2003; Gates 2005). This iterative process has been illustrated by the design spiral (Andrews 1986; Rawson and Tupper 2001) which is frequently used by naval architects to depict the ship design process. The first design spiral was produced by Evans (1959) and since then a large number of design spirals have been produced (a chronological set is shown in (Andrews et al 2009)). A typical design spiral attempts to show the way in which a naval architect deals with each topic in sequence and iteratively, until an optimum solution is reached (Evans 1959; Gale 2003). However, such a representation of the design process has been criticised by a number of authors. Design spirals depict ship design as a set of sequential processes (Watson 1998; Gale 2003), rather it is characterised as looping, nonlinear and discontinuous, therefore not as simplistic as a spiral (Brown 1986a; Andrews et al 2012b); spirals look at steps and not the design process itself (Andrews 1998); spirals are not ship type dependant (Watson 1998).

Warships have been cited as "the most complex, diverse and highly integrated of any engineering systems" produced today on a regular basis (Graham 1982) for a variety of reasons. For example, they are designed for multiple roles (Brown 1986a; Brown and Tupper 1989), it is difficult to quantify many aspects of their performance (Brown and Tupper 1989), they are distinctively large and complex (Andrews et al 1997), they operate in a hostile environment (Gates 2005), they must provide a hospitable environment for the crew (Gates 2005) and they consist of a number of high risk areas (Heather 1990). Heather (1990) suggested that the main high risk areas in warship design are: development of complex weapon items and software (which are often delivered late or fail to perform), bottom-up weight estimates for novel designs, auxiliary power generation requirements and layout of key spaces. The extent of design complication and risk increases with degree of novelty. Andrews (1986) categorised different types of design processes based on ship novelty: type-ship variation, evolution, historical, synthesis, radical configuration (e.g. SWATH), radical technology (requiring prototypes). A designer has to address a variety of often conflicting aims, always under a tight budget (Brown and Tupper 1989) and almost always without the assurance of a prototype (due to size and complexity, cost, and time factors) (Andrews 2011) which further complicates the task. 
The phases of the warship design process, gradually moving from ship-level to system-level (Heather 1990), can be summarised as follows for UK MOD practice (Andrews 1992) (for commercial practice refer to (Watson and Gilfillan 1976)):

1. Concept;

2. Feasibility;

3. Design Definition;

4. Build Contract Definition;

5. Detailed Ship Design.

The first phase, where the designer attempts to understand the customers' needs and convert them into a technical solution (Brown 1986a; Gale 2003), has been further broken down for major programmes into: concept exploration, concept studies and concept design (Andrews 1993; Andrews et al 2012a). In the concept phase, the designer explores a number of alternatives and selects the most promising in terms of size, cost and capability (Brown 1986a; Andrews and Pawling 2007). The time and resources spent during concept or preliminary design are only a fraction of those spent during the entire project (Andrews 1987). However, most major decisions are taken and trade off studies carried out early in the design process and will determine the final solution (Brown and Andrews 1980; Andrews and Pawling 2009), therefore, committing the largest part of the future costs (Andrews 2011). The cost of applying corrections to a problematic design at the later design stages, or even construction stages is disproportionally high (Goossens 1992), and costs can only be reduced by withdrawing equipment rather than considering alternative designs (Andrews and Brown 1982). (Although Knight (2012a; 2012b) commented that in some cases regarding certain shipyards "the onerous change process imposes more effort to modify a 3D model than making the change directly on the inbuilt product"). The importance of concept design is enhanced by the fact that realistically novelty can only be introduced here (Brown 1986a). It is clear that the early stages of design are the most critical, and this can be summarised by "the often quoted truism that $90 \%$ of the major design decisions have been made when less than $10 \%$ of the design effort has been expended" (Andrews et al 2006).

\subsubsection{Traditional Preliminary Ship Design}

Traditionally, naval architects have focused on the areas of speed, seakeeping, stability and strength, otherwise known as $S^{4}$ (Brown and Andrews 1980). These were 
regarded as the driving issues in preliminary ship design; in contrast, style related issues were examined at later stages (Andrews 1984) (contributing towards the relative formalisation and maturity in disciplines such as marine hydrodynamics and marine structures compared to marine design (Andrews 2012)). In general, the process was perceived as one with discreet sequential steps and feedbacks, similar to design spirals (Andrews and Dicks 1997; Andrews et al 2006); this sequential process is depicted in Figure 2.1.

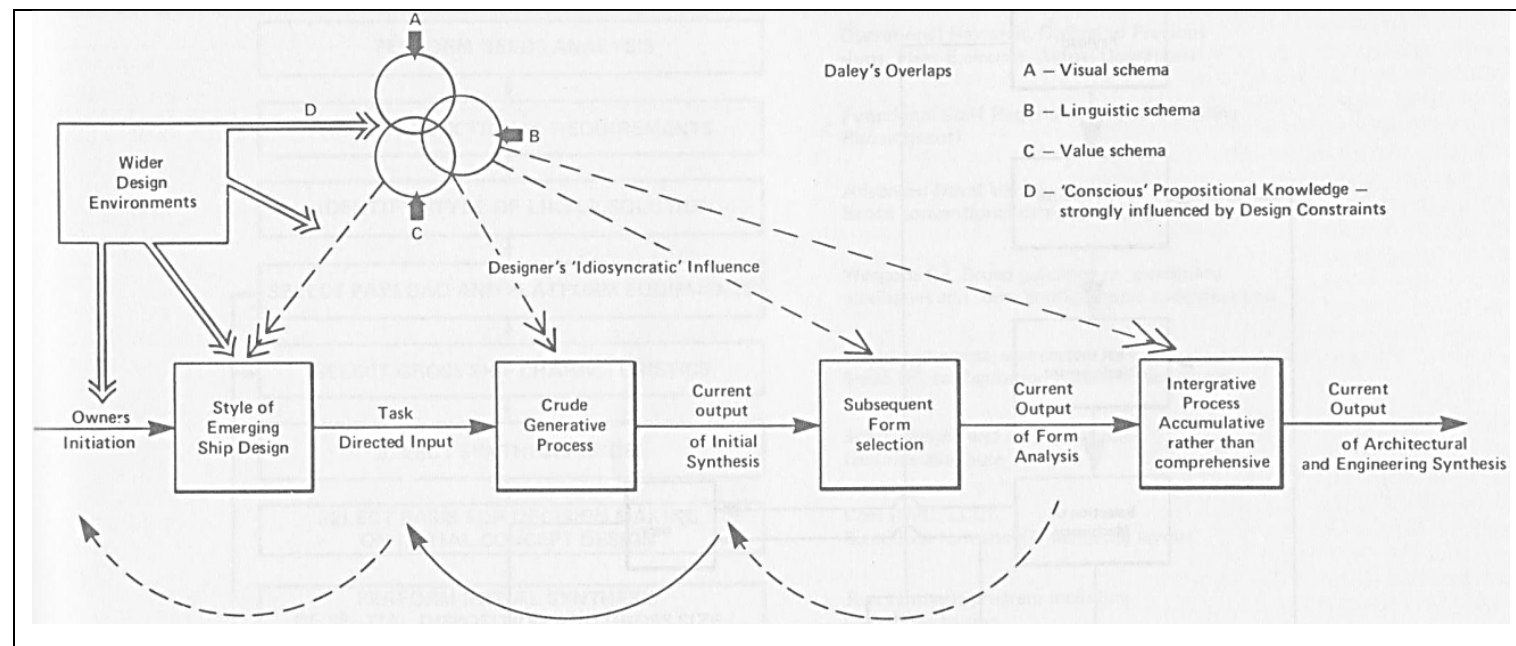

Figure 2.1: A Summary Representation of Current Sequential Synthesis (Andrews 1986)

Initial sizing (or generative process), relied extensively on existing ship data and scaling ratios to get first estimates of weight and space requirements and costs (Brown and Andrews 1980) before the first iteration commenced (Andrews 1981; Cooper et al 2007). Figure 2.2 illustrates a simplified initial sizing process showing the assumptions and sources for each step. 


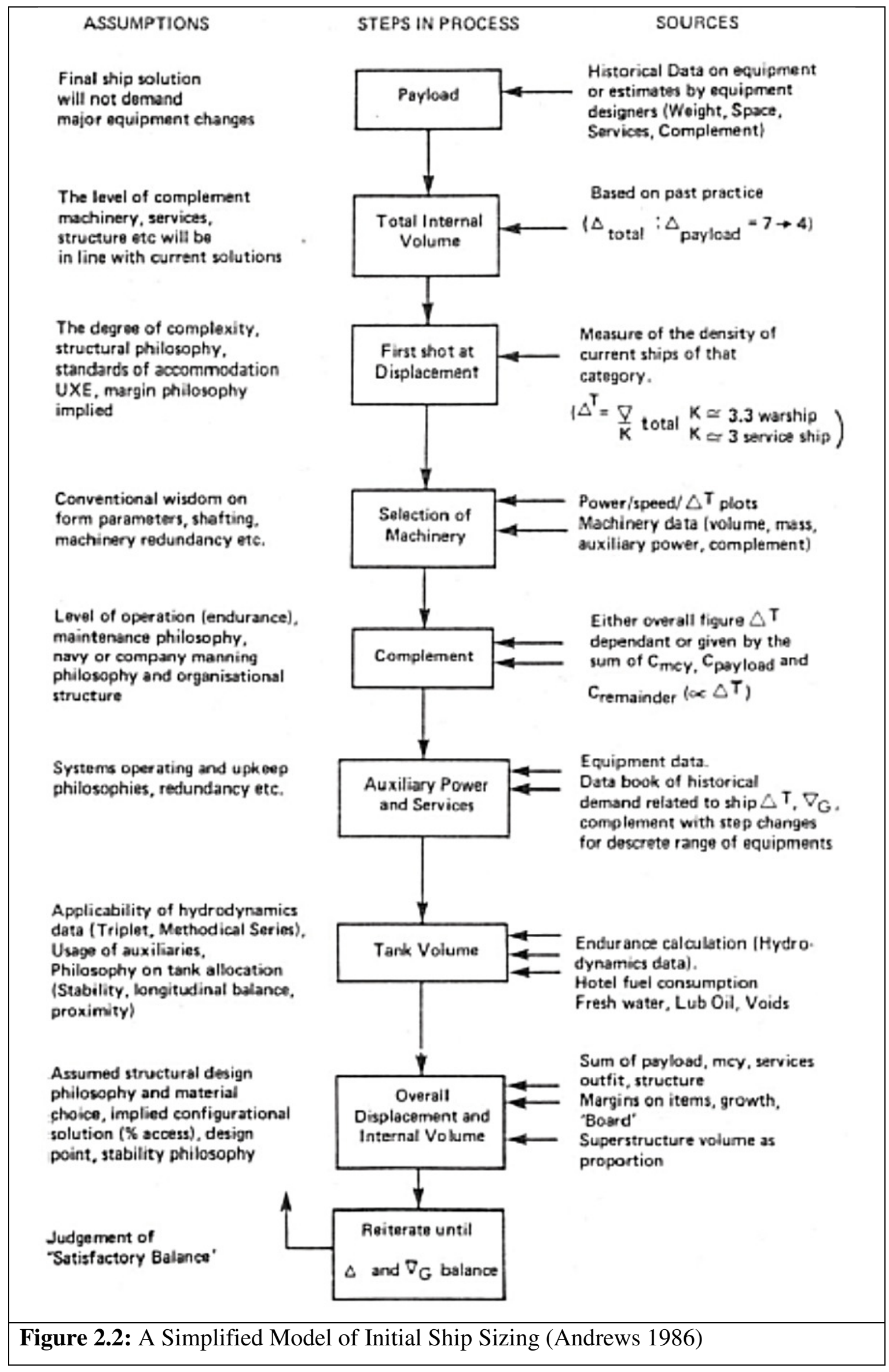


Initial sizing was followed by parametric/form selection and finally the development of the ship's layout was considered (Andrews 1986). This traditional preliminary design process limited the use of computers to the first two numerically based steps, initial sizing and parametric selection (Andrews et al 2006).

This process has been criticised for various reasons as it does not represent a fully integrated design process, limiting creativity and innovation. The fact that the process is essentially sequential means that the layout/architecture of the design (the last step in the sequence) has to be developed within a predefined hullform (Andrews 1981; Andrews et al 2006) therefore limiting the designers creative input (Andrews 1986). Issues such as bulkhead locations are governed by damage stability and structural continuity requirements (Brown 1987) further constraining the architectural evolution (Andrews 2003). Topics such as crewing, ship operations, personnel evolutions can only be examined at later stages in the design process (within the constraints previously observed) where alterations to the design are lengthy and costly (Andrews et al 2008). Another area of risk characterising the traditional approach is that, since the initial sizing step is purely numerical it relies on the availability of existing ship data (Gates 2005; UCL 2010a; UCL 2010b) and the ability of the designer to produce pragmatic scaling ratios (Cooper et al 2007). Add to this, the parametric selection stage is disconnected from the architecturally driven aspects of a ship (Andrews 1986). Andrews (1986) concludes that traditional synthesis is progressive, not comprehensive or integrative and comments that, given the forward momentum in design development, the feedback loops in Figure 2.1 are optimistic, thereby limiting the iterative nature of preliminary ship design to just getting a numeric balance rather than for exploration of the design space.

It is worth noting that, traditionally ships and their components are described by means of a weight breakdown (Garzke and Kerr 1985; MOD 2010). The UK MOD (2010) convention for warship weight groups, similar to that used in the UCL MSc Ship Design Project (UCL 2010a) is as follows: hull and superstructure, propulsion, electrical, control and communication, ancillary systems, outfit and furnishings, armament and variable load. This type of ship description, although offering the prospect of comparing a new ship design to existing ships (Andrews and Dicks 1997) has a number of limitations. It is mainly aimed towards the ship construction industry (Garzke and Kerr 1985; Andrews and Pawling 2009), thus discouraging novel/unconventional concepts (Andrews and Dicks 1997) and it is not appropriate in 
investigating the overall effect on the ship of adding or removing a certain capability (Andrews and Dicks 1997)

It is evident that insisting on the traditional approach to preliminary ship design inhibits innovation and evolution, which means most new designs are based closely on existing ships (Andrews 1981). However, with the rapid development in computer graphics over recent years (radically altering design processes (Andrews 2011)) it is possible to generate a fully integrative ship design synthesis, where architectural factors can influence major decision making from the start of the process (Andrews 1986; Andrews et al 2006). Innovation in both ship designs and the design process itself is seen to be the way of meeting the challenge of increased capability with decreasing resources (Brown 1993).

\subsubsection{Architecturally Orientated Preliminary Ship Design}

The architecture of a warship is an area often disregarded by naval architects in favour of topics such as hydrodynamics and structures (Brown 1993; Andrews et al 2006). However, Brown (1987) defined a warship as an "assembly of multifunctional interacting spaces located within an envelope set by hydrodynamic and other considerations, partition arrangement governed by structural continuity and containment of damage, more than onshore buildings" thereby placing architecture at the centre of the equation. Andrews (1986) took a further step by suggesting that certain ship types should be designed by arranging the layout such as to optimise the ship's primary function, followed by wrapping an efficient hullform around this layout. This is enhanced by the fact that most warship types are rarely driven by hydrodynamic or structural aspects (Brown 1987), especially during the early design stages (Chalmers 1993a). The importance of a ship's architecture is highlighted by the fact that it affects vital features such as operational effectiveness, survivability, economy, crew efficiency and comfort and ease of production (Andrews and Brown 1982; Brown 1987) and all that in its highly constrained, dynamic environment (Brown 1993). However it is difficult to evaluate the effectiveness of a particular layout due to the multiple and conflicting requirements that exist for warships and the interactive nature of ship architecture (e.g.: upperdeck arrangement is affected by the machinery disposition via uptakes and downtakes) (Andrews and Brown 1982; Brown 1993). The above led Purvis (1974) to the conclusion that warships are not weight or space limited, but architecture limited. 
Since warships are predominantly architecturally driven, it has been suggested that a configurationally orientated preliminary design process would reveal design drivers and risk areas (Andrews 2003). Also, this would increase the scope for creativity and enable the designer to contribute in the divergent and imaginative early stage design process; therefore, leading to innovative solutions (Andrews 1981) and better deal with complexity (Andrews 2003). In addition, the adoption of such a preliminary design process could lead to an increase in the number of alternatives considered, aiding decision making at the crucial early design stages, therefore decreasing the risk of errors in expensive and infrequent projects and assisting trade-offs and cost estimation (Andrews 1986). By integrating the three traditional stages of the design process, i.e. initial sizing, parametric selection and development of the general arrangement, a more complete solution will be presented (Andrews 2003). Since the hullform will not be set during the layout evolution stage (Andrews 1986) the dimensions of the ship can be treated as flexible and dependant on an efficient architecture (Andrews et al 2006), rather than assuming that an increase in ship size is always penalising, as is the case with numeric sizing (Andrews 1981) such as shown in Figure 2.2. By positioning warship architecture at the centre of the preliminary design process, certain design features and interactions can then be explored, which were not possible with the traditional one-dimensional numerically based design process (Andrews 1981; Andrews et al 2012a). For example, a designer can better integrate the combat system (Andrews 1992) and take into account issues such as zoning, vulnerability, modularity, access philosophy, habitability and space margins (Andrews 1986). This leads to the conclusion that design options can be compared on the basis of operational performance rather than merely cost (Andrews 1986), although questions have been raised as to how to evaluate characteristics such as survivability and habitability (Garzke and Kerr 1985). Finally, an early configurational representation of a design would enhance the dialogue between the parties involved, particularly with the requirements owner (Gale 2003).

However, space is not only dependant on principal dimensions but also on location and shape (Brown 1987). Therefore, in order to successfully implement a new fully integrated, more creative and flexible architecturally driven design philosophy, it is essential to take advantage of powerful CAD (Andrews 1981). During recent decades, developments in computer techniques were rapid and impressive, revolutionising the early ship design process (Munoz and Forrest 2002; Andrews 2011). This has led designers to be able to produce a large number of alternative designs in a relatively short time with less errors, reducing costs, (Gale 2003) and explore and assess the 
alternative layout arrangements, confidently making important decisions even at the earliest stages of the design process (Crow 2001). When such CAD tools are combined with numerical/analytical naval architectural tools, a fuller integration in the preliminary design phase can be achieved (Andrews 2003). The designer can carry out a variety of tasks which were usually carried out at later design stages (Cooper et al 2007), such as estimating cost and weight (Andrews and Brown 1982), assessing vulnerability and structural continuity (Andrews 1993; Crow 2001), investigating personnel and freight flow (using simulation techniques) (Andrews and Pawling 2009) and investigating congested areas by 3D modelling (Gale 2003). Pegg et al (2012) discuss the challenges related to integrating the various early ship design simulation tools now available in a single design and simulation environment; in particular they examine strength, hydrodynamic, shock and blast, signatures, operational and cathodic protection simulation models. Such techniques could partially address Garzke and Kerr's (1985) remark on the difficulty of evaluating and optimising certain ship characteristics. It has also been suggested that advances in computer techniques have decreased the need for physical mock-ups (Gale 2003) which are expensive and time consuming to build and modify. It is then clear that the impressive software developments of the last years have made preliminary design more creative and innovative (Andrews and Pawling 2003), producing designs which are more complete and descriptive, therefore aiding decision making (Andrews 1993), when the design is still sufficiently flexible to more easily apply changes (Andrews et al 2008).

Andrews' (1981) proposal to integrate ship architecture with the traditional numerical techniques was followed by the demonstration of 'creative synthesis' (Andrews 1984) which was presented in a paper entitled 'An Integrated Approach to Ship Synthesis' (Andrews 1986). A hull form generator was used to produce a frigate hull and output deck plans on which compartments were positioned. After auditing, space and weight data were extracted and reiterated. This way, the initial sizing step was not carried out in the traditional purely numerical manner, but it was incorporated with layout considerations ("disposition of the principal spaces"), therefore producing a much more comprehensive solution. Figure 2.3 shows a revised initial sizing process, which this time takes into account spatial factors, while Figure 2.4 is a description of fully integrated design synthesis in contrast to the sequential traditional synthesis shown in Figure 2.1. 


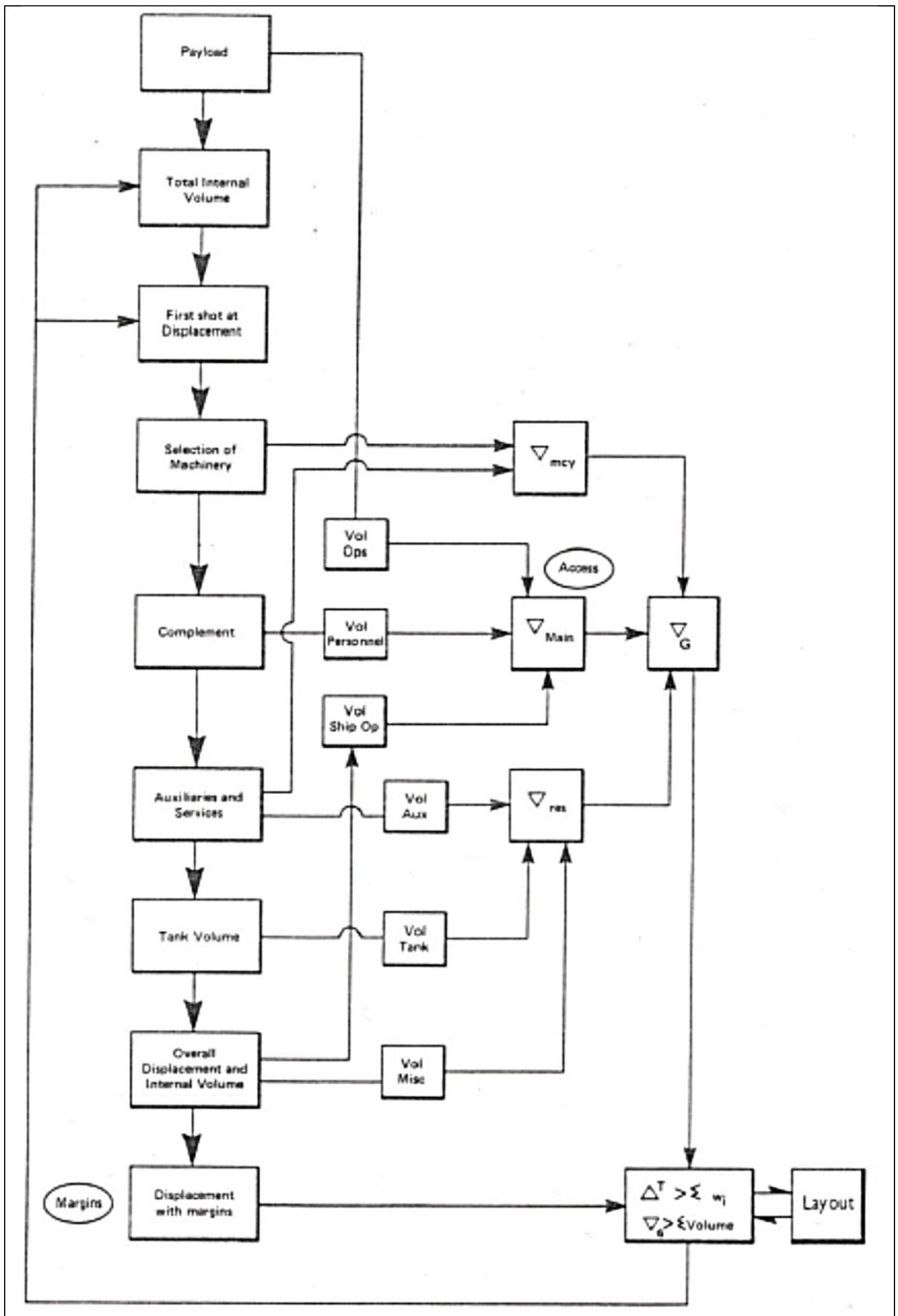

Figure 2.3: A Schematic Modification of the above Initial Ship Synthesis with Spatial Totals which are Laid Out Graphically before Reiterating (Andrews 1986) 


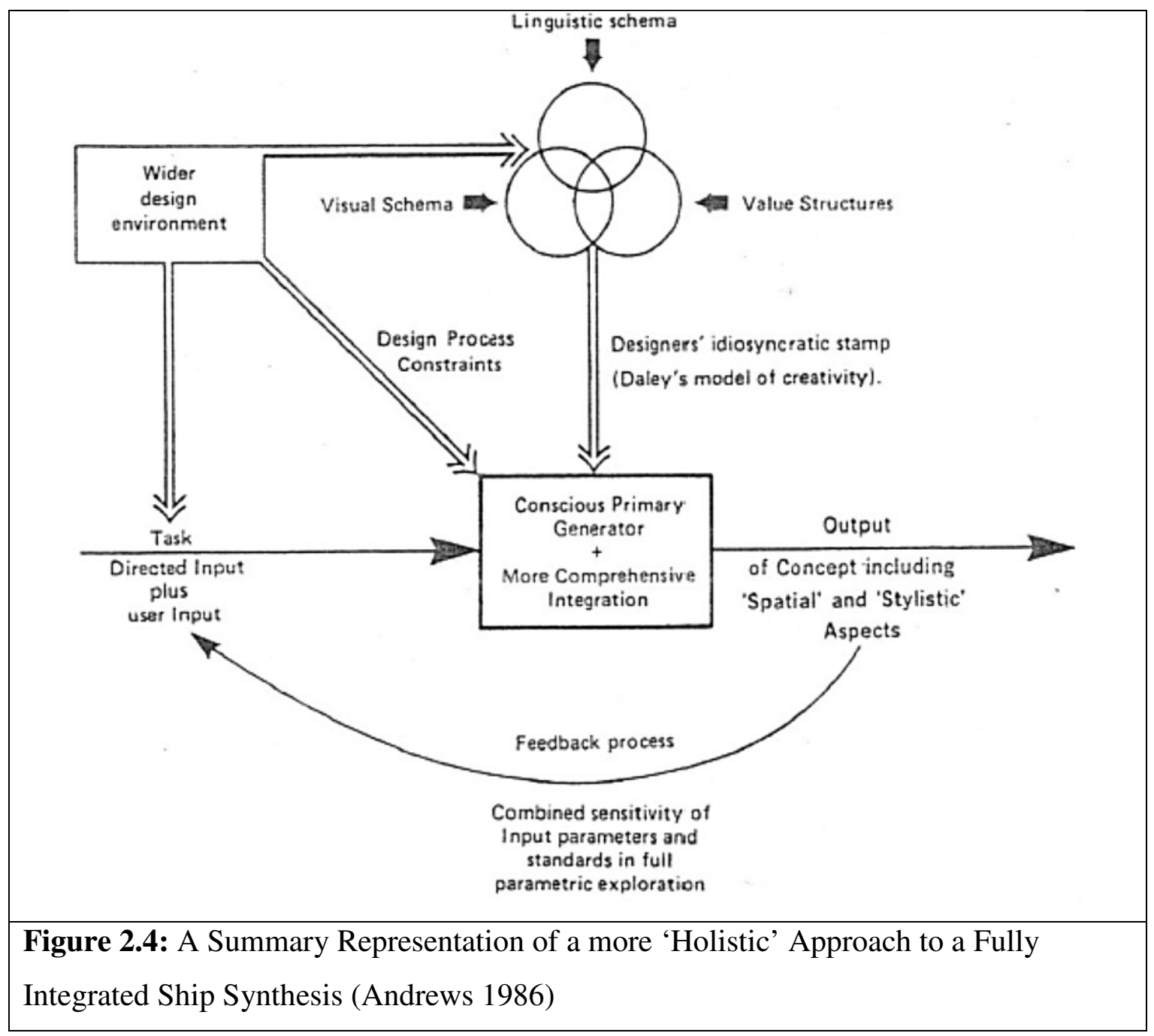

However, the initial hullform and, therefore, deck plans, had to be developed under default hull coefficient values due to the limited capabilities of computers during the 1980s (Andrews 1986), restricting the demonstration of applicability of the method only to frigates and leaving out unconventional hullforms (Andrews 1998). From this work, a new approach, namely the Design Building Block approach (Andrews and Dicks 1997) was developed and implemented, taking advantage of the impressive CAD developments previously described, first for submarines (Andrews et al 1996) and later for surface ships (Andrews and Pawling 2003). This is discussed further in Section 2.1.5.

In order to appropriately position a ship's principal compartments and build up its architecture, Andrews $(1984 ; 1986)$ proposed the method of 'circles of influence'. Figure 2.5 shows how this method could be used in order to accomplish full integrated synthesis and come up with a three dimensional representation of a frigate, around which an efficient hullform could be wrapped (Andrews 1986; Andrews 2003). 


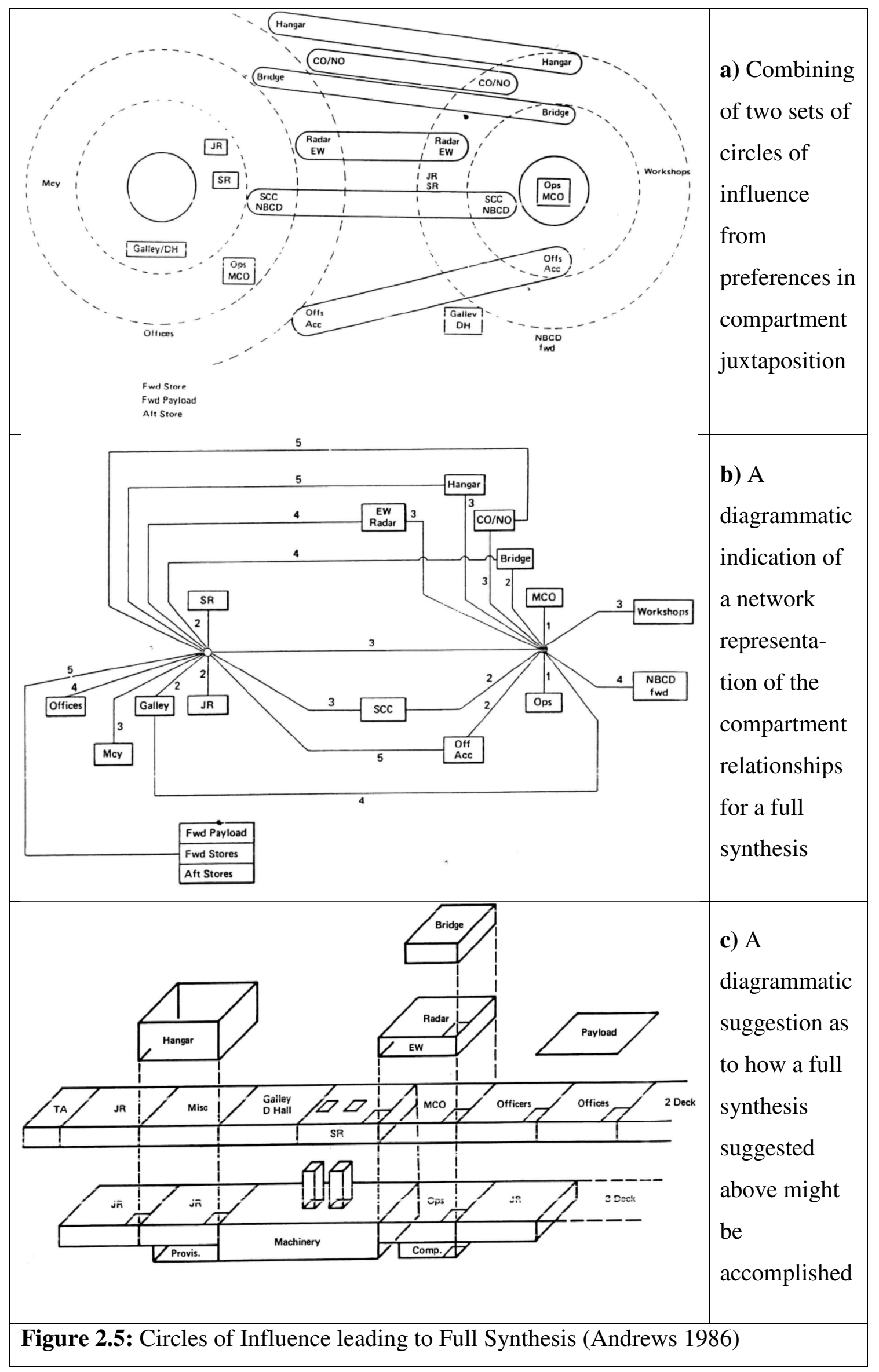


There are many obvious compartment relations around which 'circles of influence' could be built, such as dining spaces should be close to stores and in a position with low vertical accelerations (Brown 1987). Also, some compartments are more important or constrained than others (such as machinery spaces, Operations Room and accommodation spaces (Andrews 1986)) so they should occupy central roles in the 'circles of influence'. An efficient layout, locating related spaces adjacently, would also lead to minimising the extent of main passageways (Brown 1986a). Further work on 'circles of influence' and more detailed diagrams were produced by Dicks (2000).

It was previously mentioned that the weight breakdown description of traditional ships led to numerous limitations. Therefore, it was suggested that this was another area for reconsideration in order to achieve more creative ship design. The broad objectives of a warship have been determined as: 'to float, to move, to fight' (Brown and Tupper 1989), and these three categories "represent a ship in material and cost terms" (Andrews 1987). Therefore, it would make sense to apply a functional breakdown using these three groups (Float, Move, Fight) at top level with the addition of an Infrastructure group as argued by Andrews et al (1996). Many advantages of a functional descriptive breakdown have been reported: the impact of adding or removing a particular capability on the whole ship could be assessed (Andrews 2003) (therefore leading to more costeffective solutions and improving communication with the customer (Garzke and Kerr 1985)); new ship designs would no longer be solely based on assumptions from existing ships and thus would encourage innovation (Andrews 2003); a more meaningful cost description of the ship could be achieved (Andrews and Pawling 2003) (if resources devoted to Infrastructure are not taken into account, Fight accounts for approximately $70 \%$ of the UPC of a typical combatant, followed by Move and Float (Andrews and Brown 1982)).

\subsubsection{The Design Building Block Approach to Preliminary Ship Design}

It was previously mentioned that the research carried out during the 1980s concerning full integration of ship synthesis (Andrews 1986) led to the DBB approach, which is described in (Andrews and Dicks 1997). Since then, numerous types of ships have been designed using this approach to preliminary ship design, the work being carried out in the UCL DRC which was established in 2000 as part of the Marine Research Group of UCL (http://www.ucl.ac.uk/mecheng/research/marine). This CAD 
focused group makes use of the GRC's (QinetiQ 2012) Paramarine Preliminary Ship Design Software in order to investigate innovative preliminary design.

The basic idea behind the DBB approach is for the designer to separate the ship's functions and sub-functions into discrete elements (Design Building Blocks) and position them appropriately, putting architectural factors in the centre of the process, in contrast to the traditional sequential design process (Andrews and Dicks 1997). This method allows a more thorough exploration of alternative designs to meet the particular requirement, as well as encouraging novel solutions (Andrews and Pawling 2009). This is because it is not type ship based and removes the limitations of the traditional numerical sizing process, which leads to conventional configurations (Andrews and Dicks 1997). An outline of the DBB approach is given by Andrews and Dicks (1997) and Andrews and Pawling (2003) (see Figure 2.6):-

- "A need for a new conceptual design is conceived and an idea of the likely design style to meet that broad requirement is suggested;

- Drawing on novel ideas or historical data a series of building blocks are defined in a computer system. Each building block contains geometric and technical attributes regarding the functions of that building block;

- A design space is generated and the Design Building Blocks are configured as desired or required within the design space;

- Overall naval architectural balance and principal performance aspects of the design are investigated using simple and flexible algorithms and, if necessary, using analysis programs external to the main system;

- The configuration is then manipulated until the designer is satisfied;

- Decomposition of the Design Building Blocks to greater levels of detail is undertaken, as necessary to increase confidence in the design solution." 


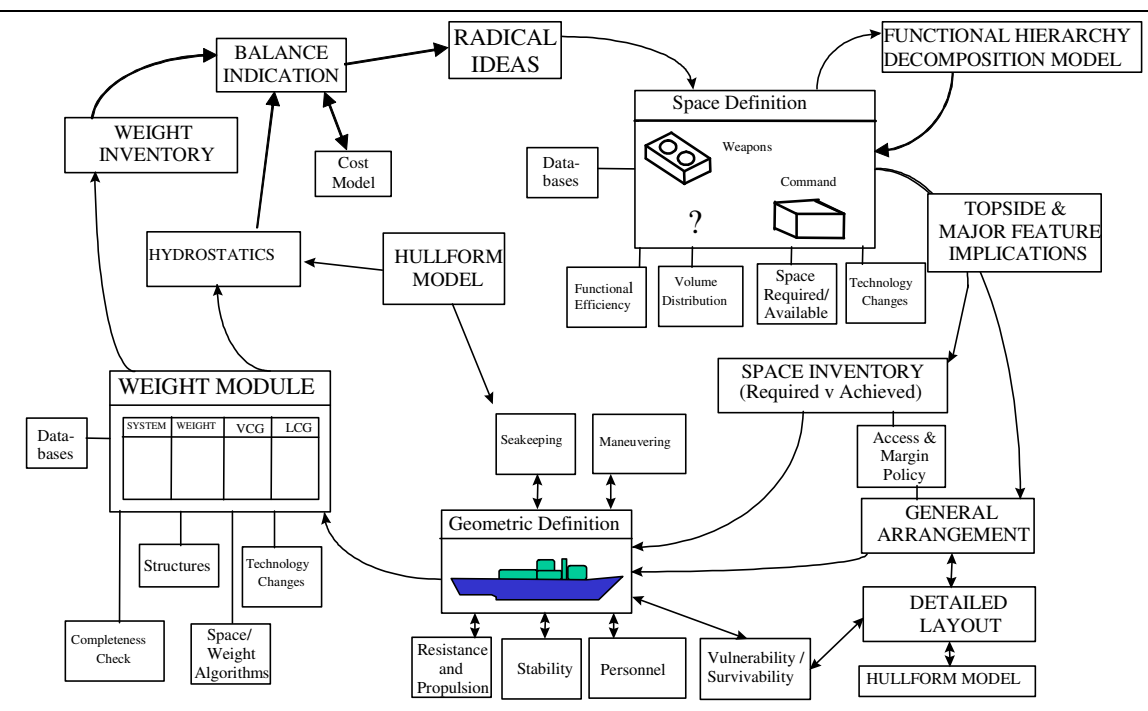

Figure 2.6: Building Block Design approach applied to Surface Ships (Andrews and Dicks 1997)

The DBB approach is characterised as: 'open/glass box', allowing the designer to input their experience and creativity, therefore, increasing their contribution and responsibility (Andrews 2006); 'soft', so that the process can be updated and improves (e.g. changing algorithms); and it allows margins (see Appendix 1) to be appropriately assessed in the course of the process rather than using historical values at global or just main weight and space group level (Andrews and Dicks 1997).

The DBB design phases are summarised in Figure 2.7.

\begin{tabular}{|c|}
\hline Design Preparation \\
\hline Selection of Design Style \\
\hline Topside and Major Feature Design Phase \\
\hline Design Space Creation \\
\hline Weapons and Sensor Placement \\
\hline Engine and Machinery Compartment Placement \\
\hline Aircraft Systems Sizing and Placement \\
\hline Superstructure Sizing and Placement \\
\hline Super Building Block Based Design Phase \\
\hline Composition of Functional Super Building Blocks \\
\hline Selection of Design Algorithms \\
\hline Assessment of Margin Requirements \\
\hline Placement of Super Building Blocks \\
\hline Design Balance \& Audit \\
\hline Initial Performance Analysis for Master B.B. \\
\hline Building Block Based Design Phase \\
\hline Decomposition of Super Building Blocks by function \\
\hline Selection of Design Algorithms \\
\hline Assessment of Margins and Access Policy \\
\hline Placement of Building Blocks \\
\hline Design Balance \& Audit \\
\hline Further Performanee Analysis for Master B.B. \\
\hline General Arrangement Phase \\
\hline Drawing Preparation \\
\hline
\end{tabular}

Figure 2.7: Building Block Design Steps (Andrews and Dicks 1997) 
As the design is evolved, the level of detail increases and at each stage the design is assessed in order to result to an appropriately balanced ship (Andrews and Pawling 2003). This is done by the design tool reporting the design state to the designer (after auditing the configuration to check if it is balanced in weight, space, stability and powering). The designer then has to judge what changes are needed to achieve a necessary level of balance (Andrews and Pawling 2003).

Since this is an architecturally orientated approach, changes can be implemented to the ship configuration at the early stages of design, thereby giving the ship designer the opportunity to investigate many aspects of the design while its configuration is still amenable to change (Andrews and Pawling 2006b). Such aspects include: distributed system details, margin philosophy, access, combat system integration (Andrews 1998), human factors, '-ilities' (Andrews and Pawling 2009), topside design (Andrews and Dicks 1997), through life costing, health and safety issues, environmental issues (Andrews and Pawling 2009). Many can be considered while also accounting for the traditional naval architectural areas of powering, hydrodynamics and structures (Andrews and Pawling 2006b). Therefore, a more detailed yet adjustable description of the design is possible at the earliest stages of design where potential conflicts can be addressed and decisions made (e.g. structural continuity (Andrews and Dicks 1997), weight and space balance, powering and stability performance, personnel flow (Andrews 2004)). It is worth noting that Andrews and Dicks (1997) designed to the same requirement a frigate following both the conventional and the DBB process. The DBB approach designed ship met all the requirements whereas the conventionally designed one did not. This example showed that further iterations were needed after the end of a conventional preliminary design, which were very likely to lead to a larger ship downstream with resultant programme consequences.

The above procedure to preliminary ship design was developed and implemented following the rapid developments in computer capability, initially for submarines (through the SUBCON implementation (Andrews et al 1996)) and subsequently for surface ships through GRC's SURFCON implementation (Munoz and Forrest 2002). By implementing the DBB approach through the SURFCON module in Paramarine, the DBB approach is now a well-established commercial preliminary ship design module (Andrews and Pawling 2003). This way, SURFCON can draw on all the naval architectural analytical tools (such as stability, powering, seakeeping, vulnerability, manoeuvring and structural analysis) available in Paramarine (Andrews and Pawling 2003). Consequently, a fully integrated preliminary design process, 
architecturally centred and combined with traditional naval architectural numerical analysis techniques to achieve balance (Andrews et al 2008) is now possible through SURFCON and has been shown to be applicable to a wide range of conventional and unconventional ships (Andrews and Pawling 2003). The three dimensional nature of SURFCON can be used to adequately investigate for naval vessels those configuration related factors previously mentioned, as well as other issues, such as design for production, adaptability, sustainability, ease of access and spatial margins.

In SURFCON, each DBB is a placeholder (folder) representing a particular function and containing all relevant information, e.g.: numerical, constraint, parametric, geometric and descriptive data (Andrews and Pawling 2003). As the design evolves, Paramarine uses the above data to update the graphical display and perform the relevant calculations. The cumulative characteristics of each DBB listed above are brought together in a Master Building Block (MBB) which provides the numerical description of the design. The MBB contains data such as the overall ship requirements, ship characteristics and overall margins. These characteristics are constantly being audited by Paramarine's analytical tools and the designer then uses the data given to judge what steps are necessary to achieve balance. A screenshot of Paramarine is reproduced in Figure 2.8.

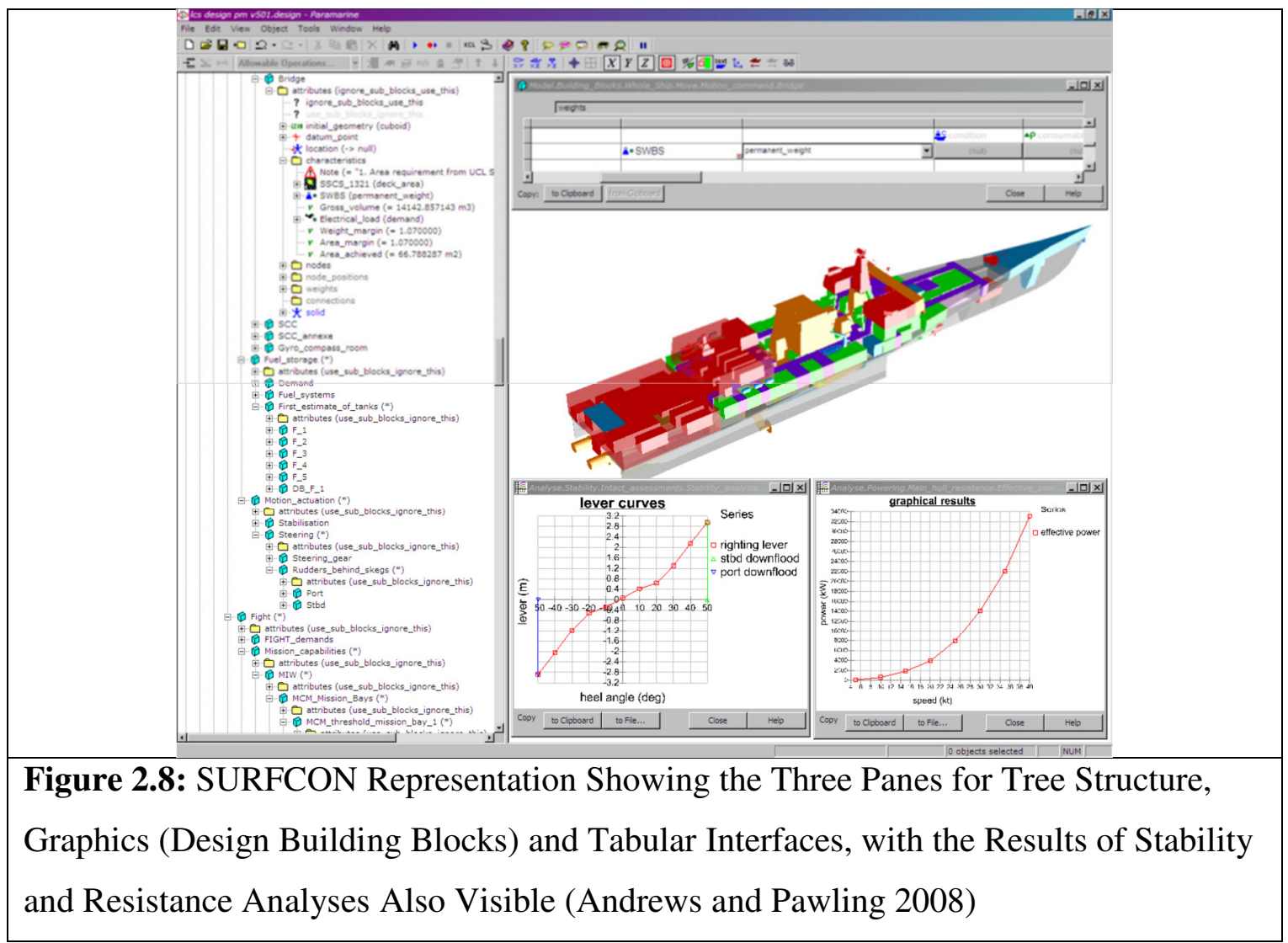


In view of the fact that one of the central elements of the DBB approach is to separate the ship's functions, it is only natural for SURFCON to describe a ship by means of the functional breakdown described in Section 2.1.4 (i.e. Float, Move, Fight, Infrastructure) (Andrews and Pawling 2003). The four top level groups gradually lead to lower level, more detailed groups. It has been recognised that the Fight and Move groups tend to drive a design to a great extent, whereas Float and Infrastructure are largely dependent groups (Andrews and Dicks 1997). This is because of the directly defined requirements and constrained nature of the Fight and Move groups. However, advancements such as IFEP and VLS could increase the adaptability of the two design driving groups (Andrews and Dicks 1997).

Since its conception and realisation through SURFCON, the DBB approach has been used for a range of projects in UCL, such as a trimaran Littoral Combat Ship (LCS) (Andrews and Pawling 2006a), a Joint Support Ship (JSS) (Andrews and Pawling 2007), personnel flow simulation (Andrews 2006; Andrews et al 2008), implications of all electric ships on their configuration (Andrews et al 2004), and several others listed in (Andrews and Pawling 2006b; Andrews and Pawling 2009). For the ship design projects, the DBB's were sized using the algorithms provided in the UCL MSc Ship Design Exercise (UCL 2010a; UCL 2010b). This UCL work has proved the applicability of the approach and realised its advantages, which are outlined in the above references. These are seen to be: identifying design drivers and interactions; producing believable concepts for unconventional designs; rapidly producing and comparing alternative designs; exploring different configurations; assessing designs in a variety of ways at the early design stages; identifying areas of uncertainty; and providing increased confidence for concept development.

Appendix 2 is a summary of the literature regarding the major design drivers of various presently common naval ship types. Combatants, with both conventional and unconventional hull configurations, and replenishment ships are investigated.

\subsection{Ship Costing}

It is well established that ship costing is a vital part of the (concept) ship design process (UCL 2010b) with optimal cost effectiveness being a key objective of ship designers (Rawson 1973). Cost estimates are required in order for governments to justify and allocate funding and project management teams "to avoid budget overruns and its consequences" (Rudius 2012; Gerdemann et al 2012). Dirksen (1996) observed 
that defence inflation over the last century has been as much as $9 \%$ above inflation. This has occurred due to military competition and the associated requirement for increased capability (Dirksen 1996) which has been realised by the technological developments (accompanied by increased complexity and maintenance) in weapons and other equipment (Rawson 1973). Since defence inflation in recent decades for Western Powers has not been complemented by a corresponding increase in defence budgets (as a percentage of GNP), the only two realistic solutions have been to reduce vessel numbers or design smaller and cheaper warships; further cost reductions would imply reduce capability per ship (Dirksen 1996). This is because, as Dirksen (1996) advised, warship capabilities are set by various characteristics in terms of speed, seakeeping, structure, stability, style and stealth, which are the major cost drivers. Carreyette (1977), from a merchant ship stance, included further variables, such as managerial, financial, political and temporal variables, which impact on ship cost. However, Knight (2012a; 2012b) separated warship cost into costs associated with people (design, project management and procurement, construction/fabrication/integration) and equipment (major equipment and steel, commodity items). His definition focuses solely on the UPC. NATO (2009) identified that Whole Life Cost (WLC) (or Life Cycle Cost (LCC)) estimation is critical, being one of the criteria (along with operational need and government constraints) assisting the decision making process regarding cost effectiveness and choice between alternatives.

NATO (2003b) defined LCC as representing "all the costs that will be borne during the life of a System (Main System and Support System) to acquire, operate, support it and eventually dispose of it". In addition, Rawson (1973) suggested that project LCC should:-

- "Expose the costs known to be significant;

- Retain relevance to the elements of effectiveness;

- Be forecastable;

- Embrace the whole system affected by the project."

UCL (2010a; 2010b) for MSc level ship design studies, considers warship WLC as being the sum of FOC costs, UPC, TLC and disposal costs, for an entire class. FOC costs include office setup, design, drawings and recruitment costs and administrative costs (UCL 2010b). UPC is the individual ship (including its weapons, machinery and maritime equipment) construction cost (i.e. material, labour costs and overheads for shipbuilding) (Dirksen 1996; UCL 2010b). TLC adds on the costs to run each ship through its operational life (i.e. daily operating costs plus maintenance costs) (UCL 
2010b). Rawson (1973) identified the main TLC drivers as complement, operations and maintenance and repair. Complement costs include basic pay, earning related national income contributions, pensions and gratuities, additional special service pay and training costs for trade and rank; operations costs include fuel (the consumption of which is a function of the operating profile and propulsion and electric power generation configuration) and spares and consumables (e.g.: propulsion equipment, auxiliaries, ordnance, paint and NBCD equipment); maintenance and repair costs include docking and refits (Dirksen 1996). The above definitions are illustrated in Figure 2.9.

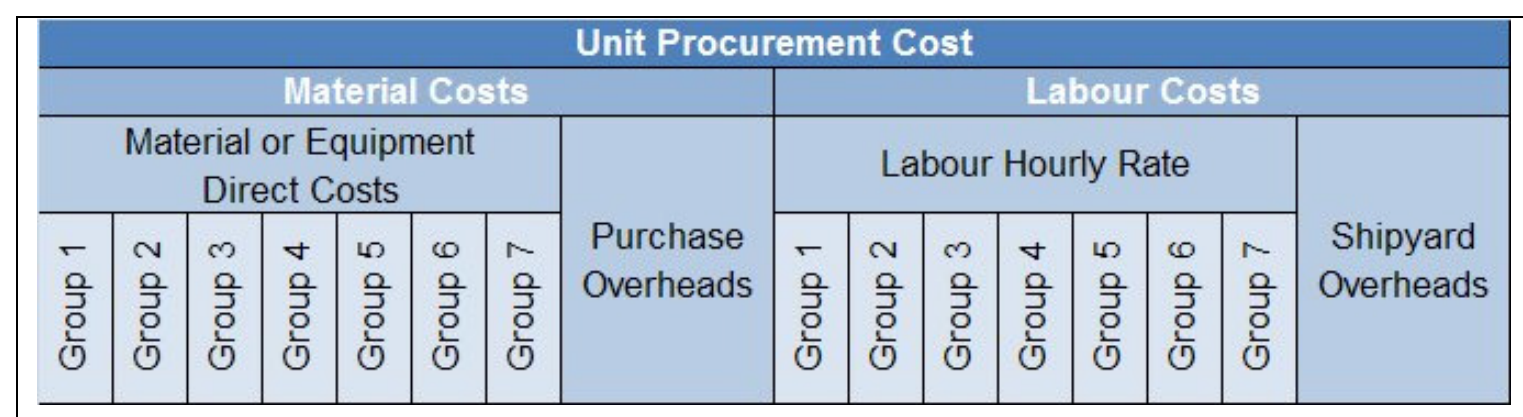

\begin{tabular}{|c|c|c|c|c|c|c|c|c|c|}
\hline \multicolumn{10}{|c|}{ Through Life Costs } \\
\hline \multicolumn{7}{|c|}{ Operating } & \multicolumn{3}{|c|}{ Maintenance } \\
\hline $\begin{array}{l}\bar{\Phi} \\
\text { ய }\end{array}$ & $\frac{3}{\frac{3}{U}}$ & 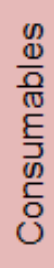 & 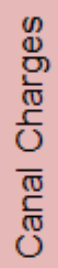 & 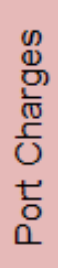 & 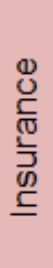 & 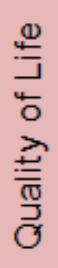 & $\stackrel{\vec{\Phi}}{\vec{己}}$ & 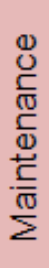 & $\begin{array}{l}+\frac{\omega}{4} \\
\stackrel{4}{\alpha}\end{array}$ \\
\hline
\end{tabular}

\begin{tabular}{|c|c|c|c|c|c|c|c|c|c|c|c|c|}
\hline \multicolumn{13}{|c|}{ Whole Life Coste } \\
\hline \multirow{7}{*}{$\begin{array}{l}\text { Design } \\
\text { Costs }\end{array}$} & \multicolumn{4}{|c|}{ Build Costs } & \multicolumn{4}{|c|}{ Whrough Life coste } & \multicolumn{4}{|c|}{ Disposal Costs } \\
\hline & $\sigma$ & $N$ & $m$ & ष & $\sigma$ & $\mathrm{N}$ & $m$ & $\forall$ & \multirow{6}{*}{ 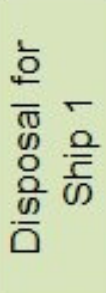 } & \multirow{6}{*}{ 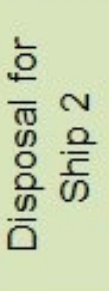 } & \multirow{6}{*}{ 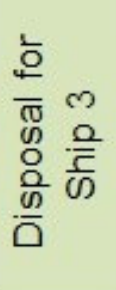 } & \multirow{6}{*}{ 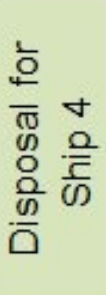 } \\
\hline & 을 & 을 & 을 & 을 & 으 & 으 & 으 & 으 & & & & \\
\hline & $\frac{\bar{c}}{(S)}$ & $\frac{\bar{C}}{(S)}$ & $\frac{\bar{c}}{(S)}$ & $\frac{\bar{c}}{\Theta}$ & $\frac{\bar{c}}{\infty}$ & $\frac{\bar{c}}{0}$ & $\frac{\bar{c}}{\infty}$ & $\frac{\bar{c}}{\infty}$ & & & & \\
\hline & 는 & 는 & ᄂั은 & 는 & 은 & 送 & 送 & 送 & & & & \\
\hline & $\circlearrowleft$ & 0 & $\circlearrowleft$ & 0 & 0 & 0 & 0 & 0 & & & & \\
\hline & 号 & & & & F & F & F & F & & & & \\
\hline
\end{tabular}

Figure 2.9: Unit Procurement Cost, Through Life Cost and Whole Life Cost Breakdown (UCL 2010b)

An alternative LCC breakdown is presented in Figure 2.10. It includes typical cost percentages of each LCC element. 


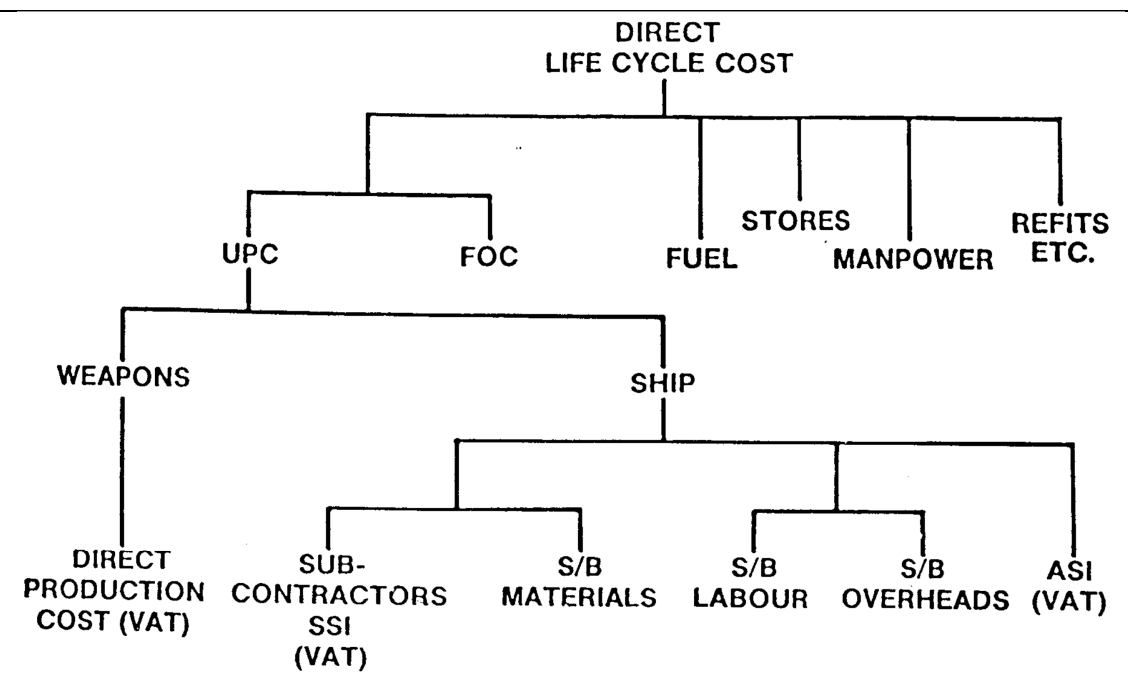

SSI: Shipbuilders Supplied Items

ASI: Admiralty Supplied Items

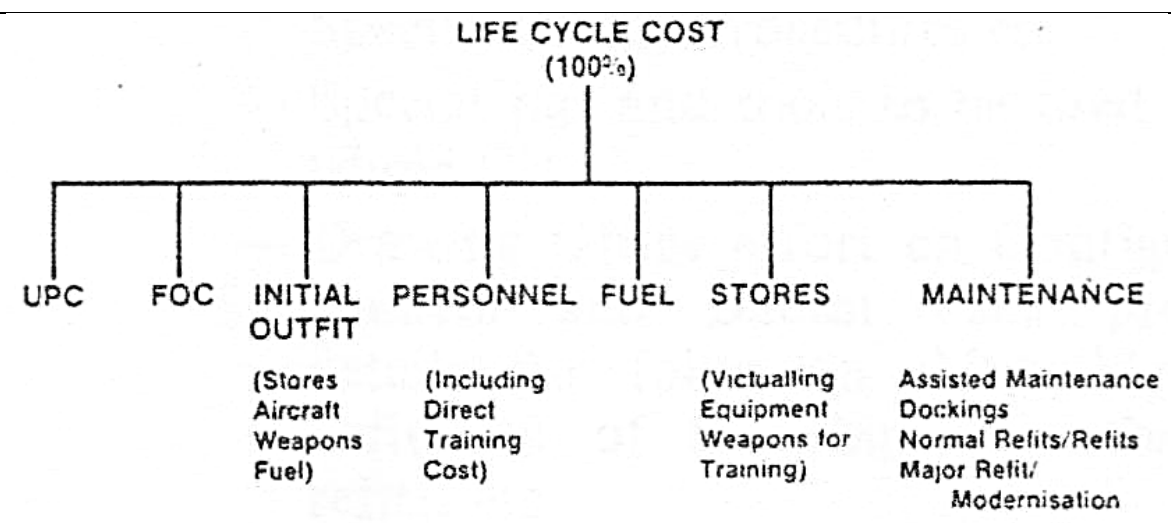

$\begin{array}{llllll}(20 \% 3) & (5 \%) & (5 \%) & (10 \%) & (10 \%) & (20)\end{array} \quad(30 \%)$

Figure 2.10: Breakdown of Warship Cost and Typical Example of the Breakdown in Life Cycle Cost for a Warship (Brown and Andrews 1980)

Dirksen (1996) updated an illustration presented in (Brown and Andrews 1980), giving typical cost percentages of each UPC element. This is shown in Figure 2.11.

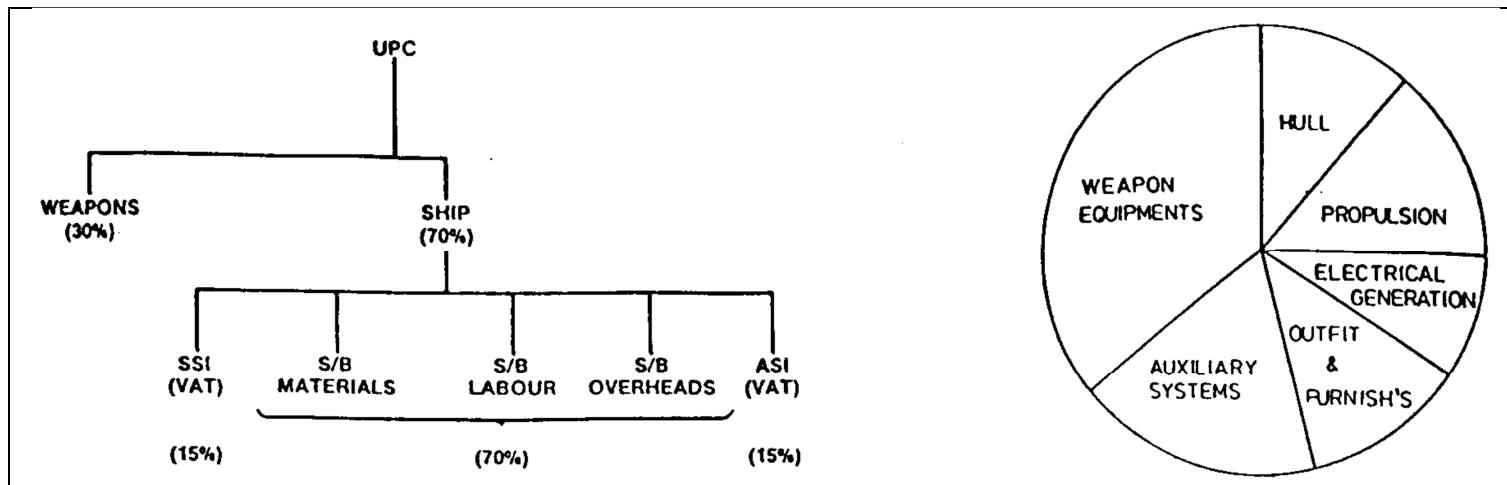

Figure 2.11: Typical Warship UPC Breakdowns (Dirksen 1996) 
Finally, Rawson (1973) illustrated the (undiscounted) LCC of a typical frigate, built at a procurement cost of $£ 12 \mathrm{M}$ in 1971 prices. This is reproduced below.

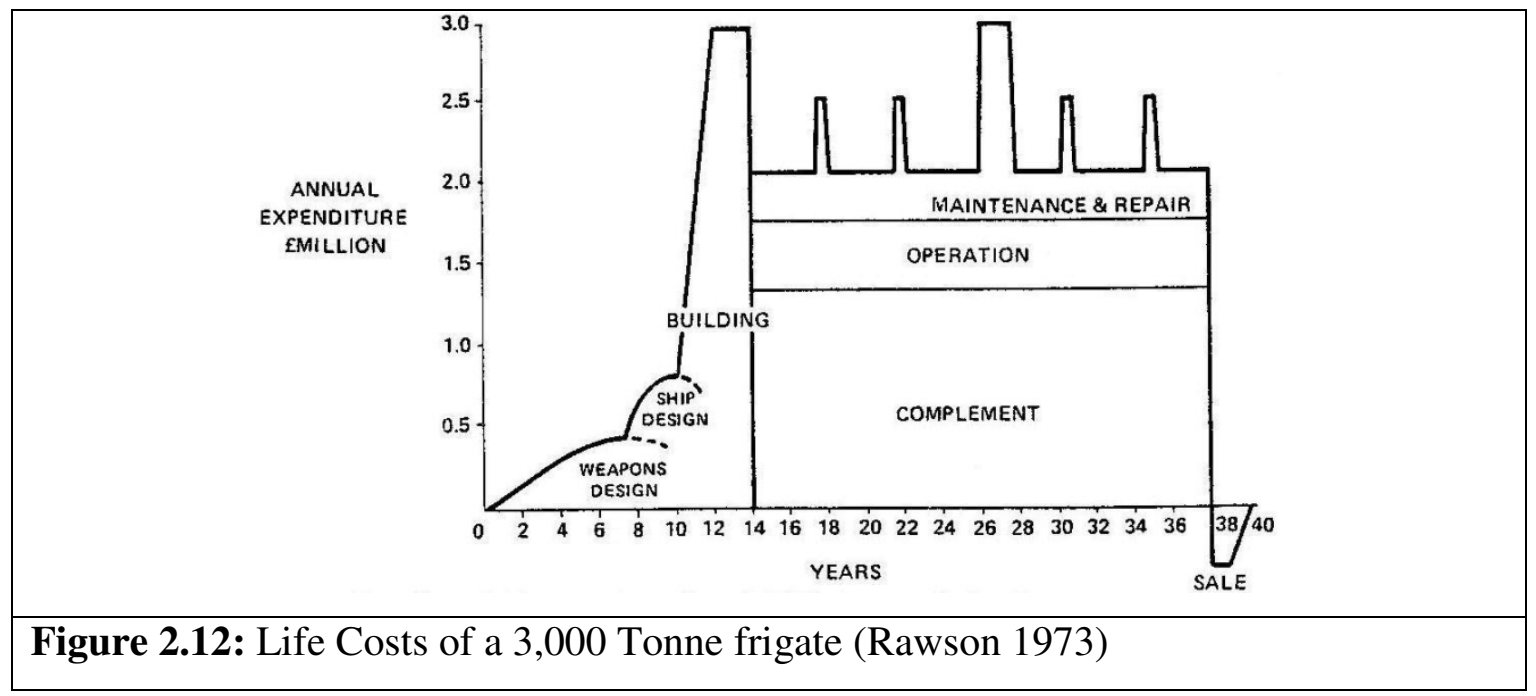

Traditionally, more attention has been paid to UPC, rather than TLC and WLC. The main reasons being the greater political significance of UPC (due to its short term impact) (Dirksen 1996; UCL 2010a), the larger uncertainties involved in TLC estimation (since future costs, spanning across multiple decades, have to be considered), the greater effort required for TLC analysis and the ability to postpone TLC elements, through for example, reduced operation (Dirksen 1996). However, it is agreed that only through the investigation of the WLC would the cost of ownership be realistically assessed (Dirksen 1996; UCL 2010a). Moreover, selection between alternative designs would be more efficient through WLC consideration (where UPC increases could be accepted provided related TLC reductions could be demonstrated) (Dirksen 1996) and through the mere consideration of minimising UPC, difficulties in the maintenance and modernisation of the ship can result (Rawson 1973).

NATO (2009) defined the LCCing as "the discipline or process of collecting, interpreting and analysing data and applying quantitative tools and techniques to predict the future resources that will be required in any life cycle stage of a system of interest". It is generally agreed that such an approach should be carried out throughout the operational life of a system (NATO 2009). However, it is also established that "the greatest opportunity to reduce life cycle costs usually occurs during the early stages of a programme" (NATO 2009) (i.e. the concept design stage) since "by the time that that a warship has been designed most of its upkeep characteristics and therefore its life costs have irrecoverably been committed" (Rawson 1973). This is summarised in illustrations found in (Andrews 1993; NATO 2009), the latter of which is replicated below. 


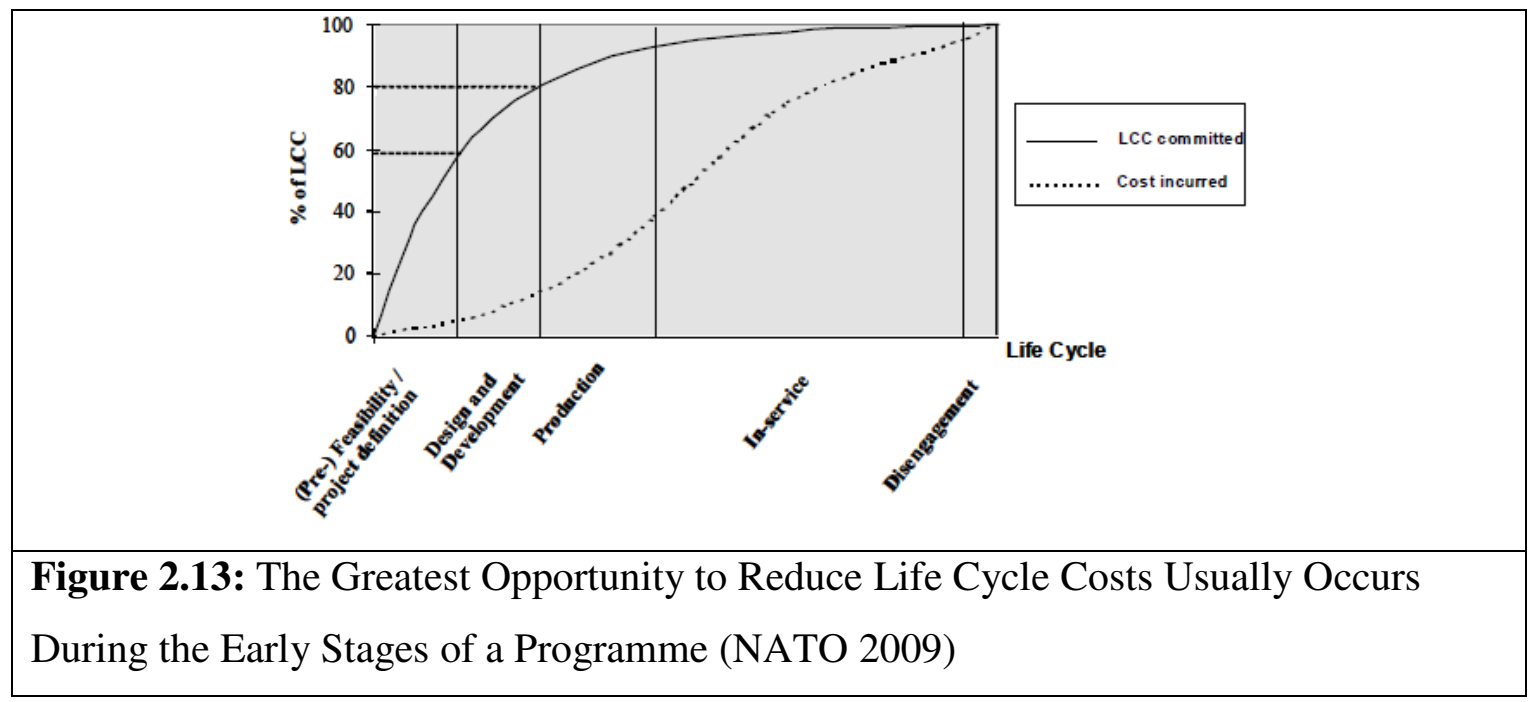

The application of LCC estimation techniques early in the design process would enable the investigation of trade-offs (UCL 2010a), the effect of varying the principal characteristics while the design is still flexible (Carreyette 1977), the evaluation of alternative solutions, the evaluation of cost reduction opportunities, the evaluation of areas of financial risk and uncertainty (NATO 2009). For example, Havron et al (2012) presented a case study where the propulsion system of the RN MARS Tanker was selected taking WLC into consideration, which identified that major drivers are fuel consumption and maintenance costs.

Being data driven, the methods used to assess the WLC of a system largely depend on the availability and quality of data (NATO 2009). An illustration reproduced in Figure 2.14 shows various cost estimating methods and their applicability throughout the programme life cycle. Further detail on these and other methods can be found in (NATO 2007).

\begin{tabular}{|l|l|l|l|l|l|}
\hline \multicolumn{5}{|c|}{ Programme Life Cycle } \\
\hline Concept & Development & Production & Utilization & Support & Retirement \\
\hline $\begin{array}{l}\text { Pardmetric } \\
\text { (Thispattem holds) }\end{array}$ & & & $\begin{array}{c}\text { Extrapolattion } \\
\text { (of actual costs) }\end{array}$ & $\begin{array}{l}\text { Analogy } \\
\text { (Similarites) }\end{array}$ \\
\hline Figure 2.14: Cost Estimating Methods (NATO 2009) & & \\
\hline
\end{tabular}


Dirksen (1996) identified two broad costing techniques, bottom-up approaches (which "require estimates of labour hours and materials for each element and subelement of work which are then accumulated to obtain the total cost estimate" and are used at later design stages) and top-down or parametric approaches (which "use historical data from previous designs to estimate costs of new projects" and are used during the early design stages with less product definition). NATO (2009) lists the following techniques as relevant during the concept stage: Bayesian, parametric, analogous, expert opinion and rule of thumb techniques. Of these, parametric costing methods are most widely utilised during the concept ship design stages; with a definition of such techniques suggested by NATO (2007) as: "the parametric method estimates costs based upon various characteristics or measurable attributes of the system, hardware and software being estimated. It depends upon the existence of a causal relationship between system costs and these parameters. Such relationships, known as CERs (Cost Estimating Relationships), are typically estimated from historical data using statistical techniques. If such a relationship can be established, the CER will capture the relationship in mathematical terms relating cost as the dependent variable to one or more independent variables. Examples would be estimating costs as a function of such parameters as equipment weight, vehicle payload or maximum speed, number of units to be produced or number of software lines of code to be written. The CER describes how a product's physical, performance and programmatic characteristics affect its cost and schedule". Similar definitions have been given by Gerdemann et al (2012) and UCL (2010a). Advantages of parametric costing methods include the output of sensible estimates despite the many unknowns (including systems and equipment) and limited information at that design stage (UCL 2010a; NATO 2007) and the ability to perform rapid sensitivity analysis (Gerdemann et al 2012). Disadvantages are seen to be the inability to capture and reflect low level design variations and the large amount of data required to derive the complex relationships (NATO 2007; Gerdemann et al 2012). Other parametric costing methods have been published. Carreyette (1977) proposed a parametric method based on historic data for assessing merchant ship initial build cost in the early design stages and investigating how changes in dimensions, weights, powering and carrying capacity might affect this cost. UCL (2010a; 2010b) describes a parametric method (largely based on the work done by Dirksen (1996)) for the estimation of WLC and all of its elements during the preliminary ship design stage, mainly applicable to naval ships. For UPC estimation, cost data are generally calculated through cost/tonne relationships for both material and labour (UCL 2010b). This costing 
model has been used during various projects, including by Bradbeer and Andrews (2012a).

Presentation of costing data is also a vital area of LCCing, in order to realise the full advantages presented by chosen methods since they have significant impact on decision making and selection of alternative designs. Two common graphical presentations are reproduced in Figure 2.15.

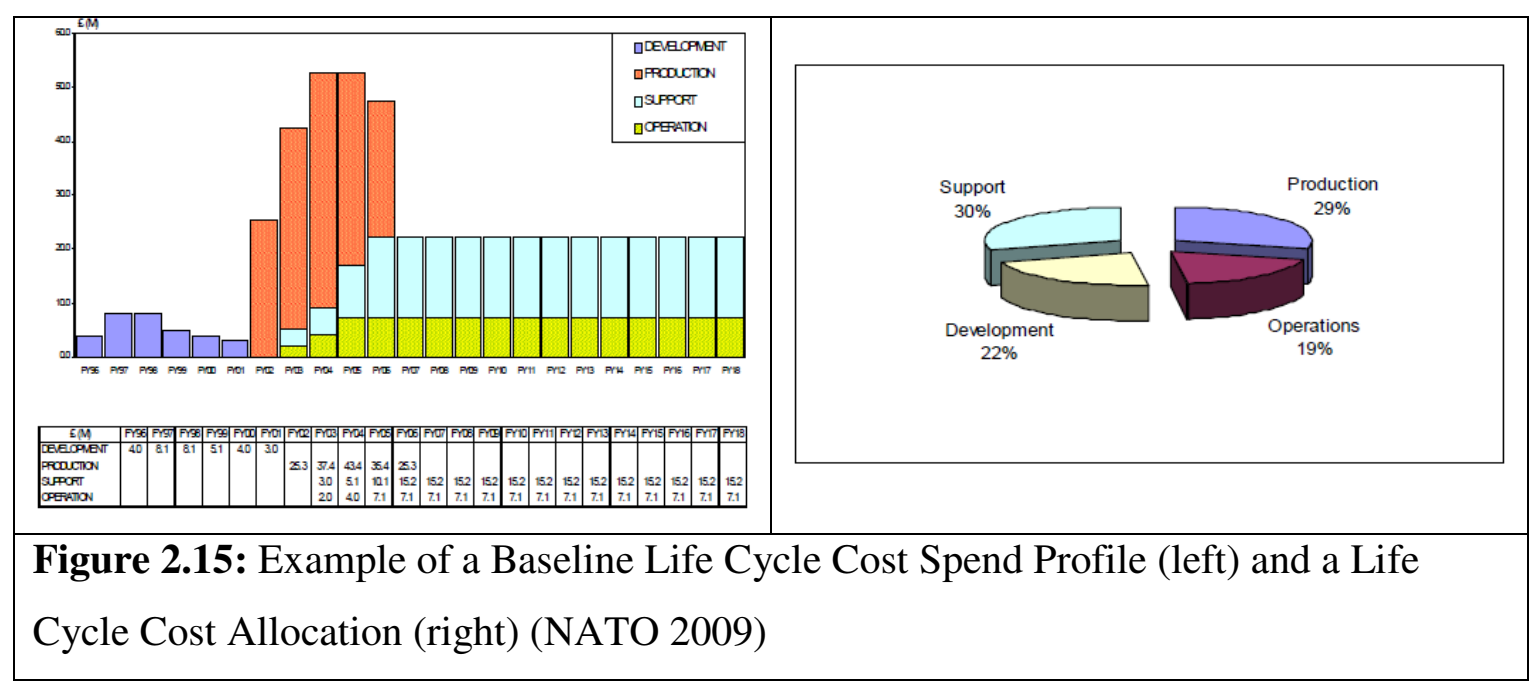

A similar spend profile was used by Rawson (1973), reproduced in Figure 2.12.

The relationship between the level of assumptions required for the cost estimation of a project and the project life cycle stage is illustrated in Figure 2.16.

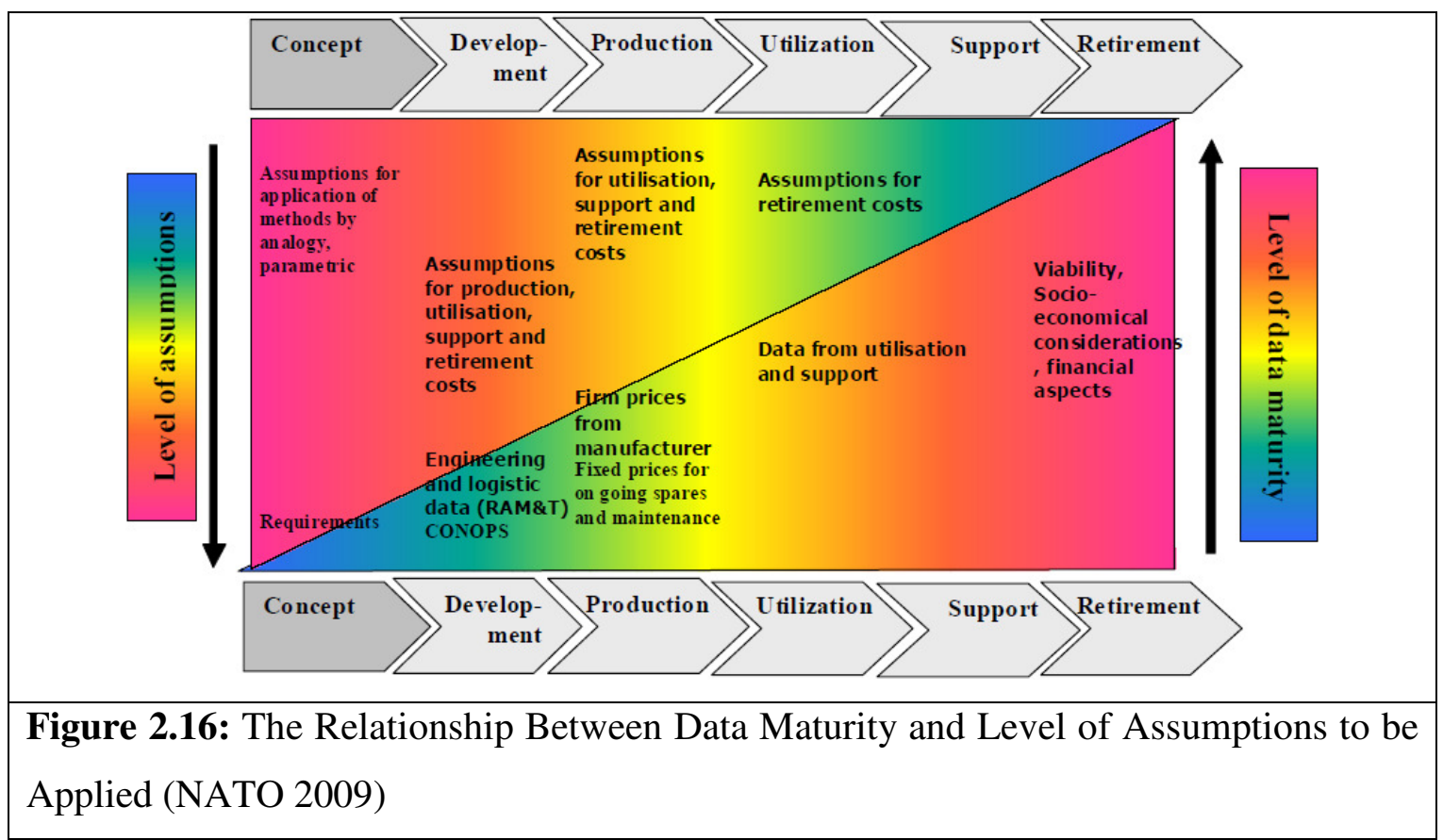


It is evident that numerous assumptions have to be made when conducting costing analysis (often by means of parametric techniques) during the early design stages, given limited design definition. This then leads to the conclusion that LCCing is far from being an exact science (NATO 2009). Necessary assumptions include:-

- FOC costs as a proportion of UPC (Dirksen 1996; UCL 2010a);

- $\quad$ Shipyard selection (Dirksen 1996);

- Material and labour costs (UCL 2010b; Gerdemann et al 2012) (noting that shipbuilding remains labour intensive compared to most other heavy engineering (Usher and Dorey 1982));

- Shipyard purchasing overhead factors (Dirksen 1996; Gerdemann et al 2012);

- Learning curves (UCL 2010b; Collins and Ward 2012) (however two warships of same class are rarely identical leading to difficulties in establishing appropriate learning curves (Usher and Dorey 1982));

- Degree of modularity (UCL 2010b; Gougoulidis 2012);

- Extent of external sub-contractor involvement (Dirksen 1996; UCL 2010b);

- When estimating UPC, factors such as the variance of wages are usually not considered, leading to the assumption that the ship is constructed in a day (Carreyette 1977);

- Inflation and discounting factors (making future cost predictions questionable) (Dirksen 1996; UCL 2010b);

- Fuel inflation (which could affect support, reliability, availability and maintainability of future vessels) (UCL 2010b; Collins and Ward 2012);

- Frequency of refits (especially extent of update and effect of obsolescence) (UCL 2010b);

- Cost margins for design and industrial uncertainty (Dirksen 1996; UCL 2010a). When this extensive list of assumptions is coupled with the difficulties associated with obtaining (often limited) appropriate historical cost data (Dirksen 1996; Rudius 2012) and the reluctance of parties to publish data that they perceive as confidential (Carreyette 1977) in order to derive the parametric relationships, ship costing during the preliminary design stage presents a major challenge. Especially since the quality of the cost estimates depends on the quality of this data (Gerdemann et al 2012). In addition, many variables (such as labour and material costs) are influenced by factors out of the shipbuilders (and ship operators) control, such as inflation, interest rates and relative currency strengths (Usher and Dorey 1982). All of the above factors generate large 
uncertainties in cost estimates. These uncertainties should be quantified in order to assess project cost risk (Rudius 2012).

Despite these problems, ship costing during the early design phases has always been essential, the more so given the increased defence inflation without a commensurate increase in defence budgets. Such costing techniques would enable the investigation of factors such as quality vs. quantity, personnel reduction, innovative design, adoption of commercial standards and life extension programmes (Dirksen 1996).

\subsection{Conclusions on Ship Design Issues}

The recent developments in warship costs accompanied by the declining defence budgets can be countered through innovation in the ship design process and in individual ship's design evolutions. Innovations are best explored in the early design stages, where the resources expended are relatively minimal, but most major decisions are taken. Recent progress in computer technology can be utilised to explore such innovations. Since most modern warships are architecturally constrained, practitioners have used computers to develop architecturally orientated preliminary design methods (e.g.: the UCL DBB approach). This has aided early stage decision making, increased designer confidence, eased the identification of design drivers and risks and provided the ability to compare alternative designs regarding their operational performance. All these can be investigated while ship configuration is still amenable to change and leads to increasing the detailing of early stage ship designs.

Cost cutting is mainly aimed towards aspects which are difficult to quantify, such as survivability. This could lead to unfeasible designs given the increased complexity of modern naval operations, mainly focused in littoral waters, where a wide range of threats are possible. A number of survivability assessment tools currently exist and are outlined in the next chapter. 


\section{Chapter 3: Background to Survivability}

This chapter, consisting of four main sections, provides the survivability related background and context for the research. The first two sections of this chapter outline survivability by giving detailed definitions, highlighting the importance of survivability in naval ship design and operations and summarising current threats faced by navies. The third section, comprising the bulk of this chapter, identifies and details current survivability assessment techniques and their applicability in preliminary naval ship design. The final section provides the main conclusions of the research review related to survivability. The gaps in current survivability assessment techniques are identified, as is the importance of combining such methods with architecturally orientated design approaches in the preliminary ship design process. Thus an indication as to the way forward is given.

\subsection{Introduction to Survivability}

NATO has adopted the following survivability definitions (NATO 2003a):-

- "Survivability is the capability of a weapon system to continue to carry out its designated mission(s) in a combat environment. Survivability is a function of both susceptibility and vulnerability.

- Susceptibility is the combination of factors that determine the probability of hit by a given threat (such factors include the susceptibility to detection, classification, targeting, attack, being hit, ...).

- Vulnerability is the extent of degradation of a system after having been subjected to combat threat(s), that is, the degree of mission impairment as a result of sustaining finite levels of damage caused by weapon hits."

The above is summarised in Figure 3.1. 


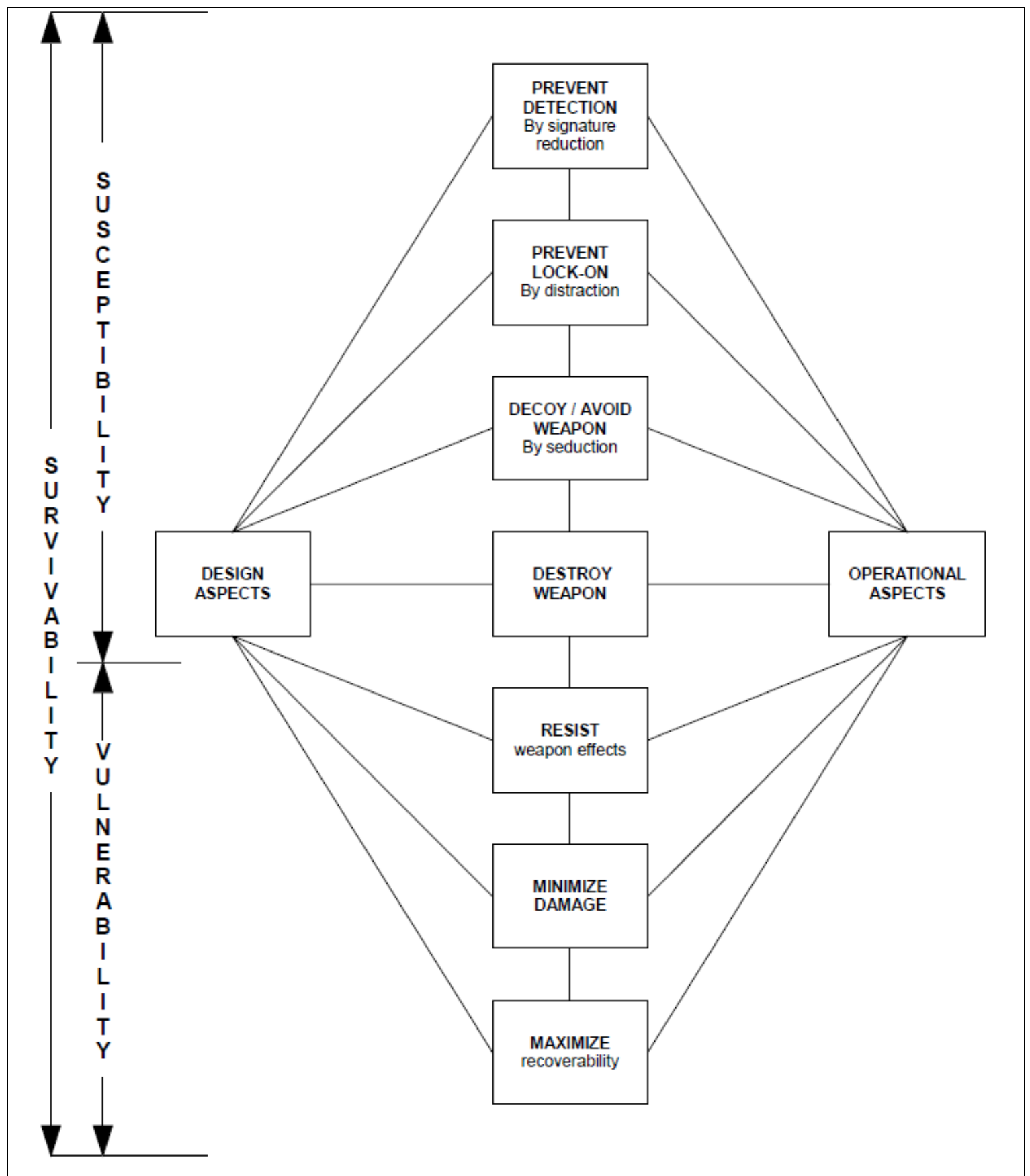

Figure 3.1: Survivability is a Subject, Which Depends on the Interrelation of Many Subjects. Not Only Design but Also Operational Effects Can play an Important Role in Ship Survivability (NATO 2003a)

However, the above definitions refer to a weapon system rather than a ship and fail to consider recoverability separately from vulnerability (as is often the case with many authors). For this reason, an extensive list of definitions (including illustrations) of survivability and its constituents, from several authors, is given in Appendix 3.

It is a commonly accepted fact that the main operational difference between commercial and naval ships is that the latter will be deliberately placed in harm's way and should therefore be able to survive much harsher conditions than those normally 
imposed by the marine environment. In order to survive such conditions, the inherent survivability due to a ship's construction needs to be complemented by additional survivability features (Belcher 2008). These requirements (such as signature management and structural design) constitute the main distinction between commercial and naval ship design. Such requirements not only increase the complexity of naval ships (Hudson et al 1996) but are also difficult to quantify (Cooper et al 2007).

Since the collection and analysis of wartime data is one of the most important methods of studying ship behaviour due to conflict, there are a number of published documents which present such data and statistics, mainly from WWII, but also from the Falklands and Persian Gulf conflicts for both naval (Korotkin 1960; Brown and Brown 1986; Brown 1997; Barton 2009; Manley 2012) and commercial ships (Ling 1985). There have been a number of recent incidents (e.g.: USS Belknap - 1975, near fatal damage in collision with CV-67; Falklands Conflict - 1982 where six RN ships sunk and twelve damaged; USS Stark - 1986, struck by two Exocets in the Persian Gulf) which have raised questions about modern combatant survivability (Carter 1988; Manley 2012). This perceived vulnerability of modern warships can be attributed to a number of causes. For example, the lack of armour of modern combatants (when compared to their WWII equivalents) and the increase in complicated and sensitive sensors and electronic equipment (shift from enhanced armour to sensor capability), the increasing diversity of threats faced by such ships, the reduced manning levels, (Papanikolaou and Boulougouris 1998), the declining defence budgets leading to smaller fleets (Robb et al 2010) and the adoption of commercial standards and equipment in warships (Martin 1998; Rattenbury 2004). The above have led Papanikolaou and Boulougouris (1998) to stress the need for a new naval ship design philosophy aiming at enhanced survivability.

The need for a new design philosophy is further justified by the fact that the successful incorporation of survivability design features is one of the prime aims of the designer (Brown and Tupper 1989) and influences the combat system effectiveness of the overall warship to a great extent (Sajdak and Karni 2006). The recognised importance of survivability in recent years has led to the inclusion of the subject in ship rules (such as the Rules for Classification and Construction of Germanischer Lloyd (Petersen 2006)) and the publication of several documents together with the implementation of survivability management strategies by major navies, in order to guide designers (Said 1995; Reese et al 1998; Robb et al 2010). However, such 
documents usually invoke feature-based rather than performance based specifications for survivability.

The absence of performance based specifications and the difficulties in quantifying overall survivability means that survivability features are harder to justify, therefore presenting a seemingly attractive area for cost cutting (Brown 1986b; Reese et al 1998). Thornton et al (2007) have also criticised the way in which survivability is costed and incorporated. They suggests that in many cases (e.g.: shock protection) it is the existence of a laid down level of survivability and not the level of protection required that has then caused the increase in cost. Aspects in the ship capability, such as defensive weapons, electronic warfare, TLC and manning are usually not taken into account in the cost of survivability (Thornton et al 2007). Martin (2007) argues that many survivability features serve more than one role, and therefore their contribution to assessing their impact on the cost of survivability is difficult. Also, survivability features such as reducing radar susceptible microgeometry by placing equipment behind bulwarks or below decks leads to cost benefits by better protecting the equipment and easier maintenance (Friedman 1991; Thornton et al 2007). Knight (2012a; 2012b) remarked that "of all the drivers in the design of warships, it is survivability which is often quoted as having the greatest impact upon the cost"; however, he also concluded that "simple survivability measures can be implemented cost effectively" and that omission of certain such measures "may have no effect on cost or may indeed have the opposite effect". In addition, the UK National Warship Survivability Committee (2008) concluded that since there is not a good understanding regarding the cost of survivability, these costs have traditionally been (possibly wrongly after experience from the Type 45 Destroyer procurement process) assumed to be unrealistically high. Another limitation of the current procurement process is that procuring warships on the basis of functional requirements and therefore, seemingly to pass risk to industry, which may not have a good understanding of survivability, will probably lead to increased UPC (since industry deals with risk by applying contingency costs) and to degrade capability (Thornton et al 2007; National Warship Survivability Committee 2008). The National Warship Survivability Committee (2008) recommended that the MOD should "retain the risk associated with survivability requirements" and that the MOD and the warship prime contractor should attempt to better understand survivability related costs and explore alternative survivability procurement processes.

It is increasingly acknowledged that the required survivability level should be specified during the development of the operational requirements (Phillips 1998) with 
survivability analysis commencing at the early design stages (Randles 2009). However, rather than the traditional feature based specification, the requirements should be set in terms of specific threats and outcomes defined by the customer, given validated analysis tools (Reese et al 1998; Doerry 2007). This would make survivability an integral part of the design process (Ball and Calvano 1994) and therefore assist in achieving a balance between the three survivability components and other design features (Brown 1990; Phillips 1998). Also, by setting operationally orientated requirements, the operator could be better informed about the capabilities of the ship (Reese et al 1998).

Balancing ship features across the three survivability components is seen to be of vital importance (Turner et al 2006; Harney 2010). After all, it is extremely impractical to design an insusceptible or invulnerable ship (MOD 2001). Logically, it could be argued that such a balance should not only be sought in a single ship design, but also across the fleet (MOD 2001; Harney 2010). It is argued that by considering the three together a more cost effective solution will be obtained (Sajdak and Karni 2006; Thornton et al 2006). In order to exploit these perceived advantages, a new separate design discipline, Total Ship Survivability, was developed in the early 1990s by the USN (Said 1995).

Robb et al (2010) investigated the cost of various survivability features for a 5,000 te surface combatant. As is the case with most design features, survivability features are much cheaper if considered and implemented at the earliest design stages; if left for the later stages they are not only more expensive, but sometimes impossible to add on (Brown 1986b; Robb et al 2010). Early consideration also allows a more effective integration of such elements with other ship systems (Belcher 2008; Said 1995). In addition, survivability has an impact on initial sizing (Andrews 1987) and layout determination (Brown 1993; Andrews et al 2004), therefore further justifying the need to consider it at early stages.

Various commonly accepted survivability enhancement design rules, relevant to the early ship design stages, have been established from past experience and from survivability analyses. These include:

- Reducing microgeometry, shaping the hull, superstructure and certain equipment, and avoiding certain shapes for RCS reduction;

- Cooling the exhaust by mixing it with cooler ambient air and hiding or masking hot metal parts for IR signature reduction; 
- Mounting engines high up in the ship, avoiding underwater exhausts and eliminating gearboxes (through the adoption of IFEP) for noise signature reduction;

- Including degaussing systems (and non-magnetic materials) for magnetic signature reduction;

- Incorporating layered defence systems including soft-kill and hard-kill;

- Hardening through the inclusion of shock resistant mounts (which also reduce the noise signature);

- Adding armour to selected systems and critical compartments;

- Locating critical equipment and compartments low in the ship to protect from abovewater weapons;

- Protecting critical spaces by placing them inboard, shielded by less critical spaces or side passageways;

- Employing concentration, separation and duplication of systems;

- Increasing the number of WT blast, fire and smoke resistant bulkheads and doors for damage containment as well as improved damage stability;

- Separating the ship into semiautonomous zones;

- Including sufficient damage detection and suppression equipment and systems (e.g.: NBCD stores and firepumps);

- Adopting a suitable access philosophy for improved crew evolutions;

- Providing with adequate spare parts to repair damage.

The above survivability reduction features, in addition to other such features more relevant to the later design stages, are described in greater detail in Appendix 4. Moreover, the above characteristics were largely incorporated in the ship design studies carried out during this research, reported in Chapter 5.

\section{$\underline{3.2 \text { Threats }}$}

The threat faced by warships has traditionally been considered to continually increase in magnitude, variety and complexity (Brown and Tupper 1989). The main reason for this is the rapid improvements in weapon technology (Barnett 1998). Warships are threatened by air, surface and underwater weapons with multiple sophisticated guidance methods (Papanikolaou and Boulougouris 1998). This is enhanced by the recent shift of maritime operations from blue water to green water (Surko 1994; Harney 2010). 
While operating in blue waters, submarines (torpedoes and missiles), aircrafts (bombs and missiles) and surface ships (missiles and guns) constituted the main threats, operating in the littoral, warships may encounter all of the above as well as coastal guns, mines, fighter aircraft, attack helicopters and small patrol boats (Barnett 1998). In addition, operations closer to the shoreline decrease reaction times (Barnett 1998; Harney 2010), which (when coupled with the degraded performance of ship sensors in such environments (Braid et al 2009)) may lead to unsophisticated weapons prevailing against more sophisticated and expensive ones, therefore increasing susceptibility and vulnerability (Barnett 1998; Phillips 1998). The trend towards littoral operations has led to the development of a new type of warship, the LCS, with high speed and adaptability as its main characteristics, to exploit rapid reaction and reduce exposure to threats (Andrews and Pawling 2006a).

Asymmetric threats are also present close to the shorelines; such threats are characterised by their short range (where stealth does not play an important role) and short duration (where weapon system recoverability is of secondary importance) (Belcher 2008).

However, naval ships are not only threatened by enemy and terrorist actions but also accidental threats such as fire, collision and grounding (Brown 1986b). During the late 80s, approximately ten large and medium fires occurred every year in RN ships, half of which started in machinery rooms therefore leading to significant damage to expensive equipment. Other potential accidents include collisions and groundings which could lead to sinking (Brown 1986b).

Threat types are generally categorised in two groups, abovewater (e.g.: missiles, bombs, shells and bullets) and underwater (e.g.: torpedoes, mines and depth charges) (Begg et al 1990). It is also useful to categorise each threat in terms of their effects, e.g.: flooding, fire, magazine explosion, structural failure, shock, impact, blast (Brown 1986b). These are discussed in greater detail in Appendix 4.2.

Modern combatants face a variety of threats, ranging from massive missile attacks to terrorist attacks with unsophisticated weapons (Dicker 1986) leading to a layered defence strategy (Brown 1997). It is clear that such ships should be designed against a number of threat scenarios, where adversary, weapon type, delivery method and weapon effects should be clearly identified (Belcher 2008). Figure 3.2 summarises some of the major threats and weapon effects encountered by naval ships. 


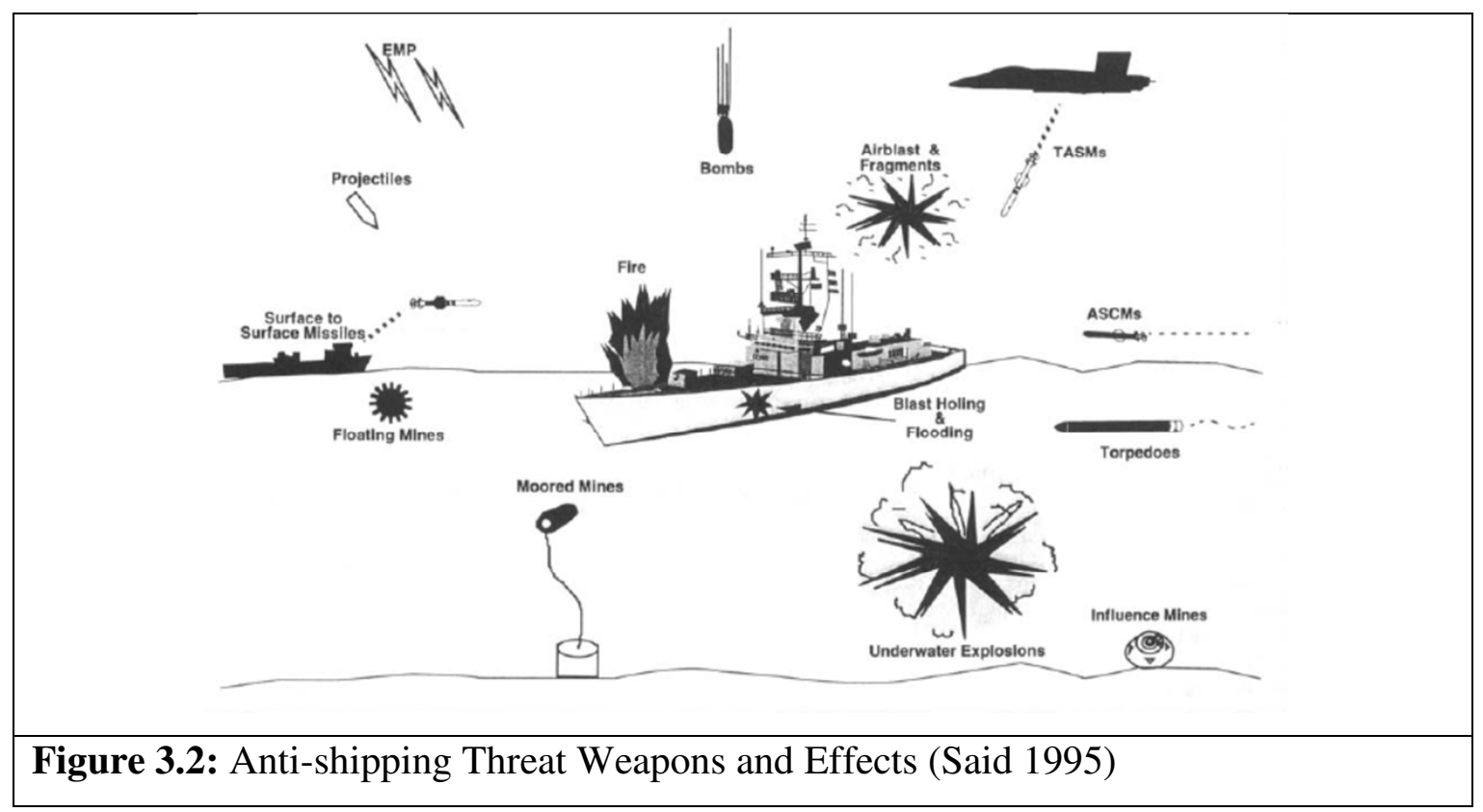

Appendix 4 is a summary of the literature regarding each of the three survivability constituents and how they affect ship design and current naval operations.

\section{$\underline{3.3 \text { Survivability Assessment }}$}

\subsubsection{Introduction}

"Survivability assessment is the systematic description, delineation, quantification, and statistical characterisation of the survivability of a ship" (NATO 2003a). In the past, the evaluation of most ship characteristics including survivability was difficult (Garzke and Kerr 1985). In recent decades (following on from the Falklands conflict), considerable progress has been made regarding survivability assessment methods; techniques such as simulation and virtual demonstrators are seen to potentially have a large impact in future ship design (Papanikolaou and Boulougouris 1998). Various current survivability assessment methods can be used to audit a design, suggest improvements, improve communication between designers, builders and operators and justify survivability enhancement measures (Heywood and Lear 2006; Robb et al 2010). Turner et al (2006) and Randles (2009) suggest that survivability assessment should not merely be an audit function, but should be carried out from the outset of the design process. By integrating such techniques in the design process survivability assessment becomes another input in this iterative process (MOD 2001; Holmberg and Kotiranta 2012) (see Figure 3.3). 


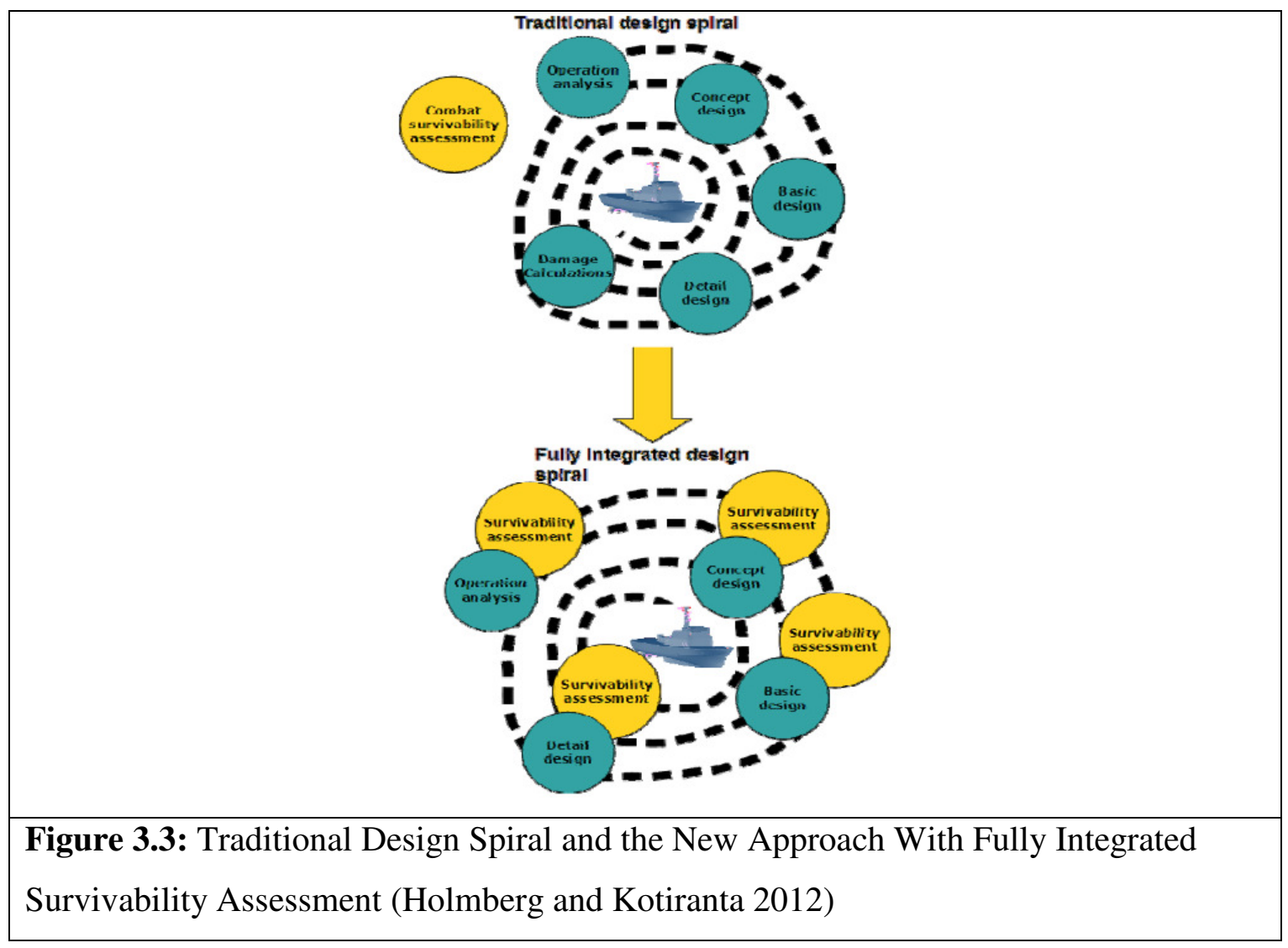

Effective use of survivability assessment methods would enable designers to find a balance between the contributing components of survivability, assess weight and cost implications (Brown and Tupper 1989) and assess the comparison of different, competing, ship designs (Brown 1987). It has been suggested that survivability goals for individual ships should be established and met, therefore, departing from traditional rule-based ship design (Martin 1998). This has been made possible due to recent computer developments and it was identified during the early 1990s that requirements concerning percentages of remaining capability following specific threats would soon be definable (Brown 1993). High level Survivability statements could be included in the URD and detailed requirements (e.g. vulnerability probabilities after a given incident), in the SRD (MOD 2001). Therefore, scenarios, required survivability levels and survivability level definitions would need to be clearly identified. However, including survivability in the requirements of a naval ship introduces further constraints (Boulougouris and Papanikolaou 2004) to what is already a very complicated process (Papanikolaou and Boulougouris 1998). To deal with this Papanikolaou and Boulougouris $(1998 ; 2004)$ regard the existence of methodologies quantifying the survivability of a ship early in the design process essential. Robb et al (2010), in Figures 10 and 11 of their publication, show the difficulty and indicate associated likely costs of implementing survivability measures throughout a ship's lifecycle. 
Confirmation that a ship has met its (measurable) survivability requirements could include (MOD 2001):

- "Analysis of the design (e.g.: using quantitative methodologies);

- Material state assessment (e.g.: survey of completed vessel);

- Trials (e.g.: FOC shock trials, signature measurements)."

However, none is that definitive for what is a complex aspect of a complex system of systems.

\subsubsection{Susceptibility Assessment}

A number of susceptibility assessment methods currently exist in order to simulate the large variety of threats faced by modern naval ships. In addition, they can be used throughout the ship design process given that susceptibility enhancement techniques are largely independent of the level of design detail (and can be added even after construction through, for example, RAM, infrared signature suppression devices and low emission paints (Boulougouris and Papanikolaou 2012)); therefore, such tools specifically aimed at preliminary design are unnecessary. However, most susceptibility assessment tools rely on classified information (such as weapon performance data) and therefore, such tools (and their mechanisms) are not readily available to the public. A limited amount of information on current tools is presented below.

Numerical models and computer programmes are used to estimate complex shape RCS (Foxwell 1990a). MOD signature estimation software include SPECTRE (for RCS prediction), ODIN and TMSS (for torpedo and mine activation respectively), SIREX (for IR signature prediction) and HVME (used to monitor platforms self-noise and take reduction measures) (Turner et al 2006; Martin 2007). Similar software can be used in order to produce lengthwise hit probability distribution, since hit location depends on the ship characteristics (signatures) and threat characteristics (homing method) (Boulougouris and Papanikolaou 2004). Turner et al (2006) accounted for the combination of SPECTRE with SURVIVE (a vulnerability assessment tool which will be described further on) to give likely ASM hit locations, and presented a case study involving a Leander class frigate. Similarly, SIREX can be employed when considering threats with IR homing devices (Martin 2007). However, factors such as environmental conditions, ship motions and manoeuvring and variations in weapon performance even out the hit probability distribution, making such methods questionable in general vulnerability analysis (MOD 2001). An alternative would be to assume a basic 
mathematical distribution, such as a linear or normal distribution, thereby connecting susceptibility to vulnerability (Boulougouris and Papanikolaou 2012) and recoverability. Furthermore, Liwang et al (2012) presented a probabilistic risk assessment method combined with numerical simulation for an example transit of a naval ship through a minefield. Simulated parameters with corresponding statistical data included environmental factors, threat, ship and sensor characteristics and organisation and decision making (Liwang et al 2012).

Defensive systems (hard-kill) effectiveness can be estimated through the CSEE which is utilised by UCL as part of its MSc in Naval Architecture course (McDonald 2010). The CSEE is able to calculate probabilities of layered defence success against missile attack scenarios; however it is very simplified and relies on various assumptions. Budgets, scenarios and unclassified weapons data (such as range, maximum burst time for CIWS, firing rates, reaction times, kill assessment times, velocities and single shot kill probabilities) are given in order to determine reaction times and success probabilities (McDonald 2010). Furthermore, MOD uses the ThreeDim code for hard-kill performance which relies on data from signature models, sensor models and weapon system performance models (Thornton et al 2006). Since the 1980s a Monte-Carlo simulation package for the engagement of ASMs by defensive missile systems had been developed for MOD use (Adams 1988).

Barnett (1998) attempted to assess susceptibility through an operational risk management method. Different scenarios and hazards (weapons) were identified and applied to various ships at different time periods. The hazards were assessed in terms of likelihood and consequence and placed in a matrix to convert them to risks (see Figure $3.4)$.

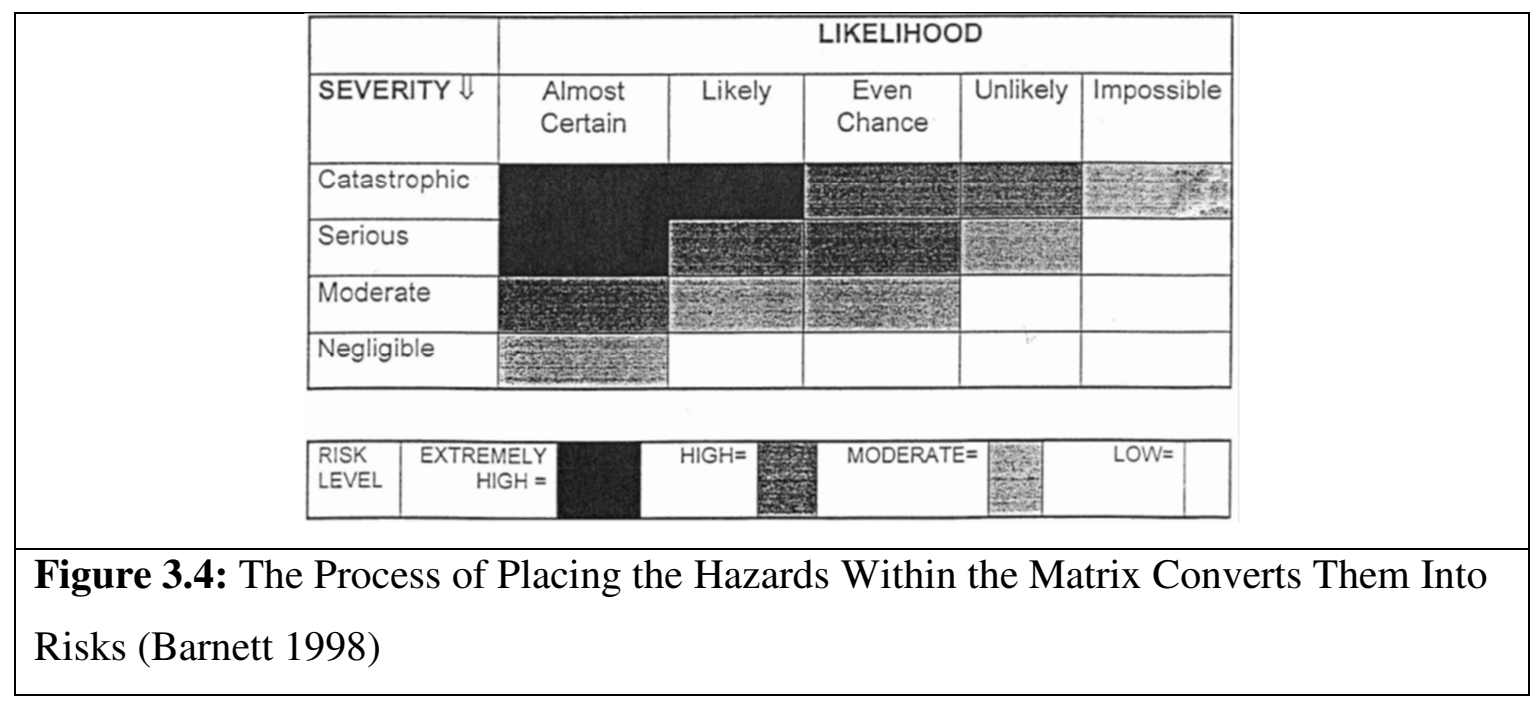


However, the drawback of this method compared to the ones detailed above is its purely qualitative nature.

\subsubsection{Vulnerability Assessment}

Extensive development of vulnerability assessment methods has been carried out during the last decades in order to assess designs (Begg et al 1990) against different weapons and appraise probabilities of preserving capability (Brown and Tupper 1989). Nowadays, multiple vulnerability assessment models have been developed; Figure 3.5 lists various software used by a range of navies.

\begin{tabular}{|l|l|l|}
\hline \multirow{4}{*}{} & Navy & Model \\
\cline { 2 - 3 } & Australia & \\
\cline { 2 - 3 } & Canada & XVAM, CVAM \\
\cline { 2 - 3 } & France & GVAM, SLAMS \\
\cline { 2 - 3 } & Germany & MINERVE \\
\cline { 2 - 3 } & Italy & REMOS \\
\cline { 2 - 3 } & Netherlands & SAVIUS \\
\cline { 2 - 3 } & Norway & PROGRESS, RESIST, TARVAC \\
\cline { 2 - 3 } & United Kingdom & SURVIVE, PREVENT \\
\cline { 2 - 3 } & United States & ASAP \\
\cline { 2 - 3 }
\end{tabular}

Figure 3.5: Vulnerability Assessment Models by Navy (Ashe et al 2006)

During the 1990s, the main vulnerability assessment tools employed by the MOD were the REVS code for abovewater threats and the SSVUL code for underwater attack (Tozer 1993; Turner et al 2006). REVS included algorithms to model primary weapon effects (blast, fragmentation, kinetic) caused by missiles and shells. The ship was described by a geometrical model including system and equipment distribution; the ship's systems were modelled by dependency tree diagrams; and the weapons were described by means of warhead mass, velocity, direction, fusing and fragment detail (Tozer 1993). This system definition method allowed the breakdown of top level functions (Float, Move and Fight) into more detailed systems such as full power, minimum power, AAW and ASW (Schofield 2006). Hit grids were then superimposed on the geometrical model, compartments affected, and by what level, were established, and through system fault trees, vulnerability probabilities were obtained. Typically 200300 compartments with 600-800 equipment items were modelled per ship (Tozer 1993). Figure 3.6 shows examples of Tozer's (1993) output. 


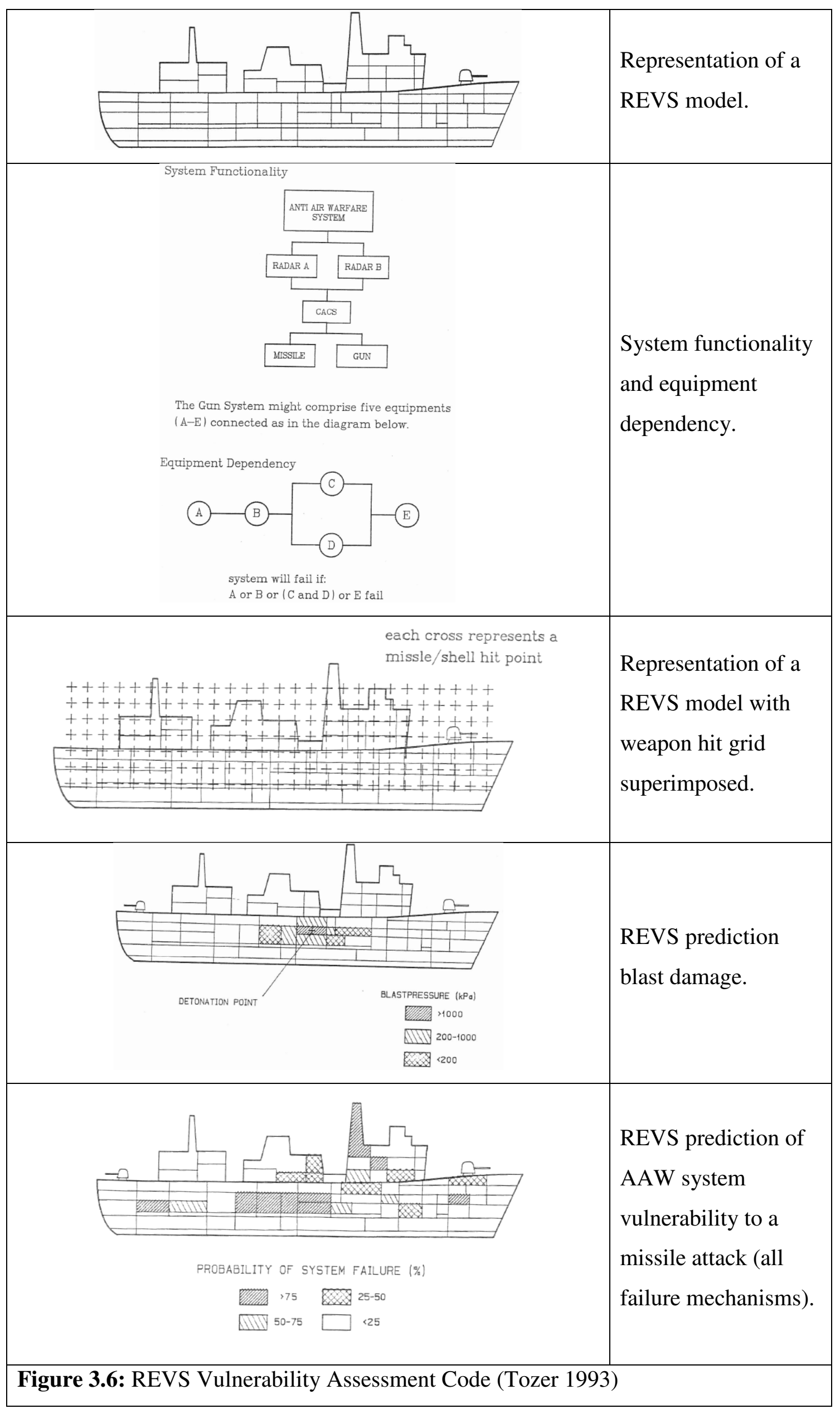


SSVUL operated under similar principles, but different weapon effect algorithms were used to model under water weapons, such as torpedoes and mines causing shock and whipping. Damage probabilities were then calculated through the Monte-Carlo technique (Tozer 1993).

During the late 1990s, REVS and SSVUL were combined into QinetiQ's SURVIVE software (Martin 1998; Turner et al 2006). SURVIVE is currently the principal vulnerability assessment code of the UK MOD simulating above and underwater weapons and their effects (such as kinetic energy, blast, fragmentation, shaped charge jets, shock, hull girder whipping) using a single ship model. It can simulate cumulative damage caused by multiple hits and can model novel hullforms, such as trimarans (Martin 1998). The ship's structural features, its systems and the weapon threats are defined in a similar manner to its predecessors. Probabilities of retaining a particular system/capability for a given weapon threat constitute the final output. The algorithms used to predict the various weapon effects have been validated through small and full scale tests (Schofield et al 2012). Typical SURVIVE equipment layout views are shown in Figure 3.7, Figure 3.8 and Figure 3.9.

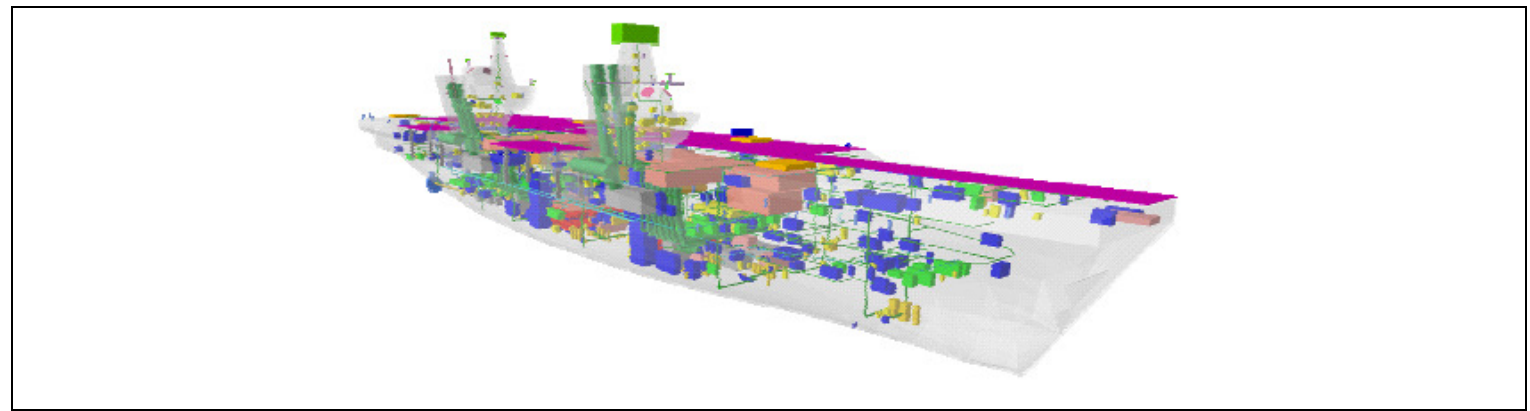

Figure 3.7: SURVIVE Equipment Layout (Turner et al 2006)

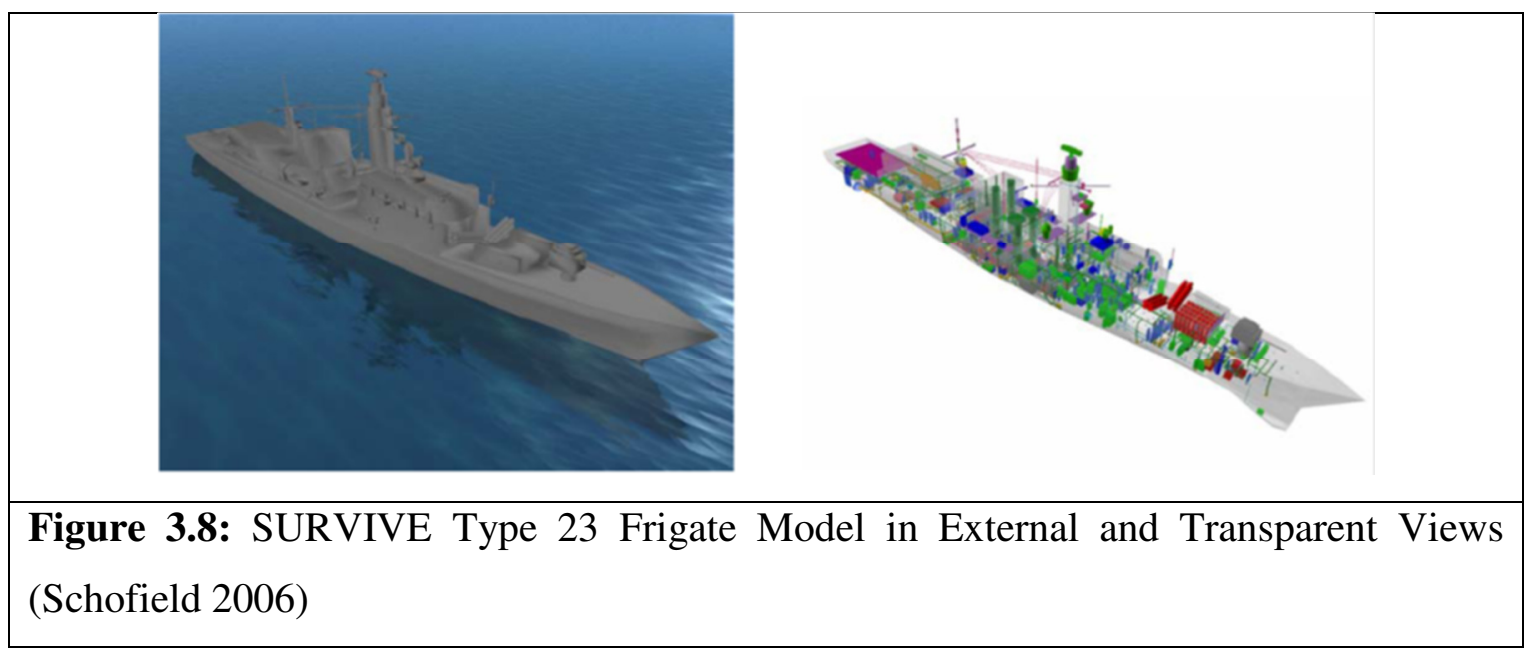




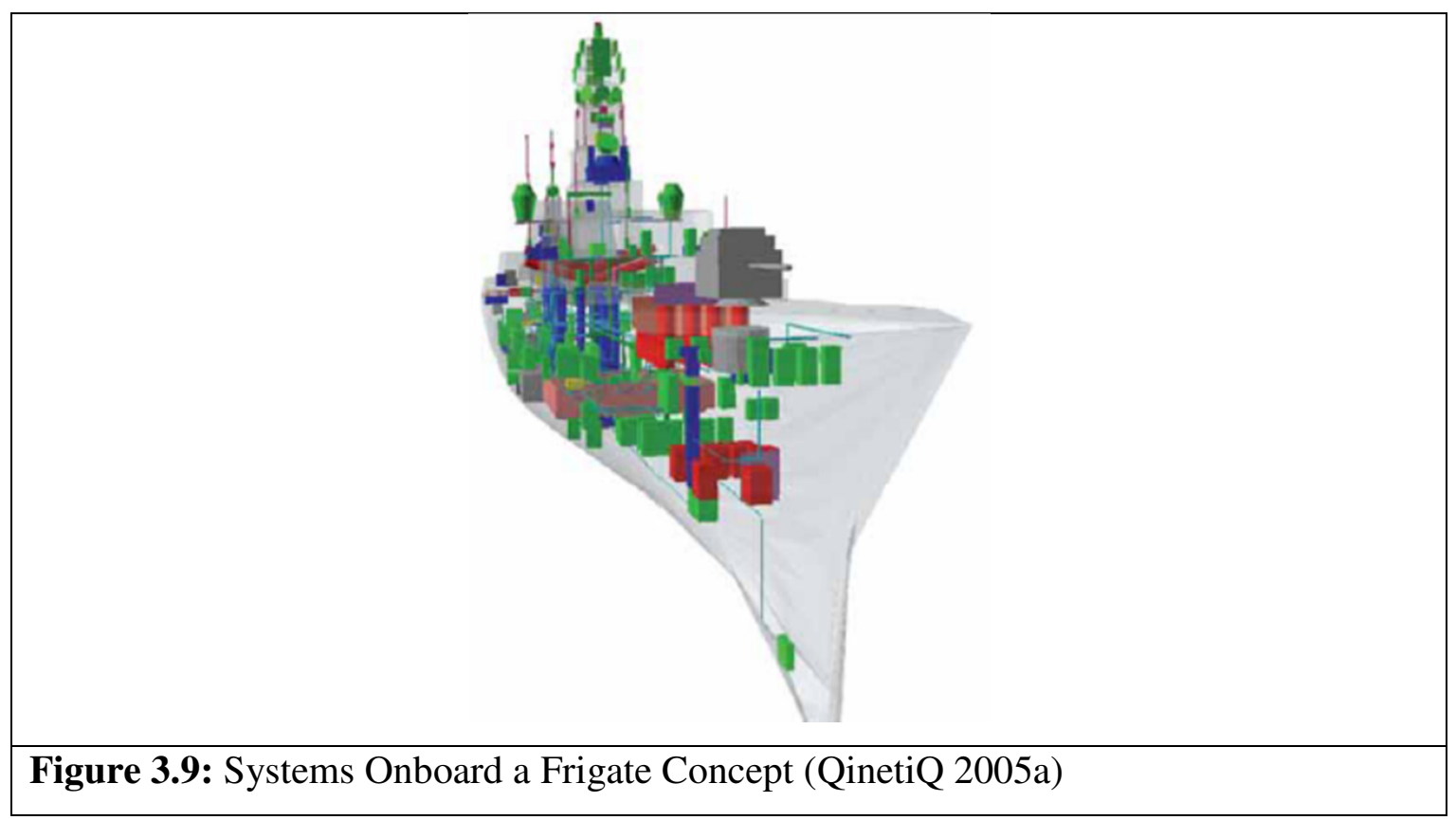

As previously discussed, vulnerability assessment has usually been carried out at the detailed design stages, as an audit function, and design corrections (such as altering bulkhead positions to improve blast response) were often too expensive to be implemented. Furthermore, the designer lacked confidence regarding the achievement of vulnerability reduction levels until the final design stages (Heywood and Lear 2006). Therefore, the need for software able to carry out such analysis at all design stages, including preliminary design, was identified (Pugh 2006).

The PREVENT software, developed by BMT Defence Services, is designed for use at concept stage, with limited ship definition (Heywood and Lear 2006; Jones and Kimber 2012). PREVENT models the effects of various abovewater and underwater weapons (such as blast, fragments and shock). The ship's structure is modelled and system components are located throughout the vessel; the ship is visualised in twodimensional slices and the systems are, again, defined by means of tree structures. Figure 3.10 shows the vulnerability of the propulsion power generation system, for a separated layout (the percentages represent the vulnerability of the system when hit at that cell). 


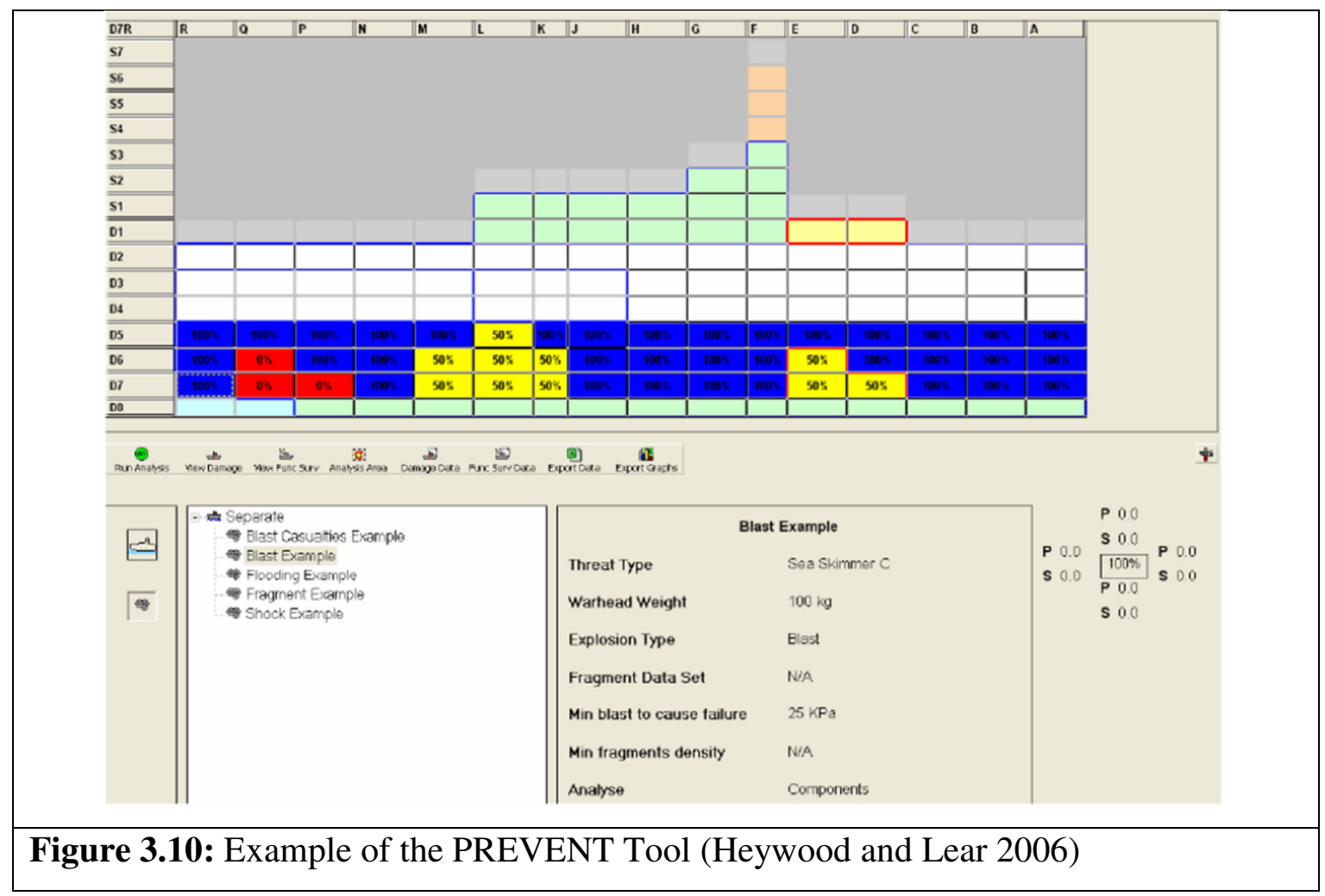

A version of SURVIVE, specifically aimed at early design stages, SURVIVE Lite, has also been developed (Pugh 2006; Turner et al 2006). Unlike PREVENT, SURVIVE Lite does not model the ship in two-dimensional slices, but in three dimensions, identically to SURVIVE. SURVIVE Lite inputs include the ships main structure and top level systems (e.g.: propulsion, steering and weapon systems), while lower level systems such as chilled water and high pressure air are neglected. Furthermore, weapon information does not have to be detailed; for example, generic fragmentation patterns are sufficient. Weapon hit locations are provided in the form of three-dimensional underwater or two-dimensional abovewater grid positions. The development of SURVIVE Lite, being fully compatible with SURVIVE, has allowed vulnerability to be managed throughout the design process (Pugh 2006). See Figure 3.11 and Figure 3.12 for a sample attack grid and SURVIVE Lite analysis output.

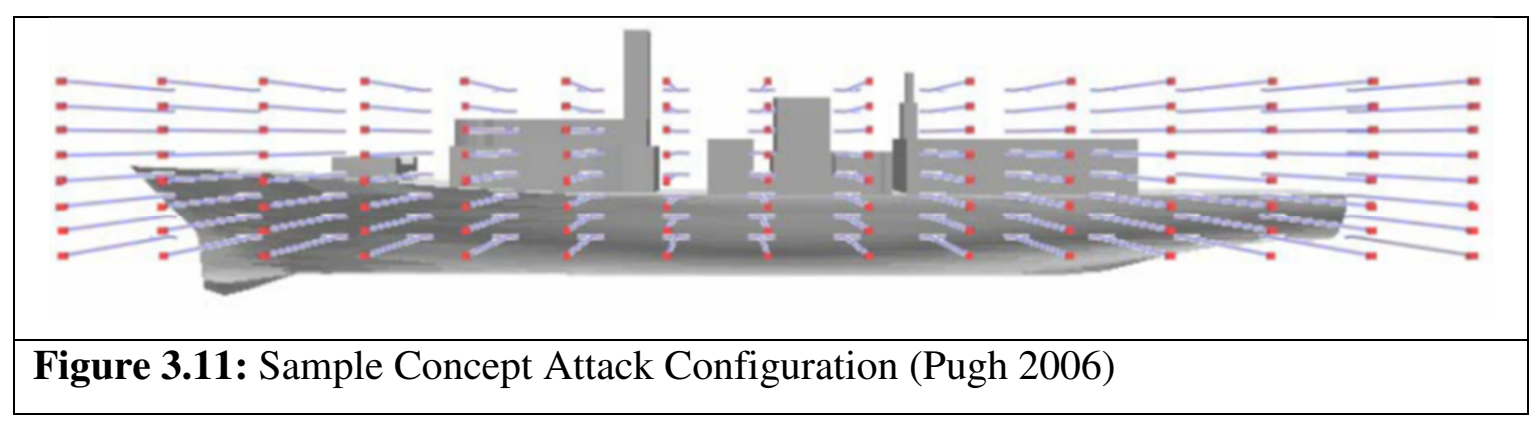




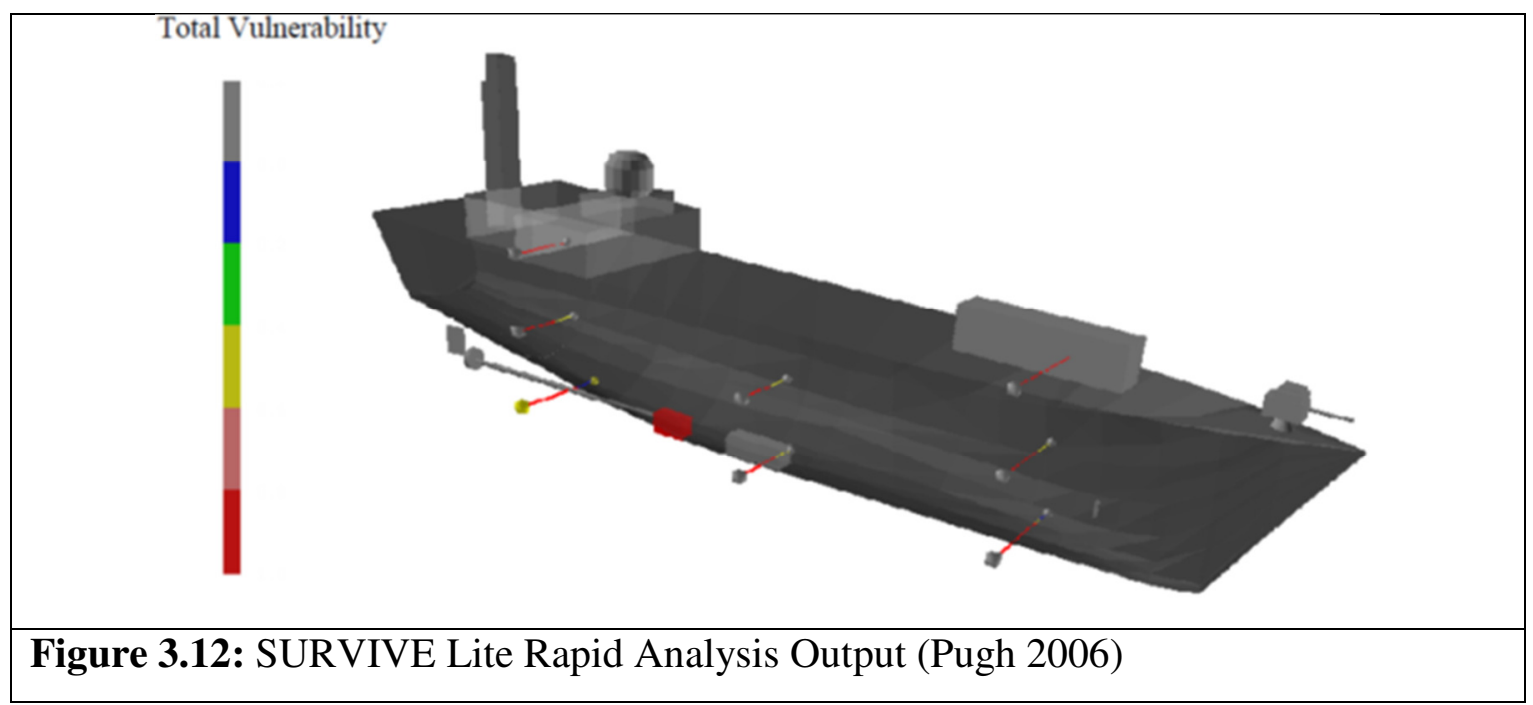

There have been efforts to link SURVIVE Lite to concept design tools such as SURFCON (Section 2.1.5) in order to avoid the need and lengthy procedure of remodelling the design; however, such links are still at an immature stage (Coote 2010).

Increasing levels of design detail also increase vulnerability estimation accuracy (Reese et al 1998) (increased detail leads to additional modes of failure). In addition, Coote (2010) reported the difficulties in simulating the zoning effects of concept level ship designs in vulnerability assessment tools aimed at such designs, due to the incomplete system models. Van Oers et al (2012) criticise the neglecting of detailed system architectures and the routing of connections in concept stage design and present a method which automatically produces alternative ship system routings, given the main component positions, which can be incorporated in vulnerability assessment software. The above problem can also be addressed by the utilisation of vulnerability margins (MOD 2001; Pugh 2006), saving computation time on the generation of connection routings, which will possibly be altered as the design evolves. Furthermore, it is easier and cheaper to implement vulnerability reduction measures on connectivity items rather than altering system layout later in the design process (Heywood and Lear 2006).

Further to the above tools, methods of estimating specific areas of naval ship vulnerability have been published. For example, Ren et al (2008) presented a method to estimate the residual strength of a damaged warship; Bradbeer and Andrews (2012a; $2012 b$ ) presented a comparative simulation study on the underwater shock response of different structural styles. However, the clear disadvantage of such methods is the fact that they focus on a very specific part of the problem, neglecting, for example, system vulnerability. 


\subsubsection{Recoverability Assessment}

Most assessment methods consider primary weapon effects (e.g.: equipment damage due to blast and shock), rather than secondary, time dependant effects such as fire and flood (Brown and Tupper 1989). Modelling and measuring recoverability performance is considerably more demanding, not only due to the dynamic nature, but also due to the critical reliance on human performance that is out of the designer's control (Brown and Tupper 1989; Thornton et al 2006). Boulougouris and Papanikolaou (2012) defined recoverability as "mainly an operational aspect relying mainly on the sufficient training of the crew although it may still pose several requirements to the designer". Knight (2012a; 2012b) described recoverability as a "critical balance between systems and features implemented by the designers, and damage control practices and procedure implemented by the ships staff". Databases on fire spread, flooding and crew actions are weak, limiting modelling capability, therefore, further research is seen to be required (Reese et al 1998). Methods such as the Delphi Method, which relies on expert advice, could be employed when sparse data is available (Harmathy 1982). Jones and Kimber's (2012) comment describing a concept design that "the level of design information produced did not support a recoverability assessment" thus implied that a greater level of design definition was required than that appropriate for preliminary designs. The MOD (2001) suggests that an interim solution would be to incorporate recoverability by modelling the vulnerability of the whole DC system, without, however, considering progressive damage. It is generally agreed that recoverability analysis is still at its infancy (Thornton et al 2006; Schofield et al 2012) in contrast to vulnerability analysis.

The majority of the literature concerning secondary effects focuses on the hazards of fires. Not only is it the most frequent hazard on ships (Azzi et al 2011), information can also be read across from buildings and infrastructure. A number of references concerning the spread of fire from compartments have been published, e.g.: (Thomas 1967; Walton and Thomas 1995), which present both statistical and physical models. Some literature on flooding has also been published, such as Jasionowski et al (2007) who propose a quantitative measure for ship vulnerability to flooding. However, fire and flood modelling techniques only assess a specific part of recoverability and therefore, cannot be considered as total recoverability tools. In addition, it is questionable if such tools (especially fire modelling techniques) are applicable to the preliminary ship design stages with a low level of design definition (where material 
types, locations of doors and portholes and detailed access arrangements are not yet detailed).

Safety and risk analysis techniques have been used to evaluate the characteristics of infrastructure, and could relatively straightforwardly be applied to naval ships. However, they would be limited in assisting the designer (possibly as a checklist) as to what safety features (e.g. fire mitigation systems, DC equipment) to include in the ship. Relying on expert judgment these techniques are subjective, qualitative and fail to evaluate recoverability as a whole. Furthermore, they fail to investigate how the layout of a ship would affect its recoverability. Some examples are given below.

Chow (2002) proposed a 15-point EB-FSRS for assessing the fire safety provisions in high-rise buildings. The proposed ranking system is reproduced in Figure 3.13 .

\begin{tabular}{|c|c|c|c|}
\hline \multirow[b]{2}{*}{ Area } & \multirow[b]{2}{*}{ Safety parameter } & \multicolumn{2}{|l|}{ FSRS } \\
\hline & & Criterion & Points \\
\hline \multirow{10}{*}{$\begin{array}{l}\text { Passive building } \\
\text { construction }\end{array}$} & Building height & $\cdot$ Height $\leqslant 50 \mathrm{~m}$ & 1 \\
\hline & & - Otherwise & 0 \\
\hline & Evacuation route & - Number of exits $>$ New BDs requirement & 1 \\
\hline & & - Number of exits < New BDs requirement & -1 \\
\hline & Width of staircase & - Width of staircase satisfying BDs requirement & 1 \\
\hline & & - Otherwise & -1 \\
\hline & Smoke door provided & - Yes & 1 \\
\hline & & $\cdot$ No & -1 \\
\hline & Provision of FRC for every construction element & - Satisfying BD's requirement (e.g. $1 \mathrm{~h}$ for domestic buildings) & 1 \\
\hline & & - Otherwise & -1 \\
\hline \multirow[t]{16}{*}{ FSI } & Fire hydrant hosereel system (must be provided) & - Provided in good condition & 0 \\
\hline & & - Otherwise & -1 \\
\hline & Fire alarm system (must be provided) & - Provided in good condition & 0 \\
\hline & & - Otherwise & -1 \\
\hline & Fire detection system & - Provided in good condition & 1 \\
\hline & & - Otherwise & 0 \\
\hline & Sprinkler system & - Provided in good condition & 1 \\
\hline & & - Otherwise & 0 \\
\hline & Fire extinguishers (must be provided) & - Provided in good condition & 0 \\
\hline & & - Otherwise & -1 \\
\hline & Smoke control system & - Provided in good condition & 1 \\
\hline & & - Otherwise & 0 \\
\hline & Emergency lighting & - Provided in good condition & 1 \\
\hline & & - Otherwise & -1 \\
\hline & Exit signs & - Provided in good condition & 1 \\
\hline & & - Otherwise & -1 \\
\hline \multirow{4}{*}{$\begin{array}{l}\text { Control of risk } \\
\text { parameters } \\
\text { appeared in local } \\
\text { fire codes }\end{array}$} & FLD: Movable items & $\cdot \mathrm{FLD} \leqslant 1135 \mathrm{MJm}^{-2}$ & 3 \\
\hline & & $\cdot \mathrm{FLD}>1135 \mathrm{MJm}^{-2}$ & -3 \\
\hline & Occupancy & $\begin{array}{l}\text { - Usable floor area per person satisfying MoE requirement (e. } \mathrm{g} \text {. } \\
9 \mathrm{~m}^{2} / \text { person for offices: } 3 \mathrm{~m}^{2} / \text { person for tenement houses) }\end{array}$ & 2 \\
\hline & & - Otherwise & -2 \\
\hline \multicolumn{4}{|c|}{ BD: Buildings Department } \\
\hline \multicolumn{4}{|c|}{ FRC: Fire Resistance Construction } \\
\hline \multicolumn{4}{|c|}{ FSI: $\quad$ Fire Services Installation } \\
\hline \multicolumn{4}{|c|}{ FLD: Fire Load Density } \\
\hline
\end{tabular}


The EB-FSRS was proposed to quantify how far the fire safety provisions in existing buildings deviate from recent fire codes (based on their score) and to design suitable fire safety management schemes for individual buildings (Chow 2002).

Harms-Ringdahl (2003) developed the method of Safety Function Analysis in order to model, evaluate and propose improvements to safety characteristics at industrial installations. Safety functions were defined as functions that can reduce the probability and/or consequences of accidents and other unwanted events in a system. The method consists of hazard identification, safety function analysis (based on expert judgment) and examination of results (Harms-Ringdahl 2003).

Developments in computer technology led to simulation software for fire spread, flooding, personnel flow and DC training, which are subject to the limitations mentioned above (i.e. they are specialised in a very specific part of recoverability and are not particularly suitable for designs with a low level definition of detail). A further limitation of simulations is the relatively lengthy computation times required for accurate results. Some current simulation techniques are summarised below.

A fire simulation tool example is the LUCIFER software, which was specifically developed for the French nuclear powered aircraft carrier (Le Garsmeur and d'Anselme 1998). In addition, Patil and Kar (2012) describe the use of the fire simulation software, Fire Dynamic Simulator, in order to examine the smoke effects on alternative escape routes in passenger ships. Moreover, Gillespie (2004) combined the same fire simulation tool with the SURFCON concept design tool (see Section 2.1.5) in an attempt to study smoke, heat and fire progression in a naval ship defined at a preliminary design stage level of detail. However, items such as doors, hatches, interior accommodation passageways and HVAC/cable routings, normally not modelled in the early ship design stages, had to be included.

An example of ship personnel flow simulation software is MaritimeEXODUS. A collaborative project was carried out between UCL and the University of Greenwich in an attempt to use the software, in conjunction with Paramarine, to assess HF performance of naval ship design. A number of references regarding the project have been published, such as (Andrews et al 2008; Casarosa 2011; Deere 2012). In order to evaluate a ship layout using the two above software, a number of evaluation scenarios and Performance Measures (PMs) were developed. PMs helped in simplifying and collecting the relevant information for HF performance assessment. PM scores were normalised to allow meaningful comparison, and weighted according to their importance. A combination of a particular evaluation scenario and a set of PMs resulted 
in a HPM, used to compare the human performance capabilities of competing vessels. Alternatively, a specific standard could be defined in order to evaluate a ship against that standard rather than comparing it to a competing design. Figure 3.14 lists various PMs.

\begin{tabular}{|c|c|}
\hline $\begin{array}{l}\text { Specific performance } \\
\text { measure }\end{array}$ & Description \\
\hline $\mathrm{C}_{1}$ & $\begin{array}{l}\text { Congestion criteria } \\
\text { The number of locations in which the population density exceeded } 4 \mathrm{p} / \mathrm{m}^{2} \text { for more than } 10 \% \text { of the overall } \\
\text { scenario time }\end{array}$ \\
\hline $\mathrm{C}_{2}$ & $\begin{array}{l}\text { The maximum time that the population density exceded the regulatory maximum of } 4 \mathrm{p} / \mathrm{m}^{2} \text { for } 10 \% \text { of the } \\
\text { simulation time }\end{array}$ \\
\hline$\cdots$ & $\begin{array}{l}\cdots \\
\text { Environmental criteria }\end{array}$ \\
\hline$E_{1}$ & The number of fatalities \\
\hline$\cdots$ & $\begin{array}{l}\cdots \\
\text { General criteria }\end{array}$ \\
\hline $\mathbf{G}_{1}$ & Average of the time required by each individual to complete all of their assigned tasks \\
\hline $\mathrm{G}_{2}$ & Average of the time spent in transition for each individual; i.e. moving from one location to another \\
\hline $\mathrm{G}_{3}$ & Time to reach final state \\
\hline $\mathrm{G}_{4}$ & Average time spent in congestion \\
\hline $\mathrm{G}_{5}$ & Average distance travelled \\
\hline$\cdots$ & $\begin{array}{l}\text { Procedural criteria } \\
\text { Prota }\end{array}$ \\
\hline $\mathbf{P}_{1}$ & The total number of operations completed by the FG \\
\hline $\mathbf{P}_{2}$ & The average number of operations completed per active member of FG \\
\hline $\mathbf{P}_{3}$ & The average time per task to complete the FGs assigned tasks' \\
\hline$\cdots$ & $\begin{array}{l}\text { Population criteria } \\
\text { Poplatis }\end{array}$ \\
\hline $\mathrm{U}_{1}$ & The FG population size \\
\hline $\mathrm{U}_{2}$ & Percentage of inactive population compared to the FG population size \\
\hline$\cdots$ & Geometric criteria \\
\hline $\mathbf{M}_{1}$ & The number of WTD used during the scenario \\
\hline $\mathrm{M}_{2}$ & The number of hatches used during the scenario \\
\hline $\mathrm{M}_{3}$ & The number of times the FG moved between decks \\
\hline $\mathrm{M}_{4}$ & Average number of components used by each crew member in FG during the ES \\
\hline $\mathrm{M}_{5}$ & The most number of times a WTD was operated \\
\hline$M_{6}$ & The most number of times a hatch was operated \\
\hline$\cdots$ & $\cdots$ \\
\hline
\end{tabular}

Figure 3.14: Example List of Performance Measures (Deere et al 2009)

Similarities between this method and fire ranking system methods exist; however, a major difference is that rather than relying on expert opinion, scenario simulation output results were used as PMs. However, weightings are likely to be based on expert opinion and therefore need to be sensitivity tested. The project was aimed at preliminary stage ship design, nevertheless, personnel movement studies require additional design detail, such as connectivity between spaces, which are not normally modelled at this stage (Andrews and Pawling 2009).

Azzi et al (2011) examines the integration of fire simulation (Fire Dynamic Simulator software) and evacuation modelling (EVI evacuation model) in order to assess passenger ship safety performance during fire scenarios. Vassalos (2012) also combined EVI with fire growth software. In addition, he describes a flooding survivability analysis case study of the cruise ship 'Project Genesis' using evacuation and dynamic flooding simulation software such as PROTEUS3. The ship model used 
(Figure 3.15) consisted of 717 compartments and 1,160 openings, clearly suggesting that the amount of detail required is not relevant in concept stage design.

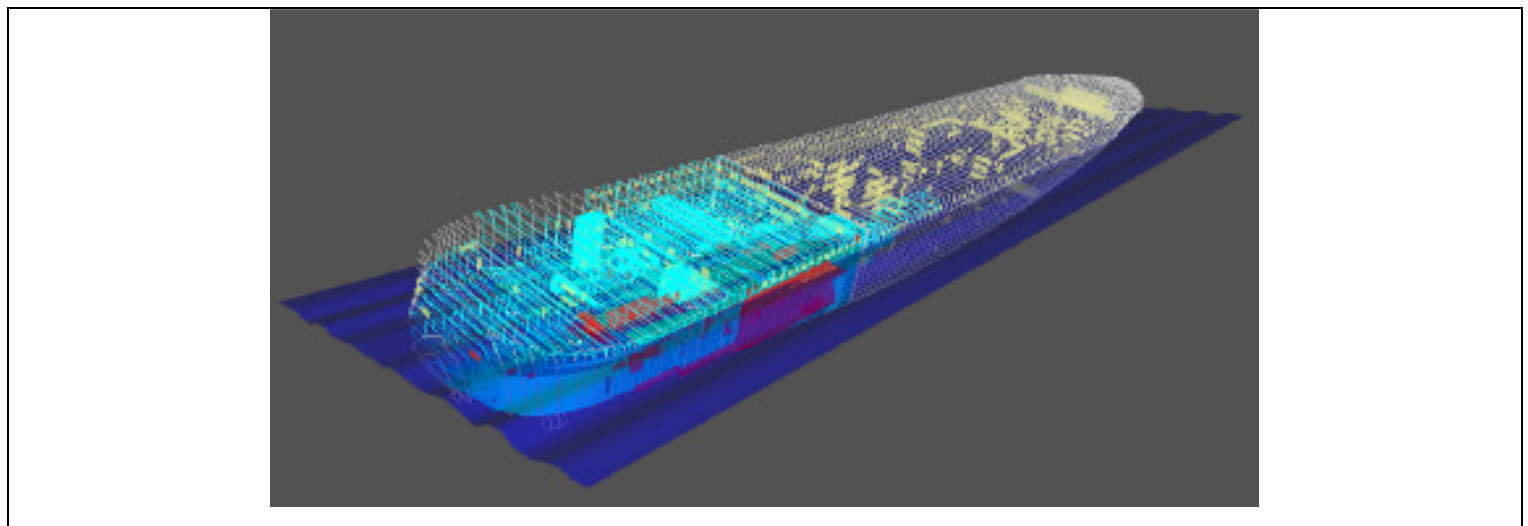

Figure 3.15: Flooding Analysis Model for Project Genesis (Vassalos 2012)

FIREPROOF was a project funded by the European Commission aimed at developing a risk-based design framework for on-board fire safety (Fireproof 2013; Mermiris et al 2012). The method includes probabilistic models for ignition, numerical (CFD) models for the growth and impact of fires, evacuation simulation methods and probabilistic descriptions of the reliability and effectiveness of fire detection and suppression systems. Mermeris et al (2012) comment that the use of CFD and evacuation simulations are necessary when historical data and analytical tools are not available, leading to "a cumbersome process considering the substantial number of simulations and the extended processing time that is needed for deriving the necessary fatality rates". Figure 3.16 shows the level of detail necessary for fire and evacuation analysis.

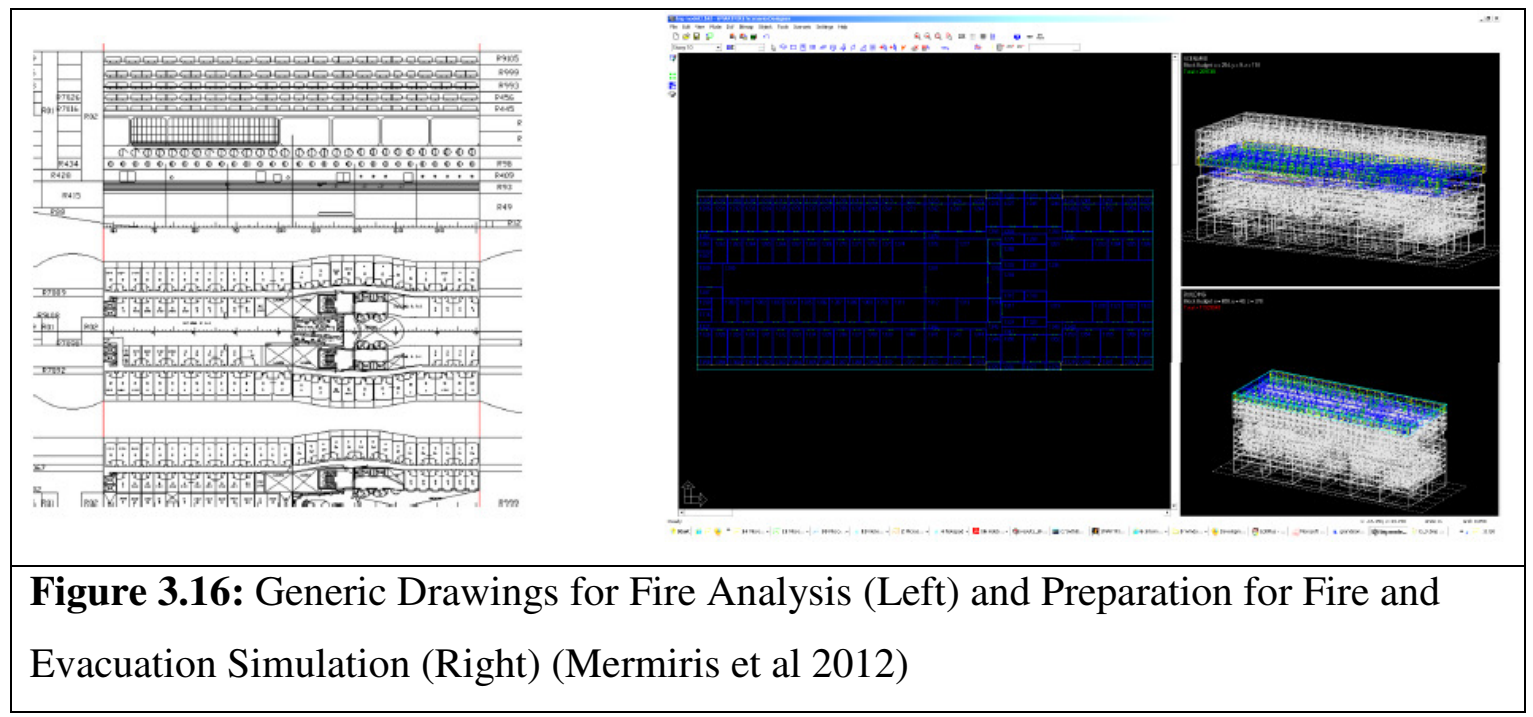


The aim of the above work is to develop tools to examine safety performance of ships against fire and flooding which account for $90 \%$ of mercantile ship serious accidents (Mermeris et al 2012), especially ones incorporating innovative layouts, for which current prescriptive rule-based design is inadequate (Azzi et al 2011; Vassalos 2012).

Simulation programmes are also used by various navies for DC training purposes. For example, the DC-Train tool is the current USN DC simulator, capable of modelling a wide range of scenarios, including fire, flood, smoke, equipment failures and personnel modelling (Grois et al 1998; Bulitko and Wilkins 1999). DC is exceptionally challenging and stressful and since real-life training is impractical, such simulators (which are also installed on board for afloat training) are aimed at improving crew performance. However, such DC simulation techniques are only effective on ship designs with full design definition, if not already constructed. Moreover, the plethora of probable DC scenarios would overcomplicate a DC simulation tool, therefore, questioning its relevance in early stage ship design. An example scenario, aimed at mercantile ships, was developed by Garre et al (2010) who compared an integrated bridge layout to a traditional one, by modelling a ship-to-ship collision. Nevertheless, it is accepted that such simulation methods can assess human performance.

The use of VR technology for DC simulation has been recognised as an important future development since the 1990s (Mayfield and Morrissey 1998). Fire, smoke and flood scenarios could be incorporated in VR using CFD, and other relevant models. In addition, through three-dimensional walkthrough, the effect of damage on compartment appearance and smoke on reduced visibility can be demonstrated. During design VR techniques can be used to assist survivability related decisions and during crew familiarisation of the ship, to look at DC approaches (Mayfield and Morrissey 1998).

UK MOD has considered extending survivability modelling to include recoverability, with work being carried out to include this aspect into the SURVIVE software (Bain 2006; Turner et al 2006). The software's algorithms have been updated to model secondary damage effects (such as fire and flood); the starting point is the output of SURVIVE's vulnerability analysis, which is then passed on to modules modelling crew numbers, movements, locations and skills, DCFF procedures and equipment layout and availability, post-damage automatic system reconfiguration and spares and external assistance, all of which have been included in the software (QinetiQ 2005a; Bain 2006). The software is relevant for use on various infrastructures (QinetiQ 
2005b; Schofield 2006); however, it is limited to models with a level of detail not associated to preliminary stage design. Figure 3.17 depicts the node network (for crew movements) within SURVIVE, Figure 3.18 shows locations of DCFF equipment and Figure 3.19 and Figure 3.20 illustrate SURVIVE views of equipment, systems and functions pre and post-damage respectively.
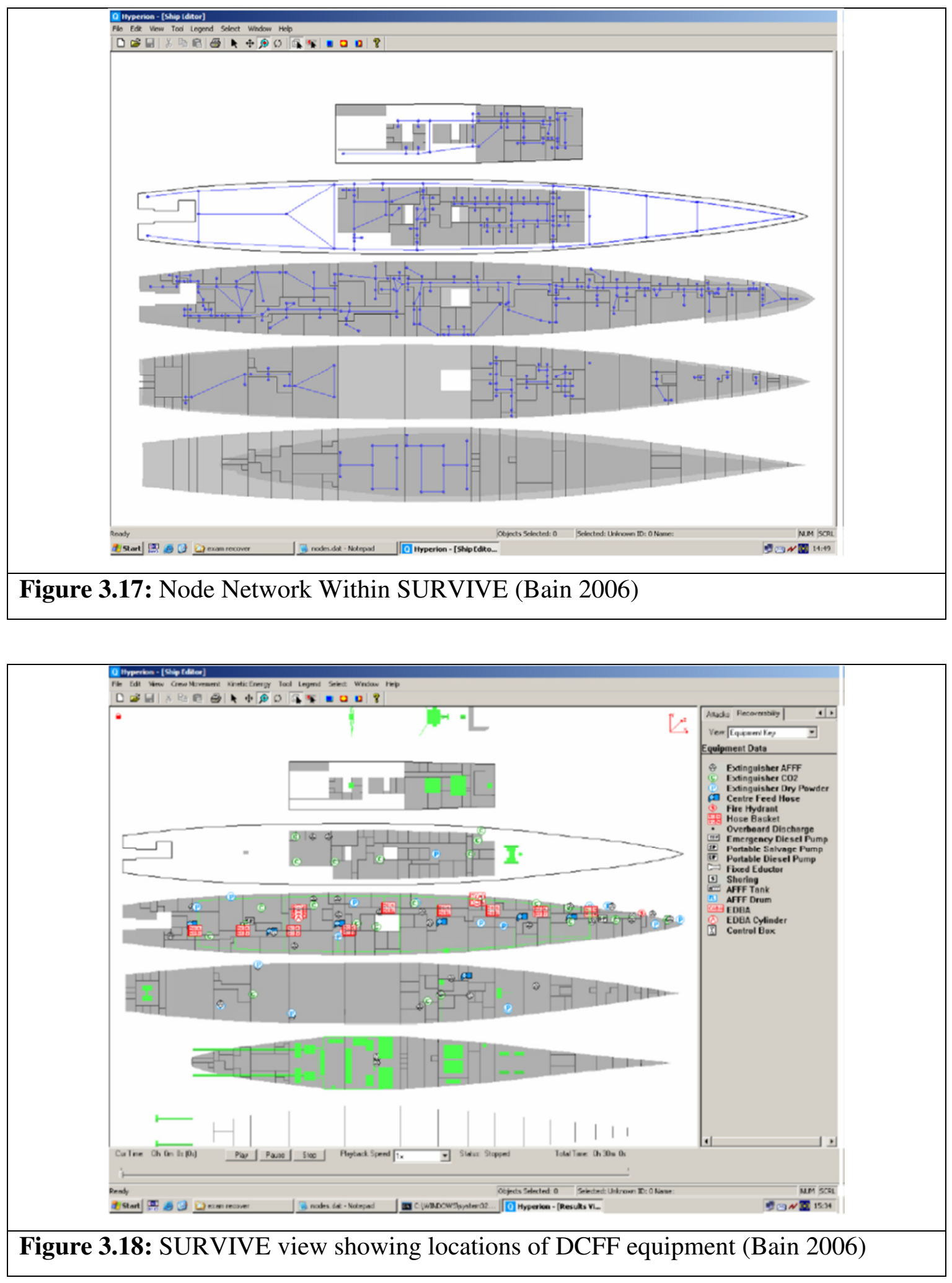


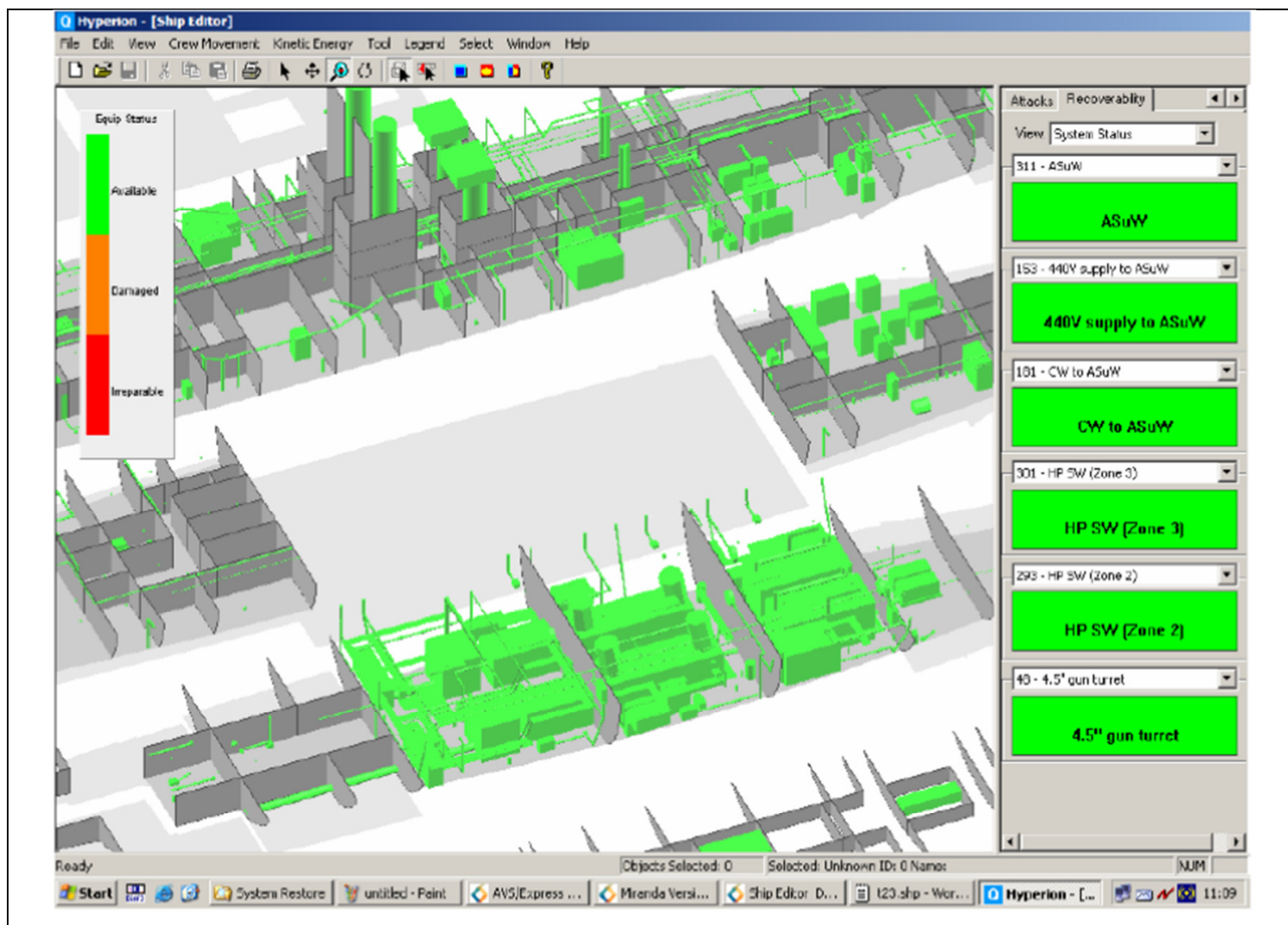

Figure 3.19: Equipment Systems and Functions Pre-damage (Bain 2006)

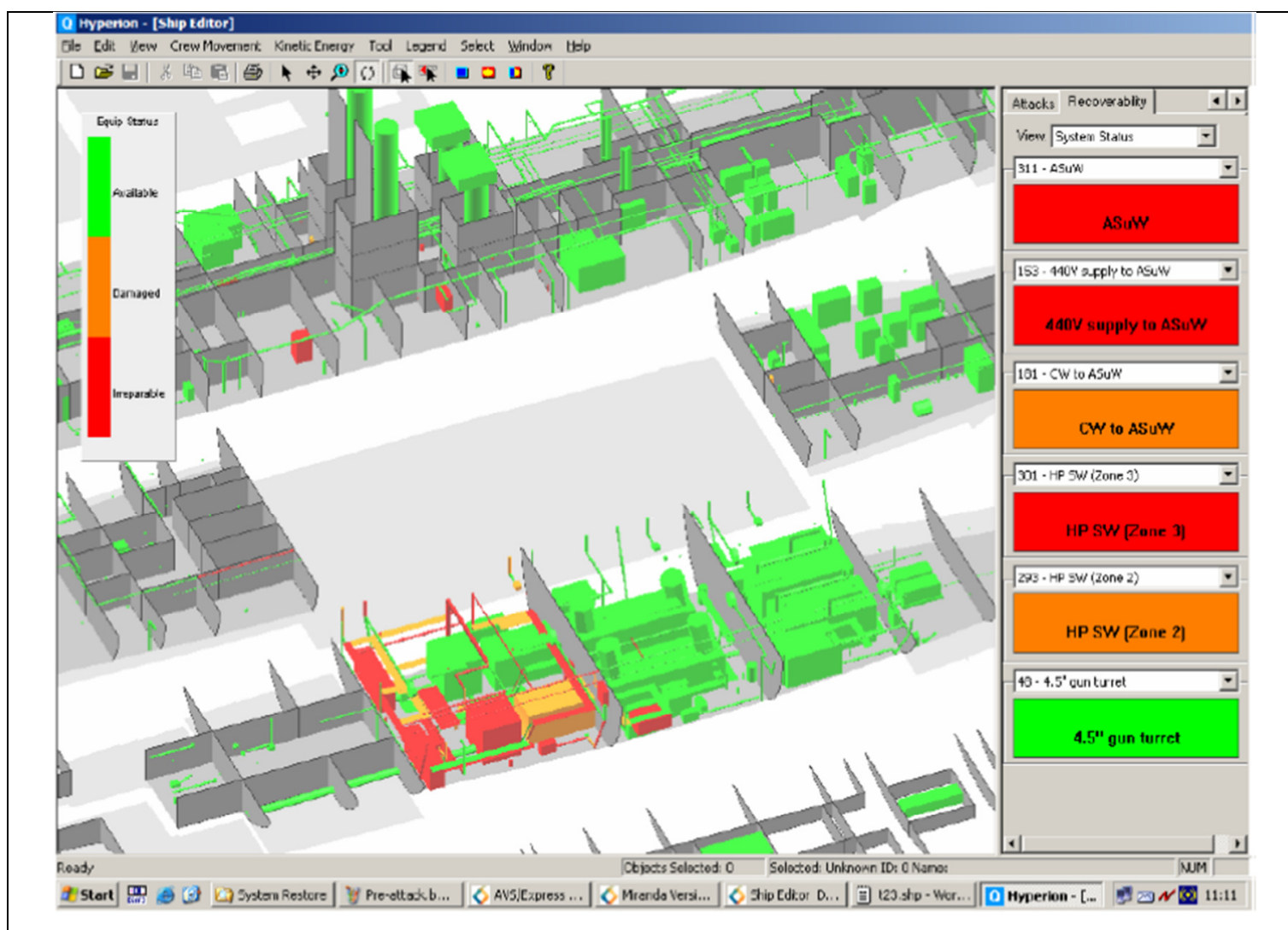

Figure 3.20: Equipment Systems and Functions Post-damage (Bain 2006) 
SURVIVE's fire and smoke spread modelling module is relatively complex and includes heat release rates, compartment and plate temperatures and smoke densities over time (Sharp 2011), therefore, requiring a lengthy setup procedure. In order to counter this, fast and simple 'Fast Fire' algorithms were included in SURVIVE Lite, requiring greatly reduced design detail. The algorithms do not rely on fluid dynamic models or crew tracking information, but function by considering the initial size of the fire, the position of zone boundaries and the presence of fixed fire fighting systems. Fast Fire methods have been developed for small, medium and large fires; it is assumed that for ignitions after ship hits by weapons with warheads less than $30 \mathrm{~kg}$, the small fire algorithm should be used, and for larger warheads, the medium fire algorithm (Sharp 2011). Although this level of simulation would be appropriate for concept stage designs, the accuracy of the results is uncertain, given the disregarding of numerous variables. In addition, the fast fire method cannot simulate fire spread; it simply outputs the vulnerability percentage of an item given that it was exposed to a fire.

Departing from detailed simulations of specific events, Kang et al (2012) proposed an early design ship safety assessment model for mercantile ships, consisting of hierarchical elements such as function (e.g.: manoeuvrability), system (e.g.: propulsion, steering and navigation) and sub-system (e.g.: engines, navigation radars and steering control panels, with more sub-systems being included at later design stages). By mapping the location of the above items, assuming several damage scenarios to various sections of the ship and assuming flooding times of these sections versus the time required to stop flooding, the ship safety is assessed (Kang et al 2012). The above process could be applied to naval ships by including the various fight capabilities as functions, using vulnerability assessment software to identify the damaged sub-systems after a given encounter and using equipment repair times to assess the recoverability of the ship functions. It also has the advantage of accounting for the ship architecture; however, further development would be required to account for factors such as access to damaged areas.

\section{$\underline{\text { 3.3.5 Total Survivability Assessment }}$}

Work has been carried out in order to integrate the three survivability constituents and obtain a single, meaningful, estimation of warship survivability. When defining survivability quantification, Ball and Calvano (1994) presented the relationship of various probability measures shown in Figure 3.21. 


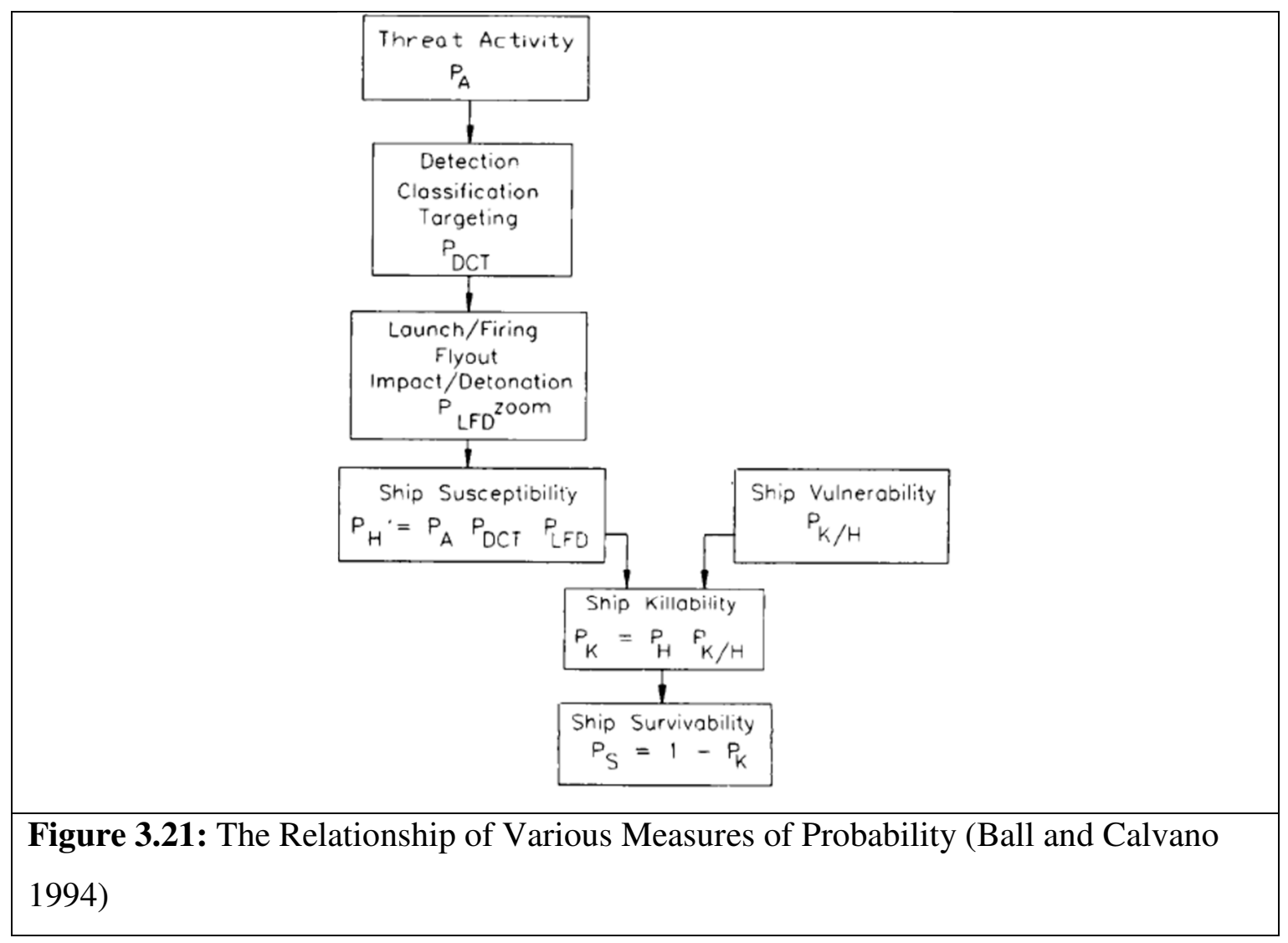

The following relationship was adopted:

$$
\mathrm{Ps}=1-(\mathrm{Ph} * \mathrm{Pk} / \mathrm{h})
$$

Ps: $\quad$ Probability of ship survivability;

Ph: Probability of ship susceptibility;

$\mathrm{Pk} / \mathrm{h}$ : Probability of ship vulnerability (including recoverability considerations).

Beyond proposing an approach to survivability on this basis, Ball and Calvano (1994) did not present any detail on how this should be populated. In addition, recoverability was neglected.

Sajdak and Karni (2006) presented a derivation of fundamental statistics regarding integrated survivability measurement. They identified the following three steps:-

- Susceptibility analysis: signature analysis for threat detection, tracking, targeting and homing in examination, and assessment of countermeasures, environmental conditions and tactics to get probability that the threat will impact the ship.

- System vulnerability analysis: examination of damage mechanisms, residual strength, system functionality after damage. 
- System recoverability analysis: assessment of systems' ability to regain (through reconfiguration, repair and replacement) lost capability.

The standard survivability equation such as that by Ball and Calvano (1994) was criticised since statistical independence between the three constituents is assumed and time is not well defined (Sajdak and Karni 2006; Thornton et al 2006). A relationship in the form of:

$$
P[\text { survivability }]=f(\text { susceptibility, vulnerability, recoverability })
$$

was suggested, eventually leading to the derivation of the MOTISS equation presented by Sajdak and Karni (2006). However, as before, no detail on how this equation should be populated was presented.

Papanikolaou and Boulougouris (1998) introduced a method aiming to transform current deterministic standards into rational probabilistic approach criteria for naval and commercial ship damage stability. Performance analysis was based on a sequence including detection (function of threat sensors, range, signatures and environmental conditions), classification, target acquisition, launch of attack, ships response to jam, deceive or destroy enemy weapon. Probability of hit at a specific location was calculated, followed by probable damages and damage extent given a hit at that location and finally, probability of ship's survival given the hit point and extent. The developed survivability performance analysis flowchart is reproduced in Figure 3.22.

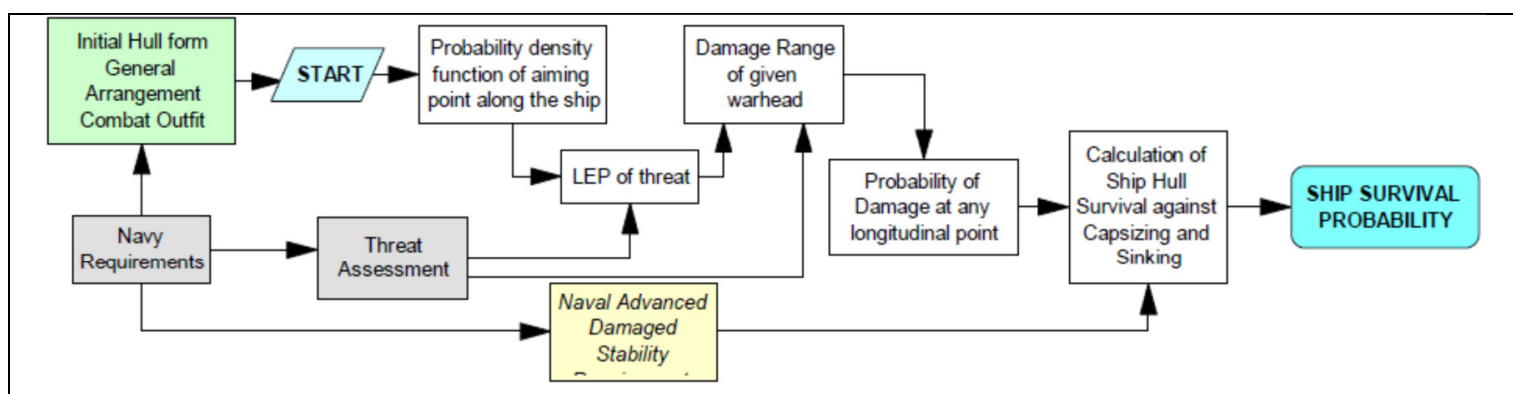

LEP: Linear Error Probability.

Figure 3.22: Survivability Performance Analysis Flowchart (Papanikolaou and Boulougouris 1998)

Boulougouris and Papanikolaou (2004) subsequently developed a damage stability optimisation method, using genetic algorithms as the optimisation process, for early ship design; outputs included survivability index, weight, shaft line length and 
mobility. Successively, numerical simulation tools in order to examine the ship's behaviour after flooding were incorporated (Boulougouris and Papanikolaou 2012). However, the above methods are limited to survivability against damage stability and do not look at, for example, system survivability.

Richards et al (2008) looked at the survivability assessment of aerospace platforms. They proposed six susceptibility and eleven vulnerability design principles (Figure 3.23) which assist in the concept generation of survivable systems.

\begin{tabular}{|l|l|l|}
\hline \multicolumn{2}{|l|}{ Type I (Reduce Susceptibility) } \\
\hline 1.1 & prevention & suppression of a future or potential future disturbance \\
\hline 1.2 & mobility & relocation to avoid detection by an external change agent \\
\hline 1.3 & concealment & reduction of the visibility of a system from an external change agent \\
\hline 1.4 & deterrence & dissuasion of a rational external change agent from committing a disturbance \\
\hline 1.5 & preemption & suppression of an imminent disturbance \\
\hline 1.6 & avoidance & maneuverability away from disturbance \\
\hline & & \\
\hline 2.1 & hardness & resistance of a system to deformation \\
\hline 2.2 & redundancy & duplication of critical system functions to increase reliability \\
\hline 2.3 & margin & allowance of extra capability for maintaining value delivery despite losses \\
\hline 2.4 & heterogeneity & variation in system elements to mitigate homogeneous disturbances \\
\hline 2.5 & distribution & separation of critical system elements to mitigate local disturbances \\
\hline 2.6 & $\begin{array}{l}\text { failure mode } \\
\text { reduction }\end{array}$ & $\begin{array}{l}\text { elimination of system hazards through intrinsic design: substitution, } \\
\text { simplification, decoupling, and reduction of hazardous materials }\end{array}$ \\
\hline 2.7 & fail-safe & prevention or delay of degradation via physics of incipient failure \\
\hline 2.8 & evolution & alteration of system elements to reduce disturbance effectiveness \\
\hline 2.9 & containment & isolation or minimization of the propagation of failure \\
\hline 2.10 & replacement & substitution of system elements to improve value delivery \\
\hline 2.11 & repair & restoration of system to improve value delivery \\
\hline
\end{tabular}

Figure 3.23: Baseline Set of Survivability Design Principles (Richards et al 2008)

Systems are analysed by mapping survivability features into the design principal categories to produce matrices giving a qualitative survivability description. The authors recognised that the next step would be to provide a quantitative implementation of the design principles through simulation in order to compare the survivability performance of competing designs (Richards et al 2008).

In an effort to integrate all survivability constituents, the MOD has produced the MISSION programme (White and Allwood 2011; Parry 2012; Parry and White 2012). Schofield et al (2012) defined MISSION as a discreet event Monte-Carlo model of a maritime mission. Apart from modelling the survivability elements and their interactions (by incorporating software previously mentioned, such as ThreeDim (AAW 
and ASuW), ANSWER and ODIN (ASW and torpedoes) and TMSS (mine warfare) for susceptibility; and SURVIVE for vulnerability and recoverability), MISSION has the ability of modelling the mission objectives, the ships within the context of and the interdependencies within the blue force and the threat environment (i.e. red force). This is because it operates on a maritime mission level with given task groups, objectives and attacks over a period of time, therefore, being able to be used as a war-gamming tool (Thornton et al 2006; Martin 2007). In fact, the main output of MISSION is the Probability of Mission Success (White and Allwood 2011; Parry 2012; Parry and White 2012). The framework of the programme is given by Thornton et al (2006) and reproduced in Figure 3.24.

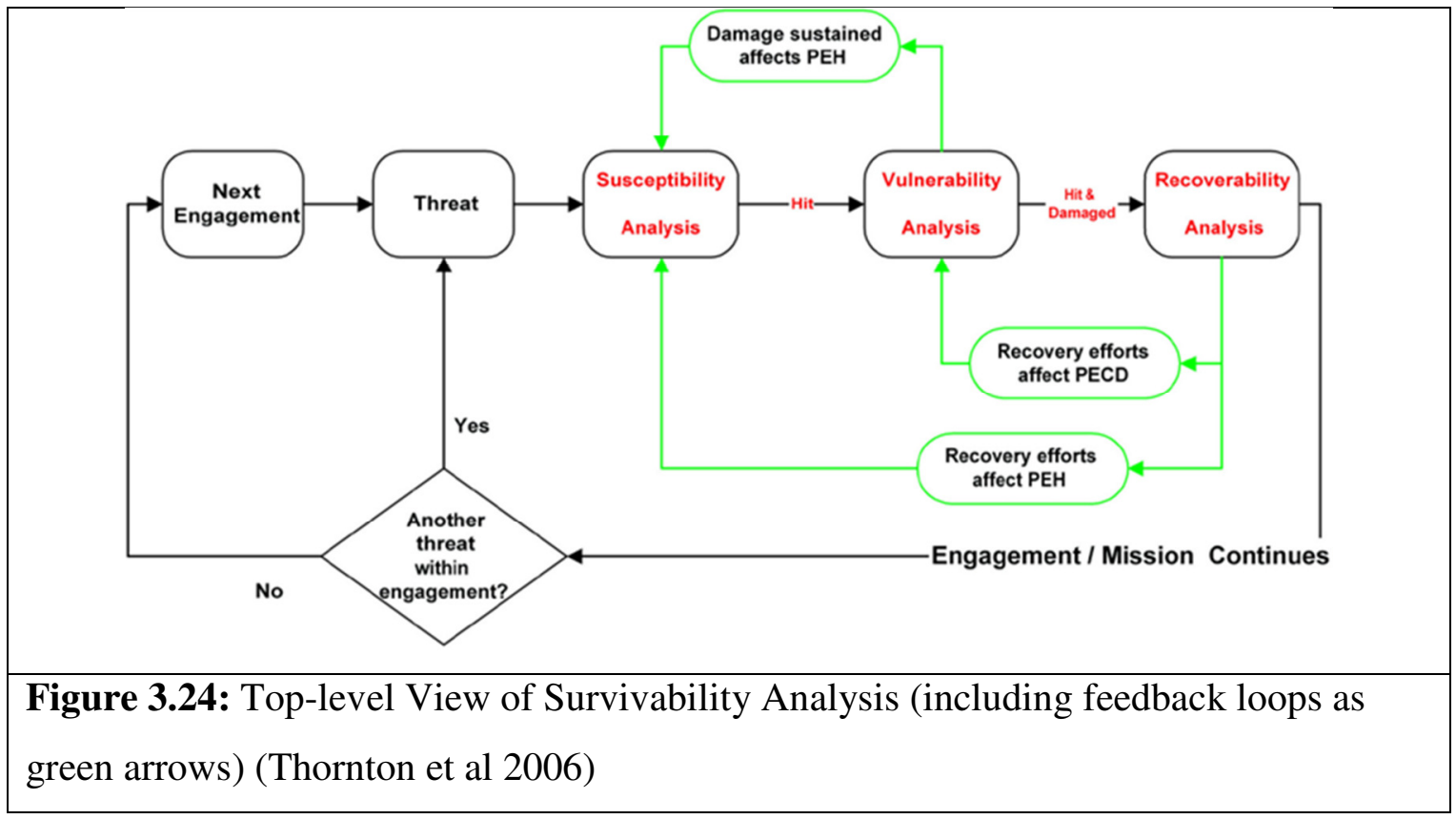

MISSION can thus be summarised as "an event simulation tool designed to simulate a maritime mission" (White and Allwood 2011; Parry 2012; Parry and White 2012) as opposed to a tool designed to integrate survivability assessment in the preliminary ship design process; also, being relatively complex, it requires a large number of input detail. Further information on MISSION is presented in Section 7.6.7.

\subsubsection{Survivability Requirement Specification}

Recent developments in survivability assessment methods have led many to believe that performance-based, rather than prescriptive, feature-based, requirements could be set for a ship design project. In addition, survivability assessment should 
commence during the early ship design stages in order to influence the ship layout (Randles 2009). Since the early 1990s it was identified that, theoretically, it would be possible to specify in the requirements, statements in the form of probability of retaining a specific percentage of a given capability after a specified threat encounter (Brown 1990). The specified threat scenario is shown in Figure 3.25.

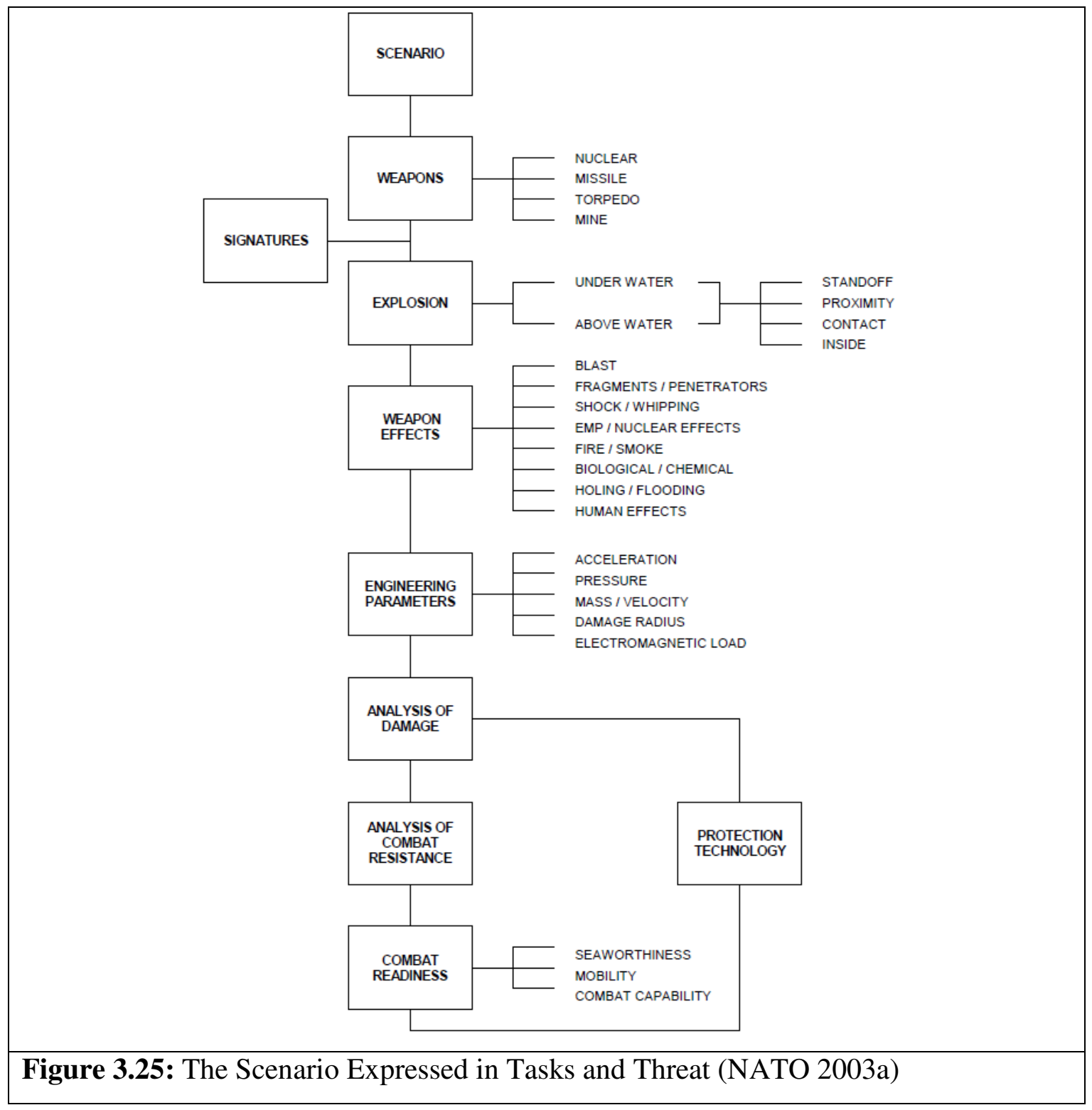

It was also clear that recoverability related requirements were the most complex to set (Reese et al 1998). This departure from the traditional approach and the move towards survivability requirements was clear during the CNGF project. The CNGF, which was assessed on REVS and SSVUL, was the first concept ship that included specific vulnerability targets, on major systems (Martin 1998). Percentage system vulnerability targets were also set for recent RN designs (the Type 45 Destroyer, Queen Elizabeth Class Aircraft Carrier (Thornton et al 2007) and Type 26 Frigate (Schofield et 
al 2012)). For these projects SURVIVE was used throughout the design development to assess vulnerability and optimise reduction measures (Thornton et al 2007; Schofield et al 2012). Similarly, RCS targets were set and SPECTRE was used to confirm that they had been met (Thornton et al 2007). The significance of such assessment methods becomes clear when considering that full prototypes are not possible for ships, therefore requirements and targets must be met from the start (Thornton et al 2007). Similar vulnerability performance requirements were first implemented in the USN during the development of what was then the DD 21 (Reese et al 1998). Reese et al (1998) presented a possible format (replicated in Figure 3.26) for probabilistic Operationally Oriented Vulnerability Requirement (OOVR) specification.

\begin{tabular}{|c|c|c|}
\hline \multicolumn{3}{|c|}{ Internal/contact detonation of a __-sized, anti-ship missile (e.g., __ } \\
\hline \multirow[b]{2}{*}{ Metric } & \multicolumn{2}{|c|}{$\begin{array}{l}\text { Ship vulnerability level } \\
\text { probabilities to be met }\end{array}$} \\
\hline & Threshold & Objective \\
\hline $\begin{array}{l}\text { Ship loss probability less than (A } \\
\text { or B) }\end{array}$ & A & $B$, where $B<A$ \\
\hline $\begin{array}{l}\text { Probability of maintaining Anti- } \\
\text { Air Warfare (AAW) primary mis- } \\
\text { sion capability greater than (C } \\
\text { or D) }\end{array}$ & $\mathrm{C} / \geq \mathrm{M} 3 /$ one hit & $\begin{array}{l}D / \geq M 3 / \text { one hit, } \\
\text { where } D>C\end{array}$ \\
\hline $\begin{array}{l}\text { Probability of maintaining Mo- } \\
\text { bility (MOB) primary mission ca- } \\
\text { pability greater than (E or F) }\end{array}$ & $\mathrm{E} / \geq \mathrm{M} 3 /$ one hit & $\begin{array}{l}\mathrm{F} / \geq \mathrm{M} 3 \text { / one hit, } \\
\text { where } \mathrm{F}>\mathrm{E}\end{array}$ \\
\hline \multicolumn{3}{|c|}{ Proximity detonation of a __ -sized torpedo or mine (e.g., ___ $)$} \\
\hline $\begin{array}{l}\text { Ship structure, protection from } \\
\text { hull whipping resulting from a } \\
\text { proximity detonation equivalent } \\
\text { to a keel shock factor (KSF) of } G \\
\text { or J with a ship loss probability } \\
\text { less than } \mathrm{H} \text { or } \mathrm{K}\end{array}$ & $\begin{array}{l}\mathrm{KSF}=\mathrm{G} / \text { proba- } \\
\text { bility less than } \mathrm{H}\end{array}$ & $\begin{array}{l}\mathrm{KSF}=\mathrm{J} / \text { probabil- } \\
\text { ity less than } \mathrm{K} \text {, } \\
\text { where } \mathrm{J}>\mathrm{G} \text { and } \mathrm{K} \\
<\mathrm{H}\end{array}$ \\
\hline $\begin{array}{l}\text { Under the these conditions for } \\
\text { ship structure, probability of } \\
\text { maintaining AAW primary mis- } \\
\text { sion capability greater than (L. } \\
\text { or M) }\end{array}$ & $\mathrm{L} / \geq \mathrm{M} 3 /$ one hit & $\begin{array}{l}M / \geq M 3 / \text { one hit, } \\
\text { where } M>L\end{array}$ \\
\hline $\begin{array}{l}\text { Under the these conditions for } \\
\text { ship structure, probability of } \\
\text { maintaining MOB primary mis- } \\
\text { sion capability greater than ( } \mathrm{N} \\
\text { or O) }\end{array}$ & $\mathrm{N} / \geq \mathrm{M} 3 /$ one hit & $\begin{array}{l}\mathrm{O} / \geq \mathrm{M} 3 / \text { one hit, } \\
\text { where } \mathrm{O}>\mathrm{N}\end{array}$ \\
\hline
\end{tabular}

Figure 3.26: Probabilistic Approach to Specifying OOVRs for a Surface Ship (Reese et al 1998)

Furthermore, the MOD (2001) suggested that vulnerability requirements should be in the form of function versus threat weapon tables, such as the one reproduced in Figure 3.27, containing required minimum probabilities. 


\begin{tabular}{|c|c|c|c|c|c|c|c|c|c|}
\hline \multirow[b]{2}{*}{ Function } & \multicolumn{9}{|c|}{ Threat } \\
\hline & $\begin{array}{c}\text { Sea } \\
\text { Skimming } \\
\text { Missile }\end{array}$ & $\begin{array}{l}\text { High Diving } \\
\text { Missile }\end{array}$ & $A R M$ & $N B C D$ & Small Arms & $\begin{array}{l}\text { Hull Lethal } \\
\text { Shock }\end{array}$ & $\begin{array}{l}\text { System Level } \\
\text { Shock }\end{array}$ & $\begin{array}{c}\text { Contact Mine/ } \\
\text { Torpedo }\end{array}$ & $\begin{array}{c}\text { Non Contact } \\
\text { Mime/ } \\
\text { Torpedo }\end{array}$ \\
\hline $\begin{array}{l}\text { Residual } \\
\text { Stability }\end{array}$ & 100 & 100 & 100 & 100 & 100 & 100 & 100 & 100 & 100 \\
\hline $\begin{array}{l}\text { Residual } \\
\text { Strength }\end{array}$ & 100 & 100 & 100 & 100 & 100 & & 100 & 100 & 100 \\
\hline $\begin{array}{l}\text { Equipment } \\
\text { capthity }\end{array}$ & & & & & & 100 & 100 & & 100 \\
\hline$D C F F$ & 100 & 100 & 100 & 100 & 100 & 100 & 100 & 100 & 100 \\
\hline $\begin{array}{l}\text { Full Power and } \\
\text { sterrage }\end{array}$ & 75 & 75 & 95 & 100 & 100 & & 100 & 50 & 50 \\
\hline $\begin{array}{l}\text { Minimum Power } \\
\text { and sterage }\end{array}$ & 95 & 95 & 100 & & & & 100 & 85 & 85 \\
\hline$A 4 W$ & 75 & 75 & 85 & 100 & 100 & & 100 & 75 & 75 \\
\hline$A s u W$ & 75 & 75 & 85 & 100 & 100 & & 100 & 75 & 75 \\
\hline$c^{\prime}$ & 85 & 85 & 85 & 100 & 100 & & 100 & 75 & 75 \\
\hline CIIIS & 85 & 85 & 85 & 100 & 100 & & 100 & 85 & 85 \\
\hline
\end{tabular}

Note: these are example figures and should not be taken as representing actual requirement levels.

Figure 3.27: Example Threat / Function Table (MOD 2001)

\subsection{Conclusions on Background to Survivability}

A number of survivability assessment tools currently exist; however, most are either aimed at a single survivability constituent (or even at a specific aspect of a single survivability constituent), being unable to balance between survivability reduction features (e.g.: SPECTRE and SURVIVE) and/or are qualitative therefore inadequate for quantifying requirement specification (e.g.: safety and risk analysis) and/or are aimed towards detailed designs where implementing changes is impractical (e.g.: personnel flow and fire simulation tools), as outlined in Section 3.3.

Therefore, the need for a simple and rapid method for fully integrating and quantifying naval ship survivability has been identified. Since survivability is layout sensitive, it is proposed that the method should take advantage of architecturally orientated ship design processes, integrating survivability in the iterative design process. It should also be applied to early stage designs where there is minimum detail, which is amenable to change. The proposed method will combine a number of tools used by UCL and the MOD, as well as a new approach to quantify recoverability. This approach will attempt to overcome the difficulties of recoverability modelling (such as the lack of data, human performance and time dependence) by using weighted performance measures. The quantification of a certain aspect of warship performance would also simplify the design of "the most complex, diverse and highly integrated of any engineering systems" produced today (Graham 1982). 


\section{Chapter 4: Survivability Assessment Method Development}

This chapter gives a detailed description of the survivability assessment method that was developed. It includes a combination of various existing and new tools. A summary of the proposed method was published by Piperakis et al (2012), which is reproduced in Appendix 5. This chapter outlines the assessment of each of the three linked survivability aspects in turn - with particular emphasis on the last (recoverability) before the overall survivability assessment method is outlined in Section 4.4.

\subsection{Susceptibility Assessment}

It is evident that a number of susceptibility (and vulnerability) assessment methods currently exist. Moreover, susceptibility assessment techniques can be used throughout the ship design process. Therefore, the development of a new susceptibility assessment method was judged unnecessary. However, before deciding on which susceptibility tools to utilise, a representative threat scenario should be developed. In order for the survivability assessment method to be valid, it should work with a range of different scenarios. However, in order to demonstrate the proposed approach, it was decided that a clear single threat scenario should be adopted. The scenario selected was that of a naval ship being attacked by radar homing (at $15 \mathrm{GHz}$ ), sea-skimming ASMs. The above scenario is demonstrated in Figure 4.1.

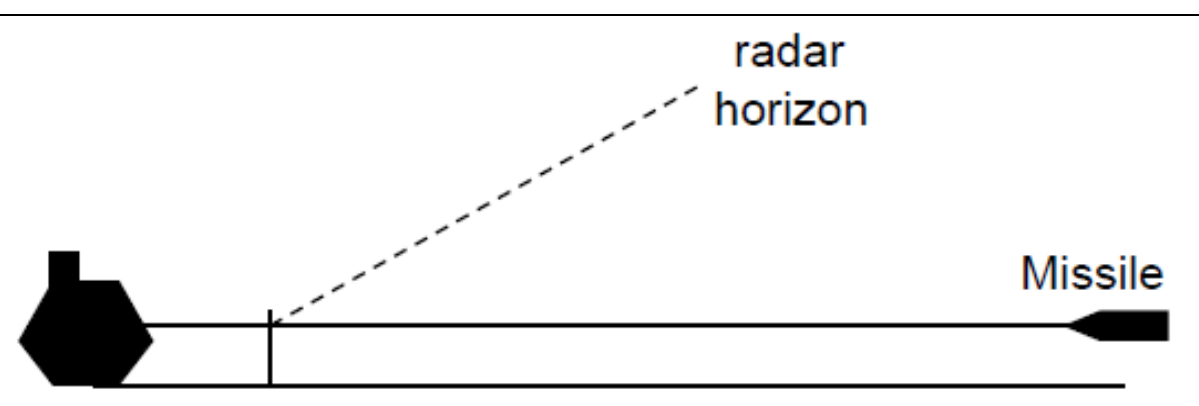

Figure 4.1: Threat Scenario (McDonald 2010)

The ship being attacked would attempt to defend itself using both its soft-kill systems (chaff decoys) and hard-kill systems (CIWS, PDMS, ADMS). These are the main defence strategies against ASMs as described by (Longworth 1983). This threat 
scenario was selected as ASMs are seen to be a principal threat faced by modern warships (Longworth 1983; Surko 1994). In addition, since the survivability assessment method would have to function with a variety of threat scenarios, it is thought that the above scenario can relatively easily be altered in order to represent different attack situations.

Three probabilities were calculated for the susceptibility part of the survivability assessment method. The probability of the ship being detected and identified, P(di), the probability that the missile locks on the ship, $\mathrm{P}(\mathrm{l})$, and the probability of the ship being hit by at least one ASM, $\mathrm{P}(\mathrm{h})$. These probabilities correspond to the four necessary steps in order for a ship to avoid being hit, as identified by Belcher (2008), and spelt out in Appendix 4.1. It was decided that $\mathrm{P}(\mathrm{di})$ would be taken to be unity for simplification purposes, given that it depends more on operational circumstances rather than the ship design itself, on which this method focuses.

In order to calculate $\mathrm{P}(\mathrm{l})$, it was decided to utilise the SPECTRE software (the current UK MOD RCS prediction tool (Turner et al 2006; Martin 2007), described in Section 3.3.2) and easy access to SPECTRE was provided by the sponsor of this research, UK MOD Dstl (https://www.dstl.gov.uk/). The ship designs to which the method was applied were sent to Dstl, who then ran them on SPECTRE in order to obtain RCS results. Given that if the RCS of the decoys launched by the ship (soft-kill) is equal to that of the ship, there is a $50 \%$ chance of the ASM locking onto the ship (Papanikolaou and Boulougouris 1998), P(l) values were obtained by assuming a simplistic (and unclassified) linear relationship (see Figure 4.2).

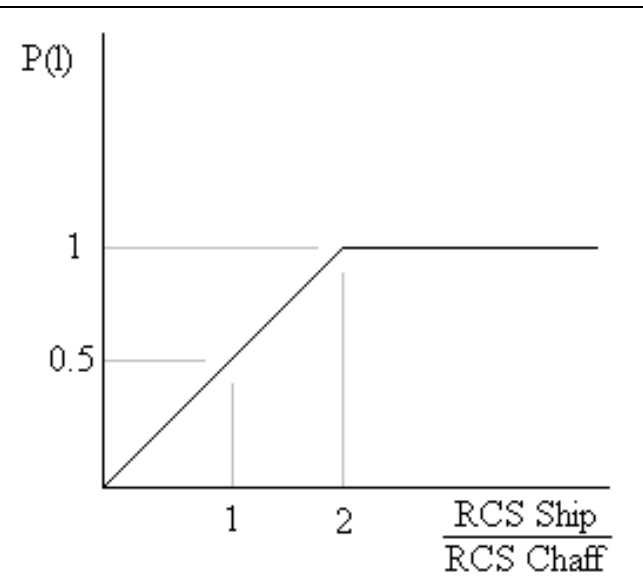

Figure 4.2: Relationship Between the Probability That the Missile Locks on the Ship, $\mathrm{P}(\mathrm{l})$, Against the Ratio of (Ship RCS)/(Chaff RCS) 


$$
\begin{aligned}
& \text { Namely, } \\
& \text { If (RCS ship) }<(2 \times \text { RCS chaff }), P(1)=0.5 \times((\text { RCS ship }) \div(\text { RCS chaff })) \\
& \text { If (RCS ship) } \geq(2 \times \text { RCS chaff }), P(1)=1
\end{aligned}
$$

While the chaff RCS should normally exceed that of the vessel being defended, it was assumed that all ship designs studied employed the same decoys. The chaff RCS was assumed to be $2,000 \mathrm{~m}^{2}$ between $10-20 \mathrm{GHz}$ (Friedman 2006), although even higher values were given in that reference and by Cherming (2006; 2011). The average ship $\mathrm{RCS}$, around $360^{\circ}$, at an elevation of $0^{\circ}$ and frequency of $15 \mathrm{GHz}$ was used in the $\mathrm{P}(1)$ equations (Equations 3 and 4). This was done as the threat selected is sea-skimming and a specific orientation of the ship to the threat was not assumed, therefore avoiding unnecessary complexities in the threat scenario. However, SPECTRE is capable of outputting ship RCS data at different elevation angles and specific azimuth angles.

$\mathrm{P}(\mathrm{h})$ was then calculated through the CSEE method (McDonald 2010). The CSEE, operated by UCL as part of its MSc in Naval Architecture course, has been used to estimate defensive system (hard-kill) effectiveness of an LPD against threat scenarios. As discussed in Section 3.3.2, the CSEE is able to calculate probabilities of layered defence success against missile attack scenarios; however it is relatively simple and relies on various assumptions. Unclassified but representative weapons data (such as range, maximum burst time for CIWS, firing rates, reaction times, kill assessment times, velocities and single shot kill probabilities) are specified to determine combat system reaction times and success probabilities (McDonald 2010). McDonald (2010) also gives other key assumptions:-

- Ship movement/manoeuvring ignored;

- $100 \%$ reliable/available systems;

- Constant (i.e.: range, RCS, speed, manoeuvrability, environment independent) kill probabilities (of incoming missile by a given defensive system) assumed;

- Investigates single ships rather than task groups;

- Incoming missile profiles are given;

- Environmental conditions are ignored;

- Constant missile velocities assumed (a typical defensive missile velocity profile is likely to follow something similar to that illustrated in Figure 4.3). 


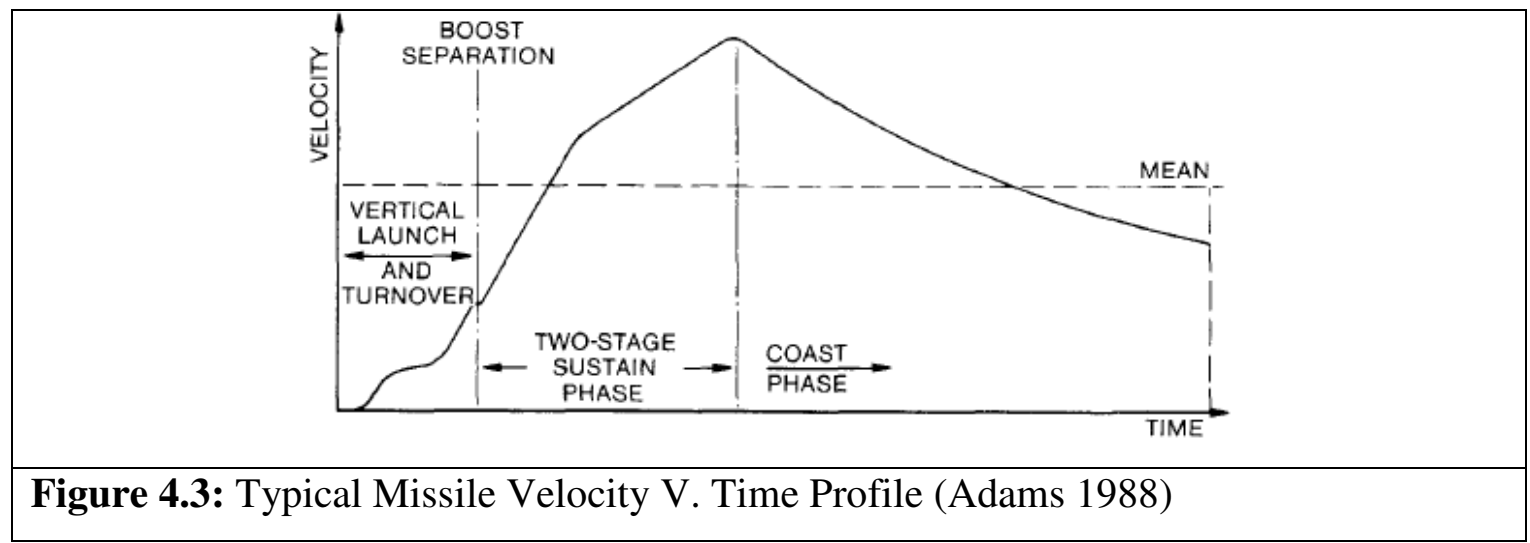

Given the above data and assumptions, event (combat system reaction) timelines can be generated, in order to calculate $\mathrm{P}(\mathrm{h})$. In addition, although the tool was originally intended for LPD type ships, it can easily be applied to other naval ships, such as combatants. The final susceptibility results were obtained by multiplying $\mathrm{P}(\mathrm{di}), \mathrm{P}(\mathrm{l})$ and $\mathrm{P}(\mathrm{h})$ for each ship design investigated. This product gives the probability that a ship was successfully detected, identified, targeted and hit by an adversary. This probability could be normalised with respect to the corresponding value for a baseline ship design, or used as an absolute value.

It is important to note that, as discussed in Section 3.3.2, the factors affecting the lengthwise hit probability on a ship are not limited to ship and threat characteristics. Factors such as environmental conditions, ship motions and manoeuvring and variations in weapon performance play an important role. Therefore, using RCS prediction software to obtain lengthwise hit probabilities is questionable (MOD 2001). Consequently, the lengthwise hit probability distribution was assumed to follow a normal distribution with a mean equal to half of the ship's overall length and a standard deviation equal to a sixth of the ship's overall length. This type of lengthwise distribution was advised by the Maritime Integrated Survivability team (part of the Maritime Systems Engineering Group in Naval Systems Department) of Dstl, who also use it in their own susceptibility analyses (in the MISSION programme) (Thornton et al 2012).

\subsection{Vulnerability Assessment}

As with susceptibility assessment, various vulnerability assessment tools currently exist, aimed at both concept and subsequent design stages. It was therefore decided that the development of a new vulnerability assessment tool was not necessary. Adding vulnerability reduction features on a warship is highly sensitive (in terms of 
design difficulty, time and cost) to the level of design detail. Since the developed survivability assessment method is aimed at preliminary ship design, it was decided that QinetiQ's SURVIVE Lite (Pugh 2006; Turner et al 2006) software, outlined in Section 3.3.3, was appropriate for these studies. This also had the advantage that SURVIVE Lite is currently the principal concept stage vulnerability assessment code of the UK MOD and can simulate a large variety of threat types, including ASMs which are examined.

It was decided that a limited amount of major ship systems/capabilities should be initially modelled; more specifically the Move and Fight related systems of the functional breakdown described in Sections 2.1.4 and 2.1.5. However, before deciding on those systems, the ship types on which the method would be applied to had to be determined. It was concluded that the method would first be applied on combatants, focusing particularly on frigate type ships, as they are the most common type of warship for most navies with blue water aspirations (Andrews et al 2004). Consequently, the systems of the ships to be investigated were:-

- Move system;

- Medium calibre naval gun system;

- ASM system;

- $\quad$ Aft SAM system;

- Fwd SAM system;

- Helicopter system.

Furthermore it was decided that the survivability assessment method should also be applied to replenishment ship designs. For this ship type, discussions with Dstl staff led to the decision that the following major systems/capabilities would be investigated (Thornton and Day 2012):-

- Move system;

- Ability to RAS AVCAT;

- Ability to RAS dieso;

- Ability to RAS dry stores;

- Ability to RAS ordnance;

- Aviation support;

- CIWS.

As noted in Section 3.3.3, in preliminary design only major system components are considered, while items such as cables and pipes connecting system components are yet to be specified beyond gross weight. SURVIVE Lite is intended of operating at this 
level of definition. System tree diagrams and system architecture illustrations from SURVIVE Lite are shown in Appendix 7.

It was decided that SURVIVE Lite's ASM hit grids (described in Section 3.3.3) should be separately applied to each WT section of each ship (for both port and starboard sides, and then averaged), as depicted in Figure 4.4 which illustrates Frigate Variant 1, detailed in Section 5.1.1. Vulnerability results (i.e. percentage vulnerabilities of the modelled equipment, compartments and systems) were obtained for a hit at each WT section. These results were multiplied by the probability that the given WT section was hit (computed through the lengthwise hit probability distribution described in Section 4.1). (The sum of the probabilities that each WT section of a ship design is hit is equal to 1 , i.e. it is given that the ship is definitely hit (susceptibility $=1$ ) which is a requirement for vulnerability analysis). Finally, by taking the sum of these results, the total system vulnerability for each system was obtained.

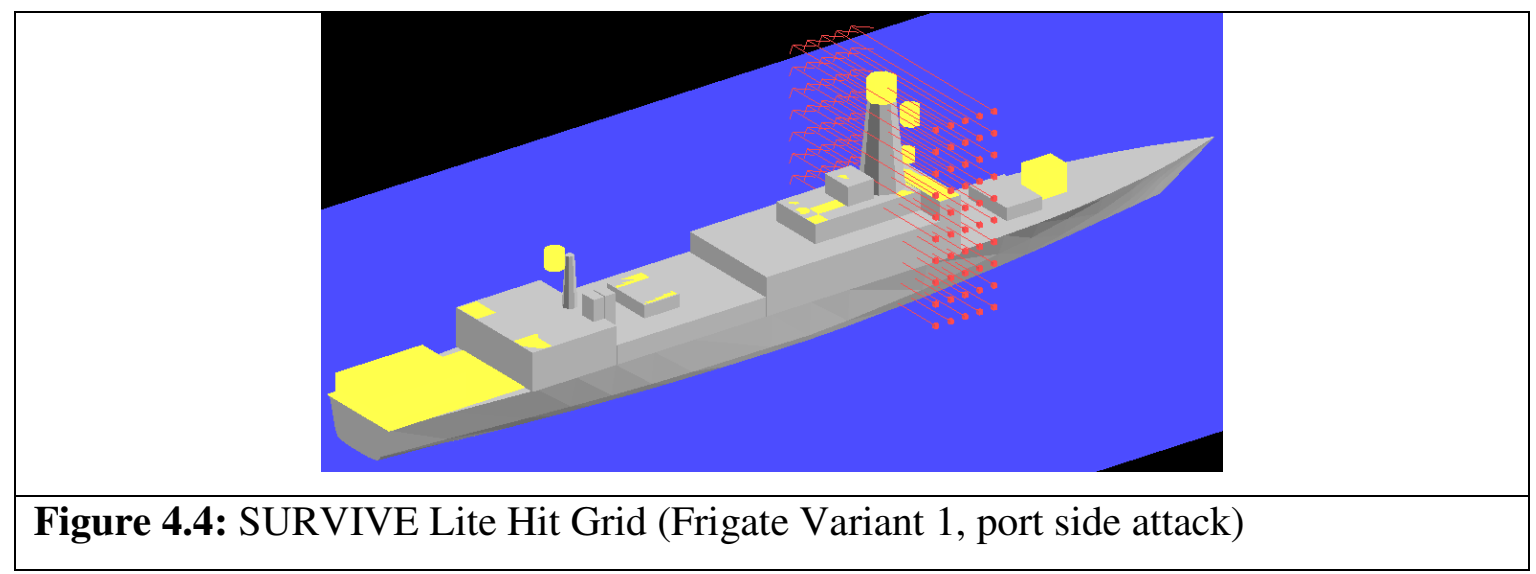

In order to keep the number of simulations and data processing to a reasonable minimum amount, only broadside attacks at an elevation of $0^{\circ}$ were simulated. However, SURVIVE Lite is capable of modelling missile hits at various azimuth and elevation angles.

The major ship systems modelled, listed above, do not have equal significance to one another and are highly dependent on the operational scenario undertaken by the ship. Therefore, weighting schemes had to be applied to the modelled systems. For the combatant designs, the weighting scheme adopted was that advised by Portuguese Navy officer, $1^{\text {st }}$ Lt. Pedro Fonseca (2011) who was an UCL MSc student at the time and had been a frigate damage control officer in his recent seagoing appointment. The proposed weighting scheme is in accordance with the warship objective hierarchy 'to float, to move, to fight' (Brown and Tupper 1989) and is shown in Table 4.1. Specifically, the 
Float group was allocated the maximum weighting of ten (this will be further detailed in Section 4.3.4 and Table 4.7), the Move group was allocated a weighting of nine, and the sum of the weightings of the systems in the Fight group was eight. Subsequently sensitivity testing on this weighting scheme was carried out and is presented in Section 6.4.1. The values of all weighting schemes applied throughout this project were between $0-10$, zero corresponding to an insignificant entity, while ten, to one of maximum importance.

Table 4.1: Combatant System Weighting Scheme

\begin{tabular}{|l|l|r|r|}
\hline MOVE & Move system & 9 & $=9$ \\
\hline \multirow{5}{*}{ FIGHT } & Medium calibre naval gun system & 2 & \\
\cline { 2 - 3 } & ASM system & 2 & \\
\cline { 2 - 3 } & Aft SAM system & 1 & \\
\cline { 2 - 3 } & Fwd SAM system & 2 & \\
\cline { 2 - 3 } & Helicopter system & & \\
& &
\end{tabular}

For the replenishment ship designs, the weighting scheme was advised by RN officer (rtd.), Lt. Cdr. Tim Day (2012) who had been a CVS NBCD officer, and was the Dstl Maritime Integrated Survivability Team Leader at the time. The proposed weighting scheme is shown in Table 4.2. From Table 4.2 (and Table 4.9 in Section 4.3.4) it is evident that the 'to float, to move, to fight' hierarchy was not utilised by Lt. Cdr. Day for the allocation of the system weightings (as was for the combatants by 1st Lt. Fonseca). On this occasion, the allocation was based on the hierarchy of capabilities for a replenishment ship as perceived by Lt. Cdr. Day.

\begin{tabular}{|l|l|r|r|}
\hline \multicolumn{2}{|l|}{ Table 4.2: Replenishment Ship System Weighting Scheme } \\
\hline MOVE & Move system & 10 & $=10$ \\
\hline \multirow{4}{*}{ FIGHT } & Ability to RAS AVCAT & 10 & \\
\cline { 2 - 3 } & Ability to RAS dieso & 6 \\
\cline { 2 - 3 } & Ability to RAS dry stores & 2 & \\
\cline { 2 - 3 } & Ability to RAS ordnance & 3 \\
\cline { 2 - 3 } & Aviation support & 39 \\
\cline { 2 - 3 } & CIWS & & \\
& &
\end{tabular}


The final vulnerability results were obtained by normalising the individual modelled system vulnerabilities with respect to the corresponding system vulnerabilities of a baseline ship design. The above weighting schemes were applied to these normalised values, and the sum taken. This procedure led to a single figure for a ship's vulnerability, similarly to the ship's susceptibility (as outlined in Section 4.1). However, unlike susceptibility, this single value has no meaning (and cannot even be derived) unless a ship design is compared to at least one, or more, other ship designs. Therefore this procedure is strictly comparative and does not output an absolute vulnerability value; rather, it outputs a vulnerability value which has been normalised with respect to a baseline ship design. However, the individual ship system vulnerabilities are absolute vulnerabilities before normalising against other systems and weighting.

In addition to the move system and major Fight systems and their components, ATU and ventilation compartments, firepumps, NBCD stores, FRP section bases, workshops, naval stores, spare gear stores and HQ1 and HQ2 were also modelled in SURVIVE Lite. These items were modelled in order to obtain vulnerability data of components necessary for recoverability, which was then used in the recoverability section of the proposed approach (see Section 4.3).

Finally, the Fast Fire algorithm of SURVIVE Lite (Sharp 2011), which is relevant to preliminary stage ship design, was employed in order to determine further system and component damage due to secondary weapon effects. Although it was suggested that the medium fire algorithm should be used for warheads above $30 \mathrm{~kg}$ (Sharp 2011), the Maritime Integrated Survivability team of Dstl suggested using the small fire algorithm (Thornton et al 2011). Damage due to flooding was not considered since only abovewater threats were examined, although SURVIVE Lite has the capability to model flooding damage to systems and components, and, therefore, the proposed approach could easily include modelling underwater threats.

\subsection{Recoverability Assessment}

As is evident from Section 3.3.4 modelling recoverability is the most demanding area of survivability assessment for a number of reasons such as the limited ability to model secondary damage and crew actions, the inadequate data available and the difficulty of incorporating crew readiness and skill levels. A number of recoverability assessment techniques exist, however, many, such as safety and risk analysis methods, are qualitative. Thus, they are not quantifiable but rather are subjective since they 
usually rely on expert judgment and, furthermore, do not take specific ship architectural features into account and so are considered to be of limited design use. They could possibly, however, be useful when deciding on what DC equipment to have on board and what procedures to follow. Numerous other methods are focused on a particular aspect of recoverability, such as fire spread or DC crew evolutions/personnel movement. This means that they rely on simulations and are therefore more appropriate to relatively detailed ship definitions. A tool aimed specifically to preliminary ship designs does not seem to exist; neither does one which specifically investigates how ship configuration/layout/architecture would affect overall naval ship recoverability (since recoverability is seen to be more heavily reliant on operational/human factors rather than design factors). For these reasons, it was decided that a new recoverability assessment method should be developed, to investigate such issues at early stage design.

By applying the proposed recoverability method to early stage design, where there is minimum detail, changes in ship layout could be relatively easily investigated. It was decided that simulations were probably not appropriate for the level of ship definition in the ship design studies produced. However, assessment of recoverability requires temporal metrics, such as the time taken to repair systems, which could be obtained from simulation. An alternative analytical method was thus required to generate this data. It was determined that the new recoverability assessment approach would work on the basis of developing a number of Performance Measures (PMs) together with an appropriate weighting scheme which might overcome the difficulties in recoverability modelling (e.g.: lack of data, human performance and time dependence). The PM method was inspired by work such as (Chow 2002) and (Casarosa 2011) (see Figure 3.13 and Figure 3.14). PMs relevant to recoverability were derived from existing work, such as the two references above and other literature on naval ship recoverability (see Appendix 4.3), as well as from interviewing 1st Lt. Fonseca, knowledgeable in the area of (frigate) damage control. Values for those PMs were obtained using the Paramarine and SURVIVE Lite tools.

\section{$\underline{\text { 4.3.1 Category } 1 \text { Performance Measures }}$}

The PMs developed were split into three categories. As before, the weighting schemes of all PMs were derived with the assistance of 1st Lt. Fonseca (2011) for the combatant design studies, and Lt. Cdr. (rtd.) Day (2012) for the AOR design studies, to ensure consistency. The three PM categories and their associated weighting schemes are 
presented in Table 4.3, Table 4.4 and Table 4.5. Sensitivity testing on this weighting scheme was carried out and is presented in Section 6.6.1. Note that the larger the value of a specific PM is, the worst its performance and that some PMs have units while others are dimensionless.

The first category, which is detailed in Table 4.3, consists of PMs related to immediate effects on DCFF, upon which the Float objective at the top of the warship hierarchy depends.

\begin{tabular}{|c|c|c|c|c|}
\hline & \multirow{2}{*}{$\mathbf{P M}$} & \multirow{2}{*}{ Software } & \multicolumn{2}{|c|}{ Weighting } \\
\hline & & & Combatant & AOR \\
\hline 1.1 & $\begin{array}{l}\text { Average distance between FRPP and } \\
\text { damaged WT section (m) }\end{array}$ & Paramarine & 7 & 4 \\
\hline 1.2 & Average number of WTD operated per FRP & Paramarine & 4 & 4 \\
\hline 1.3 & $\begin{array}{l}\text { Number of internal decks in damaged WT } \\
\text { section }\end{array}$ & Paramarine & 6 & 5 \\
\hline 1.4 & $\begin{array}{l}\text { Average total width of alternative } \\
\text { passageways (inverse) (m) }\end{array}$ & Paramarine & 7 & 2 \\
\hline 1.5 & $\begin{array}{l}\text { ATU and Ventilation (of damaged zone) } \\
\text { (man-hours) }\end{array}$ & SURVIVE Lite & 8 & 9 \\
\hline 1.6 & Firepump (of damaged zone) (man-hours) & SURVIVE Lite & 2 & 1 \\
\hline 1.7 & $\begin{array}{l}\text { Overall firepump system (man-hours/no of } \\
\text { equipment) }\end{array}$ & SURVIVE Lite & 8 & 10 \\
\hline 1.8 & NBCD stores - aft FRP section base & SURVIVE Lite & 1 & 1 \\
\hline 1.9 & NBCD stores - fwd FRP section base & SURVIVE Lite & 1 & 1 \\
\hline 1.10 & Remaining NBCD stores & SURVIVE Lite & 2 & 1 \\
\hline 1.11 & Power (of damaged zone) (man-hours) & SURVIVE Lite & 2 & 1 \\
\hline 1.12 & $\begin{array}{l}\text { Overall power system (man-hours/no of } \\
\text { equipment) }\end{array}$ & SURVIVE Lite & 8 & 10 \\
\hline 1.13 & SCC (HQ1) (man-hours) & SURVIVE Lite & 6 & 6 \\
\hline 1.14 & Bridge (HQ2) (man-hours) & SURVIVE Lite & 2 & 4 \\
\hline 1.15 & Ops. Room (man-hours) & SURVIVE Lite & 6 & 10 \\
\hline 1.16 & Aft FRPP & SURVIVE Lite & 10 & 5 \\
\hline 1.17 & Fwd FRPP & SURVIVE Lite & 10 & 5 \\
\hline
\end{tabular}


PMs 1.1-1.4 in Table 4.3 are related to the ease of access of the damaged part of the ship from the FRP section bases, i.e. they are human factor PMs. Values for those PMs were taken directly from the CAD software used for the ship design studies, Paramarine. PMs 1.1 and 1.2 are directly related to the time needed to reach the damaged WT section of the ship. For PM 1.4, which can be interpreted as the number of alternative routes between the FRP section bases and the damaged portion of the ship, the inverse is taken, in order to remain consistent with the point made above, i.e. the larger the value of a specific PM, the worst its performance in survivability terms. The term 'average' is contained in the description of PMs 1.1, 1.2 and 1.3 (Table 4.3) as it was decided that each ship design would include two FRP section bases; therefore, the corresponding values for the two bases were averaged. PM 1.3 is related to the ease of (vertical) access and personnel evolutions within the damaged section in order to deal with the damage and remove casualties. In addition, the larger the number of decks in the hit WT section, the greater extent of a resultant fire.

Values for the remaining PMs in Table 4.3 (as well as the ones in Table 4.4 and Table 4.5), all of which have the indication 'SURVIVE Lite' in the software column, were obtained by means of the following procedure: First, the output from the SURVIVE Lite models indicated if the specified item had been affected by a specific hit. All unaffected items were given a value of zero. The affected items, i.e. all items with vulnerability greater that $0 \%$ (with the exception of PMs 1.8-1.10 and 1.16-1.17) were given a PM value based on the assumed number of man-hours to repair that specific equipment. Values for the number of man-hours necessary to repair each equipment item are detailed in Section 4.3.5. PMs 1.8-1.10 (stores) and 1.16-1.17 (humans) were assumed to be unrecoverable and were, therefore, given a value of 0 if unaffected by a hit, and 1 if affected. Although SURVIVE Lite was used to indicate what equipment items had been affected by a given threat, no vulnerability percentages were used in the recoverability assessment method. This is because, in order to carry out recoverability assessment, vulnerability (and susceptibility) needed to be taken as unity. Therefore, even if SURVIVE Lite outputted a vulnerability value of $0.1 \%$ for a specific item, it was assumed that, for recoverability assessment purposes, that item had been definitively hit, i.e. vulnerability $=1$.

PMs 1.5-1.7 are related to the propagation and suppression of fire and smoke after a ship has been damaged. PM 1.5 looks at the state of the ventilation system of a ship after attack, upon which smoke spread and containment are heavily reliant. The indication 'of damaged zone' is included in the description of PM 1.5, as it was decided 
that each zone of each ship designed should have an independent non-cross-connected ventilation system. Therefore, the smoke hazards in a particular zone of the ship should be directly dependent on the state of the ventilation system of that zone after attack. PMs 1.6 and 1.7 examine the state of the fire fighting systems of a ship after attack. As with the ventilation system, each zone of each ship designed had an independent firemain system, however, the firemain systems were assumed to be cross-connected. This justifies the existence of two PMs for fire suppression, one for the damaged zone and one for the overall ship. Although a firemain system contains a number of connectivity items (in addition to the actual firepumps) those were not modelled due to the level of design detail in the concept ship design studies. As explained previously, this is a common simplification step in survivability analysis of concept stage ship designs (see Section 3.3.3). Therefore, the whole firemain system of a ship was represented exclusively by its firepumps. In PM 1.7, the number of man-hours required to repair the affected firepumps was divided by the total number of firepumps in that ship design. Thus, the more firepumps there are on the ship (increasing redundancy), the smaller the value of the PM. This was done as the firepumps were assumed to be crossconnected in parallel rather than in series, i.e. if one firepump is out of action, the ship's whole firemain system remains operational.

PMs 1.8-1.10 are related to the manual equipment necessary for DCFF, and their performance reflects on the state of the NBCD stores after a given attack. Again it was decided that each zone of each ship designed should have independent NBCD stores. Given that usually there are two FRP section bases on a modern combatant (Figure A36), the FRP section bases and the NBCD stores, in the zones where the FRP section bases were located, would be positioned appropriately (PMs 1.8 and 1.9). A separate PM for the remaining NBCD stores was created (PM 10). Note that for PM 1.10, the number of remaining NBCD stores affected by a given hit was divided by the total number of remaining NBCD stores to take redundancy into account (as with PM 1.7).

PMs 1.11 and 1.12 are related to the state of the power generating system of a ship after attack. Power is not only required for the ship to attempt to carry on its mission even if hit, but also to operate many of the DCFF equipment necessary to deal with the internal battle. In addition, complications in the power generation and distribution system could lead to hazardous situations while fire fighting. These two PMs function in an identical manner to PMs 1.6 and 1.7 respectively, as described above. 
PMs 1.13-1.15 are related to command, control and communication during the DCFF process. PM 1.13 is related to the state of the SCC (with which HQ1 is usually collocated) after a given attack. Similarly, PM 1.14 is related to the state of the Bridge (with which HQ2 is usually collocated) and PM 1.15 to the state of the Operations Room, after a given attack. The importance of these three compartments is highlighted in Appendix 4.3: in RN ships, overall DC responsibility and priority setting belongs to the $\mathrm{CO}$, who is usually at the Operations Room or bridge (Clements and Kneebone 1985). He is in direct communication with the NBCDO (usually the MEO) located at HQ1, and the WEO located in the Operations Room. The NBCDOs responsibilities include DC co-ordination (damage state information, resource availability and allocation, containment and restoration actions), while the WEOs respectively is combat system availability (Clements and Kneebone 1985). In addition to DC, the MEOs responsibilities include propulsion and power. The $\mathrm{CO}$, therefore, has to manage and balance the internal and external battles.

Finally, PMs 1.16 and 1.17 are related to the state of the FRPs after a specified attack. The FRPs are responsible for dealing with the secondary effects immediately following a hit, and containing the damage. Each ship was assumed to include two FRPs, as previously indicated, split forward and aft. More FRPs could relatively easily be incorporated by increasing the number of PMs. This would be relevant in ship types such as LPDs, LPHs and CVs.

Since the Category 1 PMs (Table 4.3) relate to the immediate actions/effects of an ASM hit, secondary damage effects (i.e. fire damage in this case) were not accounted for.

\subsubsection{Category 2 Performance Measures}

The second category of PMs, given in Table 4.4 together with the associated weighting scheme, relates to the items necessary for major system recovery (i.e. recovery support), once the secondary effects have been dealt with; whereby, 'major system recovery' refers to the modelled systems listed in Section 4.2 and Table 4.1 and Table 4.2. 


\begin{tabular}{|r|l|l|r|r|}
\hline \multicolumn{2}{|c|}{ Table 4.4: Major System Recovery Performance Measures (Category 2) } \\
\hline \multirow{2}{*}{} & PM & Software & \multicolumn{2}{|c|}{ Weighting } \\
\cline { 3 - 5 } & & & Combatant & AOR \\
\hline $\mathbf{2 . 1}$ & Aft workshops (man-hours) & SURVIVE Lite & 3 & 5 \\
\hline $\mathbf{2 . 2}$ & Fwd workshops (man-hours) & SURVIVE Lite & 3 & 5 \\
\hline $\mathbf{2 . 3}$ & Naval stores & SURVIVE Lite & 1 & 2 \\
\hline $\mathbf{2 . 4}$ & Aft spare gear stores & SURVIVE Lite & 7 & 5 \\
\hline $\mathbf{2 . 5}$ & Fwd spare gear stores & SURVIVE Lite & 7 & 5 \\
\hline $\mathbf{2 . 6}$ & SCC (updated value) (man-hours) & SURVIVE Lite & 6 & 9 \\
\hline $\mathbf{2 . 7}$ & Ops. Room (updated value) (man-hours) & SURVIVE Lite & 7 & 8 \\
\hline
\end{tabular}

Values for the above PMs would be obtained in an identical manner as the PMs in Table 4.3, with the indication 'SURVIVE Lite', under the Software column. That is to say, PM 2.3-2.5 (stores) were assumed to be unrecoverable, therefore, given a value of 0 if unaffected by a specific hit, and a value of 1 if affected. The remaining PMs were valued in terms of man-hours to repair that compartment (if affected) after a given attack (Section 4.3.5). Again, SURVIVE Lite was used to indicate which compartments have been affected by a specific missile attack.

PMs 2.1 and 2.2 attempt to characterize the state of a ship's workshops after an attack. Workshops might be necessary in order to repair major equipment, especially ones related to the power generating system of a naval ship, although it is accepted that most repair work is currently conducted on site. It was assumed that the ships to be designed would include split forward and aft integrated workshops; hence the two workshops related PMs (although, this could easily be altered in order to accommodate a different number of workshops).

PMs 2.3-2.5 are related to the state of the ship's naval and spare gear stores after a given attack. These stores contain equipment, spare parts and raw material which may be necessary in order to repair and put back into action an affected major ship system. It was assumed that the ships to be designed would include a single naval store and split forward and aft spare gear stores, although, once again, this arrangement could be altered.

Finally, PMs 2.6 and 2.7 relate to the condition of the SCC (and, hence, HQ1) and the Operations Room after a given attack. As clarified in Section 4.3.1 these two compartments are necessary for command, control and communication purposes. The NBCDO positioned in the SCC is responsible, amongst other tasks, for coordinating 
restoration efforts of the major systems damaged after the attack and the proper operation of the power and propulsion systems.

This category of PMs includes fire effects. (Therefore, PMs 2.6 and 2.7 are 'updated value' of PMs 1.13 and 1.15, including secondary effects, as indicated at the description of the PMs in Table 4.4). Fire effects are included since these PMs 'relate to the items necessary for major system recovery, after secondary effects have been dealt with'. Since no fire spread simulation techniques were incorporated, it was assumed that all equipment and compartments (only) within the hit WT section would be affected by the resulting fire. Therefore, for Category $2 \mathrm{PM}$ analysis, all items within the hit WT section, in addition to all other items affected by the missile hit (outputted by SURVIVE Lite) were assumed to be affected.

\subsubsection{Category 3 Performance Measures}

The third and final PM group (Category 3) is listed in Table 4.5, together with the associated weighting scheme. This category includes PMs relevant to the recovery of the specific major systems modelled, listed in Section 4.2 (Table 4.1 and Table 4.2). The Category 3 PMs were applied to all of those major systems, in a manner that is described in Section 4.3.4. 


\begin{tabular}{|c|c|c|c|c|}
\hline & \multirow{2}{*}{$\mathbf{P M}$} & \multirow{2}{*}{ Software } & \multicolumn{2}{|c|}{ Weighting } \\
\hline & & & Combatant & AOR \\
\hline 3.1 & $\begin{array}{l}\text { Minimum man-hours for system to } \\
\text { be functioning }\end{array}$ & SURVIVE Lite & 10 & 10 \\
\hline 3.2 & $\begin{array}{l}\text { Number of man-hours for system to } \\
\text { be } 100 \%\end{array}$ & SURVIVE Lite & 3 & 5 \\
\hline 3.3 & Access measure from naval stores & $\begin{array}{l}\text { SURVIVE Lite and } \\
\text { Paramarine }\end{array}$ & 1 & 2 \\
\hline 3.4 & $\begin{array}{l}\text { Access measure from aft spare gear } \\
\text { stores }\end{array}$ & $\begin{array}{l}\text { SURVIVE Lite and } \\
\text { Paramarine }\end{array}$ & 3 & 5 \\
\hline 3.5 & $\begin{array}{l}\text { Access measure from aft } \\
\text { workshops }\end{array}$ & $\begin{array}{l}\text { SURVIVE Lite and } \\
\text { Paramarine }\end{array}$ & 2 & 5 \\
\hline 3.6 & $\begin{array}{l}\text { Access measure from fwd spare } \\
\text { gear stores }\end{array}$ & $\begin{array}{l}\text { SURVIVE Lite and } \\
\text { Paramarine }\end{array}$ & 3 & 5 \\
\hline 3.7 & $\begin{array}{l}\text { Access measure from fwd } \\
\text { workshop }\end{array}$ & $\begin{array}{l}\text { SURVIVE Lite and } \\
\text { Paramarine }\end{array}$ & 2 & 5 \\
\hline 3.8 & $\begin{array}{l}\text { Equipment in damaged section } \\
\text { measure }\end{array}$ & $\begin{array}{l}\text { SURVIVE Lite and } \\
\text { Paramarine }\end{array}$ & 8 & 10 \\
\hline
\end{tabular}

PMs 3.1 and 3.2 attempt to give a description of the severity of damage to a major system of a ship after a specific incident and the time needed to restore it. The score of PM 3.1 would be equal to the total number of man-hours required to repair all damaged equipment items of a specific major system which are in series (tree diagrams of all major system modelled can be found in Appendix 7). Thus, this PM, by taking equipment redundancy into consideration, describes the minimum time needed to get an affected major system back into action. On the other hand, the value of PM 3.2 is equal to the total number of man-hours required to repair every single equipment item of an affected major system, whether in series or in parallel. This can be illustrated by considering the simplified system tree diagram below. 


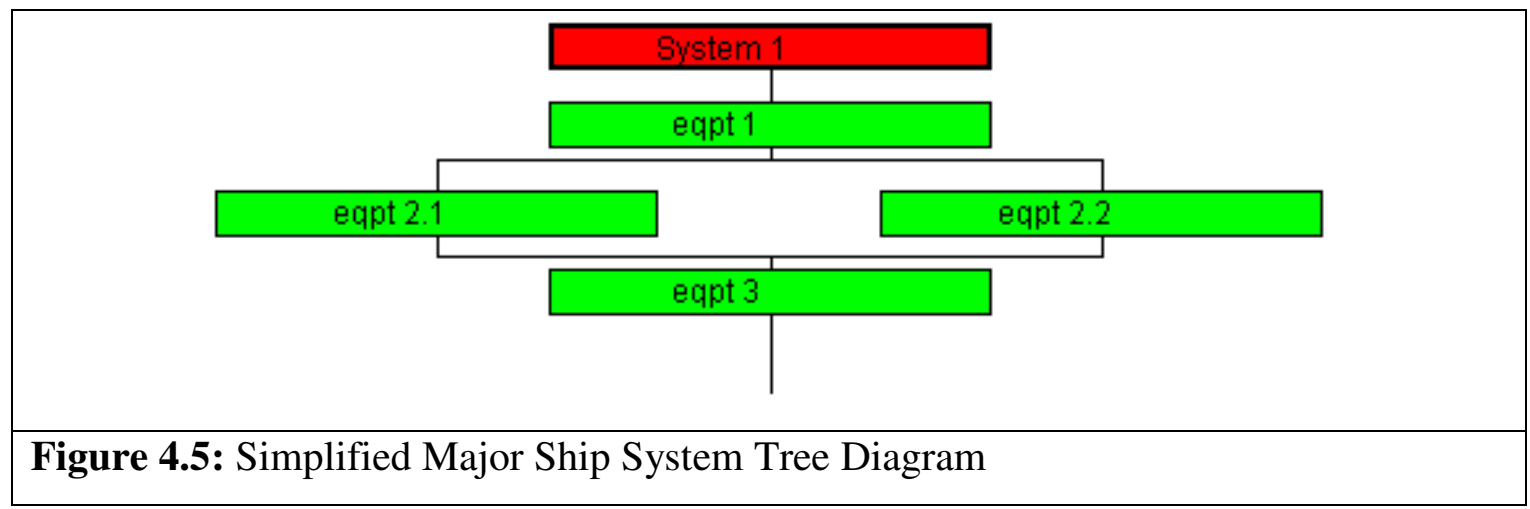

Consider that, after a particular incident, all equipment items of System 1 have been affected, putting System 1 out of action. The value of PM 3.1 would be equal to the sum of the number of man-hours needed to repair eqpt 1, eqpt 3 and either eqpt 2.1 or eqpt 2.2 (whichever is less); while the value of PM 3.2 would be the number of manhours required to repair all four damaged equipment items, therefore, not taking redundancy into account. PM 3.2 increases in importance when follow-up attacks are expected.

PMs 3.3-3.8 endeavour to reflect on the difficulty in accessing equipment which has been damaged after a specific attack and, therefore, the effort required to repair them. The significance of such factors is clear when considering that most of the repair work will generally be carried out on site. The above access measures are then quantified by multiplying the criticality of affected equipment items by the number of man-hours needed to repair that equipment item having been affected by the specific hit (as revealed by SURVIVE Lite). Criticality, in this context, is defined as:

$$
\begin{array}{r}
\text { Criticality }=1 \div \text { (number of equipment items (of a } \\
\text { specific major system) in parallel) }
\end{array}
$$

For example, if there are four propellers in a ship, all providing the same capability, each has a criticality of $1 / 4$. Alternatively, when considering System 1 in Table 4.5, eqpt 1 and eqpt 3 each have a criticality of 1 , whilst eqpt 2.1 and eqpt 2.2 have a criticality of $1 / 2$. This was done in order to denote the diminishing importance of a specific equipment item should there be an increasing number of equipment items that can carry out the same functions (i.e. redundancy/duplication) and thus, the value of the PM is proportionately decreased to commensurate with the damaged equipment's associated redundancy level. However, PMs 3.3-3.7 were only applied to damaged equipment requiring personnel to cross the damaged WT section in order to get access from the 
corresponding store/workshop. In addition PM 3.8 was obtained by multiplying the criticality (Equation 5) by the number of man-hours needed to repair each equipment item which was affected by the given hit and was located in the damaged WT section in question. Hence the software column of Table 4.5 indicates 'SURVIVE Lite and Paramarine', as Paramarine was required to locate the affected equipment items. For example, suppose that System 1 (Figure 4.5) was laid out as illustrated in Figure 4.6 in a typical frigate and all naval and spare gear stores and workshops are co-located at the compartment shown in green.

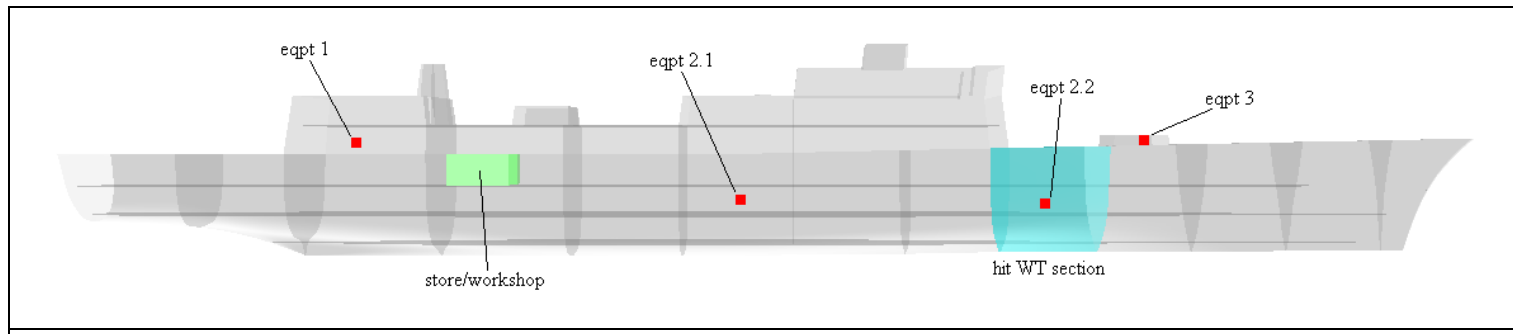

Figure 4.6: Example Architecture of Simplified Major Ship System 1

In this case, given an ASM hit at the WT section shown (and supposing that all four equipment items of System 1 have been affected by the hit), the values of PMs 3.33.7 would be identical and equal to the criticality of eqpt 3 (i.e. 1, as previously explained) multiplied by the number of man hours needed to repair that equipment item. The value of PM 3.8 would be equal to the criticality of eqpt 2.2 (i.e. $1 / 2$ ) multiplied by the number of man-hours required to repair that equipment item. Although eqpt 1 and eqpt 2.1 have also been damaged by the incident, access to them is assumed unobstructed, and, therefore, they do not contribute to those, access related, PMs. If there were more damaged equipment items of System 1 forward of the hit WT section in Figure 4.6, the values of PMs 3.3-3.7 would be the sum of the products of each item's criticality and repair time. Likewise for PM 3.8, should there been more System 1 equipment items inside the damaged WT section.

Secondary (fire) damage effects were included in PM Category 3 analysis in the same manner to that for such effects being accounted for in PM Category 2 analysis (Section 4.3.2). 


\subsubsection{Recoverability Performance Measures Matrix}

As mentioned in Section 4.2, SURVIVE Lite was run separately to simulate an ASM hit at each WT section of each ship designed, as illustrated in Figure 4.4. Therefore, values for all 32 PMs were obtained for a given hit at each WT section. PM matrices, such as the one shown in Table 4.6 (for a combatant) were then completed to analyse the recoverability of each ship design studied. This procedure is now explained in more detail. 


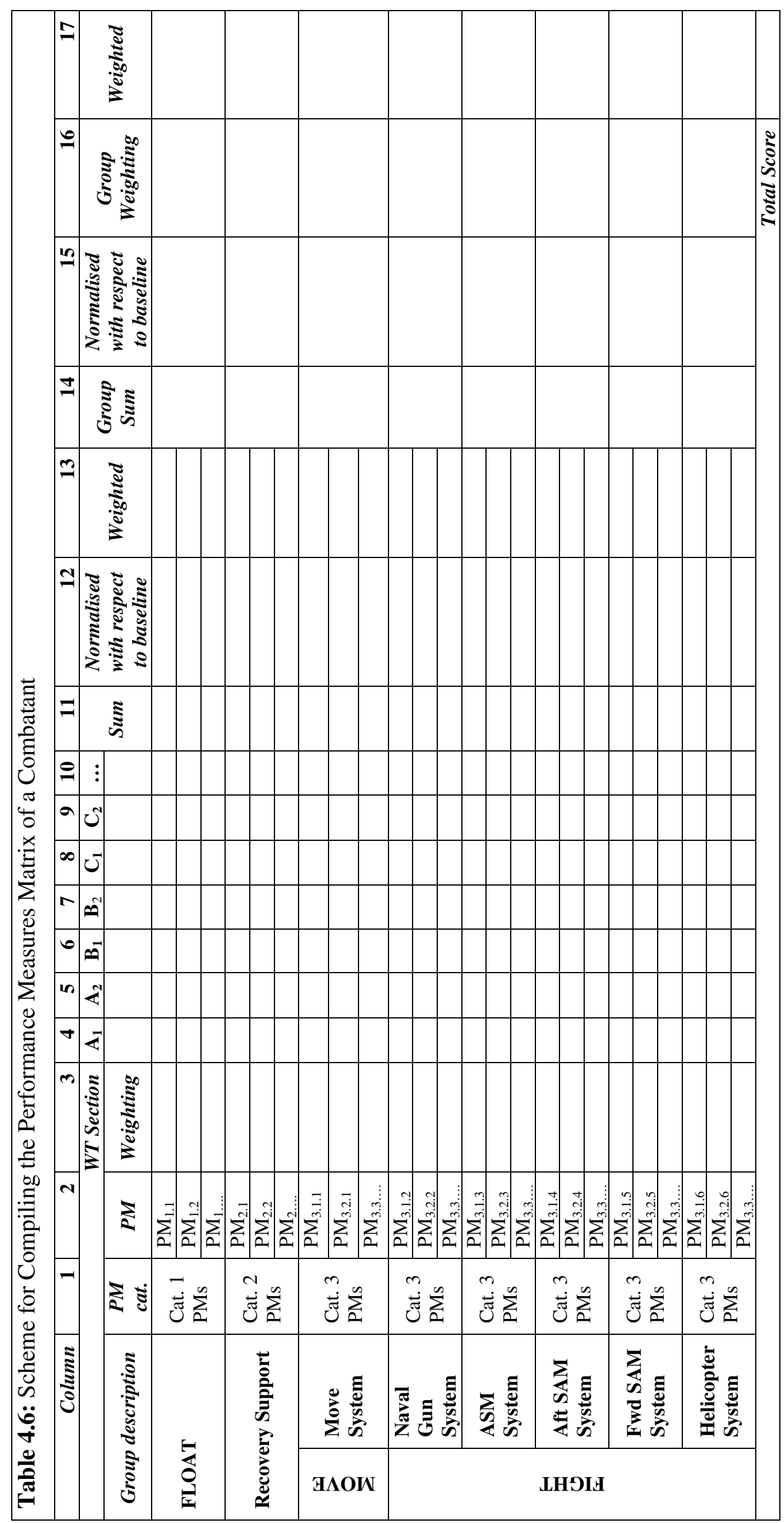


Firstly, from columns 1 and 2 it can be observed that the Category 3 PMs were applied to all of the (six in the combatant case) major systems modelled. This is because those PMs are related to the recovery of the specific major systems modelled (see Table 4.1 and Table 4.2). The weightings in column 3 are the weightings of each individual PM and are detailed in Table 4.3, Table 4.4 and Table 4.5.

Columns 4, 6, 8.. represent all of the WT section of each ship design analysed. In the cells of these columns, the values of the corresponding PMs (from column 2) were entered, (following the procedure detailed in Sections 4.3.1 to 4.3.3) giving values for all PMs for a separate hit at each WT section of each ship. The values of each PM at each WT section attack scenario were then multiplied by the probability that that WT section was hit, and the results entered in the cells of columns 5, 7, 9... The probability that a WT section was hit was computed through the lengthwise hit probability distribution described in Section 4.1, where the sum of the probabilities that each WT section of a ship design is hit was equalled to unity, i.e. it was assumed that the ship is definitely hit (susceptibility $=1$ ) which (together with vulnerability = 1) are requirements for the conduction of recoverability analysis. Thus, it can be said that each PM was weighted, not only by the importance of that PM (column 3) but also by the likelihood of hit at a specific location on the ship affecting that PM. In addition, this enabled the comparison of the recoverability performance of ship designs with differing numbers of WT bulkheads.

The next step (column 11) was to compute the sum of columns 5, 7, 9... along the entire length of the ship being analysed. Thus, summing the products of the value of each PM at each WT section attack scenario and the probability that that WT section has been hit, which gave the total ship value of each of the 32 PMs developed. In column 12, these total PM values were normalised with respect to the corresponding values of a baseline ship design, and in column 13, the normalised PM values were weighted given the weightings in column 3 (Table 4.3, Table 4.4 and Table 4.5).

The sum of the normalised and weighted PM values was then computed for each PM Category (column 14). For Category 3 PMs, a separate sum was produced for each of the major systems modelled, as shown in Table 4.6. These group sums were then once again normalised with respect to the corresponding values of the same baseline ship (column 15). Finally, these normalised group sums were weighted (column 17) with the group weightings (column 16), shown in Table 4.7 for the case of a combatant. As before, this weighting scheme was derived with the assistance of $1^{\text {st }}$ Lt. Fonseca. 


\begin{tabular}{|c|c|c|c|c|}
\hline \multicolumn{2}{|c|}{ Group Description } & PM cat. & Group Weighting & \\
\hline \multicolumn{2}{|l|}{ FLOAT } & Cat. 1 PMs & 10 & $=10$ \\
\hline \multicolumn{2}{|c|}{ Recovery support } & Cat. 2 PMs & 2 & \\
\hline MOVE & Move system & \multirow{6}{*}{ Cat. 3 PMs } & 9 & $=9$ \\
\hline \multirow{5}{*}{ FIGHT } & Naval gun system & & 2 & \\
\hline & ASM system & & 2 & \\
\hline & Aft SAM system & & 1 & \\
\hline & Fwd SAM system & & $\mathbf{1}$ & \multirow[b]{2}{*}{$=8$} \\
\hline & Helicopter system & & 2 & \\
\hline
\end{tabular}

The weightings used for each PM category is in accordance with the warship objective hierarchy 'to float, to move, to fight'. For this reason, Category 1, which consists of PMs related to immediate effects on DCFF, upon which the Float objective at the top of the warship hierarchy depends, was given the highest weighting. This was followed by the repair and recovery of the Move and then the Fight related major systems, (Category $3 \mathrm{PMs}$ ) which followed in weighting significance. In order to maintain consistency, the weighting scheme adopted for the move and fight groups was identical to that used in the vulnerability assessment element of the proposed approach (Section 4.2), listed in Table 4.1. Finally, it is worth mentioning that Category 2, consisting of PMs supporting system recovery, was considered separately from the three main warship objectives as it was seen to have an impact on all three. It is difficult to quantify the magnitude of this impact; however, an indication can be given by the (subjective) weightings that were assigned to this PM Category by various naval officers during the sensitivity tests (see Sections 6.4.1 and 6.6.1)

After obtaining all of the entries of column 17 in Table 4.6, the final step was to calculate the sum of these entries, thereby evaluating the total recoverability performance score of a particular ship design. This procedure led to a single figure for a ship design's recoverability performance (similarly to the ship's susceptibility performance, Section 4.1, and vulnerability performance Section 4.2). However, unlike susceptibility (but corresponding to vulnerability performance, Section 4.2), this single value has no meaning (and cannot even be derived) unless a ship design is compared to at least one other ship design. Therefore, as explained in Section 4.2, this procedure is strictly comparative and does not output an absolute recoverability performance value; 
rather, it outputs a recoverability performance value normalised with respect to a baseline ship design.

A PM matrix for a replenishment ship design is shown in Table 4.8 with the major systems modelled, already listed in Section 4.2 and Table 4.2. Furthermore, the group weightings (column 16 of Table 4.8) are also shown in Table 4.9. Note that the recoverability weighting scheme of a replenishment ship design was derived with the assistance of Lt. Cdr. (rtd.) Day; hence the dissimilar weighting scheme philosophy when compared to the recoverability weighting scheme of a combatant (Table 4.7), which was derived by a different officer serving in a different navy and for a ship with a different role. As in the combatant case, in order to maintain consistency, the weighting scheme of the Move and Fight groups is identical to that used in the vulnerability assessment element of the method (Section 4.2), listed in Table 4.2. Finally, the headings of columns 12 and 15 were changed from 'normalised with respect to baseline' to 'normalised with respect to worst performing'. The logic behind this is explained in Section 7.2.4. 


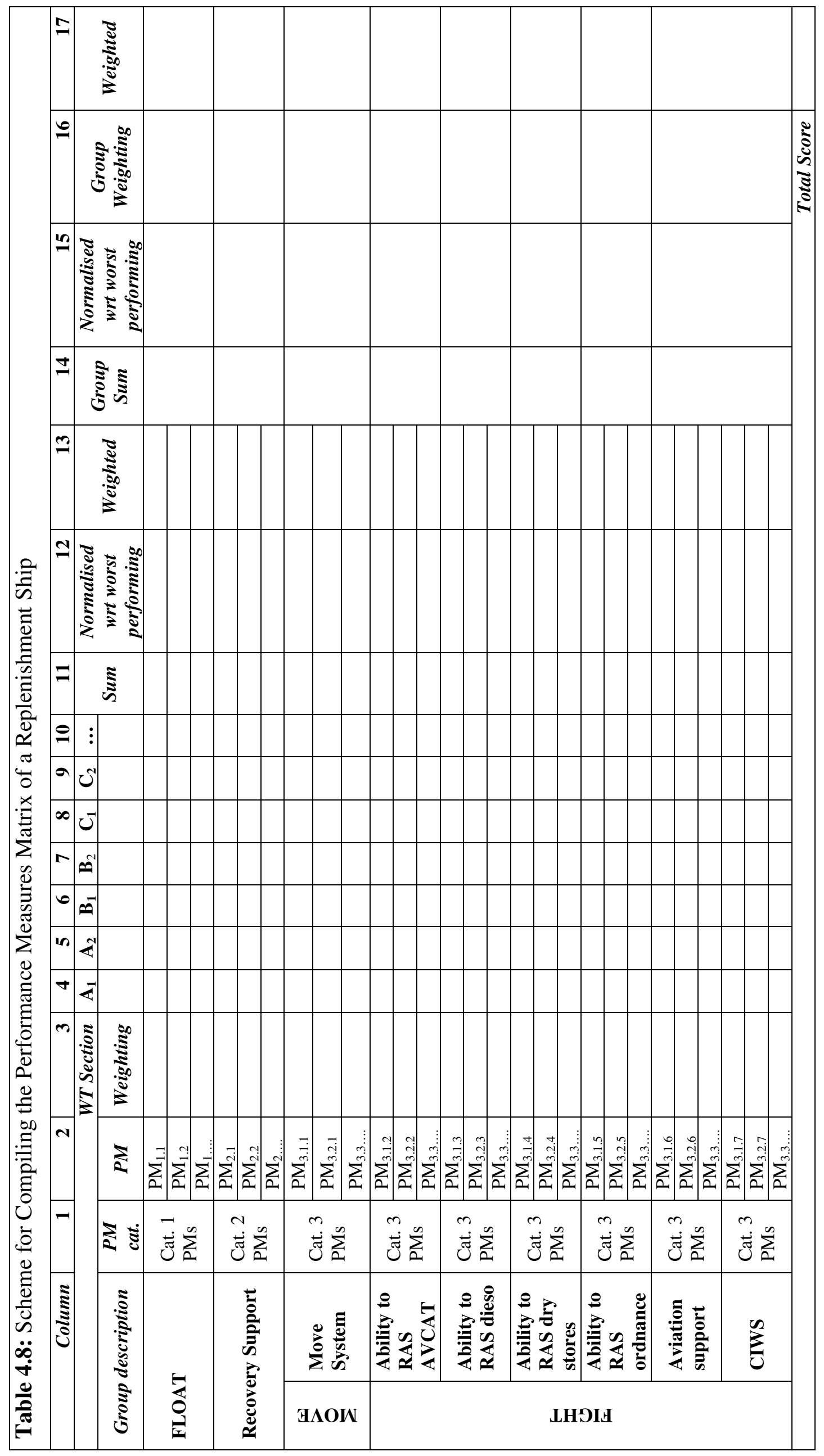




\begin{tabular}{|c|c|c|c|c|}
\hline & Group Description & PM cat. & Group Weighting & \\
\hline \multicolumn{2}{|l|}{ FLOAT } & Cat. 1 PMs & 10 & $=10$ \\
\hline \multicolumn{2}{|c|}{ Recovery support } & Cat. 2 PMs & 3 & \\
\hline MOVE & Move system & \multirow{7}{*}{ Cat. 3 PMs } & 10 & $=10$ \\
\hline \multirow{6}{*}{ FIGHT } & Ability to RAS AVCAT & & 10 & \multirow[b]{6}{*}{$=39$} \\
\hline & Ability to RAS dieso & & 10 & \\
\hline & Ability to RAS dry stores & & 6 & \\
\hline & Ability to RAS ordnance & & 8 & \\
\hline & Aviation support & & 2 & \\
\hline & CIWS & & 3 & \\
\hline
\end{tabular}

\subsubsection{Equipment Categorisation and Repair Data}

The majority of the recoverability PMs, (i.e. PMs 1.5-1.17 in Table 4.3, and all PMs in Table 4.4 and Table 4.5, which are related to specific equipment/compartments), were given values based on the assumed number of man-hours to repair a specific equipment or compartment if damaged after a given incident. It is axiomatic that, had an equipment/compartment not been affected by a specific attack, the corresponding PM would be given a value of zero. Man-hour data for the repair of various equipment categories (shown in Table 4.10) were provided by Dstl (Thornton 2011). In addition, they are the same values as were employed by QinetiQ in SURVIVE's recently developed recoverability module, with the exception of the $12^{\text {th }}$ category. The fact that humans (i.e. personnel) and stores were assumed to be unrecoverable if affected by a hit, led the necessity for this $12^{\text {th }}$ repair category. This category applied to PMs $1.8-1.10$, 1.16, 1.17 in Table 4.3 and 2.3-2.5 in Table 4.4, which, as explained in Section 4.3.1, were given a value of zero if unaffected and one if affected by a specific attack modelled. 
Table 4.10: Equipment Repair Categories

\begin{tabular}{|l|l|r|r|r|}
\hline & Category & Repair time (hrs.) & \multicolumn{1}{l|}{ Crew } & \multicolumn{1}{l|}{ Man-hours } \\
\hline 1 & Heavy engineering & 4 & 4 & 16 \\
\hline 2 & Light engineering & 2 & 2 & 4 \\
\hline 3 & Heavy electrical & 0.5 & 1 & 0.5 \\
\hline 4 & Light electrical & 5 & 3 & 15 \\
\hline 5 & Large pumps & 4 & 2 & 8 \\
\hline 6 & Small pumps & 0.5 & 2 & 1 \\
\hline 7 & Radar aerials & 0.5 & 2 & 1 \\
\hline 8 & Fluid tanks & 1.5 & 2 & 3 \\
\hline 9 & Aircraft & 3 & 1 & 1 \\
\hline 10 & Rudder/propeller & infinite & infinite & infinite \\
\hline 11 & Intake/exhaust & & & 1 \\
\hline 12 & Humans and stores & & & 2 \\
\hline
\end{tabular}

The data in Table 4.10 is remarkably simplified, since there are only 12 repair categories covering a very broad spectrum of equipment types, with different requirements, from different manufacturers, built under different standards. In addition, no reference to severity of damage was made. It is considered that more detailed and accurate data should be used, however, repair times are largely based on factors which cannot be easily quantified, such as crew skills. Another point worth noting is the fact that the categories in Table 4.10 are different to the equipment categories used by SURVIVE Lite for vulnerability assessment (QinetiQ 2011b), although both sets were developed by the same company and are used in the same software, SURVIVE.

Table 4.11 details the repair category to which each of the equipment items and compartments relevant to Category 1 and 2 PM analysis (PMs 1.5-1.17 in Table 4.3 and 2.1-2.7 in Table 4.4) was assumed to belong. Also listed are the corresponding SURVIVE Lite categories (i.e. the category to which each item was assumed to belong when building the SURVIVE Lite ship models for vulnerability assessment). The vulnerability and recoverability categorisation of all equipment items and compartments from which are comprised the major ship systems modelled (and which were used for Category 3 PM analysis in Section 4.3.3), is given in Appendix 6. Moreover, system tree diagrams are included in Appendix 7. 


\begin{tabular}{|c|c|c|c|}
\hline Relevant PMs & $\begin{array}{l}\text { Equipment / } \\
\text { compartment }\end{array}$ & $\begin{array}{c}\text { SURVIVE Lite } \\
\text { (vulnerability) } \\
\text { category }\end{array}$ & $\begin{array}{c}\text { Repair (recoverability) } \\
\text { category }\end{array}$ \\
\hline 1.5 & ATU \& ventilation & $\begin{array}{l}\text { Light engineering / } \\
\text { (silo/magazine) }\end{array}$ & Light engineering \\
\hline $1.6,1.7$ & Fire pump & Pump & Small pumps \\
\hline $\begin{array}{l}1.8,1.9,1.10 \\
2.3,2.4,2.5\end{array}$ & Stores & $\begin{array}{l}\text { Light engineering / } \\
\text { (silo/magazine) }\end{array}$ & Humans and sores \\
\hline $1.11,1.12$. & Auxiliary engine & Diesel generator & Heavy engineering \\
\hline $1.11,1.12$. & Cruise engine & Diesel generator & Heavy engineering \\
\hline $1.11,1.12$ & Genset & Diesel generator & Heavy engineering \\
\hline $1.11,1.12$. & Boost engine & Gas turbine & Heavy engineering \\
\hline $1.11,1.12$ & Alternator & Gas turbine & Heavy engineering \\
\hline $1.11,1.12$ & Intake/exhaust & Uptake / down takes & Intake/exhaust \\
\hline $1.13,2.6$. & $\mathrm{SCC}$ & Control console & Light electrical $\times 5$ \\
\hline 1.14 & Bridge & Control console & Light electrical $\times 7$ \\
\hline $1.15,2.7$ & Operations Room & Control console & Light electrical $\times 12$ \\
\hline $1.16,1.17$ & Humans & Human & Humans and sores \\
\hline $2.1,2.2$. & Workshops & $\begin{array}{l}\text { Light engineering / } \\
\text { (silo/magazine) }\end{array}$ & Light engineering \\
\hline
\end{tabular}

The vulnerability equipment category of power generating equipment is related to the power and propulsion systems selected for the ship design studies to which the method was applied, detailed in Chapter 5. Furthermore, for the replenishment ship designs it was decided not to include boost propulsion prime movers. It is observed that the repair categories for the SCC, bridge and Operations Room compartments result in a multiplication of the number of man-hours applicable to the light electrical repair category (i.e. 1/2 man-hour, Table 4.10) and 5, 7 and 12 respectively. This arose since it was decided that these compartments of the ship designs would contain 5, 7 and 12 control consoles respectively, and the time needed to repair each, if each were damaged, would have been the time given in the light electrical repair category in Table 4.11. 


\subsection{Total Survivability Assessment}

The next step, after finalising the susceptibility and vulnerability assessment approaches and developing a new, architecturally-driven recoverability assessment approach, was to combine the three constituents in order to create a total survivability assessment approach. However, as mentioned in Section 3.3.5, it has been argued that the three survivability constituents are not statistically independent (Sajdak and Karni 2006; Thornton et al 2006). Therefore, relationships such as Equation 1 in Section 3.3.5, where survivability is assumed to be directly proportional to the product of its three constituents are not thought to be valid. Sajdak and Karni (2006) suggested that total survivability should be a function of its three constituents (Equation 2), rather than the product. For the above reasons, and after studying work done by Vasudevan and Rusling (2007) on the development of a ship design tool based on multi-objective optimisation using genetic algorithms, it was decided that the output results of the three survivability constituent assessment methods detailed above were considered separately and combined (for visualisation and comparison purposes) in the form of star plots (or triangle plots in the current case, as only three variables are presently being compared). An advantage of this type of presentation of the results is that further spokes, such as cost, displacement and power can be added; a limitation, however, is that it can only depict characteristics that are currently amenable to quantification (Vasudevan and Rusling 2007). There is also the issue of how the diagram would be interpreted, with regards to the significance of the enclosed area. Visualisation and comparison methods are an area for further development. Each star plot presented by Vasudevan and Rusling (2007) represents a single ship design, and each spoke within each star represents a design parameter. A sample of star plots, as a form of results visualisation, from Vasudevan and Rusling's (2007) research is shown in Figure 4.7.

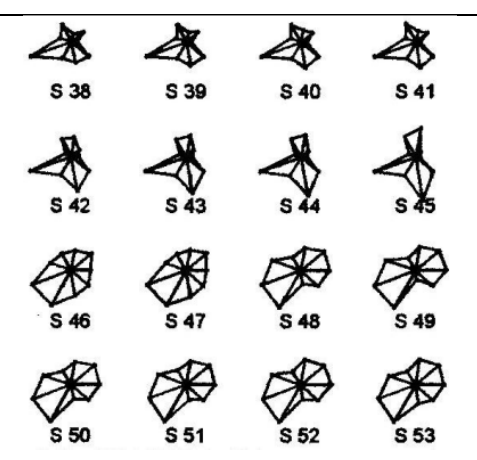

Figure 4.7: A Sample of Star Plots From (Vasudevan and Rusling 2007) 
It was also decided to examine the applicability of PM matrices, such as Table 4.6, for total survivability assessment. This was done by multiplying the percentage values of total ship susceptibility, and vulnerability of each equipment/compartment, with each PM. Therefore, unlike the approach adopted in Section 4.3, susceptibility and vulnerability were not assumed to be equal to unity. More specifically, all PMs which were derived from the Paramarine ship models (i.e., PMs 1.1-1.4, Table 4.3) and those PMs related to stores and humans (i.e., PMs 1.8-1.10, 1.16 and 1.17, Table 4.3; PMs 2.3-2.5, Table 4.4) were not only multiplied by susceptibility values of the corresponding WT section (as described in Section 4.3.4), but also by the total ship susceptibility percentage. For the remaining Category 1 and 2 PMs, values of which were based on repair man-hour data (i.e., PMs 1.5-1.7 and 1.11-1.15, Table 4.3; PMs 2.1, 2.2, 2.6 and 2.7, Table 4.4), the number of man-hours to repair the relevant item were multiplied not only by the susceptibility of the hit WT section (as described in Section 4.3.4), but also by the total ship susceptibility and the vulnerability of that item, given a hit in that WT section.

Similarly, for Category 3 PM analysis, Table 4.5 , the procedure summarised in Section 4.3.3 was followed, however, on this occasion, vulnerability data of the relevant equipment/compartments were multiplied by the number of man-hours required to repair them in order to obtain the value of each PM for each major system. These PM values were then multiplied by the total ship susceptibility, as well as that of the WT section in question. Regarding all PMs which account for secondary effects (i.e. Category 2 and $3 \mathrm{PMs}$ ), the additional vulnerability due to fire effects was included in the PM scoring procedure by using the output of SURVIVE Lite's Fast Fire method (Sharp 2011) (see Section 3.3.4). The reason why store and personnel related PMs were only multiplied by susceptibility data (without involving vulnerability) was that since the time for these items to recover was assumed to be infinite (Table 4.10), multiplication of the recovery time by the items' vulnerability resulted in an infinite solution. Therefore, once again, the PMs were given a value of zero if the item remained unaffected and unity if it were affected by a given hit. This was then multiplied by the total ship susceptibility and the WT section susceptibility, as explained above.

This proposed procedure involves multiplications of susceptibility, vulnerability and recoverability data, which is questionable. However, it was decided to carry out this process on several ship designs (see Section 6.7) for comparison purposes, and examine emergent advantages and disadvantages which are summarised in Section 7.4.2. 


\section{Chapter 5: Ship Design Studies}

This chapter gives a description of the ship designs to which the survivability assessment method was applied. In total, seven designs were developed. Five were combatant type ships and included three frigate variants (one of which adopted a trimaran hullform), a corvette and a destroyer. The remaining two design studies were AOR type ships with varying internal and external configuration.

As mentioned in Section 2.1.4, survivability is largely dependent on ship architecture. Moreover, the recoverability assessment method developed was aimed at looking specifically at how ship layout affects recovery from attack damage. It was therefore decided that the ship designs on which the survivability method should be applied would be designed by means of an architecturally orientated preliminary ship design approach. Further advantages when using a configurationally orientated preliminary design process were discussed in Section 2.1.4. It was concluded that the Design Building Block approach to preliminary ship design, through its SURFCON implementation in the Paramarine CASD software (Section 2.1.5), which has been extensively used and proven by the Design Research Centre of the Marine Research Group of the Department of Mechanical Engineering at UCL (http://www.ucl.ac.uk/mecheng/research/marine), would be the most appropriate architecturally orientated preliminary design approach to utilise. The above procedure to preliminary ship design was described in (Andrews and Pawling 2003). The method of 'circles of influence' proposed by Andrews $(1984$; 1986) in order to appropriately position a ship's principal compartments and build up its architecture, Figure 2.5, was extensively used, through specific detailed frigate related 'circles of influence' diagrams which were published by Dicks (2000).

All ship studies designed in this investigation were designed and sized according to the procedures, data and parametric relationships available for the ship design projects in the MSc in Naval Architecture at UCL (UCL 2010a, UCL 2010b) and other references such as (UCL 2006a; UCL 2006b; Saunders 2008; IAI 2009; Wartsila 2010; Vestdavit 2012). In addition all studies were designed with the intent of maximising survivability based on relevant literature summarised in Appendix 4. For example, concentration and separation of duplicated systems was incorporated and all superstructure sides were designed with a $7^{\circ}$ tumblehome.

GAs of all ships designed are included in Appendix 8. 


\subsection{Frigate Design Studies}

Frigate type ships are the most common, blue water capable, type of warship, providing a point of reference for most new developments in naval ship design. Three general purpose, general ocean going sea control frigates were designed, all of which were fitted with an identical weapon fit, listed in Table 5.1. The same table conveys the main payload equipment corresponding to the major Fight systems which were assessed in the vulnerability and recoverability methods described above. However, it should be noted that each Fight system includes further items such as sensors, control rooms and power generation units (full system diagrams are shown in Appendix 7).

\begin{tabular}{|c|c|}
\hline \multicolumn{2}{|l|}{ Table 5.1: Frigate Payload } \\
\hline Payload Equipment & FIGHT Systems \\
\hline $1 \times$ BAE Systems $155 \mathrm{~mm}$ gun & Medium calibre naval gun system \\
\hline $2 \times 4$ Harpoon launchers & ASM system \\
\hline $\begin{array}{l}4 \times \text { MBDA MICA PDMS with } 32 \text { MICA missiles } \\
(4 \times 8 \text { round VL launchers })\end{array}$ & Aft/fwd SAM system \\
\hline $\begin{array}{l}1 \times \text { Lynx (Kestrel) Helicopter }+ \text { hangar and single } \\
\text { spot flight deck for EH-101Merlin }\end{array}$ & Helicopter system \\
\hline $\begin{array}{l}2 \times \text { MSI Seahawk 30mm } \\
2 \times \text { Triple barrel torpedo tubes } \\
4 \times \text { Rheinmetall MASS Decoy Launchers } \\
1 \times \text { Surface Ship Torpedo Defence System } \\
2 \times \text { Navigation radars } \\
1 \times \text { Single Face SR STAR Surveillance Radar } \\
1 \times \text { General Purpose Electro Optical Device } \\
2 \times \text { Thales Sirius IRST } \\
1 \times \text { Raytheon AN/SLQ-32(V)3 Shipboard ESM/ } \\
\text { ECM System } \\
1 \times \text { Spherion hull mounted sonar } \\
1 \times \text { Small ship communications system }(1 \times \\
\text { communications mast }+2 \times \text { whip antennae }) \\
1 \times \text { Generic Satellite Communications System } \\
\text { with } 2 \text { antennae } \\
25 \text { Embarked forces }\end{array}$ & $\begin{array}{l}\text { Other payload (certain sensors are } \\
\text { part of the major Fight systems } \\
\text { above; for full system tree } \\
\text { diagrams see Appendix 7) }\end{array}$ \\
\hline
\end{tabular}


In addition, all three frigates had the same performance requirements, listed in Table 5.2.

\begin{tabular}{|l|l|}
\hline \multicolumn{2}{|l|}{ Table 5.2: Frigate Performance Requirements Adopted for Design Studies } \\
\hline Maximum speed & $30 \mathrm{kts}$ \\
\hline Endurance & $7,000 \mathrm{~nm}$ at 15kts \\
\hline Stores & 30 days \\
\hline Stability & $\begin{array}{l}\text { Defence Standard 02-109 (NES 109) intact and damage criteria } \\
\text { assessment (MOD 2000) }\end{array}$ \\
\hline Zoning & 4 zones with maximum independence \\
\hline
\end{tabular}

A summary of the principal particulars of the three frigate variants is given in Table 5.3, with GAs in Appendix 8. Note that Variant 3 adopted a trimaran configuration.

\begin{tabular}{|c|c|}
\hline \multicolumn{2}{|c|}{ Table 5.3: Principal Particulars of the Frigate Variants Investigated } \\
\hline \multicolumn{2}{|l|}{ Variant 1} \\
\hline Dimensions & $\begin{array}{l}132.2 \mathrm{~m}(125.8 \mathrm{~m}) \times 16.1 \mathrm{~m}(15.2 \mathrm{~m}) \times 9.7 \mathrm{~m}(\text { deep draught } 4.0 \mathrm{~m}) \text {; deep trim } 0.6 \mathrm{~m} \text {, light trim } \\
0.8 \mathrm{~m}\end{array}$ \\
\hline Hullform Parameters & $\mathrm{C}_{\mathrm{B}}: 0.487, \mathrm{C}_{\mathrm{P}}: 0.596, \mathrm{C}_{\mathrm{M}}: 0.818, \mathrm{C}_{\mathrm{W}}: 0.755$, Circular M: 8.1 \\
\hline Displacement & 3,890te deep, 3,270te light \\
\hline Maximum Speed & $30.5 \mathrm{kts}$ \\
\hline Range & $7,100 \mathrm{~nm}$ at $15 \mathrm{kts}, 6,000 \mathrm{~nm}$ at $18 \mathrm{kts}$ \\
\hline Power Plant & $\begin{array}{l}1 \times 31 \mathrm{MW} \text { GT (boost), } 2 \times 2.94 \mathrm{MW} \text { diesels (cruise), } 2 \times 2.69 \mathrm{MW} \text { diesels (auxiliary) } \\
\text { driving two FPPs on } 20 \mathrm{MW} \text { HTS motors }\end{array}$ \\
\hline Accommodation & 11 officers, 137 ratings, 25 embarked forces \\
\hline \multicolumn{2}{|l|}{ Variant 2} \\
\hline Dimensions & $\begin{array}{l}125.2 \mathrm{~m}(119.0 \mathrm{~m}) \times 16.1 \mathrm{~m}(15.3 \mathrm{~m}) \times 12.1 \mathrm{~m}(\text { deep draught } 4.4 \mathrm{~m}) \text {; deep trim } 0.4 \mathrm{~m} \text {, light } \\
\text { trim } 0.4 \mathrm{~m}\end{array}$ \\
\hline Hullform Parameters & $\mathrm{C}_{\mathrm{B}}: 0.487, \mathrm{C}_{\mathrm{P}}: 0.596, \mathrm{C}_{\mathrm{M}}: 0.816, \mathrm{C}_{\mathrm{W}}: 0.761$, Circular M: 7.6 \\
\hline Displacement & 4,060te deep, 3,450te light \\
\hline Maximum Speed & $30.4 \mathrm{kts}$ \\
\hline Range & $7,000 \mathrm{~nm}$ at $15 \mathrm{kts}, 5,900 \mathrm{~nm}$ at $18 \mathrm{kts}$ \\
\hline Power Plant & $\begin{array}{l}1 \times 31 \mathrm{MW} \text { GT (boost), } 2 \times 5.22 \mathrm{MW} \text { diesels (cruise), } 2 \times 2.69 \mathrm{MW} \text { diesels (auxiliary) } \\
\text { driving two FPPs on } 21 \mathrm{MW} \text { HTS motors }\end{array}$ \\
\hline Accommodation & 11 officers, 141 ratings, 25 embarked forces \\
\hline \multicolumn{2}{|l|}{ Variant 3} \\
\hline \multirow[t]{3}{*}{ Dimensions } & Overall: $150.3 \mathrm{~m} \times 29.2 \mathrm{~m} \times 12.3 \mathrm{~m}$ (deep draught $5.2 \mathrm{~m}$ ); deep trim $0.6 \mathrm{~m}$, light trim $0.9 \mathrm{~m}$ \\
\hline & Main hull: $150.3 \mathrm{~m}(144.2 \mathrm{~m}) \times 11.6 \mathrm{~m}(10.4 \mathrm{~m}) \times 12.3 \mathrm{~m}($ deep draught $5.2 \mathrm{~m})$ \\
\hline & Side hulls: $51.2 \mathrm{~m}(42.4 \mathrm{~m}) \times 3.7 \mathrm{~m}(1.7 \mathrm{~m}) \times 9.2 \mathrm{~m}($ deep draught $2.7 \mathrm{~m})$ \\
\hline Hullform Parameters & Main hull: $\mathrm{C}_{\mathrm{B}}: 0.532, \mathrm{C}_{\mathrm{P}}: 0.638, \mathrm{C}_{\mathrm{M}}: 0.834, \mathrm{C}_{\mathrm{W}}: 0.831$, Cir. M: 9.0 \\
\hline & Side hulls: $\mathrm{C}_{\mathrm{B}}: 0.138, \mathrm{C}_{\mathrm{P}}: 0.669, \mathrm{C}_{\mathrm{M}}: 0.207, \mathrm{C}_{\mathrm{W}}: 0.678$, Cir. M: 11.3 \\
\hline Displacement & 4,330 te deep $(4,230$ te $+2 \times 50$ te $), 3,820$ te light \\
\hline Maximum Speed & $31.3 \mathrm{kts}$ \\
\hline Range & $7,000 \mathrm{~nm}$ at $15 \mathrm{kts}, 5,900 \mathrm{~nm}$ at $18 \mathrm{kts}$ \\
\hline Power Plant & $\begin{array}{l}1 \times 31 \mathrm{MW} \text { GT (boost), } 2 \times 2.94 \mathrm{MW} \text { diesels (cruise), } 2 \times 2.69 \mathrm{MW} \text { diesels (auxiliary) } \\
\text { driving one FPP on a } 37 \mathrm{MW} \text { HTS motor and one pump-jet on a } 3.5 \mathrm{MW} \text { HTS motor }\end{array}$ \\
\hline Accommodation & 12 officers, 152 ratings, 25 embarked forces \\
\hline
\end{tabular}


The complements and accommodation requirements of the frigate design studies (breakdowns of which are given in Appendix 9.1) were sized based on current combatants' complement/displacement densities calculated from sources such as Saunders (2008).

\section{$\underline{\text { 5.1.1 Baseline Frigate }}$}

The baseline frigate (Frigate Variant 1), shown in Figure 5.1, is a typical modern frigate with a one passing deck hull and a continuous superstructure out to ship's side.

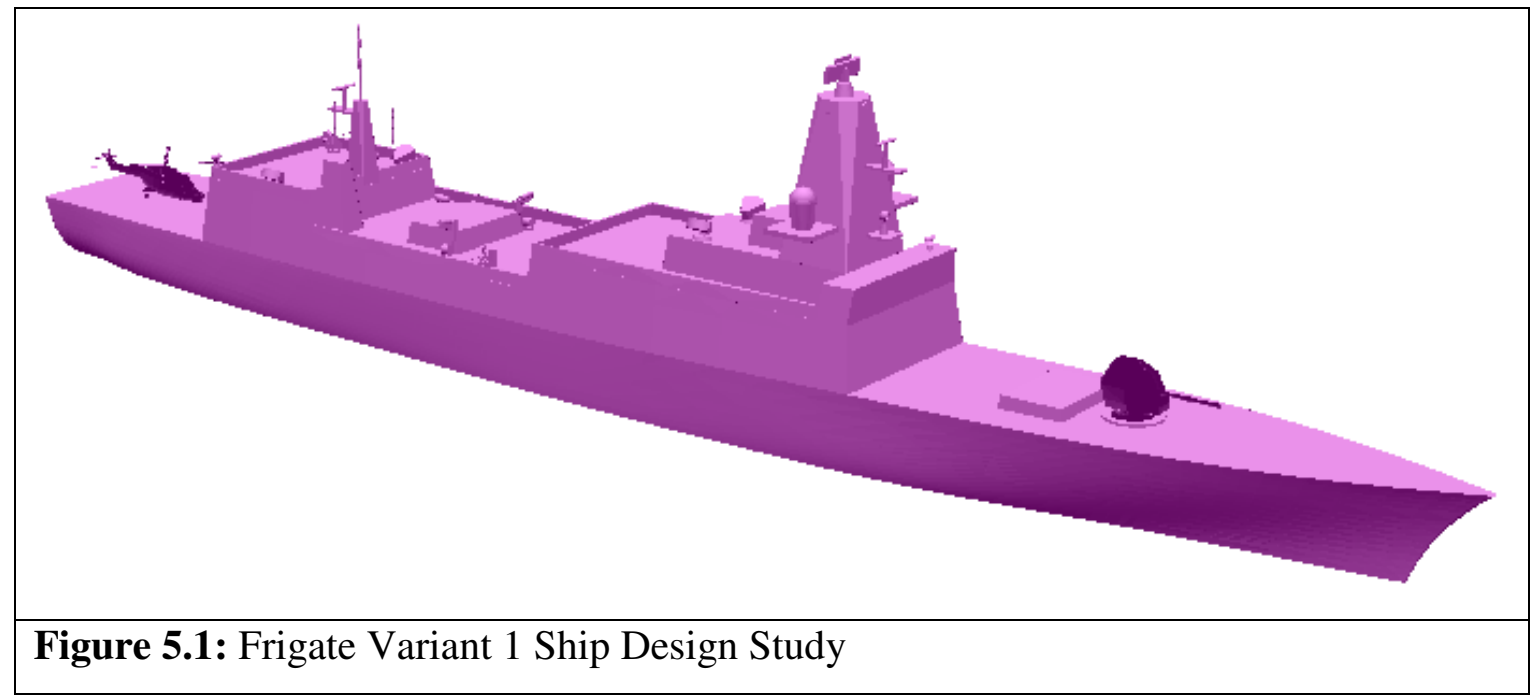

It has previously been argued (Section 2.1.4) that a functional breakdown of a naval ship is more relevant to a weight breakdown, which is the current practice. The broad objectives of a warship have been determined as: 'to float, to move, to fight' (Brown and Tupper 1989). Therefore, the functional breakdown should be expressed in terms of these three groups (Float, Move, Fight) plus the addition of an Infrastructure group as argued by Andrews et al (1996). This functional breakdown was used when designing the ships, as illustrated in Figure 5.2 for Frigate Variant 1. 


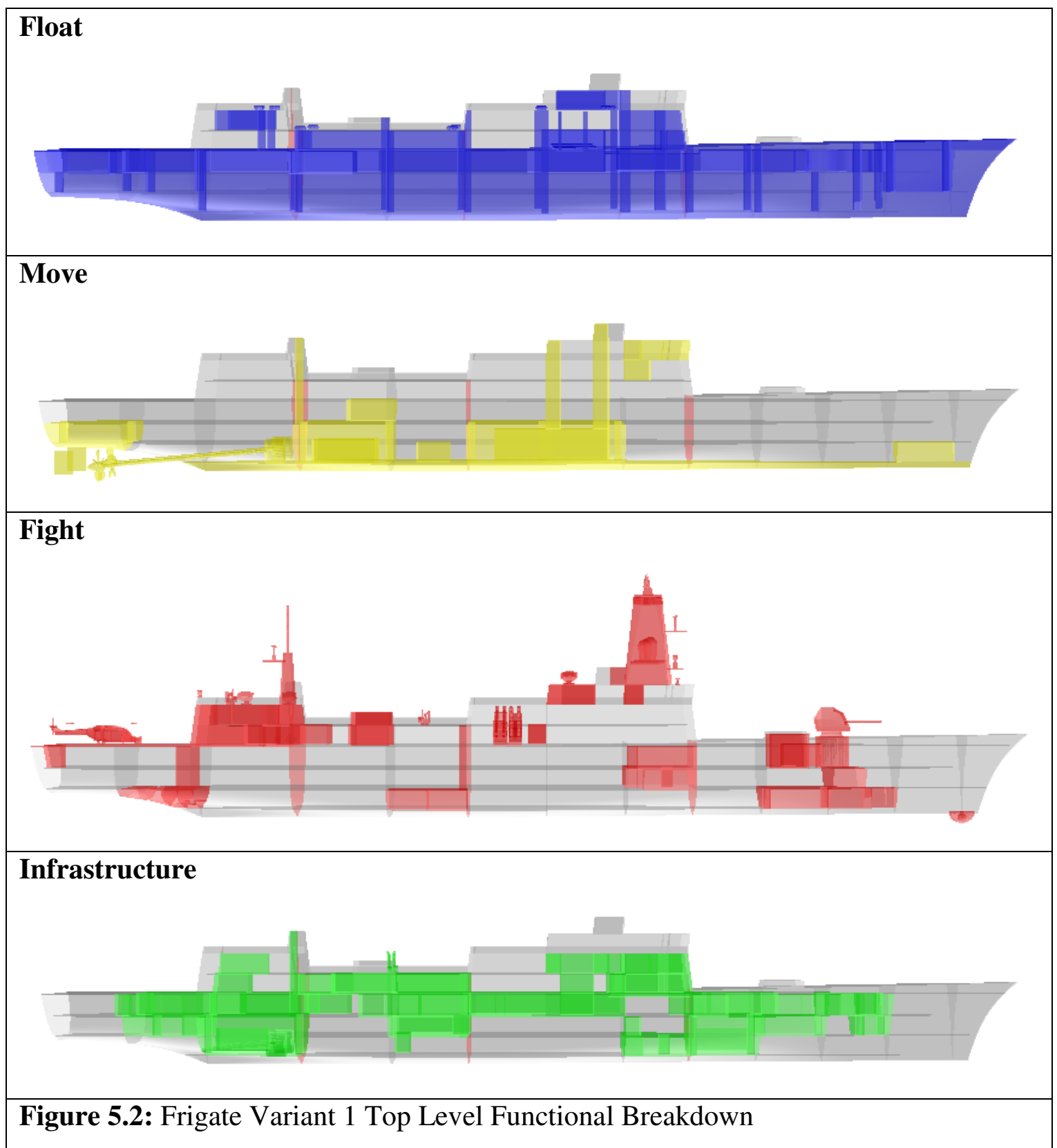

Furthermore, Frigate Variant 1 is described with respect to its top level weight breakdown in Table A8 of Appendix 9.2, and the margin philosophy adopted for this design is summarised in Appendix 9.3.

The hull of the baseline design is based on that of the Type 22 Frigate and was generated using the Quickhull object of Paramarine. The lines plan is shown in Figure 5.3 . 

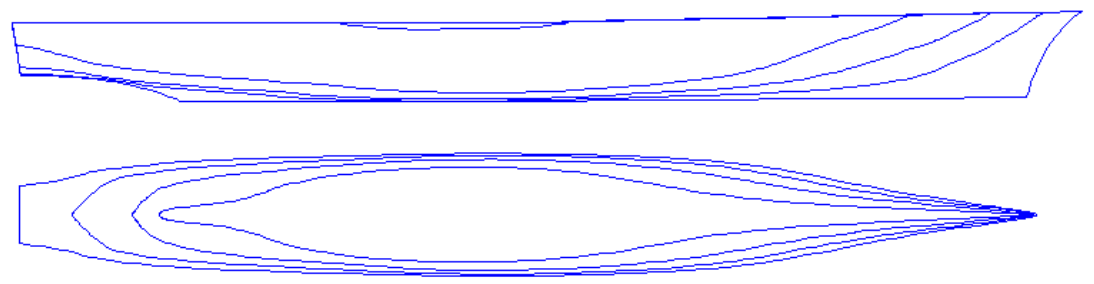

Figure 5.3: Frigate Variant 1 Lines Plan

The propulsive powering requirements were computed using the inbuilt Paramarine powering module (see Appendix 9.4). The machinery that was selected consisted of:-

- $\quad 2 \times 2.94 \mathrm{MW}$ Wartsila 9L26 diesel gensets (cruise);

- $\quad 1 \times 31 \mathrm{MW}$ Rolls-Royce MT30 marine gas turbine, electrical drive (boost);

- $2 \times 2.69 \mathrm{MW}$ Wartsila 16V200 diesel gensets (auxiliary).

The above machinery, driving two FPPs on 20MW HTS motors. Figure 5.4 illustrates the arrangement of the selected machinery, auxiliary machinery shown in green (Infrastructure functional group), and main machinery in yellow (move functional group). This is closely related to the move major system, which was modelled in the vulnerability and recoverability assessment approach, without, however, including steering control units and rudders. Whole major system architecture and tree diagrams are included in Appendix 7.

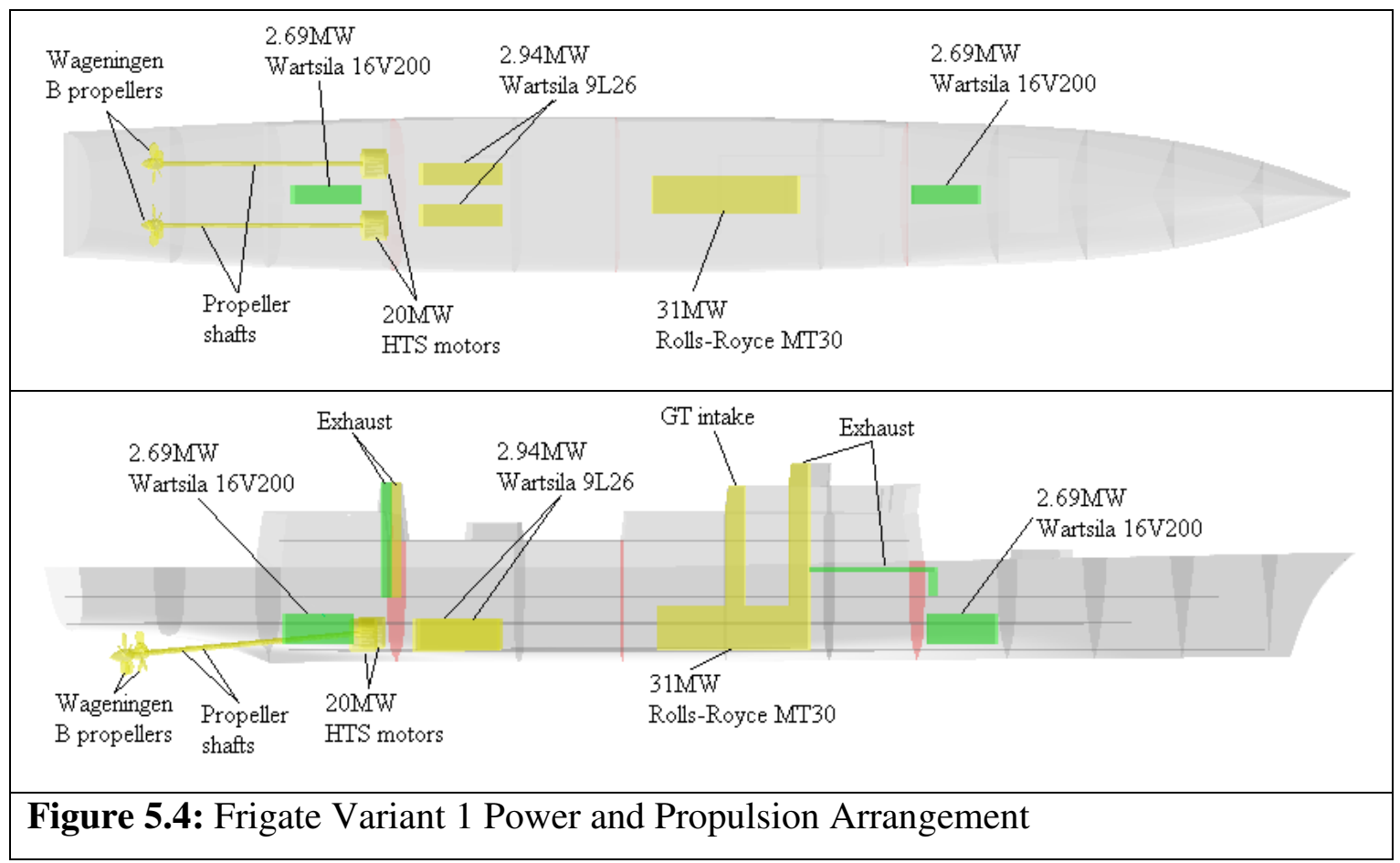


It is clear that the adoption of IFEP has led to the opportunity of widely separating the power generation units (which are in parallel) across the length of the frigate, which leads to significant vulnerability reduction, as summarised in Appendix 4.2. However, this led to the unconventional forward Wartsila 16V200 exhaust arrangement, extending through No 2 Deck until it merges with the gas turbine exhaust since no superstructure is located above this genset. Another vulnerability reduction feature is the fact that short propeller shafts are possible (although this has led to a relatively large declination angle). Removal routes for all engines were taken into account through the inclusion of soft patches in the passageways and compartments above each engine.

The hydrostatics of the baseline frigate were also computed using the inbuilt Paramarine module. Intact stability was assessed against Defence Standard 02-109 (NES 109) Stability Standards for Surface Ships (MOD 2000) until all criteria were passed. Damage stability (of both deep and light conditions) was assessed against Defence Standard 02-109 (NES 109) damage criteria assessment (MOD 2000). Hydrostatics data are given in Appendix 9.5.

It should be mentioned that seakeeping, structural and manoeuvring analysis were not carried out due to the preliminary nature of the design.

Figure 5.5 displays the twelve WT bulkheads (and thirteen WT sections) which are comprised in the hull of the baseline frigate. It should be noted that the bulkhead locations were set by taking into account zoning, structural, layout and damage stability related factors. In order to maintain structural continuity, bulkheads were located at the forward and aft superstructure ends, after cut-up, masts, $155 \mathrm{~mm}$ gun and at every location where there is a step in the superstructure.

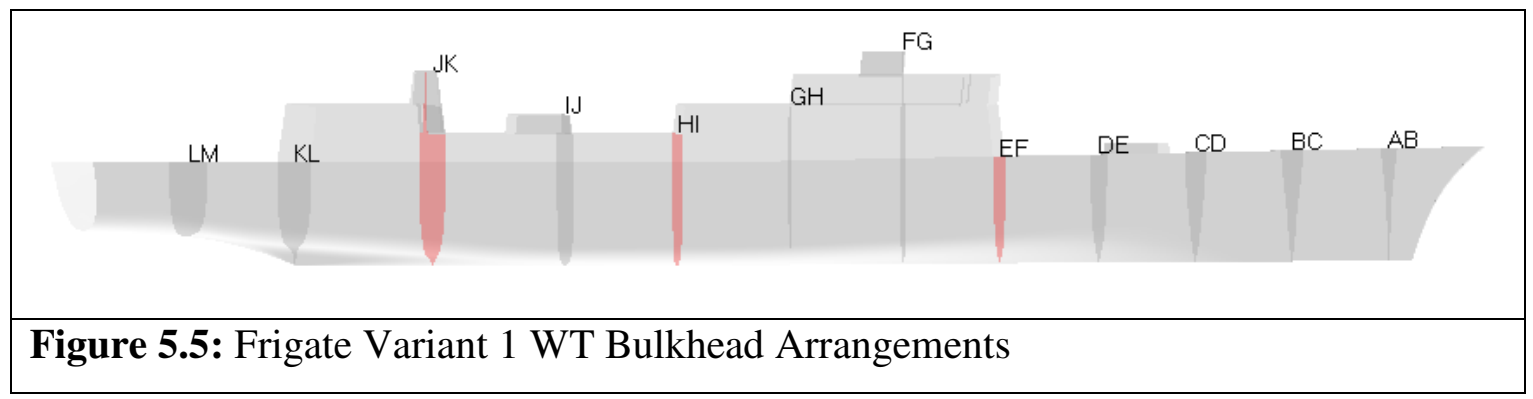

The three bulkheads shown in red in Figure 5.5 constitute the main zone boundaries. From aft to forward, each zone consisted of 3, 2, 3 and 5 WT sections respectively. Each of the four zones contains independent power generation units, CWP, 
HPAC, FP, ATU and ventilation, NBCD stores, and airlocks for access to and from the citadel/weather, as shown in Figure 5.6.

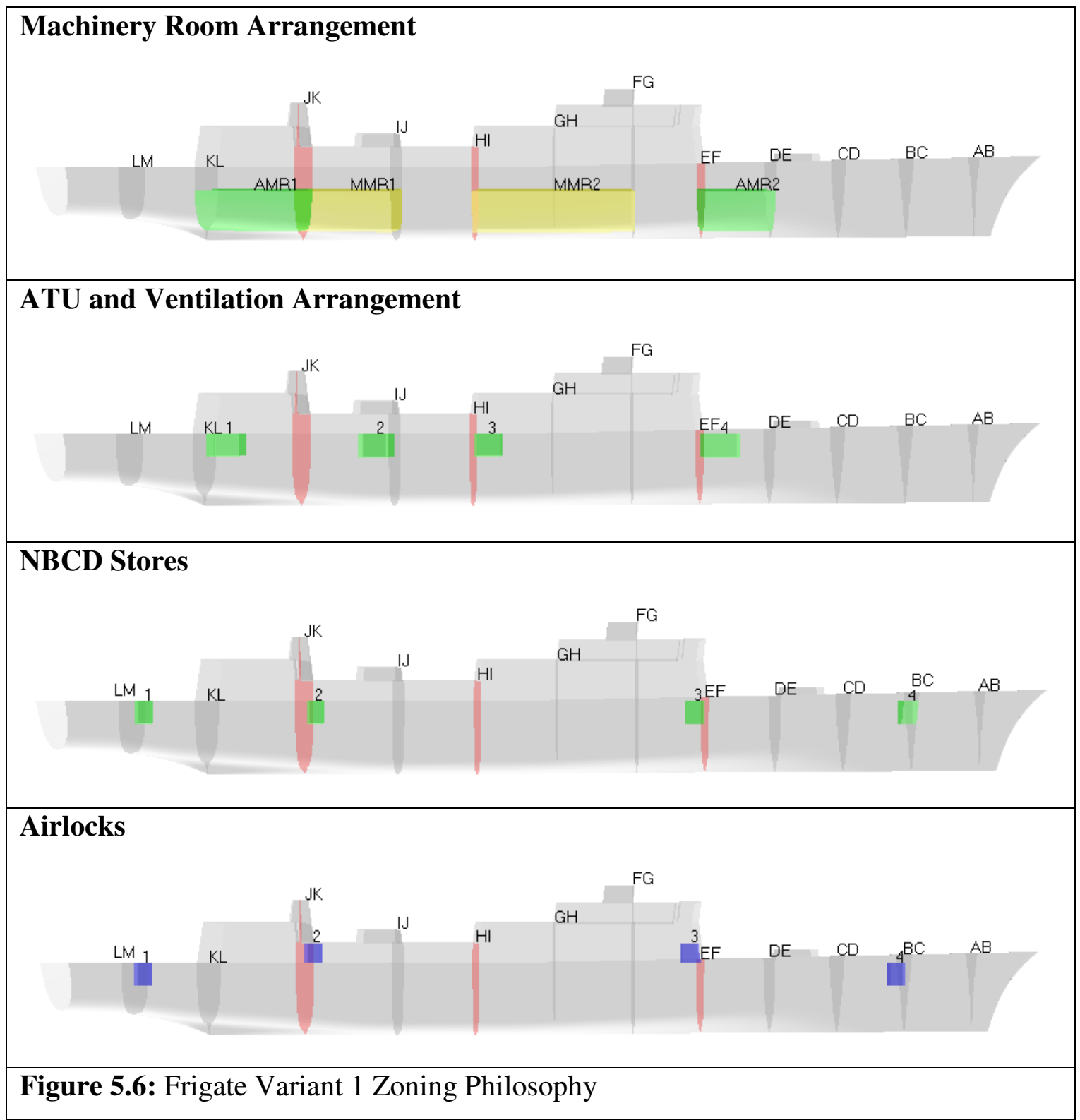

Each zones power generation unit, CWP, HPAC and FP is located in its machinery room. MMR2, in which the Rolls-Royce MT30 marine gas turbine is located (Figure 5.4), extends along the length of two adjacent WT sections since, due to the GTs size, its alternator was placed in a separate WT section from of the engine. ATU and ventilation compartments are located close to areas requiring such services, such as machinery rooms and living spaces (Appendix 8). Moreover, they are located at a reasonable distance from the waterline. NBCD stores are located in proximity to the airlocks. Airlocks 1 and 4 lead to the aft and forward mooring spaces respectively, 
airlock 2 leads to the hangar and airlock 3 leads the forward weather deck. In addition, airlocks 1 and 3 are positioned adjacent to cleansing/decontamination stations.

It was also attempted to distribute the major Fight systems (which were modelled in the vulnerability and the recoverability assessment method) in accordance to the zonal arrangements. Each zone contained the main equipment items of at least one of the five major Fight systems (medium calibre naval gun system, ASM system, aft SAM system, forward SAM system and helicopter system). This not only led to the specialisation of each zone in a specific type of warfare/operations, but also assisted in the concentration of items in series of a particular system, therefore decreasing the systems vulnerability (Appendix 4.2). This is clearly illustrated in Figure 5.7.

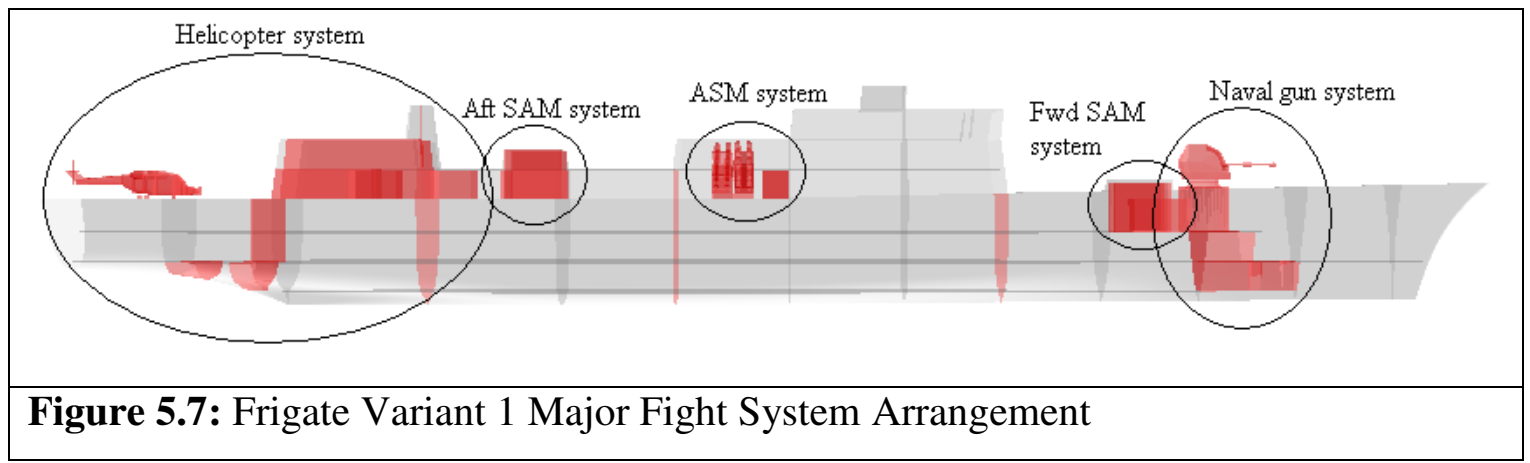

Due to the conventional nature of the baseline frigate, it was relatively easy to achieve the zonal distribution of the major Fight systems. Only the sonobuoy store of the helicopter system (the forward-most compartment of that system) was not managed to be located in the same zone as the remaining equipment and compartments of that system. However, it is important to point out that Figure 5.7 illustrates only the equipment items that are solely related to each of the major Fight systems. Items such as sensors, power generation units and centralised command and control (e.g.: Operations Room) spaces are not included. Whole major system architecture and tree diagrams are included in Appendix 7.

Being a conventional modern frigate, it was decided that the access philosophy adopted for the baseline would be that used in similar existing warships. The hull consisted of 4 decks, one of which, No 2 Deck or the DC deck, was a passing deck. The DC deck contained a centreline passageway and each WT section included two vertical accesses, one at either side of the centreline passageway and at the forward and aft end of each WT section respectively. This is summarised in Figure 5.8 below, and detailed in the GA, Appendix 8. 


\section{Deck Access}

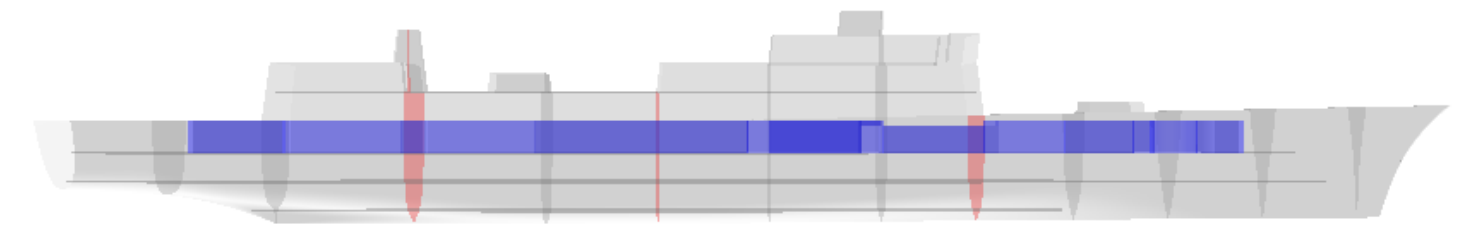

DC Deck Access and Vertical Access

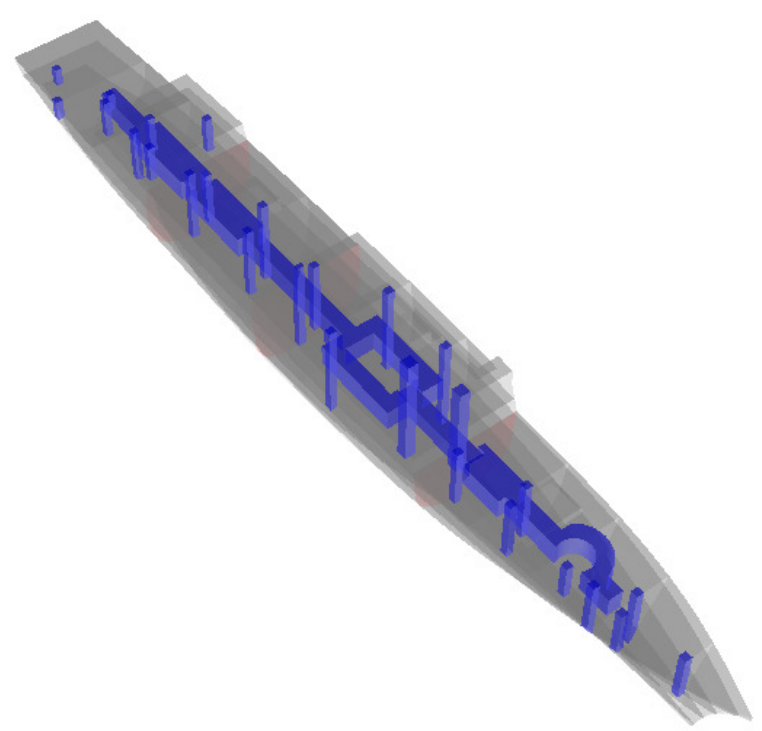

Figure 5.8: Frigate Variant 1 Access Philosophy

It is of significance to examine the location of the compartments and equipment related to each of the recoverability related PMs (Sections 4.3.1, 4.3.2 and 4.3.3). Some of the elements, e.g.: access, WTD (each WT bulkheads has one on the DC deck), ATU and ventilation and NBCD stores have been separately examined. However it would be useful to study the entirety of each PM Category. Figure 5.9 illustrates the distribution of compartments and equipment related to Category 1 PM analysis.

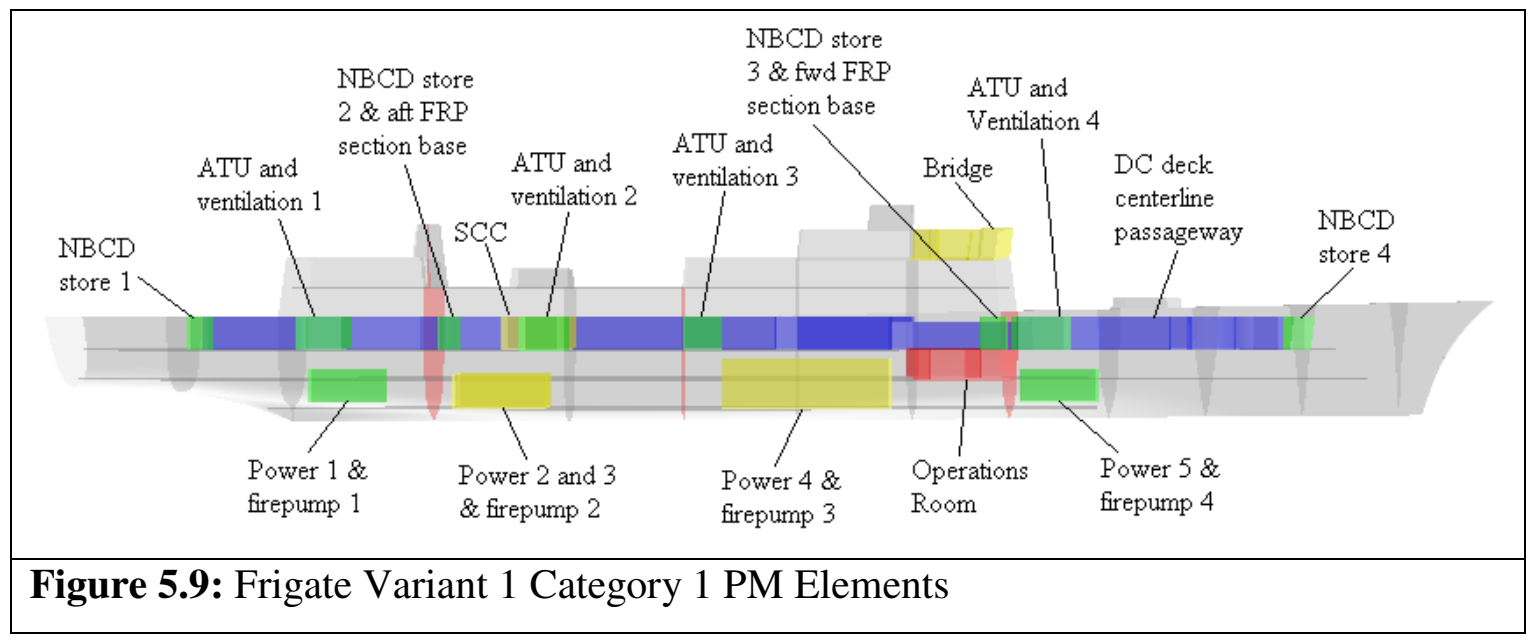


PM 1.1 will be calculated along the centreline DC passageway and PM 1.4 would be based on the width of the passageway, given that there are no alternative routes. In addition, the number of internal decks in each WT section is clear from Figure 5.9 and the value of PM 1.2 is equal to the number of WT bulkheads crossed by each FRP. The two FRP section bases are positioned conveniently to a NBCD store. Furthermore, each FRP section base is situated at a WT section bordering a main zone boundary, for quicker access to the adjacent zone. This way, it can be said that each of the two FRPs are responsible for 2 zones. Note that the firepumps have been collocated with the power generation units in Figure 5.9, since they are both positioned in the machinery rooms, as previously stated. The Operations Room is located deep in the hull (No 3 Deck as in the Type 23 Frigate (Manley 2012)) in order to decrease vulnerability to abovewater attacks; although this could lead to an increase in underwater incidents. The SCC is situated at a distance from the Operations Room so that, in case of an emergency, it could be utilised as a secondary Operations Room. Finally, the bridge and the Operations Room are located at the same WT section in order to improve access and communication between the two spaces in which the $\mathrm{CO}$ is mostly stationed. All other elements are located with respect to the zoning arrangements as described above.

Similarly, Figure 5.10 depicts the arrangement of Category 2 PM analysis (as well as access measure of Category 3 PMs) related elements.

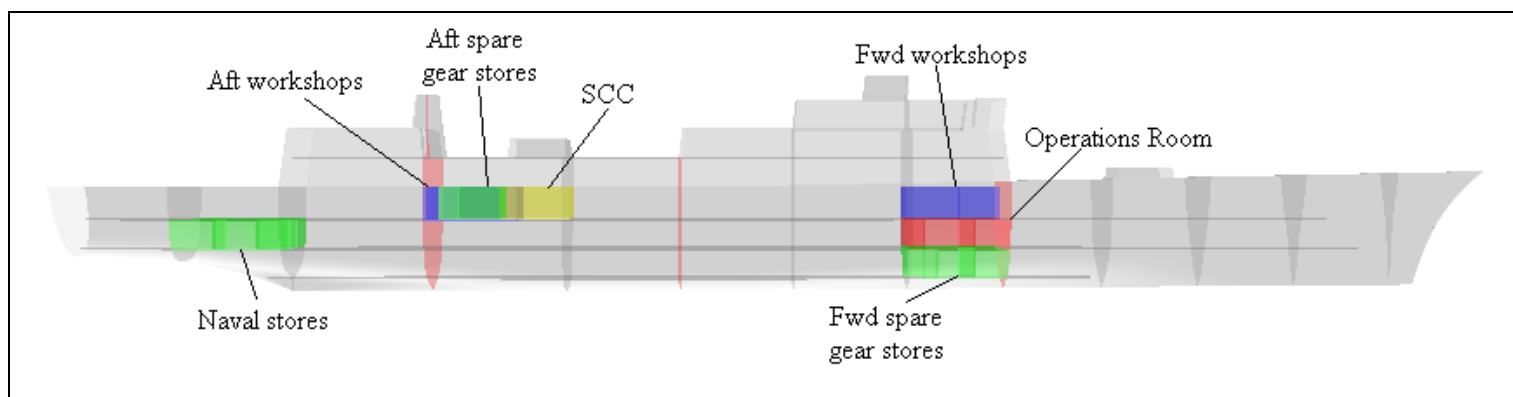

Figure 5.10: Frigate Variant 1 Category 2 PM Elements

Note the proximity of the aft and forward workshops with the aft and forward spare gear stores respectively, one of the many relationships obtained from the 'circles of influence' diagrams presented by Dicks (2000). In addition, the two groups of workshops and spare gears stores were separated as much as reasonably possible, and are located convenient to the machinery rooms for easier and quicker access. The choice of location of the naval stores was principally based on factors such as trim and available (void) space since it is not involved in any complicated architectural 
relationships. Category 3 PM analysis involves all of the components of each major ship system; system architecture illustrations are included in Appendix 7.

\subsubsection{Frigate Variants}

The baseline frigate (Frigate Variant 1) was intended to represent a typical conventional modern frigate type ship. The following two frigate variants would therefore depart from the conventional style of the baseline. Frigate Variant 2, Figure 5.11 , is characterised by the adoption of a deeper, two passing deck hull and a minimal superstructure, and was influenced by the work done by Begg et al (1990). A similar variant was designed by Percival (2010).

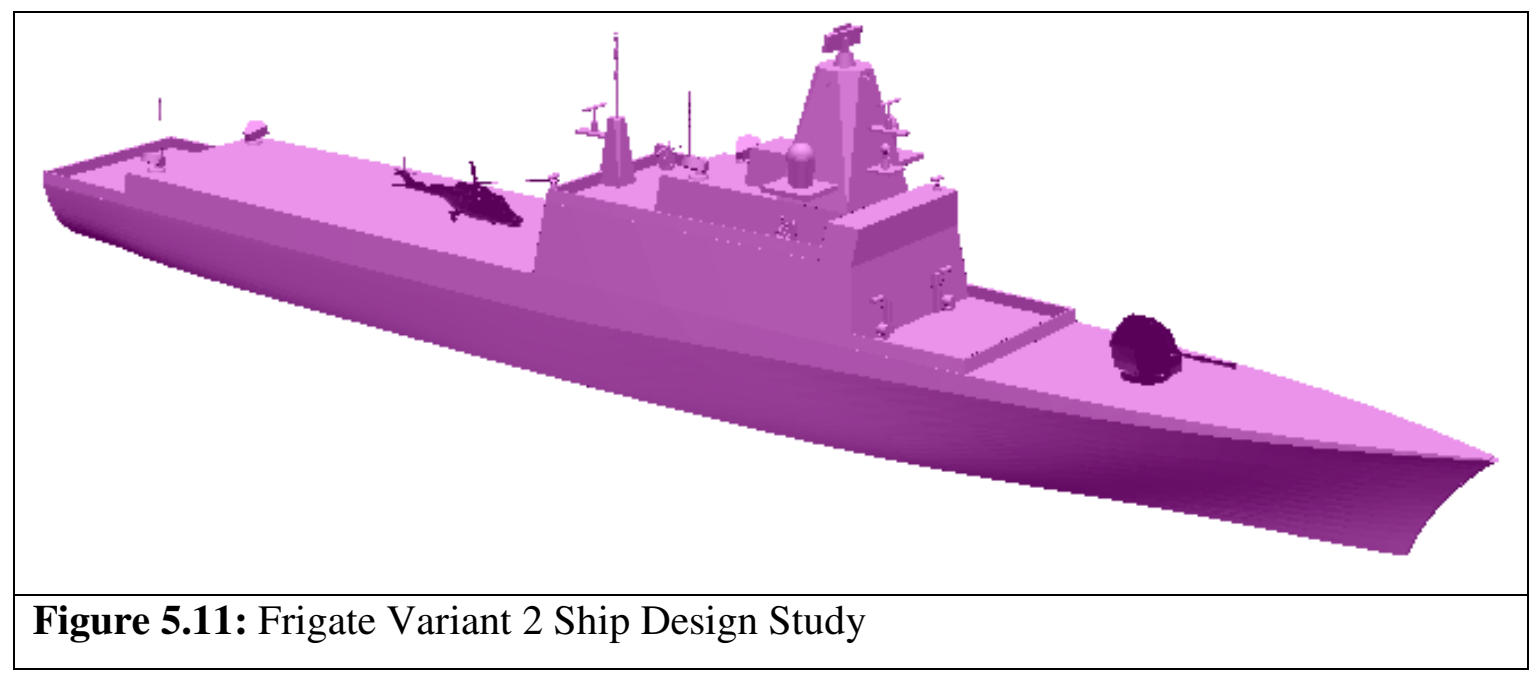

The choice of these features were aimed at the exploration of survivability related advantages of such configurations, such as improved DC due to the second passing deck, therefore providing unrestricted access over the length of the ship on two decks (Begg et al 1990), and lower RCS due to the small superstructure (Begg et al 1990). In addition, this feature also allowed the location of the flight deck close to amidships, which presents some operational advantages, since the optimum location for aviation facilities is at amidships where motions are kept to a minimum and the helicopters operational effectiveness is enhanced (Lloyd and Hanson 1985; Brown 1991) (see Appendix 2).

For the third and final variant, Frigate Variant 3, Figure 5.12, a trimaran configuration was selected, therefore, departing even more from the style of modern conventional frigates. 


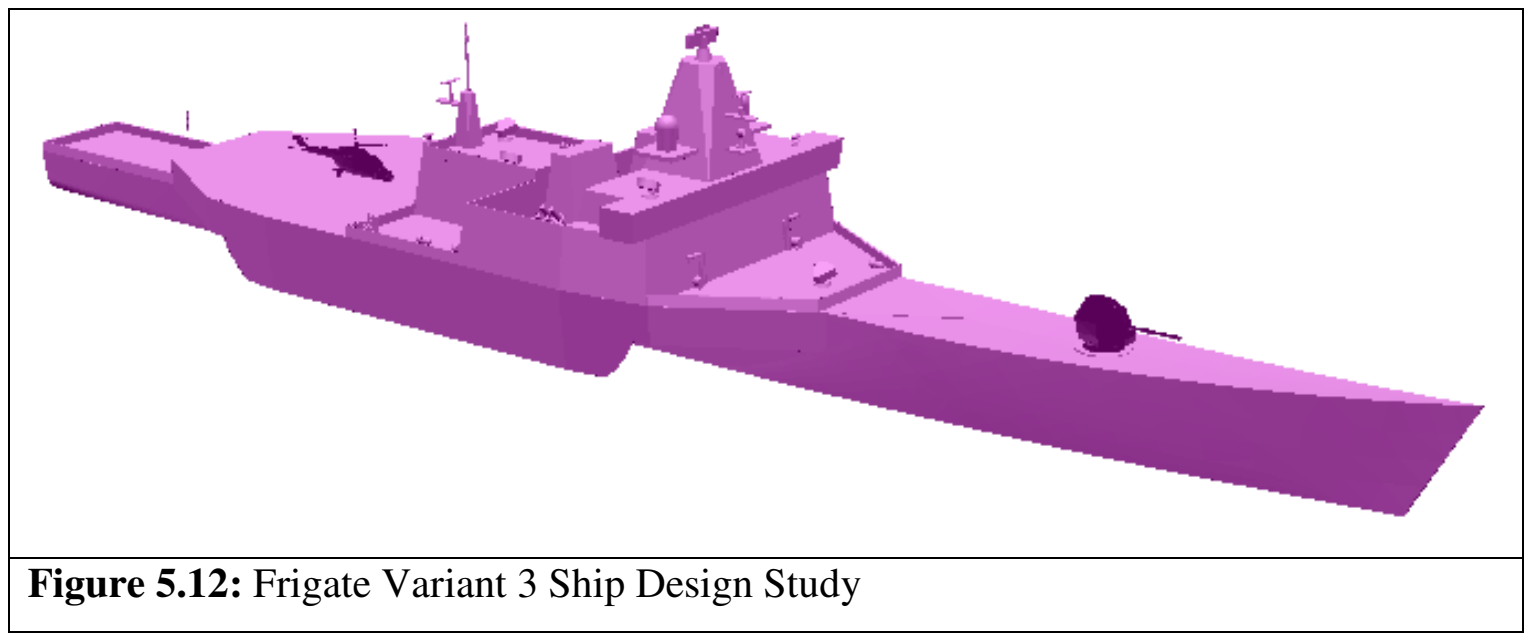

The intention was to investigate the possible advantages of this unconventional hull type. Since trimarans in the naval domain are relatively recent (with only one warship design currently in service, the USN's Independence Class LCS (Naval War College 2002)) various UCL designed trimaran warships as well as the UCL ship design procedure for trimarans (UCL 2010a) were investigated in order to overcome the problems associated with the lack of data. As observed in Figure 5.12 and Appendix 8, the trimarans superstructure is relatively minimal (due to the large internal volume of the box structure) and was split into two structurally separate blocks, one slightly aft and one slightly forward of amidships. Another interesting feature was that, due to the requirement of a $3.5 \mathrm{~m}$ minimum air gap to the underside of the box structure (arising from requirements to minimise slamming), as well as due to the narrow beam of the main hull which made the incorporation of the main machinery and systems challenging, a fifth deck (which however was not a 'through-deck') was included in this ship design study.

Figure 5.13 and Figure 5.14 describe the two frigate variants in terms of the 'Float, Move, Fight, Infrastructure' top level functional breakdown, similar to Figure 5.2 for the baseline frigate design. 


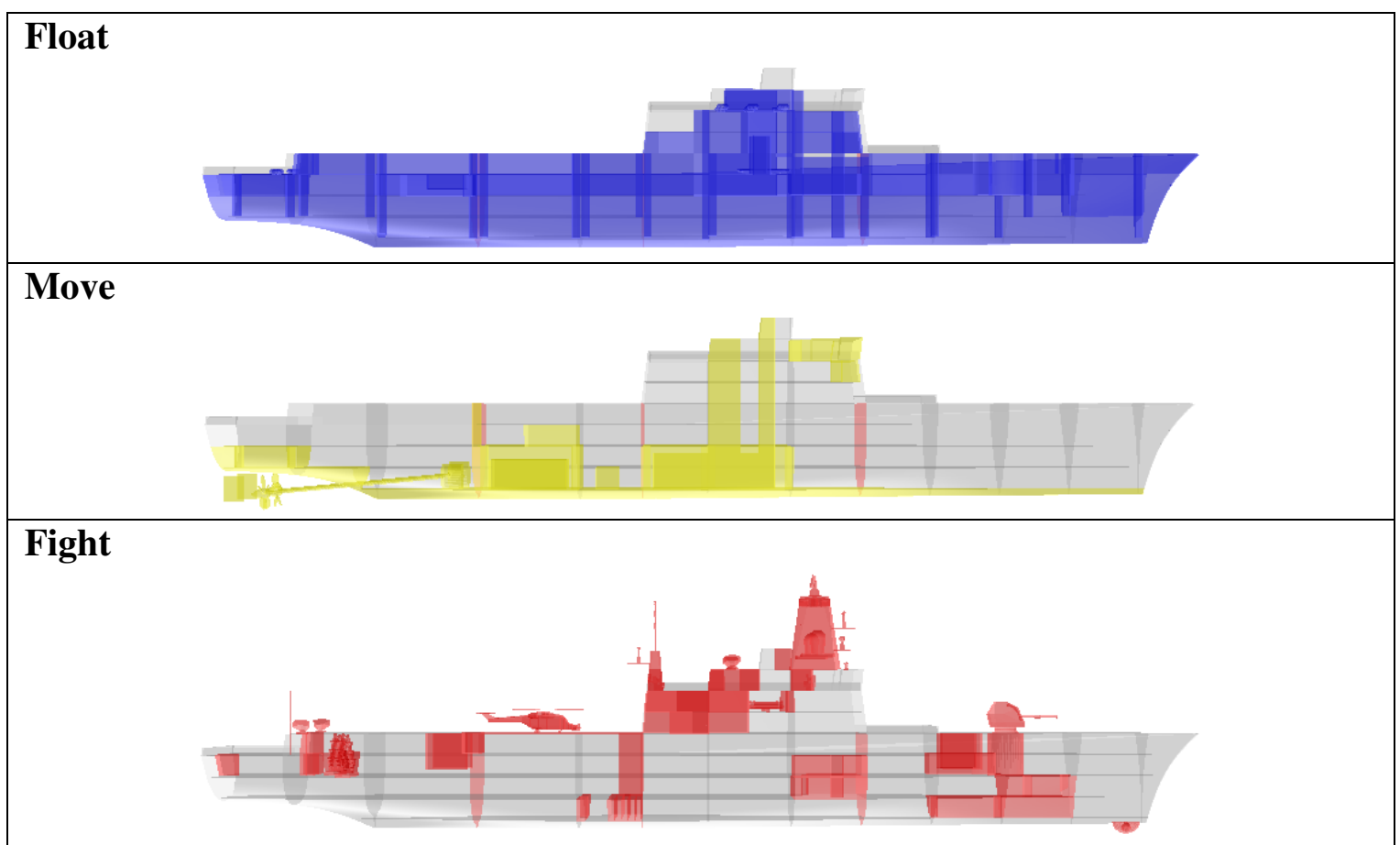

Infrastructure

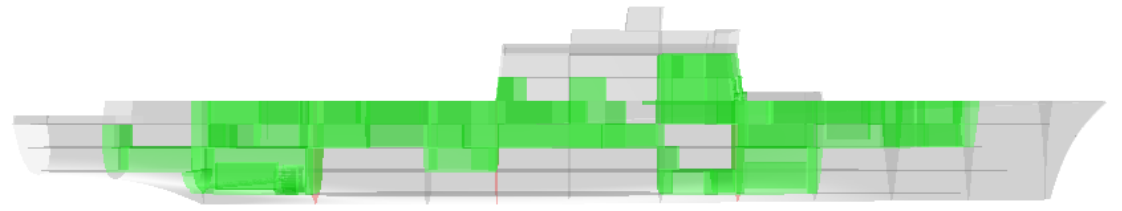

Figure 5.13: Frigate Variant 2 Top Level Functional Breakdown

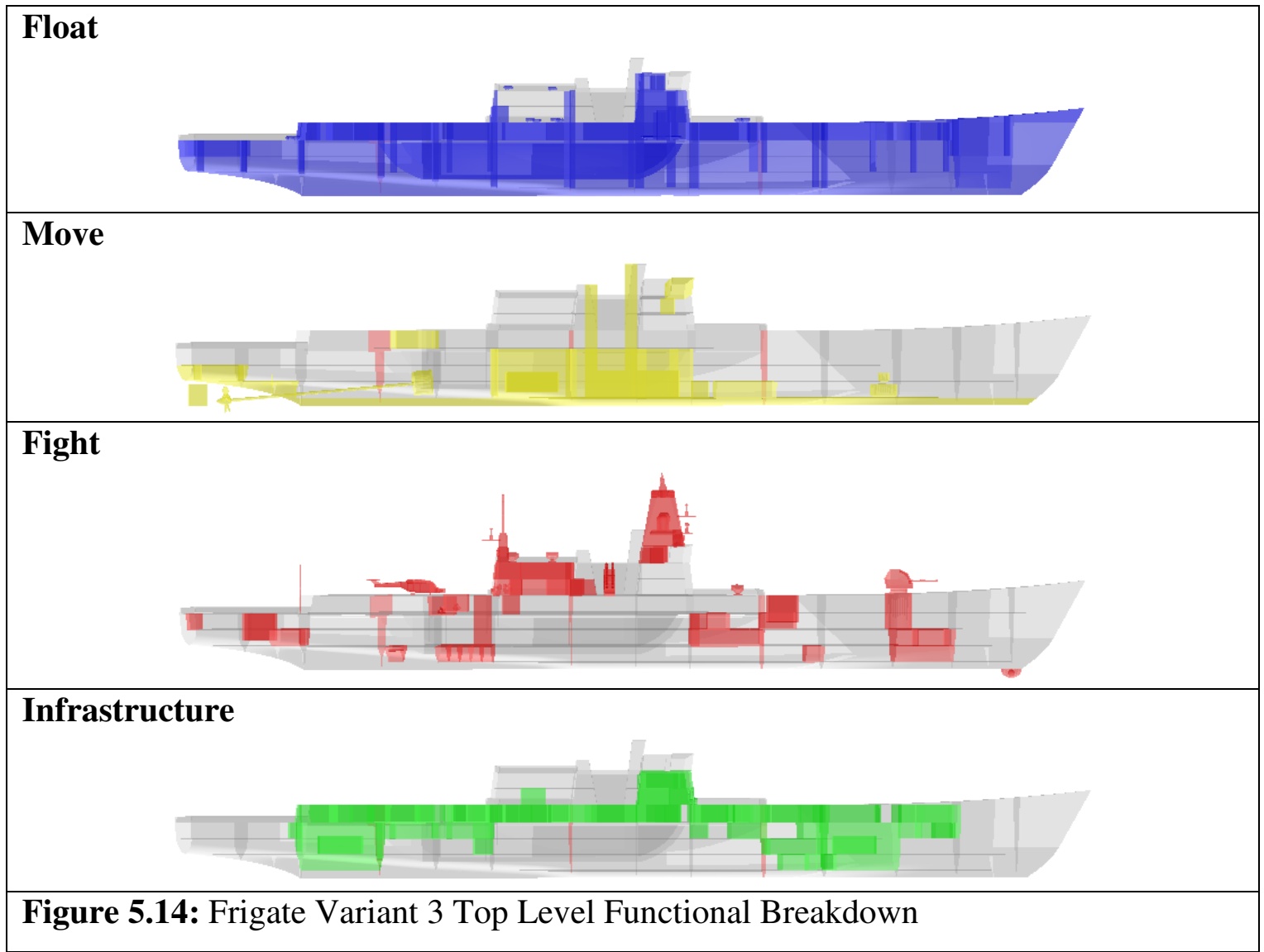


A number of observations can be made with regards to those two frigate variants. For example, the relatively small superstructures of both ship designs have made possible the location of their flight decks close to amidships. However, they have also decreased the distance between sensors (possibly causing interference problems and increasing vulnerability), caused a concentration of the fight functional group at amidships to a greater extent than the baseline frigate (which could lead to increased vulnerability) and introduced difficulties in the arrangement of exhausts and intakes. Also noticeable is that the Infrastructure functional group of the trimaran is largely concentrated in No 2 Deck, i.e. in the box structure (to a much greater extent than Frigate Variants 1 and 2). This arises from the very large overall beam of the trimaran and has simplified the architectural design of this frigate by easing the achievement of the architectural relationships of the 'circles of influence'. Furthermore, it has minimised the need for a large superstructure or an extra passing deck.

Frigate Variants 2 and 3 are described in terms of their top level weight breakdowns in Table A9 and Table A10 of Appendix 9.2 (similar to Table A8 for the baseline frigate design). In addition, the margin philosophy adopted is discussed in Appendix 9.3.

From the functional breakdown comparison above and the weight breakdown comparison in Appendix 9.2 between these three designs, it is evident that radically different conclusions can be drawn by investigating a functional (therefore, architectural) or a weight breakdown of a ship design. In Sections 2.1.3 and 2.1.4, the fact that novel hullform configuration, such as trimarans, are not encouraged when merely examining the weight breakdown of a ship design was pointed out (Andrews and Dicks 1997; Andrews and Pawling 2009), and has been observed from the three designs produced for this survivability investigation. It follows that a new ship design must be described architecturally, in terms of its functional breakdown, and also (traditionally) in terms of its weight breakdown, in order for one to be able to justify the selected design.

Similar to the baseline frigate, the hull of Frigate Variant 2 (Figure 5.15) is also based on that of the Type 22 Frigate and was generated using the Quickhull object of Paramarine. 

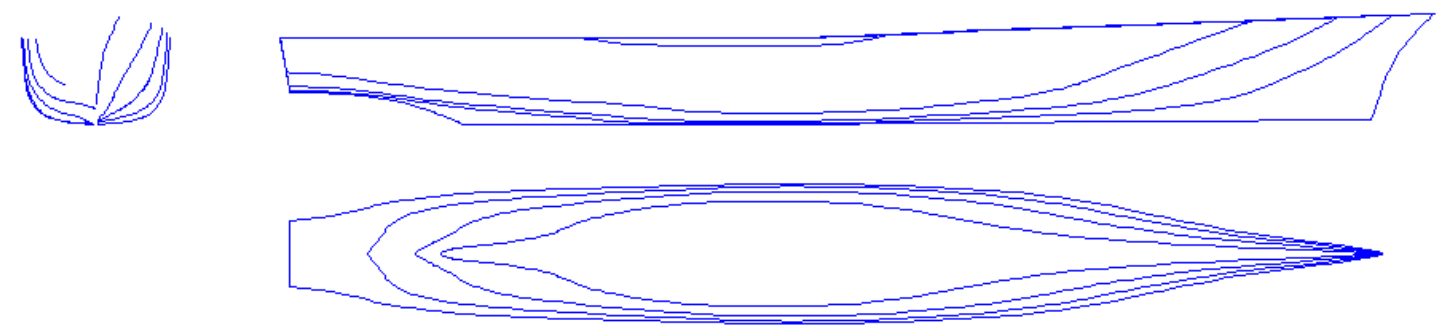

Figure 5.15: Frigate Variant 2 Lines Plan

Furthermore, the propulsive powering requirements for Frigate Variant 2 were computed in the same manner as for Frigate Variant 1 (see Appendix 9.4). The machinery selected consisted of:-

- $\quad 2 \times 5.22 \mathrm{MW}$ Wartsila 16V26 diesel gensets (cruise);

- $\quad 1 \times 31 \mathrm{MW}$ Rolls-Royce MT30 marine gas turbine, electrical drive (boost);

- $2 \times 2.69 \mathrm{MW}$ Wartsila 16V200 diesel gensets (auxiliary).

The above machinery driving two FPPs on 21MW HTS motors. The arrangement of the above Move related machinery is portrayed in Figure 5.16.

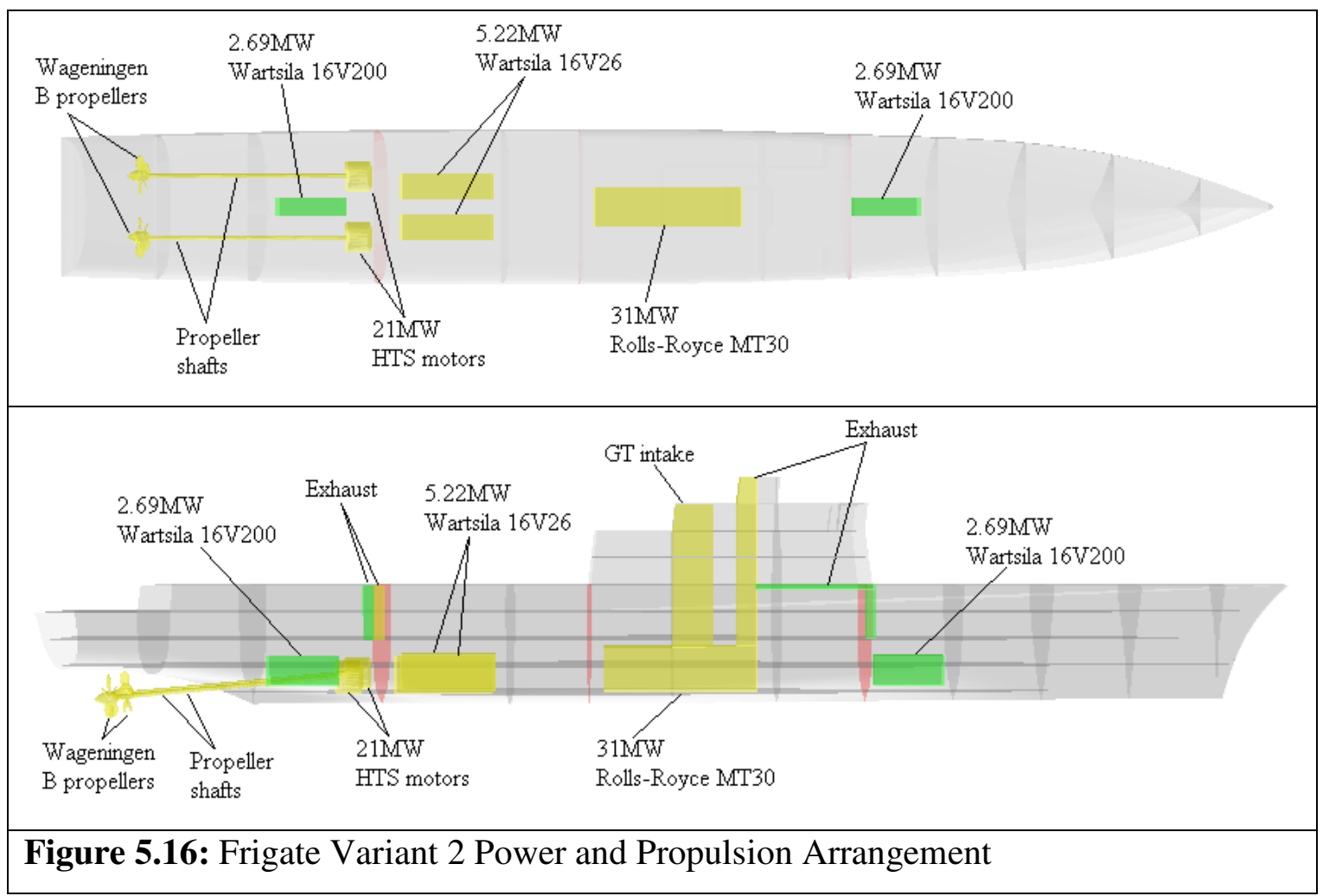

The above arrangement is almost identical to that of Frigate Variant 1 (Figure 5.4), therefore, presenting the same conclusions regarding the reduced vulnerability due to the widely separated power generation units and the short, but declined, propeller 
shafts (arising from the adoption of IFEP). The wide separation of the engines has once again led to the unconventional forward Wartsila 16V200 exhaust arrangement, due to the absence of superstructure above it. Unlike in the baseline frigate, the forward auxiliary exhaust has to extend through two decks (rather than one), before merging with the gas turbine exhaust, due to the deeper, 5-deck, hull of Frigate Variant 2. However, the only major difference is the fact that, due to the minimal superstructure of Frigate Variant 2, superstructure is absent above the aft auxiliary and both cruise gensets. This has led to the implementation of side exhausts for these three engines. The side exhausts are located starboard, just aft of the flight deck, consequently, should not interfere with helicopter operations. Moreover, since these particular engines exhaust closer to the waterline, the ships IR signature would also decrease (Dicker 1986; Afanasieff and Mabry 1994), as mentioned in Appendix 4.1. However, this could introduce problems related to the insertion of seawater through the exhausts; in addition, exhaust stains on the hull would make the ship more visible and would require more maintenance. As with the baseline design, removal routes for all engines were taken into account through the inclusion of soft patches in the passageways and compartments above each engine.

The trimaran hull of Frigate Variant 3, the lines plan of which is shown in Figure 5.17, was generated by use of a UCL developed algorithm.

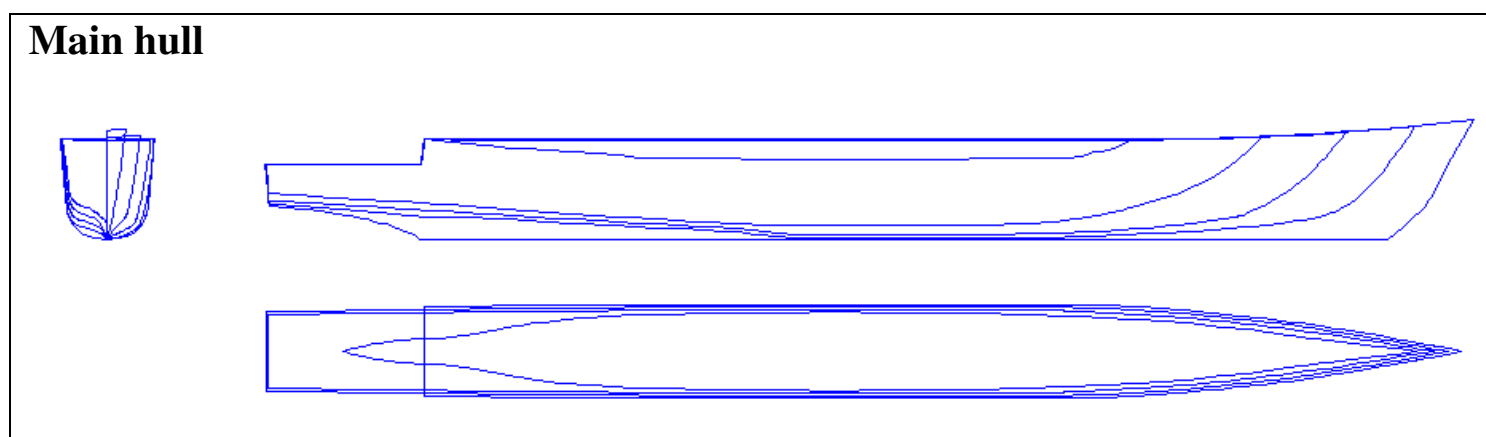

Side hulls
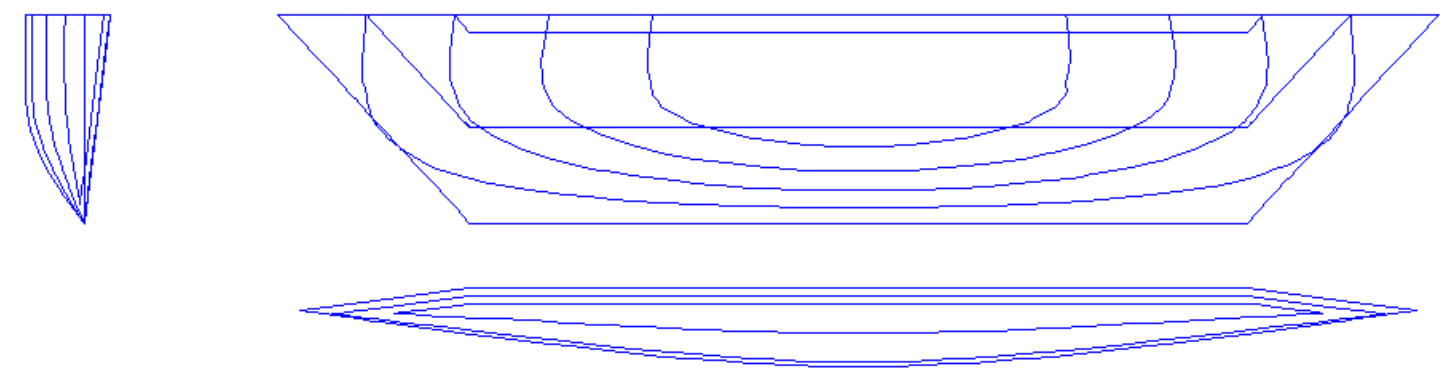

Figure 5.17: Frigate Variant 3 Lines Plan 
The propulsive powering requirements were computed using a UCL developed program based on the Holtrop \& Mennen method (see Appendix 9.4). The machinery that was selected consisted of:-

- $\quad 2 \times 2.94 \mathrm{MW}$ Warstila 9L26 diesel gensets (cruise);

- $1 \times 31 \mathrm{MW}$ Rolls-Royce MT30 marine gas turbine, electrical drive (boost);

- $2 \times 2.69 \mathrm{MW}$ Wartsila 16V200 diesel gensets (auxiliary).

A major difference in the power and propulsion system between Frigate Variant 3 and the first two variants is that, due to the narrow main hull, a double shaft (therefore, two motor) arrangement could not be facilitated. However, containing merely a single shaft was deemed unacceptable for survivability and availability reasons. Furthermore, the side hulls, being even narrower, restricted access and impeded the addition of large equipment items, such as of secondary propulsive units, in them. It was, in fact, decided to keep the space inside the side hulls void, not only for the reasons mentioned above, but also so that one side hull can be ballasted, if the other is damaged (Andrews and Zhang 1995; Andrews and Pawling 2008) as summarised in Appendix 2.2. Therefore, it was concluded that a secondary propulsive unit, and more specifically a pump-jet (Schottel 2010a), would be included in the forward end of the ship design. The machinery listed above, therefore, drove a single FPP on a 37MW HTS motor and a single Schottel SPJ 520 Pump-Jet on a 3.5MW HTS motor. Figure 5.18 illustrates the arrangement of the above machinery for the trimaran frigate variant.

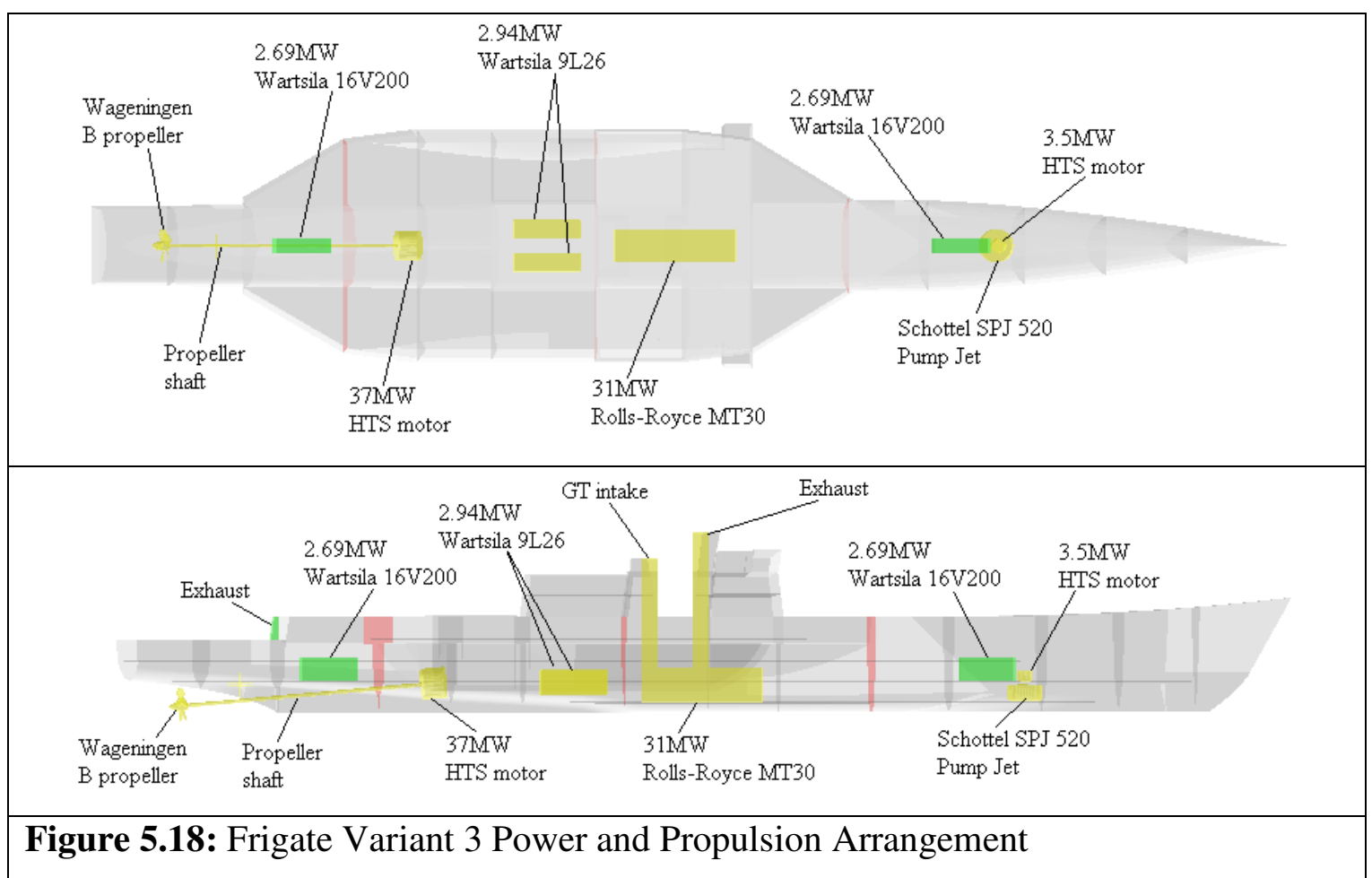


The power and propulsion arrangement of Frigate Variant 3 presents the most dissimilarities compared to the first two frigate design variants, although still benefiting from the widely separated power generation units and relatively short (but declined) shaft, therefore, reducing vulnerability, due to the IFEP system. The most noticeable difference is the separate forward and aft propulsive units. The large separation of the two parallel units should have a profound effect on the vulnerability of the move major system. Another main differences between the trimaran and the other two designs is that the two Wartsila 9L26 cruise gensets had to be moved one compartment forward, therefore, closer to amidships, adjacent to the WT sections containing the gas turbine boost engine. This is because, the narrow main hull beam of the trimaran only permitted the placement of the two relatively large engines at an amidships compartment due to volumetric constraints towards the extremities of the main hull. In addition the 37MW HTS motor (the largest motor used in all of the three design variants) could not fit in the aft auxiliary machinery compartment (where the aft Wartsila 16V200 was located) which was the practice in the first two variants, again due to the limited volume towards the ends of the trimarans main hull. The motor was thus moved one WT section forward, therefore, increasing the shaft length, but decreasing its declination angle value. The overall effect was to concentrate machinery at the amidships part of the ship to a greater extent than in the monohull designs. It could be argued that this arrangement increased the vulnerability of the power and propulsion related equipment (which are in parallel) of the trimaran, but the protection provided by the side hulls to attacks from torpedoes and missiles (Andrews and Zhang 1995; Andrews and Pawling 2008) should more than cancel this effect.

It is of interest to briefly describe the exhaust arrangements of Frigate Variant 3. This ship has the largest overall length (Table 5.3), therefore, the most WT bulkheads implying larger zones. Due to the longer zones, the forward Wartsila 16V200 auxiliary genset was placed at a large distance from the GT. Again, there is no superstructure above the forward auxiliary engine, however, the large distance from the gas turbine inhibits the combination of the exhausts of the two engines (which was the principle followed during the design of the first two variants) due to the large number of bulkhead penetrations required. This led to the solution of an underwater exhaust arrangement. The effects of this were to decrease vulnerability of the auxiliary genset (at least to abovewater threats) due to its smaller footprint and to reduce the IR signature of the ship by eliminating the exhaust plume (Heather 1990; Foxwell 1990a). However, underwater exhausts worsen the acoustic signature of ships (Heather 1990) and raise 
back pressure and corrosion related issues (Dicker 1986; Belcher 2008) as was summarised in Appendix 4.1. Other noteworthy aspects of the exhaust arrangements are that the cruise engines exhaust to the side of the main hull (between the main and the side hulls), and the aft auxiliary engine exhausts at the aft end (also due to the absence of superstructure above it). As in Frigate Variant 2, this arrangement should decrease the IR signature of the ship due to the close to waterline exhaust (Dicker 1986; Afanasieff and Mabry 1994) and the shielding effect of the side hulls regarding the cruise engines exhausts. However, it could introduce problems related to the insertion of seawater through the exhausts and would increase maintenance requirements due to exhaust stains on the hull. Nevertheless, a unique advantage of a trimaran is the ability of the side hulls to conceal the exhaust stains, therefore, not affecting the visual signature and reducing the need for maintenance. The aft auxiliary exhaust is located starboard, well aft of the flight deck, and the cruise engines exhaust plumes are shielded by the box structure, therefore, not interfering with helicopter operations. Removal routes for all engines were taken into account through the inclusion of soft patches in the passageways and compartments above each engine, as was done for the first two variants.

Similarly to the baseline frigate, the hydrostatics of Frigate Variants 2 and 3 were computed using the inbuilt Paramarine module. Intact stability was assessed against Defence Standard 02-109 (NES 109) Stability Standards for Surface Ships (MOD 2000) until all criteria were passed. Damage stability (of both deep and light conditions) was assessed against Defence Standard 02-109 (NES 109) damage criteria assessment (MOD 2000). Hydrostatics data are given in Appendix 9.5.

The WT bulkhead arrangements of Frigate Variants 2 and 3 are visible in Figure 5.19 and Figure 5.20.

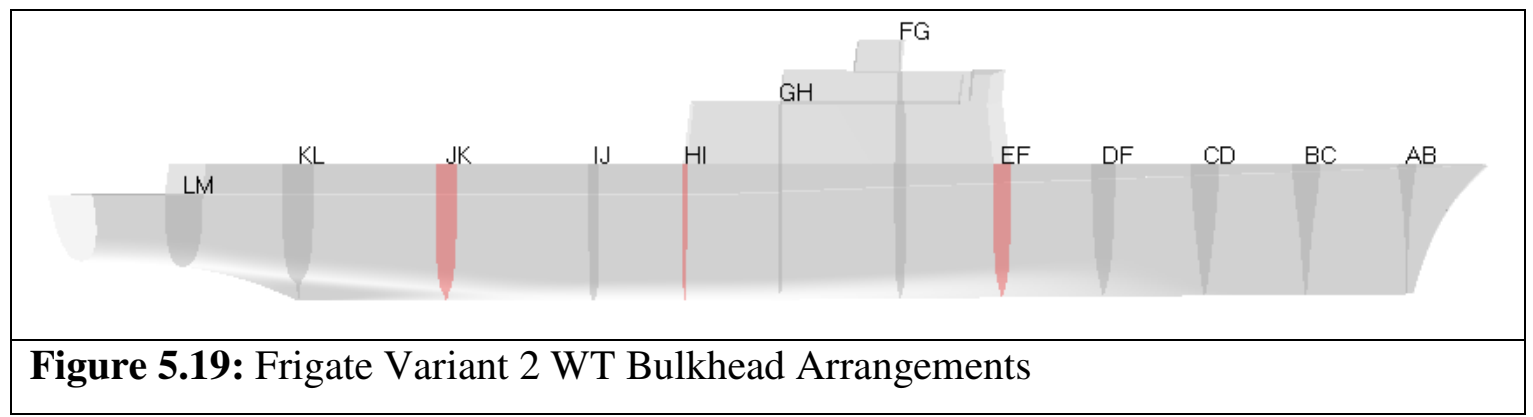




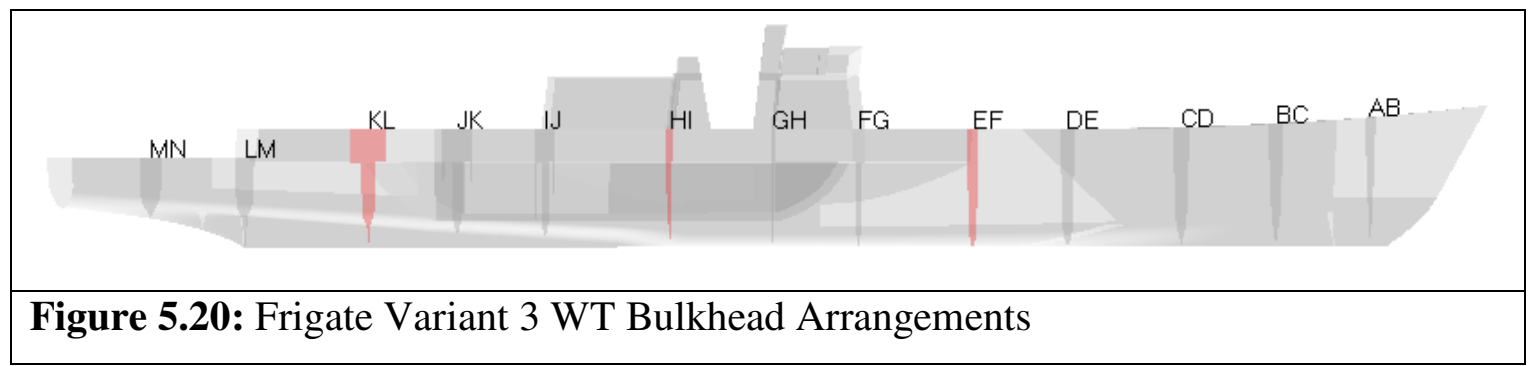

The bulkhead locations were decided upon using the same criteria as for the baseline frigate. Frigate Variant 2 included the same number of WT bulkheads as the baseline (twelve bulkheads, thirteen WT sections), while the longer hull of Frigate Variant 3 contained thirteen WT bulkheads and fourteen WT sections.

In order to maintain consistency, all frigate variants had the same number of zones, i.e. four. The red bulkheads in Figure 5.19 and Figure 5.20 represent the zone boundaries. Frigate Variant 2 is identical to the baseline in that, from aft to forward, each zone contains 3,2, 3 and 5 WT sections. The zones of the trimaran frigate on the other hand comprise of 3, 3, 3 and 5 WT sections respectively. Each of the four zones contains independent power generation units, CWP, HPAC, FP and ATU and ventilation. Due to the relatively small superstructure length of Frigate Variant 2 it proved difficult (and unreasonable since the superstructure in its totality is in the same zone) to include airlocks and NBCD stores in all four zones, so they were only fitted in three zones. Oppositely, Frigate Variant 3 included NBCD stores and airlocks for access to and from the citadel/weather in all zones, similarly to Frigate Variant 1. This is clearly shown in Figure 5.21 and Figure 5.22. 


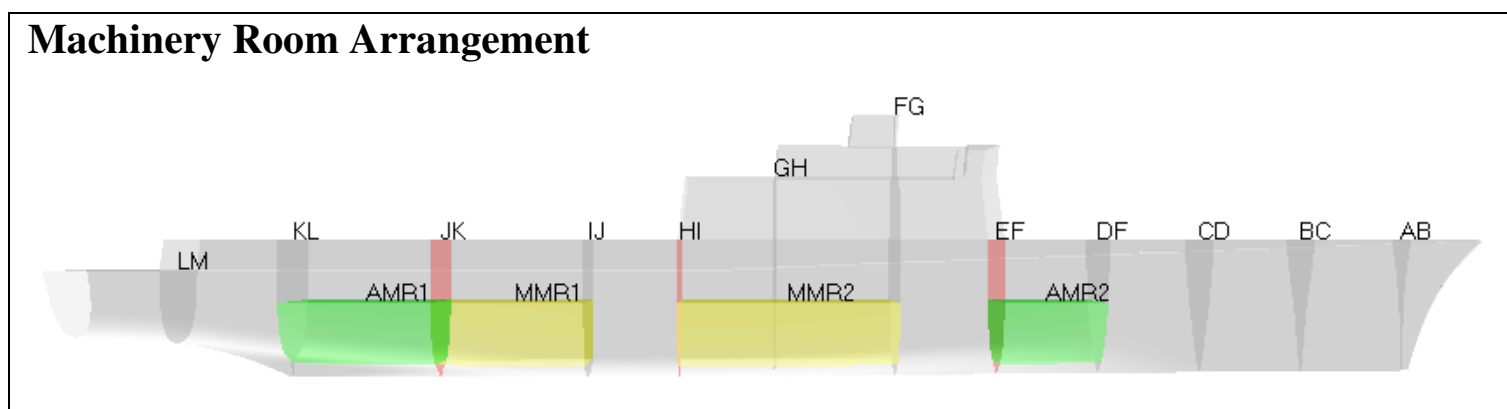

\section{ATU and Ventilation Arrangement}

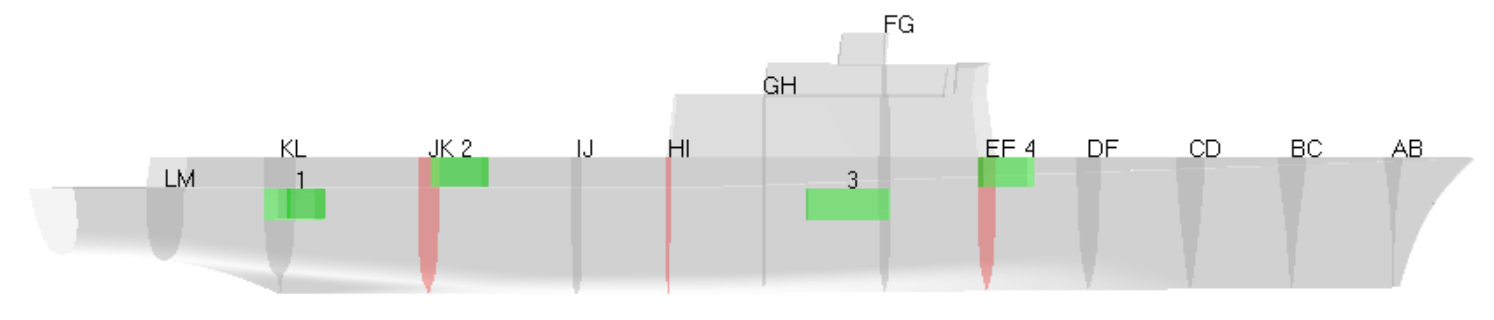

\section{NBCD Stores}

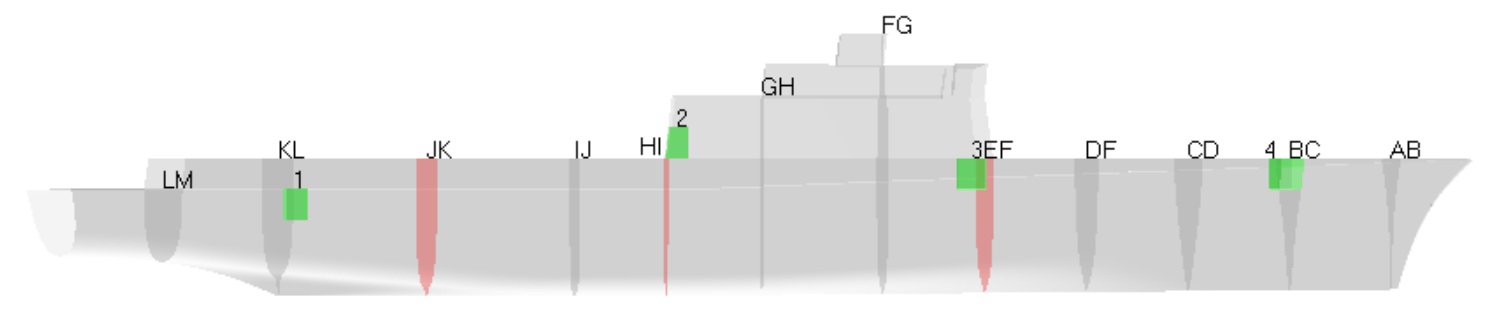

\section{Airlocks}

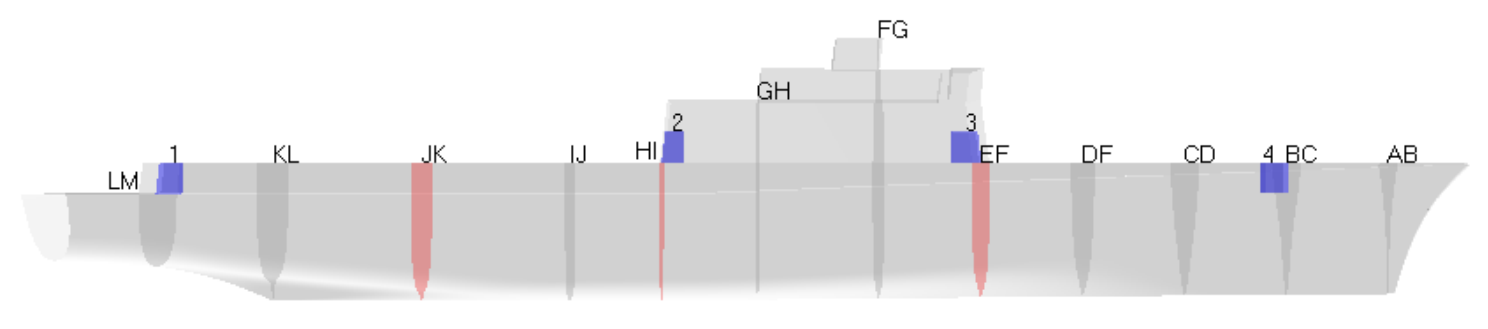

Figure 5.21: Frigate Variant 2 Zoning Philosophy

From above, it is evident that the arrangement of the MMRs and AMRs (which contain each zones power generation unit, CWP, HPAC and FP) is identical to that of the baseline frigate (Figure 5.6). MMR2 extends along the length of two adjacent WT sections in order to accommodate the engine and alternator of the large Rolls-Royce MT30 marine gas turbine (Figure 5.16); as in the baseline the alternator was placed in a separate WT section from of the engine due to size limitations. Another similarity to the baseline design is the placement of ATU and ventilation compartments close to spaces such as machinery rooms and living spaces (Appendix 8), and NBCD stores in proximity to the airlocks. However, NBCD store 1 is situated one WT section forward of airlock 1. This arrangement was chosen since the location of the ASM launchers 
(which will be examined later in this section) did not allow enough space for both compartments (Appendix 8). Furthermore, since each FRP section base is situated at a WT section bordering a main zone boundary as previously mentioned, and each section base should be adjacent to a NBCD store, the location of NBCD store 1 is convenient in that positioning the aft FRP section base adjacent to it (as will be later observed) meets both requirements. Airlock 1 leads above the aft mooring space, airlock 4 leads inside the forward mooring space, airlock 2 leads both to the hangar and the flight deck while airlock 3 leads to the forward weather deck. From Figure 5.21 it becomes observable why it was illogical to include an airlock (and NBCD store) in the second from aft zone given that the entire superstructure was in the third zone. Airlocks 1 and 3 are positioned adjacent to cleansing/decontamination stations.

\section{Machinery Room Arrangement}

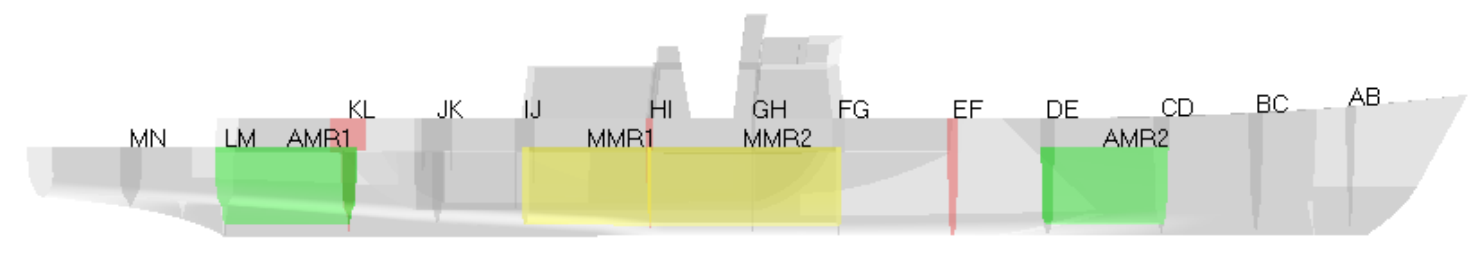

\section{ATU and Ventilation Arrangement}

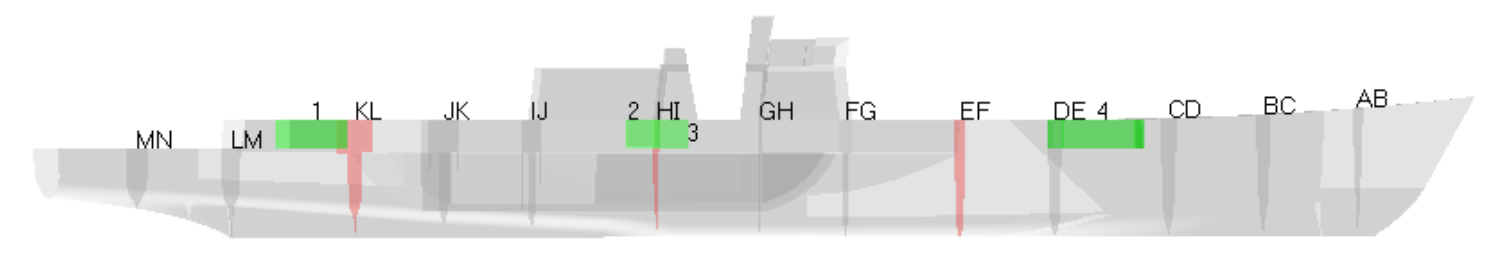

\section{NBCD Stores}

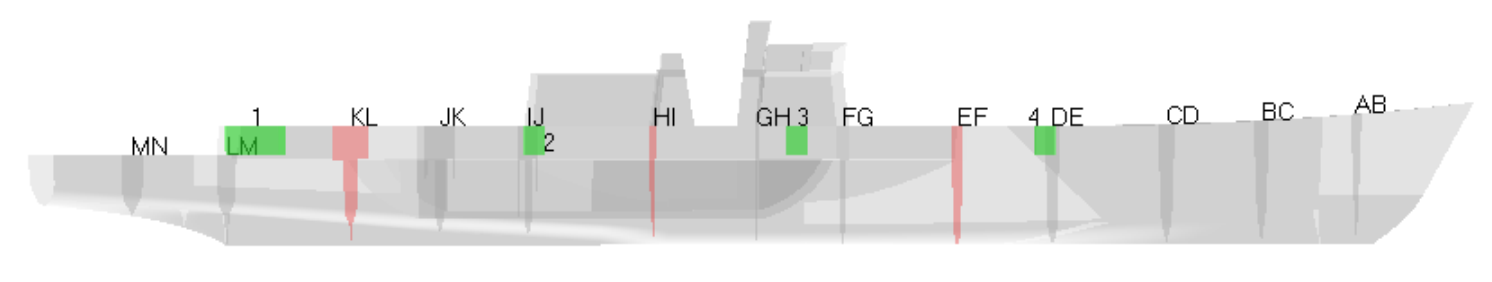

\section{Airlocks}

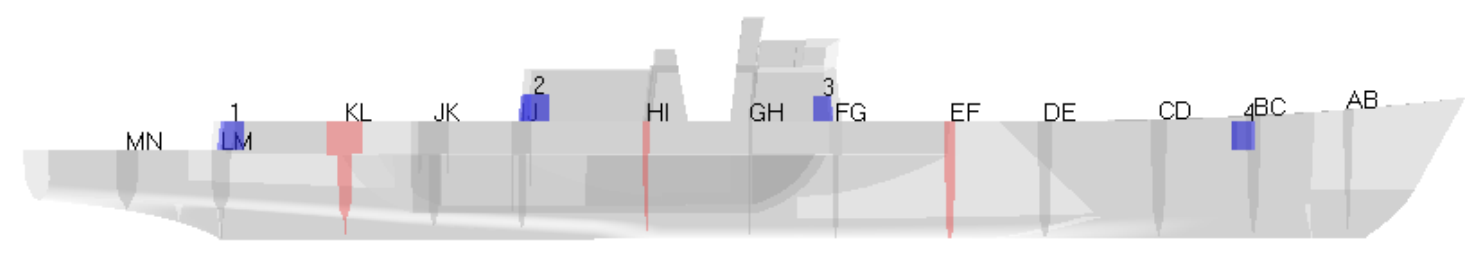

Figure 5.22: Frigate Variant 3 Zoning Philosophy 
In Frigate Variant 3 the two main machinery rooms are adjacent due to the space constraints of the narrow main hull beam mentioned above. However, the separation between the two auxiliary machinery rooms (containing smaller engines, therefore, able to be located at the ships extremities) and main machinery rooms has increased due to the presence of more WT sections in the longer trimaran and therefore, compensates for the increased vulnerability of the concentrated MMRs. As with the two other frigate variants, MMR2, in which the Rolls-Royce MT30 marine gas turbine is located (Figure 5.18), extends along the length of two adjacent WT sections, one containing the engine and the other the alternator. Moreover, each machinery room is three decks deep (as opposed to the two deck depth of the equivalent compartments in the first two variants) in order to accommodate all machinery and equipment (such as power generation units, CWP, HPAC and FP) in the narrow hull (and in order to meet the $3.5 \mathrm{~m}$ minimum air gap to the underside of the box structure requirement (Appendix 2.2). For the location of ATU and ventilation, a similar philosophy to that for the first two variants was used. This led to the positioning of ATU and ventilation 2 and 3 in adjacent WT sections. Although this gives an impression of increased vulnerability, it is important to note that the separation between the two spaces in the y direction is relatively large, due to them being placed at the port and starboard side respectively of the wide box structure connecting the three hulls (Appendix 8). However, ATU and ventilation spaces are assumed to not be cross-connected, but independent for each zone, as mentioned in Section 4.3.1 and PM 1.5, Table 4.3, therefore, their separation distances are of minor importance. A similar philosophy has also been used in the adjacency of NBCD stores and airlocks. The main point to note is that in the trimaran frigate variant NBCD store 4 and airlock 4 are separated by two WT bulkheads. This is because each FRP section base should be located adjacent to a NBCD store and a main zone boundary, and it was decided to locate the forward section base next to NBCD store 4. NBCD store 3 could also have been moved forward, by just 1 WT section, to meet the adjacency requirements. However, since one of the two cleansing and decontamination stations was located next to airlock 3 (the other being at airlock 1), it was decided that the location of its corresponding NBCD store was a higher priority. Airlock 1 leads above the aft mooring spaces, airlock 2 leads to the hangar and flight deck, airlock 3 leads to the forward weather deck and airlock 4 leads inside the forward mooring spaces.

Figure 5.23 and Figure 5.24 illustrate the arrangement of the main equipment items that are related to each of the major Fight systems which were modelled in the vulnerability and the recoverability assessment method, for Frigate Variants 2 and 3. As 
in Figure 5.7 for Frigate Variant 1, items solely related to each Fight system are shown, therefore, neglecting items such as sensors, power generation units and centralised command and control spaces.

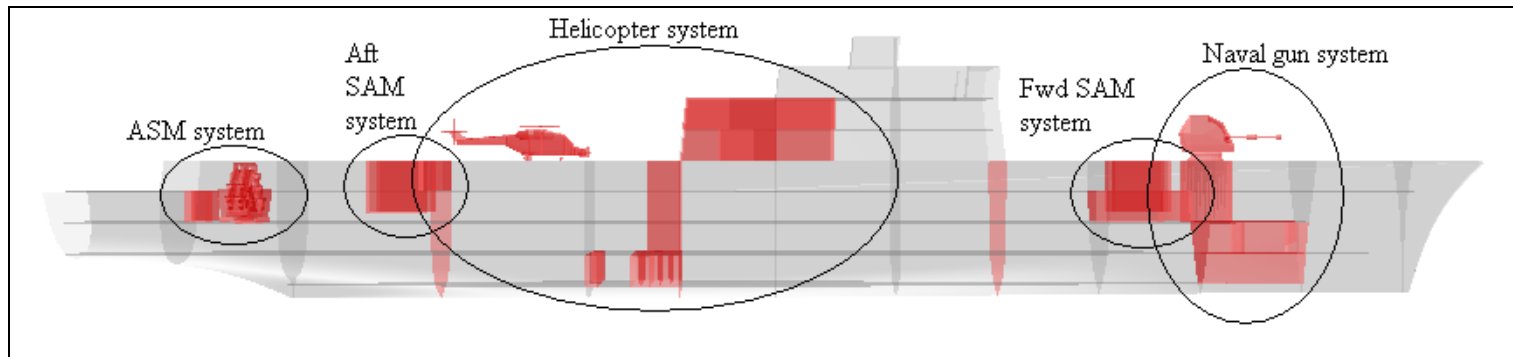

Figure 5.23: Frigate Variant 2 Major Fight System Arrangement

It is clear that for Frigate Variant 2 the distribution of the major Fight systems in accordance to the zoning arrangements has not been achieved to the same level as in the baseline. This is mainly due to the disruptions caused by the large footprint of the helicopter system, in combination with the small superstructure which is entirely in the same zone. The hangar, sonobuoy store and all helicopter maintenance and store spaces are inevitably located in the superstructure (including a below decks hangar with a lift arrangement was not considered due to cost implications). The superstructure, being in its entirety in the same zone, dictates that all other spaces (flight deck, magazine, weapon lift and AVCAT tank) have to be located in a separate, adjacent, zone. Therefore, the helicopter system extends over two adjacent zones, forcing the ASM system and aft SAM system to be located at the same aft-most zone. Moreover, the positioning of the harpoon launchers below decks, (Appendix 8) (to not obstruct helicopter operations) has led to the removal of a large portion of No 2 Deck, possibly leading to structural problems. The arrangement of the forward SAM system and medium calibre naval gun system is identical to that of the baseline, since the forward parts of both variants are largely similar. Another difficulty arising from the small superstructure configuration is the fact that the hangar (located on the port side (Appendix 8)) extends through a WT bulwark, therefore, removing approximately half of that WT bulwark above No 2 Deck. This could also lead to structural deficiencies. In addition, the positioning of the hangar amidships complicates the arrangement of uptakes and downtakes. Despite the above problems and although the specialisation of each zone in a specific type of warfare/operations has not satisfactorily been achieved, approximately the same level of concentration of equipment in series has been attained as in the baseline (see Figure 5.7). 


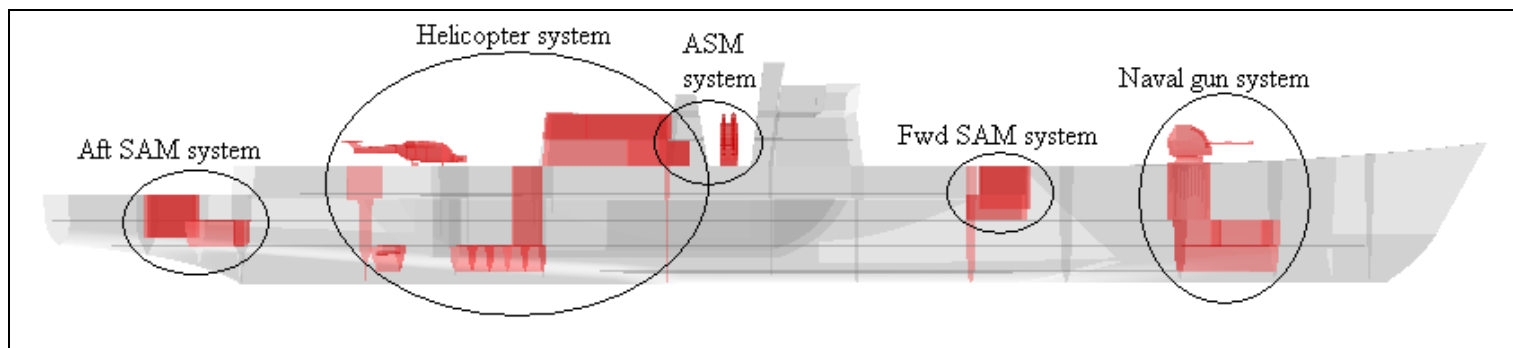

Figure 5.24: Frigate Variant 3 Major Fight System Arrangement

Frigate Variant 3 is the ship design that is capable of linking the major Fight system arrangements and the zoning arrangements to the greatest extent, despite that fact that it also incorporates a relatively small superstructure. This can easily be understood by the fact that the trimaran is the longest and widest (in terms of overall beam) of the three variants, therefore, allowing for a better distribution of systems. For example, the larger size of the second from aft zone has permitted the location of all helicopter system related equipment items in the same zone (with the exception of the aft part of the flight deck which extends over the adjacent zone, Appendix 8). (It is of interest that in Frigate Variants 1 and 3, the helicopter system is contained in a three WT section zone, while in the second variant in a two WT section zone, leading to the problems stated above). Additionally, the large overall beam has made possible the arrangement of the Harpoon launchers in a port-starboard, rather than forward-aft, arrangement, something unfeasible in the monohull variants, Appendix 8. The benefits of increased length are also observed through the separation distance between the medium calibre naval gun system and forward SAM system, which were located in adjacent WT sections in the first two variants. Thus, the trimaran configuration has allowed the specialisation of each zone in a specific type of warfare/operations, and assisted in the concentration of items in series of a particular system, therefore decreasing the systems vulnerability. The above remarks highlight the importance of increased ship length (and beam) on weapon system (upperdeck) arrangements and once again, the solutions presented by the trimaran configuration to the complications relevant in the layout of a combatant are unmistakeable.

A major modification between the three frigate design studies was the access philosophy adopted for each one. The hull of Frigate Variant 2 consisted of 5 decks, two of which, No 2 and No 3 Decks, were access decks. Each access deck contained a centreline passageway identical to the one in the baseline. In addition, similarly to the baseline, each WT section included two vertical accesses, one at either side of the 
centreline passageway and at the forward and aft end of each WT section respectively. These arrangements are depicted in Figure 5.25, and detailed in the GA, Appendix 8.

\section{Decks Access}

DC Decks Access and Vertical Access
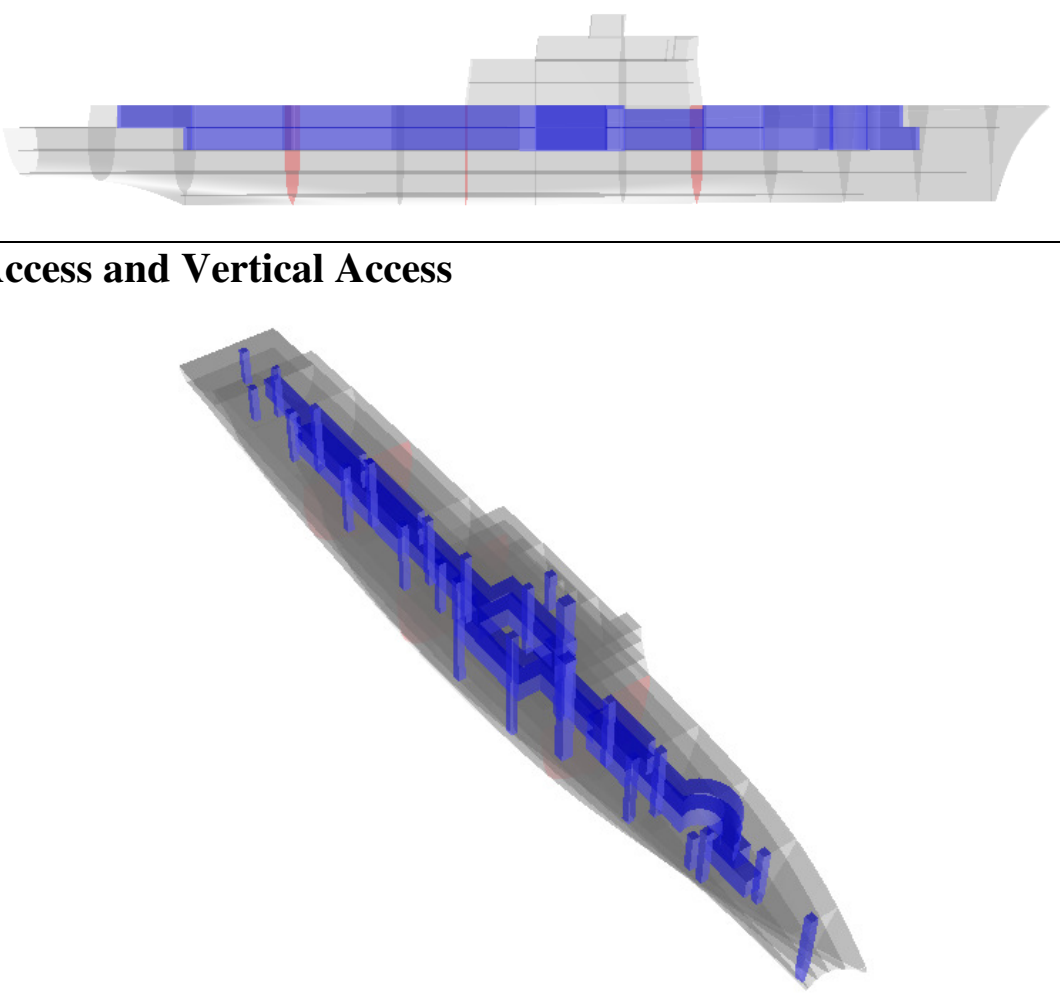

Figure 5.25: Frigate Variant 2 Access Philosophy

It is of interest to observe that the aft end of the passageway on No 3 Deck ends one WT section forward of the aft mooring space due to the location of the of the harpoon launchers (Figure 5.23).

Conversely, the trimaran variant contained two side passageways on the DC deck (No 2 Deck) in the box structure which converge to a centreline passageway in the forward section of the hull. Two side passageways were included as it would have been problematic to achieve satisfactory access between spaces with a single passageway for a design with such a large beam. Although the hull did include a fifth deck, this was not a passing deck. Each WT section included two vertical accesses, one adjacent to each of the two side passageways and at the forward and aft end of each WT section respectively. Once the side passageways converged, the vertical access arrangements were identical to those of the first two variants. This is illustrated in Figure 5.26 and detailed in the GA, Appendix 8. 


\section{Deck Access}

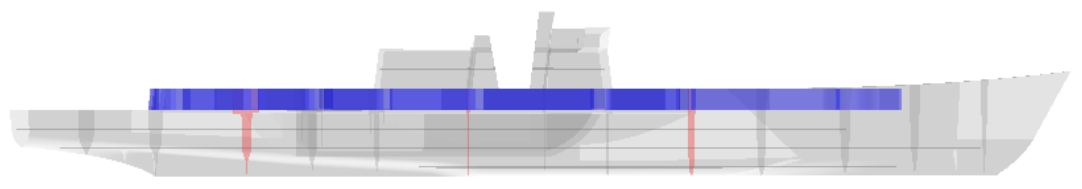

DC Deck Access and Vertical Access

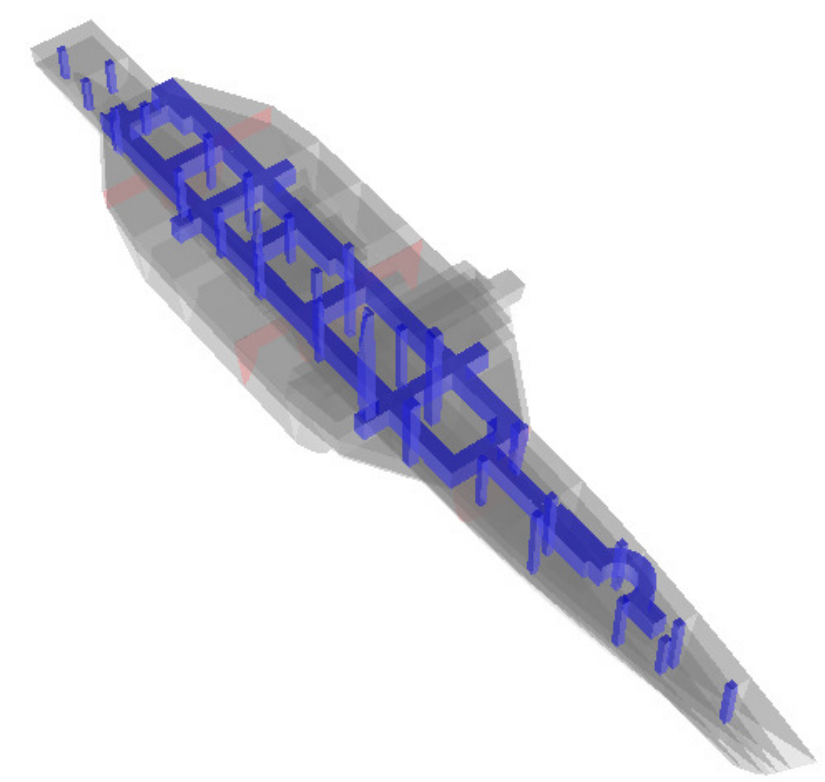

Figure 5.26: Frigate Variant 3 Access Philosophy

An interesting feature of the above access arrangements is the fact that the two side passageways are cross-connected in every WT section. This improves communication and personnel flow, however, occupies a considerable amount of space. Therefore, the access arrangements of the trimaran are not as efficient as those of a monohull (for which access between spaces is relatively easy, given the comparatively small beam) and contribute to the increased gross volume compared to its monohull equivalents. Nonetheless, side passageways do present certain advantages (described in Appendix 4.2), such as providing some protection to sensitive compartments and equipment located in between them (Begg et al 1990; Harney 2010) and providing a means of incorporating redundancy and separation of services by running pipes and cables along the side passageways (Brown 1987; Afanasieff and Mabry 1994) .

As with Frigate Variant 1 it is of interest to examine the location of all compartments and equipment relevant to the recoverability related PMs in Sections 4.3.1, 4.3.2 and 4.3.3. Figure 5.27 and Figure 5.28 below illustrate the distribution of compartments and equipment related to Category 1 PM analysis. 


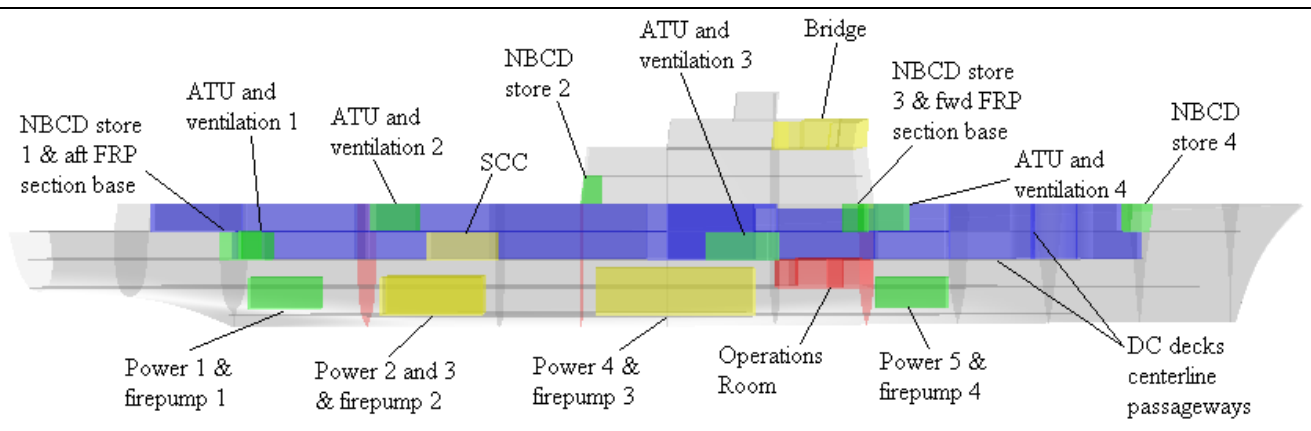

Figure 5.27: Frigate Variant 2 Category 1 PM Elements

The arrangement of the relevant compartments in Frigate Variant 2 is similar to that in the baseline. The site of the (collocated) power generation units and firepumps is identical to the corresponding arrangement in the baseline frigate designs, as is the location of the Operations Room, bridge and SCC. Once again, the Operations Room is located deep in the hull (No 4 Deck) in order to decrease vulnerability to abovewater attacks (although this could lead to an increase in underwater incidents) and the bridge and the Operations Room are located at the same WT section in order to improve access and communication between the two spaces. In addition the SCC is located at a distance from the Operations Room in order to permit its use as a secondary Operations Room in emergency. The location of the FRP section bases has already been briefly mentioned. They are both situated adjacent to an NBCD store and a main zone boundary. However, due to the absence of an NBCD store in one of the zones, the location of the aft FRP section base is slightly less efficient than that of the baseline in that it is situated relatively far aft. Though, this might be compensated by the fact that, being a shorter ship, DC crews would travel shorter distances to reach the affected areas of the ship (PM 1.2) and would have a larger choice of alternative routes (PM 1.4) given the second access deck. All other elements are located with respect to the zoning arrangements as described above.

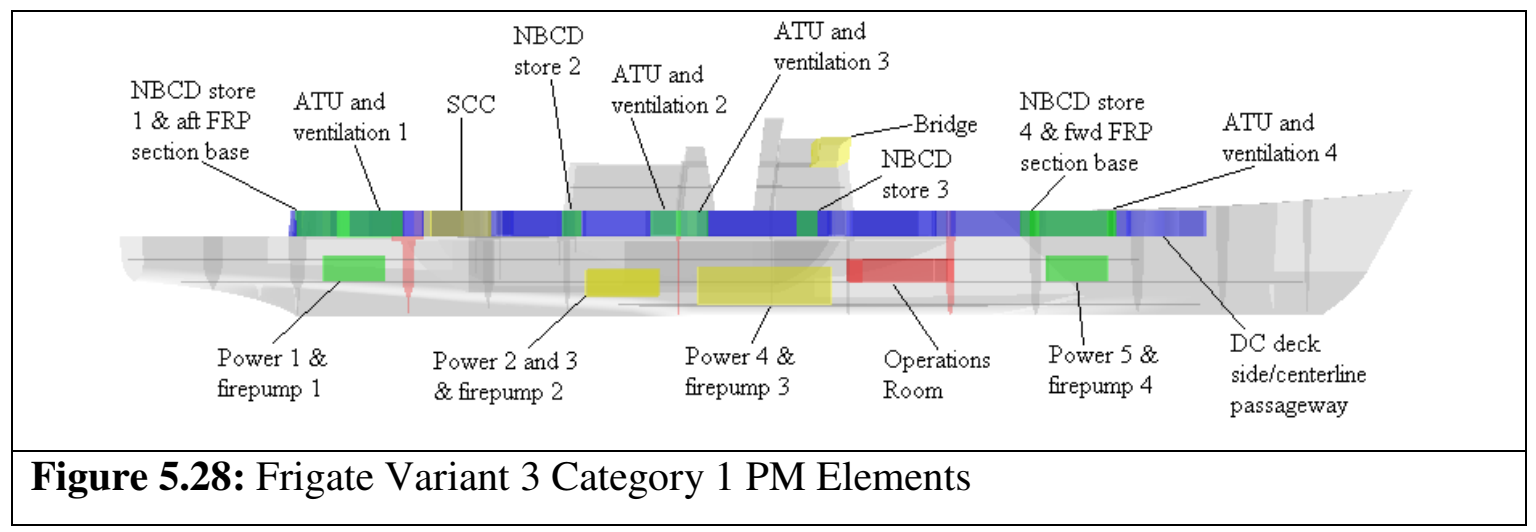


The main noticeable difference between the trimaran frigate variant and the other two ship designs, regarding compartments and equipment items related to Category 1 PM analysis, is the location of the Operations Room. Although it is again positioned deep in the hull (No 4 Deck) for protection from abovewater attacks, it is not located at the same WT section as the bridge as was the case with the first two variants. This is clearly due to the arrangement of the machinery (previously detailed) forcing the Operations Room one WT section forward of the bridge. As a general conclusion it can be said that the architectural design of a trimaran, although simpler (than a monohull) in the box structure and upperdeck, is more challenging under the DC deck, due to the comparatively narrow beam of the three hulls. Another aspect of the trimaran is that, for the FRP section bases to meet both adjacency (with a NBCD store and main zone boundary) requirements, they are less efficiently positioned than in the baseline, since they are located closer to the extremities of the hull. This arises from constraints caused by the relatively small, amidships, superstructure which contains two of the ships airlocks (Figure 5.22) to which the other two NBCD stores should be in proximity. The inefficient FRP section base locations are worsened by fact that DC crews would travel longer distances to reach the affected areas of the ship (PM 1.2) due to the ship's length (although having a larger choice of alternative routes (PM 1.4) given the two side passageways might have a balancing effect). All other elements are located using a similar reasoning as for the first two variants and with respect to the zoning arrangements described above.

Figure 5.29 and Figure 5.30 illustrate the distribution of compartments and equipment related to Category 2 (and Category 3) PM analysis.

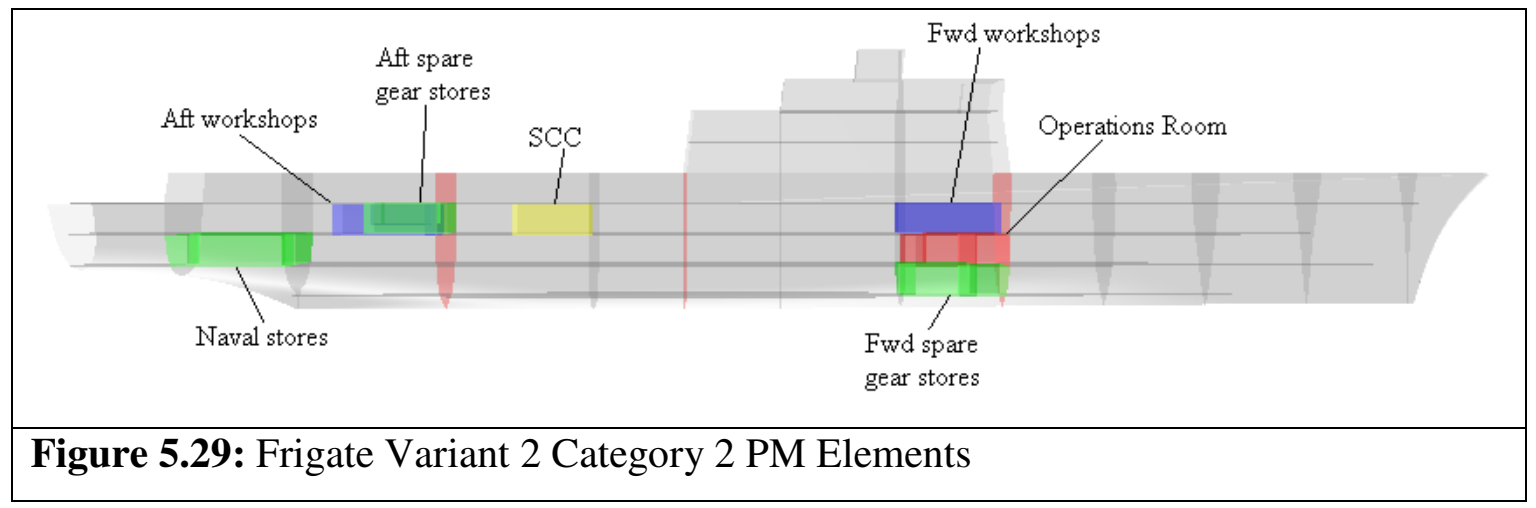




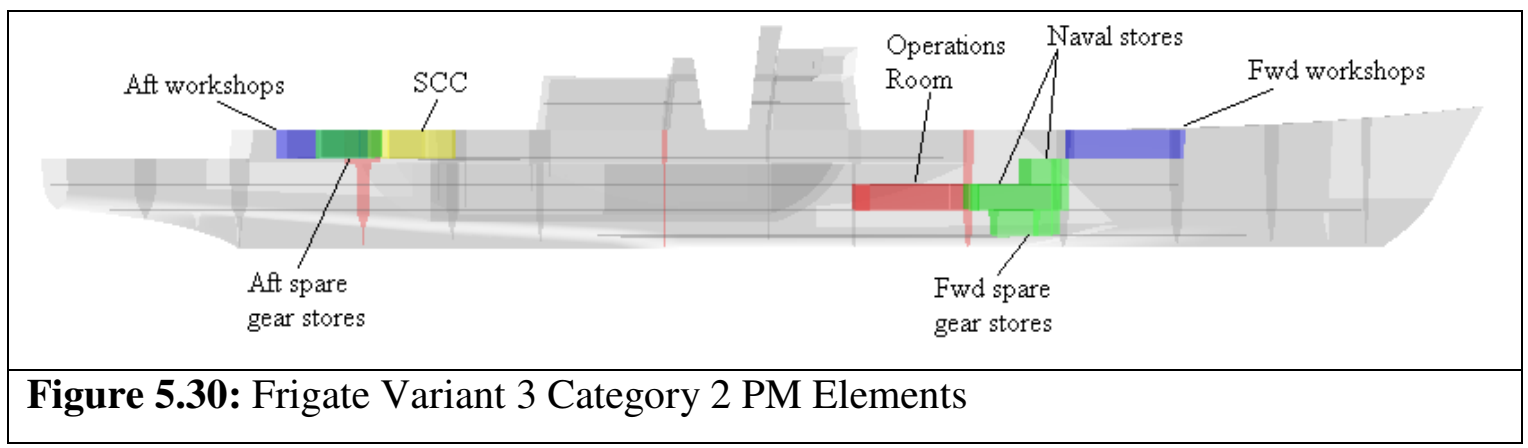

The arrangement of these spaces in Frigate Variants 2 and 3 are almost identical to that of the baseline design. Workshops have been located conveniently to their corresponding spare gear stores and to the machinery compartments. The two groups of workshops and spare gear stores have been separated as much as possible, with the longer trimaran variant presenting clear advantages in that regard. Naval stores have been located by taking into account trim and available space. A feature of interest is the distribution of the trimarans naval stores in two decks due to insufficient space; another effect of the narrow main hull beam of a trimaran configuration. Category 3 PM analysis, in addition to the above compartments, involves all of the components of each major ship system; system architecture illustrations are included in Appendix 7.

\subsection{Corvette and Destroyer Design Studies}

It was decided that it would be beneficial to apply the survivability assessment method discussed in Chapter 4 to combatants of different sizes to investigate how ship size affects survivability. An obstacle associated with comparative studies is the careful variation of only one variable at a time. It was therefore decided that the hull configuration would be constant (i.e. all ships compared would be monohulls) as would be the major move and Fight systems modelled; only ship size would vary. A further two combatant design studies were carried out, one smaller combatant (corvette) and one larger combatant (destroyer). The two above additional combatant design studies were compared to the baseline frigate by applying the survivability assessment method outlined in Chapter 4. However, it is clear that combatants of different sizes/types contain different combat systems, number of zones and performance capabilities (i.e. “interconnected variables" (Bradbeer and Andrews 2012b)). This limitation was observed by Bradbeer and Andrews (2012b) who concluded that "the best that can be achieved is to design ... which are equivalent against one metric and accept that some variables cannot be fixed", therefore, the designs "can only be considered equivalent in 
limited sense". This led to the conclusion that in order for the design studies to be realistic, certain lower level variations (all connected to the high level variable of ship size) would have to be incorporated. Therefore, the way in which each aspect of survivability is affected by ship size (including varying zoning) and level of combat system and ship performance capabilities (appropriate to the ship type) was investigated.

Two additional general purpose, general ocean going sea control combatants were designed. Table 5.4 below lists the payload of the three combatants.

\begin{tabular}{|c|c|c|c|}
\hline $\begin{array}{c}\text { Corvette Payload } \\
\text { Equipment }\end{array}$ & $\begin{array}{c}\text { Frigate Variant } 1 \\
\text { Payload Equipment }\end{array}$ & $\begin{array}{c}\text { Destroyer Payload } \\
\text { Equipment }\end{array}$ & $\begin{array}{l}\text { FIGHT } \\
\text { Systems }\end{array}$ \\
\hline $\begin{array}{l}1 \times \text { Oto Melara } 76 \mathrm{~mm} \\
\text { Naval Gun System }\end{array}$ & \multicolumn{2}{|c|}{$1 \times$ BAE Systems $155 \mathrm{~mm}$ gun } & $\begin{array}{l}\text { Medium } \\
\text { calibre naval } \\
\text { gun system }\end{array}$ \\
\hline \multicolumn{3}{|c|}{$2 \times 4$ Harpoon launchers } & ASM system \\
\hline \multicolumn{2}{|c|}{$\begin{array}{l}4 \times \text { MBDA MICA PDMS with } 32 \text { MICA } \\
\text { missiles }(4 \times 8 \text { round VL launchers })\end{array}$} & $\begin{array}{l}6 \times \text { PAAMS ADMS } \\
\text { with } 48 \text { Aster missiles } \\
\text { (SYLVER A50 VLS: } \\
3 \times 8 \text { Aster-15 and } 3 \times \\
8 \text { Aster-30) }\end{array}$ & $\begin{array}{l}\text { Aft/fwd } \\
\text { SAM system }\end{array}$ \\
\hline $\begin{array}{l}1 \times \text { Lynx (Kestrel) } \\
\text { Helicopter }+ \text { hangar } \\
\text { and single spot flight } \\
\text { deck for Lynx }\end{array}$ & $\begin{array}{l}1 \times \text { Lynx }(\text { Kestrel }) \\
\text { Helicopter }+ \text { hangar } \\
\text { and single spot flight } \\
\text { deck for EH- } \\
101 \text { Merlin }\end{array}$ & $\begin{array}{l}1 \times \text { EH-101Merlin } \\
\text { Helicopter }+ \text { hangar } \\
\text { and single spot flight } \\
\text { deck for EH- } \\
101 \text { Merlin }\end{array}$ & $\begin{array}{l}\text { Helicopter } \\
\text { system }\end{array}$ \\
\hline \multicolumn{3}{|c|}{$2 \times$ MSI Seahawk 30mm } & \multirow{15}{*}{$\begin{array}{l}\text { Other } \\
\text { payload } \\
\text { (certain } \\
\text { sensors are } \\
\text { part of the } \\
\text { major Fight } \\
\text { systems } \\
\text { above; for } \\
\text { full system } \\
\text { tree diagrams } \\
\text { see } \\
\text { Appendix 7) }\end{array}$} \\
\hline & & $\begin{array}{l}4 \times \text { Oto Melara } \\
12.7 \mathrm{~mm}\end{array}$ & \\
\hline & & $2 \times$ Phalanx CIWS & \\
\hline \multicolumn{3}{|c|}{$2 \times$ Triple barrel torpedo tubes } & \\
\hline \multicolumn{3}{|c|}{$4 \times$ Rheinmetall MASS Decoy Launchers } & \\
\hline \multicolumn{3}{|c|}{$1 \times$ Surface Ship Torpedo Defence System } & \\
\hline \multicolumn{3}{|c|}{$2 \times$ Navigation radars } & \\
\hline $1 \times$ Single Face SR ST & AR Surveillance Radar & $2 \times$ MFR Spectar & \\
\hline \multicolumn{3}{|c|}{$1 \times$ General Purpose Electro Optical Device } & \\
\hline \multicolumn{3}{|c|}{$2 \times$ Thales Sirius IRST } & \\
\hline \multirow{2}{*}{\multicolumn{3}{|c|}{$\begin{array}{c}1 \times \text { Raytheon AN/SLQ-32(V)3 Shipboard ESM/ ECM System } \\
1 \times \text { Spherion hull mounted sonar }\end{array}$}} & \\
\hline & & & \\
\hline \multicolumn{2}{|c|}{$\begin{array}{l}1 \times \text { Small ship communications system }(1 \times \\
\text { communications mast }+2 \times \text { whip antennae })\end{array}$} & $\begin{array}{l}1 \times \text { Medium ship } \\
\text { communications } \\
\text { system }(1 \times \\
\text { communications mast } \\
+4 \times \text { whip antennae }) \\
\end{array}$ & \\
\hline \multicolumn{3}{|c|}{$1 \times$ Generic Satellite Communications System with 2 antennae } & \\
\hline- & 25 Embarked forces & 50 Embarked forces & \\
\hline
\end{tabular}


The table above conveys the main payload equipment corresponding to the major Fight systems which were assessed in the vulnerability and recoverability methods described in Chapter 4. The differences in the combat systems, appropriate to the ship type, are highlighted. (Each Fight system includes further items such as sensors, control rooms and power generation units; full system diagrams are shown in Appendix 7).

The performance requirements of the three combatants are detailed in Table 5.5. The variations in issues such as zoning and ship performance capabilities appropriate to the ship size and type are visible.

Table 5.5: Corvette, Frigate Variant 1 and Destroyer Performance Requirements Adopted for Design Studies

\begin{tabular}{|l|l|l|l|}
\hline & \multicolumn{1}{|c|}{ Corvette } & \multicolumn{1}{c|}{ Frigate Variant 1 } & \multicolumn{1}{c|}{ Destroyer } \\
\hline Maximum speed & \multicolumn{3}{|c|}{$30 \mathrm{kts}$} \\
\hline Endurance & $3,000 \mathrm{~nm}$ at 15kt & $7,000 \mathrm{~nm}$ at 15kts & $8,000 \mathrm{~nm}$ at 15kts \\
\hline Stores & 10 days & 30 days & 45 days \\
\hline Stability & \multicolumn{2}{|c|}{ Defence Standard 02-109 (NES 109) intact and damage criteria } \\
& $\begin{array}{l}\text { assessment (MOD 2000) } \\
\text { Zoning }\end{array}$ & $\begin{array}{l}\text { 4 zones with } \\
\text { maximum } \\
\text { independence }\end{array}$ & $\begin{array}{l}5 \text { zones with } \\
\text { maximum } \\
\text { independence }\end{array}$ \\
\hline
\end{tabular}

In addition, increased accommodation standards from the Corvette to the Destroyer will be adopted due to the increasing range and stores endurances, implying a longer time for the crew spent at sea.

A summary of the principal particulars of the three combatants is included in Figure 5.31, with GAs in Appendix 8. 


\begin{tabular}{|c|c|}
\hline $\begin{array}{l}\text { Figure 5.31: } \mathrm{Pr} \\
\text { Investigated }\end{array}$ & pal Particulars of the Corvette, Frigate Variant 1 and Destroyer \\
\hline Corvette & \\
\hline Dimensions & $\begin{array}{l}90.7 \mathrm{~m}(84.5 \mathrm{~m}) \times 13.7 \mathrm{~m}(12.2 \mathrm{~m}) \times 8.5 \mathrm{~m}(\text { deep draught } 3.9 \mathrm{~m}) \text {; deep } \\
\text { trim } 0.5 \mathrm{~m} \text {, light trim } 0.5 \mathrm{~m}\end{array}$ \\
\hline Hullform Param. & $\mathrm{C}_{\mathrm{B}}: 0.451, \mathrm{C}_{\mathrm{P}}: 0.615, \mathrm{C}_{\mathrm{M}}: 0.733, \mathrm{C}_{\mathrm{W}}: 0.745$, Circular M: 7.0 \\
\hline Displacement & 1,830te deep, 1,640te light \\
\hline Maximum Speed & $29.7 \mathrm{kts}$ \\
\hline Range & $3,000 \mathrm{~nm}$ at $15 \mathrm{kts}, 2,500 \mathrm{~nm}$ at $18 \mathrm{kts}$ \\
\hline Power Plant & $\begin{array}{l}1 \times 24.05 \mathrm{MW} \text { GT (boost), } 2 \times 1.2 \mathrm{MW} \text { diesels (cruise), } 2 \times 1.2 \mathrm{MW} \\
\text { diesels (auxiliary) driving one FPP on a } 25.5 \mathrm{MW} \text { HTS motor and } \\
\text { one pump-jet on a 1MW HTS motor }\end{array}$ \\
\hline Accommodation & 10 officers, 64 ratings \\
\hline Frigate Variant & \\
\hline Dimensions & $\begin{array}{l}132.2 \mathrm{~m}(125.8 \mathrm{~m}) \times 16.1 \mathrm{~m}(15.2 \mathrm{~m}) \times 9.7 \mathrm{~m}(\text { deep draught } 4.0 \mathrm{~m}) \text {; } \\
\text { deep trim } 0.6 \mathrm{~m} \text {, light trim } 0.8 \mathrm{~m}\end{array}$ \\
\hline Hullform Param. & $\mathrm{C}_{\mathrm{B}}: 0.487, \mathrm{C}_{\mathrm{P}}: 0.596, \mathrm{C}_{\mathrm{M}}: 0.818, \mathrm{C}_{\mathrm{W}}: 0.755$, Circular $\mathrm{M}: 8.1$ \\
\hline Displacement & 3,890te deep, 3,270te light \\
\hline Maximum Speed & $30.5 \mathrm{kts}$ \\
\hline Range & $7,100 \mathrm{~nm}$ at $15 \mathrm{kts}, 6,000 \mathrm{~nm}$ at $18 \mathrm{kts}$ \\
\hline Power Plant & $\begin{array}{l}1 \times 31 \mathrm{MW} \text { GT (boost), } 2 \times 2.94 \mathrm{MW} \text { diesels (cruise), } 2 \times 2.69 \mathrm{MW} \\
\text { diesels (auxiliary) driving two FPPs on } 20 \mathrm{MW} \text { HTS motors }\end{array}$ \\
\hline Accommodation & 11 officers, 137 ratings, 25 embarked forces \\
\hline Destroyer & \\
\hline Dimensions & $\begin{array}{l}154.0 \mathrm{~m}(147.5 \mathrm{~m}) \times 18.9 \mathrm{~m}(17.7 \mathrm{~m}) \times 12.4 \mathrm{~m}(\text { deep draught } 4.7 \mathrm{~m}) \text {; } \\
\text { deep trim } 0.6 \mathrm{~m} \text {, light trim } 0.9 \mathrm{~m}\end{array}$ \\
\hline Hullform Param. & $\mathrm{C}_{\mathrm{B}}: 0.489, \mathrm{C}_{\mathrm{P}}: 0.592, \mathrm{C}_{\mathrm{M}}: 0.825, \mathrm{C}_{\mathrm{W}}: 0.757$, Circular M: 8.1 \\
\hline Displacement & 6,250 te deep, 5,120te light \\
\hline Maximum Speed & $29.7 \mathrm{kts}$ \\
\hline Range & $8,000 \mathrm{~nm}$ at $15 \mathrm{kts}, 7,200 \mathrm{~nm}$ at $18 \mathrm{kts}$ \\
\hline Power Plant & $\begin{array}{l}1 \times 31 \mathrm{MW} \text { GT (boost), } 2 \times 4.08 \mathrm{MW} \text { diesels (cruise), } 2 \times 5.44 \mathrm{MW} \\
\text { diesels (auxiliary) driving two FPPs on } 20 \mathrm{MW} \text { HTS motors }\end{array}$ \\
\hline Accommodation & 22 officers, 151 ratings, 50 embarked forces \\
\hline
\end{tabular}


Correspondingly to the three frigate variants described in Section 5.1, the complements and accommodation requirements of the Corvette and Destroyer designs were sized based on complement/displacement ratios of similar ships. Complement breakdowns are detailed in Appendix 9.1.

The two additional combatants represent a typical modern corvette and destroyer respectively, each with a one passing deck hull. The Destroyer (Figure 5.33) includes the typical five deck hull and a continuous superstructure out to ship's side, while the superstructure of the Corvette (Figure 5.32) is split into two structurally separate blocks and its hull consists of four decks. Since the UCL MSc Ship Design Procedure (UCL 2010a) is aimed primarily at frigate type ships, existing UCL design studies of corvettes and destroyers were investigated.
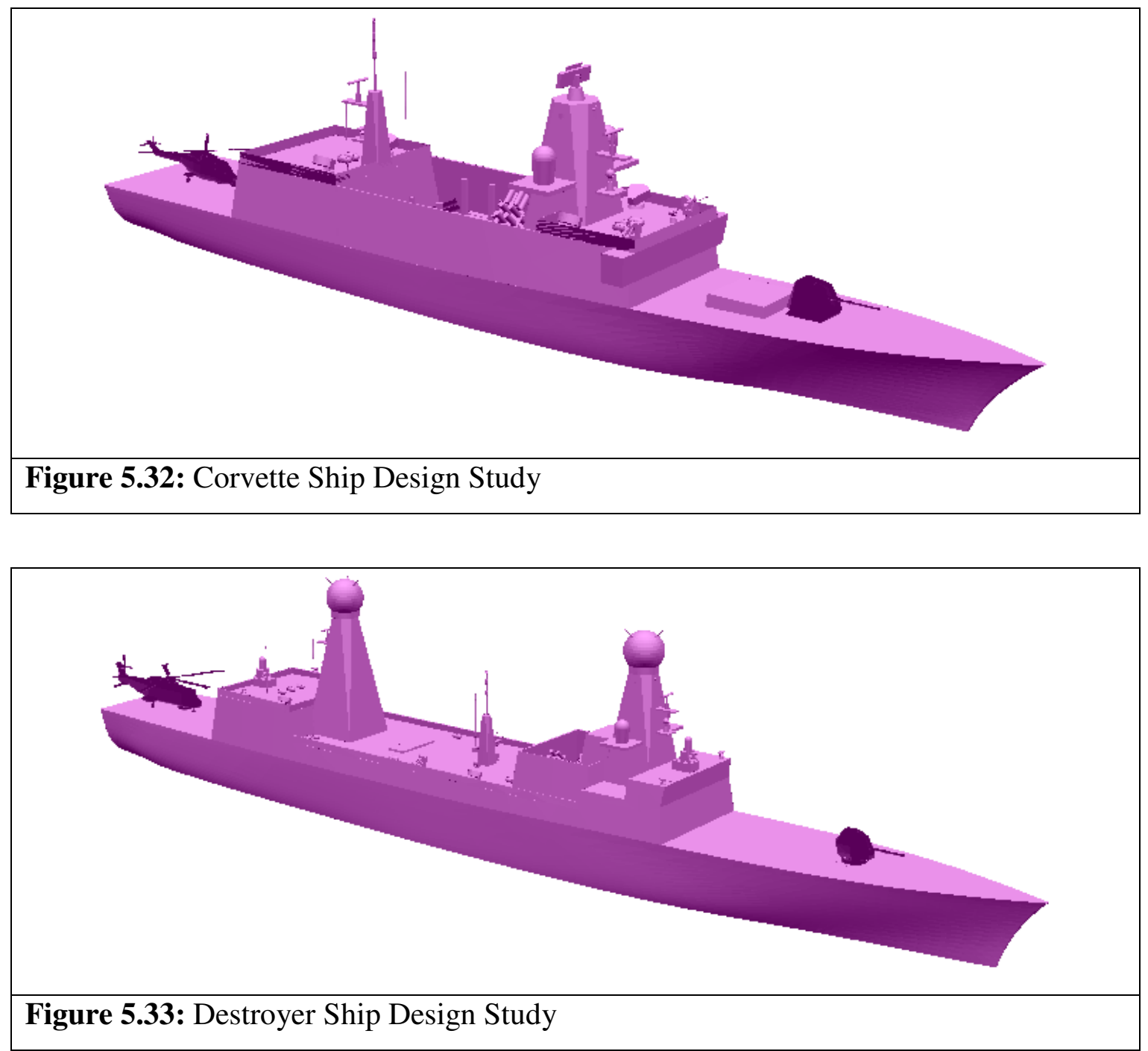

The two additional combatants are described in terms of their top level 'Float, Move, Fight, Infrastructure' functional breakdown in Figure 5.34 and Figure 5.35. 


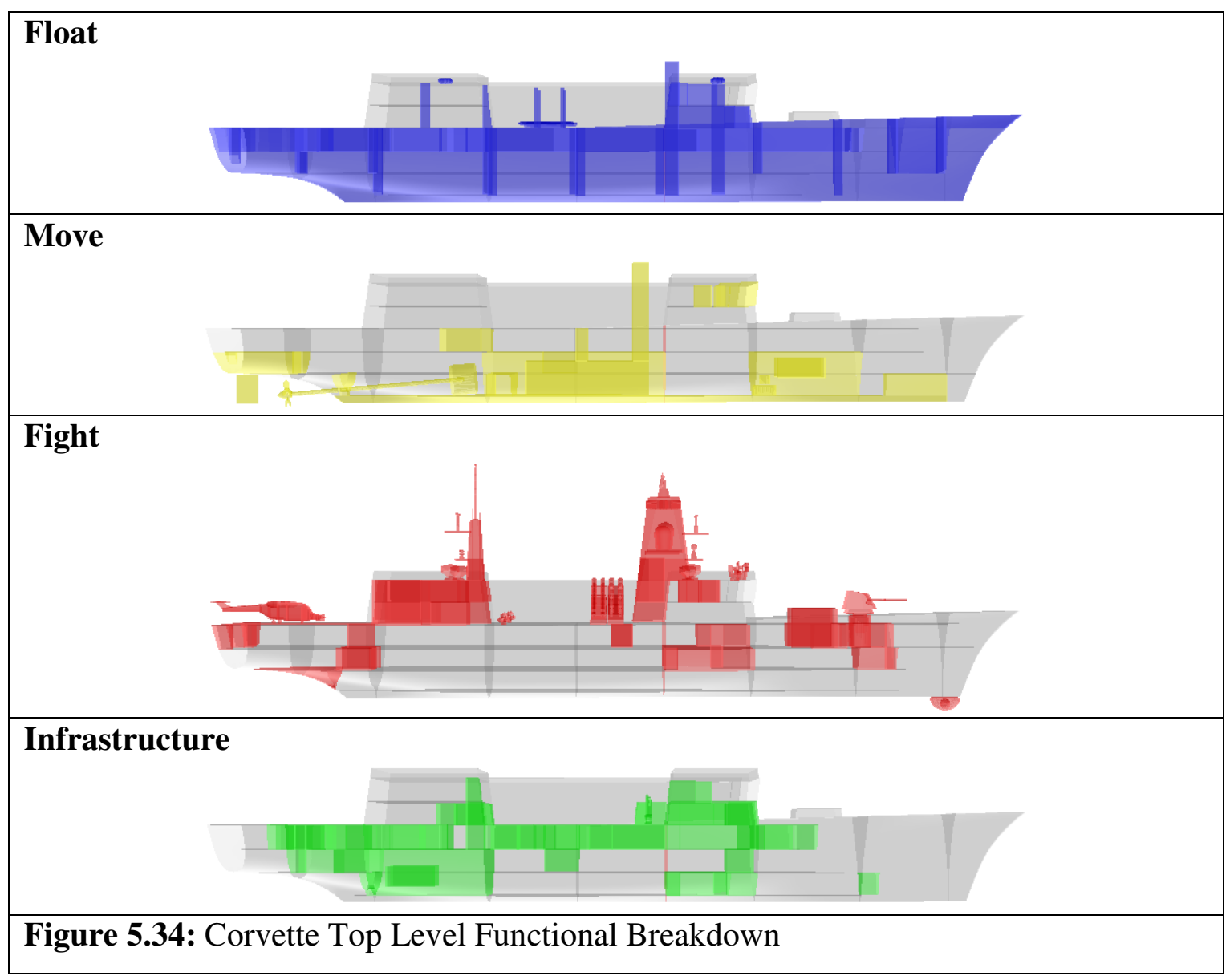

\section{Float}

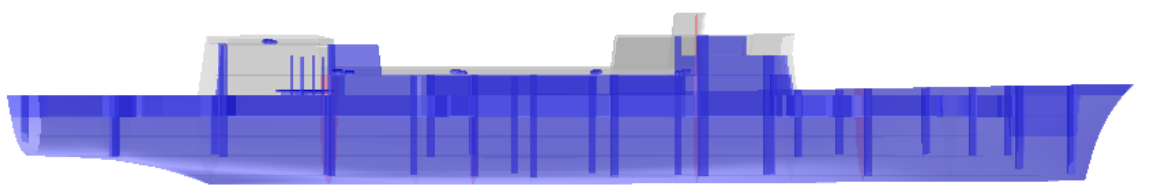

Move

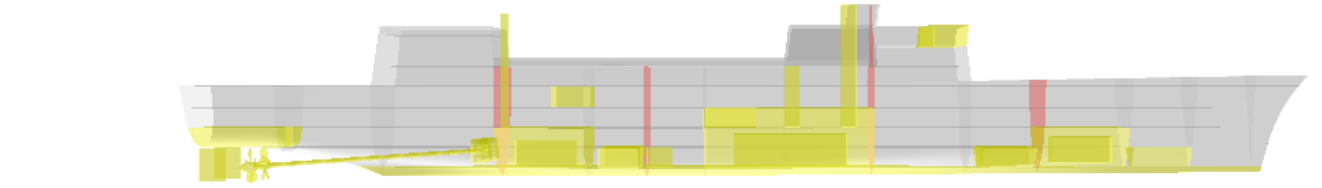

Fight

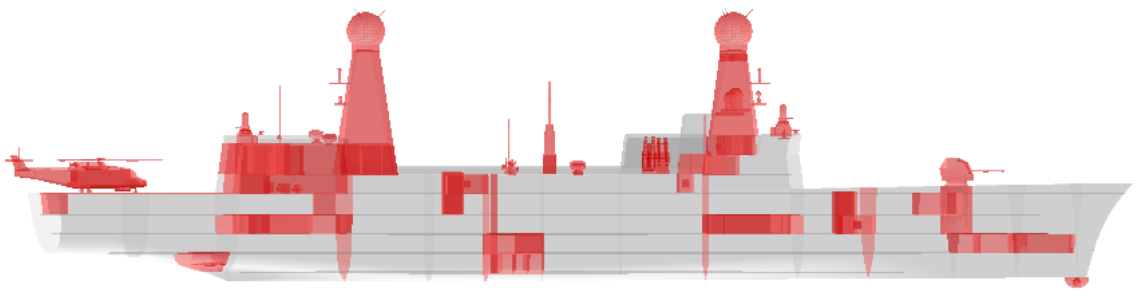

Infrastructure

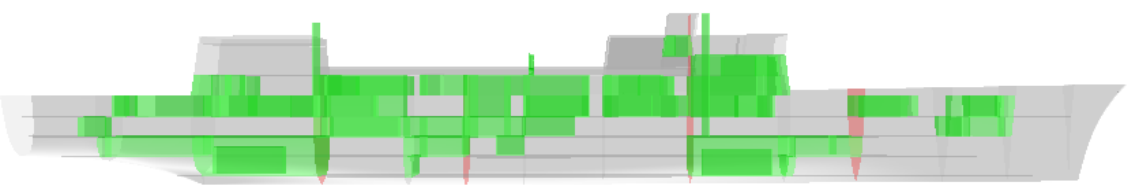

Figure 5.35: Destroyer Top Level Functional Breakdown 
Since the baseline frigate and the Corvette and Destroyer designs shown above all represent typical modern combatants, they present many similarities in the arrangement of the four top level functional groups. The main observation worth mentioning is the lack of fight related compartments in the lower deck (No 4 Deck) of the Corvette hull (Figure 5.34) with the exception of its AVCAT tanks. All other combatants contain magazines in their lower decks. This was not possible for the Corvette since machinery compartments, stores and liquids occupied the entire length of the lower deck due to the ships short overall length. Priority is given to these heavy compartments for stability considerations (Begg et al 1990; Heather 1990), as mentioned in Appendix 4.2. Magazines were, therefore, forced towards the higher decks of this ship design. Although this would improve reloading and stowing operations, it would also increase the vulnerability of the magazines, especially to threats such as fire, which could have devastating overall effects. Another effect of the Corvettes short length, noticeable in the illustrations above, is the decreased separation distances between sensors (which could result to problems similar to the ones possible, due to the small superstructures, in Frigate Variants 2 and 3, such as interference issues and increased vulnerability) and machinery compartments. Additionally, it is clear that moving from the smallest combatant to the largest, the footprint of the distribution of the Infrastructure functional group in the $\mathrm{z}$ axis (i.e. depth) increases, purely because of the increasing number of decks in the comfortable amidships area of the hull and superstructure. A final observation is the existence of different numbers of machinery compartments in each combatant. This is directly related to the zoning philosophy of each design study.

The Corvette and Destroyer designs are described in terms of their top level weight breakdowns in Table A11 and Table A12 of Appendix 9.2. The margin philosophy adopted is discussed in Appendix 9.3.

The hulls of both additional combatants were generated in an identical manner to that of Frigate Variants 1 and 2, i.e. the Quickhull object of Paramarine was used to scale a Type 22 Frigate based hull. The lines plans of the Corvette and Destroyer designs are illustrated in Figure 5.36 and Figure 5.37 respectively. The small L/B ratio and large transom beam of the Corvette design (typical in many corvettes), primarily in order to achieve good stability and adequate internal volume, is clearly visible. 

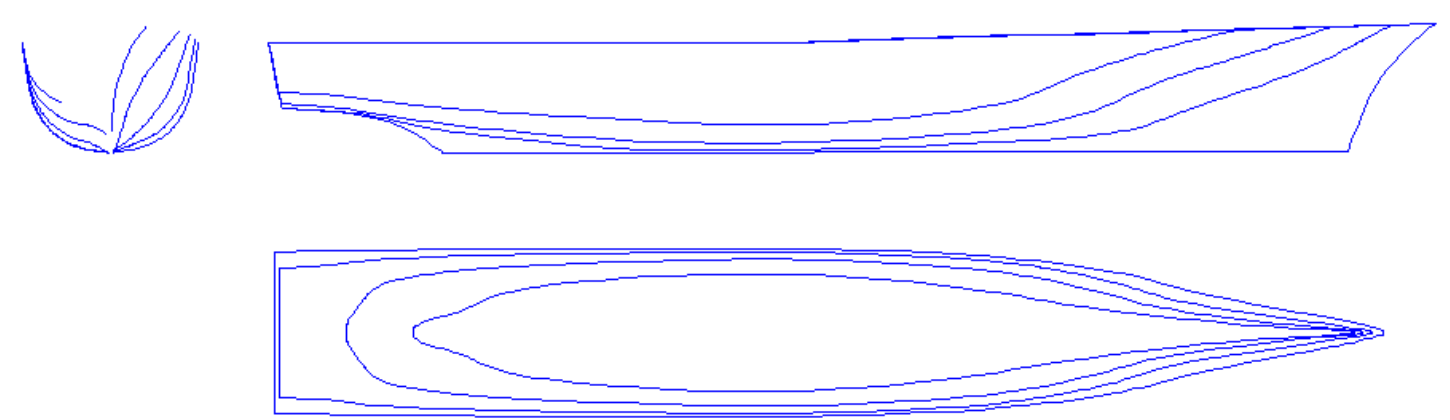

Figure 5.36: Corvette Lines Plan

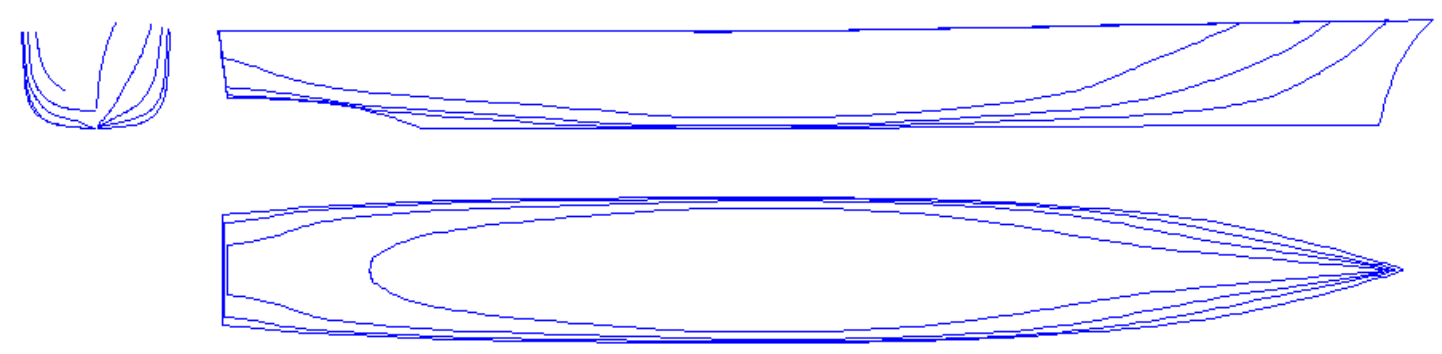

Figure 5.37: Destroyer Lines Plan

The propulsive powering requirements for the Corvette were computed using the inbuilt Paramarine powering module (see Appendix 9.4). The machinery that was selected consisted of:-

- $\quad 2 \times 1.2 \mathrm{MW}$ Warstila 6L20 diesel gensets (cruise);

- $1 \times 24.05 \mathrm{MW}$ General Electric LM2500 marine gas turbine, electrical drive (boost);

- $\quad 2 \times 1.2 \mathrm{MW}$ Wartsila 6L20 diesel gensets (auxiliary).

Similarly to the trimaran frigate variant, the small beam of the Corvette obstructed the use of a double shaft arrangement. Since a single shaft was unacceptable for survivability and availability reasons, a secondary forward propulsive unit, consisting of a motor and pump-jet arrangement (Schottel 2010a), was fitted. Therefore, the Corvettes machinery drives a single FPP propeller on a 25.5MW HTS motor and a single Schottel SPJ 220 Pump-Jet on a 1MW HTS motor. Figure 5.38 illustrates the arrangement of the above machinery, auxiliary machinery shown in green (Infrastructure functional group), and main machinery in yellow (move functional group). As previously noted, this is closely related to the move major system, which was modelled in the vulnerability and recoverability assessment approach. Whole major system architecture and tree diagrams are included in Appendix 7. 


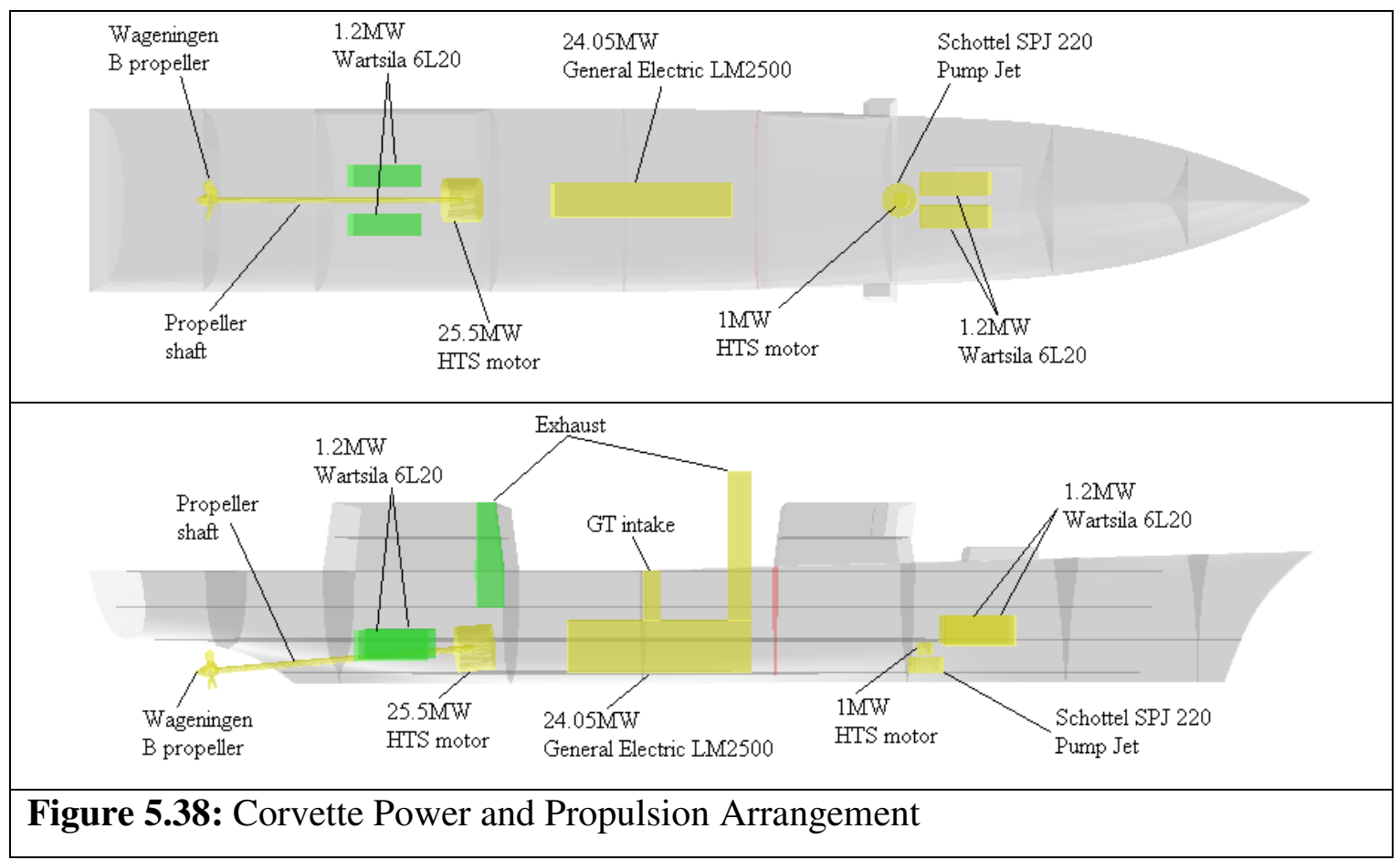

A number of dissimilarities are observed between the power and propulsion of the Corvette and the baseline frigate, such as the placement of both auxiliary engines in the same compartment. This was done due to constraints imposed by the short length of the Corvette as well as the fact that, having a two zone requirement (Table 5.5), a minimum of two machinery compartments (as opposed to the four of the frigate variants) was acceptable. Despite the decreased number of machinery compartments, the relative footprint of the power and propulsion arrangement is larger than that in the baseline frigate, indicative of the tight arrangement inflicted by the short overall length. Another noticeable difference is the split forward and aft propulsive units, similar to the trimaran arrangement (Figure 5.18), which should strongly impact on the vulnerability of the move system. Furthermore, as with all frigate variants, superstructure above the forward-most engines is absent. An underwater exhaust arrangement of the forward cruise engines (similar to the trimaran) was preferred over the linkage of their exhaust with that of the gas turbine. This was the preferred option as it was evaluated inefficient to extend the exhaust of the (two) cruise engines through the relatively short $(2.7 \mathrm{~m}$, as opposed to the baselines $3 \mathrm{~m}$, deck head height) and unique through deck of the Corvette, through which connectivity items (such as pipes and cables) usually traverse. The implications of underwater exhausts were previously discussed (Appendix 4.1) and include a reduction in the ships IR signature (Heather 1990; Foxwell 1990a), the worsening of the ships acoustic signature (Heather 1990) and the danger of confronting back pressure and corrosion related issues (Dicker 1986; Belcher 2008). In addition, the 
gas turbine exhausts through the forward mast (see Appendix 8) and the two auxiliary engines exhaust at the forward starboard corner of the aft superstructure, therefore, not interfering with helicopter operations. The main similarity between the power and propulsion arrangements of the Corvette and baseline frigate is the reduction in vulnerability due to the wide separation of the (parallel) power generation units (Appendix 4.2) and the relatively short (but declined) shaft, arising from the IFEP system selected. However, the choice of this style of arrangement, in combination with the short hull of the Corvette, has led to the positioning of the two cruise engines quite far forward which could raise issues with their operation under high pitch motions. Removal routes for all engines were taken into account through the inclusion of soft patches in the passageways and compartments above each engine.

The propulsive power requirements of the Destroyer were also estimated through the inbuilt Paramarine module (see Appendix 9.4). The machinery selected consisted of:-

- $\quad 2 \times 4.08 \mathrm{MW}$ Wartsila 12V26 diesel gensets (cruise);

- $1 \times 31 \mathrm{MW}$ Rolls-Royce MT30 marine gas turbine, electrical drive (boost);

- $2 \times 5.22 \mathrm{MW}$ Wartsila 16V26 diesel gensets (auxiliary).

The above machinery driving two FPPs on 21MW HTS motors. The arrangement of the main (yellow) and auxiliary (green) machinery described above is illustrated in Figure 5.39.

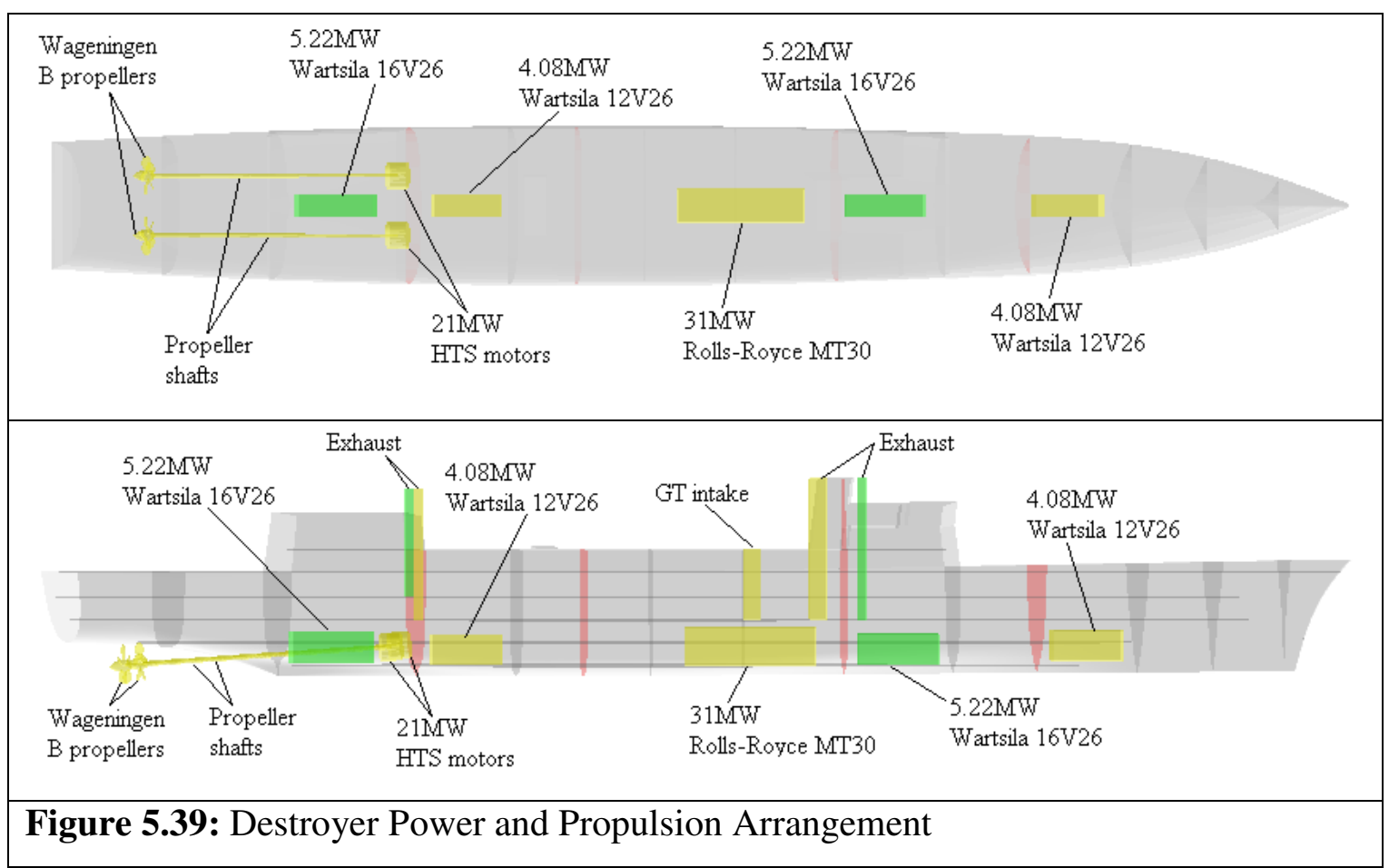


The power and propulsion arrangements of the Destroyer and the baseline frigates present many similarities. The main difference is the fact that all power generation units are located in separate machinery compartments. This was done in order to match the number of engines to the number of zones, since both ship designs included five engines, but the Destroyer had a five zone requirement as oppose to the four zones of the frigate design. This has led to a better distribution of the (parallel) engines along the ship's length and should, therefore, decrease vulnerability of the move system. It is evident that the lengthwise distribution of engines was only possible due to the adoption of an IFEP system, as was the use of relatively small shafts. However, similarly to the Corvette design, despite the greater length of the Destroyer, the increased separation requirements of the power generation units have led to the placement of the forward Wartsila 12V26 engine relatively far forward, therefore, possible arising concerns with the engines operation under high pitch motions. Another difference between the two ship deigns is the underwater exhaust of the forward cruise engine, the implications of which have been discussed above and in Appendix 4.1. The uptakes of the aft auxiliary and aft cruise engines merge and exhaust through the aft mast, as do the uptakes of the gas turbine and the forward auxiliary engine, in the forward mast; this is clearer in the GA, Appendix 8. Identically with all ship design studies, removal routes for all engines were taken into account through the inclusion of soft patches in the passageways and compartments above each engine.

The hydrostatics of the Corvette and Destroyer designs were computed using the inbuilt Paramarine module. As in the frigate variants, intact stability was assessed against Defence Standard 02-109 (NES 109) Stability Standards for Surface Ships (MOD 2000) until all criteria were passed. Damage stability (of both deep and light conditions) was assessed against Defence Standard 02-109 (NES 109) damage criteria assessment (MOD 2000). Hydrostatics data are given in Appendix 9.5.

The transverse subdivisions of the Corvette and the Destroyer designs are illustrated in Figure 5.40 and Figure 5.41, with the WT bulkheads constituting the main zone boundaries shown in red. 

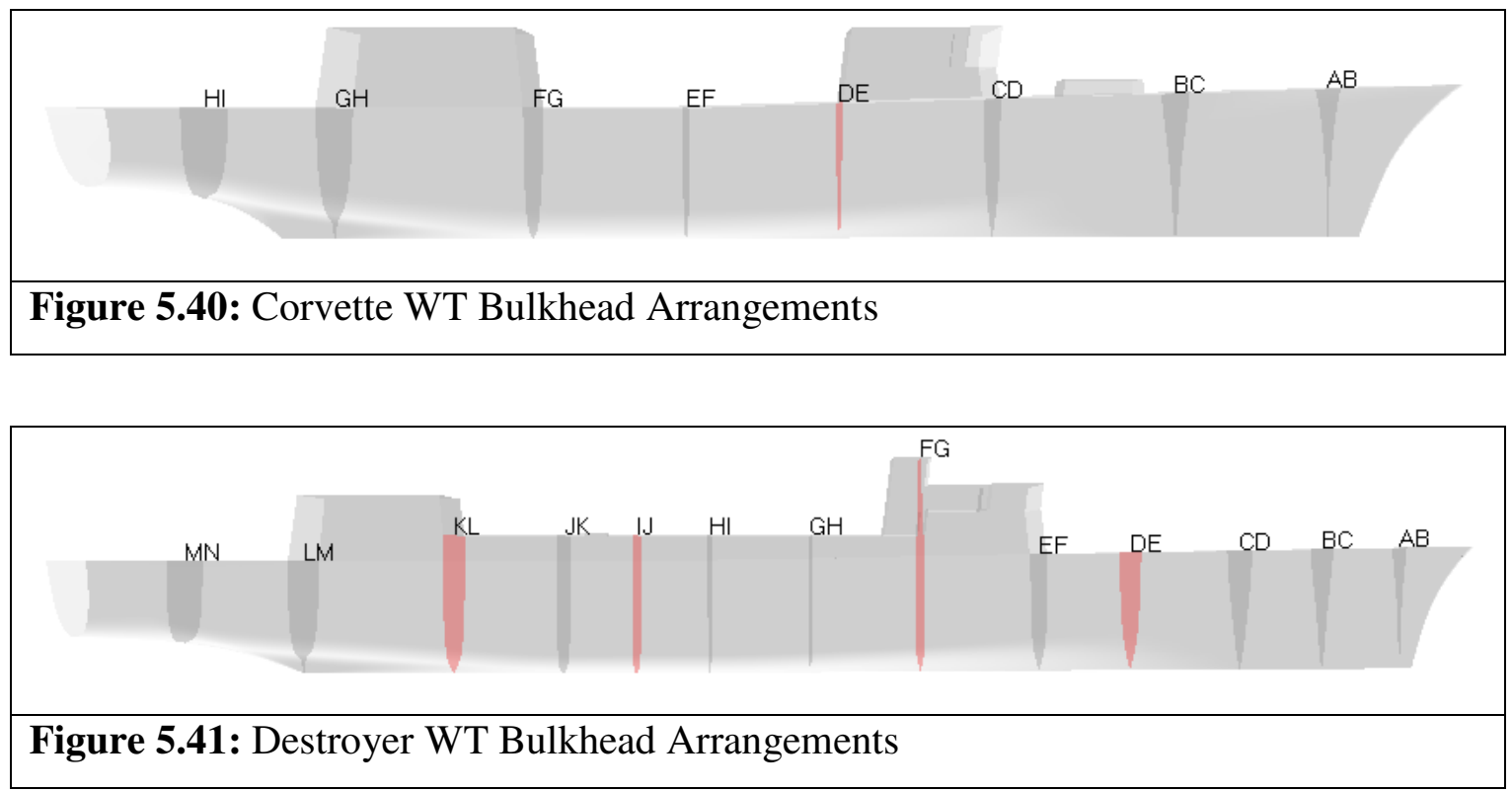

As with the frigate variant designs, bulkheads locations were dictated through zoning, structural, layout and damage stability considerations. For structural continuity, bulkheads were positioned at the forward and aft superstructure ends, masts, medium calibre naval gun and at every location where there is a step in the superstructure. Note that in the Corvette design a bulkhead was not included at the after cut-up because of the inability of the hull generator to produce a realistic hull, had the after cut-up been shifted further forward. However, the selected arrangement, with the after cut-up half way between two bulkheads is acceptable and frequently occurring. The Corvette design contained eight WT bulkheads (and nine WT sections), four less than the baseline frigate; the Destroyer design contained thirteen bulkheads (and fourteen WT sections), two more than the baseline frigate.

The Corvette encompassed two zones (the aft zone including 5 WT sections and the forward zone, 4) and the Destroyer contained five zones (with 3, 2, 3, 2 and 4 WT sections respectively). The zone boundary of the Corvette is slightly forward of amidships in order for the whole gas turbine (engine and alternator) to be contained in the same zone. Each of the zones contains independent power generation units, CWP, HPAC, FP, ATU and ventilation, NBCD stores and airlocks for access to and from the citadel/weather, as shown in Figure 5.42 and Figure 5.43. 


\section{Machinery Room Arrangement}

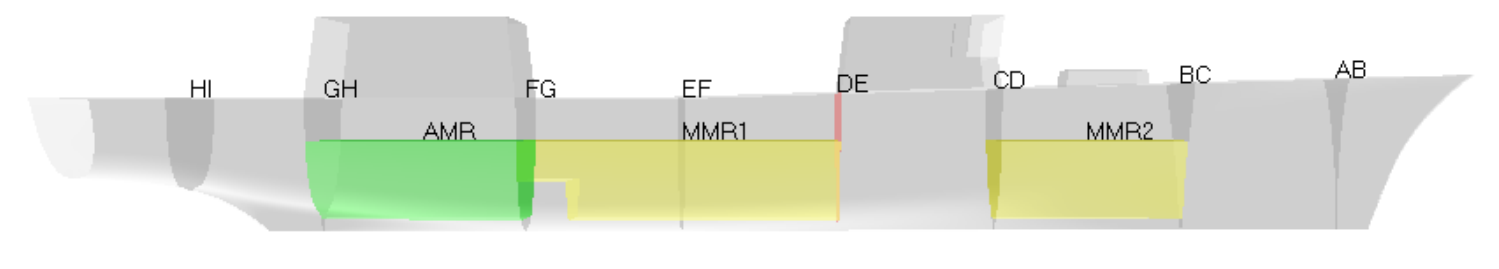

ATU and Ventilation Arrangement

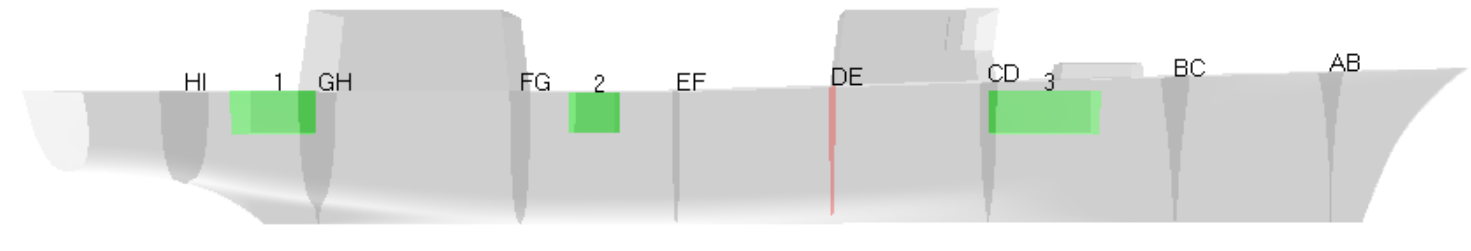

NBCD Stores

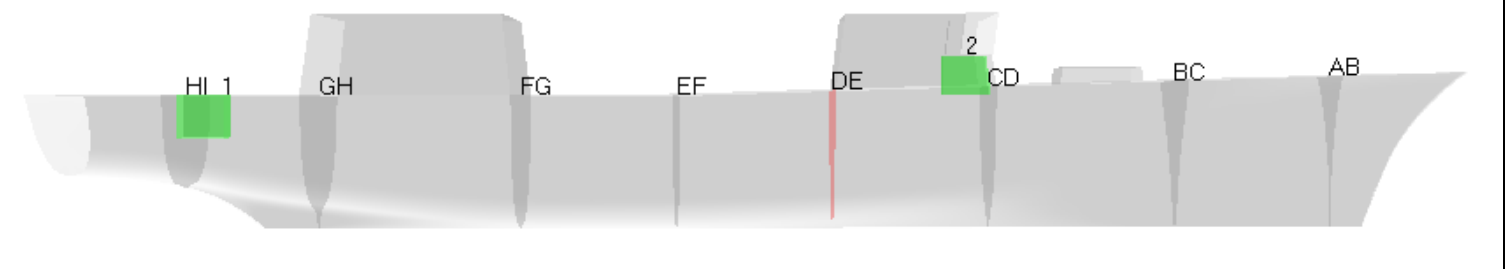

Airlocks

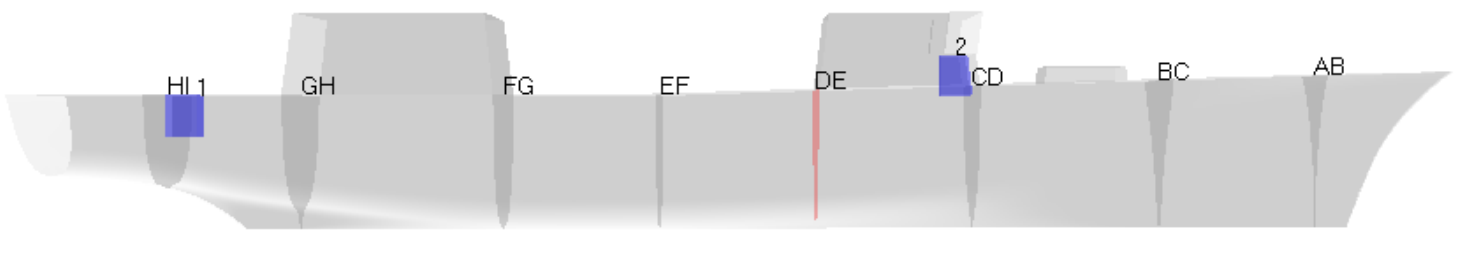

Figure 5.42: Corvette Zoning Philosophy

A number of differences between the Corvette and baseline frigate zoning arrangements arise due to halving the number of zones. For example the Corvette is the only ship design to contain only one auxiliary machinery room. Since the requirement was that each zone should have an independent power generation unit, two machinery compartments would be sufficient for this ship design study. However, since the two auxiliary gensets, two cruise gensets and the gas turbine could not fit inside two such compartments, a total of three machinery rooms were included, two main and one auxiliary. Consequently, the Corvette was the only design to include more than one machinery room in one of its zones (aft zone includes two) and more than two engines in one of its zones (aft zone contains two diesels and one gas turbine). This possibly suggests that a three zone philosophy was more suitable and efficient for the above design. As in all other design studies, the gas turbine, although smaller than the RollsRoyce MT30 used in the frigates, was split between two WT sections due to its large size, Figure 5.38. The AMR and MMR2 each encompassed a CWP, HPAC and FP in 
order to meet the zonal requirement with a maximum separation distance between the equipment. Due to the size of the aft zone, which houses three of the five engines and the living spaces of the bulk of the crew, it was decided to split its ATU and ventilation compartments into two units, aft and at amidships. Therefore the aft zone contained $2 / 3$ of the ships ATU and ventilation capacity, again encouraging a three zone philosophy. Each zone contained an airlock, located conveniently to an NBCD store. Airlock 1 led to the aft mooring space and airlock 2 to the forward part of the weather deck; both airlocks were adjacent to cleansing and decontamination stations for access to the weather.

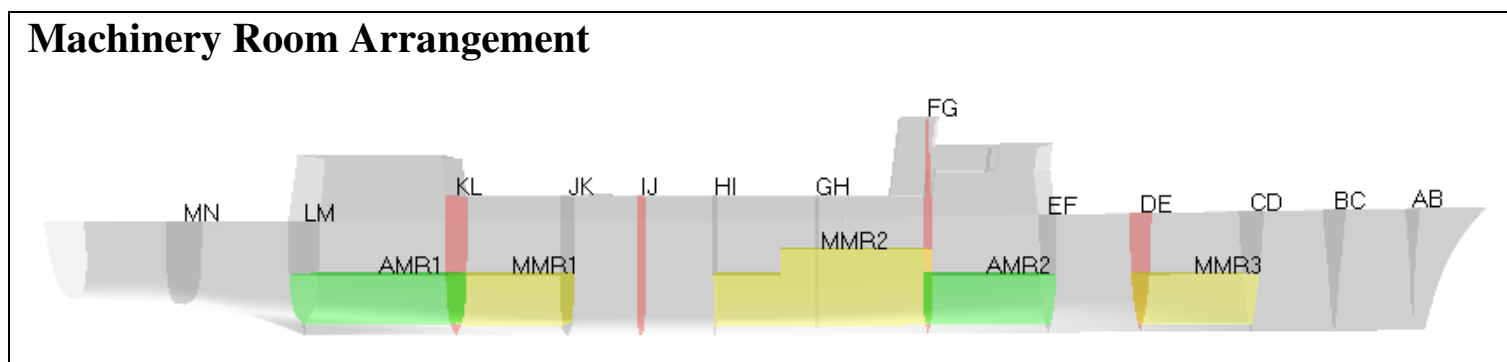

\section{ATU and Ventilation Arrangement}

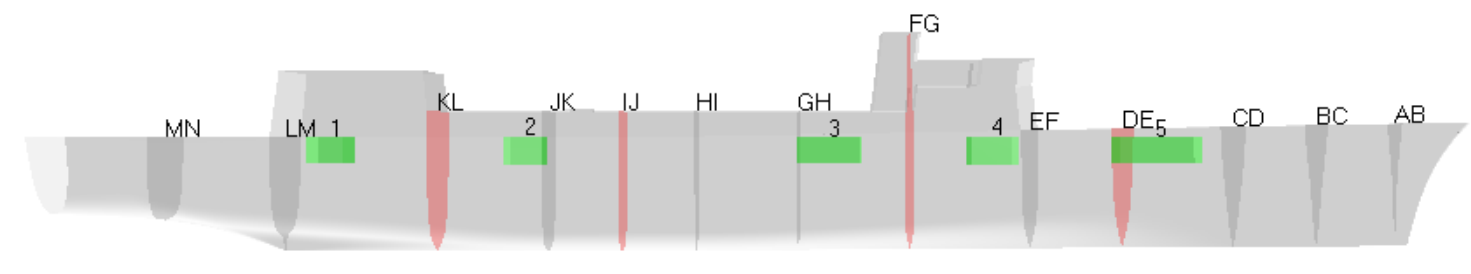

\section{NBCD Stores}

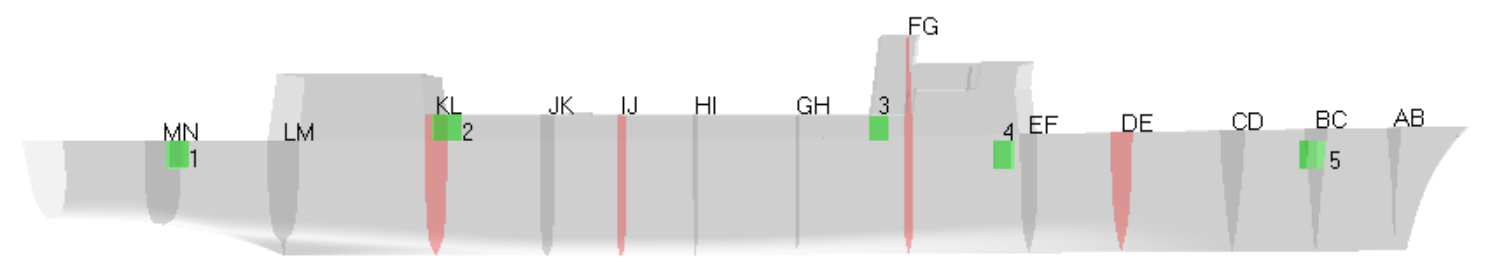

\section{Airlocks}

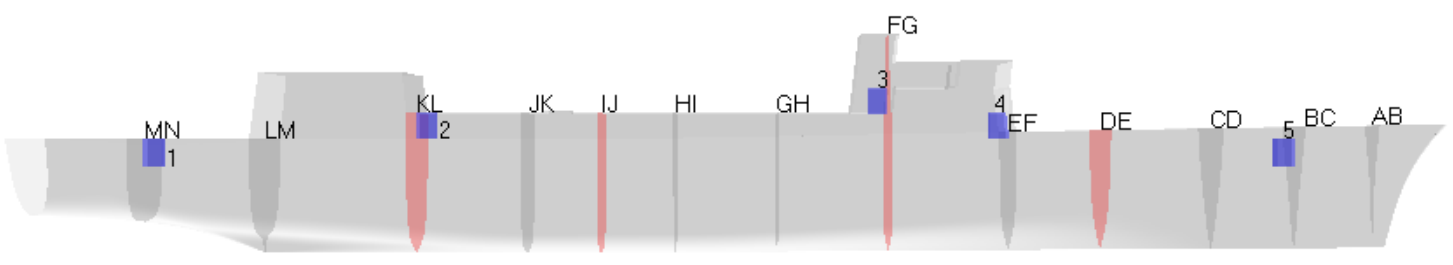

Figure 5.43: Destroyer Zoning Philosophy

The Destroyer includes one more of each of the replicated (in accordance to the zoning philosophy) equipment items compared to the baseline frigate design (due to the presence of an added zone), apart from power generation units. As observed in Figure 
5.4 and Figure 5.39, both ship designs include five engines, the only difference being that the Destroyer contains each engine in a different machinery compartment (and zone), therefore, achieving the maximum number of zones possible for the given configuration and requirement. Consequently, the main difference between the baseline frigate and the Destroyer designs in this regard is the presence of an extra machinery room in the Destroyer, each room containing one engine, CWP, HPAC and FP. Once again, MMR2 has been extended along two WT sections to accommodate (in separate WT sections) the alternator and engine of the large gas turbine. Note that a portion of MMR2 is three decks deep due to the adequate volume provided by the large 5-deck hull of the Destroyer. The ATU and ventilation units are located in proximity to the living spaces and engine rooms, reasonably above the waterline. The airlocks are situated close to the NBCD stores, as in all previous design studies. Airlock 1 leads to the aft mooring space, airlock 2 to the hangar, airlock 3 above the amidships section of the superstructure, airlock 4 to the forward section of the weather deck and airlock 5 to the forward mooring space. Cleansing and decontamination stations are located adjacent to airlocks 1 and 4 .

Figure 5.44 and Figure 5.45 illustrate the arrangement of the main equipment of the major Fight systems which were modelled in the survivability assessment method. As before, items solely related to each Fight system are shown, therefore, neglecting sensors, power generation units and centralised command and control. (For full system tree diagrams and architectures see Appendix 7).

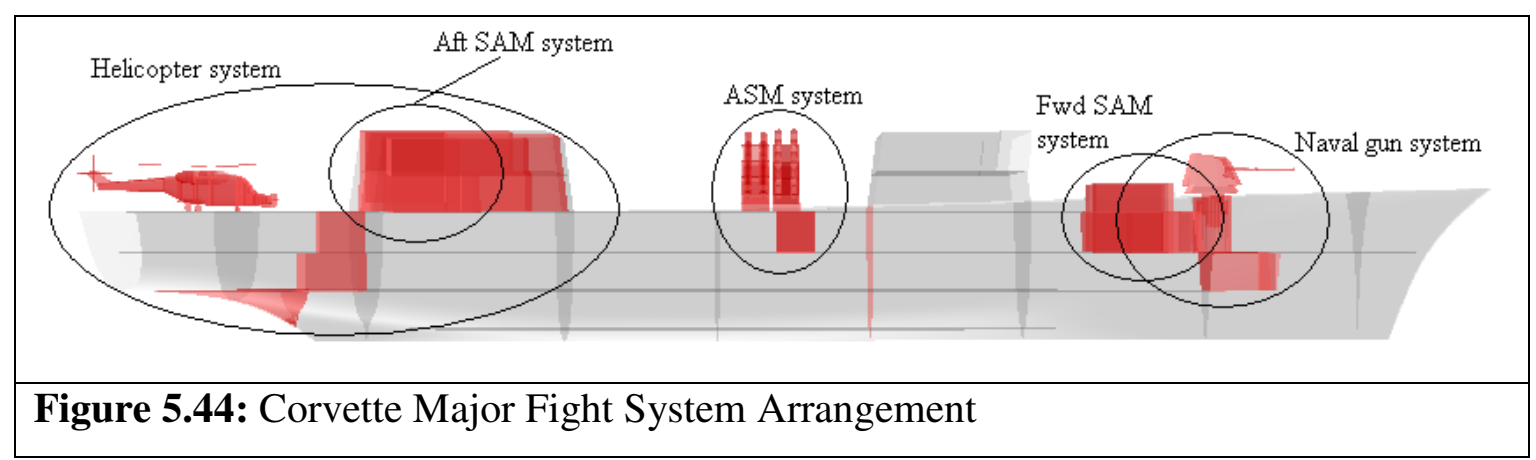

It is obvious that in the case of the Corvette it was not realistically possible to link the arrangement of the five major Fight systems to the zoning philosophy, due to the presence of only two zones. However, each system (the items of which are in series) was attempted to be concentrated as much as possible in a particular area of the ship to decrease vulnerability. That is evident from Figure 5.44, as is the overlapping of certain Fight systems (to a much greater extent than in the frigate variants) due to the tight 
arrangements imposed by the short hull. This verifies the strong link between ship length and weapons and sensors (upperdeck) layout (Andrews and Brown 1982; Brown 1987) identified in Appendix 2 and in the description of the major Fight system arrangement of the trimaran, Figure 5.24.

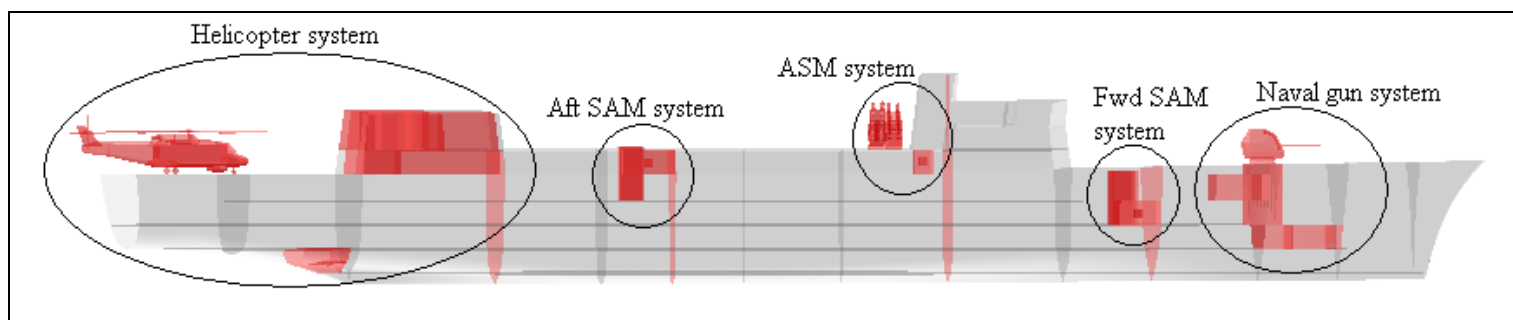

Figure 5.45: Destroyer Major Fight System Arrangement

The Destroyer, on the other hand, is the only ship design study to have achieved full linkage between the distribution of the main equipment of the major Fight systems and the zoning arrangement. This was possible due to the equal number of Fight systems and zones (once again implying that the maximum number of zones for the given configuration and requirement has been reached) and the large overall ship length, allowing for a comfortable upperdeck layout. From Figure 5.45 it is clear that none of the footprints of any Fight system overlap with another one and all main equipment of all Fight systems are within the same zone, therefore achieving the specialisation of each zone with a particular type of warfare/operations.

The Corvette design study included 4 decks in its hull, with a conventional centreline passageway on the DC deck (No 2 Deck), common in such ship types. In addition, each WTs section included two vertical accesses, one at either side of the centreline passageway and at the forward and aft end of each WT section respectively. Therefore, identical access arrangements were observed as for the baseline frigate, the only difference being the reduction of the DC deck passageway width from $2 \mathrm{~m}$ (used in all other ship design studies) to $1.5 \mathrm{~m}$ due to the smaller size of the ship. The above arrangements are shown in Figure 5.46. 


\section{Deck Access}

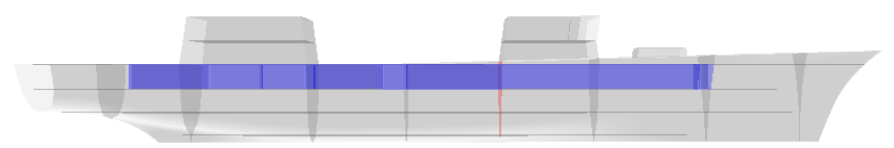

DC Deck Access and Vertical Access

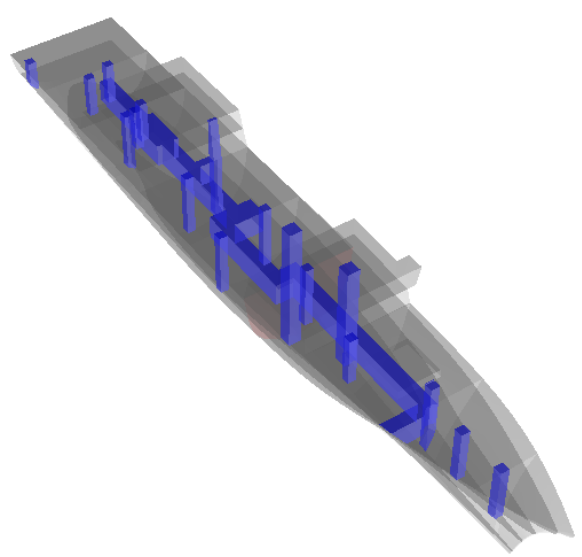

Figure 5.46: Corvette Access Philosophy

The Destroyer design also includes one through deck (No 2 Deck) although its hull consists of 5 decks. Being a conventional destroyer, it was decided to implement a double, side, passageway arrangement, Figure 5.47.

\section{Deck Access}

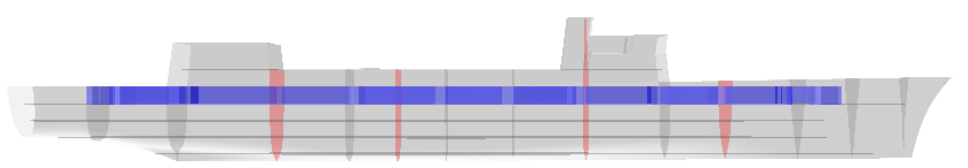

DC Deck Access and Vertical Access

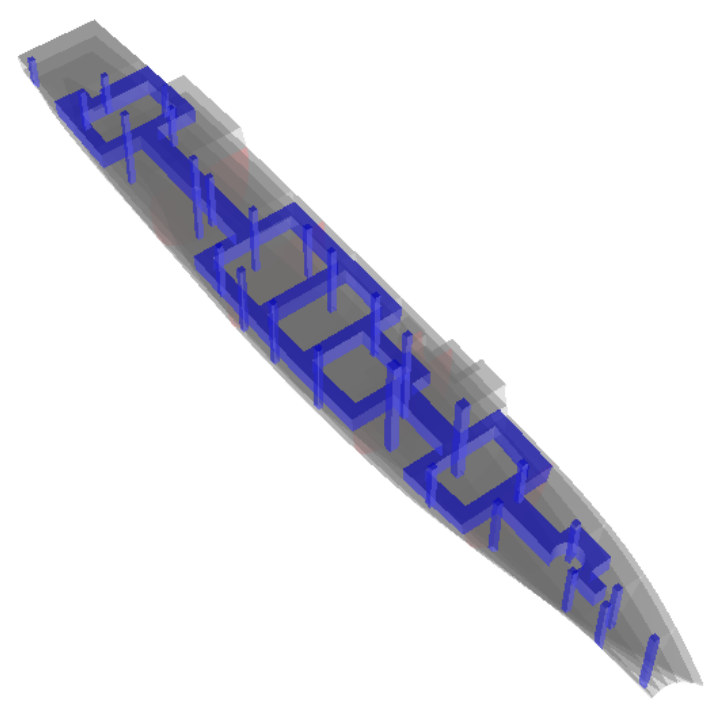

Figure 5.47: Destroyer Access Philosophy 
The two side passageways converged to a single centreline access strip above all machinery compartments (with the exception of the gas turbine compartment) in order to facilitate engine removal, and at the narrow forward end of the hull. The WT sections with a centreline passageway include two vertical accesses, one at either side of the passageway and at the forward and aft end of each WT section respectively; the WT sections with side passageways include two vertical accesses, one adjacent to either passageway and at the forward and aft end of each section respectively. A crossconnecting passageway is included at all WT sections with side passageways, therefore, improving communication and personnel flow, but occupying a considerable amount of space, as in the trimaran frigate design. Advantages of side passageways were examined (see Appendix 4.2) and include the protection of sensitive compartments and equipment located in between them (Begg et al 1990; Harney 2010) and the ability to run pipes and cables along them for the redundancy and separation of services (Brown 1987; Afanasieff and Mabry 1994). More detailed access arrangements are presented in the GAs, Appendix 8.

Figure 5.48, Figure 5.49, Figure 5.50 and Figure 5.51 illustrate the distribution of all equipment and compartments relevant to the recoverability PMs (Sections 4.3.1, 4.3.2 and 4.3.3) employed in the recoverability assessment method previously described. The first two illustrations show the arrangement of items related to Category $1 \mathrm{PM}$ analysis.

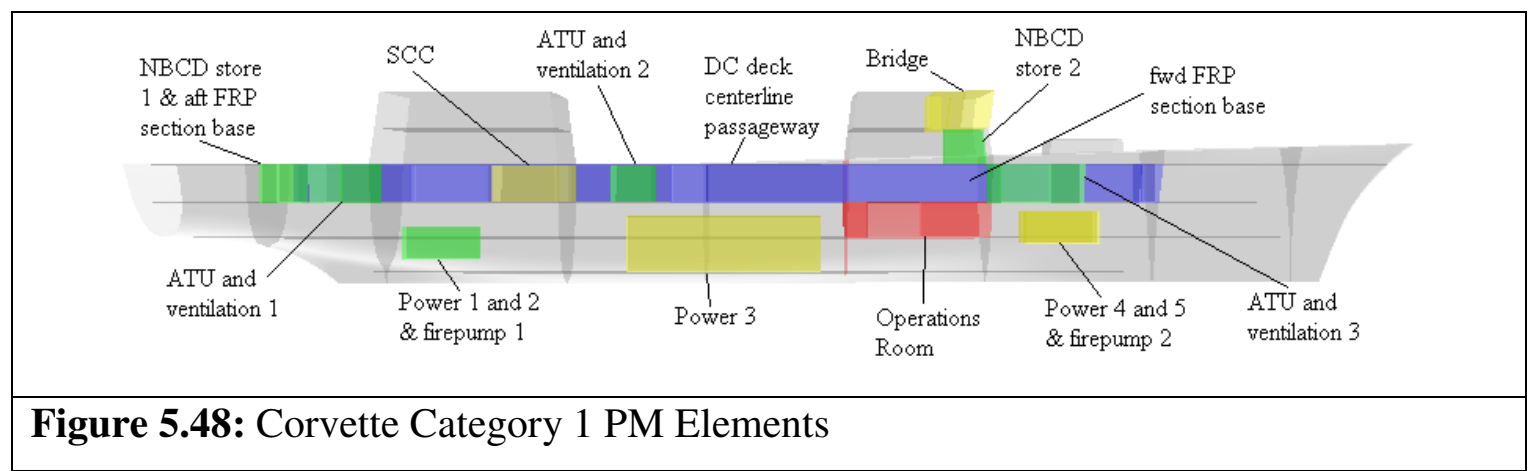

As before, the calculation of MPs 1.1, 1.2 and 1.3 is relatively straightforward from the figure above, as is that of PM 1.4, given there is only one route (of smaller width than in the frigate variants and Destroyer designs). For the location of items related to all other category 1 PMs (which are estimated by use of SURVIVE Lite, as explained in Section 4.3.1), a similar philosophy to that for the frigate designs was used; i.e. the Operations Room was located deep in the hull for reduced abovewater threat vulnerability, conveniently to the bridge for improved crew evolutions and at a distance 
from the SCC (positioned close to machinery compartments, although the use of a widely distributed IFEP system created constraints) to enable its use as a secondary Operations Room in emergency. All other equipment and compartments were located through zoning considerations. A noticeable difference between the Corvette and frigate arrangements is the positioning of one of the FRP section bases at a different deck from its corresponding NBCD store, although the adjacency requirement has still been met. This was a result of the decision to locate the NBCD store adjacent to the forward airlock and cleansing and decontamination station (Figure 5.42), which were located in the forward superstructure in order to direct to the weather (forward weather deck); and the requirement for all FRPs to be stationed on the DC deck, being the only deck with unrestricted access the ship's length. Another difference with the baseline frigate is the fact that not all FRP section bases were located adjacent to main zone boundaries. With only two zones and one main zone boundary, such a decision would effectively group the two section bases together, therefore, practically eliminating one of them and increasing their vulnerability. Instead, the two FRP section bases were separated in a forward and aft arrangement.

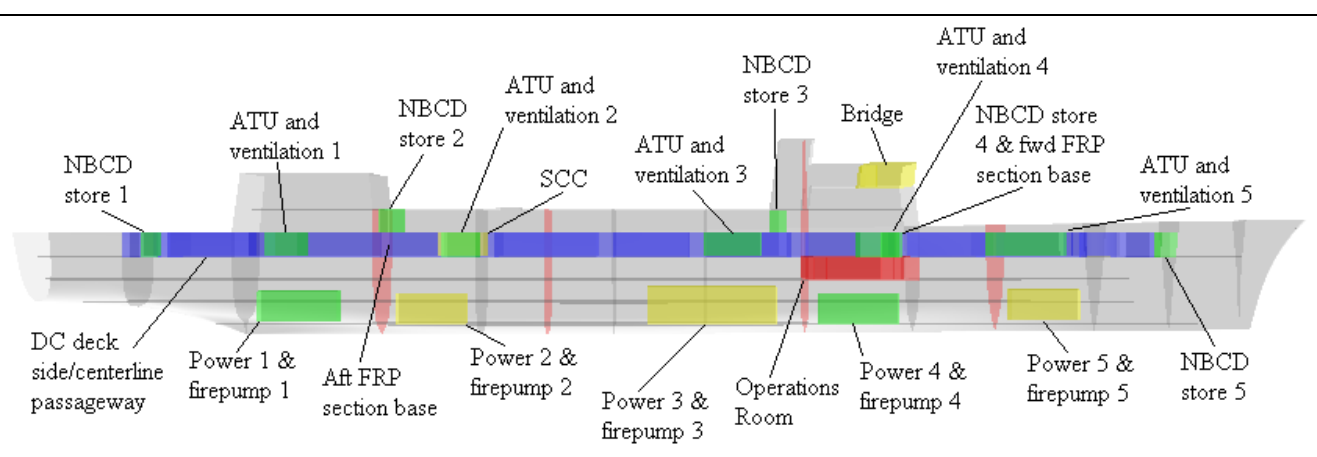

Figure 5.49: Destroyer Category 1 PM Elements

The logic used for the location of the Category 1 related PM spaces in the Destroyer design was the same as in the remaining combatants described above. There are three main differences worth commenting. First, the side passageway arrangement across a substation length of the hull would affect PM 1.4 by providing a larger number of alternative routes to reach the affected compartment, therefore, improving DC. Second, the aft FRP section base has been placed adjacent to, but one deck below its corresponding NBCD store (which in turn is located beside the airlock leading to the hangar, Figure 5.43). A final, but significant variation is the placement of the Operations Room above a machinery compartment. This arrangement is generally undesirable due to the compulsory provision of removal routes for engines, which has 
led to the incorporation of a generous margin in the Operations Room space requirement in order to remove the engine below it with minimal disruptions. This complicated arrangement was a result of the 5 zone requirement (each with independent power generation) which led to relatively small zones; the zone in which the Operations Room is located being only 2 WT sections long. It could be argued that the Operations Room could be moved one WT section forward (as in the trimaran frigate variant); however, due to the presence of the forward SAM vertical launchers at that WT section (visible in Figure 5.45, and in Appendix 8) there was not an adequate provision of space.

Figure 5.50 and Figure 5.51 below depict collectively the distribution of Category 2 (and Category 3) PM related items on the Corvette and Destroyer designs.

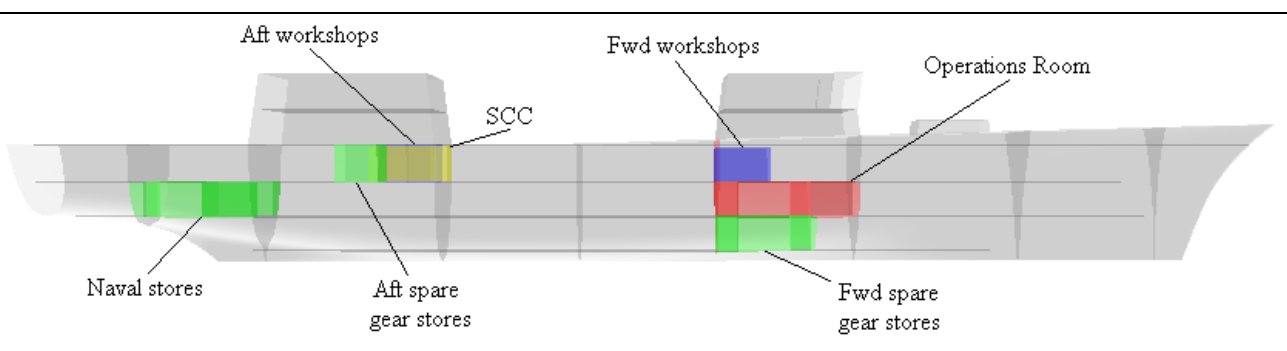

Figure 5.50: Corvette Category 2 PM Elements

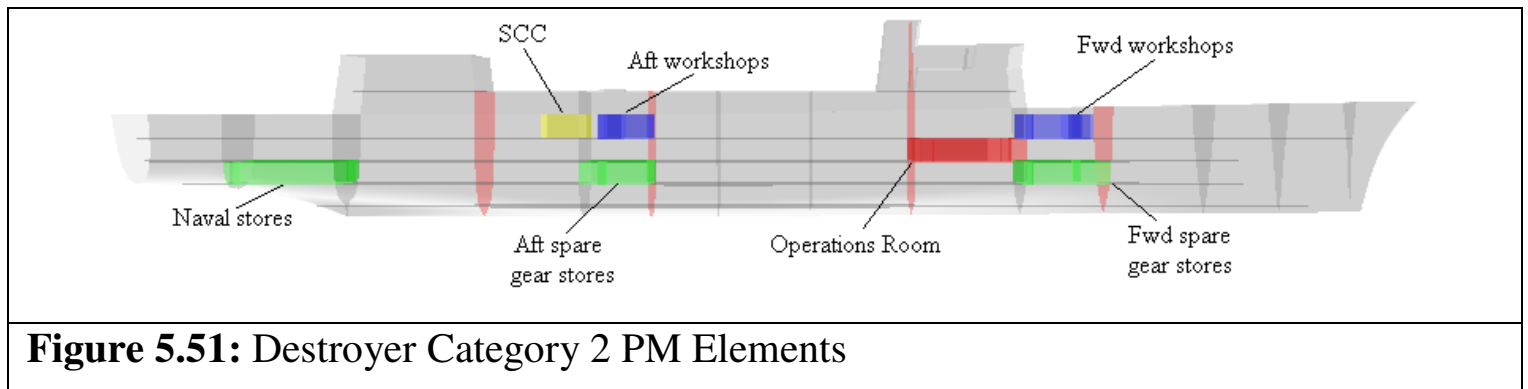

By comparing the above two illustrations with Figure 5.10 it becomes clear that the arrangement of Category 2 PM related compartments is almost identical for all three Corvette, baseline frigate and Destroyer design studies. Workshops and spare gear stores have been split in two groups for redundancy, arranged in a forward and aft orientation (separated by only two WT sections in the smaller Corvette design). Each workshop has been located conveniently to its corresponding spare gear store and to the machinery rooms. The Operations Room and SCC have been separated by a reasonable distance for redundancy reasons and the naval stores have been located primarily by taking into account stability considerations. In addition to the above compartments, 
Category 3 PM analysis involves all of the items of each major ship system; system architecture illustrations are included in Appendix 7.

\subsection{Replenishment Ship Design Studies}

In Section 4.2 it was mentioned that the current survivability assessment method would be applied to AOR type ships in order to demonstrate the applicability of the method on non-combatant ship types. The increased significance of such ships is examined in Appendix 2.3, where the need for global military presence (despite declining defence budgets and fleet sizes) and new IMO regulations were identified as the main motives for many navies to require improved underway replenishment capabilities (Bricknell and Vedlog 2007; Andrews and Pawling 2007).

Two AOR variants were designed, again using the DBB approach through the SURFCON module in Paramarine, Section 2.1.5. To familiarise with designing such a ship type, past UCL as well as industry developed designs were studied (Project Management Office JSS 2005a; Project Management Office JSS 2005b; BMT Defence Services 2012). Information about specialised equipment such as RAS equipment and cranes was taken from (Rolls-Royce 2008; TTS Marine 2010; Flensburger 2012). The requirement was for a ship capable of accomplishing the roles of cargo transportation, replenishment at sea, aviation support and provision of medical facilities. The aim was that two of the AOR ship designs would be part of a task group, consisting of one CVF, two AAW Destroyers, four ASW Frigates, with a maximum range of 15,000nm in addition to a 30 day period for the conduction of operations. The AOR cargo capacity was calculated primarily through the work done by Martin (2001) as well as by comparing to existing similar ships. Table 5.6 describes the required cargo capacity breakdown and Table 5.7 lists the performance requirements for the two AOR variants.

\begin{tabular}{|l|r|}
\hline \multicolumn{2}{|l|}{ Table 5.6: AOR Required Cargo Capacity } \\
\hline AVCAT (m3) & 11,600 \\
\hline Dieso (m3) & 7,100 \\
\hline Dry Stores (m3) & 4,850 \\
\hline Ordnance (m3) & 2,600 \\
\hline Water (m3) & 400 \\
\hline Lube oil (m3) & 100 \\
\hline TEU & Minimum 8 \\
\hline
\end{tabular}




\begin{tabular}{|l|l|}
\hline \multicolumn{2}{|l|}{ Table 5.7: AOR Performance Requirements Adopted for Design Studies } \\
\hline Maximum speed & $18 \mathrm{kts}$ \\
\hline Endurance & $15,000 \mathrm{~nm}$ at 15kts \\
\hline Stores & $42+30$ days \\
\hline Stability & $\begin{array}{l}\text { Defence Standard 02-109 (NES 109) intact and damage criteria } \\
\text { assessment (MOD 2000) }\end{array}$ \\
\hline Zoning & 2 zones with maximum independence \\
\hline
\end{tabular}

In addition, Table 5.8 lists the weapons and sensors, as well as the RAS infrastructure, incorporated in the AOR designs. Identical equipment was used in both variants.

\begin{tabular}{|c|c|}
\hline $\begin{array}{l}\text { Port, starboard and astern refuelling } \\
4 \times \text { RAS posts } \\
1 \times \text { Crane }\end{array}$ & RAS Infrastructure \\
\hline $\begin{array}{l}1 \times \text { EH-101 Merlin Helicopter + single spot flight deck for EH- } \\
101 \text { Merlin and hangar for four EH-101Merlins } \\
2 \times \text { Raytheon SeaRAM Weapon System } \\
2 \times \text { MSI Seahawk } 30 \mathrm{~mm} \\
4 \times \text { Oto Melara } 12.7 \mathrm{~mm} \\
4 \times \text { NATO Standard Decoy Launching System } \\
2 \times \text { Navigation radars } \\
1 \times \text { Single Face SR STAR Surveillance Radar } \\
1 \times \text { Surface Ship Torpedo Defence System } \\
2 \times \text { Thorn-EMI 'Guardian' Type } 675 \text { Jammer } \\
1 \times \text { Medium ship communications system }(1 \times \text { communications } \\
\text { mast }+4 \times \text { whip antennae }) \\
1 \times \text { Generic Satellite Communications System with } 2 \text { antennae }\end{array}$ & Weapons and Sensors \\
\hline
\end{tabular}

The SeaRam Weapon System was preferred to a conventional CIWS (e.g.: Raytheon Phalanx CIWS amongst which SeaRAM is based) due to its higher effectiveness (Harney 2010). 
A summary of the principal particulars of the two AOR variants is included in Table 5.9, with GAs in Appendix 8.

\begin{tabular}{|c|c|}
\hline \multicolumn{2}{|c|}{$\begin{array}{l}\text { Table 5.9: Principal Particulars of the AOR Variants Investigated } \\
\text { Variant 1 }\end{array}$} \\
\hline Dimensions & $\begin{array}{l}205.4 \mathrm{~m}(198.8 \mathrm{~m}) \times 28.2 \mathrm{~m}(25.5 \mathrm{~m}) \times 20.4 \mathrm{~m}(\text { deep draught } 12.0 \mathrm{~m}) \\
\text { deep trim } 0.7 \mathrm{~m} \text {, light trim } 1.2 \mathrm{~m}\end{array}$ \\
\hline Hullform Param. & $\mathrm{C}_{\mathrm{B}}: 0.616, \mathrm{C}_{\mathrm{P}}: 0.738, \mathrm{C}_{\mathrm{M}}: 0.835, \mathrm{C}_{\mathrm{W}}: 0.861$, Circular M: 6.0 \\
\hline Displacement & 38,450te deep, 23,620te light \\
\hline Maximum Speed & $18.3 \mathrm{kts}$ \\
\hline Range & $15,300 \mathrm{~nm}$ at $15 \mathrm{kts}, 11,400 \mathrm{~nm}$ at $18 \mathrm{kts}$ \\
\hline Power Plant & $\begin{array}{l}4 \times 5.76 \mathrm{MW} \text { diesels (cruise and auxiliary), } 1 \times 0.685 \mathrm{MW} \text { diesel } \\
\text { (emergency) driving two } 10 \mathrm{MW} \text { pods and one pump-jet on a } 1 \mathrm{MW} \\
\text { HTS motor. }\end{array}$ \\
\hline Accommodation & 30 officers, 183 ratings \\
\hline \multicolumn{2}{|l|}{ Variant 2} \\
\hline Dimensions & $\begin{array}{l}205.4 \mathrm{~m}(198.7 \mathrm{~m}) \times 28.2 \mathrm{~m}(25.4 \mathrm{~m}) \times 20.4 \mathrm{~m}(\text { deep draught } 11.9 \mathrm{~m}) \\
\text { deep trim } 0.6 \mathrm{~m} \text {, light trim } 1.6 \mathrm{~m}\end{array}$ \\
\hline Hullform Param. & $\mathrm{C}_{\mathrm{B}}: 0.615, \mathrm{C}_{\mathrm{P}}: 0.737, \mathrm{C}_{\mathrm{M}}: 0.835, \mathrm{C}_{\mathrm{W}}: 0.860$, Circular M: 6.0 \\
\hline Displacement & 37,850te deep, 23,360te light \\
\hline Maximum Speed & $18.3 \mathrm{kts}$ \\
\hline Range & $15,600 \mathrm{~nm}$ at $15 \mathrm{kts}, 11,600 \mathrm{~nm}$ at $18 \mathrm{kts}$ \\
\hline Power Plant & $\begin{array}{l}4 \times 5.76 \mathrm{MW} \text { diesels (cruise and auxiliary), } 1 \times 0.685 \mathrm{MW} \text { diesel } \\
\text { (emergency) driving two } 10 \mathrm{MW} \text { pods. }\end{array}$ \\
\hline Accommodation & 30 officers, 183 ratings \\
\hline
\end{tabular}

The complements of both ships were identical and were calculated based on work done by Martin (2001). Complement breakdown and accommodation requirements are given in Appendix 9.1.

The two AOR design variants are illustrated in Figure 5.52 and Figure 5.53. 


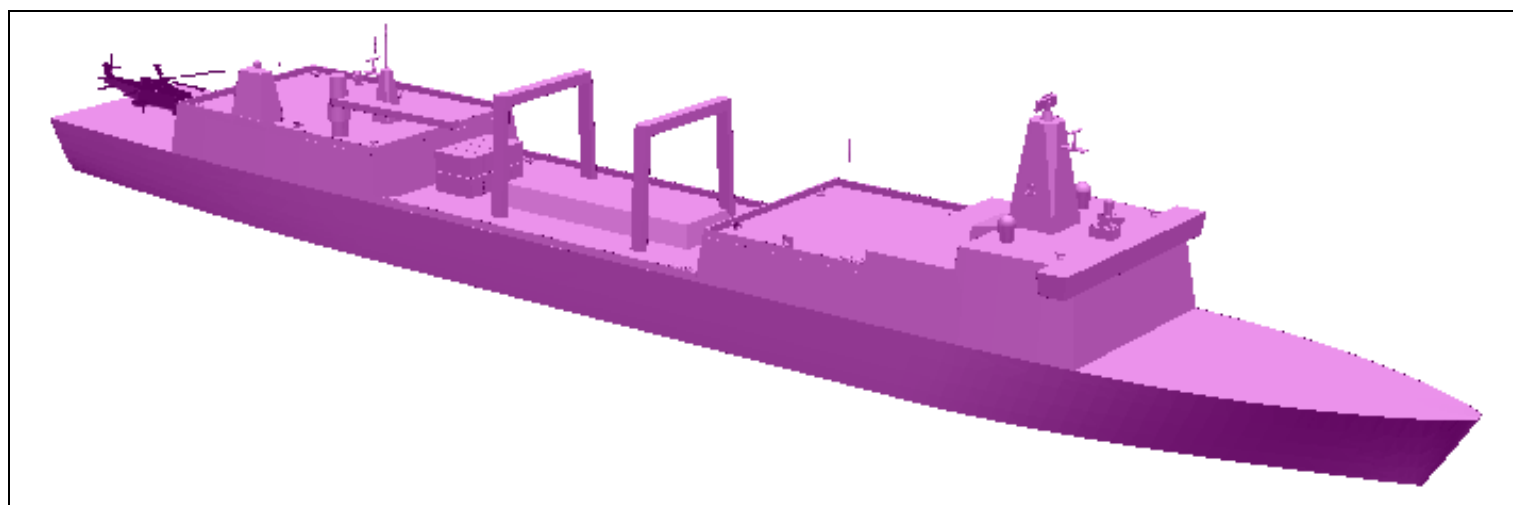

Figure 5.52: AOR Variant 1 Ship Design Study

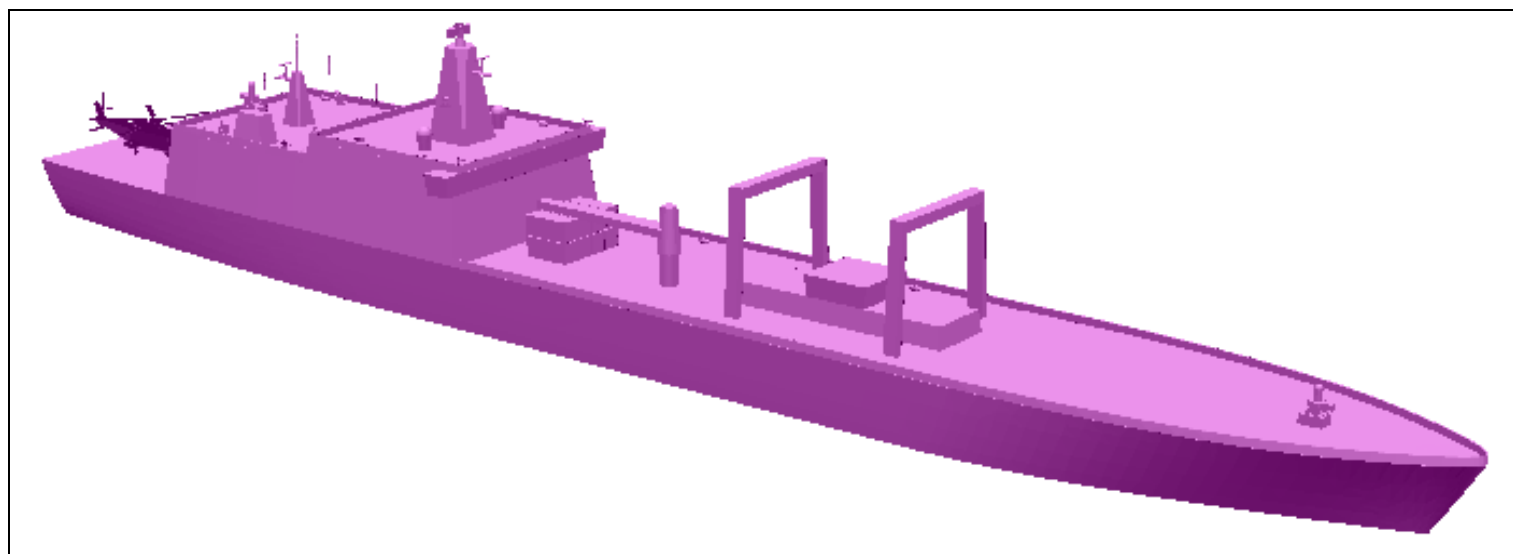

Figure 5.53: AOR Variant 2 Ship Design Study

It becomes evident that two naval replenishment ship designs vary in configuration, one including a split forward and aft superstructure arrangement with the RAS infrastructure located between the two blocks (analogous to the Fort Victoria class replenishment oiler); the other including a single aft superstructure block, with the RAS infrastructure slightly forward of amidships (comparable to the Wave class tankers). These two configurations were chosen as they are the most common in current naval auxiliary ships. Therefore, an opportunity to investigate the survivability implications of the two choices was presented.

The top level functional breakdown of the AOR variants is illustrated in Figure 5.54 and Figure 5.55. 


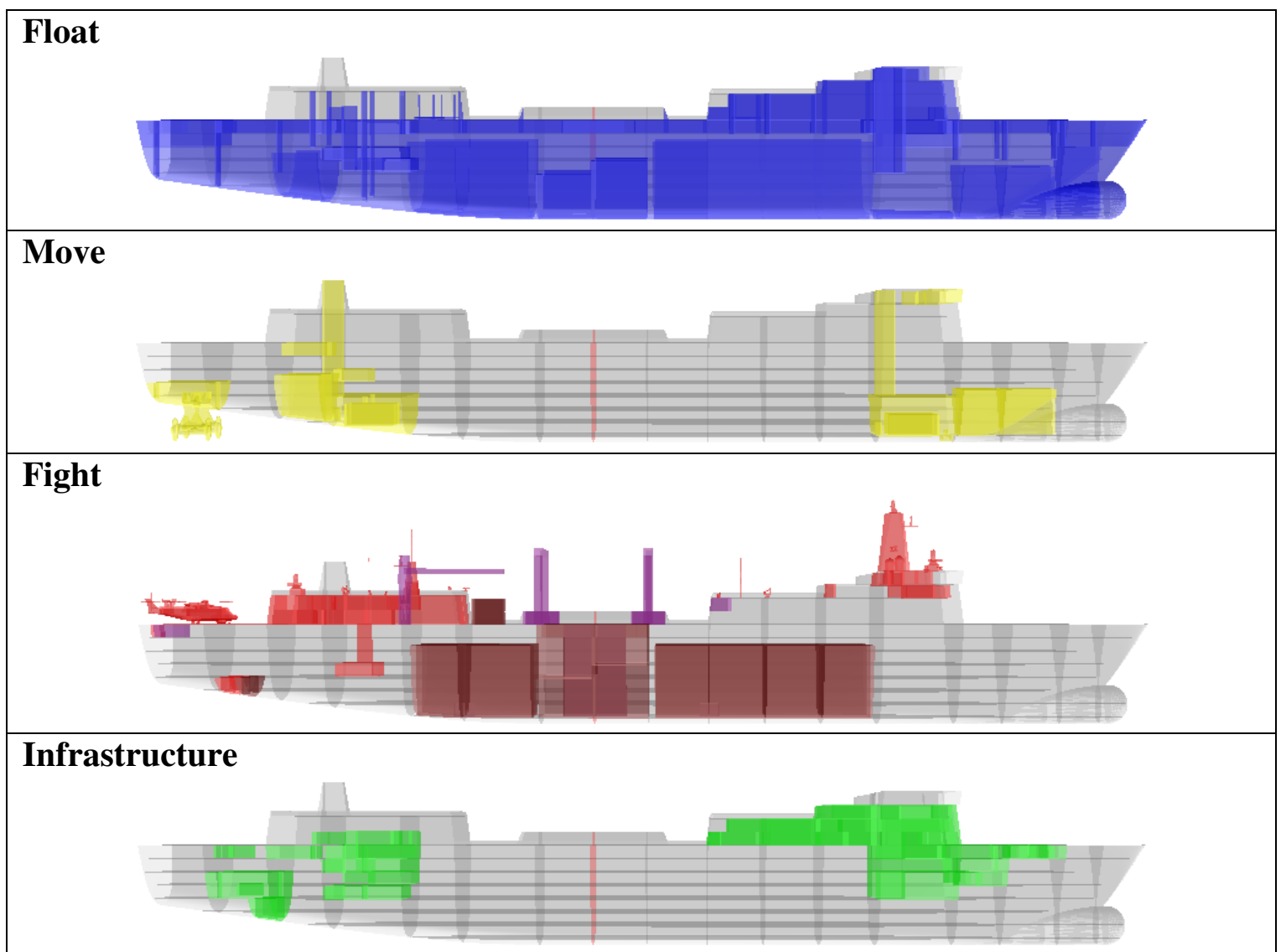

Figure 5.54: AOR Variant 1 Top Level Functional Breakdown

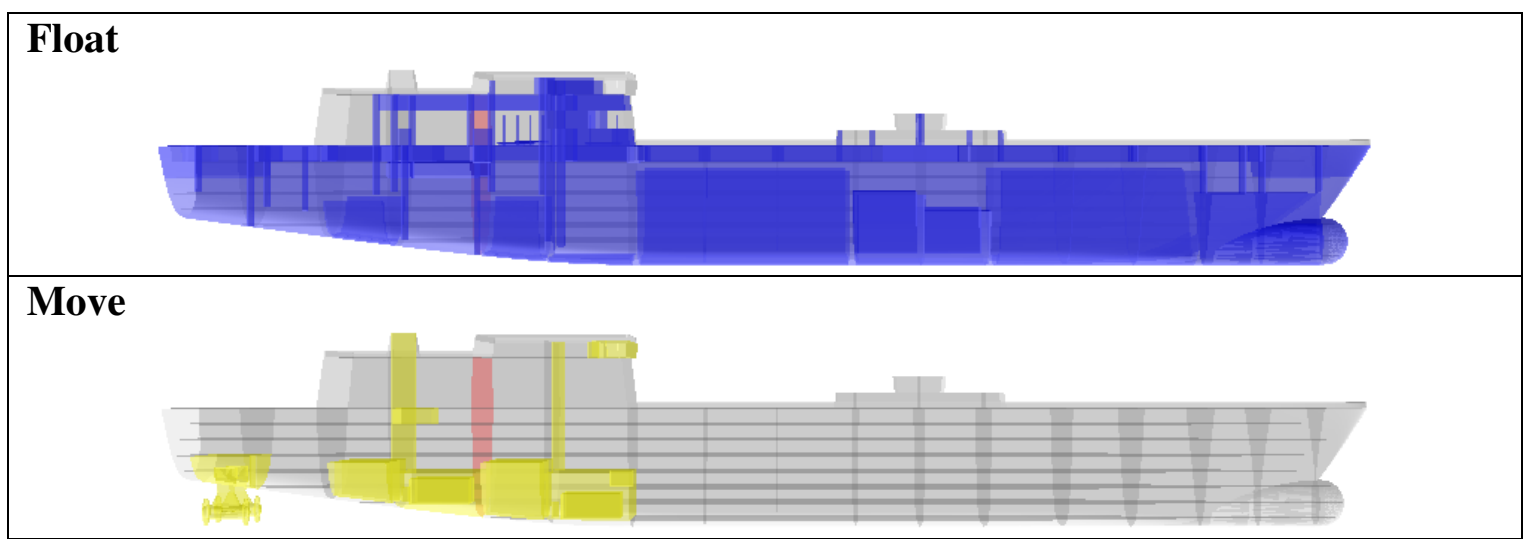

Fight

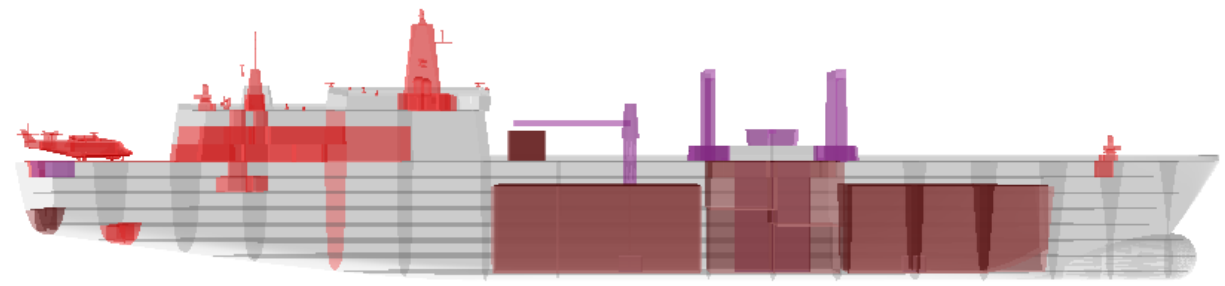

\section{Infrastructure}

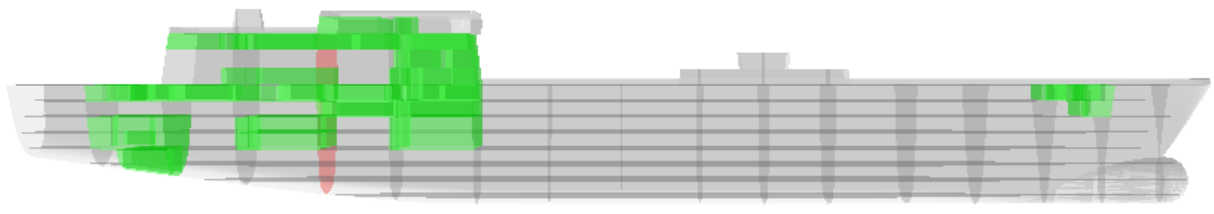

Figure 5.55: AOR Variant 2 Top Level Functional Breakdown 
The most obvious effects of the configurational variations between the two design are the split nature of the move functional group (which resulted from the decision to apply an IFEP system, similarly to the combatant designs) and the Infrastructure functional group, as well as some of the sensors in the fight functional group in AOR Variant 1, as opposed to the concentrated nature of those groups in the second variant. This gives the impression of AOR Variant 1 being a less vulnerable design. A further point of interest is the fact that, unlike in the combatant design studies, the Fight group of the replenishment ship designs is represented in three different colours, each signifying a lower level Fight sub-group. In red are the ships weapons, sensors and related spaces (e.g.: hangars and LCUs); in brown is the ship's cargo (although water tanks are depicted as part of the Infrastructure functional group as they have been combined with the ships own water tanks); and purple represents the RAS equipment. This colour variation assists in distinguishing that, the fact that all move and most Infrastructure related compartments and equipment were pushed aft in AOR Variant 2, has resulted in moving the cargo spaces (and RAS equipment) slightly forward. However, in Appendix 2.3 it was recognised that it is beneficial to locate the stores and tanks in the wide central part of the ship, therefore increasing cargo capacity and minimising ballast requirements when operating in light conditions (Andrews and Pawling 2007). Therefore, the configuration of AOR Variant 2 has led to a slight decrease in cargo capacity (more clearly represented in the GAs, Appendix 8) compared to AOR Variant 1 (see Table 5.10), although both designs meet the volume requirements listed at Table 5.6.

\begin{tabular}{|l|r|r|}
\hline \multicolumn{2}{|l|}{ Table 5.10: AOR Achieved Cargo Capacity } \\
\hline & AOR Variant 1 & AOR Variant 2 \\
\hline AVCAT (m3) & 12,800 & 12,240 \\
\hline Dieso (m3) & 7,800 & 7,220 \\
\hline Dry Stores (m3) & 5,040 & 5,040 \\
\hline Ordnance (m3) & 2,670 & 2,670 \\
\hline Water (m3) & 505 & 505 \\
\hline Lube oil (m3) & 105 & 105 \\
\hline TEU & 8 & 8 \\
\hline
\end{tabular}

A final point worth commenting on is that the observation of Cooper et al (2007), that that the main design drivers for such ships are the aviation requirements, 
the accommodation requirements (i.e. Infrastructure) and cargo storage (although RAS rig location is vital for the efficient operation of replenishment ships, their placement is not usually a design driver due to the large abundant deck area above the cargo tanks) summarised in Figure A3 of Appendix 2.3, is roughly confirmed from the functional group description of the above two AOR design variants.

The two AOR Variants are described with respect to their top level weight breakdown in Table A13 and Table A14 of Appendix 9.2. In addition, the margin philosophy adopted for these design studies is summarised in Appendix 9.3.

The Intellihull object of Paramarine was used to generate the AOR hulls, due to its ability to produce auxiliary ship hullforms; the bulbous bow was sized based on current similar ships. Both variants were based on identical (8-deck) hulls, the lines plan of which is shown in Figure 5.56.

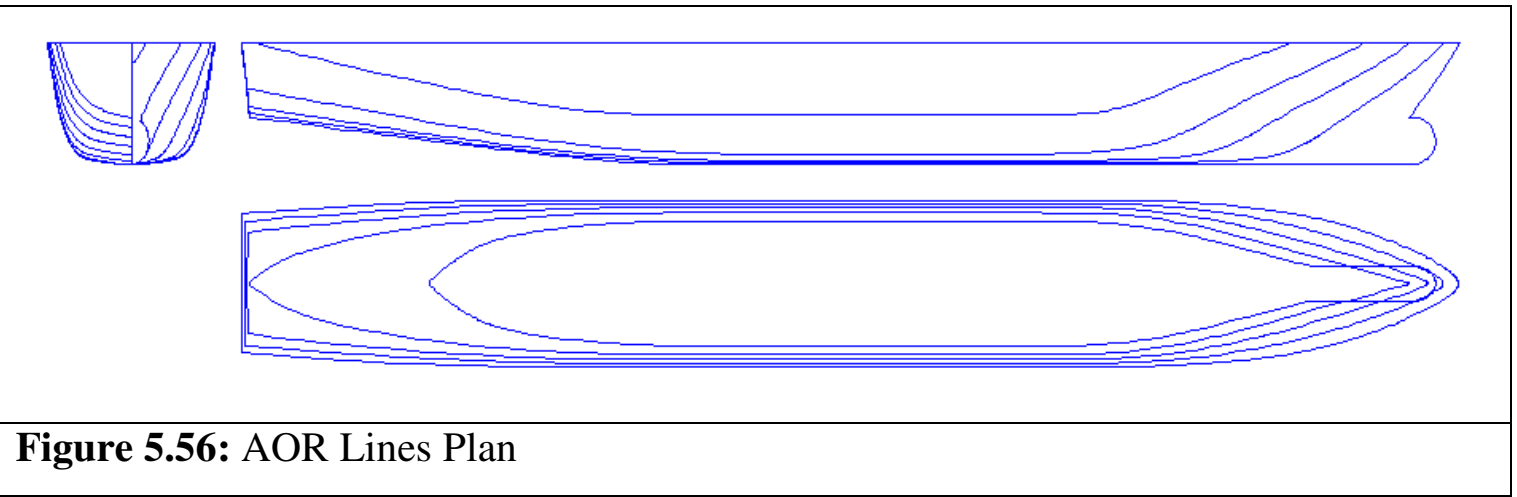

The propulsive powering requirements for AOR Variant 1 were computed through the inbuilt Paramarine powering module. It was decided to include a podded drive arrangement in the AOR variants. The main reason for this, discussed in Appendix 2.3, was that the use of podded propulsors requires reduced internal volume, therefore, providing more storage and tankage space (Andrews and Pawling 2007). After conducting the powering analysis (see Appendix 9.4), the machinery that was selected consisted of:-

- $\quad 4 \times 5.76 \mathrm{MW}$ Wartsila 12V32 diesel gensets (cruise and auxiliary);

- $\quad 1 \times 0.685 \mathrm{MW}$ Wartsila 4L20 diesel genset (emergency).

In order to take full advantage of the split move system, visible in Figure 5.54, it was chosen to include a secondary forward propulsor in AOR Variant 1. Similarly to Frigate Variant 3 and the Corvette design studies, a pump-jet (Schottel 2010a) was the selected choice. Therefore, the machinery above drove two stern mounted SSP10 10MW pods and one Schottel SPJ 220 Pump-Jet on a 1MW HTS motor. The layout of the above 
Move related machinery is illustrated in Figure 5.57, with whole major system architecture and tree diagrams included in Appendix 7.

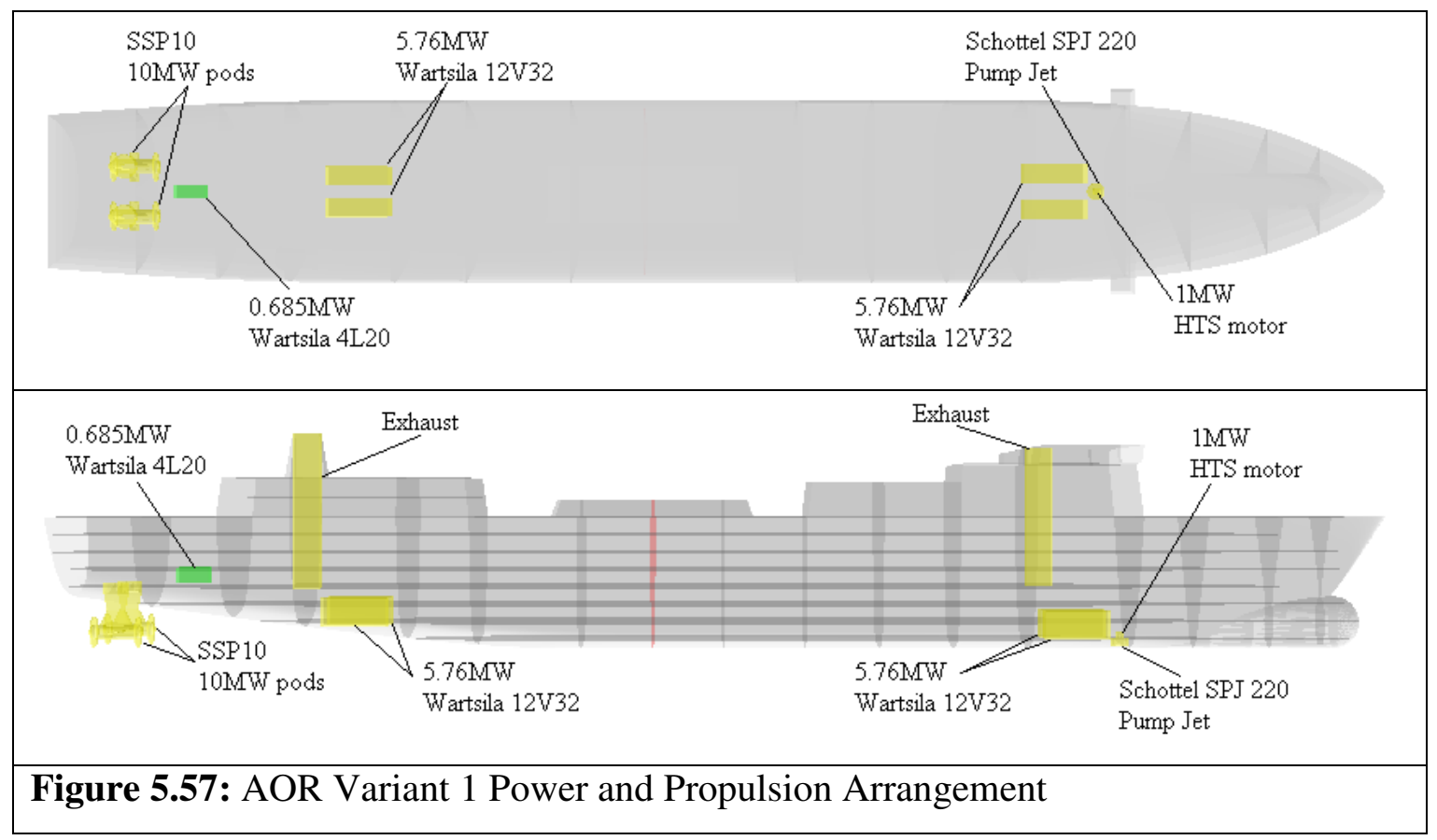

The scale and significance of the separation distance between the two sets of main machinery, which could have only been achieved through the use of an IFEP system, are clear in Figure 5.57. As a result, vulnerability (Appendix 4.2) of the power generation sub-system and, therefore, move system is greatly reduced, especially once taking into account the secondary propulsive unit. This is located at the forward machinery room, along with the forward set of gensets, therefore, providing a highly concentrated arrangement of equipment in series. The above IFEP arrangement has also allowed the positioning of cargo tanks and stores in the wide central part of the hull (Andrews and Pawling 2007), the advantages of which have already been examined (Appendix 2.3). The aft main machinery exhausts through a funnel on the starboard side, therefore, not interfering with helicopter operations, whereas the forward main machinery exhausts through the forward mast. The emergency genset exhausts from the stern, again to avoid interference with helicopter operations. Removal routes for all engines were taken into account through the inclusion of soft patches in the passageways and compartments above each engine.

The propulsive powering requirements for AOR Variant 2 were also computed through the inbuilt Paramarine powering module (see Appendix 9.4). The machinery that was selected consisted of:-

- $\quad 4 \times 5.76 \mathrm{MW}$ Wartsila 12V32 diesel gensets (cruise and auxiliary); 
- $\quad 1 \times 0.685 \mathrm{MW}$ Wartsila 4L20 diesel genset (emergency).

For AOR variant 2, due to the relatively concentrated (towards the aft end) Move functional group (Figure 5.55) it was decided to not include a forward propulsor. Moreover, the survivability implications of the two different arrangements could be investigated and compared after applying the survivability assessment method. Therefore, the above machinery drove exclusively two stern mounted SSP10 10MW pods. The layout of the above machinery is illustrated in Figure 5.58, and is very similar to the move system architecture (see Appendix 7) which was investigated in the vulnerability and recoverability assessment methods.

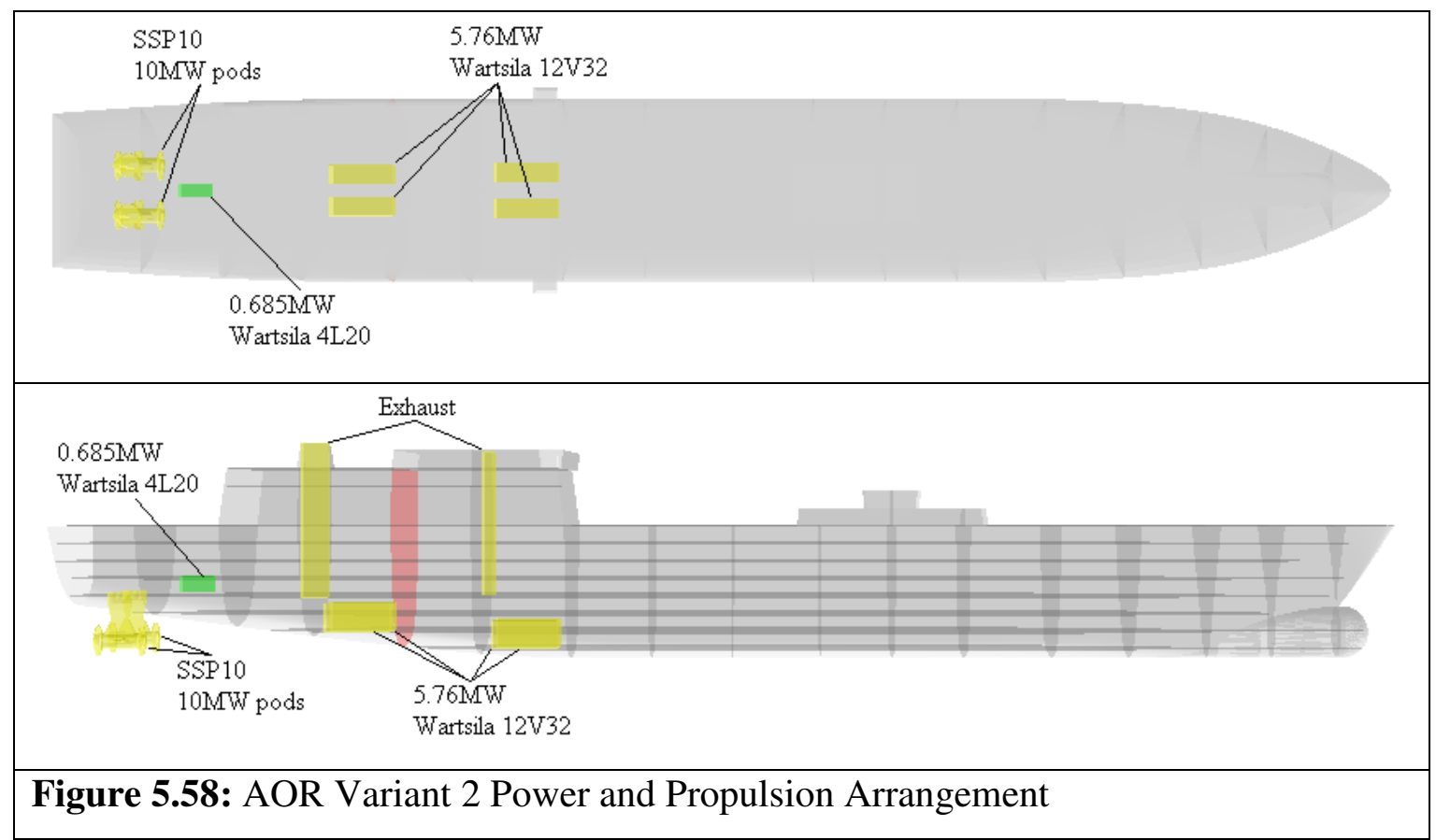

The above illustration undoubtedly shows a much more concentrated arrangement than that of AOR Variant 1 (Figure 5.57) and justifies the scepticism behind the inclusion of a secondary forward propulsor unit. However, as a vulnerability reduction measure, the IFEP system has allowed the two sets of main machinery to be separated by one WT section, in which a (ships own) dieso tank is located. Equally to the first AOR variant, the aft main machinery exhausts through a funnel on the starboard side (not interfering with helicopter operations), the forward main machinery exhausts through the forward mast and the emergency genset exhausts from the stern. Furthermore, consistently with all ship design studies, removal routes for all engines were taken into account through the inclusion of soft patches in the passageways and compartments above each engine. 
Hydrostatic analysis for the two AOR design studies was conducted through the inbuilt Paramarine module, as with all other ship designs. Intact stability was assessed against both Defence Standard 02-109 (NES 109) Stability Standards for Surface Ships (MOD 2000) and the Code on Intact Stability for all Types of Ships Covered by IMO Instruments (IMO 1993), until all criteria were passed. Damage stability for both replenishment ship variants was assessed against Defence Standard 02-109 (NES 109) damage criteria assessment (MOD 2000). Hydrostatics data are given in Appendix 9.5.

The two auxiliary ship variants each contain sixteen WT bulkheads (and seventeen WT sections), the arrangement of which is shown in Figure 5.59 and Figure 5.60 .
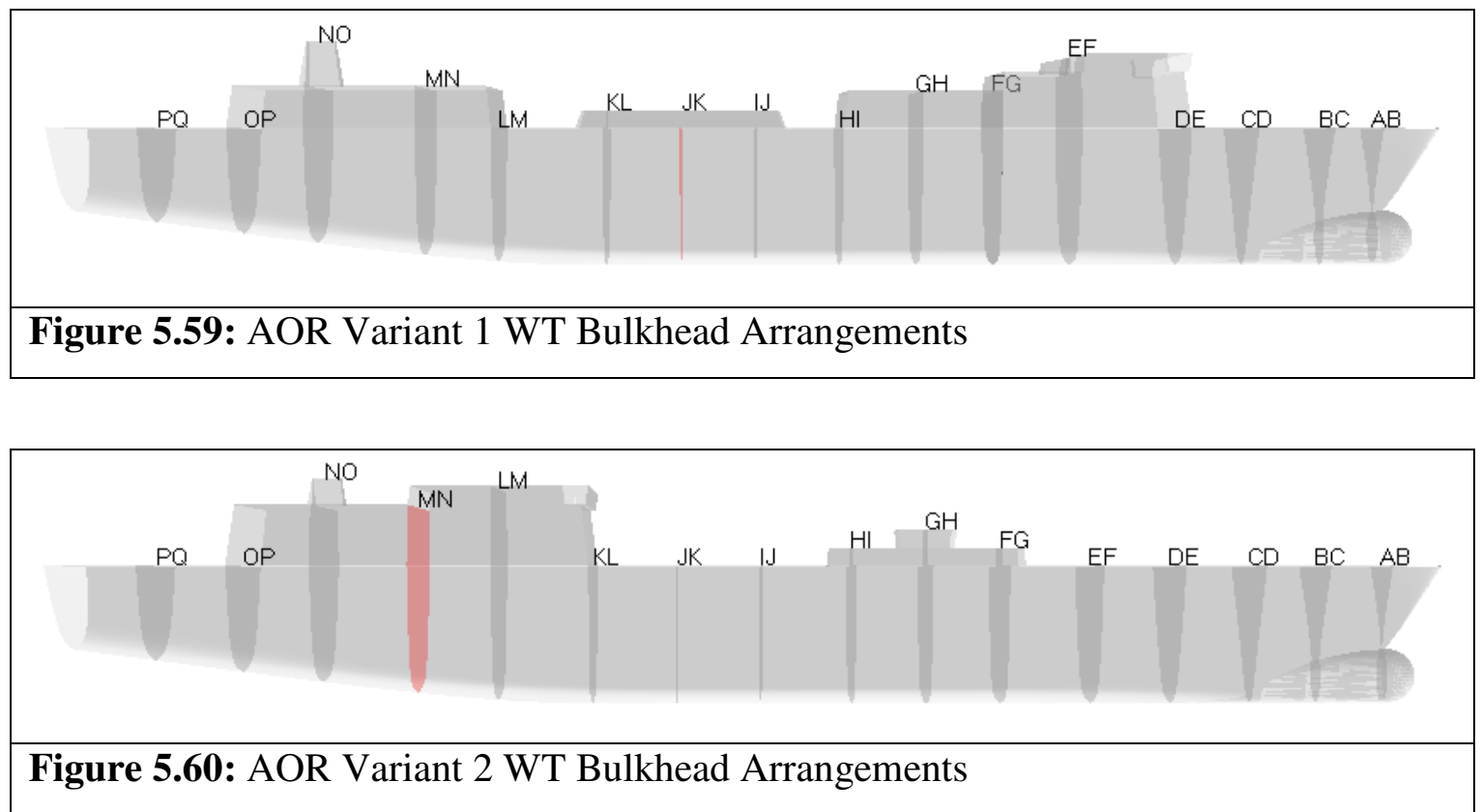

As with the combatant designs, bulkhead locations were decided upon by taking into account zoning, structural, layout and damage stability related factors. In order to maintain structural continuity, bulkheads were located at superstructure steps and ends, masts, cargo cranes and RAS high points.

Given the non-military role of the auxiliary ship designs it was determined that a double zone philosophy is adequate. The zone boundaries are the red WT bulkheads in the illustrations above. AOR Variant 1 included seven WT sections in the aft zone and ten in the forward zone since the zone boundary was approximately at amidships, which suited the split forward and aft superstructure style. Conversely, the fact that the forward part of AOR Variant 2 was almost exclusively used for cargo storage, pushing most compartments and services to the aft section (Figure 5.55), led to the unusual 
zoning arrangement illustrated in Figure 5.60. The aft zone includes four WT sections and the forward one extends across thirteen WT sections. Each of the two zones contain independent power generation units, 2 CWPs, 2 HPACs, 2FPs, independent ATU and ventilation, 2 NBCD stores, 2 airlocks for access to and from the citadel/weather and a cleansing/decontamination station. The zoning philosophy is clearly depicted in Figure 5.61 and Figure 5.62.

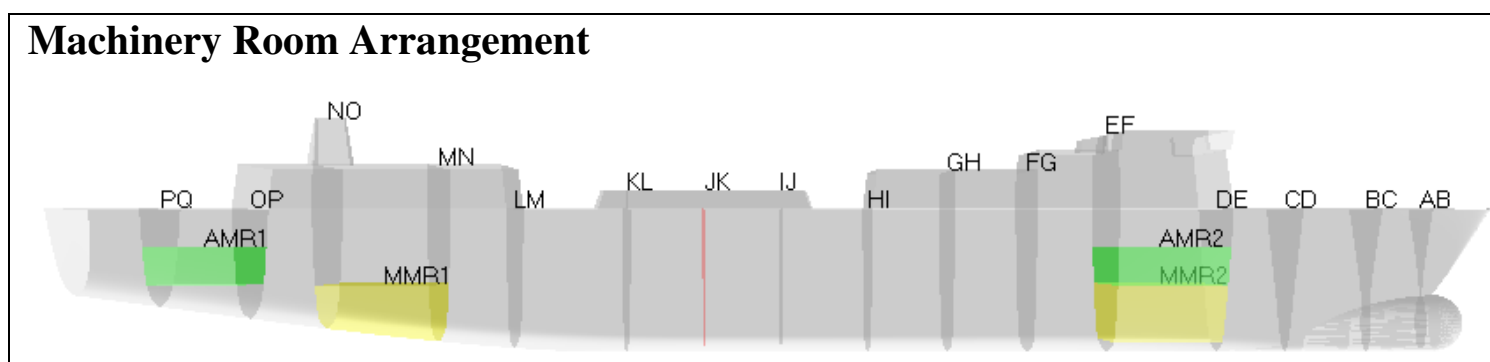

\section{ATU and Ventilation Arrangement}

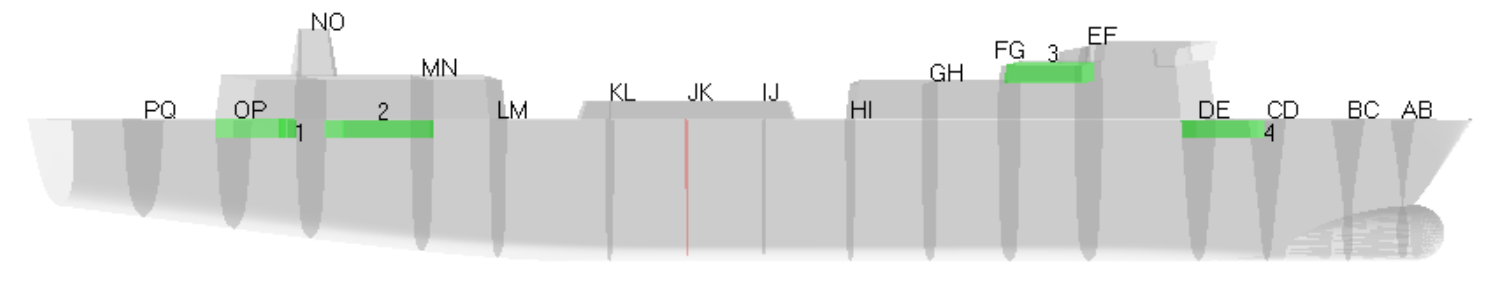

\section{NBCD Stores}

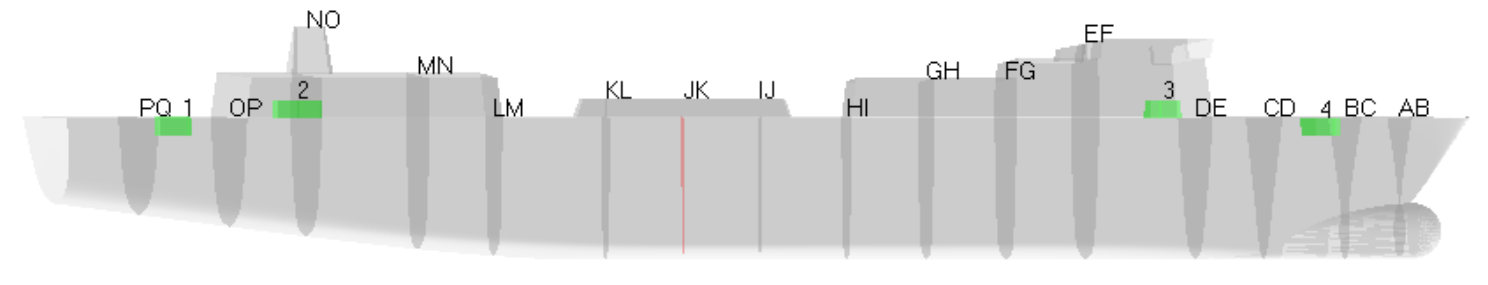

\section{Airlocks}

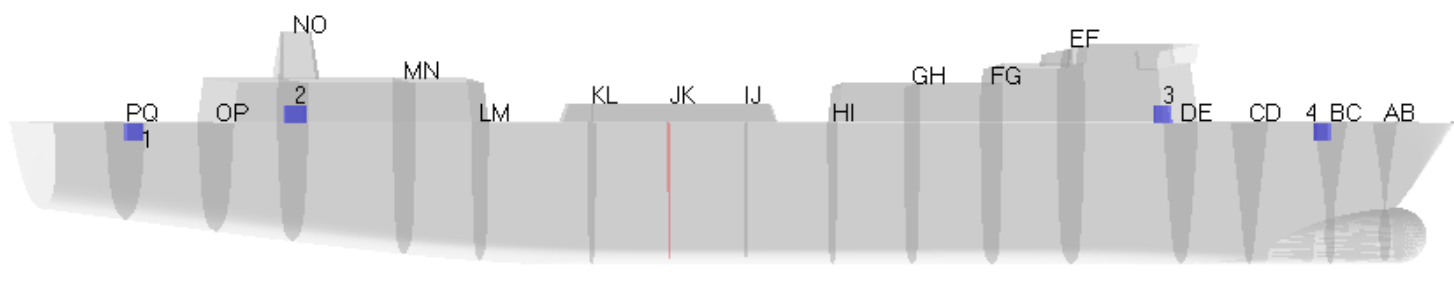

Figure 5.61: AOR Variant 1 Zoning Philosophy

From above it is evident that each zone has one main and one auxiliary machinery room. Each machinery room includes a CWP, a HPAC and a FP, while MMR1, MMR2 and AMR1 contain power generation units (Figure 5.57). Due to the large ship size, the ATU and ventilation compartments of each zone were split into two units in an effort to spread this service more efficiently across the ship's length. These 
units are located in proximity to spaces such as machinery rooms and accommodation areas and well above the waterline, correspondingly to the combatant designs. Another similarity with the combatant design studies is the adjacency of NBCD stores and airlocks. Airlocks 1 and 3, which are adjacent to cleansing/decontamination stations, lead to the aft mooring space and forward weatherdeck respectively. Airlock 2 leads to the hangar and airlock 4 , to the forward mooring space.

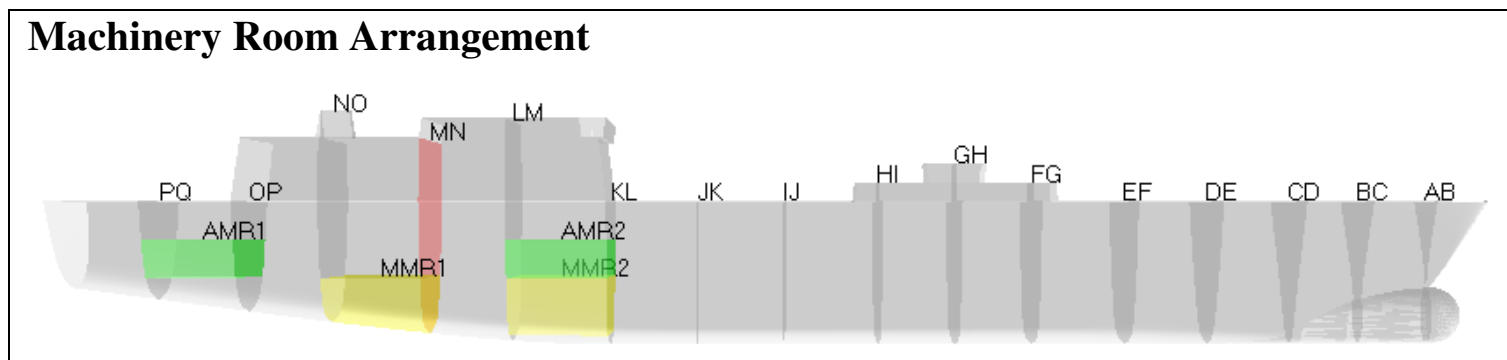

\section{ATU and Ventilation Arrangement}

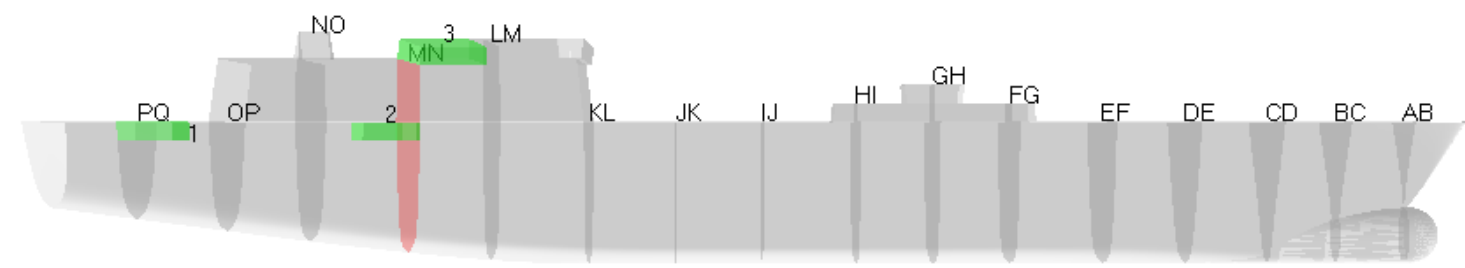

\section{NBCD Stores}

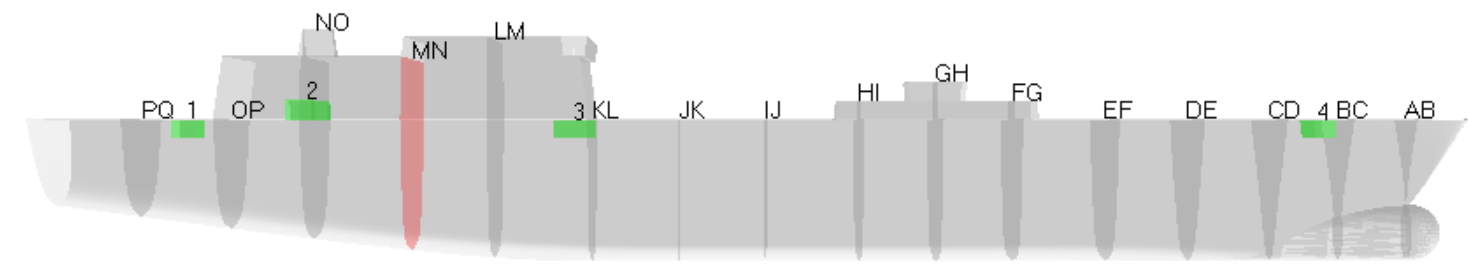

\section{Airlocks}

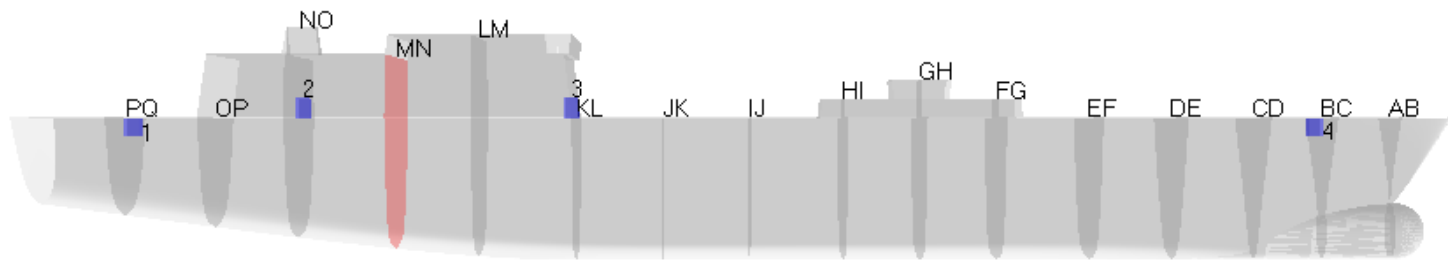

Figure 5.62: AOR Variant 2 Zoning Philosophy

AOR Variant 2 included identical equipment in its corresponding machinery rooms. A modification compared to the first variant is the fact that the forward zone ATU and ventilation arrangements are not split. This is because essentially only two full WT sections of that zone are dedicated to compartments and services other than cargo stores and tanks (Figure 5.55). Therefore, the necessity to spread the ventilation 
arrangements in the forward zone was minimised. Other than that, the main difference between the zoning arrangements of the two auxiliary ships is the fact that in AOR 2 all replicated items (with the exception of airlock 4 and NBCD store 4 which lead to the forward mooring space) are concentrated at the aft end of the ship. An outcome that was expected given the arrangement of cargo and non-cargo related entities. This would probably lead to increased vulnerability, due to the relative concentration of parallel equipment. For example, the separation between all machinery rooms which include power generation units is only one WT section. As in AOR Variant 1, airlocks 1 and 3, which are adjacent to cleansing/decontamination stations, lead to the aft mooring space and forward weatherdeck respectively, while airlock 2 leads to the hangar. Due to the much larger size of the replenishment ship designs and the requirement for only two zones, the general observation was that it was considerably easier to achieve the minimum zone requirements compared to the combatant designs.

Figure 5.63 and Figure 5.64 illustrate the arrangement of the main items of the major Fight systems (Table 4.2) which were modelled in the vulnerability and recoverability assessment methods. Equipment which are solely related to each Fight system are depicted, with other necessary items which have functions additional to the capabilities modelled, such as engines, sensors and centralised command and control spaces, not included. Full major systems architecture and tree diagrams are shown in Appendix 7.

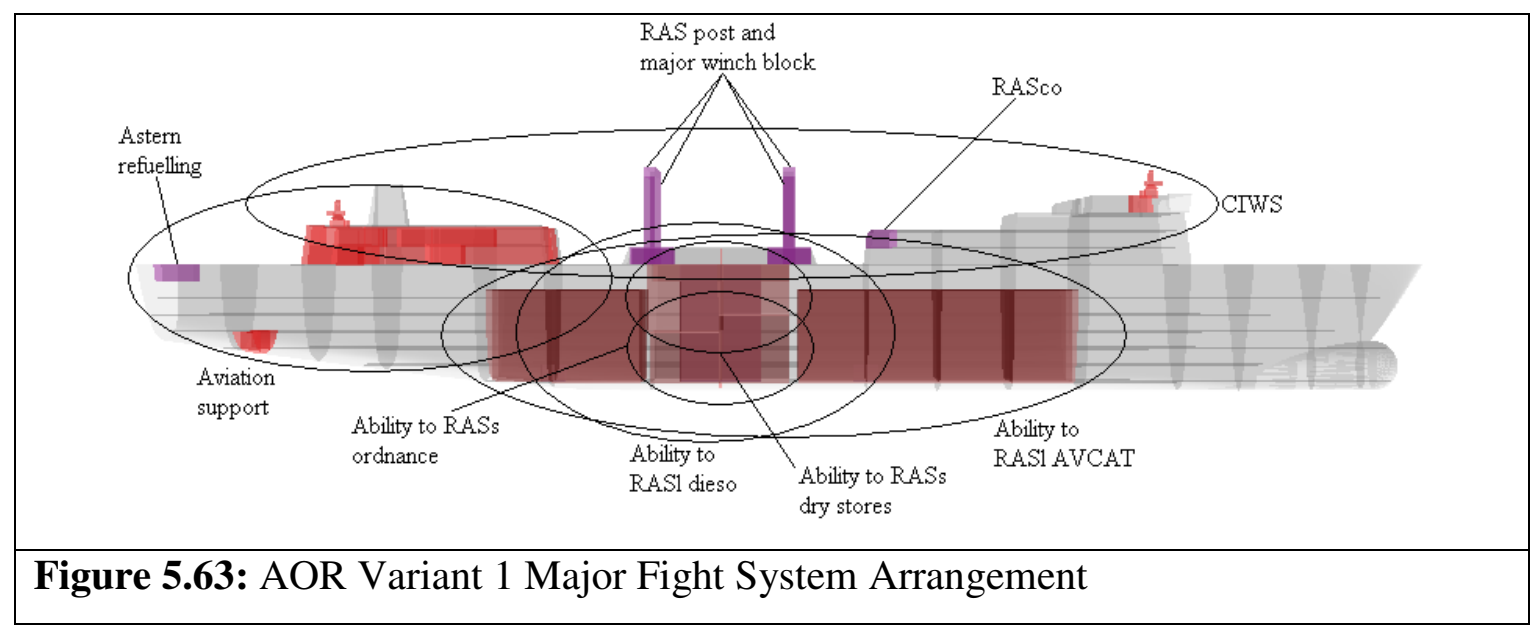

From the above image it is evident that, unlike in the combatant design studies, each major Fight system has a much larger footprint (as a proportion of the ship's length) and overlapping of different systems occurs to a much larger extent. This gives the impression that it is impractical to link Fight system arrangements with the zoning philosophy. However, by studying the major system tree diagrams (Appendix 7) one 
can easily comprehend that (with the exception of the aviation support capability), all other major systems include equipment/compartments mainly arranged in a parallel pattern, rather than in series as in the combatant designs. That is to say that the major system tree diagrams of the replenishment ships are arranged in a horizontal configuration to a much greater extent than the combatant major system tree diagrams. It is, therefore, advantageous for most systems have large footprints, indicating a large separation (and therefore reduced vulnerability, Appendix 4.2) of items in parallel. For example, the two CIWS launchers shown in Figure 5.63 (the CIWS having the largest footprint) are in parallel; therefore, the large separation distance between them would cause that system to be almost invulnerable. Thus, it can be said that in the case of the replenishment ship design, the principal aim was to spread each major Fight system to as great an extent as possible (and in different zones) rather than to achieve high degrees of concentration as in the combatant design studies. The zoning arrangements of AOR Variant 1 have allowed separating AVCAT cargo tanks (three tanks in aft zone and nine in forward zone) and dieso cargo tanks (three tanks in each zone), as well as dry stores and ordnance cargo (approximately 50\% in each zone); this is more clearly shown in the GA, Appendix 8. On the other hand, the aviation support system was attempted to be concentrated as much as reasonably possible, with the large footprint of the flight deck plus hangar constraining the minimum length to five WT sections. Importantly, the aviation requirement on such ships was identified as a design driver (Cooper et al 2007) in Appendix 2.3 and Figure A3.

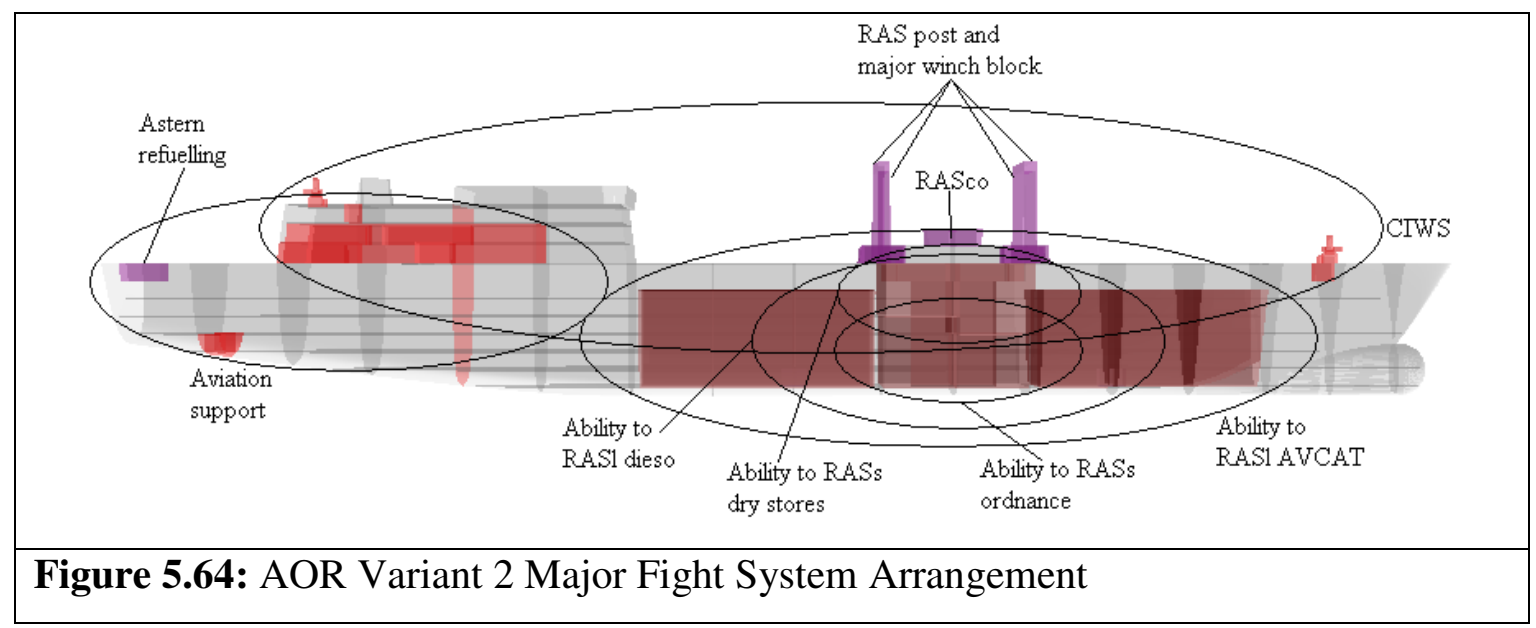

The comparison between the major Fight system arrangements of the two auxiliary ship designs shows that, although separation of the ability to RAS capabilities with respect to zoning has not been achieved, the footprints (and, therefore, separation between parallel items) of these major systems are approximately identical. This 
suggests that zoning is not as critical in such ships, as it is in ship designs where equipment concentration is pursued. However, the unorthodox zoning arrangements of the second variant, in terms of the relative zone sizes, have as a consequence the hangar crossing the zone boundary. Since this was deemed unacceptable, it was decided to move the zone boundary one WT section forward in No 1 Deck and 01 Deck, proving that the zoning arrangements of this variant are not as efficient as in the first AOR design variant. A further dissimilarity is that the RAS posts in AOR Variant 2 are located at the middle of the cargo spaces, whereas in the first variant they are located just aft of the centre. The AOR Variant 2 arrangement is advantageous in that cargo transference routes are minimised, whereas the AOR Variant 1 arrangement is superior in that RAS posts are located closer to amidships which is regarded as the ideal location. However, the main reason for moving the RAS posts slightly forward in AOR Variant 2 was in order to have an adequate deck area between the superstructure and RAS equipment (i.e. aft of amidships) to locate heavy items (such as the cargo crane and containers) in order to reduce trim (and, therefore, ballast requirements) at the deep condition (see Appendix 9.5). An important similarity between the two variants is the positioning of the ordnance (and dry stores) cargo directly below the RAS posts (see GAs in Appendix 8). This was done to minimise required routes for RAS operations with hazardous materials (Andrews and Pawling 2007; Scott 2010), as suggested in Appendix 2.3. Figure 5.63 and Figure 5.64 also illustrate the arrangement of RAS equipment and compartments in the two AOR variants. The major variance between the two arrangements concerns the location of the RAS control space (RASco) compartment. In AOR Variant 1 this is situated at the aft end of the forward superstructure, therefore, providing good visibility towards the RAS equipment. However, RAS operations could be supervised from any other area of the amidships weatherdeck, reducing the importance of that compartment. In AOR Variant 2, RASco is located between the two sets of RAS posts, also providing good visibility. However, it is also located forward of the single superstructure block, therefore, providing visibility to the bow. This means that the RASco of the second variant could be used as a secondary bridge in emergency situations, reducing the vulnerability of an otherwise unduplicated service.

Both designs were based on an identical, 8 deck hull. One of the decks (No 2 Deck) is the access, or DC deck. In both designs the DC deck includes a roughly centreline passageway. Figure 5.65 and Figure 5.66 elucidate the access arrangement of the two AOR variants. 


\section{Decks Access}

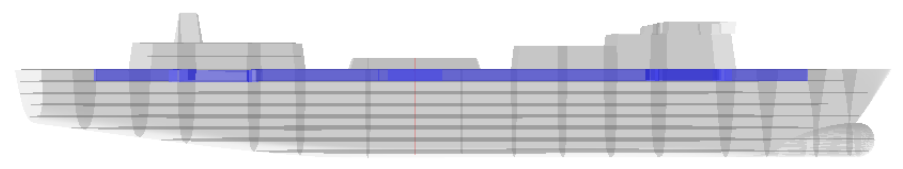

DC Decks Access and Vertical Access

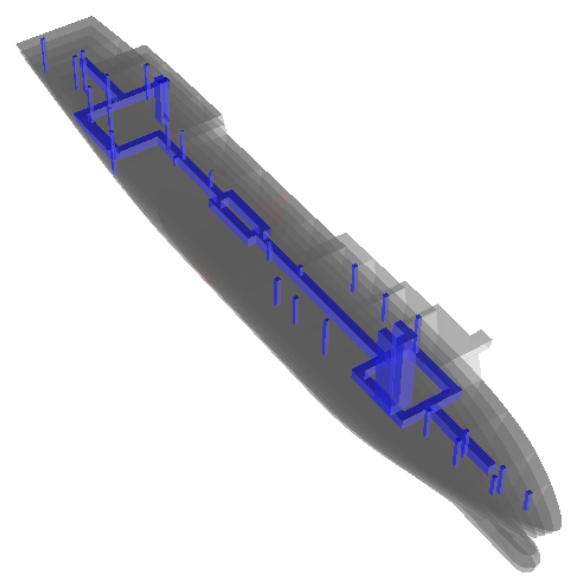

Figure 5.65: AOR Variant 1 Access Philosophy

\section{Deck Access}

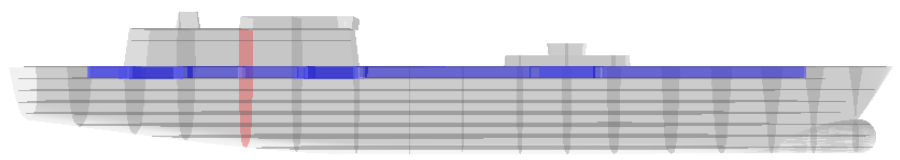

\section{Deck Access and Vertical Access}

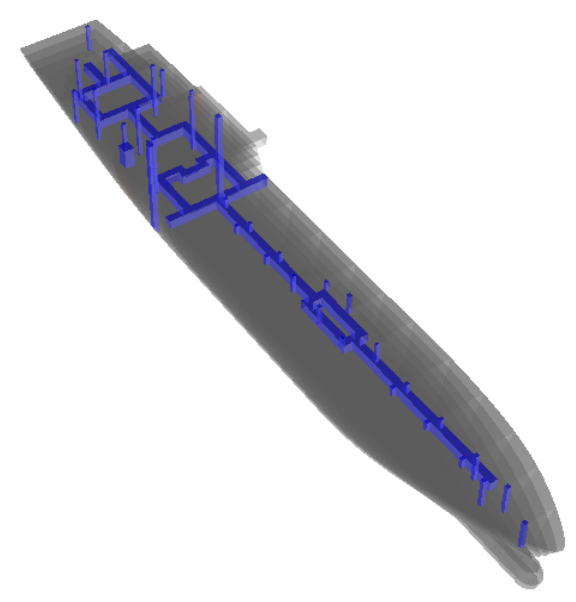

Figure 5.66: AOR Variant 2 Access Philosophy

Although it was attempted to accommodate a centreline main passageway (resulting to reduced volume and easier access (Ferreiro and Stonehouse 1994)), from the above two figures it is evident that numerous irregularities had to be incorporated. The main reasons for this were so that the access arrangements complied with certain 
layout related constraints, such as the requirement for a large (almost through-going) medical centre, as well as the requirement for RFA crew cabins to include portholes (encouraging a side passageway arrangement). Furthermore, the access arrangements of the through deck were further constrained by the fact that they should integrate with the corresponding arrangements in the higher (superstructure) decks, which mainly contained accommodation spaces. These aspects (which are clearer in the GA, Appendix 8) led to an alternation between centreline and side passageway (to a much greater extent than in the combatant designs). This also affected the vertical access arrangements, with each WT section including two vertical accesses, one at either side of the centreline passageway and at the forward and aft end of each WT section respectively, or one adjacent to either side passageway and at the forward and aft end of each WT section respectively, depending on the longitudinal access style. This inconsistent access arrangement, present in both AOR design variants, could prove problematic during DC efforts. It also highlights the increasing difficulty of designing access arrangements with increasing ship size and possibly ascertains that a double side passageway system becomes more efficient for larger ships.

The following figures explore the distribution of compartments and equipment relevant to the recoverability related PMs in Sections 4.3.1, 4.3.2 and 4.3.3. The first two illustrations (Figure 5.67 and Figure 5.68) examine the arrangements of compartments and equipment related to Category 1 PM analysis in AOR Variant 1 and AOR Variant 2 respectively.

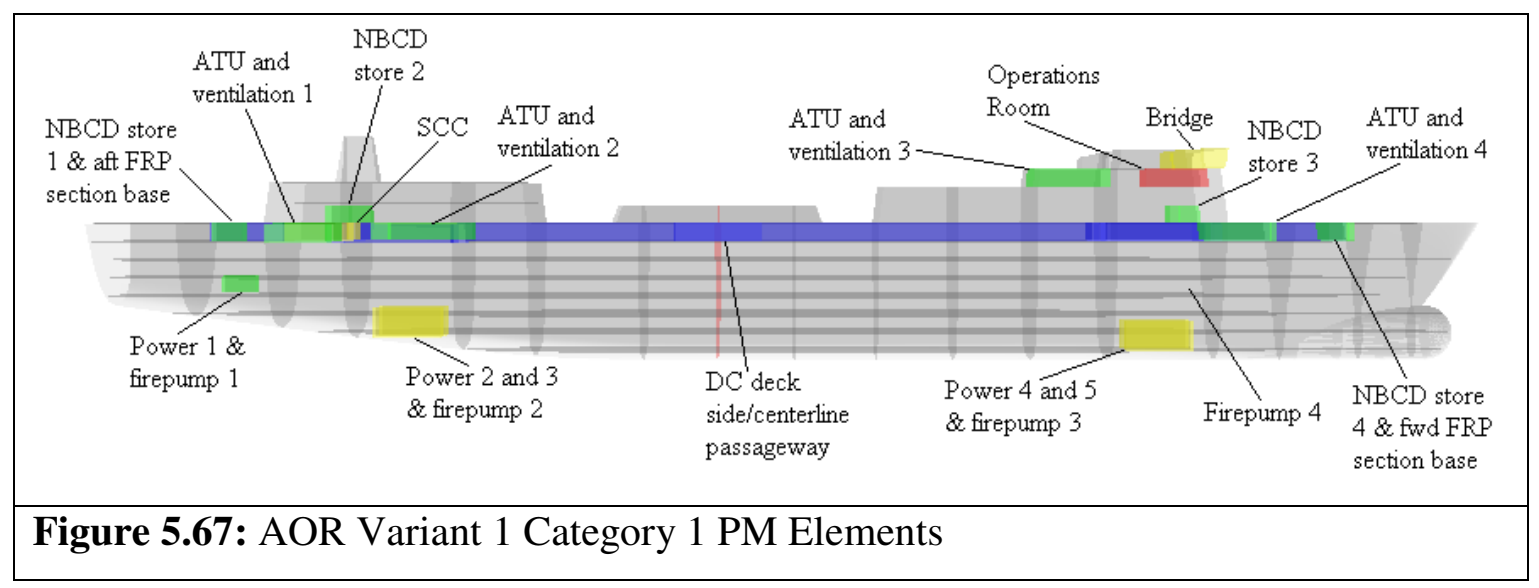

From Figure 5.67 it is evident that all redundant items are split between the forward and aft superstructure blocks, therefore, providing low levels of vulnerability (Appendix 4.2). For example the two FRPs (note that the same number of FRP section bases, positioned conveniently to NBCD stores, as in the combatant design studies was 
selected, as suggested in Appendix 4.3 and Figure A36) are stationed at the extremities of the hull, therefore, minimising the probability of both FRPs being affected by a hit. However, being far from amidships (the ideal location in terms of minimising transference time and facilitating access to both zones ), their locations are rather inefficient in that the probability for DC crews to travel long distances (and operate many WT doors) to reach the affected section would increase, therefore, producing large values for PMs 1.1 and 1.2. Another similarity between the AOR designs and the combatant designs is the large distance between the Operations Room and the SCC, the roles of which could be interchanged in emergency situations, and the adjacency between the bridge and the Operations Room to improve communication. However, in the auxiliary designs it was decided that the Operations Room would be located high in the superstructure (02 Deck), increasing its vulnerability to abovewater attacks but decreasing it to underwater attacks. This decision was taken principally due to the largely civilian crew, usually managing the ship from the bridge. Had the Operations Room been located deep in the hull, at a distance from the bridge, there is a possibility that it would be understaffed. In addition, given the minor military role of the vessel, the increase in abovewater threat vulnerability of this arrangement was deemed of minor importance, compared to the argument above.

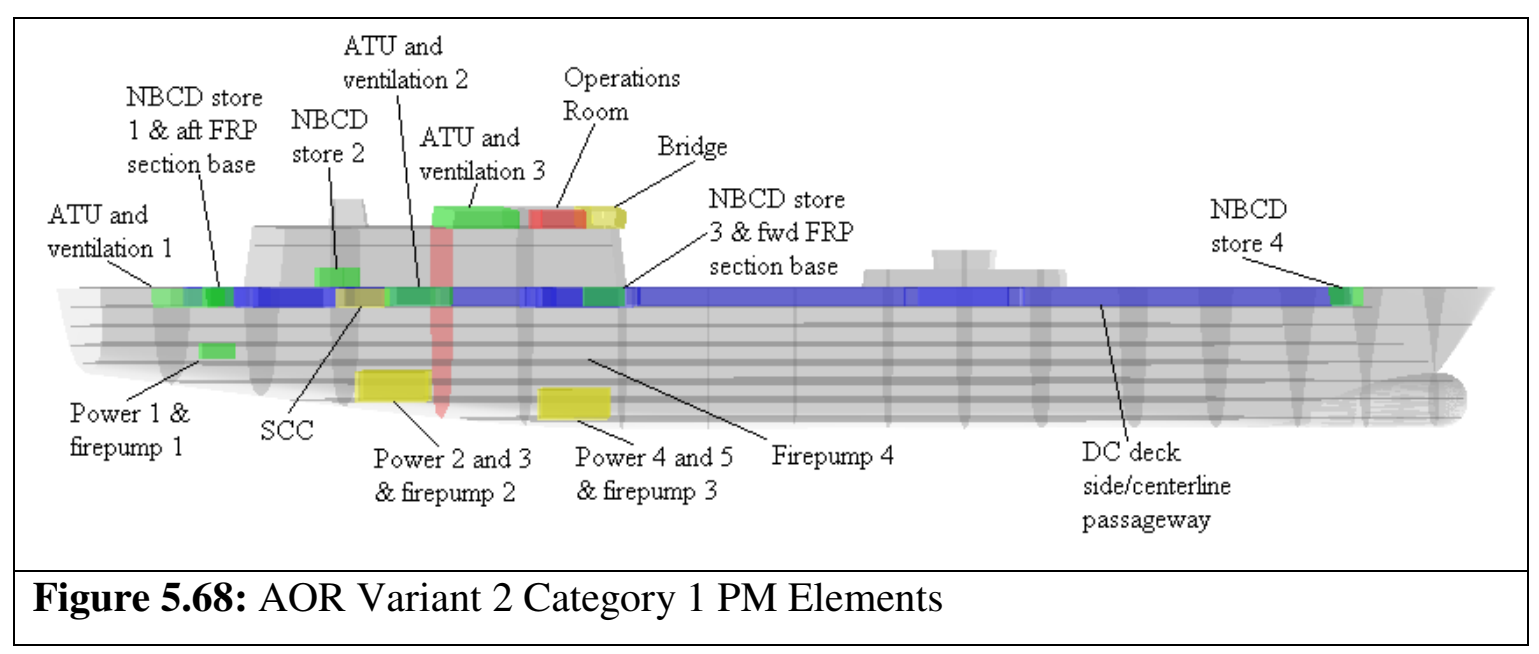

By studying Figure 5.68 one can easily conclude that the main difference between the two auxiliary ship variants is the extensive concentration of compartments and equipment at the aft end in AOR Variant 2. (This, for example, has led to the inclusion of only three ATU and ventilation units, contradictorily to the four units in the first AOR design variant). This concentration of redundant (i.e. parallel) items should result to an increase in overall system vulnerability. However, the fact that the forward 
FRP section base is located more efficiently (i.e. proximate to amidships) should introduce some advantages in crew DCFF efforts (PMs 1.1 and 1.2). In addition, encompassing only one superstructure block, the number of internal decks in most WT section of AOR Variant 2 is less than in the first variant, therefore, improving PM 1.3. Namely, one would expect that overall AOR Variant 2 should ease DCFF crew efforts, although, a constant threat would probably lead to a greater number of items/systems being affects and needing recovery. The logic behind the location and arrangement of all other equipment and compartments is similar to that in AOR Variant 1 and in consistency with the zoning arrangements described above.

Similarly, Figure 5.69 and Figure 5.70 examine the arrangements of compartments and equipment related to Category 2 (and Category 3) PM analysis in AOR Variant 1 and AOR Variant 2 respectively

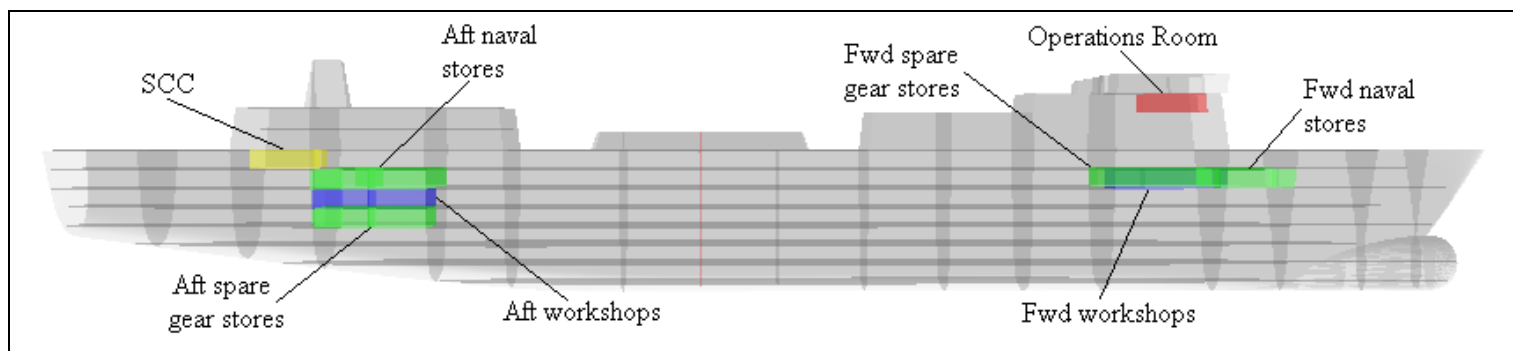

Figure 5.69: AOR Variant 1 Category 2 PM Elements

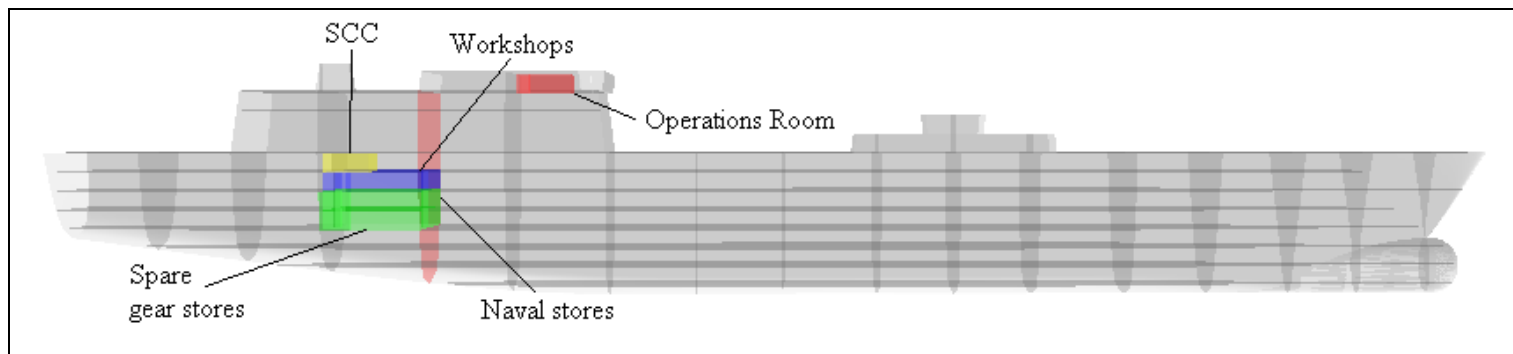

Figure 5.70: AOR Variant 2 Category 2 PM Elements

There are two major aspects worth commenting on regarding the distribution of Category 2 (and 3) PM analysis. First, AOR Variant 1 adopts a split stores and workshops philosophy, including duplicated naval stores, in contrast to the combatant design variants. This is because the larger size of the auxiliary ship designs dictate larger naval stores (the parametric relationship used for naval stores required weight and space scaled with hull gross volume (UCL 2010b)) and the distance between the two superstructure blocks where most compartments and systems are located is relatively large; therefore, it was reasoned advantageous to select a split configuration. It follows 
that the first auxiliary ship variant has two groups of workshops, spare gear stores and naval stores, each positioned adjacently to a main machinery room, therefore, tying in with the zoning arrangements. The presence of the second naval stores compartment necessitates alterations in the Category 2 PMs (PM 2.3, Table 4.4, Section 4.3.2) and Category 3 PMs (PM 3.3, Table 4.5, Section 4.3.3), which are specified in Section 6.5.3. The second major aspect is that in AOR Variant 2, none of the above stores and workshops are split. There is only one workshop, spare gear store and naval store, all located adjacently and reasonably close to the two main machinery rooms. It is clear that this configuration was chosen as, given the compact arrangement of most non-cargo related spaces at the ship designs aft end, there was not much reason behind the selection of a split configuration. This arrangement would also require modifications to a number of Category 2 and 3 PMs (PM 2.1, 2.2, 2.3, 2.4, 2.5, Table 4.4, Section 4.3.2 and PM 3.3, 3.4, 3.5, 3.6, 3.7, Table 4.5, Section 4.3.3). This non split arrangement may increase the vulnerability of the three compartments but should also lead to a susceptibility reduction. As with all previous design studies, category 3 PM analysis also involves all of the components of each major ship system, with system architecture illustrations included in Appendix 7. 


\section{Chapter 6: Results of Applying Proposed Survivability Assessment Approach}

This section presents the full set of results which were obtained after applying the survivability assessment method detailed in Chapter 4 to the ship design studies described in Chapter 5. Results (including various sensitivity tests) are initially presented separately for each survivability constituent, followed by total survivability results. Finally, a brief section on ship costing is included, providing costing information of the ships designed.

\subsection{Susceptibility Assessment Results}

The procedure described in Section 4.1 was followed in order to obtain susceptibility results for all ship design studies.

\section{$\underline{6.1 .1 \text { Frigate Variants (Including Baseline) }}$}

The scenario selected was that of a naval ship being attacked by four radar homing (at $15 \mathrm{GHz}$ ) sea-skimming ASMs (Figure 4.1). At this stage, the probability of each ship being detected and identified, $\mathrm{P}(\mathrm{di})$, was assumed equal to unity. In order to calculate decoy effectiveness (i.e., the probability that the missile locks on the ship, $\mathrm{P}(1)$ ) the three frigate design variants were run in the SPECTRE RCS prediction software, since the attacking missiles were assumed to be radar homing. The RCS estimations for the three frigate designs are shown in Figure 6.1.

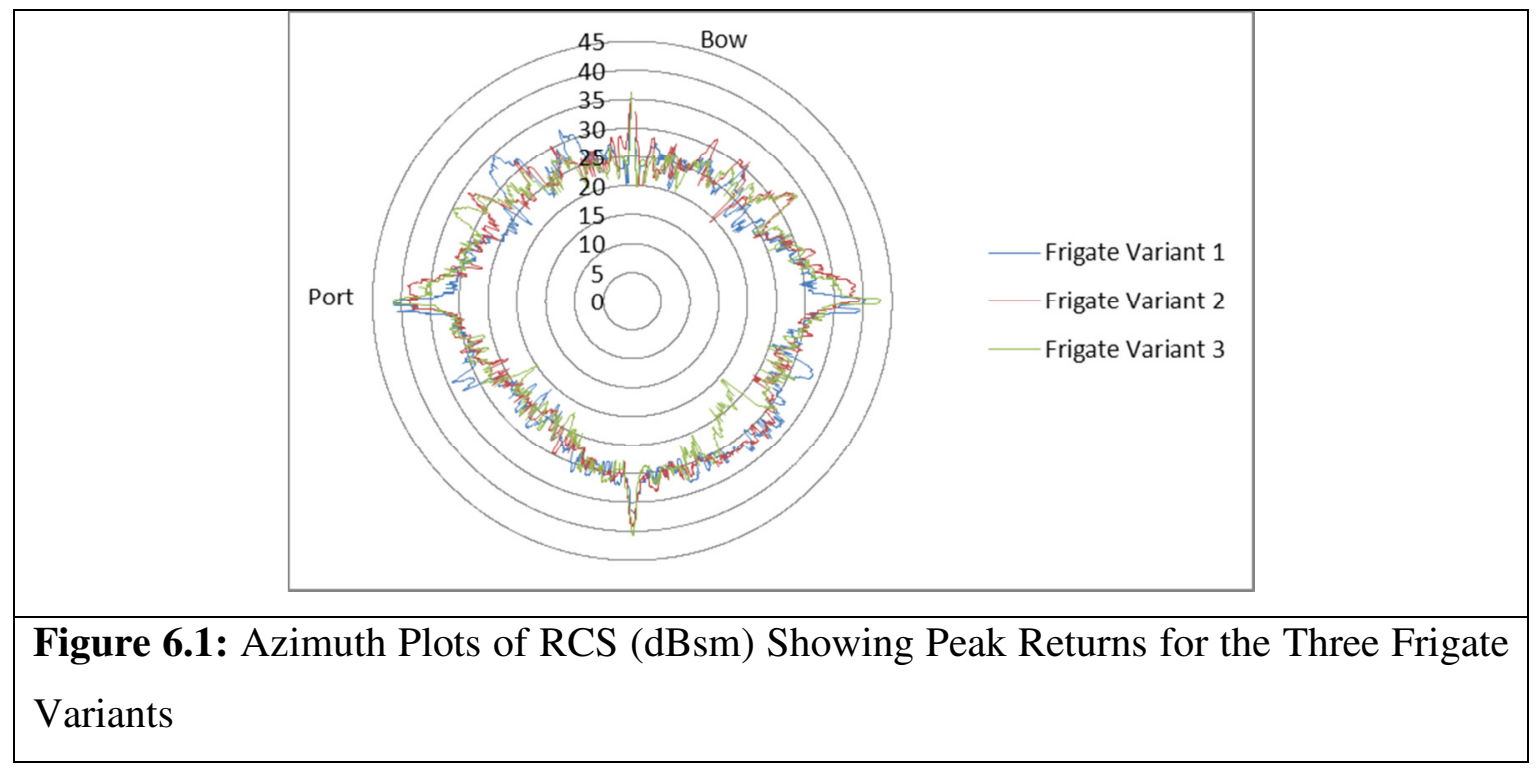


This illustration above gives RCS at an elevation of $0^{\circ}$ and frequency of $15 \mathrm{GHz}$. The relationship given in Figure 4.2 and Equations 3 and 4 (see Section 4.1) was then used to calculate $\mathrm{P}(1)$. The ship RCS value used in the equations is equal to average ship RCS. Note that RCS values at $90^{\circ}$ (starboard), $180^{\circ}$ (stern), $270^{\circ}$ (port) and $360^{\circ}$ (bow) $\pm 4^{\circ}$ were neglected due to the exceptionally large and, therefore, misleading, RCS returns. These are quite clear in Figure 6.1. As mentioned in Section 4.1 the chaff RCS used in Equations 3 and 4 was assumed to be 2,000 ${ }^{2}$ between 10-20GHz (Friedman 2006).

The CSEE method (McDonald 2010) was then used in order to estimate defensive system (hard-kill) effectiveness (i.e., the probability of the ship being hit by at least one ASM, P(h)). The timeline of events, from the launch of the first ASM until the last one hits the ship, is presented in Figure 6.2 and Table A33, using the weapon system data shown in Table A31 (see Appendix 10.1). Since all frigates had identical payload (Table 5.1), their event timelines were identical.

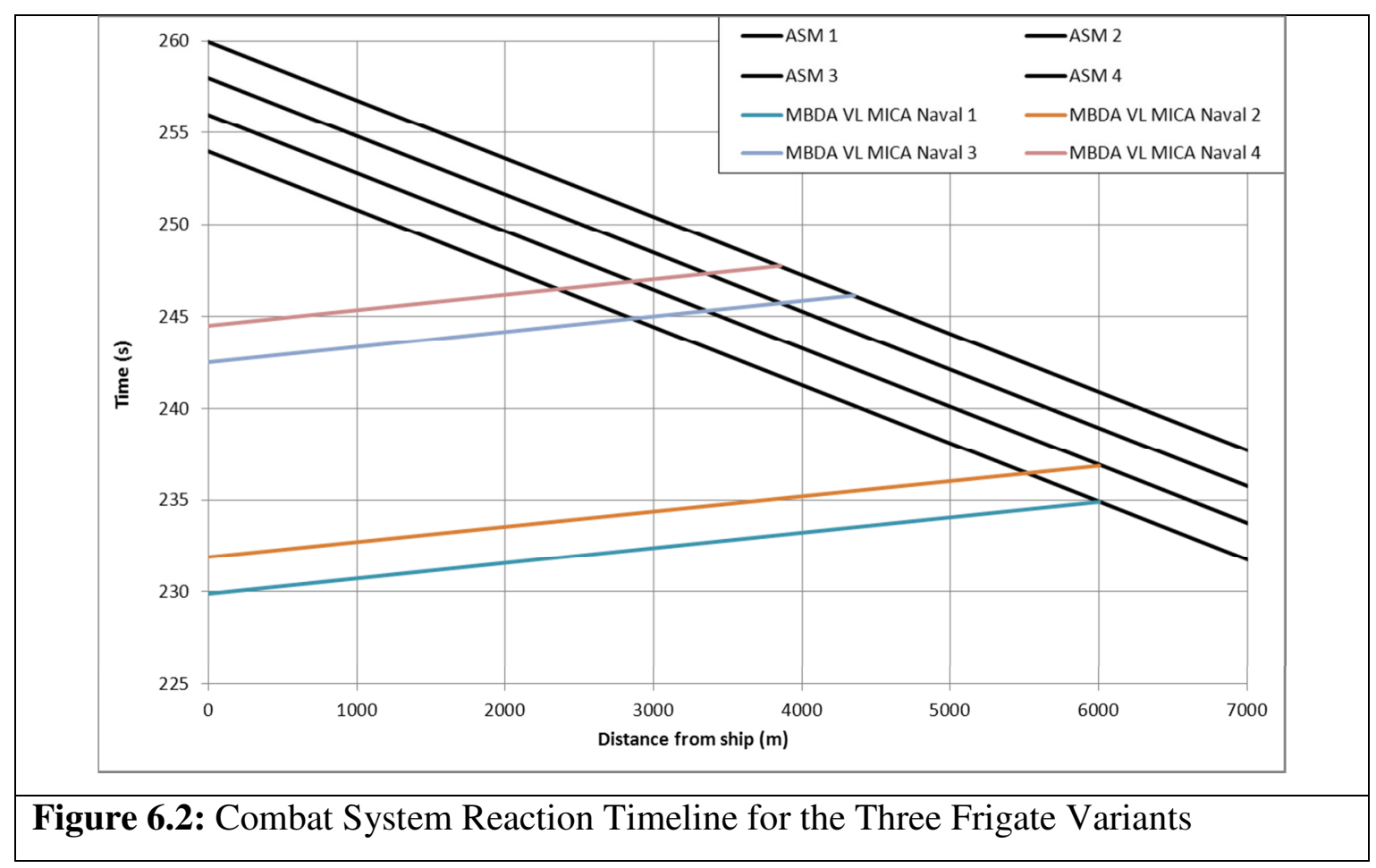

From Figure 6.2 it is clear that each frigate variant had enough time to fire four defensive missiles against the four attacking ASMs. For simplicity, the defensive missiles were assumed to have a probability of kill, $\mathrm{P}(\mathrm{k})$, equal to 0.73 (McDonald 2010) (although, as explained in Appendix 4.1, $\mathrm{P}(\mathrm{k})$ varies with the angle between the attacking and defensive missiles). In addition, a simplified and unclassified jammer with a $\mathrm{P}(\mathrm{k})$ of 0.25 (McDonald 2010) was assumed. It was chosen not to calculate jammer 
effectiveness using a separated method (as was done with decoy effectiveness) in order to avoid unnecessarily complications (reflecting the multifaceted mechanism characterising jammers) for the level of detail of the chosen ship design studies; a constant jammer effectiveness value for all designs was presumed adequate. Given that each pair of defensive missiles targets the same attacking missile, it follows that the first two attacking missiles were each targeted by two defensive missiles (as well as the jammer), while the last two attacking missiles only had the jammer to overcome. Therefore, the $\mathrm{P}(\mathrm{k})$ of each attacking missile was calculated using equation 6 ; the results are shown in Table 6.1.

$$
\mathrm{P}(\mathrm{k}) \text { Overall }=1-(1-\mathrm{P}(\mathrm{k}) \text { Defensive Missile }) \times(1-\mathrm{P}(\mathrm{k}) \text { Jammer })
$$

\begin{tabular}{|l|r|r|r|}
\hline \multicolumn{5}{|c|}{ Table 6.1: Probabilities of Kill of Attacking ASMs for the Three Frigate Variants } \\
\hline & P(k) Defensive Missile & P(k) Jammer & P(k) Overall \\
\hline Attacking ASM 1 & 0.927 & 0.250 & 0.945 \\
\hline Attacking ASM 2 & 0.927 & 0.250 & 0.945 \\
\hline Attacking ASM 3 & 0 & 0.250 & 0.250 \\
\hline Attacking ASM 4 & 0 & 0.250 & 0.250 \\
\hline
\end{tabular}

From the data given above it is relatively straightforward to calculate the three susceptibility related probabilities mentioned in Section 4.1, the probability of the ship being detected and identified, $\mathrm{P}(\mathrm{di})$, the probability that the missile locks on the ship, $\mathrm{P}(\mathrm{l})$, and the probability of the ship being hit by at least one ASM $\mathrm{P}(\mathrm{h})$. These, as well as each frigates total susceptibility (being equal to the product of the three above probabilities and yielding the probability that a ship was successfully detected, identified, targeted and hit by the adversary) are given in Table 6.2.

Table 6.2: Susceptibility Results for the Three Frigate Variants

\begin{tabular}{|l|r|r|r|}
\hline & Frigate Variant 1 & Frigate Variant 2 & Frigate Variant 3 \\
\hline Average RCS (m2) & $1,011.7$ & $1,045.9$ & 928.8 \\
\hline P(di) (\%) & 100 & 100 & 100 \\
\hline P(l) (\%) & 25.3 & 26.2 & 23.2 \\
\hline P(h) (\%) & 94.4 & 94.4 & 94.4 \\
\hline P(susc.) (\%) & $\mathbf{2 3 . 9}$ & $\mathbf{2 4 . 7}$ & $\mathbf{2 1 . 9}$ \\
\hline
\end{tabular}


The main points worth mentioning are that all three variants have almost identical RCS values (also clear from Figure 6.1) leading to very similar P(1). (Note that the RCS results are generally lower than those of existing comparable current frigates (typically having an RCS of approximately $10,000 \mathrm{~m}^{2}$ ) due to factors such as the perfectly smooth sides outputted by the Paramarine CAD software (before a design is imputed in SPECTRE) and the very little microgeometry included due to the minimum (preliminary design stage) level of detail. However, since a comparison is being made between concept ship designs, as long as the above variables are kept constant, the end results should be representative. Moreover, the fact that $\mathrm{P}(\mathrm{di})$ has been assumed equal to 1 for all ship designs and $\mathrm{P}(\mathrm{h})$ is equal for all frigate variants due to their identical payload (and, therefore, defensive systems) has led to all frigate variants outputting very similar susceptibility results. This is evident in Figure 6.3 which shows total susceptibility results (i.e. the last row of Table 6.2) normalised with respect to the baseline frigate (hence the susceptibility value of Frigate Variant 1 in Figure 6.3 is equal to 1$)$.

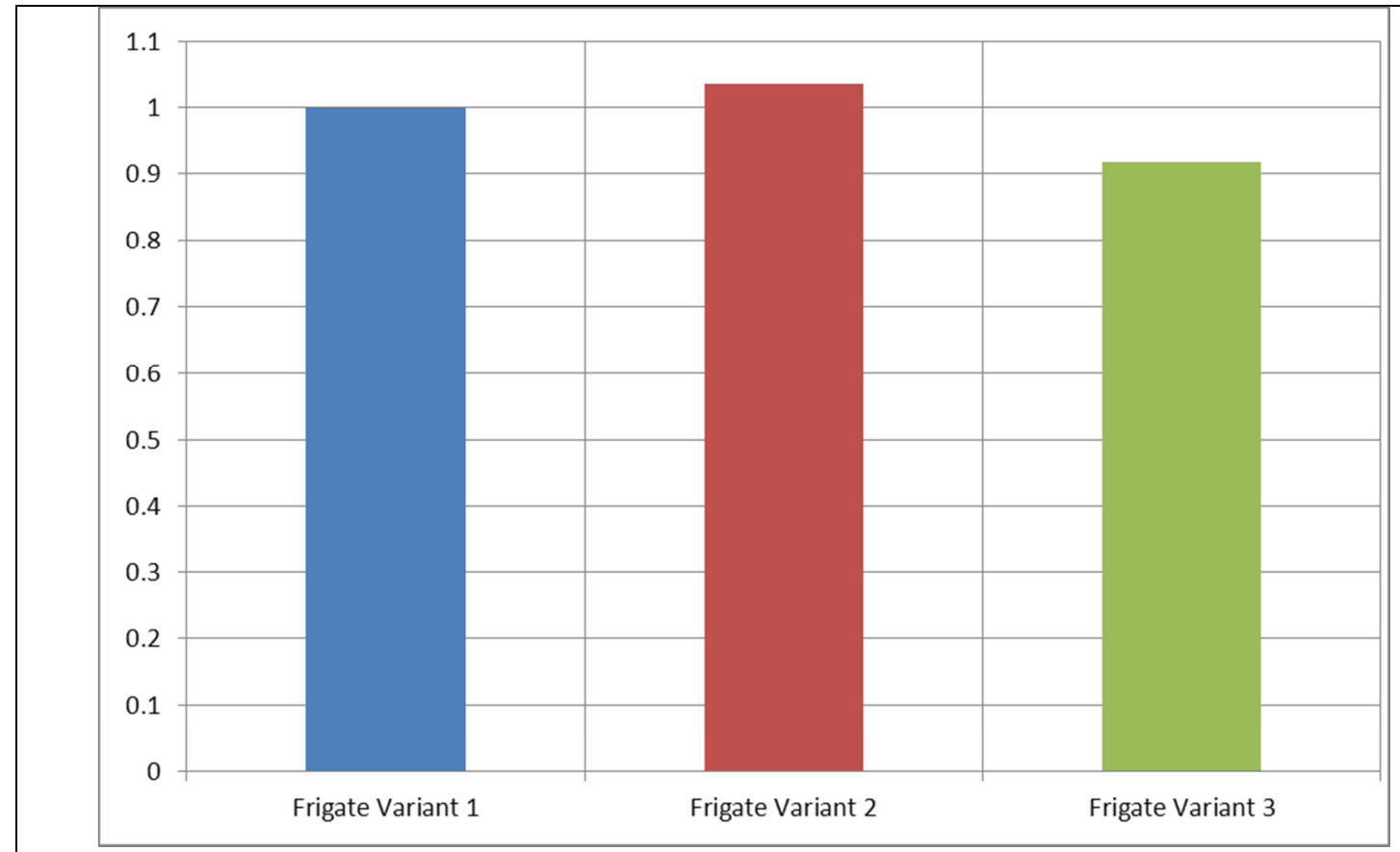

Figure 6.3: Normalised Plot of Susceptibility for the Three Frigate Variants

The above results indicate the relative total ship susceptibility, not the susceptibility characteristics of each of the designs. However, as summarised in Section 3.3.2 and 4.1 the factors affecting the lengthwise hit probability on a ship are not limited to ship and threat characteristics, but are affected by environmental conditions, ship 
motions and manoeuvring and by variations in weapon performance (MOD 2001). Therefore, rather than using an RCS prediction software to obtain a (dubious) lengthwise hit probability distribution, it was assumed to follow a normal distribution with a mean equal to half of the ship's overall length and a standard deviation equal to a sixth of the ship's overall length (Section 4.1), as advised by the Maritime Integrated Survivability team of Dstl (Thornton et al 2012). These distributions are shown in Figure 6.4.

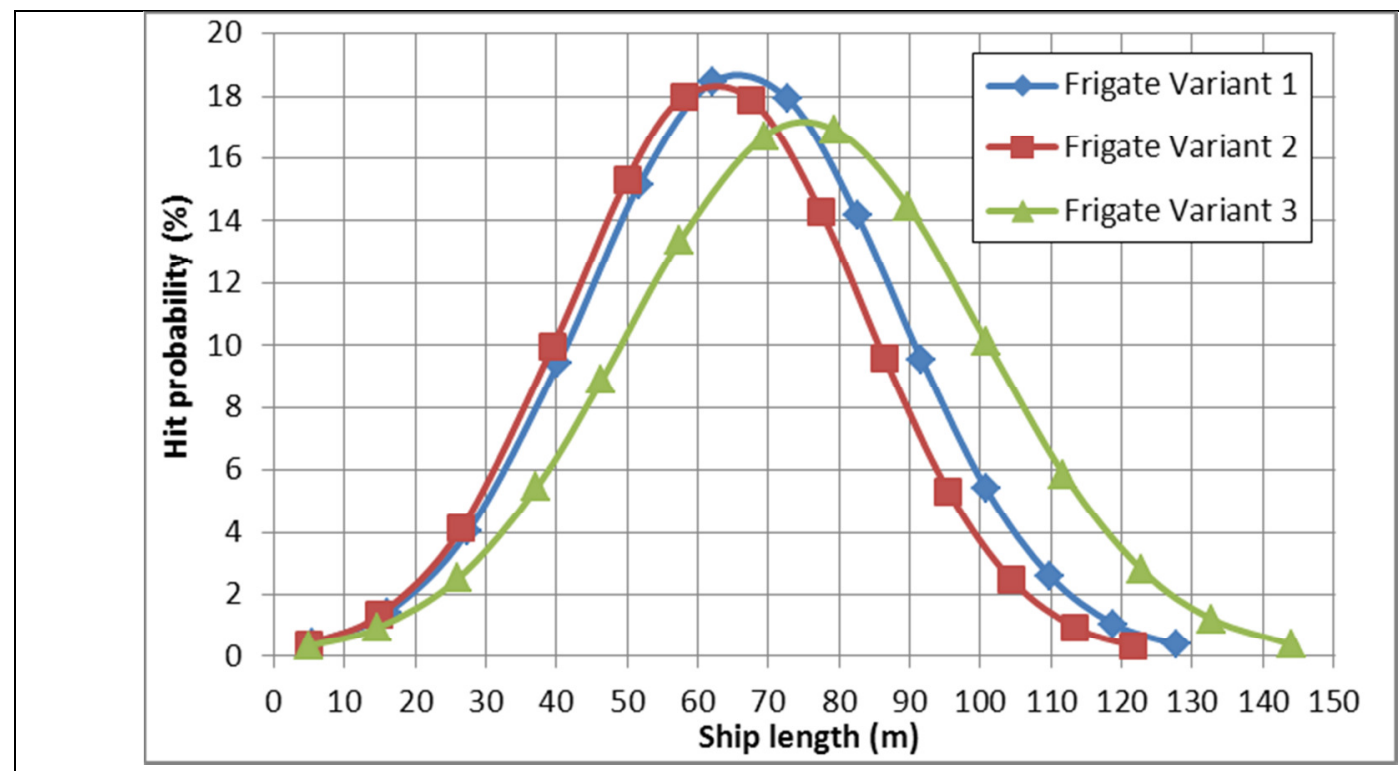

Figure 6.4: Lengthwise Probability Hit Distribution for the Three Frigate Variants

The data points shown in Figure 6.4 represent the hit probability of the centroid of each WT section of each frigate design variant and aggregate to $100 \%$ for each ship.

\subsubsection{Corvette, Baseline Frigate and Destroyer}

For the comparative survivability investigation of the Corvette, baseline frigate and Destroyer, the identical threat scenario to that for the three frigate variants was assumed. Once again, $\mathrm{P}(\mathrm{di})$ was equalled to $1 ; \mathrm{P}(1)$ was calculate by obtaining RCS data from SPECTRE, substituting the average ship RCS in Equations 3 and 4 and assuming a decoy RCS of 2,000 $\mathrm{m}^{2}$ at $15 \mathrm{GHz}$. The SPECTRE generated RCS data (at an elevation of $0^{\circ}$ and frequency of $15 \mathrm{GHz}$ ) for the three combatant designs are shown in Figure 6.5. 


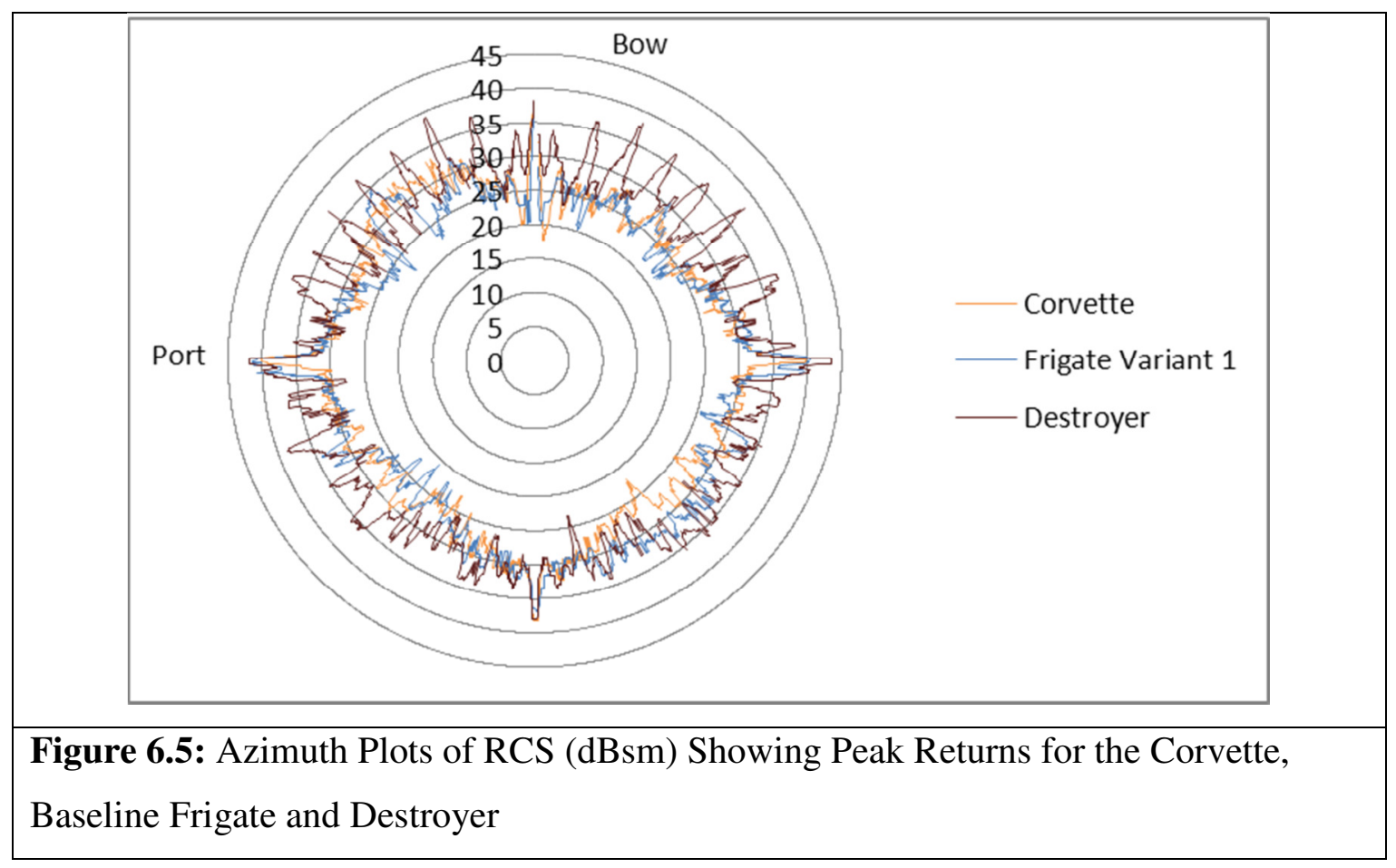

The CSEE method was then utilized in order to estimate defensive system effectiveness and obtain $\mathrm{P}(\mathrm{h})$ values. Both the Corvette design and the baseline frigate design had identical AAW systems (MBDA MICA PDMS) to counter the threat (Table 5.4). Therefore, by using the data displayed in Table A31, an identical combat system reaction timeline as for the frigate design variants, illustrated in Figure 6.2 and Table A33, was obtained, with both ships having enough time to fire four defensive missiles against the attacking missiles.

Since the Destroyer design had a different, more advanced AAW capability (Table 5.4), the CSEE method was reapplied in order to calculate the corresponding $\mathrm{P}(\mathrm{h})$ value. The weapon system data shown in Table A35 was used to produce the combat system reaction timeline illustrated in Figure 6.6 and the sequence of events shown in Table A36 (see Appendix 10.2) for the scenario of the Destroyer being attacked by four ASMs. It is important to note that it was initially assumed that only Aster-15 (rather than Aster-30) SAMs would be deployed (together with the Phalanx CIWS). 


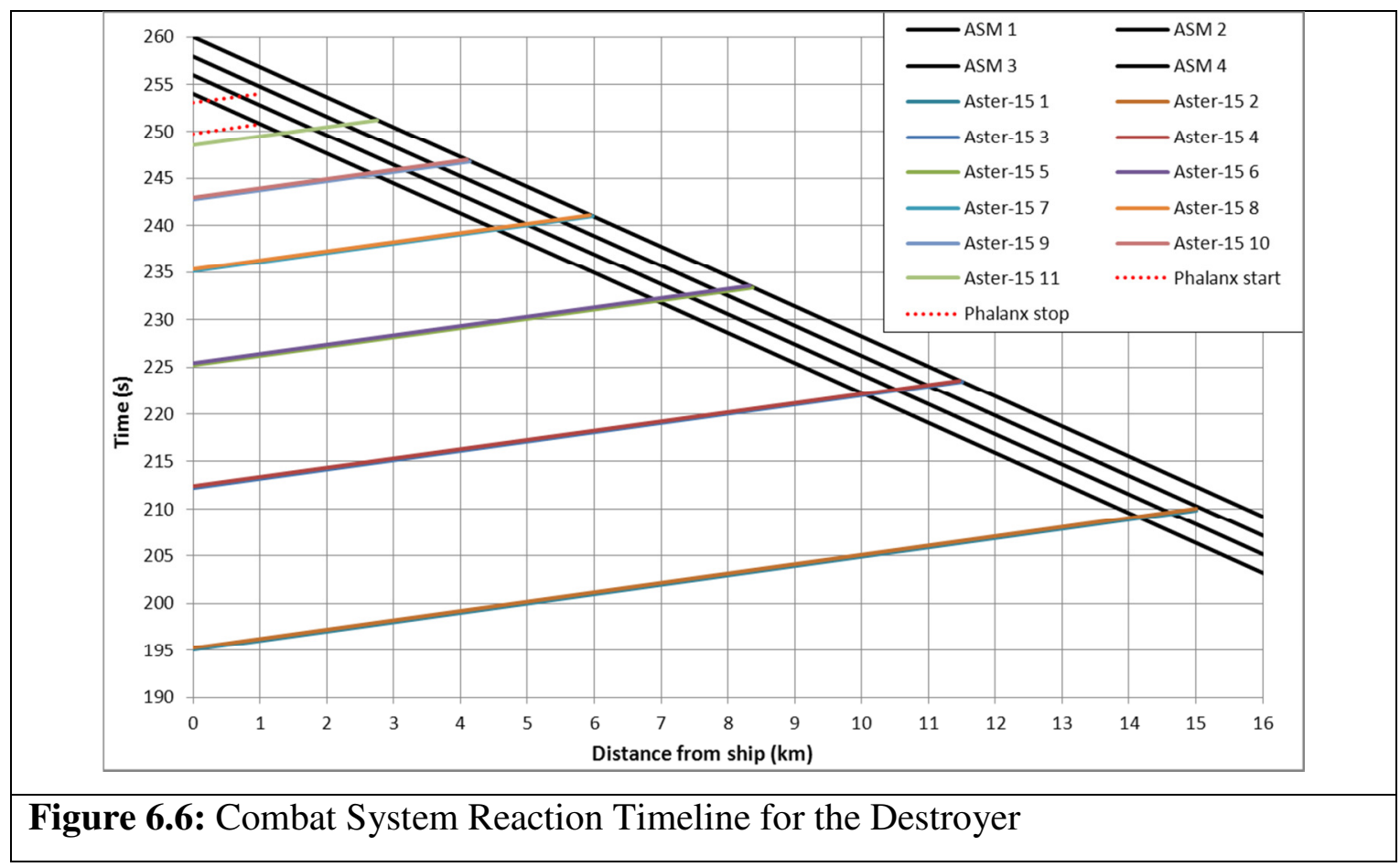

From the above data, the much more capable AAW system of the Destroyer had enough time to fire eleven defensive missiles before being hit. As with the frigate variants and the Corvette, the defensive missiles were assumed to have a probability of kill, $\mathrm{P}(\mathrm{k})$, equal to 0.73 (although this is an underestimate, given the increased capability) and the jammer a $\mathrm{P}(\mathrm{k})$ of 0.25 (McDonald 2010). In addition, the Phalanx CIWS P(k) was taken as 0.78 (McDonald 2010). Again, each pair of defensive missiles targets the same attacking missile. Therefore, the first attacking missile was targeted by four defensive missiles, $2+2$, (as well as the jammer), the second attacking missile was targeted by three defensive missiles, $2+1$, (as well as the jammer), while the last two attacking missile were targeted by two defensive missiles (and the jammer) each. Furthermore, the CIWS could only fire one burst, targeting only one of the four incoming missiles. The $\mathrm{P}(\mathrm{k})$ of each attacking missile is presented in Table 6.3 (while the corresponding Corvette and baseline frigate data is presented in Table 6.1).

\begin{tabular}{|l|r|r|r|r|}
\hline Table 6.3: Probabilities of Kill of Attacking ASMs for the Destroyer \\
\hline & $\begin{array}{c}\text { P(k) Defensive } \\
\text { Missile }\end{array}$ & P(k) CIWS & $\begin{array}{c}\text { P(k) } \\
\text { Jammer }\end{array}$ & P(k) Overall \\
\hline Attacking ASM 1 & 0.995 & 0.780 & 0.250 & 0.999 \\
\hline Attacking ASM 2 & 0.980 & 0 & 0.250 & 0.985 \\
\hline Attacking ASM 3 & 0.927 & 0 & 0.250 & 0.945 \\
\hline Attacking ASM 4 & 0.927 & 0 & 0.250 & 0.945 \\
\hline
\end{tabular}


From the data above, the total susceptibility results for the three combatants were calculated and presented in Table 6.4.

Table 6.4: Susceptibility Results for the Corvette, Baseline Frigate and Destroyer

\begin{tabular}{|c|c|c|c|}
\hline & Corvette & Frigate Variant 1 & Destroyer \\
\hline Average RCS (m2) & 910.0 & $1,011.7$ & $3,924.6$ \\
\hline $\mathbf{P}(\mathrm{di})(\%)$ & 100 & 100 & 100 \\
\hline $\mathbf{P}(\mathbf{l})(\%)$ & 22.8 & 25.3 & 98.1 \\
\hline $\mathbf{P}(\mathbf{h})(\%)$ & 94.4 & 94.4 & 12.0 \\
\hline P(susc.) $(\%)$ & 21.5 & 23.9 & 11.8 \\
\hline
\end{tabular}

Although the Corvette and baseline frigate present similar susceptibility results, due to analogous radar signatures $(\mathrm{P}(\mathrm{l}))$ and an identical AAW system $(\mathrm{P}(\mathrm{h}))$, the Destroyer is distinctly less susceptible (see Figure 6.7 displaying total susceptibility results normalised with respect to the baseline frigate).

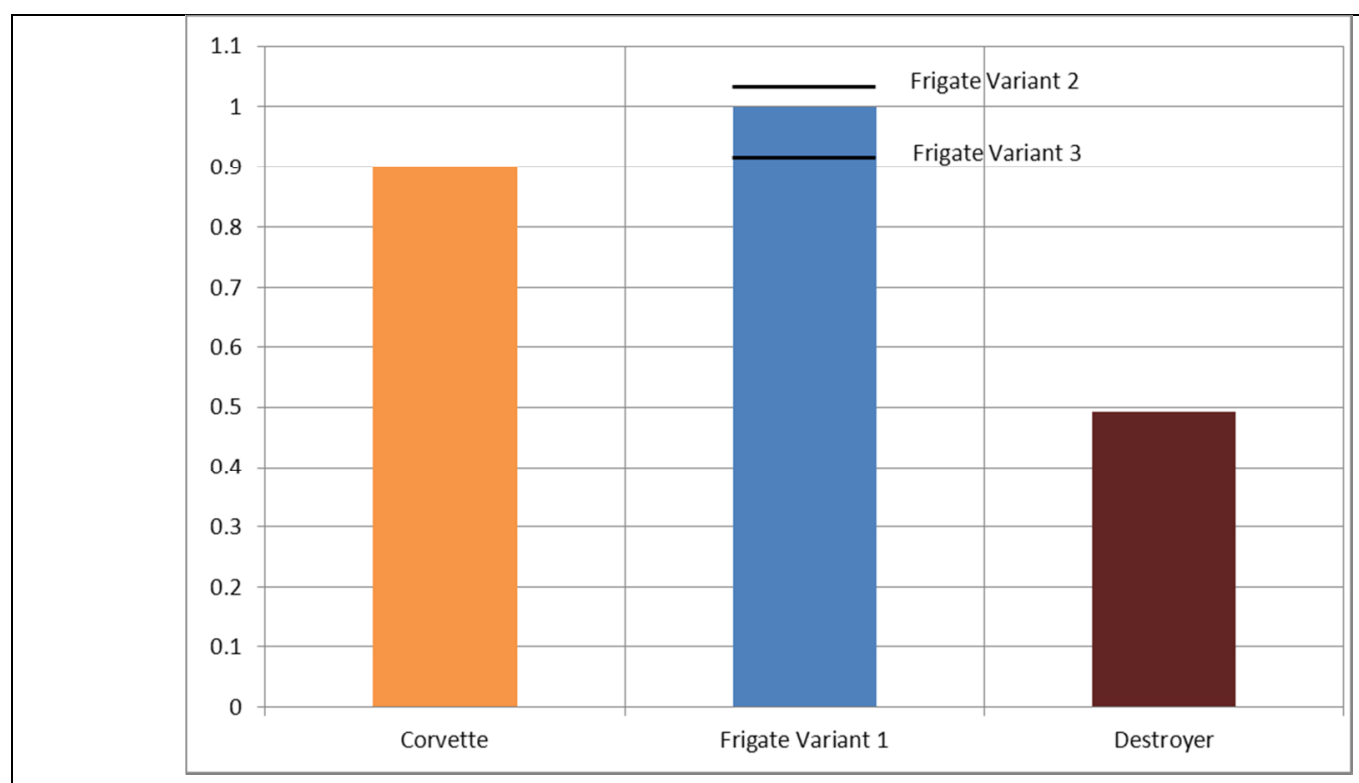

Figure 6.7: Normalised Plot of Susceptibility for the Corvette, Frigate Variants and Destroyer

The lengthwise hit probability distribution of the Corvette and Destroyer designs were assumed to follow a normal distribution with the same characteristics as for the three frigate Variants. The Corvette and Destroyer distributions, together with that of the baseline frigate design, are shown in Figure 6.8. 


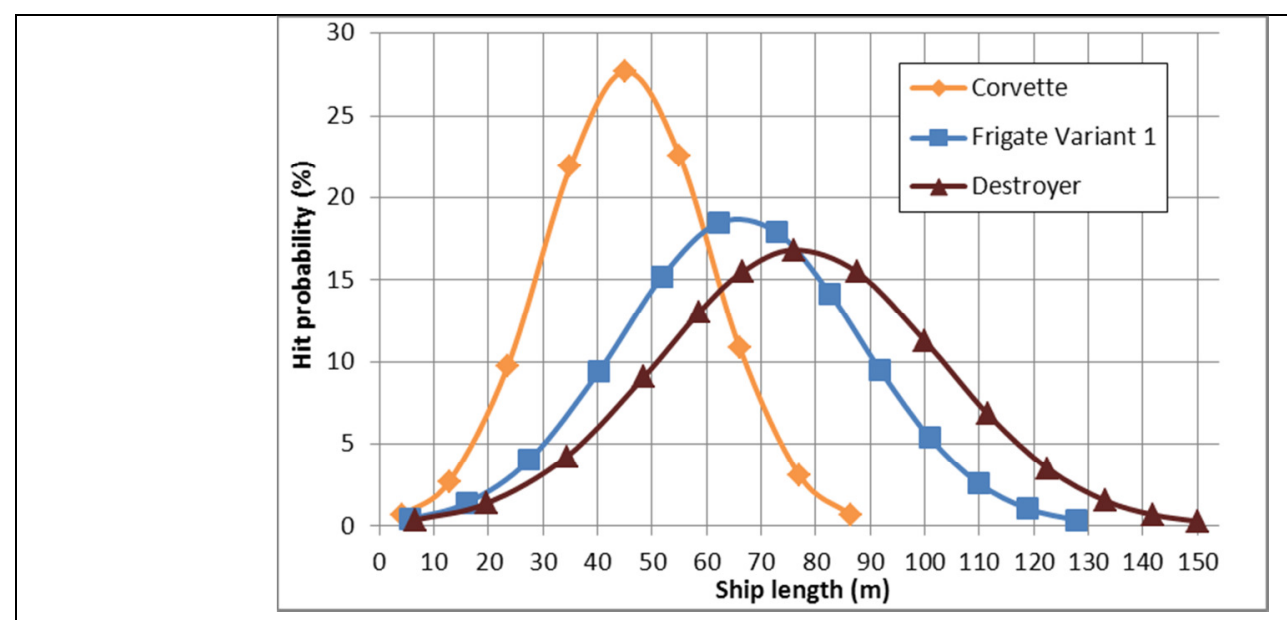

Figure 6.8: Lengthwise Probability Hit Distribution for the Corvette, Baseline Frigate and Destroyer

The data points in the figure above represent the hit probability of the centroid of each WT section of each ship design and sum up to $100 \%$ for each ship.

\section{$\underline{6.1 .3 \text { AOR Variants }}$}

For the final comparative survivability investigation, between the two AOR variants, the same threat scenario, of the ships being attacked by four radar homing seaskimming ASMs, was assumed. $\mathrm{P}(\mathrm{di})$ was equalled to 1 , while decoy effectiveness, $\mathrm{P}(\mathrm{l})$, was calculate by obtaining RCS data from SPECTRE and using Equations 3 and 4. The results are shown in Figure 6.9.

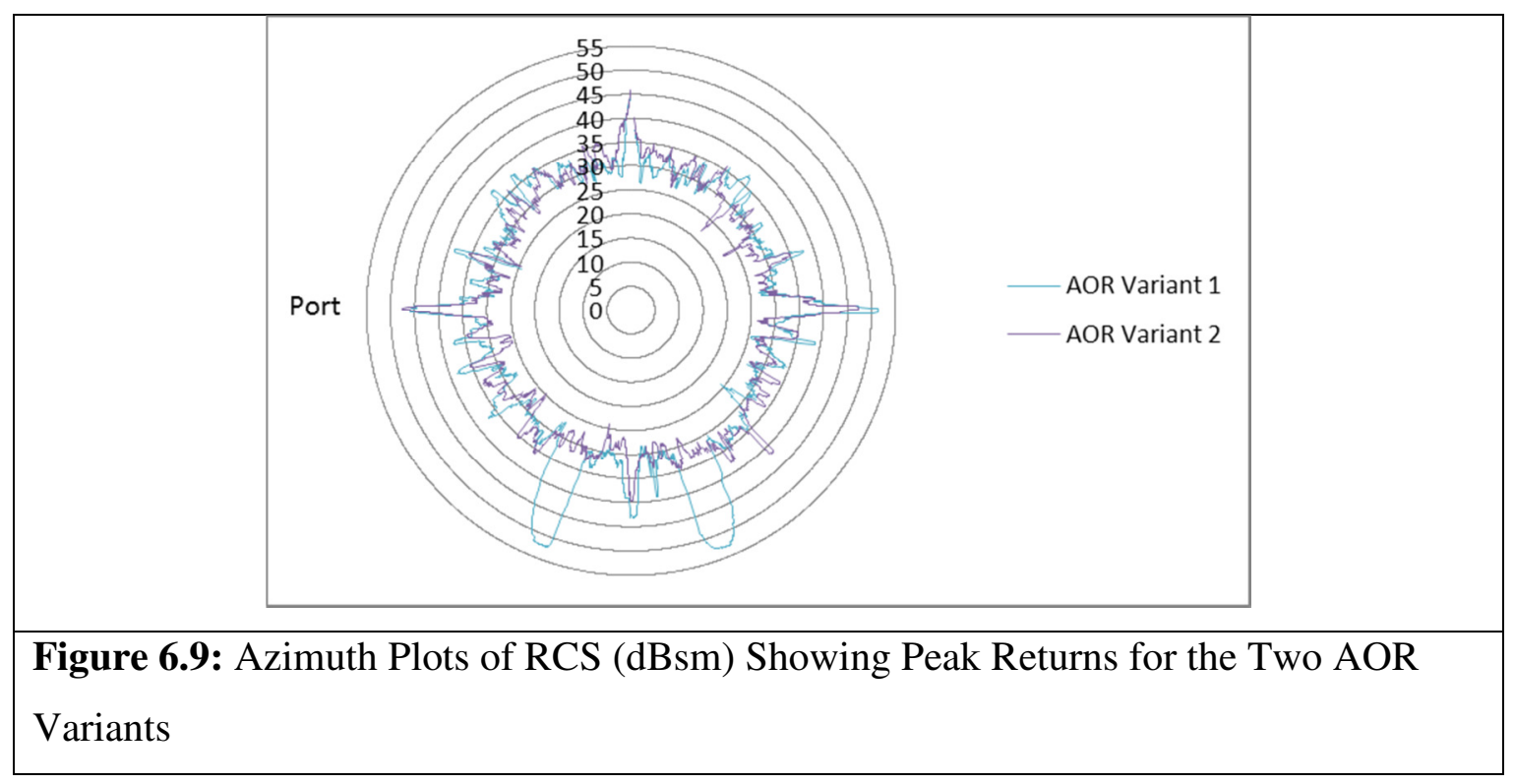


The CSEE method was then used in order to estimate defensive system effectiveness against the above threat scenario and obtain $\mathrm{P}(\mathrm{h})$ values. Both AOR design variants incorporated identical weapon systems (Table 5.8), therefore resulting to identical event timelines. Data for the incoming missiles (which were identical to the ones assumed in the combatant scenarios) as well as the AAW capability of the auxiliary ships are given in Table A37. This data was then used in order to obtain the sequence of events shown in Figure 6.10 and Table A39 (see Appendix 10.3).

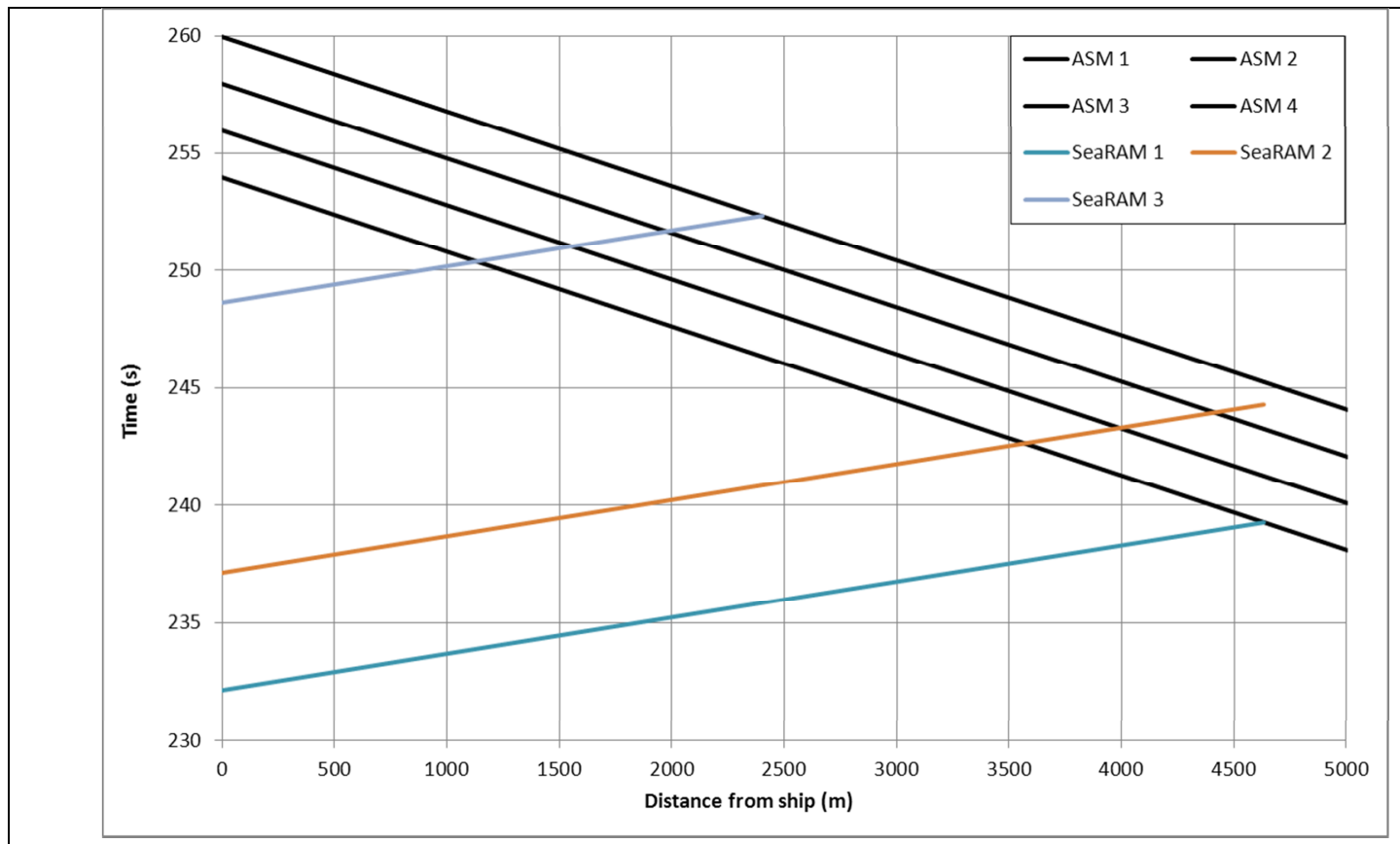

Figure 6.10: Combat System Reaction Timeline for the Two AOR Variants

The above data yields that the AOR design variants, with the relatively weak, purely self-defensive (given the limited military role of the vessels) AAW capability, had enough time to fire three SeaRAM missiles to the four attacking ASMs. The defensive SeaRAM missiles were assumed to have a probability of kill, $\mathrm{P}(\mathrm{k})$, equal to 0.72 (McDonald 2010) and the jammer was given a $\mathrm{P}(\mathrm{k})$ of 0.25 (McDonald 2010), as in the combatant cases. Each pair of defensive missiles targets the same attacking missile, therefore, the first attacking missile was targeted by two defensive missiles (as well as the jammer), the third attacking missile was targeted by only one defensive missile (and the jammer), while the last two attacking missiles had only to overpower the jammer. The $\mathrm{P}(\mathrm{k})$ results of each attacking missile are presented in Table 6.5. 
Table 6.5: Probabilities of Kill of Attacking ASMs for the Two AOR Variants

\begin{tabular}{|l|r|r|r|}
\hline & \multicolumn{1}{|c|}{ P(k) Defensive Missile } & P(k) Jammer & P(k) Overall \\
\hline Attacking ASM 1 & 0.922 & 0.250 & 0.941 \\
\hline Attacking ASM 2 & 0.720 & 0.250 & 0.790 \\
\hline Attacking ASM 3 & 0 & 0.250 & 0.250 \\
\hline Attacking ASM 4 & 0 & 0.250 & 0.250 \\
\hline
\end{tabular}

The above data was then processed in a similar manner to the previous comparative survivability investigations in order to obtain total susceptibility results for the AOR variants, Table 6.6.

Table 6.6: Susceptibility Results for the Two AOR Variants

\begin{tabular}{|l|r|r|}
\hline & AOR Variant 1 & \multicolumn{2}{|c|}{ AOR Variant 2 } \\
\hline Average RCS (m2) & $11,248.5$ & $3,069.6$ \\
\hline P(di) (\%) & 100 & 100 \\
\hline P(l) (\%) & 100 & 76.7 \\
\hline P(h) (\%) & 95.4 & 95.4 \\
\hline P(susc.) (\%) & $\mathbf{9 5 . 4}$ & $\mathbf{7 3 . 2}$ \\
\hline
\end{tabular}

From above it is evident that both AOR variants output similar susceptibility characteristics. Furthermore, they are much more susceptible than the combatant designs, given a constant threat (mainly due to increased signatures, but also due to a less capable AAW system when compared to the Destroyer design). However, a large difference is observed between the RCSs of the two variants (deducing that the launch of decoys with an RCS of $2,000 \mathrm{~m}^{2}$ from the first variant is of no use and therefore, $\mathrm{P}(\mathrm{l})$ is equal to 1) which could change the similar susceptibility characteristics observation previously mentioned, given improved decoy systems. Figure 6.11 shows total susceptibility results for the two AOR design variants. The results are normalised with respect to the worst performing design (i.e. AOR Variant 1, Table 6.6), rather than a baseline design, the reasoning behind which is explained in Section 7.2.4. 


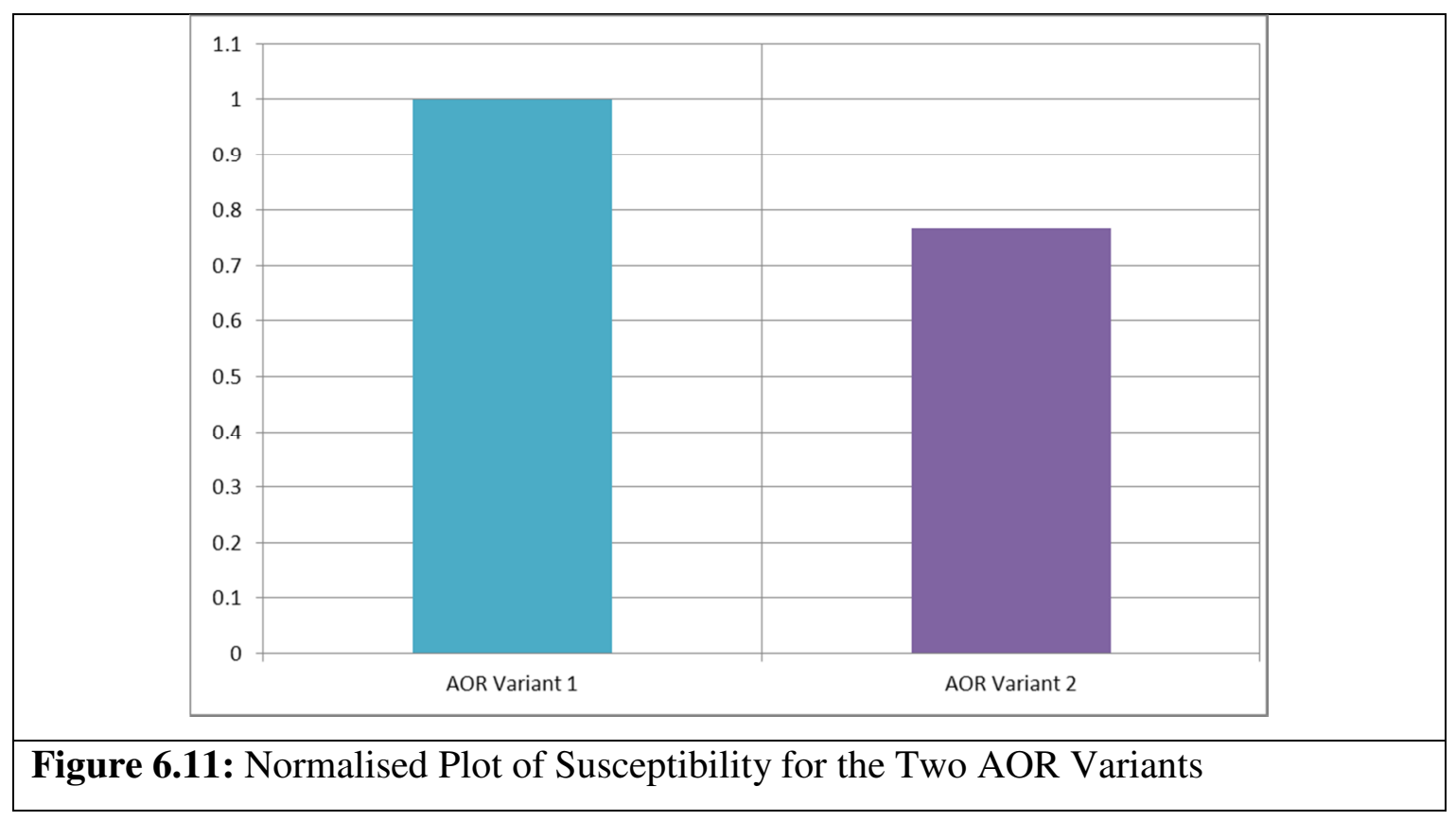

The lengthwise hit probability distributions of the AOR design variants (see Figure 6.12) were assumed to follow a similar, normal distribution, pattern as in the combatant design studies.

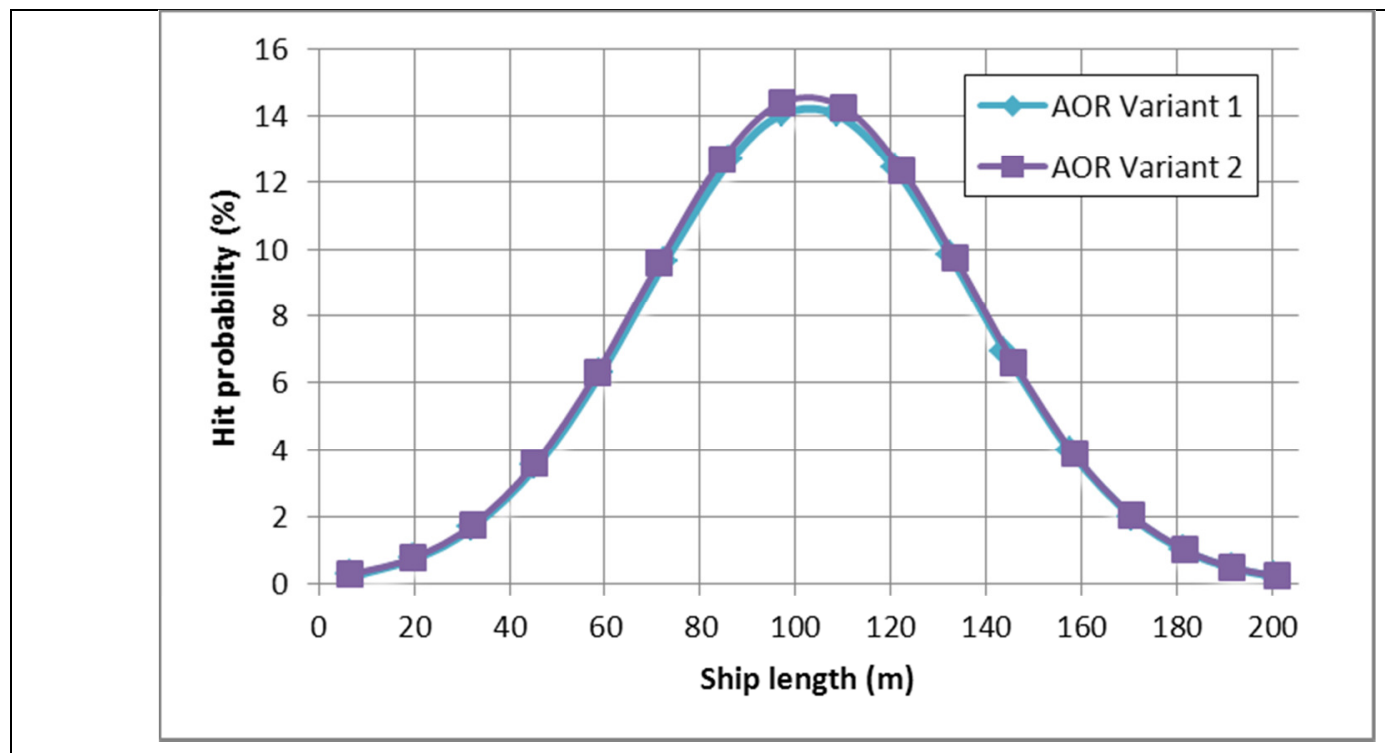

Figure 6.12: Lengthwise Probability Hit Distribution for the Two AOR Variants

\subsection{Susceptibility Assessment Sensitivity Studies}

Four sensitivity studies were carried out in the susceptibility part of the survivability assessment method presenting some interesting results:-

- Selected external features on certain design variants were altered in order to investigate the effects on RCS; 
- The defensive missile targeting pattern in the CSEE was altered;

- The effect of changing the SAM type of the Destroyers PAAMS ADMS was studied;

- The lengthwise probability hit distribution on the frigate variants was modified.

\subsubsection{External Features}

Certain external features of Frigate Variant 1 and Frigate Variant 3 were altered in order to examine their effect on the RCS signature. The first (microgeometry related) feature was the rotation of the RAS high points in the baseline frigate (located at approximately amidships, port and starboard, Figure 5.1) by $7^{\circ}$ on the $\mathrm{x}$-axis. This is clearly illustrated in Figure 6.13, where the before configuration is shown in clear light blue and the after, in opaque purple.

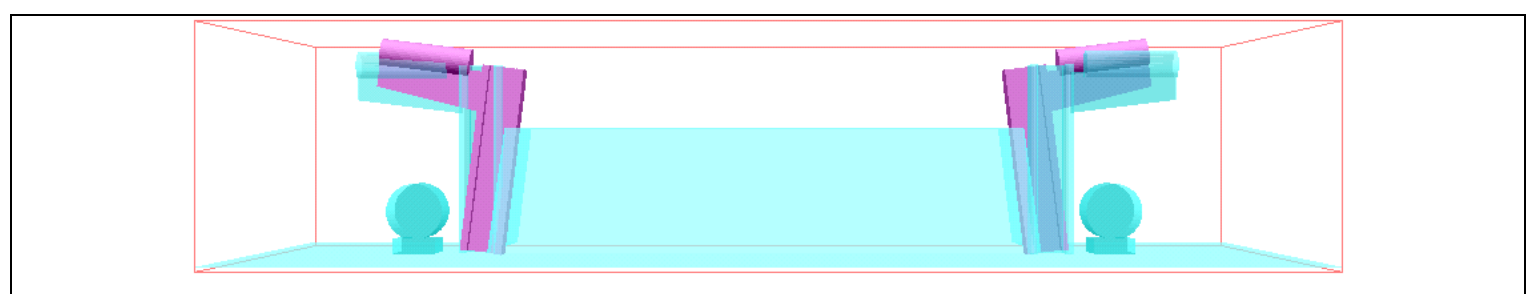

Figure 6.13: Baseline Frigate Microgeometry Signature Reduction Feature

This minor alteration led to a significant decrease in RCS, illustrated in the before and after plots of RCS showing peak returns, Figure 6.14.

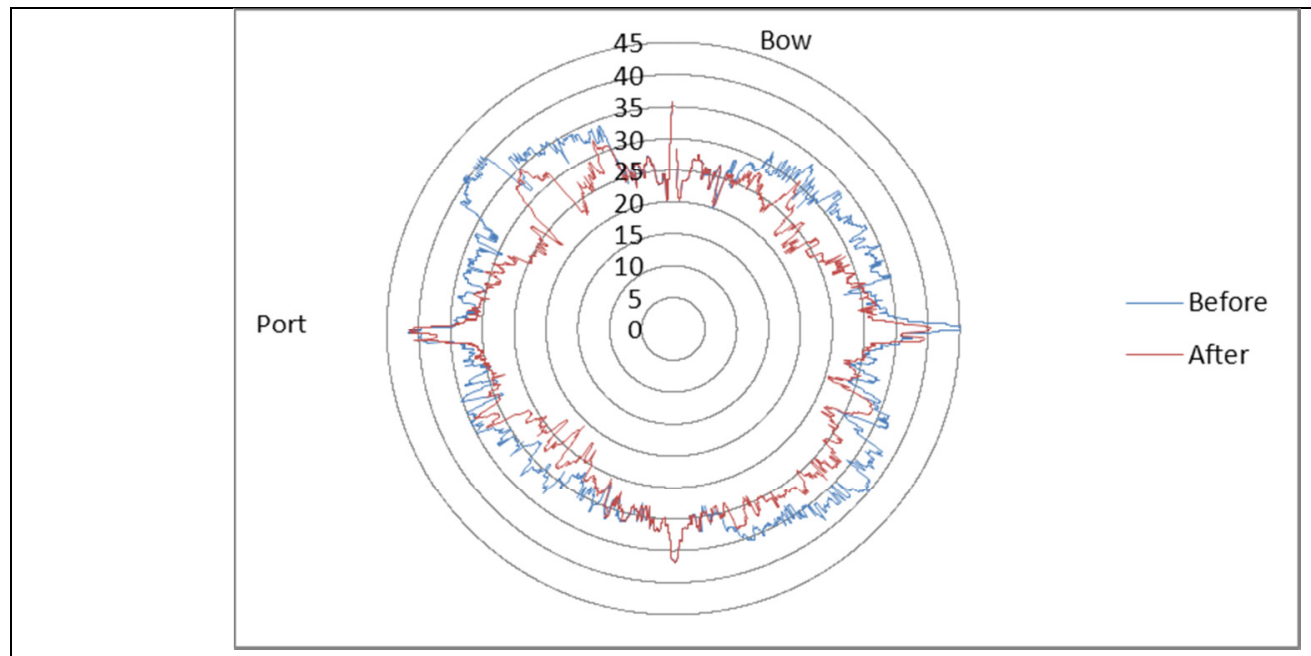

Figure 6.14: Before and After Azimuth Plots of RCS (dBsm) Showing Peak Returns for the Baseline Frigate 
Following this find, all RAS high points were inclined by the same angle in all combatant design studies.

The second (shaping related) feature varied was the application of a $7^{\circ}$ flare on the structure connecting the third frigate variants main hull to its two side hulls, highlighted (in red) in Figure 6.15.

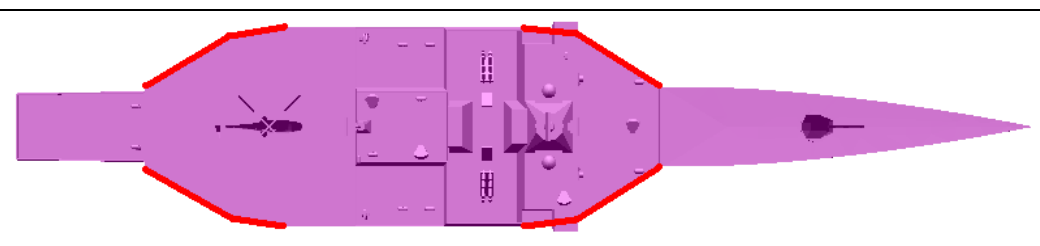

Figure 6.15: Cross-connecting Structure in Trimaran Frigate Variant

This is illustrated in Figure 6.16, where the before configuration is shown in clear light blue and the after, in opaque purple. For each of the four locations highlighted above, there are two sections where flare was applied, one on the side hull itself and the other on the box structure.

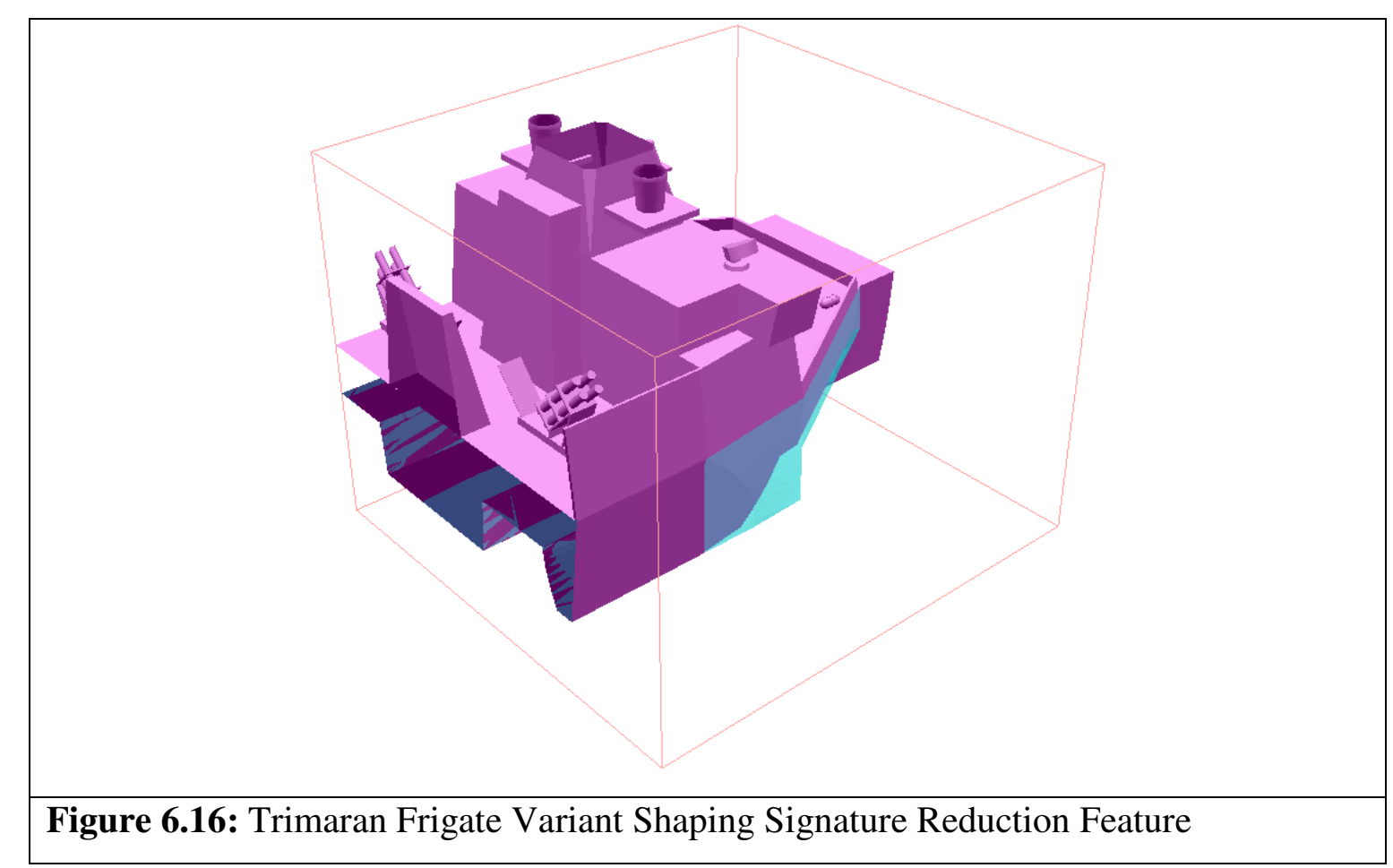

The incorporation of this feature also led to an impressive RCS reduction (Figure 6.17). However, it also caused a slight reduction in the design's fluid GMt due to a portion of the side hull being removed, visible in Figure 6.16. This stability loss could be relatively easily regained by moving the side hulls further outboard. This is an advantage of the trimaran configuration due to powering and stability considerations 
being decoupled (Andrews and Zhang 1995; Andrews 2004). The result of the above feature is illustrated in Figure 6.17, where the eight additional RCS peaks arising from the two sections of each of the four cross-connecting structures before the flare was applied are clearly visible.

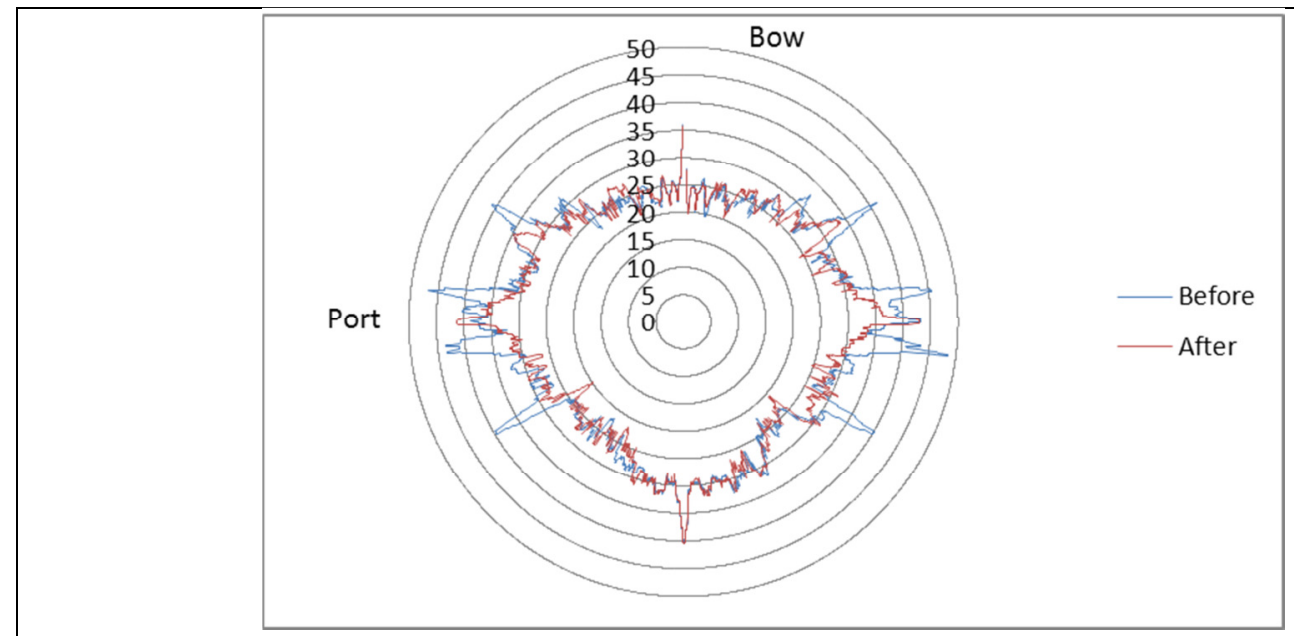

Figure 6.17: Before and After Azimuth Plots of RCS (dBsm) Showing Peak Returns for the Trimaran Frigate Variant

The effect of these alterations on the probability that the attacking missile avoids the decoys launched and locks on the targeted ship $(\mathrm{P}(1)$, calculated through Equations 3 and 4) was to reduce it from $66.7 \%$ and $100 \%$ for Frigate Variants 1 and 3 respectively, to the current $25.3 \%$ and $23.2 \%$ (Table 6.2); i.e. an improvement of approximately $60 \%$ and $75 \%$ respectively, as illustrated in Figure 6.18.

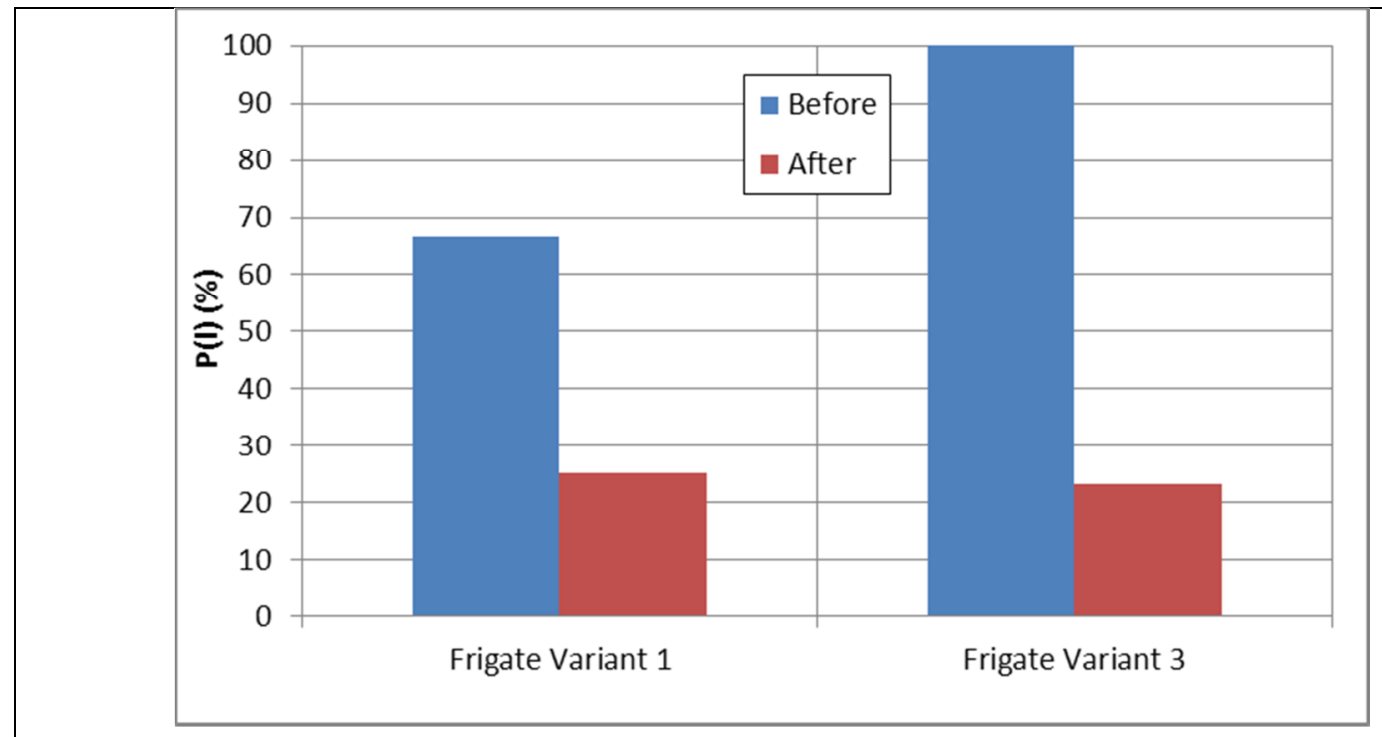

Figure 6.18: Before and After P(l) Values for Frigate Variants 1 and 3 


\subsubsection{Defensive Missile Targeting Pattern}

In Section 6.1 it was mentioned that while applying the CSEE method to the ship design studies, each pair of defensive missiles launched by the targeted ship could only home in on the same attacking ASM, resulting in the data given in Table 6.1, Table 6.3 and Table 6.5. A further sensitivity test was carried out where the above assumption was dropped, therefore, allowing each defensive missile to independently target an attacking missile. In the cases of the three frigate variant and Corvette designs where four defensive missiles were launched, it was assumed that each defensive missile targeted one of the four attacking missiles; therefore, resulting in equal $\mathrm{P}(\mathrm{k})$ values for all attacking missiles, shown in Table 6.7.

Table 6.7: Probabilities of Kill of Attacking ASMs for the Three Frigate Variants and Corvette (Sensitivity Test)

\begin{tabular}{|l|r|r|r|}
\hline & $\mathbf{P}(\mathbf{k})$ Defensive Missile & P(k) Jammer & P(k) Overall \\
\hline Attacking ASMs & 0.730 & 0.250 & 0.798 \\
\hline
\end{tabular}

For the Destroyer, which launched eleven Aster-15 missiles, it was assumed that the first three attacking missiles were targeted by three defensive missiles and the last attacking missile by two defensive SAMs, Table 6.8 .

Table 6.8: Probabilities of Kill of Attacking ASMs for the Destroyer (Sensitivity Test)

\begin{tabular}{|l|r|r|r|r|}
\hline & $\begin{array}{c}\text { P(k) Defensive } \\
\text { Missile }\end{array}$ & P(k) CIWS & \multicolumn{1}{c|}{$\begin{array}{c}\text { P(k) } \\
\text { Jammer }\end{array}$} & P(k) Overall \\
\hline Attacking ASM 1 & 0.980 & 0.780 & 0.250 & 0.997 \\
\hline Attacking ASM 2 & 0.980 & 0 & 0.250 & 0.985 \\
\hline Attacking ASM 3 & 0.980 & 0 & 0.250 & 0.985 \\
\hline Attacking ASM 4 & 0.927 & 0 & 0.250 & 0.945 \\
\hline
\end{tabular}

Finally, in the scenario of the AOR design variants being attacked by four ASMs, the three SeaRAM missiles that were launched each targeted one of the attacking missiles, therefore, the last attacking missile was only countered by the jammer, Table 6.9. 
Table 6.9: Probabilities of Kill of Attacking ASMs for the Two AOR Variants (Sensitivity Test)

\begin{tabular}{|l|r|r|r|}
\hline & $\mathbf{P}(\mathbf{k})$ Defensive Missile & $\mathbf{P}(\mathbf{k})$ Jammer & $\mathbf{P}(\mathbf{k})$ Overall \\
\hline Attacking ASM 1, 2 and 3 & 0.720 & 0.250 & 0.790 \\
\hline Attacking ASM 4 & 0 & 0.250 & 0.250 \\
\hline
\end{tabular}

The effect of the above alterations was to decrease the probability of the ship being hit by at least one ASM, $\mathrm{P}(\mathrm{h})$, in all cases examined. More specifically, $\mathrm{P}(\mathrm{h})$ for the frigate variants and Corvette design was reduced to $59.5 \%$, for the Destroyer design to $8.5 \%$ and for the AOR design variants to $87.7 \%$. The magnitude of the enhancement in performance is depicted in Figure 6.19.

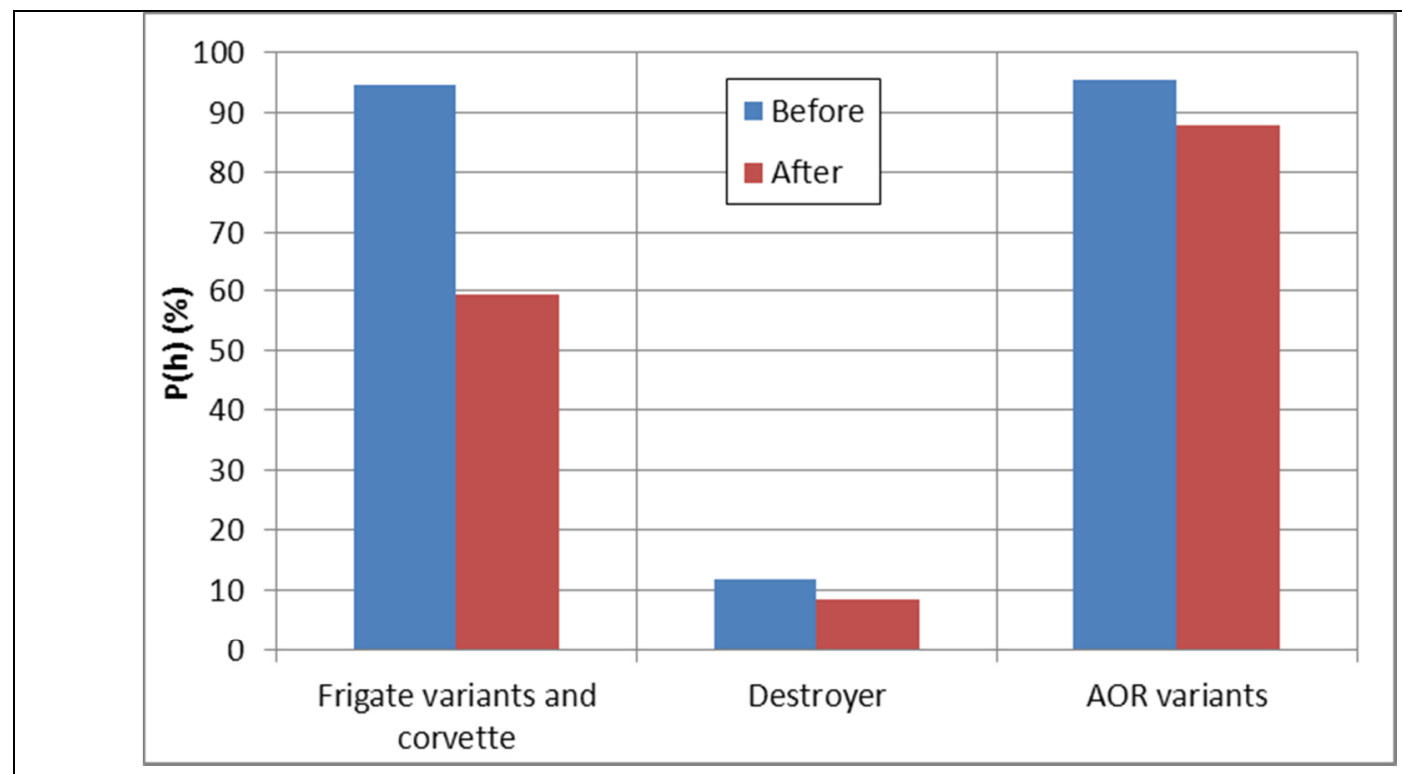

Figure 6.19: Before and After P(h) Values for all Ship Designs

The largest effect in the above sensitivity study was observed in the frigate variants and Corvette design, where the $\mathrm{P}(\mathrm{h})$ decreased to $59.9 \%$, an improvement of almost $40 \%$. The performance of the Destroyer improved by approximately $30 \%$, but for the AOR variants, the above assumption did not have a significant outcome.

\subsubsection{Defensive Missile Type}

Table 5.4 conveys that the PAAMS ADMS of the Destroyer design is able to contain a combination of both Aster-15 and Aster-30 missiles. However, while applying the CSEE method to the Destroyer (Section 6.1.2) it was assumed that the ship only 
defended itself with Aster-15 missiles. It was, therefore, decided to examine the effects of changing the missiles to the more capable Aster-30, against the same threat scenario (four sea-skimming ASMs targeting the ship). Performance data for the Aster-30 missile is identical to that for the Aster- 15 shown in Table A35, with the exception of an increase in maximum range against sea-skimming ASMs to $30,000 \mathrm{~m}$, minimum range to $3,000 \mathrm{~m}$ and velocity to $1,530 \mathrm{~m} / \mathrm{s}$. The threat, as well as the Phalanx CIWS characteristics, are consistent with the initial scenario and are summarised in Table A35. From this data, it is evident that the Destroyers MFR horizon (Table A34) is lesser than the Aster-30s maximum range against sea-skimming ASMs. It was decided to apply the CSEE method taking both, the Destroyer's radar horizon and the SAMs maximum range, as the limiting factors. The sequences of events for both cases are listed in Table A40 and Table A41 of Appendix 10.4 and the combat system reaction timelines are illustrated in Figure 6.20 and Figure 6.21.

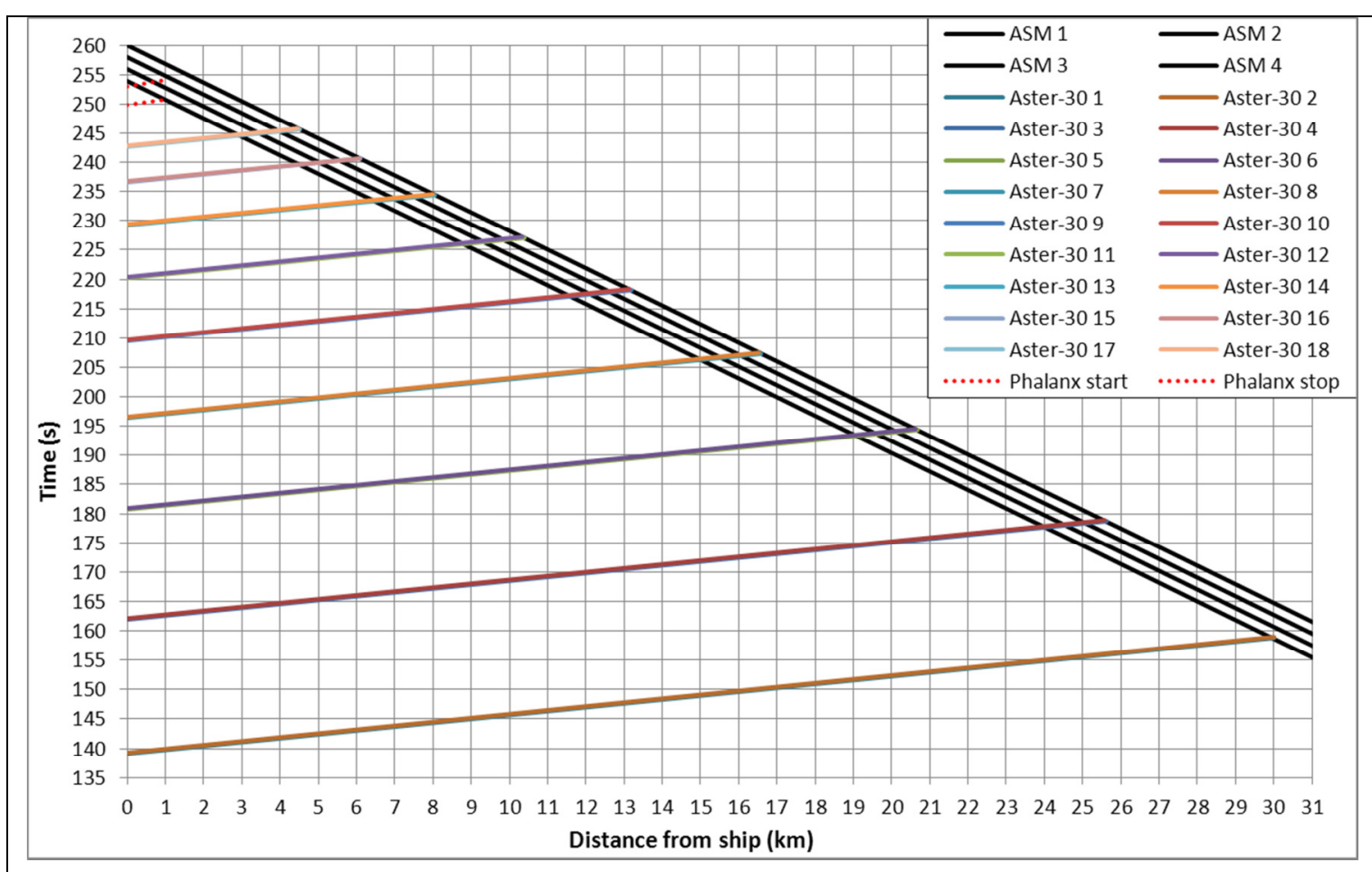

Figure 6.20: Combat System Reaction Timeline for the Destroyer (Aster-30, Maximum Range limited) 


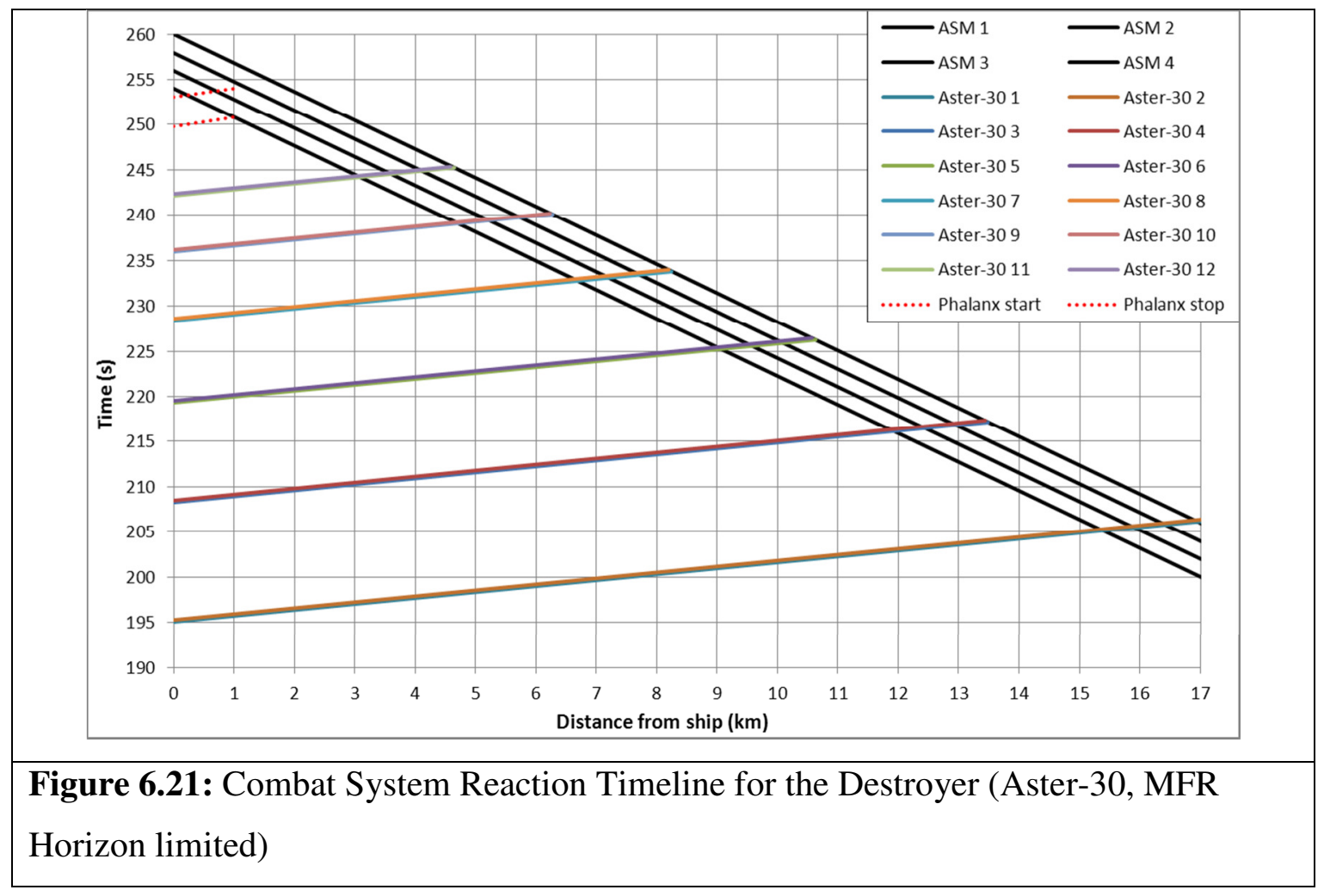

It is observed that if Aster-30 missiles were used rather than Aster-15s, the attacked ship would be able to launch one additional defending missile (i.e. twelve in total); if the limiting factor was the maximum range of the AAW system against seaskinning threats, this figure would increase to eighteen missiles. By assuming the same defensive missile targeting pattern and defensive missile, CIWS and jammer $\mathrm{P}(\mathrm{k})$ as before (Section 6.1.2), the probability of the ship being hit by at least one ASM, P(h), is decreased to $11.1 \%$ and $1.2 \%$ respectively. The comparative magnitude of this improvement is shown in Figure 6.22.

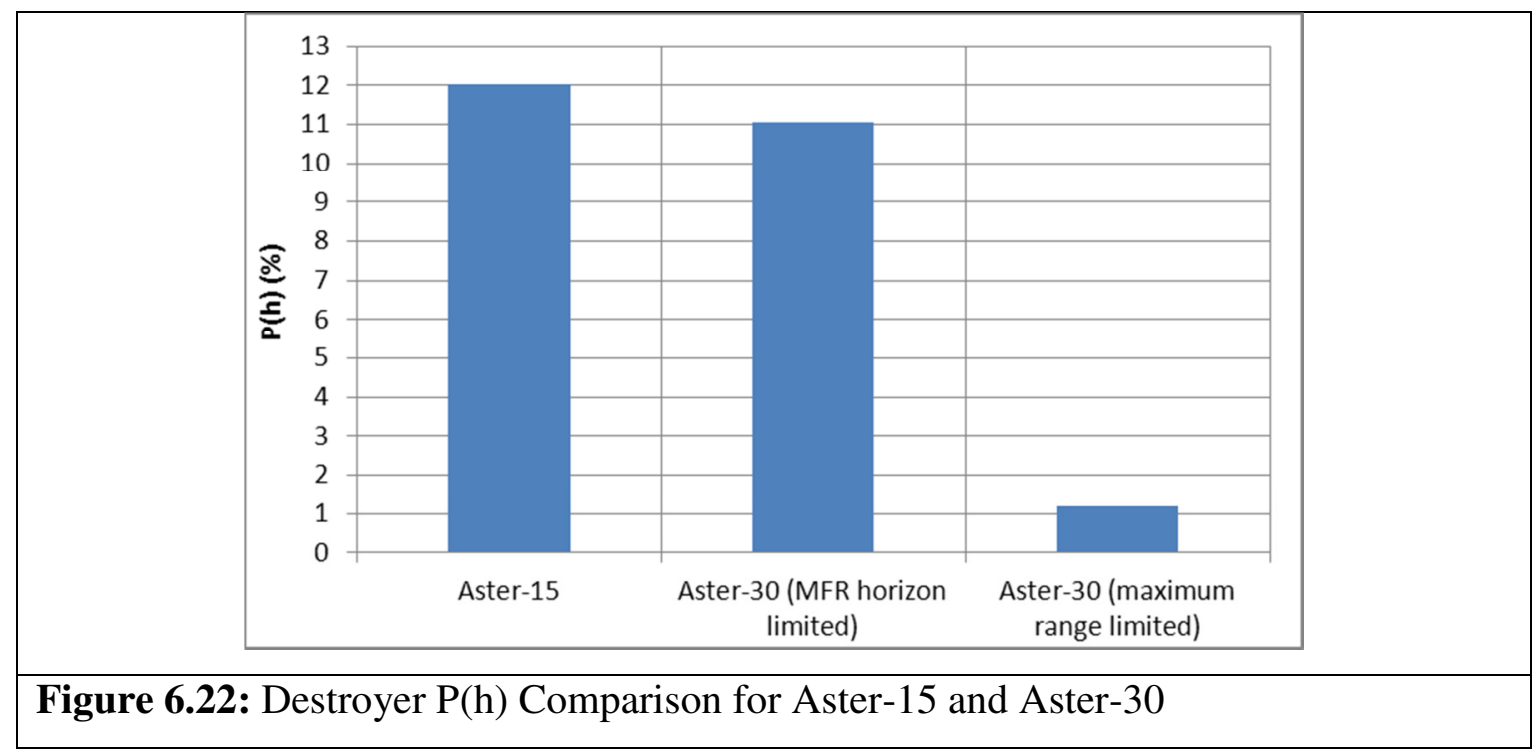


Although theoretically the use of Aster-30 missiles could vastly improve performance, by approximately 90\%, practically, given the limiting, against seaskimming targets, radar horizon, the performance of both missile types is virtually constant.

\subsubsection{Lengthwise Probability Hit Distribution}

The final susceptibility related sensitivity study was to assume a linear lengthwise probability hit distribution on the frigate design variants (rather than the normal distribution shown in Figure 6.4). This distribution is shown in Figure 6.23.

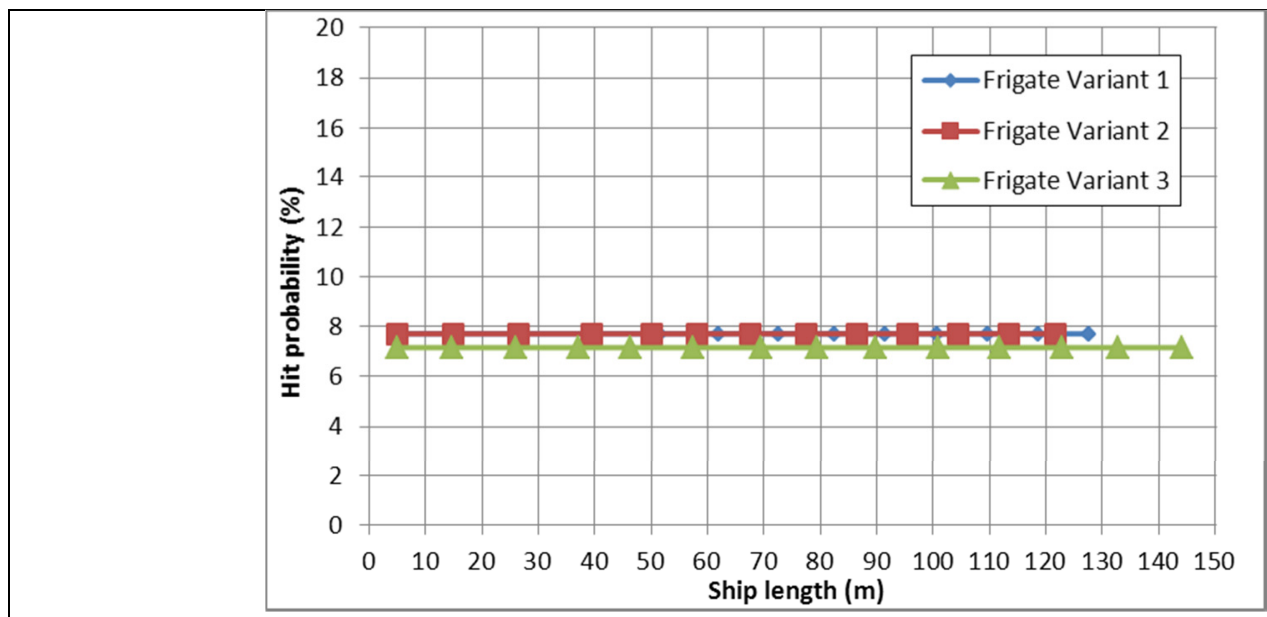

Figure 6.23: Linear Lengthwise Probability Hit Distributions for the Frigate Design Studies

As before, the data points shown above represent the hit probability of the centroid of each WT section of each ship design study and aggregate to $100 \%$ for each ship. The effects of changing the lengthwise probability hit distribution from normal to linear is further investigated in Sections 6.4 and 6.6.

\subsection{Vulnerability Assessment Results}

The procedure described in Section 4.2 was followed in order to obtain vulnerability results for all ship design studies. QinetiQ's SURVIVE Lite (Pugh 2006; Turner et al 2006) vulnerability assessment software for concept stage design was used for all comparative survivability studies, therefore, the first step was to develop 
SURVIVE Lite models for all ship design studies. Certain features were kept constant in all SURVIVE Lite models produced for this research:-

- The hull thickness was set equal to $10 \mathrm{~mm}$;

- $\quad$ The superstructure thickness was set as $4 \mathrm{~mm}$;

- The hull, superstructure, decks, WT bulkhead and passageway material was set as Mild Steel (Grade A) 40A;

- The frame spacing was equalled to $0.7 \mathrm{~m}$;

- Deck thickness was assumed to be $10 \mathrm{~mm}$ and all decks were fire insulated;

- WT bulkhead thickness was set as $6 \mathrm{~mm}$ and all bulkheads were fire insulated;

- Longitudinal partition (i.e. passageway) thickness was assumed 3mm without fire insulation;

- Main zone boundaries were also main fire boundaries;

With the exception of manually establishing main zone boundaries as main fire boundaries, all above settings are the default SURVIVE Lite v2.3.3 settings. It should be noted that in the two AOR variants, in addition to the main zone boundaries (i.e. WT bulkhead JK in Figure 5.59 and WT bulkhead MN in Figure 5.60 respectively), WT bulkheads EF and MN in AOR Variant 1 and WT bulkhead KL in AOR Variant 2 (i.e. the WT bulkheads separating the main cargo spaces from the remaining spaces (see Figure 5.54 and Figure 5.55) were also set as main fire boundaries.

The next step was to cross-model all the required equipment items and compartments from the Paramarine ship design models (GAs of which are included in Appendix 8) to the SURVIVE Lite ship models. The equipment and compartments modelled included all those belonging to the major ship systems to be studied (Table 4.1 and Table 4.2) as well as the recoverability related items listed in Section 4.2. System tree diagrams and system architecture illustrations for all ships are included in Appendix 7. In addition, the SURVIVE Lite equipment category to which each modelled equipment and compartment (belonging to the major ship systems investigated) was set, is given in Table A1, Appendix 6. The SURVIVE Lite equipment category (as well as the repair category) of recoverability related equipment and compartments is listed in Table 4.11.

Before running the models, the following threat characteristics were inputted, consistent in all ship design runs:-

- Threat Type: Missile;

- Charge Weight: 165kg;

- Fragment Pattern: Medium; 
- Attack Direction: Side Grid;

- Vector Direction, Azimuth: $90^{\circ}$ (port) and $270^{\circ}$ (starboard);

- Vector Direction, Elevation: $0^{\circ}$ (sea-skimming).

As mentioned in Section 4.2, hit grids were applied to each WT section of each ship (for both port and starboard sides) separately, as illustrated in Figure 4.4 for Frigate Variant 1. The lengthwise spacing between hit locations was approximately $2 \mathrm{~m}$ and in the $\mathrm{z}$ (depth) direction each deck of each ship included one row of hit locations.

\subsubsection{Frigate Variants (Including Baseline)}

After running the missile hit simulations for the three frigate variant designs, the results listed in Table A42, Table A43 and Table A44 (see Appendix 11.1) were obtained. The vulnerability of each major system given an ASM hit at a WT section was then multiplied by the probability that that WT section was hit. Namely, the vulnerabilities in Appendix 11.1 were multiplied with the corresponding WT section susceptibility given in Figure 6.4, as outlined in Section 4.2. The sum of the above product was taken for each system in order to obtain the total major ship system vulnerabilities for the three frigate designs, shown in Figure 6.24.

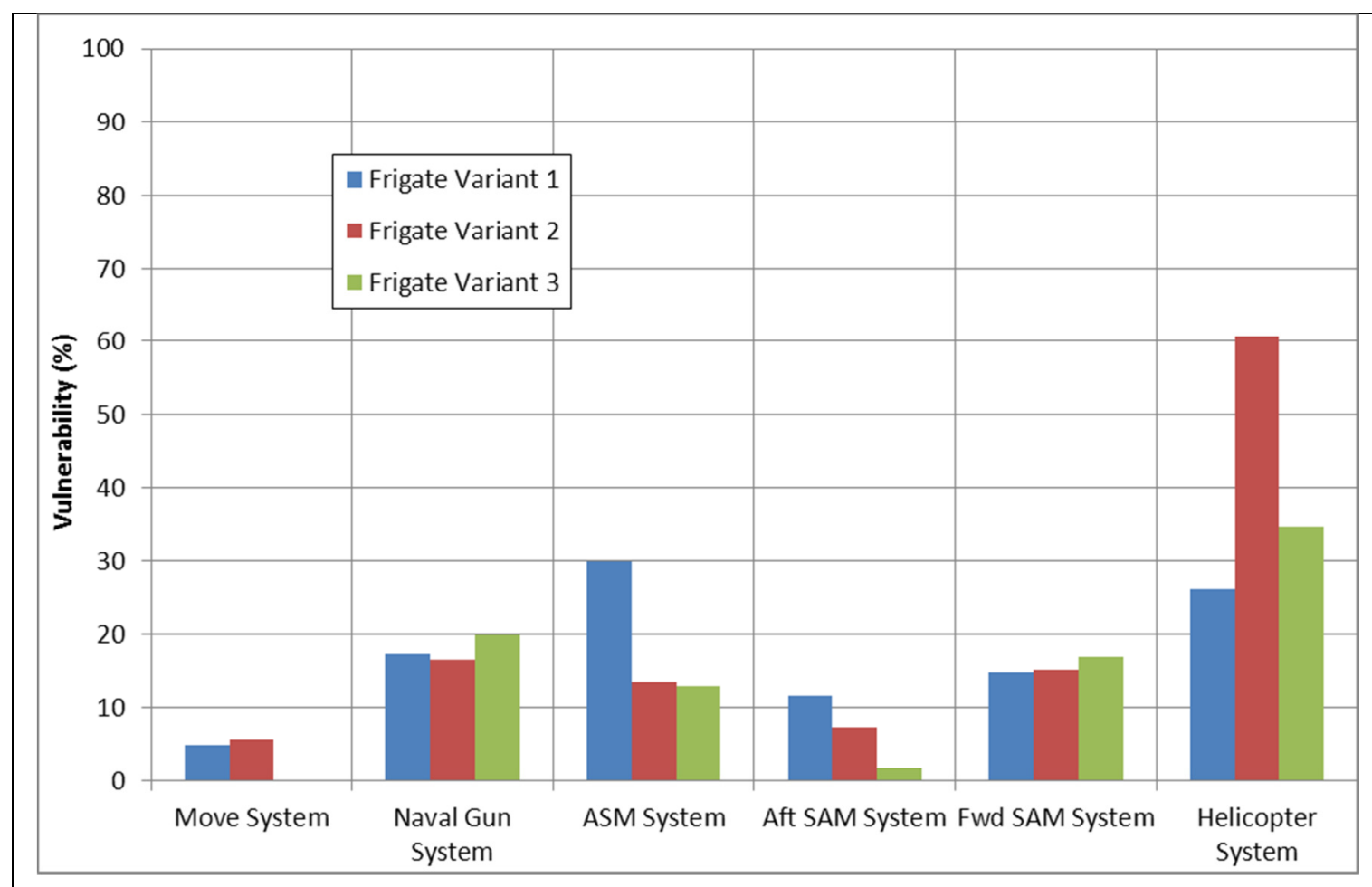

Figure 6.24: Total Major Ship System Vulnerability for the Frigate Design Studies 
The system vulnerabilities presented in Figure 6.24 were normalised with respect to the baseline (Frigate Variant 1) and weighted given the weightings in Table 4.1. The sum of the normalised and weighted system vulnerabilities were taken for each ship design, and again normalised with respect to the baseline (hence Frigate Variant 1 has a vulnerability of 1), outputting the results illustrated in Figure 6.25.

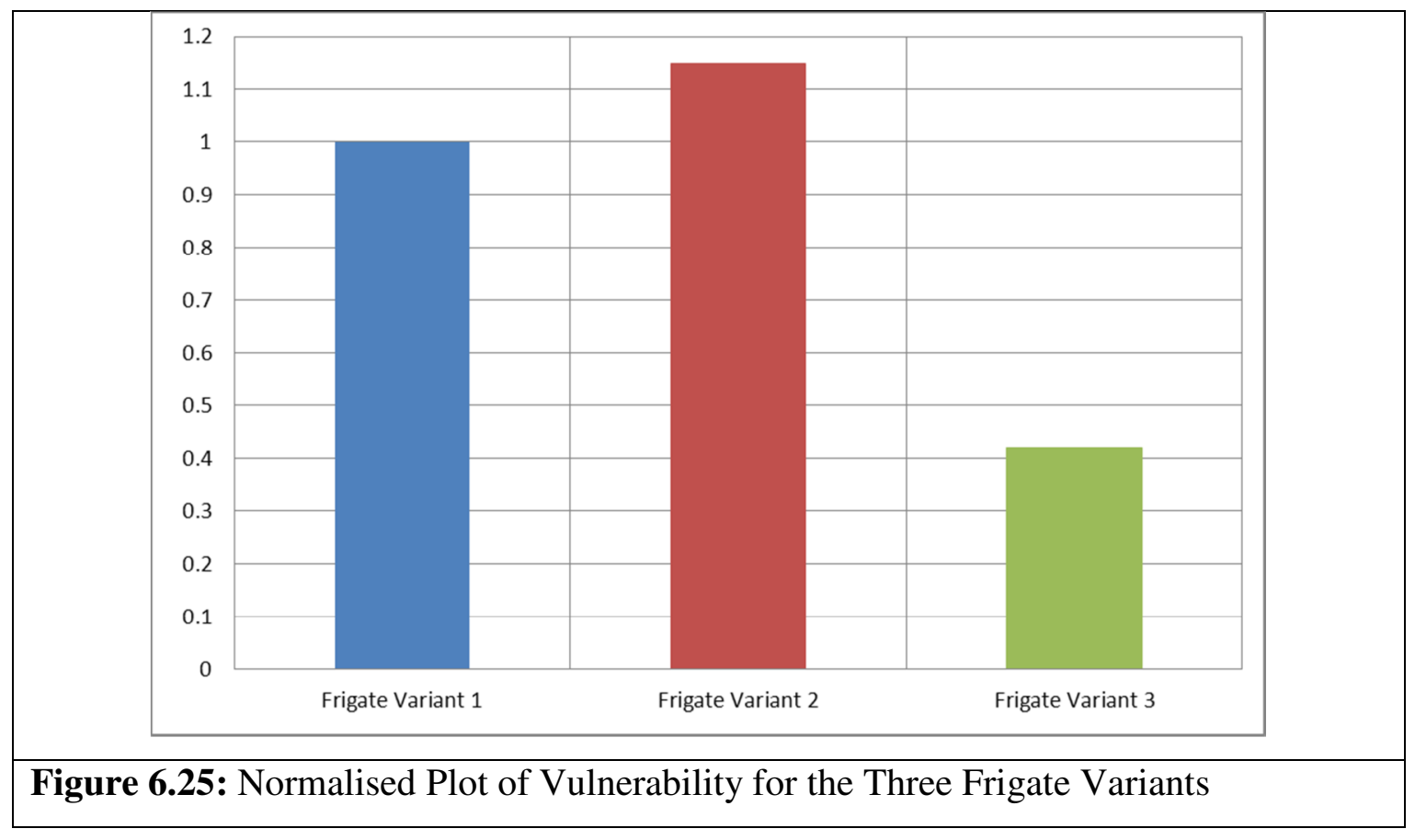

From above it is clear that although the first two variants present similar survivability levels, the trimaran frigate design is more than $50 \%$ less vulnerable than both its counterparts. This is largely due to its invulnerable move system (Figure 6.24), which was given an incomparably larger weighting than the other modelled systems (Table 4.1).

\subsubsection{Corvette, Baseline Frigate and Destroyer}

As with the frigate variant design studies, the Corvette and Destroyer designs were run in SURVIVE Lite, simulating the identical threat scenario. The vulnerability results obtained are presented in Table A45 and Table A46 (see Appendix 11.2). This data was combined with corresponding WT section susceptibility data, given in Figure 6.8, as outlined in Section 4.2. Total major ship system vulnerabilities for the combatant designs were then estimated in a similar manner to the frigate variants. The system vulnerability results, together with the corresponding results of the baseline frigate, are presented in Figure 6.26. 


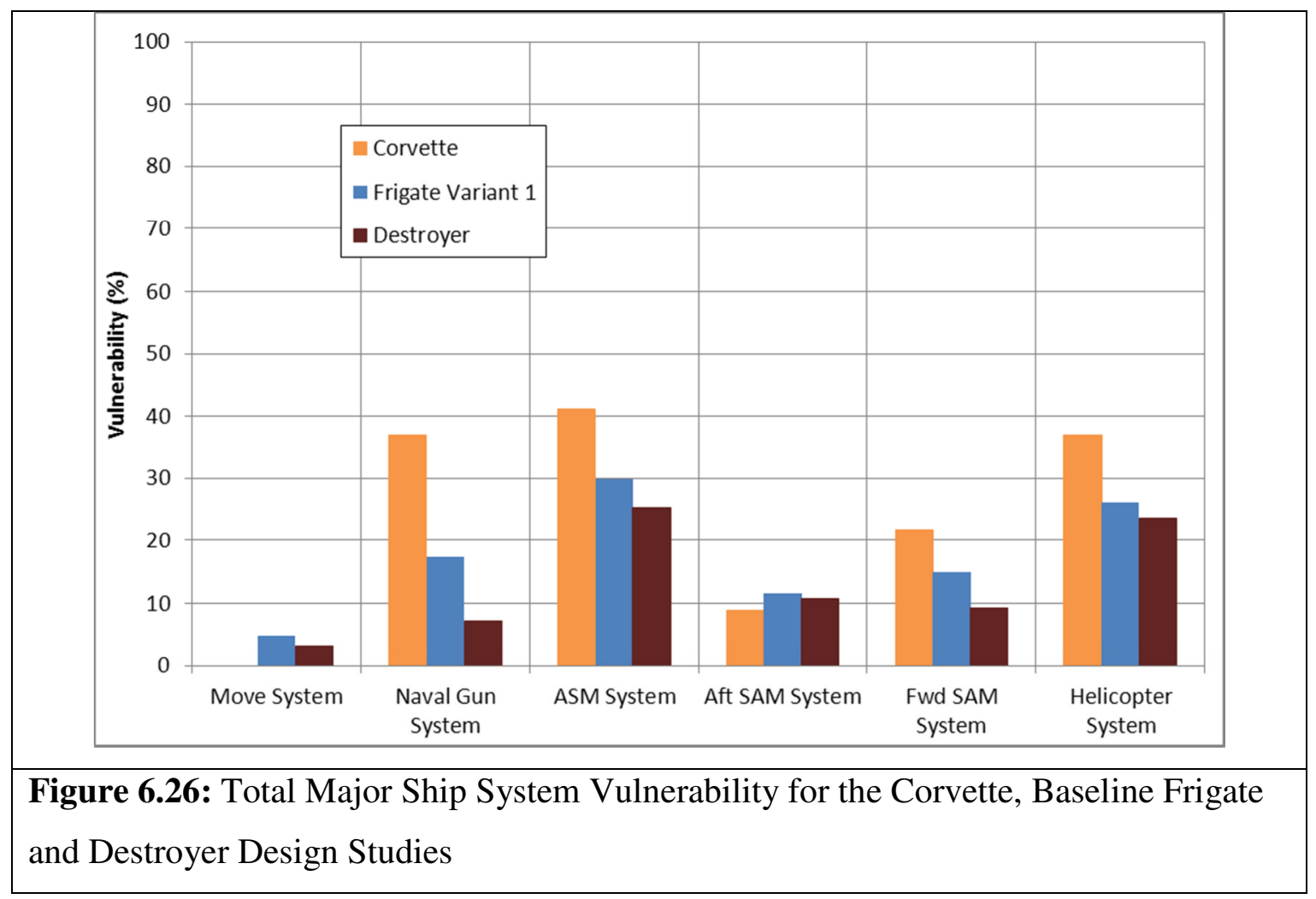

After normalising the above vulnerability results with respect to the baseline frigate and weighting given the weightings in Table 4.1 (following an identical procedure to the vulnerability assessment of the frigate variants), the results illustrated in Figure 6.27 were obtained.

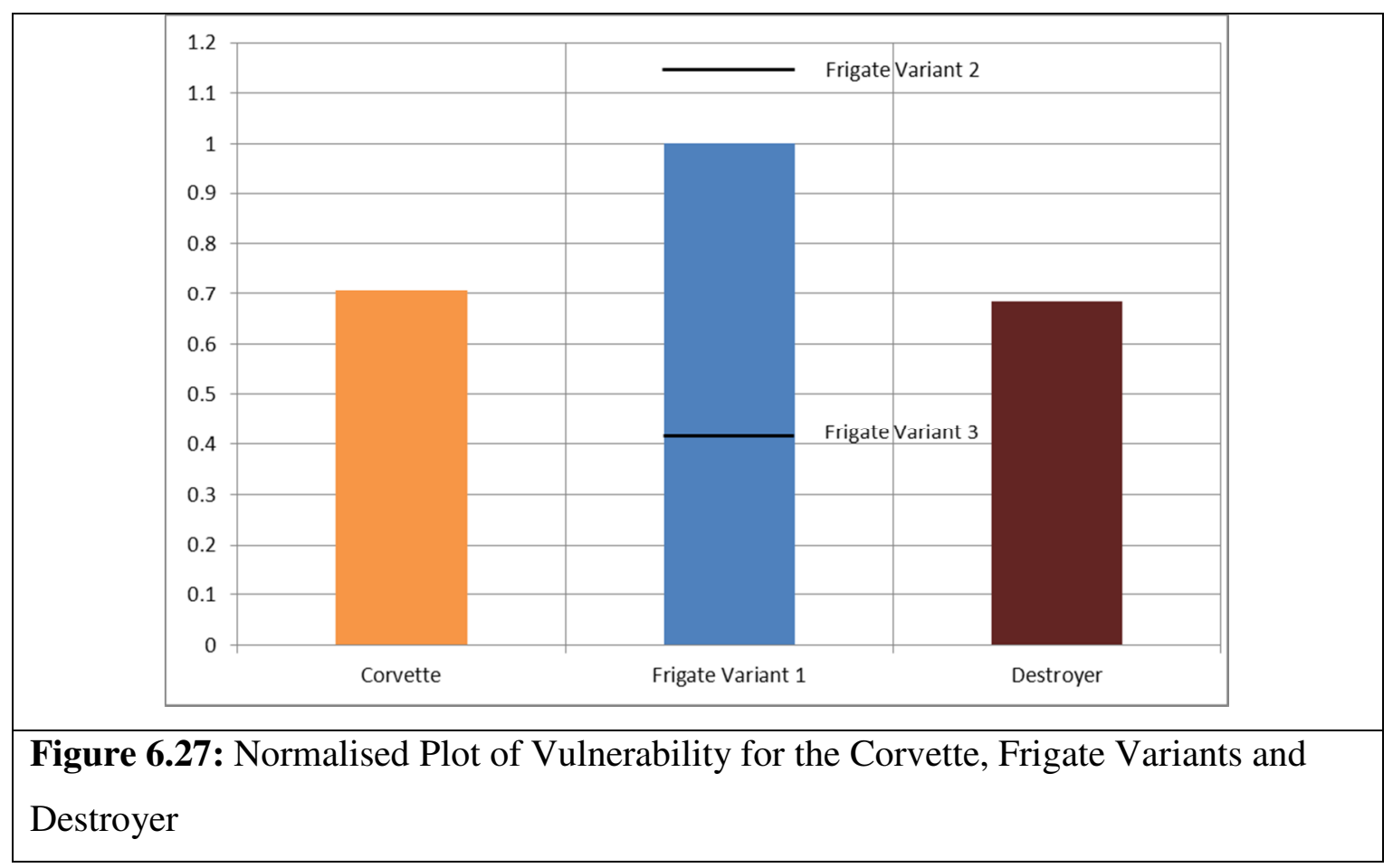


Figure 6.27 shows that the Destroyer is the least vulnerable ship, not unexpectedly since it is the largest of the three ship designs. However, a remarkable observation is that the smaller Corvette design is less vulnerable than the baseline frigate. This is entirely due to its invulnerable move system (Figure 6.26), which was given a much larger weighting than all other major systems (Table 4.1) as is discussed in Section 7.2.2.

\section{$\underline{\text { 6.3.3 AOR Variants }}$}

The final SURVIVE Lite ASM simulation runs were carried out for the two AOR variants. The vulnerability results outputted are presented in Table A47 and Table A48 (see Appendix 11.3). These system vulnerability data given an ASM hit at a WT section were multiplied by the probability of hit of that WT section, Figure 6.12. The sum for each system was taken, as in the previous cases, and the total major ship system vulnerability results shown in Figure 6.28 were obtained.

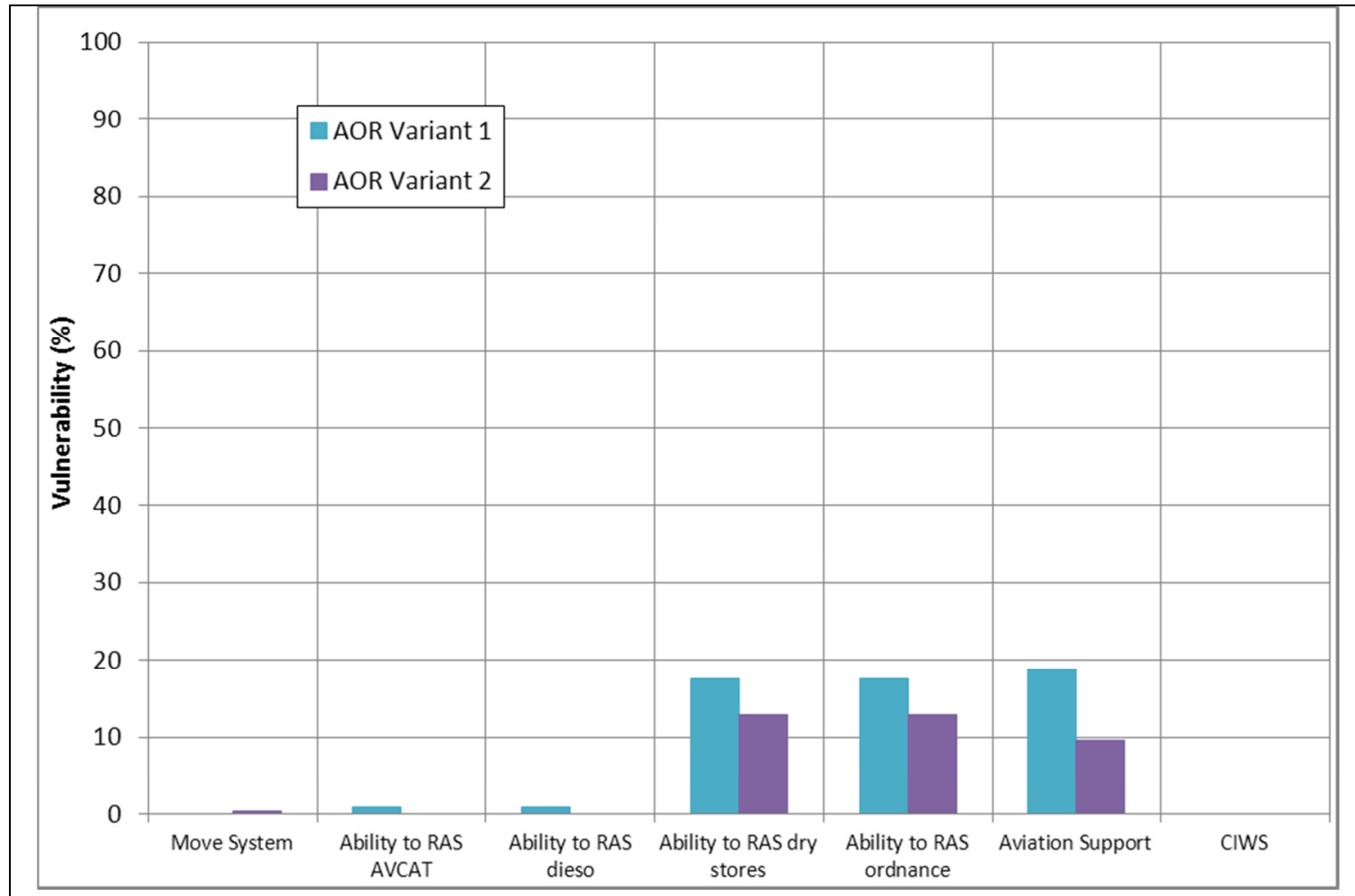

Figure 6.28: Total Major Ship System Vulnerability for the AOR Design Studies

The above system vulnerability results were normalised with respect to the corresponding vulnerabilities of the worst performing design (i.e. the move system vulnerability was normalised with respect to AOR Variant 2, RAS related capabilities 
and aviation support were normalised with respect to AOR Variant1 and the CIWS, which was invulnerable in both designs, was given a value of zero). As with susceptibility assessment, in the case of the AOR design studies normalisations were performed with respect to the worst performing design, rather than a baseline, the reasoning behind which is explained in Section 7.2.4. The normalised vulnerabilities values were then weighted given the weightings in Table 4.2 and summed as before in order to obtain the results illustrated in Figure 6.29. Yet again, the total vulnerability results were normalised with respect to the worst performing design, i.e. AOR Variant 1 , hence the total vulnerability value of that variant is equal to 1 .

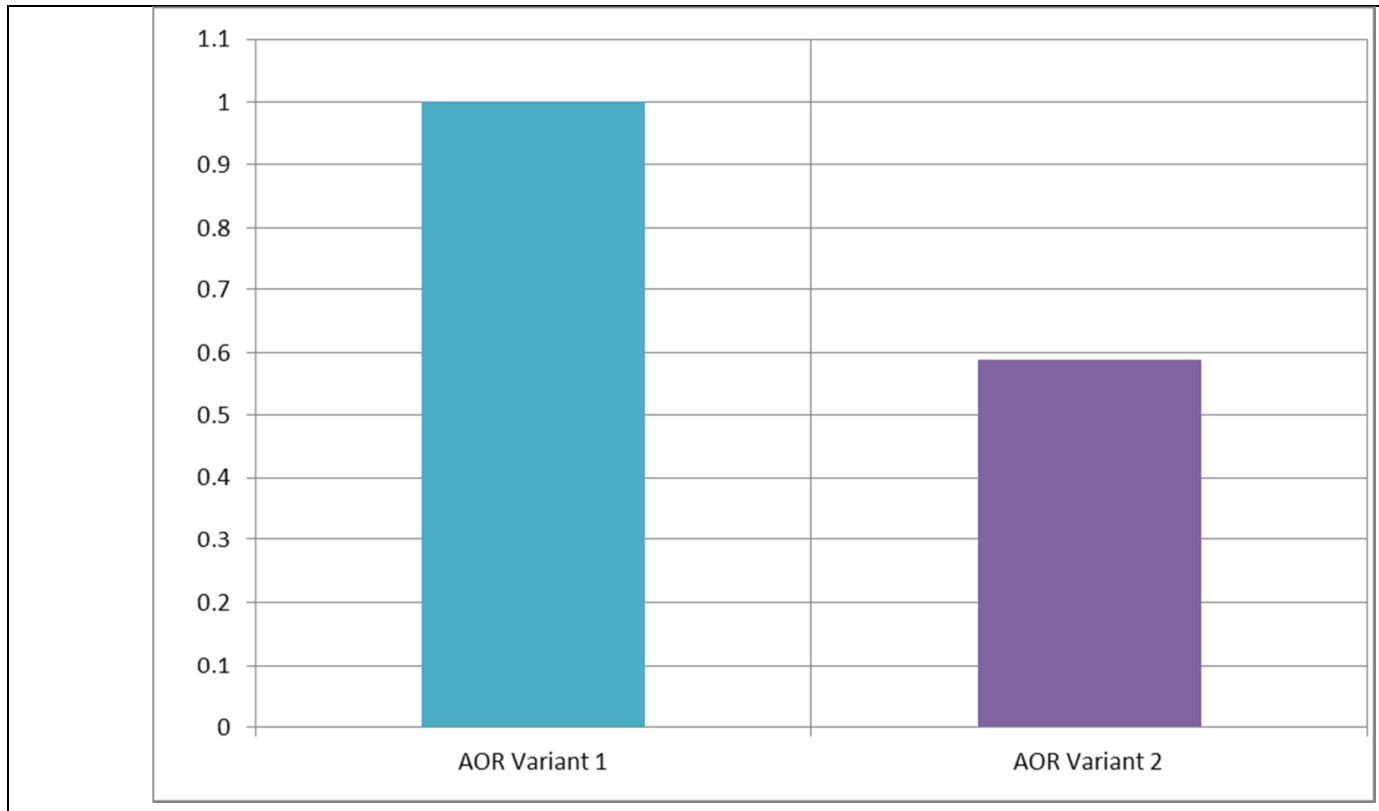

Figure 6.29: Normalised Plot of Vulnerability for the Two AOR Variants

The overall observations from the vulnerability data above are that the vulnerabilities of the systems modelled in the AOR designs (Figure 6.28) are generally considerably lower than the vulnerabilities of the systems modelled in the combatant designs (Figure 6.24 and Figure 6.26), probably as a result of the much greater size of the auxiliary ships (although different systems were modelled, therefore, a direct comparison cannot be made); in addition an improvement of more than $40 \%$ in terms of vulnerability reduction was observed in the second AOR variant, due to enhanced performance in RAS related capabilities and aviation support.

As mentioned in Section 4.2, SURVIVE Lite was also used to investigate the vulnerability (to the same threat) of certain features required for recovery. This data, being more relevant to the recoverability part of the survivability assessment method, is presented in the PM matrices in Section 6.5. 


\section{$\underline{6.4 \text { Vulnerability Assessment Sensitivity Studies }}$}

Three main sensitivity studies were carried out for the vulnerability part of the survivability assessment method:-

- The application of different system weighting schemes was examined;

- The effect on system vulnerability, given a different lengthwise probability hit distribution on the frigate variants was investigated;

- The consequence of varying the angle of ASM attack (rather than merely considering broadside attacks at $0^{\circ}$ elevation) was studied.

\subsubsection{System Weighting Schemes}

In order to examine the sensitivity of the vulnerability assessment method to the weighting scheme applied, a number of $\mathrm{RN}$ officers working at Dstl were interviewed (Mant 2012; Hood 2012; Day 2012; Sutcliffe 2012; Boughton 2012; Koheeallee 2012; O'Brien 2012; Kadinopoulos 2012). They were asked to fill in a form (see Appendix 12) suggesting alternative weighting schemes. In contrast to the original (scenario independent) weighting scheme (Table 4.1) provided by $1^{\text {st }}$ Lt. Fonseca, the interviewed officers were asked to provide a weighting scheme for three different scenarios. These are summarised in Table 6.10. (It should be noted that this sensitivity test was only carried out for the three frigate design variants).

\begin{tabular}{|l|l|}
\hline \multicolumn{2}{|l|}{ Table 6.10: Scenarios for System Weighting Schemes of the Frigate Design Studies } \\
\hline Scenario 1 & $\begin{array}{l}\text { Air and submarine } \\
\text { Blue water }\end{array}$ \\
\hline $\begin{array}{l}\text { Threats present } \\
\text { Location }\end{array}$ & \multicolumn{2}{|l|}{} \\
\hline Scenario 2 & Lit, shore based missiles and gun batteries \\
\hline $\begin{array}{l}\text { Threats present } \\
\text { Location }\end{array}$ & $\begin{array}{l}\text { Air and surface (missile firing) } \\
\text { Scenario 3 }\end{array}$ \\
\hline $\begin{array}{l}\text { Threats present } \\
\text { Location }\end{array}$
\end{tabular}


As with the original weighting scheme, the naval officers were asked to weigh each ship system between $0-10$, ten being the most important. In addition, the interviewed officers were not exposed to previous weighting schemes so that their responses remain unaffected. The weighting schemes suggested by the interviewed officers are shown in Table 6.11. 


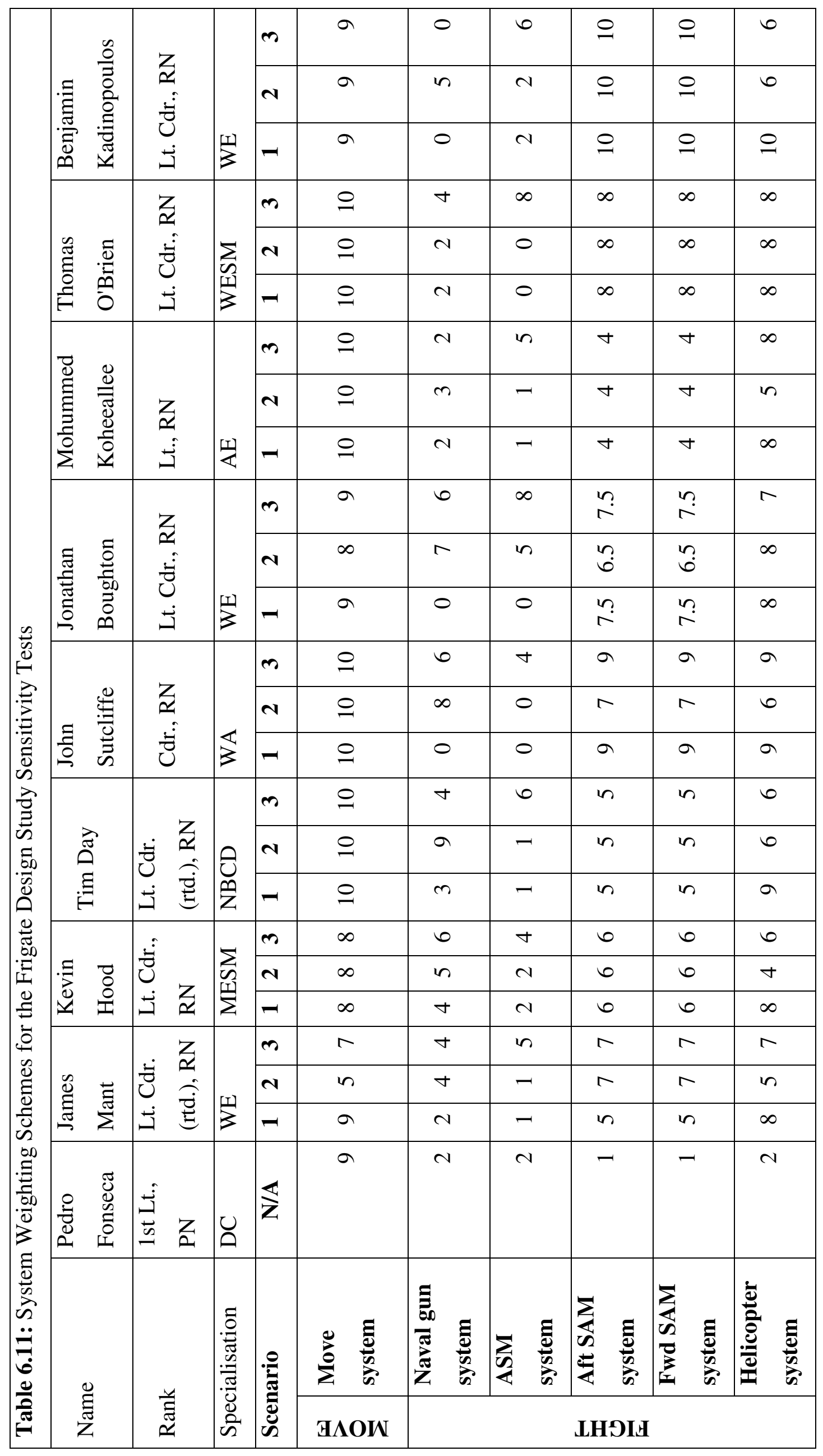


It is important to mention that Lt. Cdr. Boughton proposed that the duplicated aft and forward SAM systems should have different weightings given that they provide the same function (Boughton 2012). However, since the decision of which of the two systems would be given higher priority depends on purely operational factors, the two weightings given by that officer were averaged; thus the decimal fraction form of the relevant weightings. This would have the same effect as interchanging the higher priority SAM system, obtaining the vulnerability results for both cases and averaging. Lt. Koheeallees (2012) remark that: "These weightings are based on self-protection and survival of own ship. If the role of own ship is also to provide force protection (over naval assets or land), weightings could be different. In fight section, consider adding external communications and surveillance systems", is noteworthy. The above weighting schemes were plotted in a line graph (Figure 6.30) in order to compare the different weighting scheme philosophies of the interviewed officers.
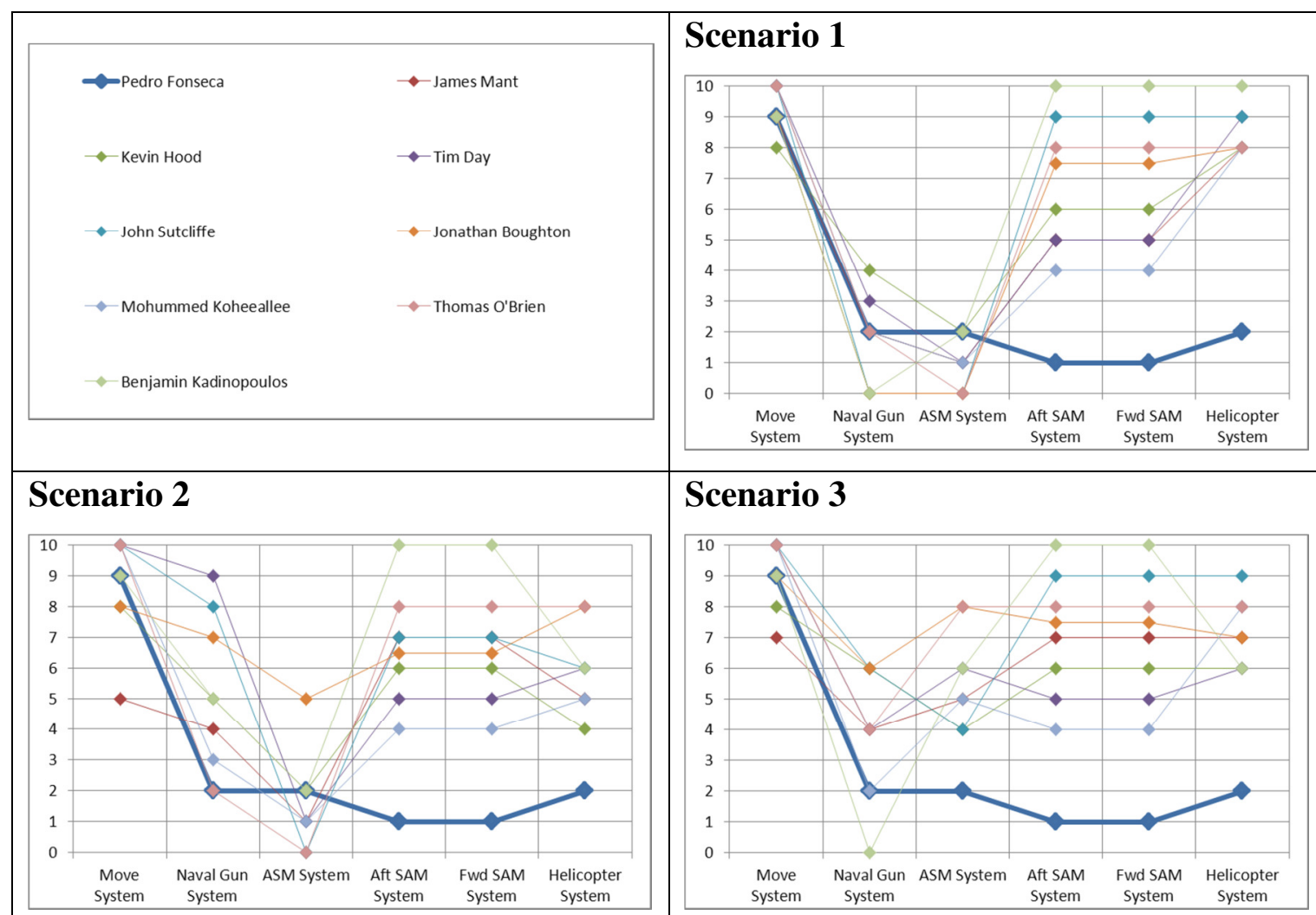

Figure 6.30: System Weighting Scheme Philosophies for the Sensitivity Tests on Frigate Vulnerability

Broad patterns can be observed in Figure 6.30, something unsurprising since all officers (with the exception of $1^{\text {st }} \mathrm{Lt}$. Fonseca) served in the same navy and, therefore, underwent similar training. However, each officer reflects on his own experiences and 
knowledge, accounting for the differences in the schemes above. In addition, the different weighting scheme philosophies between the RN officers and $1^{\text {st }}$ Lt. Fonseca are evident since the latter officer provided a scenario independent scheme. The weighting schemes in Table 6.11 were applied to the data provided in Figure 6.24 in an identical manner as described in Sections 4.2 and 6.3; the output results are presented in Figure 6.31, Figure 6.32 and Figure 6.33 for the three scenarios respectively.
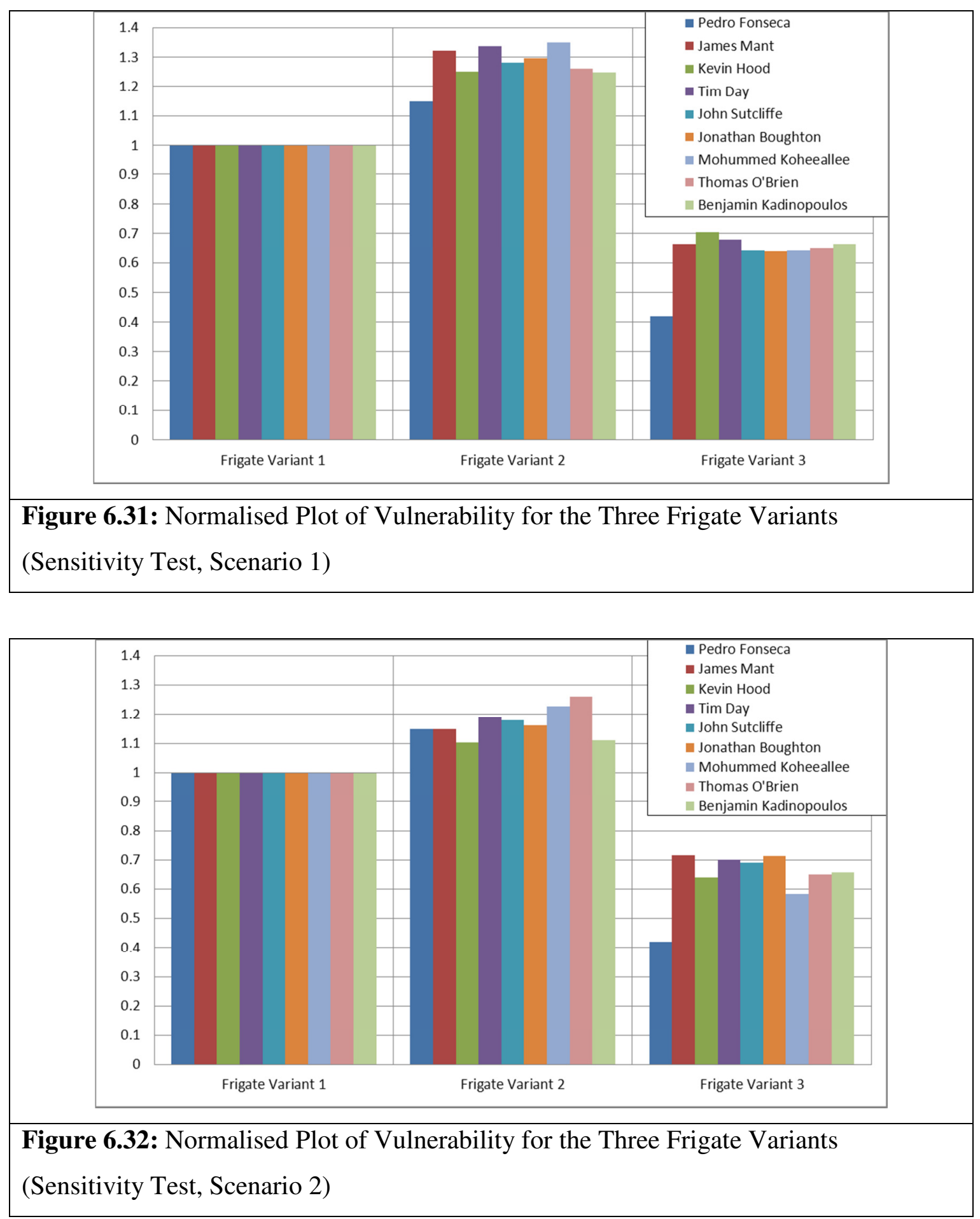


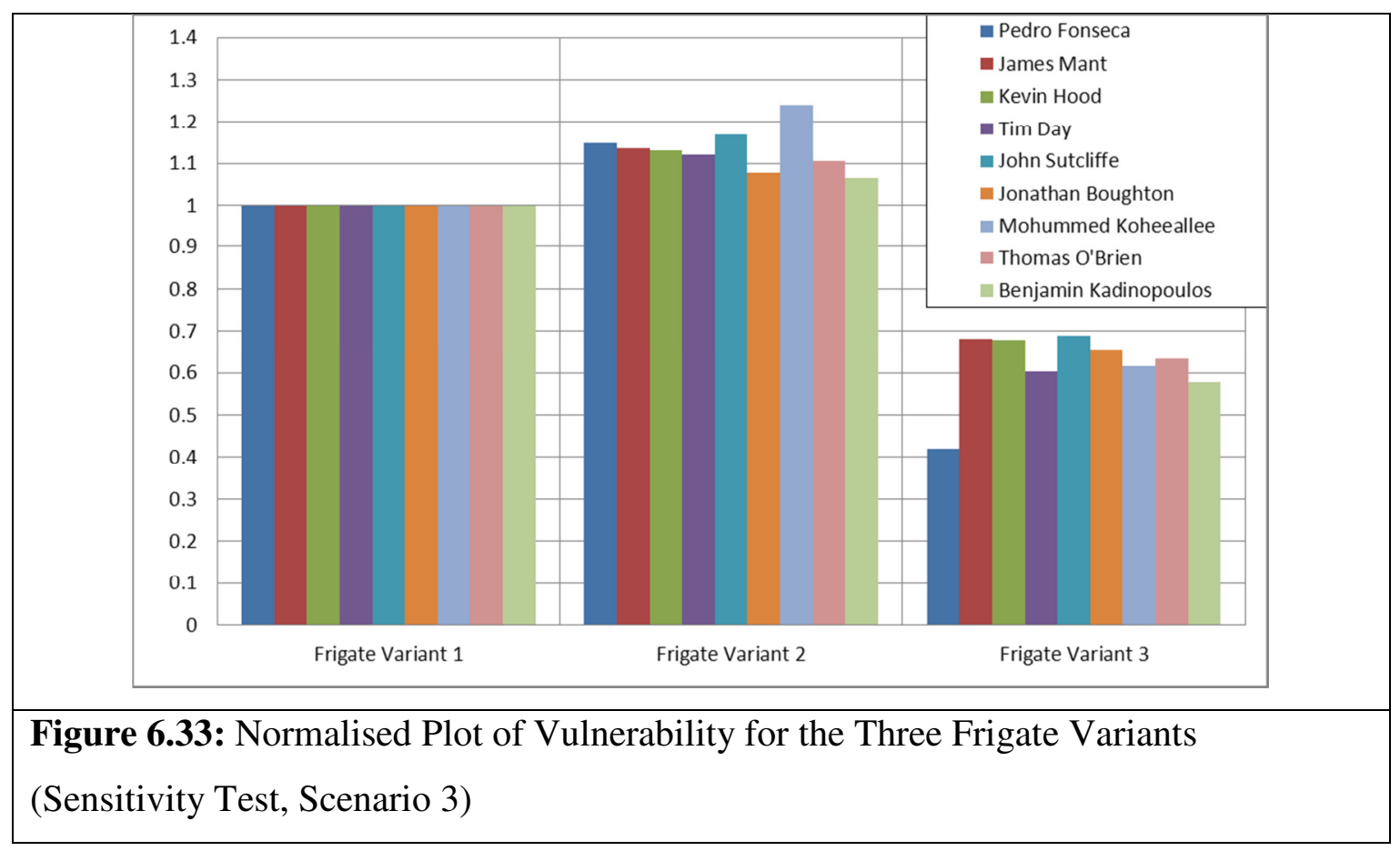

The main observation from the three illustrations above is that vulnerability is to a significantly greater extent dependant on (i.e. sensitive to) the design itself, rather than the scenario or the weighting scheme applied. Another point of interest is the substantial difference in the total vulnerability results for the trimaran frigate design using $1^{\text {st }} \mathrm{Lt}$. Fonseca's weighting scheme and those of all RN officers. This is largely caused by the relative weighting given to the move system. A final remarkable result is that, although Frigate Variant 2 is the worst performing in all cases, the margin by which it fails in the first scenario is increased compared to the other two scenarios, principally due to the location of its aviation facilities.

\subsubsection{Lengthwise Probability Hit Distribution}

The next sensitivity study was related to the lengthwise probability hit distribution. As described in Section 6.2.4, a linear lengthwise probability hit distribution (rather than the normal distribution shown in Figure 6.4 which was used in all above vulnerability data processing) was assumed. This linear lengthwise probability hit distribution (Figure 6.23) was applied to the data given in Table A42, Table A43 and Table A44 in the manner outlined in Sections 4.2 and 6.3. The vulnerability of each major system given an ASM hit at a WT section was multiplied by the probability that the given WT section was hit and by taking the sum of these results, the total vulnerability for each system was obtained. This sensitivity study was also only applied 
to the three frigate design variants. The effect of changing the lengthwise hit probability pattern from a normal to a linear distribution is shown in Figure 6.34, Figure 6.35 and Figure 6.36.

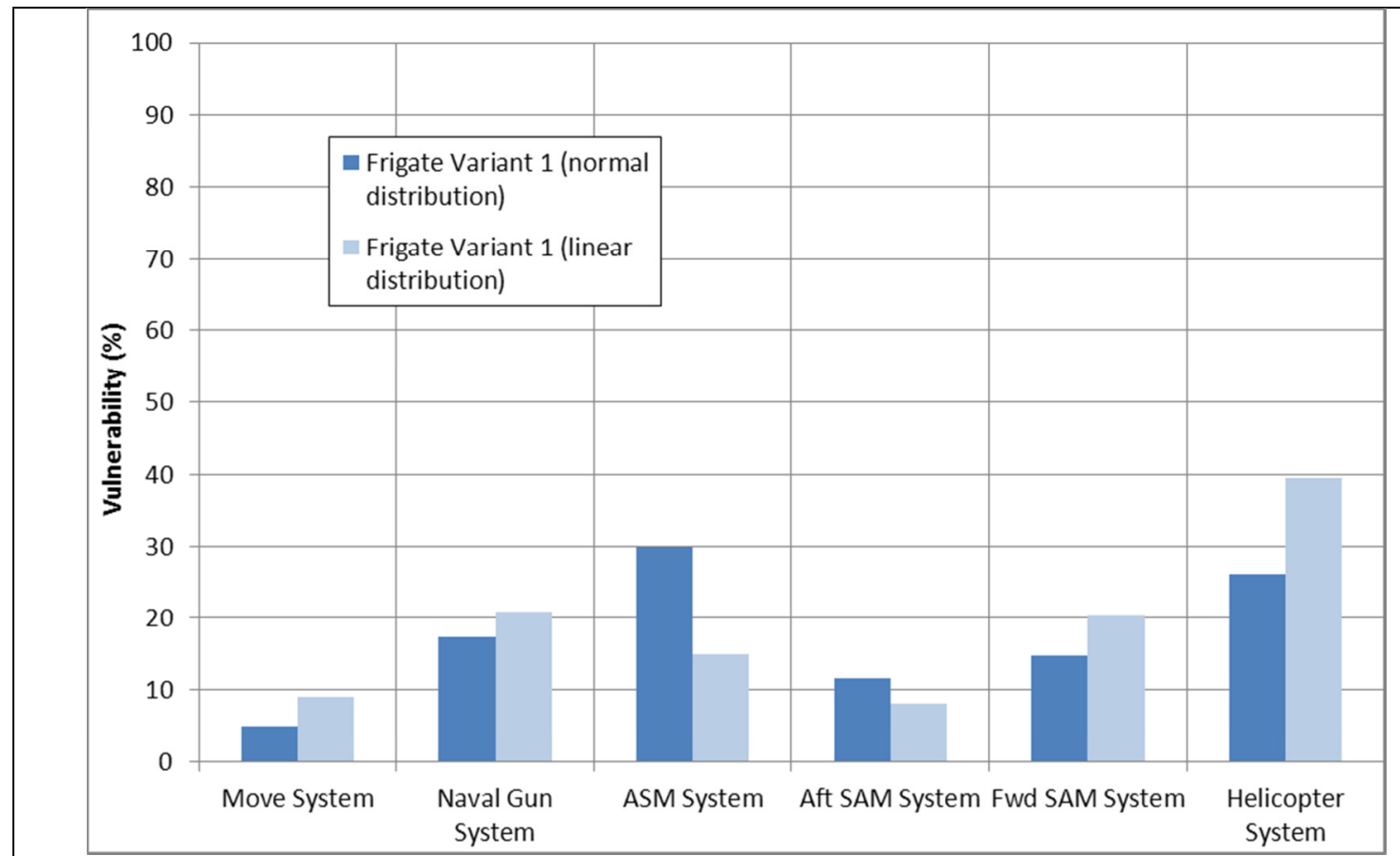

Figure 6.34: Total Major Ship System Vulnerability for Frigate Variant 1 (Varying Lengthwise Hit Distribution)

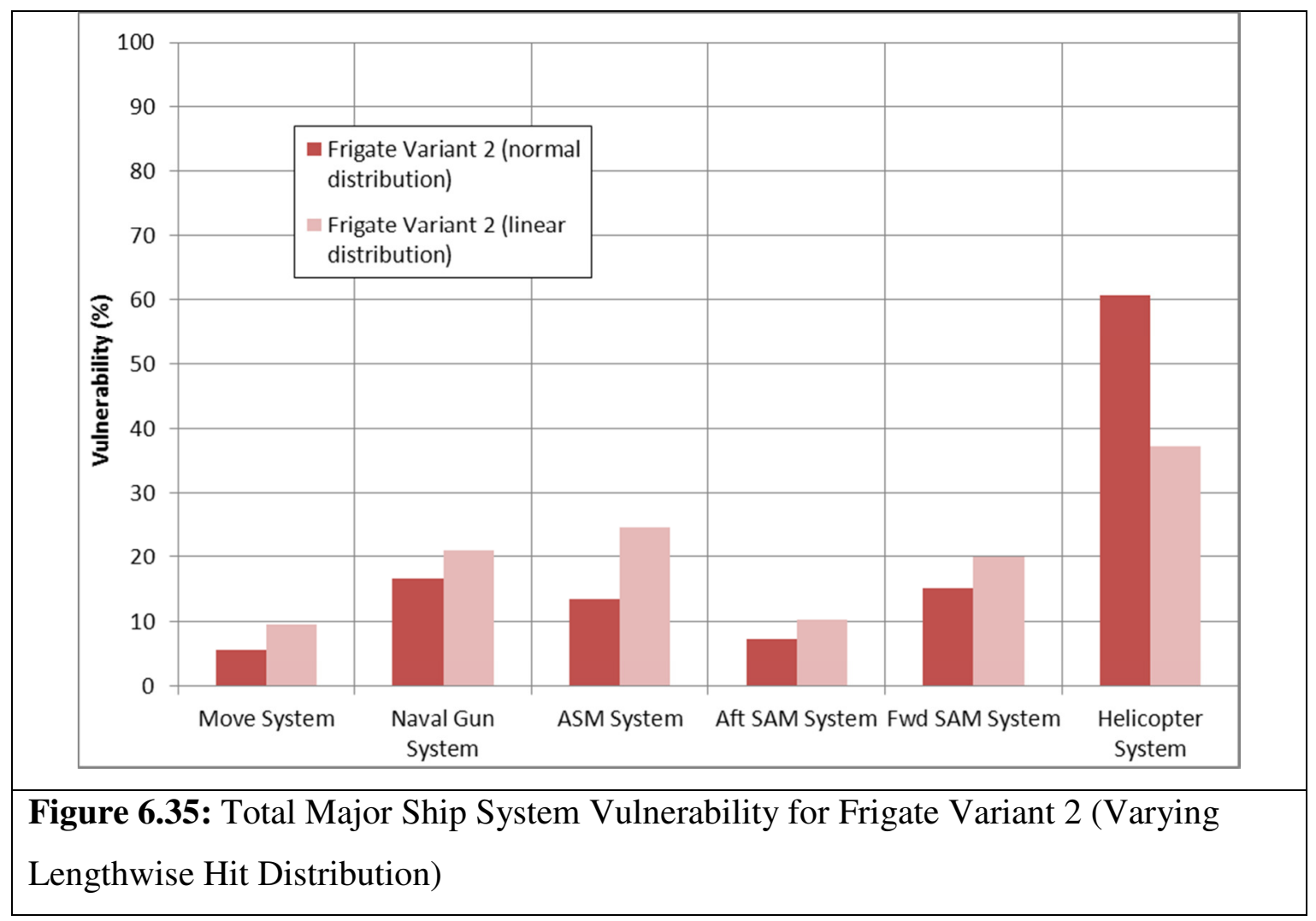




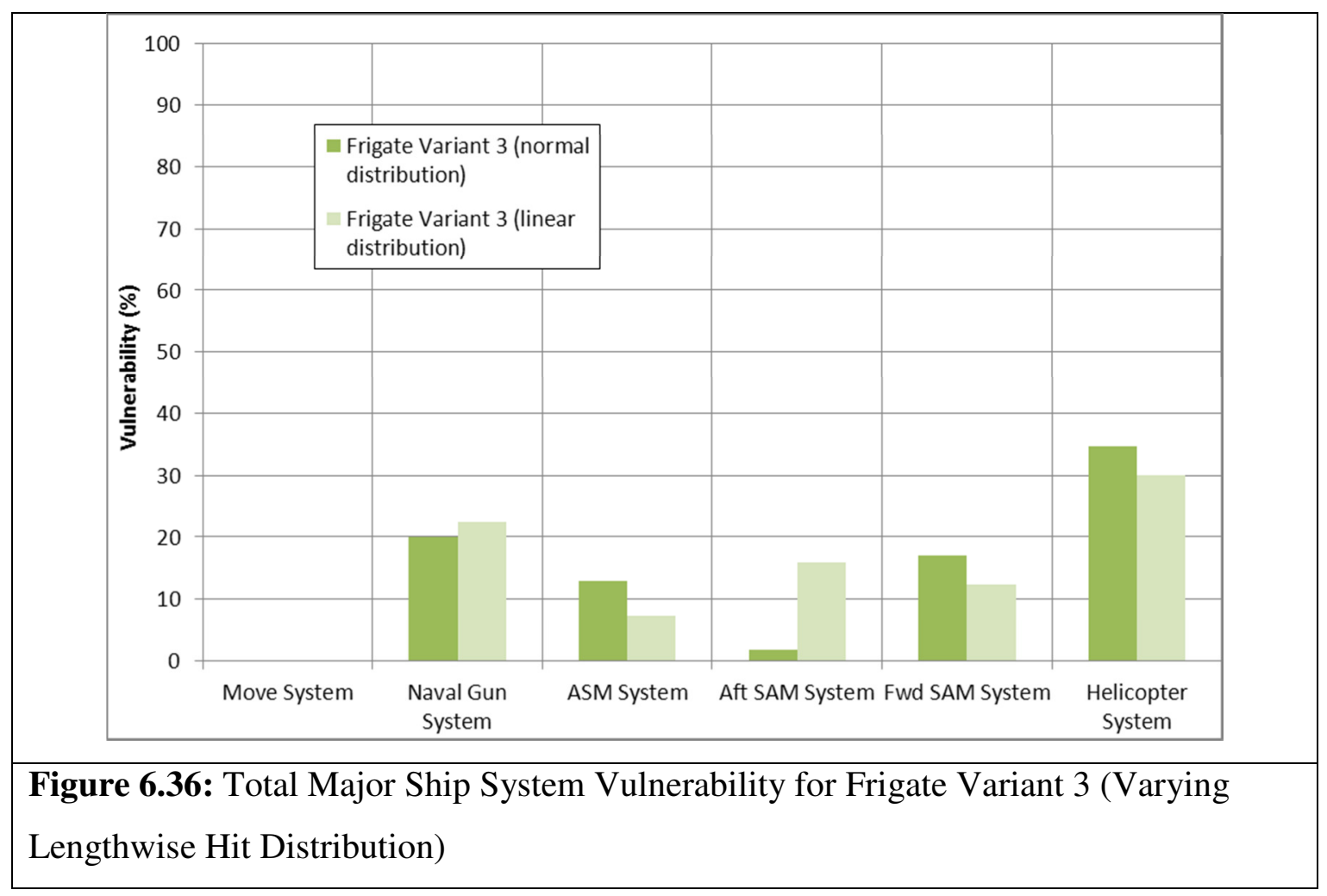

Evidently, the selected pattern of the lengthwise hit distribution of the ASM on the ship has a large effect on the vulnerability of the modelled major ship systems to the ASM. Some systems present vulnerability fluctuations of $50 \%$ or more. After normalising the data above with respect to the baseline (Frigate Variant 1), weighing given the weightings in Figure 4.1 and summing the outcomes, the total vulnerability results presented in Figure 6.37 were obtained.

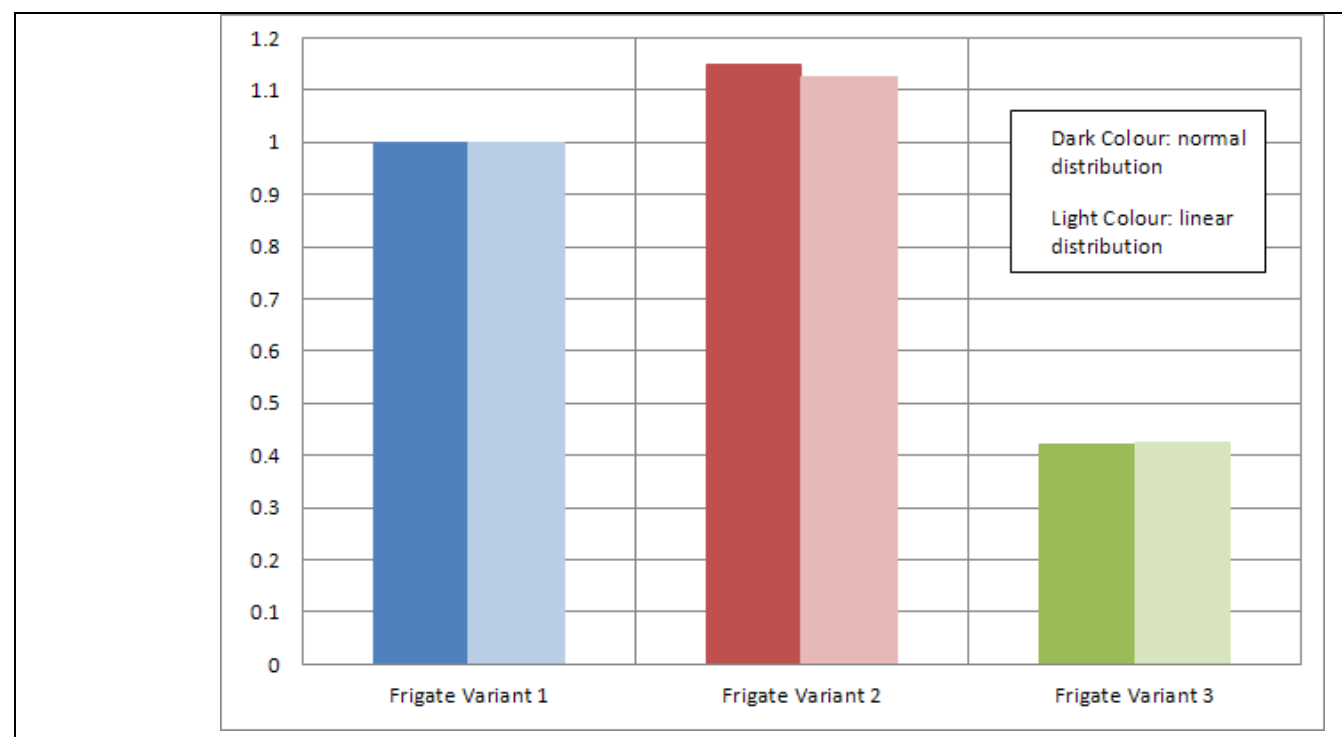

Figure 6.37: Normalised Plot of Vulnerability for the Three Frigate Variants (Varying Lengthwise Hit Distribution) 
Surprisingly, despite the large vulnerability variances previously observed the normalised total vulnerability results illustrated in Figure 6.37 are virtually unaffected by the choice of the lengthwise pattern of ASM hit probabilities.

\subsubsection{ASM Attack Angle}

All vulnerability data presented above were obtained by simulating broadside missile attacks, with an elevation of $0^{\circ}$, in SURVIVE Lite. It was deemed appropriate to perform a brief investigation on the effect of varying the angle of attack. This investigation was carried out on the SURVIVE Lite model of the Destroyer design, simulating the effect of the same ASM with an $165 \mathrm{~kg}$ warhead and a medium fragment pattern on the same ship systems listed in Table 4.1. However, in order to reduce computation and data processing time, it was decided to apply a single hit grid across the entire dimension of the design, rather than applying separate hit grids on each WT section (Figure 4.4) as was the norm in all previous investigations. The angles of attack considered, as well as the system vulnerability outputs, are summarised in Table A49 (see Appendix 11.4) and Figure 6.38. The first number in the legend of Figure 6.38 is the ASM attack azimuth $\left(0^{\circ} / 360^{\circ}\right.$ representing the stern and $90^{\circ}$ the port side $)$ and the second number is the ASM attack elevation, both in degrees.

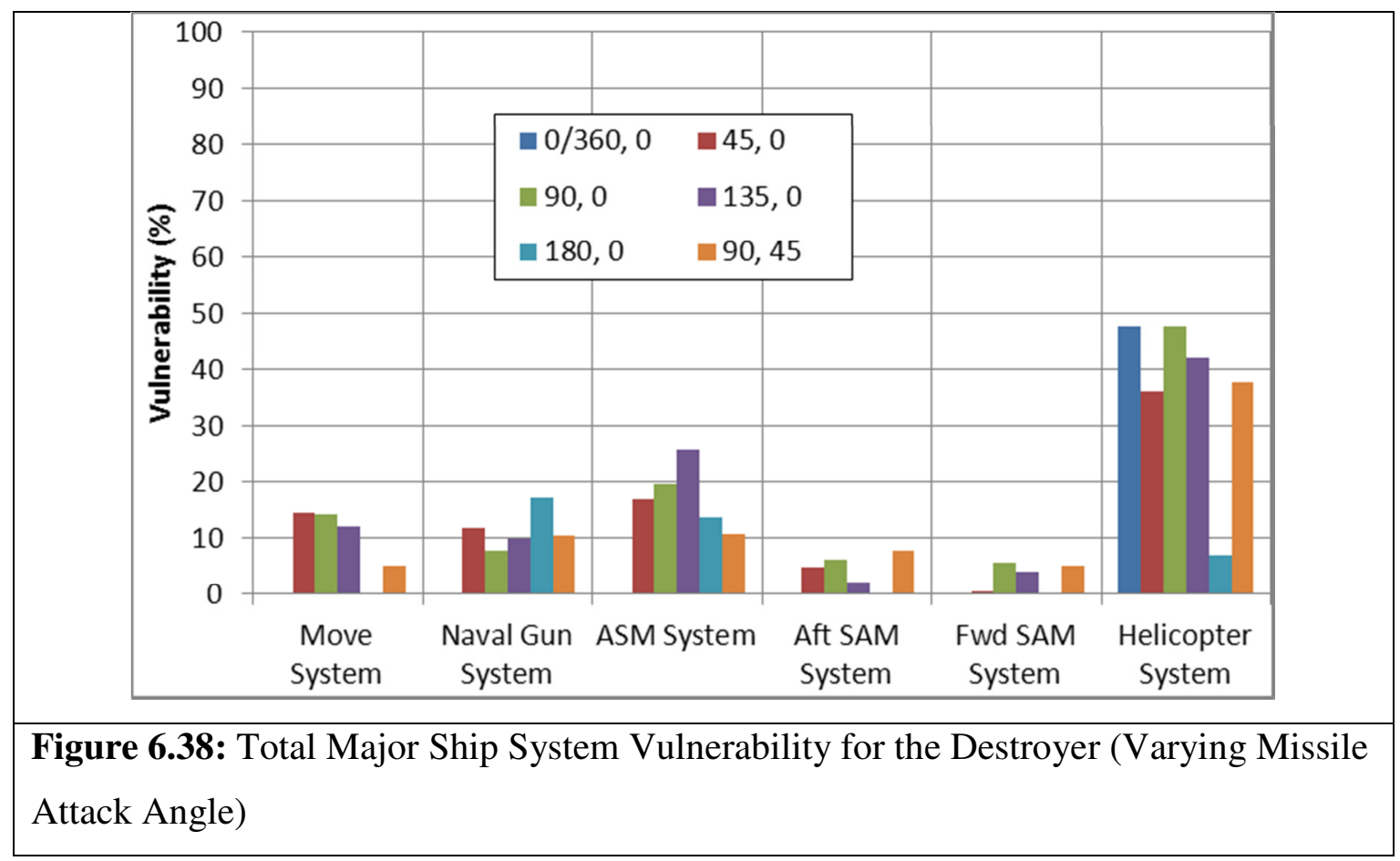


Although an overall pattern is observed in Figure 6.38, all systems investigated present vulnerability fluctuations of more than $50 \%$ depending on the angle of ASM attack. This suggests that in survivability assessment it is important to not only examine different hit locations (of a specific weapon), but also different threat trajectories. (Note that this sensitivity study was conducted on the Destroyer rather than Frigate Variant 1 since it was carried out during a four week placement at Dstl, during which access only to the Destroyer SURVIVE Lite model was available).

\subsection{Recoverability Assessment Results}

The procedure detailed in Section 4.3 was followed in order to obtain recoverability results for all ship design studies. The Paramarine models of all ships were completed and the SURVIVE Lite models were run as described in Section 6.3 in order to obtain the PM results presented in this section.

\section{$\underline{\text { 6.5.1 Frigate Variants (Including Baseline) }}$}

The recoverability performance matrices for the three frigate designs are presented in Table 6.12, Table 6.13 and Table 6.14. The matrices are identical to Table 4.6. The weightings (in red) are the ones presented in Table 4.3, Table 4.4 and Table 4.5 (PM weightings for each PM Category) and Table 4.7 (group weightings). The numerals in black are the raw PM data given an ASM hit at each WT section (averaged port and starboard hit), while the numerals in blue are equal to the same raw PM data multiplied by the probability that the corresponding WT section is hit (i.e. Figure 6.4), as outlined in Section 4.3.4. All normalisation have been conducted with respect to Frigate Variant 1, consequently all values in the two columns with 'normalised wrt baseline' data are equal to 1 in Table 6.12 . 


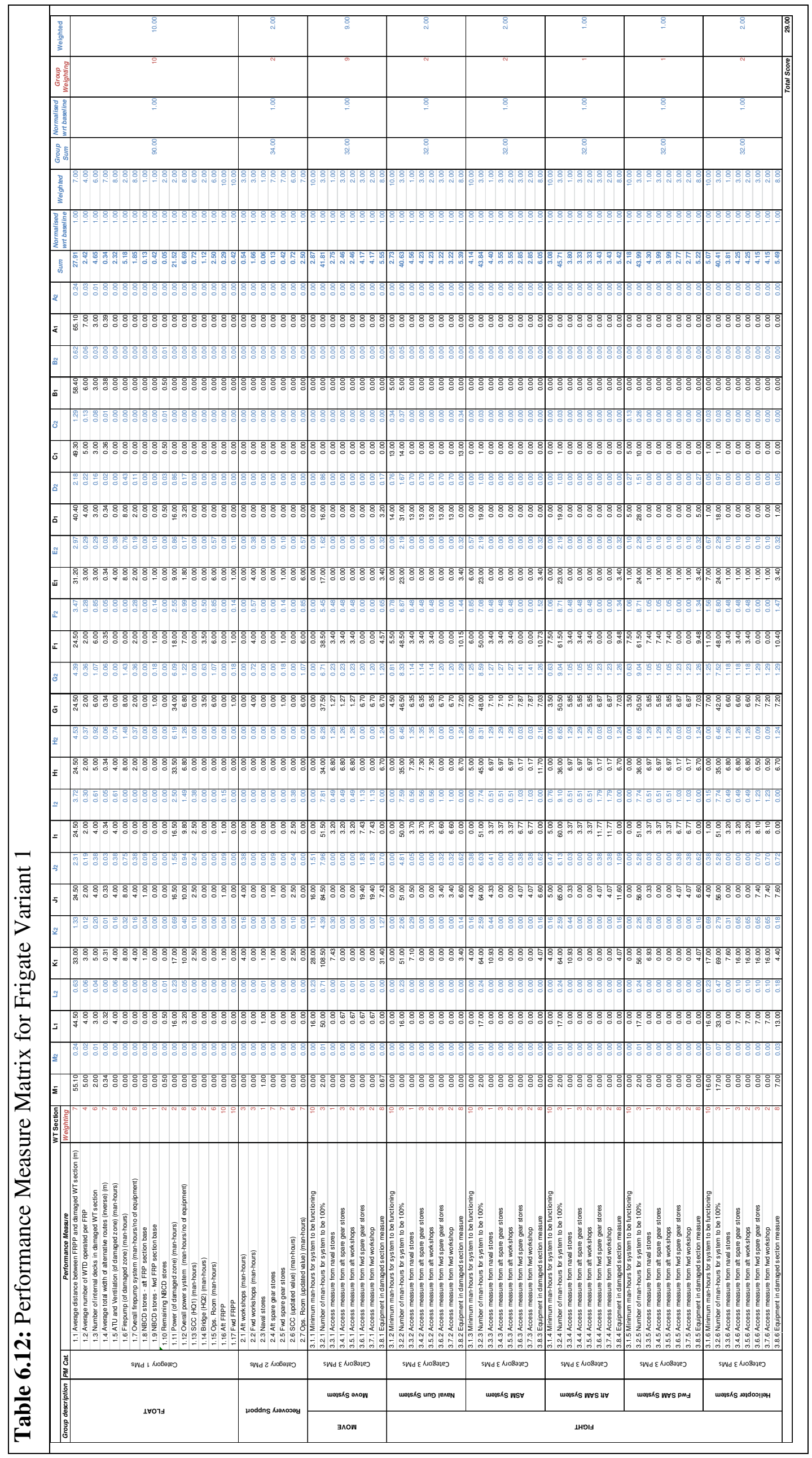




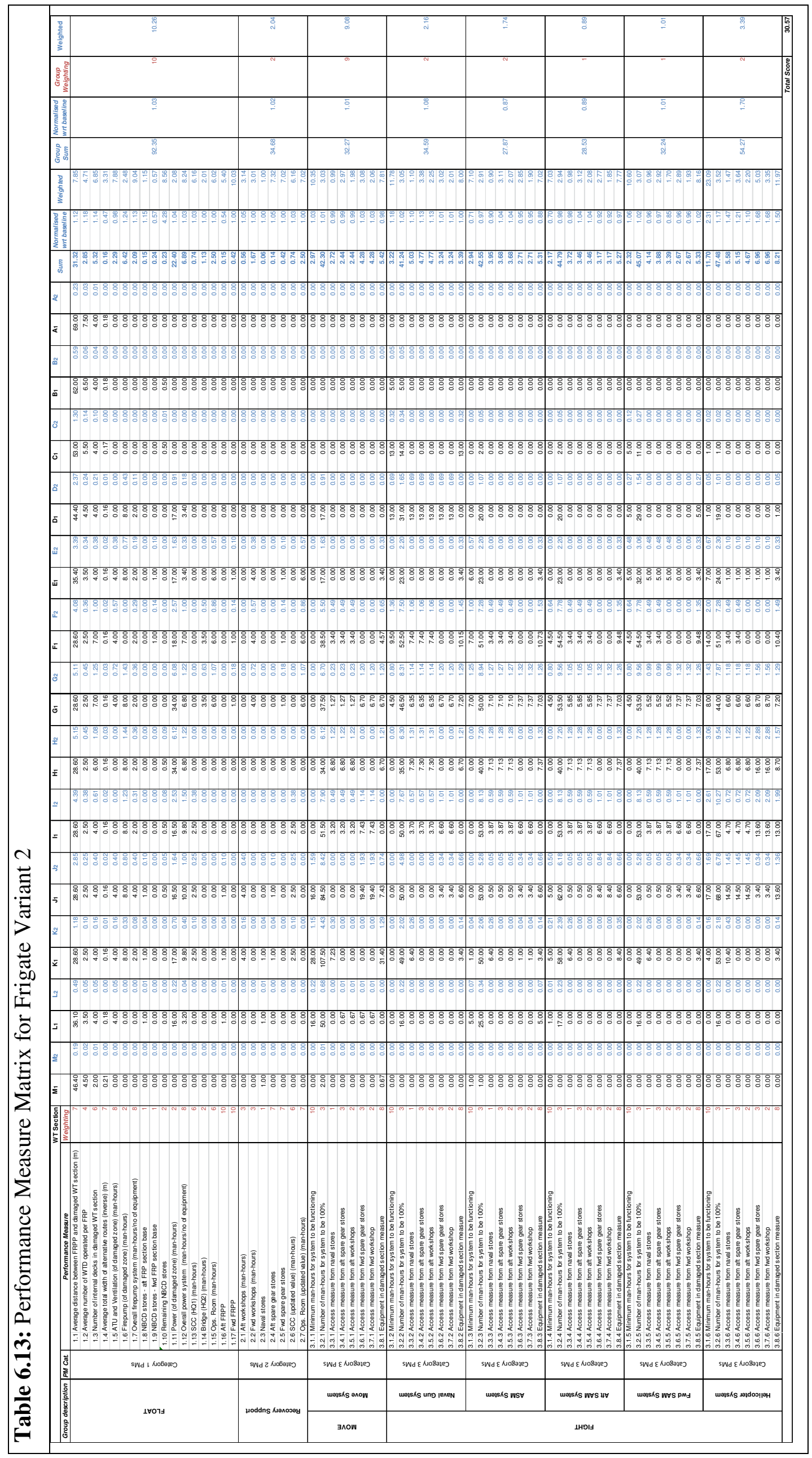




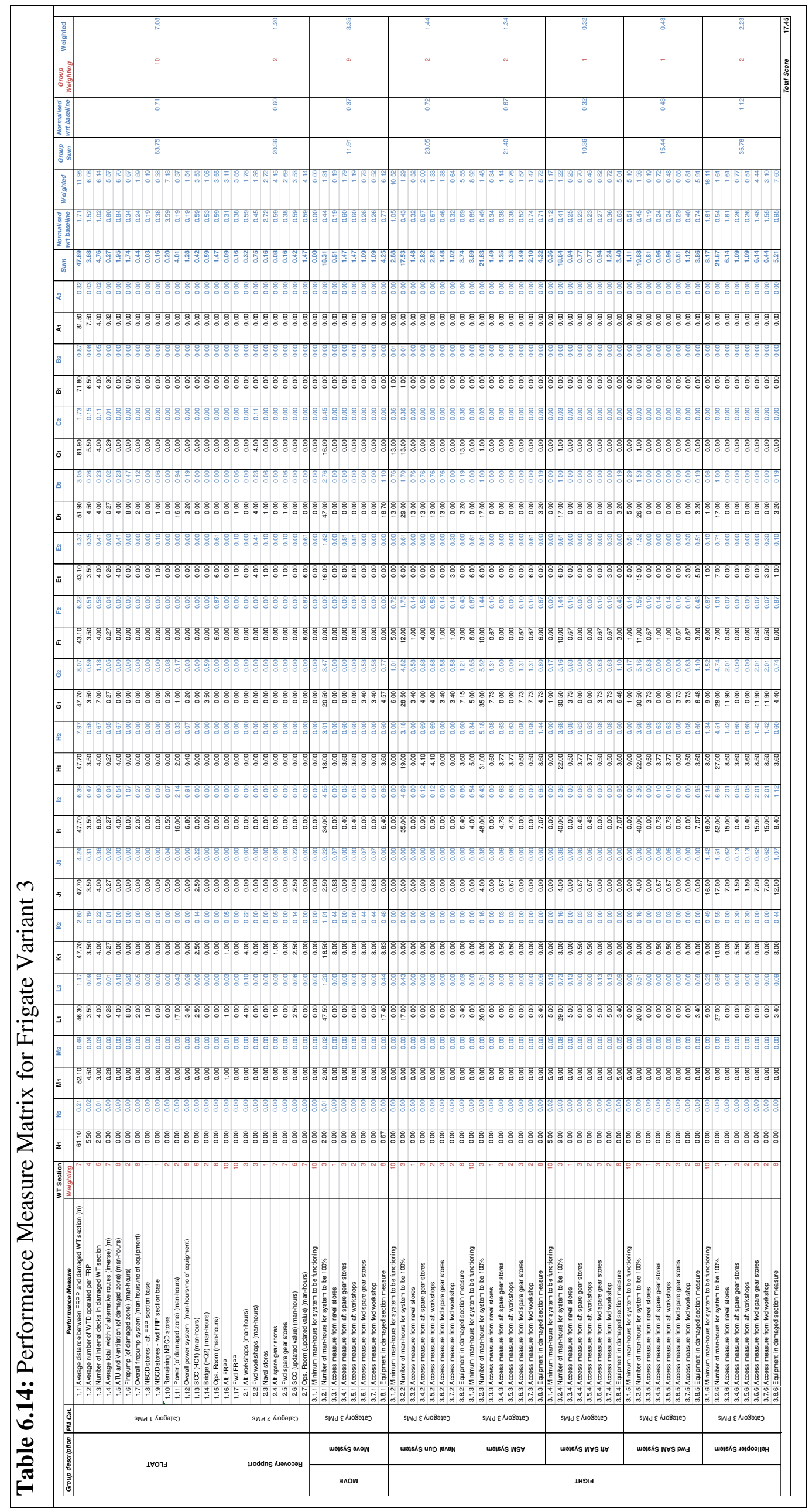


The above total scores for each ship design were normalised with respect to the baseline total score (hence Frigate Variant 1 has a recoverability performance of 1) in order to obtain the total recoverability performance results shown in Figure 6.39. The higher the score, the worse the performance and, therefore, in effect the difficulty of recoverability in a given design is shown by the relative magnitude.

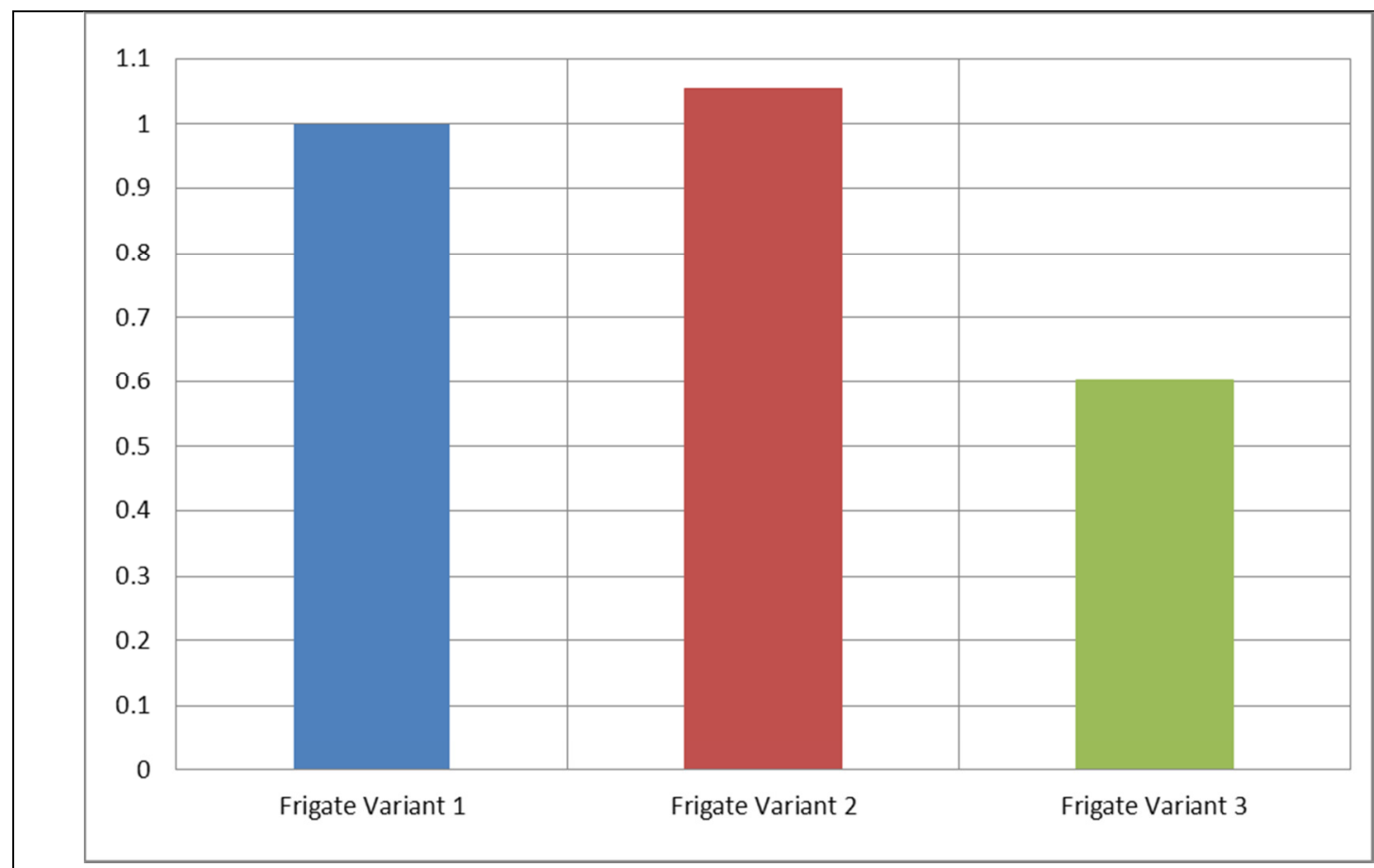

Figure 6.39: Normalised Plot of Difficulty of Recoverability for the Three Frigate Variants

The main observation from Figure 6.39 is that the two monohulls portray very similar recoverability characteristics. Conversely, the trimaran frigate design appears to be approximately $40 \%$ more recoverable than its two counterparts. This is further discussed in Section 7.3.1.

\subsubsection{Corvette, Baseline Frigate and Destroyer}

The identical procedure was followed with the intention of investigating the recoverability performance of the Corvette and Destroyer designs. The recoverability performance matrices for the two additional combatant designs are presented in Table 6.15 and Table 6.16. As before, all normalisation have been conducted with respect to Frigate Variant 1, Table 6.12. 


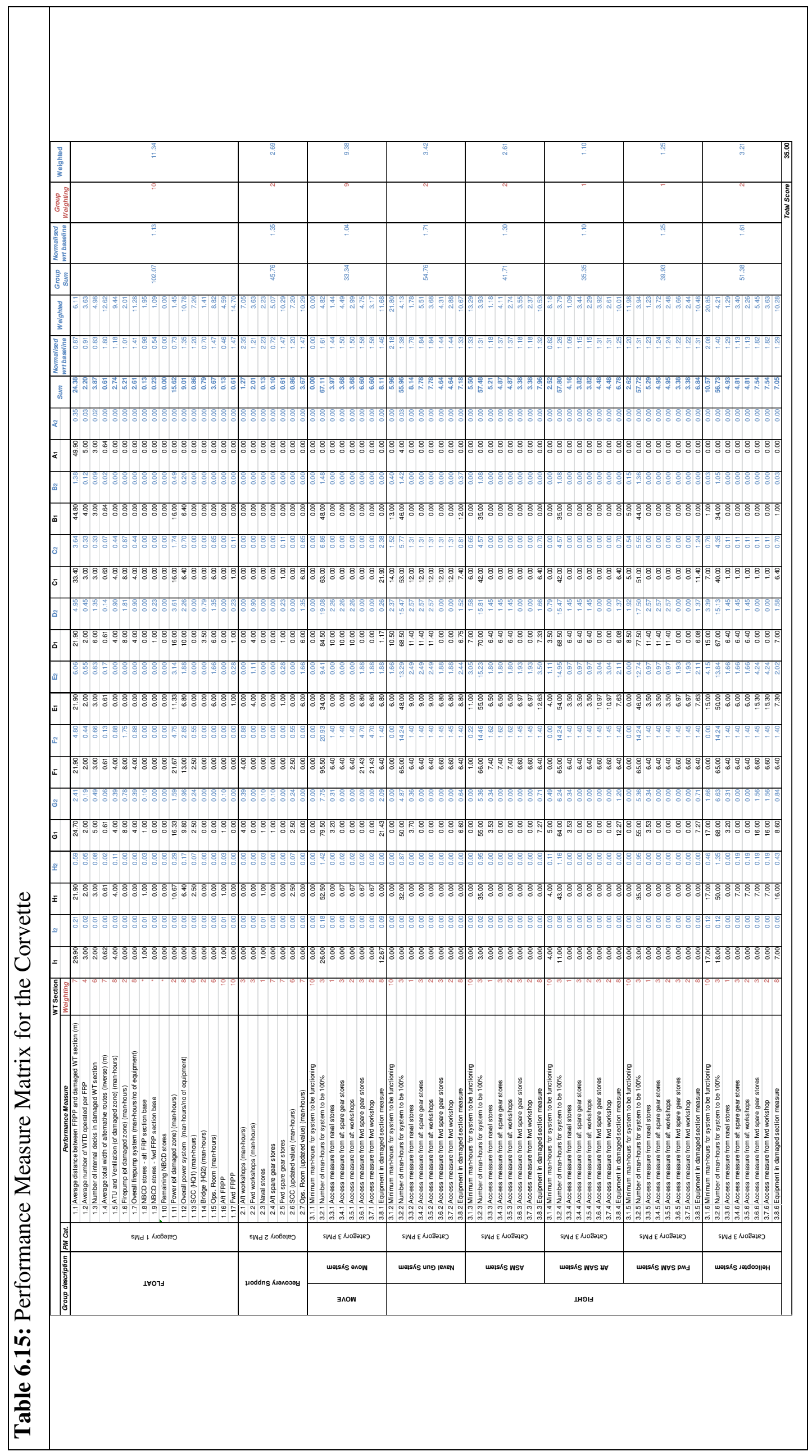




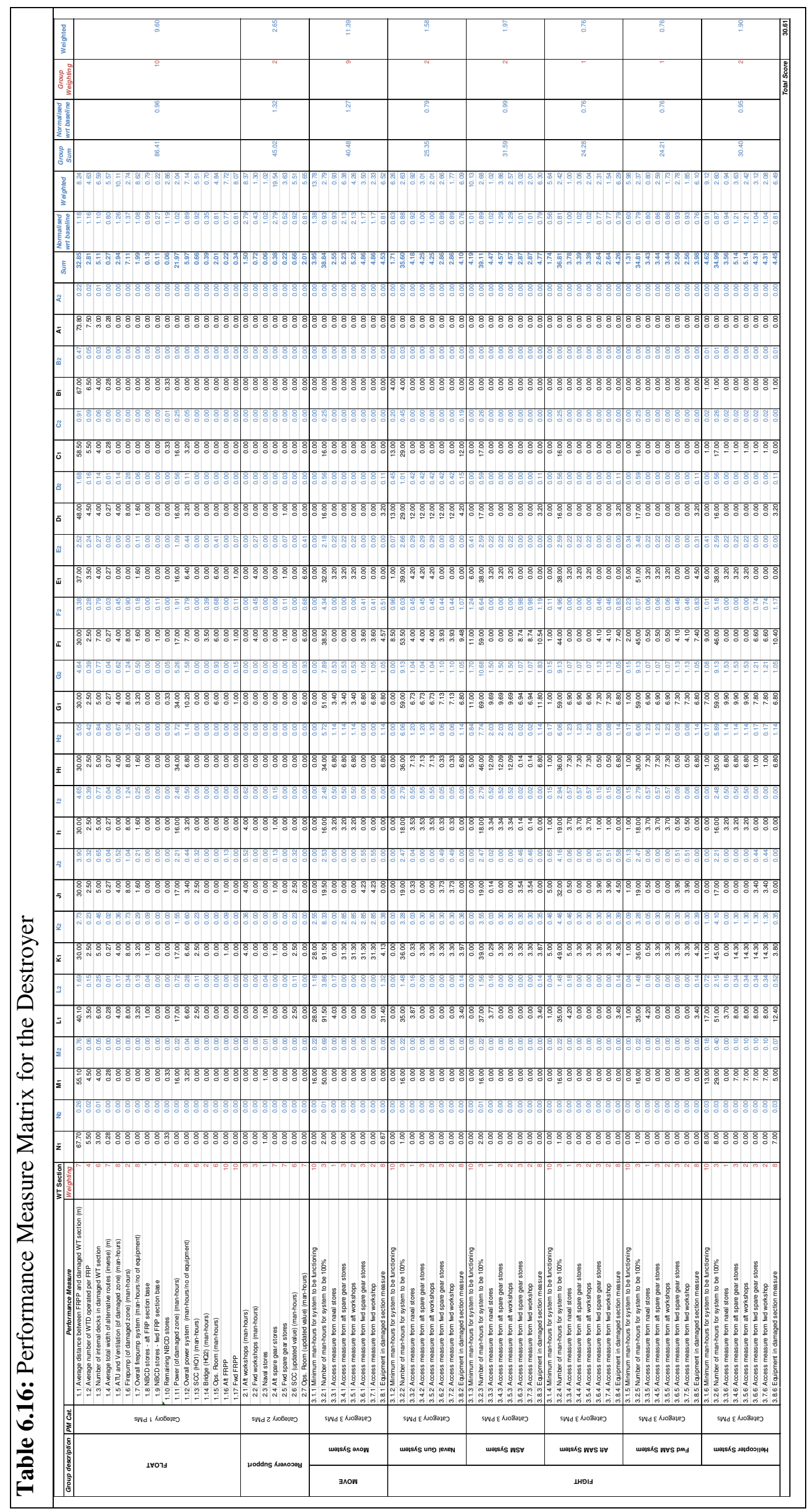


In Table 6.15 and Table 6.16 the weighting values of PMs 1.8-1.10 (i.e. the NBCD store related PMs) are absent. This is due to the different number of NBCD stores each combatant contained. As summarised in Section 5.2 the baseline frigate included four NBCD stores (Figure 5.6 and Figure 5.9), the Corvette contained two (Figure 5.42 and Figure 5.48) and the Destroyer five (Figure 5.43 and Figure 5.49). It was decided that the total weighting of all NBCD stores suggested by 1st Lt. Fonseca (Table 4.3), i.e. 4 , would be divided between the quantity of NBCD stores. Table 6.17 shows the weighting scheme adopted for the NBCD stores for the Corvette, baseline frigate (same as before) and Destroyer design studies.

Table 6.17: NBCD Store Weighting Scheme for the Corvette, Baseline Frigate and Destroyer

\begin{tabular}{|l|l|r|r|r|}
\hline \multirow{2}{*}{} & \multirow{2}{*}{ PM } & \multicolumn{3}{|c|}{ Weighting } \\
\cline { 3 - 5 } & & Corvette & Frigate Variant 1 & Destroyer \\
\hline $\mathbf{1 . 8}$ & NBCD stores - aft FRP section base & 2 & 1 & 0.8 \\
\hline $\mathbf{1 . 9}$ & NBCD stores - fwd FRP section base & 2 & 1 & 0.8 \\
\hline $\mathbf{1 . 1 0}$ & Remaining NBCD stores & 0 & 2 & 2.4 \\
\hline \multicolumn{2}{r}{ Total } & $\mathbf{4}$ & $\mathbf{4}$ & $\mathbf{4}$ \\
\hline
\end{tabular}

After normalising the total recoverability scores of the Corvette and Destroyer designs with respect to the baseline total score, the results shown in Figure 6.40 were obtained.

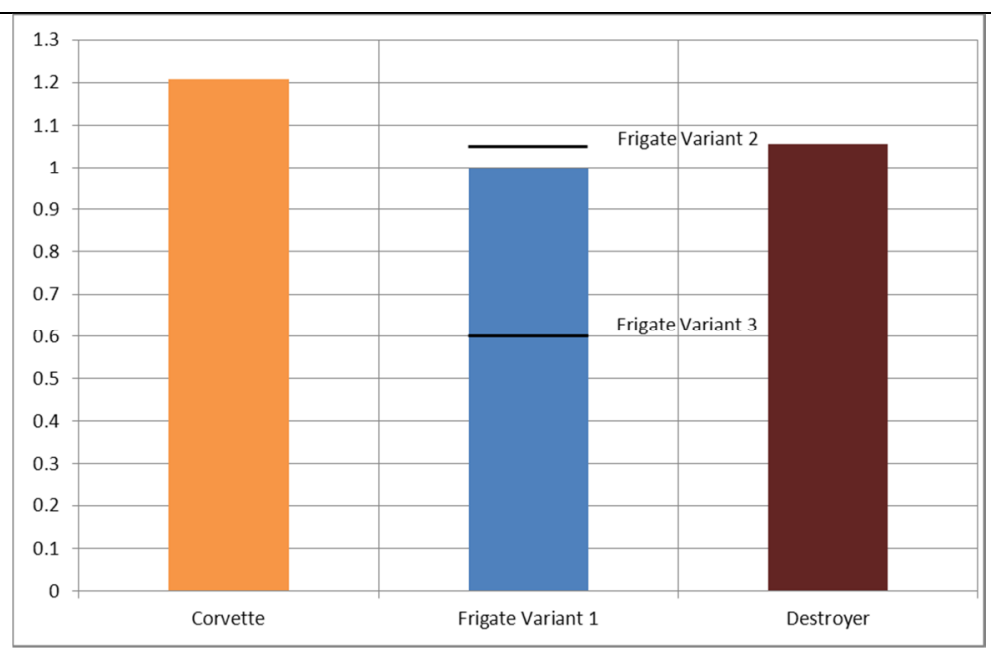

Figure 6.40: Normalised Plot of Difficulty of Recoverability for the Corvette, Frigate Variants and Destroyer 
From Figure 6.40 it can be deduced that recoverability performance is more sensitive to hullform configuration than to ship size. In addition, one could assume that recoverability performance worsens with decreasing ship size, which could be expected. However, the fact that the Destroyer design performs marginally worse than the baseline frigate suggests that the above statement requires further exploration, which is addressed in Section 7.3.2.

\section{$\underline{\text { 6.5.3 AOR Variants }}$}

Lastly, the procedure previously described was reproduced in order to investigate the recoverability performance of the two replenishment ship designs. The corresponding recoverability performance matrices are shown in Table 6.18 and Table 6.19 and are identical to Table 4.8. A different weighting scheme to the above combatant design studies (detailed in Table 4.3, Table 4.4 and Table 4.5) was applied. However, this was necessary for consistency reasons since the weighting scheme proposed for the AORs major systems (Table 4.9) was given by a different officer, Lt. Cdr. (rtd.) Day. It was, therefore, deemed necessary that the PM weighting scheme should also be proposed by the same officer. Note that PM and group normalisation have been performed with respect to the corresponding value of the worst performing design variant. As with susceptibility and vulnerability assessment, in the case of the AOR design studies, normalisations were performed with respect to the worst performing design, rather than a baseline, the reasoning behind which is explained in Section 7.2.4. 


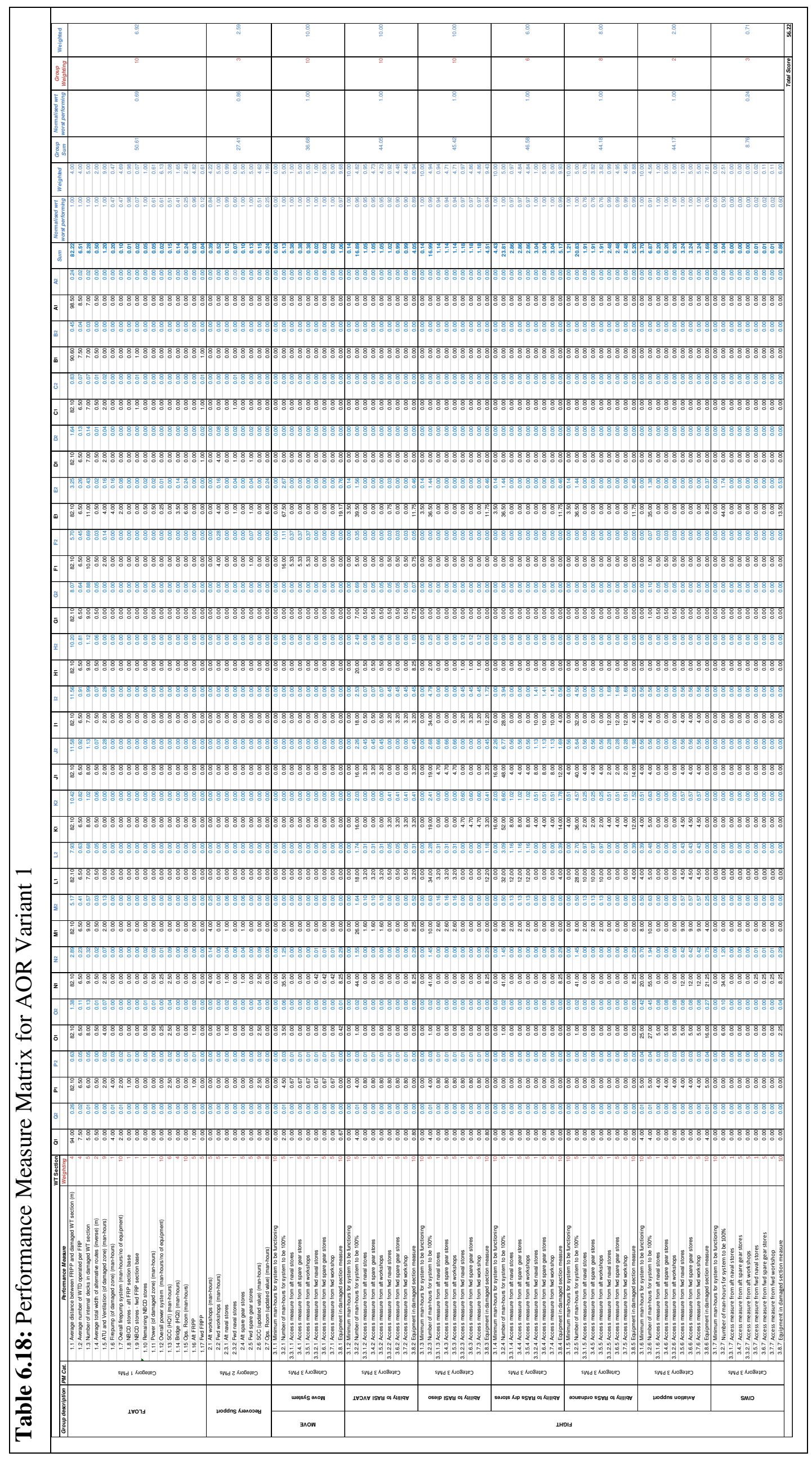




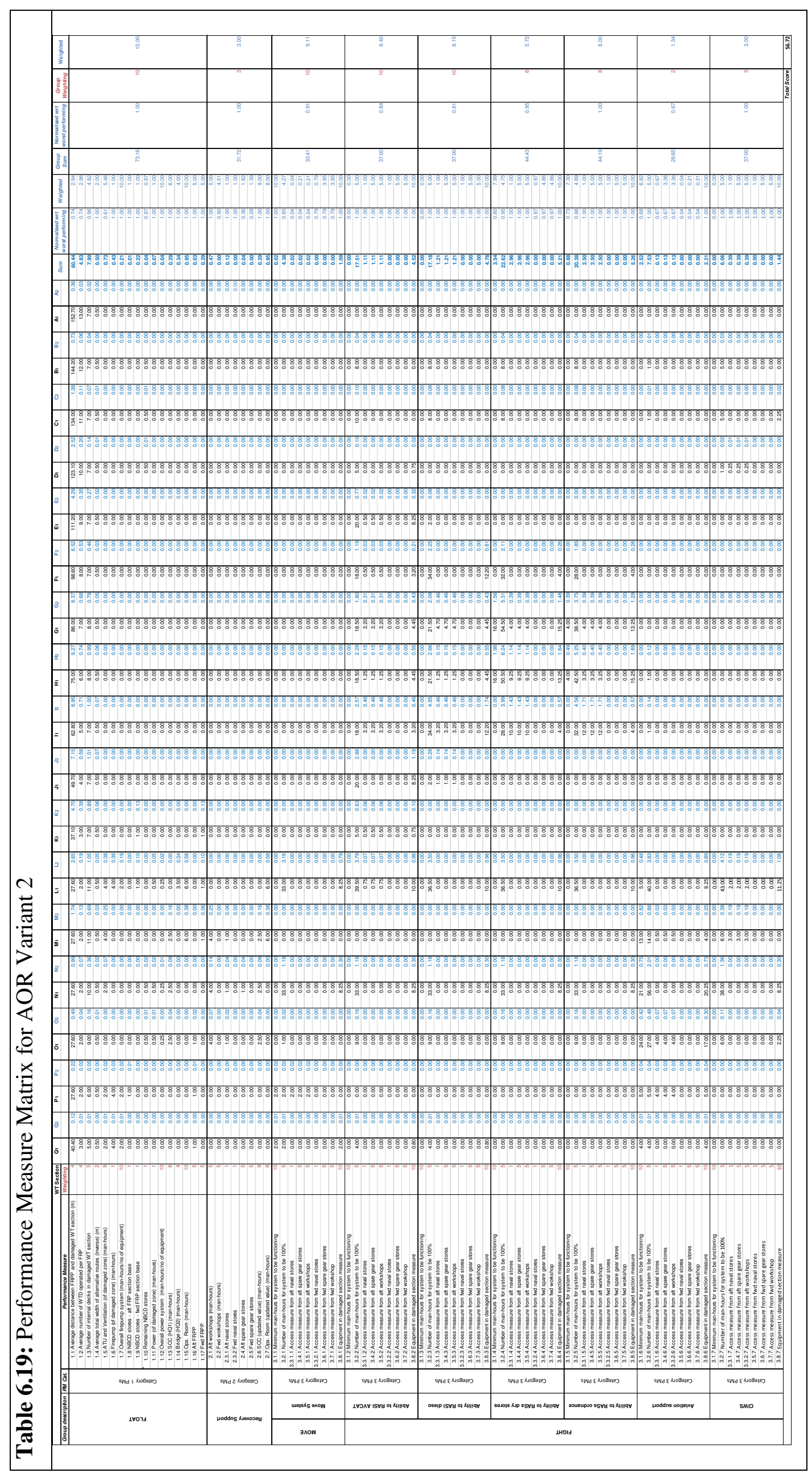


By inspecting the two PM matrices above it became evident that certain modifications were required in order to apply the overall recoverability method to the AOR design studies. Firstly, the presence of two naval stores in AOR Variant 1 (Figure 5.69) necessitated the alteration of two PMs and the creation of two new PMs; namely PMs 2.3.1 and 2.3.2 (aft naval stores and fwd naval stores respectively) and PMs 3.3.1 and 3.3.2 (access measure from aft naval stores and access measure from fwd naval stores respectively). The weightings of the previous PMs 2.3 and 3.3 (both of which were equal to 2, see Table 4.4 and Table 4.5) were equally split between the new PMs, with all four PMs having weightings of unity each.

Secondly, AOR Variant 2 included a single workshop, spare gear store and naval store (Figure 5.70), in contrast to the duplicated above compartments in the first variant. It was assumed that, given the identical requirements of both ship designs, the total workshops and stores in both variants would contain identical systems, equipment and material (and, therefore, the total weight and volume of these compartments was equal in both design studies). It was therefore, decided that for AOR Variant 2, PMs 2.3.1 and 2.3.2, PMs 3.3.1 and 3.3.2 (mentioned above) as well as PM pairs 2.2 and 2.1, 2.4 and 2.5, 3.4 and 3.6 and finally 3.5 and3.7 (i.e. all workshops, spare gear stores and naval stores related PMs, see Table 4.4 and Table 4.5) would be given the same value (which would be equal to the PM values for the single workshop, spare gear store and naval store respectively, calculated as described in Section 4.3.2 and 4.3.3). After normalising the total scores of the AOR variant (Table 6.18 and Table 6.19) with respect to the worst performing design, the total recoverability performance results shown in Figure 6.41 were obtained.

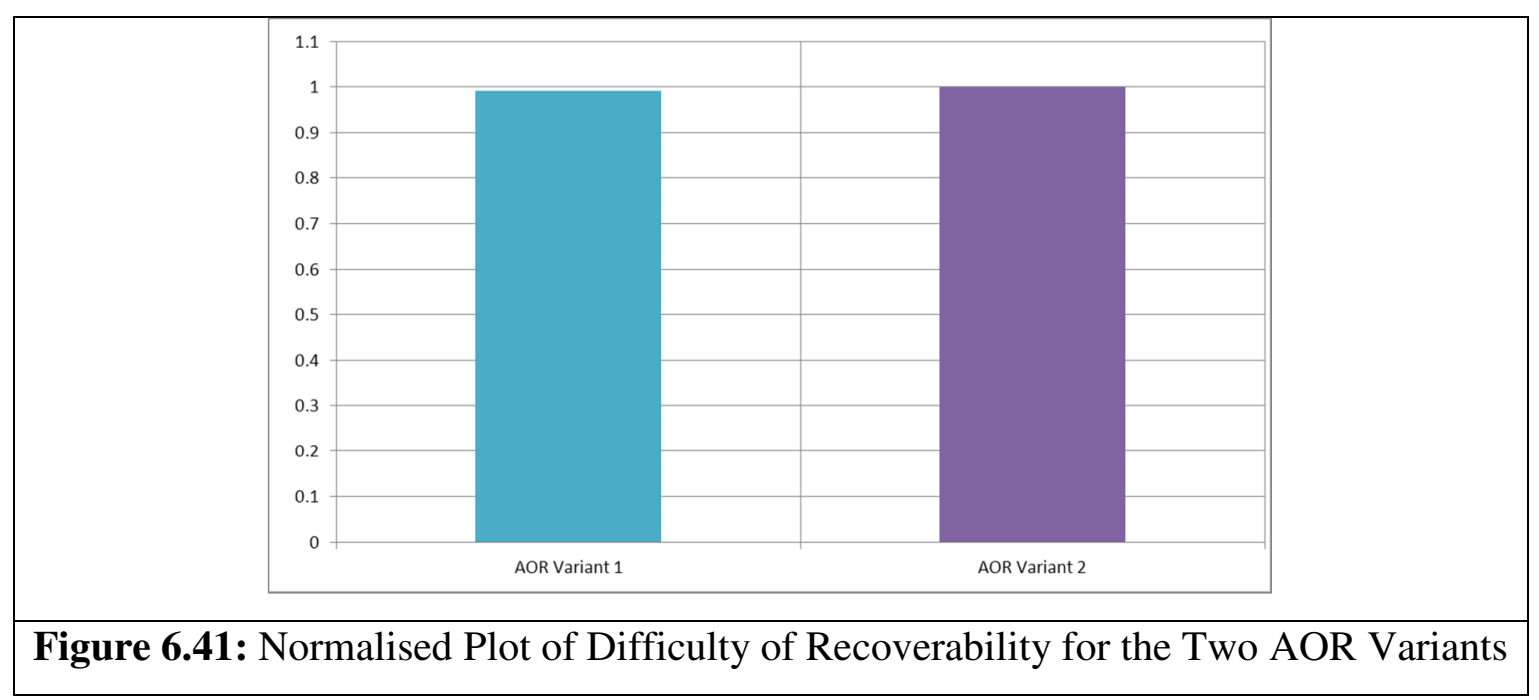


From above it is evident that both AOR design variants present near identical recoverability characteristics despite the different configurations adopted.

\subsection{Recoverability Assessment Sensitivity Studies}

Sensitivity studies were also carried out in the recoverability part of the survivability assessment method. Three sensitivity studies were conducted:-

- The application of different weighting schemes was examined;

- The effect on recoverability, given a different lengthwise probability hit distribution on the frigate variants was investigated;

- The effect of decoupling the proposed recoverability assessment approach from SURVIVE Lite was explored.

\subsubsection{Weighting Schemes}

The initial investigation, as in the vulnerability assessment approach (Section 6.4.1), was on the sensitivity of the recoverability assessment method to the weighting scheme applied. The same RN officers listed in Table 6.11 were interviewed and asked to fill in a form (Appendix 12) suggesting alternative weighting schemes (Mant 2012; Hood 2012; Sutcliffe 2012; Boughton 2012; Koheeallee 2012; O'Brien 2012; Kadinopoulos 2012). Consistently, the naval officers were asked to weigh each PM between $0-10$, ten being the most important and they were not exposed to previous weighting schemes so as to not affect their responses. Their proposed weighting schemes, including the ones suggested by $1^{\text {st }}$ Lt. Fonseca and Lt. Cdr. (rtd.) Day which have already been reported (Table 4.3, Table 4.4 and Table 4.5), are presented in Table 6.20, Table 6.21 and Table 6.22. Unlike the system weighting schemes in the vulnerability assessment part of the method (Table 6.11) the PM weighting schemes are scenario independent. This sensitivity test was only carried out to the three frigate design variants. 


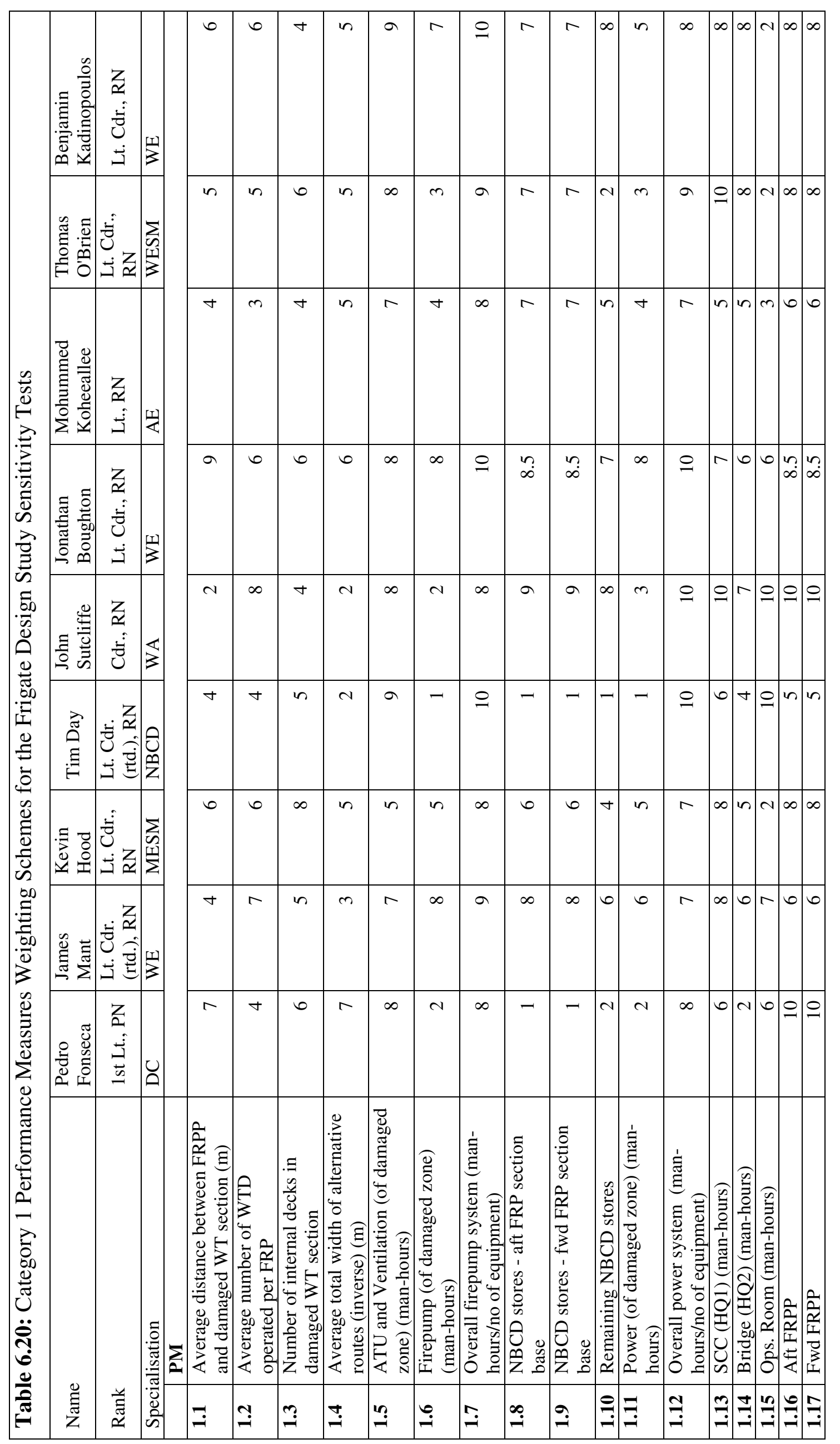


It is worth mentioning some of the remarks made by the above officers on the Category 1 (immediate DCFF) PMs whilst filling in the weighting scheme form. 1st Lt. Fonseca (2011) commented that DC equipment is usually distributed through the ship (mainly on the DC deck, in proximity to the FRPPs), whereas NBCD stores merely contain maintenance material for the DC equipment. Hence the high weighting proposed by this officer for PM 1.16 and PM 1.17 and the low weightings given to NBCD store related PMs (PMs 1.8-1.10). Besides, he recommended the addition of Category 1 PMs describing the status of fixed firefighting systems, detection equipment, communications and the sickbay (for personnel recovery) after successful attack. Lt. Cdr. (rtd.) Mant (2012) suggested the addition of a Category 1 PM reflecting on the condition of smoke curtains (to prevent spread of smoke) after attack. Lt. Cdr. Hood (2012) recommended Category 1 PMs related to training as well as situational awareness and command and control (although it can be argued that command and control is incorporated in PMs 1.13-1.15). Moreover he gave a very low weighting to PM 1.15, Operations Room, since DCFF is associated with an automatic response, therefore, theoretically does not require substantial input from the CO. Lt. Cdr. Boughton (2012) mentioned that the addition of a Weapon Section Base Category 1 PM would be beneficial, therefore, linking the external and internal battles. He also proposed that the duplicated forward and aft FRPPs, as well as their corresponding NBCD stores, should have different weightings given that they provide the same function. However, since the decision of which of the two section bases and stores would be given higher priority depends on the $\mathrm{CO}$ and the operational circumstances, the two weightings given by that officer were averaged; thus the decimal fraction form of the relevant weightings. This would have the same effect as interchanging the higher priority FRPP and corresponding NBCD store, obtaining the recoverability results for both cases and averaging. Finally, Lt. Koheeallee (2012) suggested a Category 1 PM on the state of internal communications after the hit. 


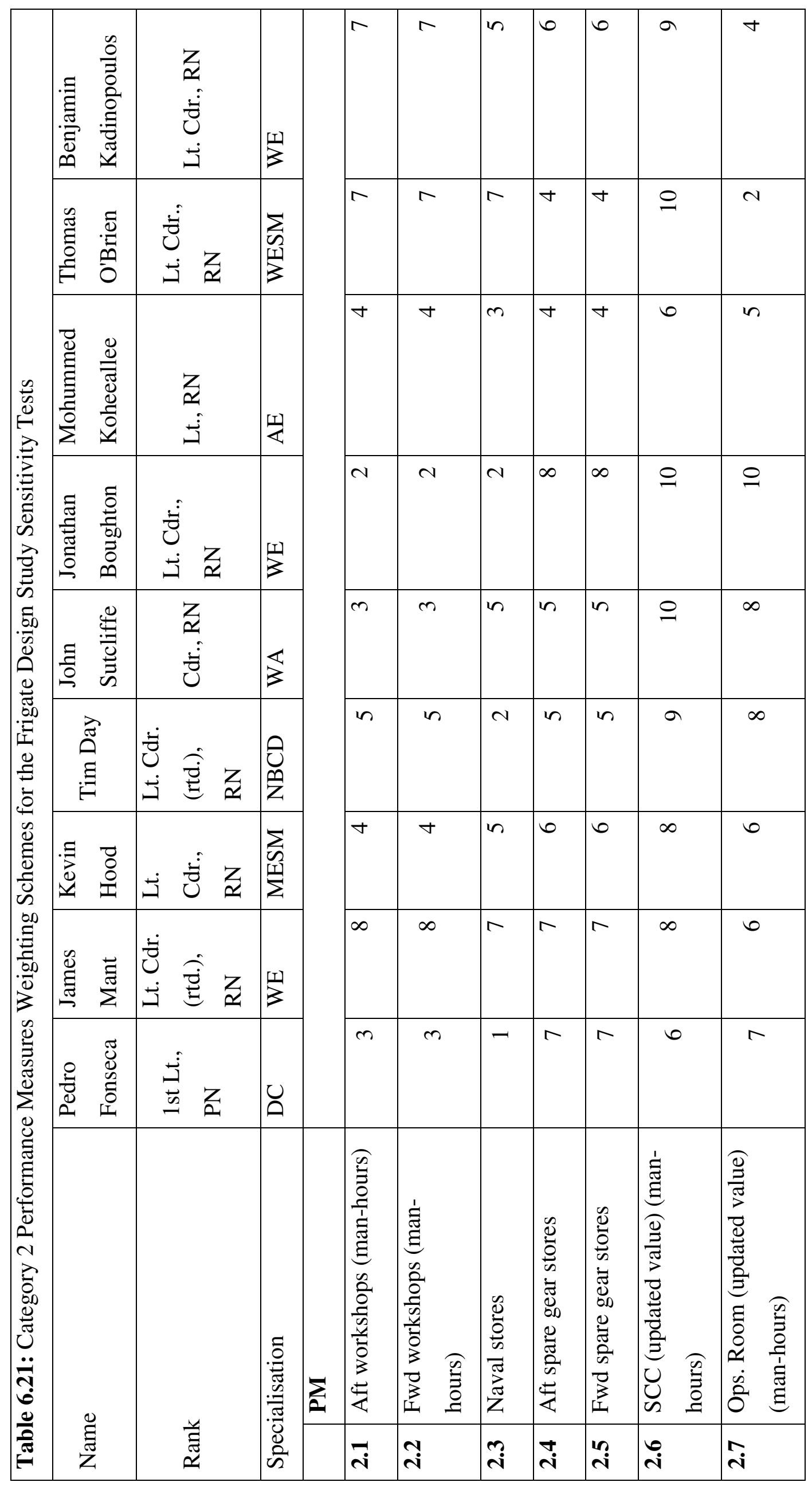

$\stackrel{ }{\sim}$ 
Regarding the Category 2 (major system recovery) PMs, there was some uncertainty as to what the naval and spare gear stores contained. According to $1 \mathrm{st} \mathrm{Lt}$ Fonseca (2011) and Lt. Cdr. Hood (2012) the naval stores contained raw material, whereas the spare gear stores included spare parts. For this reason, given that most equipment and systems in modern frigates are electronic, raw material is of decreased worth, therefore, 1st Lt. Fonseca proposed a diminutive weighting for PM 2.3, in contrast to the relatively high weightings of PMs 2.4-2.5. However, Lt. Cdr. Hood did not agree with the above argument, therefore, suggesting almost equal weightings for both naval and spare gear store related PMs. 1st Lt. Fonseca further justified the large weightings for PMs 2.4-2.5 since there is no spares duplication. Moreover, he reasoned that most repair activities are performed on site; therefore, rationalising the relatively small weighting of the workshop related PMs. Lt. Cdr. Hood also proposed the addition of Category 2 PMs reflecting on then availability of people and their skills, necessary for system repair and recovery. Once again, Lt. Cdr. Boughton (2012) proposed the addition of a Weapon Section Base Category 2 PM. Furthermore, Lt. Koheeallee (2012) suggested that an updated bridge Category 2 PM is beneficial. 


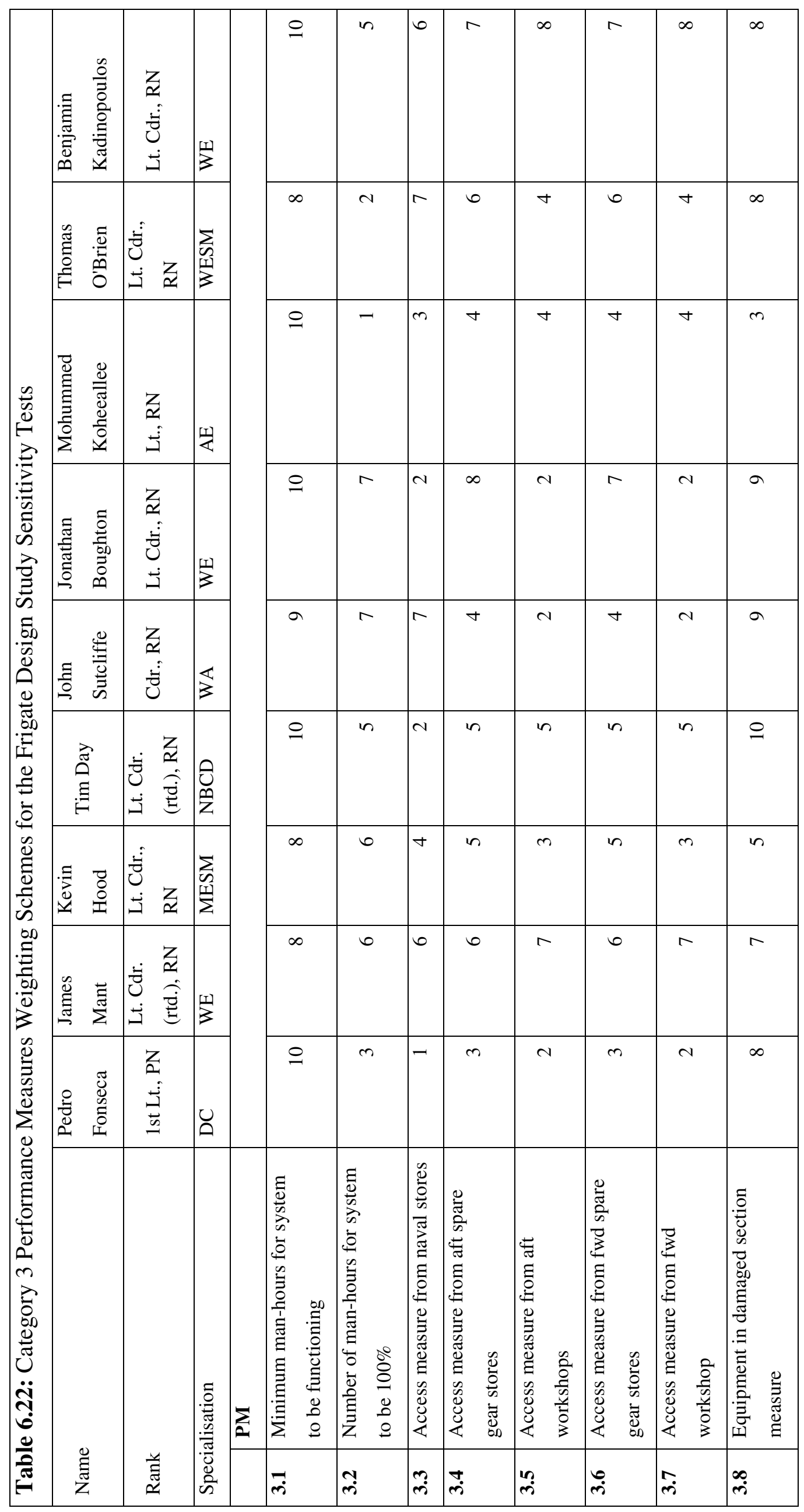


Regarding the Category 3 (individual major system recovery) PMs, form Table 6.22 it is clear that the officers ranked the importance of access from the workshops and stores in a corresponding order to their importance for major system recovery (Table 6.21). In addition, they generally agreed that access to the damaged WT section is more important than access of damaged equipment and compartments from the stores and workshops and that to have a system functioning due to redundant equipment (PM 3.1) is of greater significant than to have all, including redundant, equipment of a system functioning (PM 3.2). The PM weighting schemes were plotted in a line graph (Figure 6.42) in order to compare the different weighting scheme philosophies of the interviewed officers.

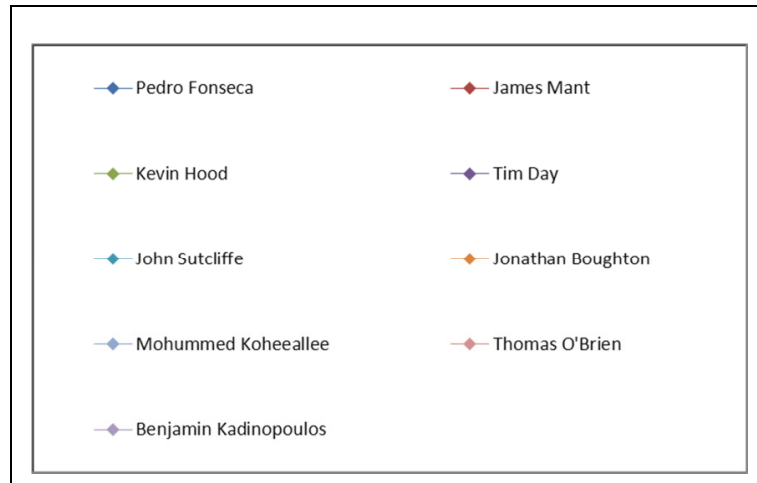

\section{Category 1}

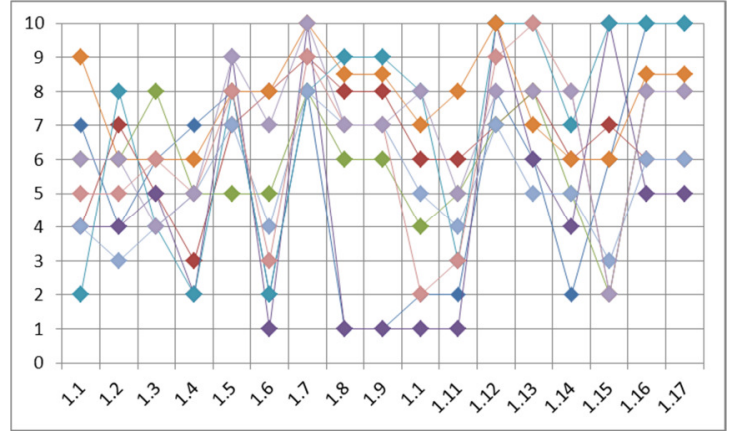

\section{Category 2}

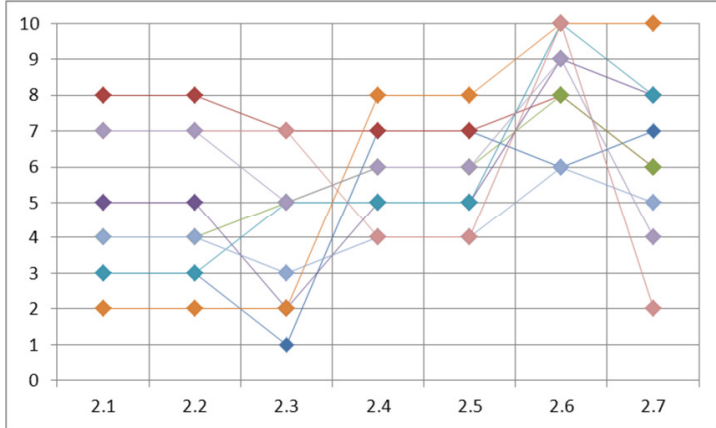

\section{Category 3}

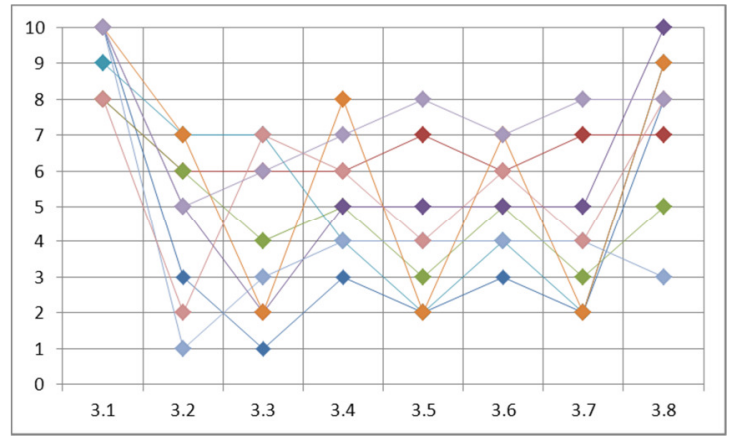

Figure 6.42: Performance Measures Weighting Scheme Philosophies for the Sensitivity Tests

Some very broad patterns may be distinguished, although, the fact that each officer reflects on his own experiences and knowledge and is specialised in a different area, has led to significant variations in certain PMs. It is observed that the PM weighting schemes are more diverse than the major system weighting schemes proposed by the same officers, presented in Figure 6.30. This could be due to the fact that the 
major system weighting schemes are scenario dependant and, therefore, probably rely to a significant extent on the response that the officers have been trained to execute in such situations (which should be similar, given that most of them served in the same navy and underwent similar training). The PM weighting schemes also rely on procedures to which the officers have been trained, however, the internal threat is much more visible and direct than the external threat, therefore, possibly the interviewed staff input elements of their own response to the internal battle, judged on the picture of it that they have created.

The weighting schemes in Table 6.20, Table 6.21 and Table 6.22 were substituted in the column headed 'Weighting' in Table 6.12, Table 6.13 and Table 6.14. In addition, for consistency reasons, the values in the 'Group Weighting' column were substituted by the major system weighting schemes proposed by the corresponding officers, Table 6.11, therefore, obtaining recoverability performance scores for the three scenarios summarised in Table 6.10. However, Table 6.11 includes weightings for the move and Fight groups, but not for the float and recovery support groups (refer to Table 4.7). Therefore, the naval officers were also asked to propose weightings for the two remaining groups, which are presented in Table 6.23. 


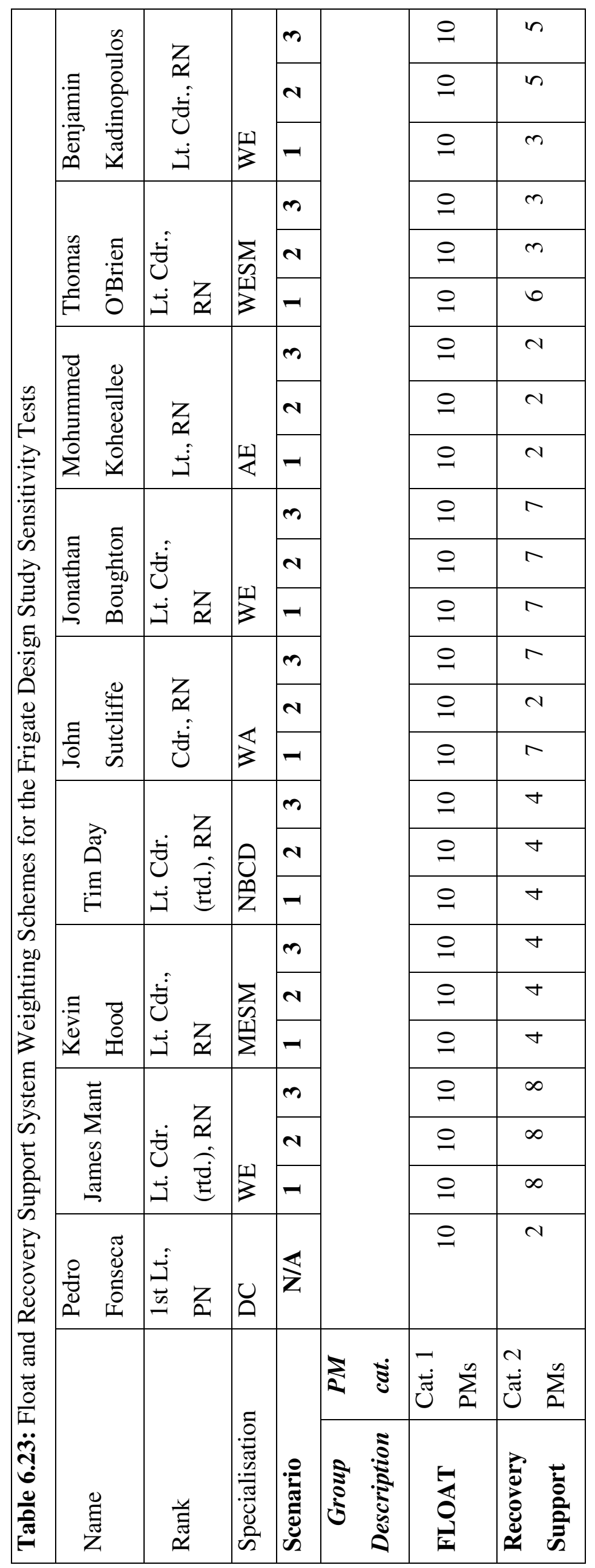


All officers gave the maximum weighting, 10, to the float group, for all scenarios. Moreover, Cdr. Sutcliffe (2012) and Lt. Cdr. O’Brien (2012) proposed a smaller recovery support group weighting for Scenario 2 and for Scenarios 2 and 3 respectively. This probably reflects on the littoral location of the threats (Table 6.10), providing the ships with the opportunity to withdraw and proceed towards onshore repair bases rather than carrying out the repair and recovery activities on board during the scenario's evolutions. The threat in Scenario 2 is shore based, therefore, cannot pursue the ship if it withdraws. However, Lt. Cdr. Kadinopoulos (2012) proposed a lower weighting for the recovery support group in the first (blue water) scenario, possibly implying that repair and recovery is increasingly difficult far from base, therefore, the crew should focus efforts in other mission areas.

Once the weighting scheme corresponding to each officer and scenario was substituted in the PM matrices, the data was processed in an identical manner as described in Section 4.3 and 6.5. The results illustrated in Figure 6.43, Figure 6.44 and Figure 6.45 were produced for the three scenarios respectively.

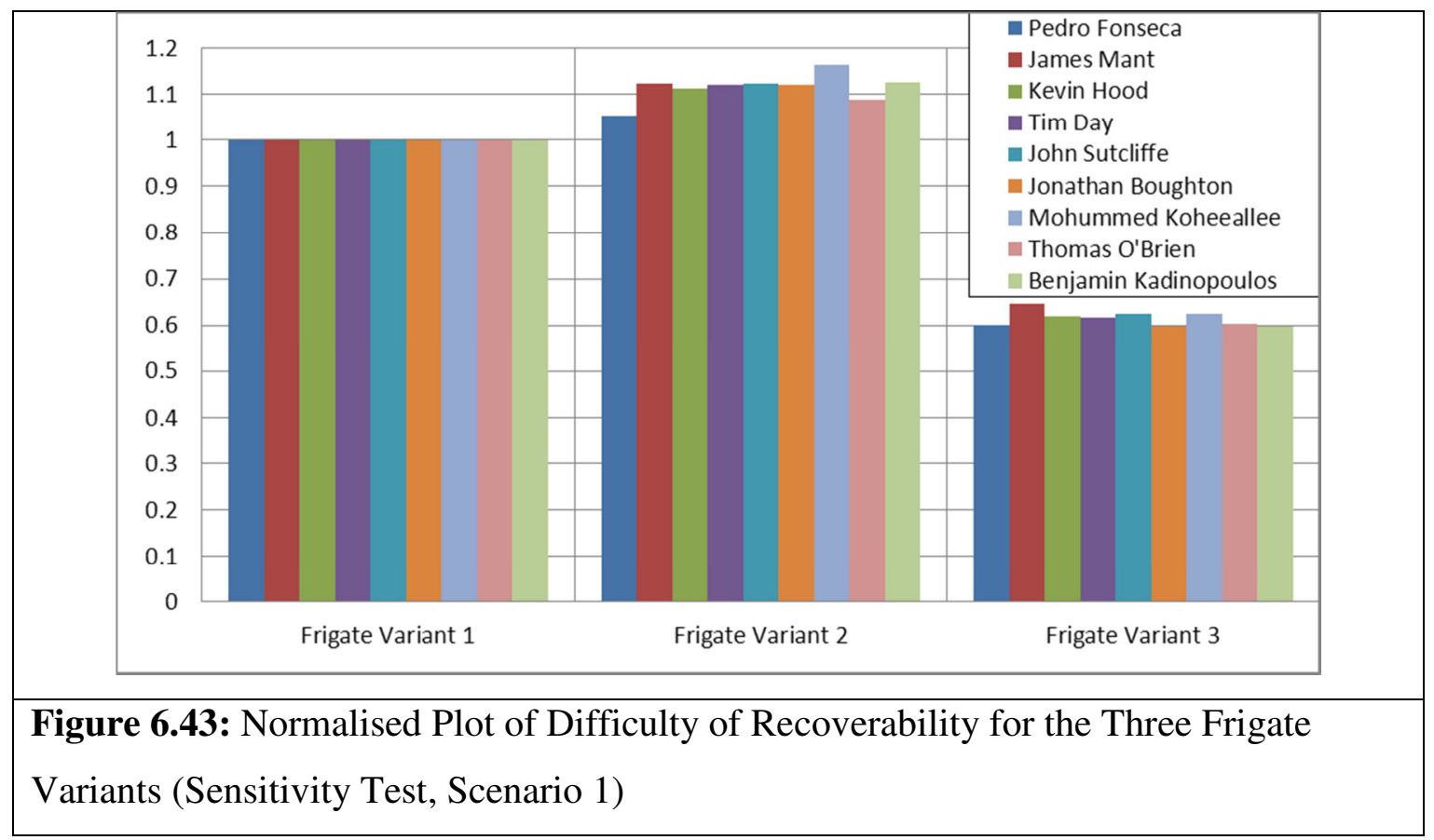



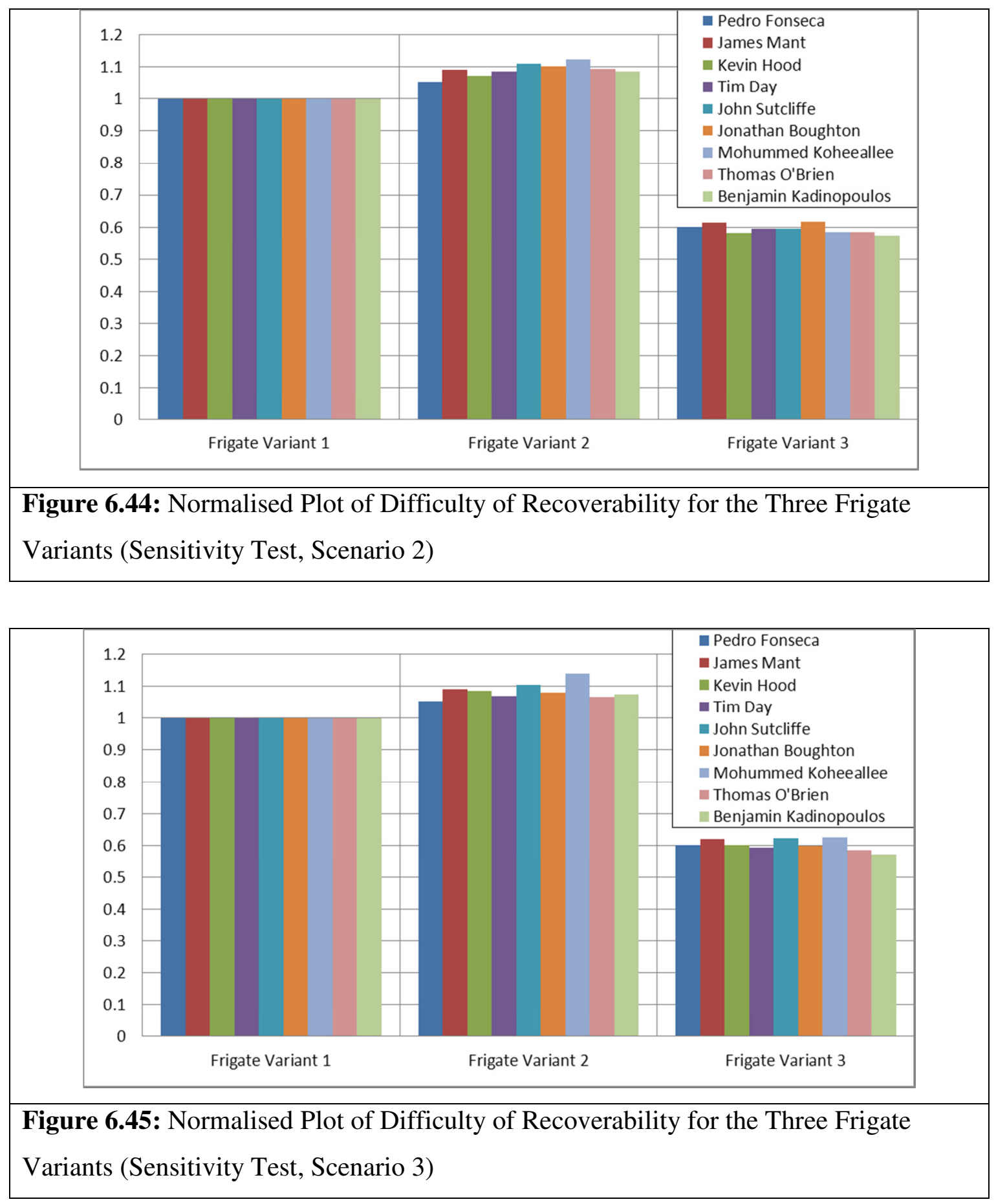

As in the study of the sensitivity of the vulnerability weighting scheme, the main observation from the three investigations above is that recoverability is dependent to a significantly greater extent on the characteristics of the ship design itself, rather than the scenario or the weighting scheme applied. Furthermore, despite the scenario independence of the weighting scheme provided by $1^{\text {st }} \mathrm{Lt}$. Fonseca, the results outputted are consistent with those that applied the RN officers' proposed weighting schemes. 


\subsubsection{Lengthwise Probability Hit Distribution}

Similarly to the vulnerability assessment approach sensitivity studies (Section 6.4.2), the next investigation regarding the recoverability approach concerned the examination of the sensitivity of the results with respect to the lengthwise probability hit distribution. This was done by using the linear lengthwise probability hit distribution, illustrated in Figure 6.23, in place of the normal distribution (Figure 6.4). This data was therefore substituted in Table 6.12, Table 6.13 and Table 6.14 and the procedure summarised in Sections 4.3 and 6.5 was followed. That is, raw PM data, given an ASM hit at each WT section (averaged port and starboard hit), was multiplied by the probability that the corresponding WT section is hit and after a series of normalisations, weightings and summations, the results illustrated in Figure 6.46 were obtained. This sensitivity study was also applied only to the frigate design variants.

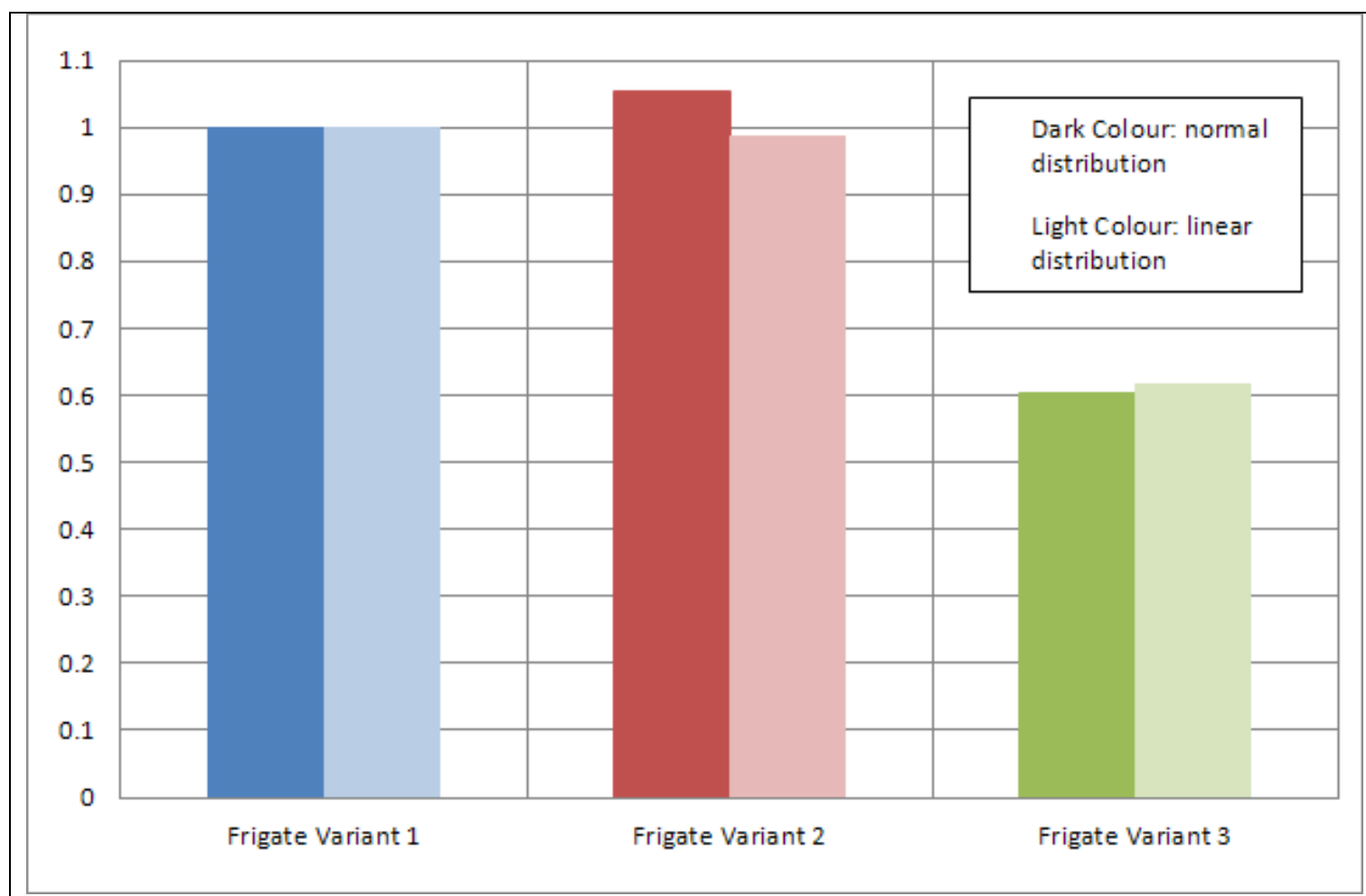

Figure 6.46: Normalised Plot of Difficulty of Recoverability for the Three Frigate Variants (Varying Lengthwise Hit Distribution)

From above it is evident that the lengthwise probability hit distribution does not significantly affect the recoverability performance of the frigate designs. The only point worth mentioning is that, with a linear distribution, the second variant improves its 
performance by approximately 7\%, although the trimaran variant still presents the most promising results by a significant margin (approximately $70 \%$ better).

\subsubsection{SURVIVE Lite Decoupling}

As detailed in Section 4.3, the SURVIVE Lite ship design models were used in order to indicate if a specified item (corresponding to a PM) had been affected by a specific hit; if it had, the PM would be given a value based on the assumed number of man-hours to repair that specific equipment, otherwise it would be equal to zero. One could argue that through this procedure vulnerability is incorporated in the recoverability assessment method (and, therefore, double-counted) rather than measuring recoverability independently. (Although, as indicated in Section 4.3, no vulnerability percentages were used in the recoverability assessment method since, in order to carry out recoverability assessment, vulnerability (and susceptibility) is required to be equal to unity.) For this reason, it was decided to repeat the recoverability assessment procedure for the three frigate design variants, this time assuming that an ASM hit at any WT section of the ship would affect all equipment and compartments modelled (with the exception of underwater equipment, i.e. propellers, rudders and hull mounted sonars, which were assumed to be unaffected by the abovewater threat studied). This then decoupled the recoverability method from the SURVIVE Lite vulnerability assessment software. Other than the above alteration, an identical procedure to that described in Sections 4.3 and 6.5 was followed in order to produce the PM matrices shown in Table 6.24, Table 6.25 and Table 6.26. As before, the weightings (in red) are the ones proposed by 1 st Lt. Fonseca for combatant type ships. The numerals in black are the raw PM data given an ASM hit at each WT section (assuming all items are affected), while the numerals in blue give the same raw PM data multiplied by the probability that the corresponding WT section is hit. All normalisation have been conducted with respect to Frigate Variant 1. 


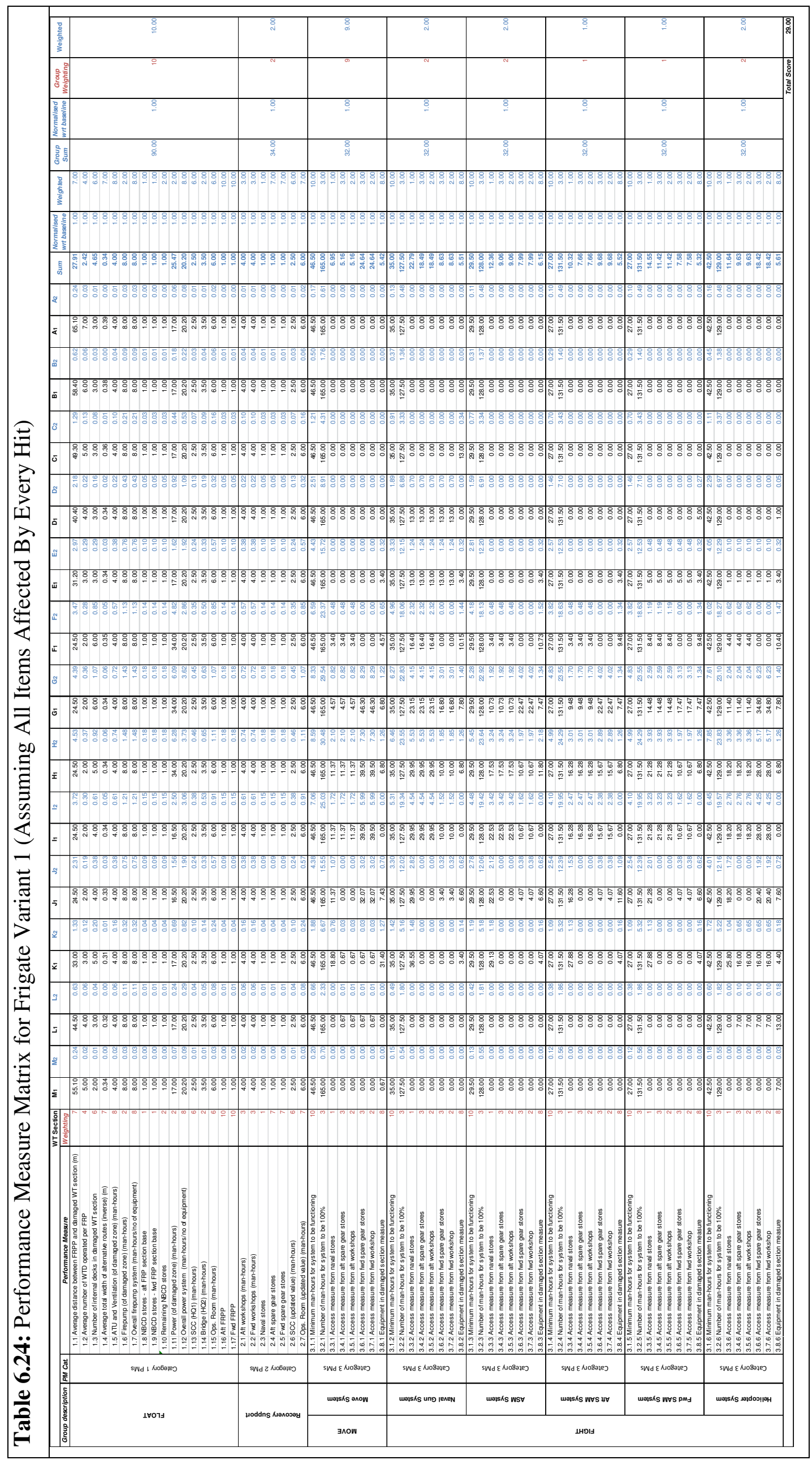




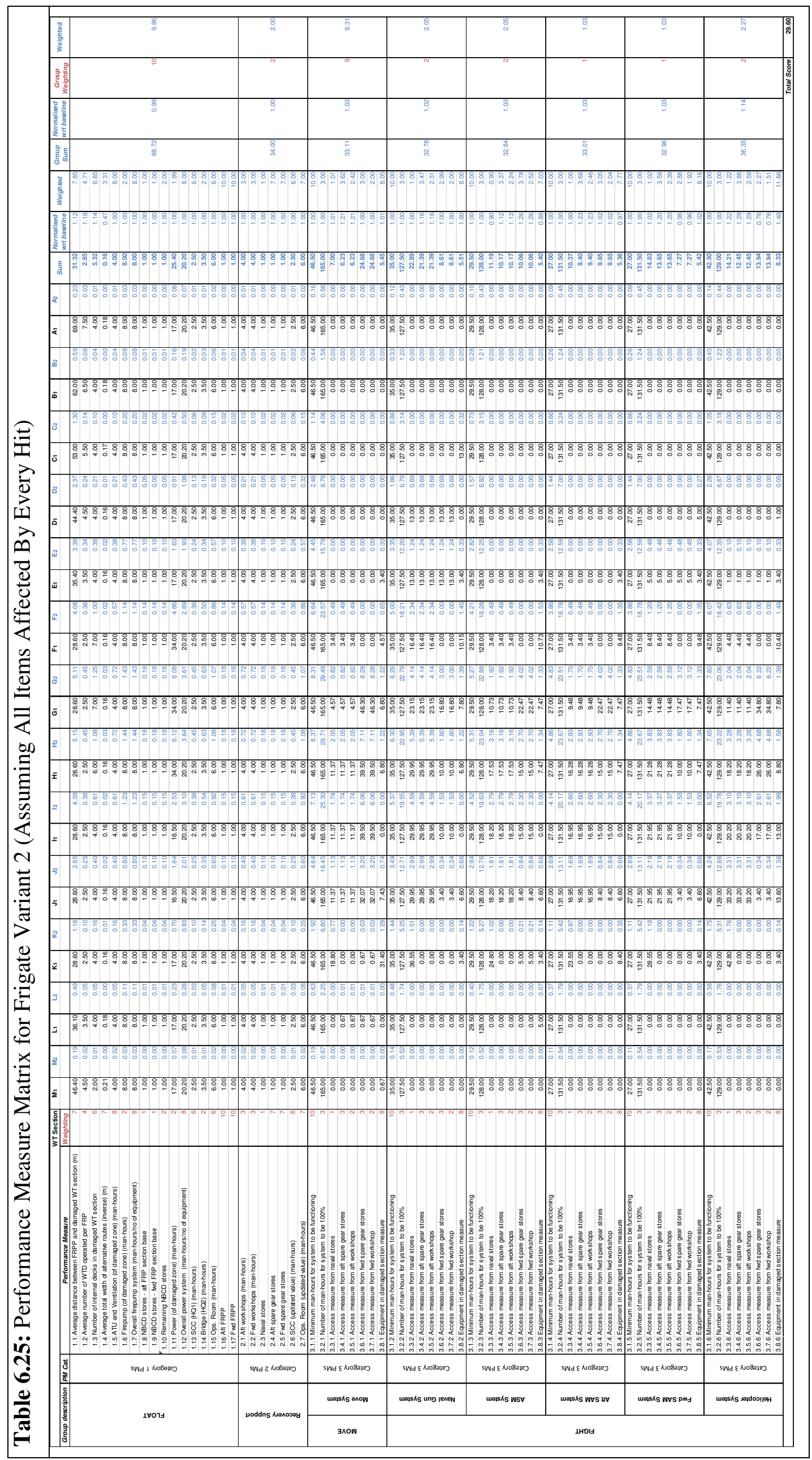




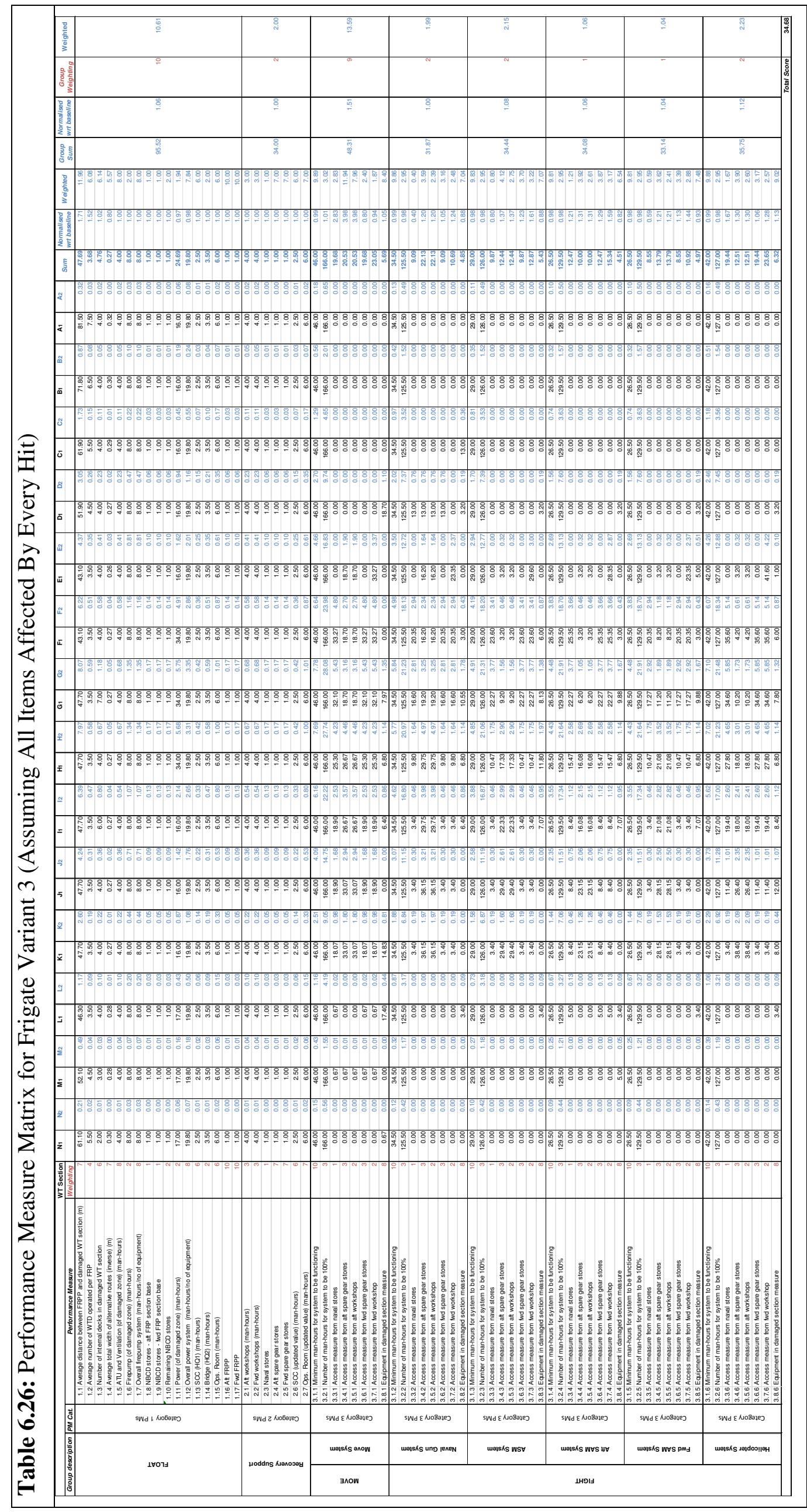


After the total score for each ship design was normalised with respect to the baseline total score, the total recoverability performance results shown in Figure 6.47 were obtained.

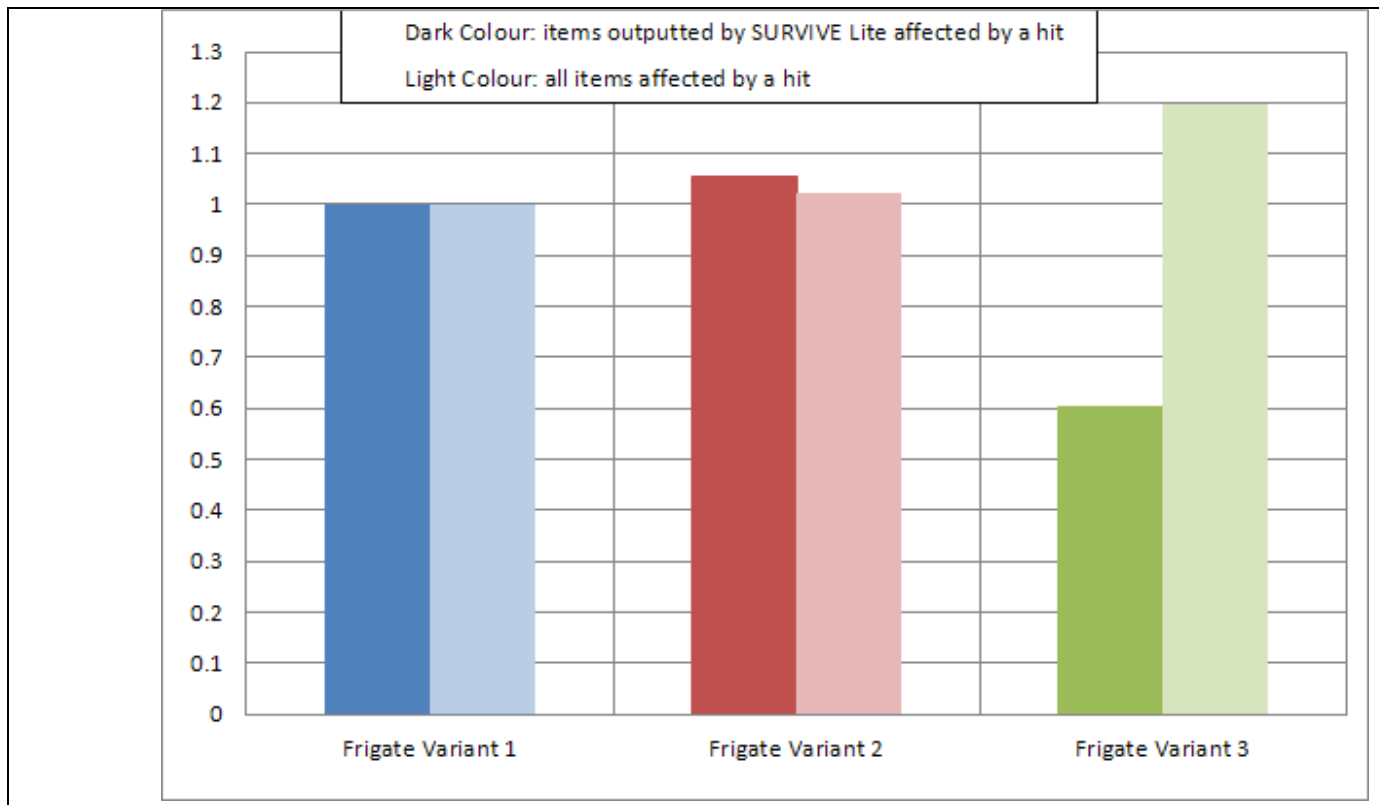

Figure 6.47: Normalised Plot of Difficulty of Recoverability for the Three Frigate Variants (Assuming All Items Affected By Every Hit)

Interestingly, this modification caused a $100 \%$ reduction in the recoverability performance of the trimaran frigate design, whereas the first two variants are very similar. Therefore, Frigate Variant 3 is now the worst performing design by approximately $20 \%$. Given that all three variants have identical systems and near identical system architectures (Appendix 7), the main cause for the trimaran resulting in worse results than the two monohulls is associated with the location of its stores and workshops in relation to the move system. However, the procedure followed above raises some scepticism linked to the assumption that a given threat would affect (and, therefore, recovery would be dependent on) the same items in two different designs.

\subsection{Total Survivability Assessment Results}

As mentioned in Section 4.4, it was decided to combine the results of the three survivability constituents in the form of star plots. The total survivability star plot for the three frigate design studies is illustrated in Figure 6.48. The data presented below are identical to the survivability constituent results presented in Figure 6.3, Figure 6.25 and Figure 6.39 and are, therefore, normalised with respect to the baseline frigate. Each 
star (triangle in this case) represents a specific ship design and the smaller the area of the triangle, the more survivable against an ASM threat that ship design is.

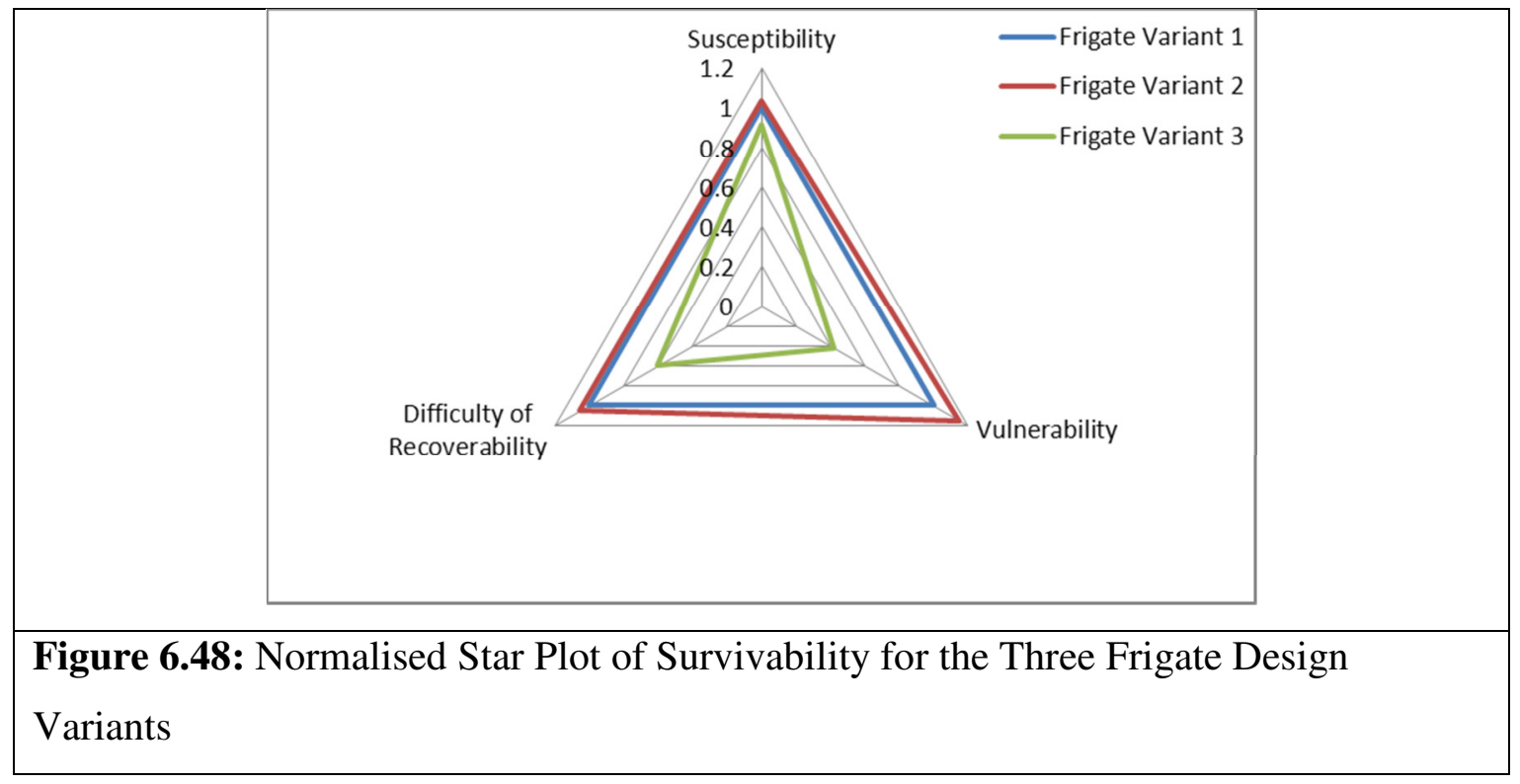

Similarly, Figure 6.49 is the total survivability star plot of the Corvette and Destroyer design studies, including Frigate Variant 1 against which the results (Figure 6.7, Figure 6.27 and Figure 6.40) were normalised.

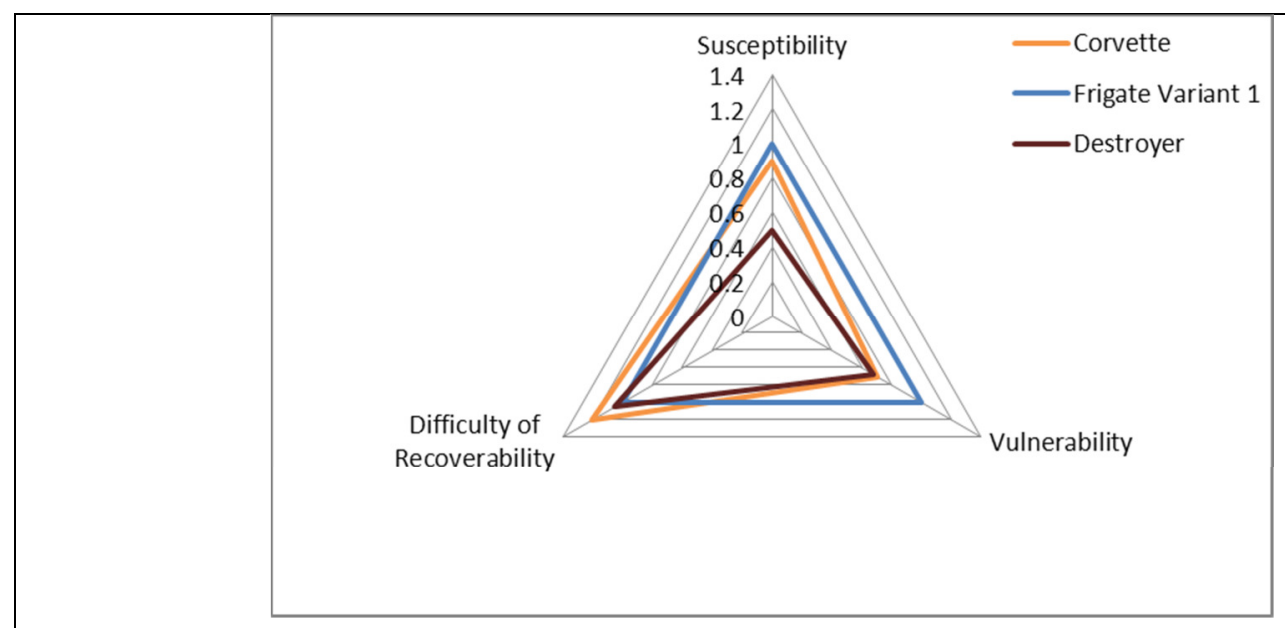

Figure 6.49: Normalised Star Plot of Survivability for the Corvette, Baseline Frigate and Destroyer

Finally, the total survivability star plot of the two auxiliary ship designs is illustrated in Figure 6.50. As already pointed out, the survivability results of these two design studies (Figure 6.11, Figure 6.29 and Figure 6.41) have been normalised with respect to the worst performing, rather than a baseline design. 


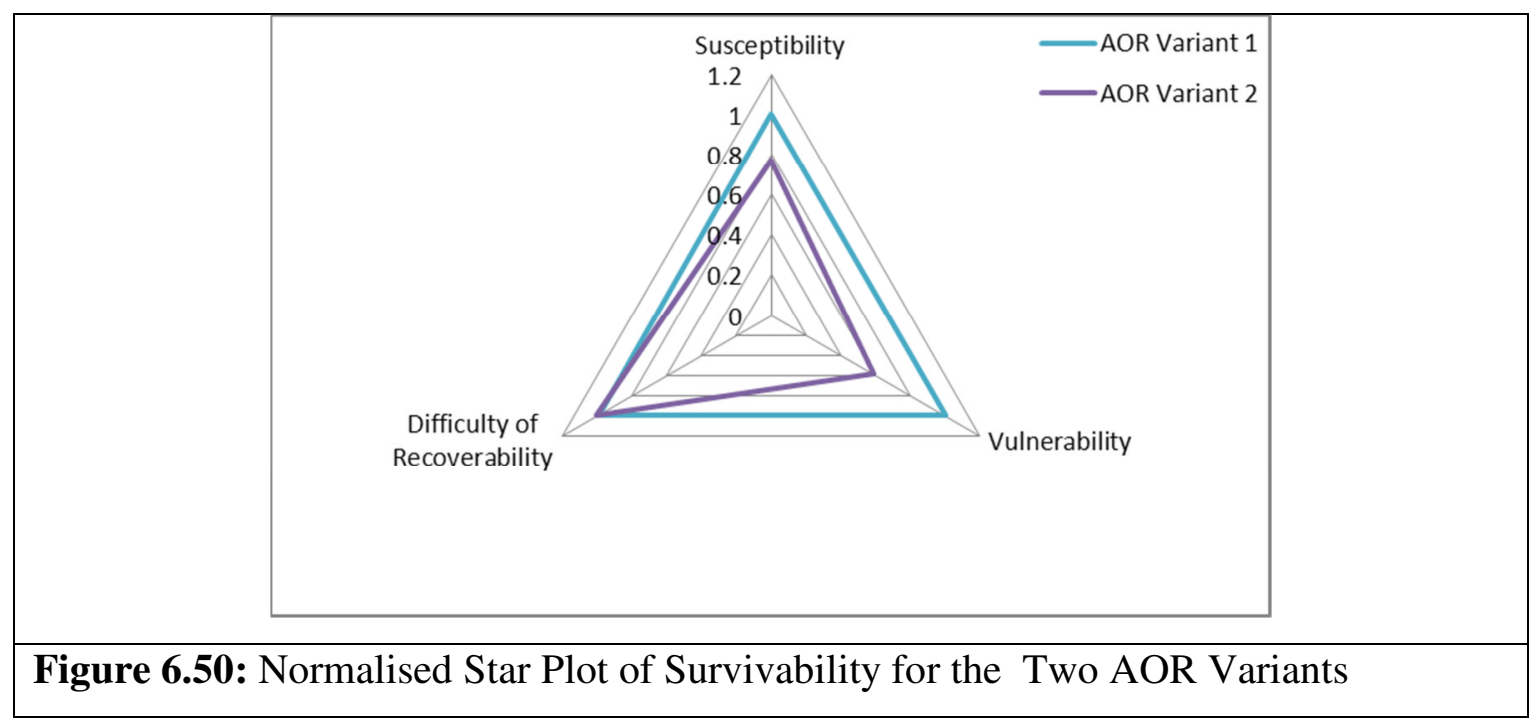

Moreover, it was decided to investigate the applicability of PM matrices for total survivability assessment. The procedure followed, detailed in Section 4.4, is similar to that for the recoverability assessment method, although total ship susceptibility and equipment vulnerability data were combined. This procedure was only carried out for the three frigate variant designs, and the results are illustrated in Figure 6.51. In Figure 6.51 , the higher the score, the worse the performance, consequently, the difficulty of a given design to survive a given threat is measured; furthermore, all results have been normalised with respect to the baseline frigate as was the norm.

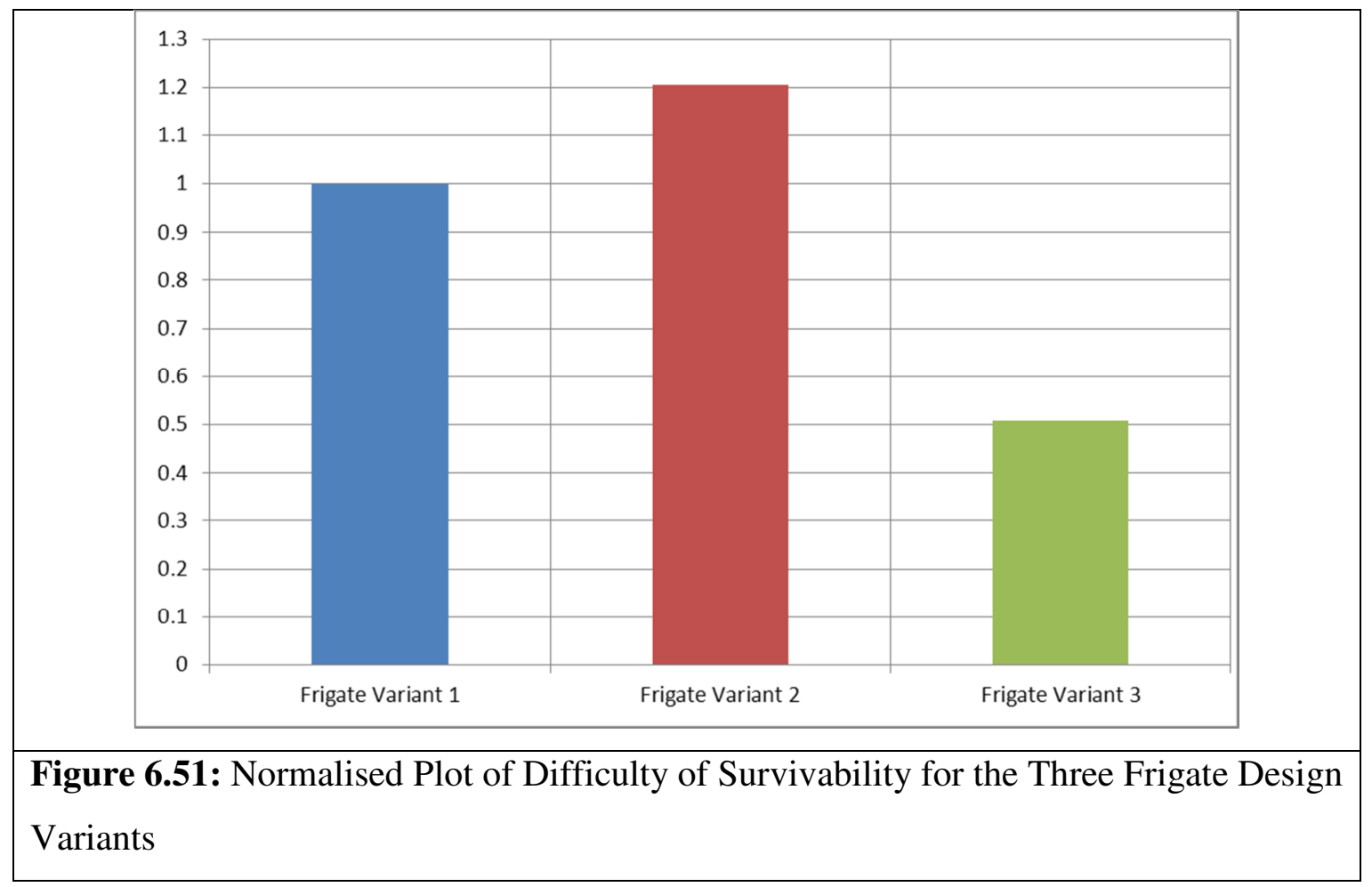


Although the results revealed above are in agreement with the frigate designs' total survivability star plot in Figure 6.48, the appropriateness of such a procedure to estimate total ship survivability is questionable. Not only because of the multiplications involved between the survivability constituents connoting statistical independence (refer to Sections 3.3.5 and 4.4) which is not the case in the star plots, but also the PM matrices were specifically designed to examine recoverability, rather than total survivability.

\subsection{Ship Costing Results}

Cost analysis for the three frigate variants was undertaken in order to give an indication as to how survivability gains could be measured against ship cost. A brief discussion on ship costing is given in Section 2.2. Since it was beyond the scope of this research to create a new or modify a current naval ship costing method, it was decided to follow the procedure and use the parametric relationships and costing data given by UCL (2010a; 2010b).

\section{$\underline{\text { 6.8.1 Unit Procurement Cost }}$}

The parametric naval ship UPC relationships used are specific to the $4^{\text {th }}$ ship of a class of twelve, designed to RN standards in 2008 prices and are in accordance with the UCL weight and space classification system (UCL 2010b). It should be noted that all cost analyses were carried out using 2008 prices (and, therefore, the results presented are in 2008 prices). The equation used in order to calculate the cost of a weight group, $\mathrm{C}_{\text {grpA, }}$ (with the exception of specific items, i.e. engines, electric motors and payload items) was (UCL 2010b):

$$
\mathrm{C}_{\text {grpA }}=1.15 \times \mathrm{MC}_{\text {grpA }} \times \mathrm{W}_{\text {grpA }}+\mathrm{HR} \times \mathrm{LR}_{\mathrm{grpA}} \times \mathrm{W}_{\text {grpA }}
$$

1.15 is the purchasing overhead factor used, $\mathrm{MC}_{\mathrm{grpA}}$ is the material and equipment cost in $£ /$ te, $W_{\text {grpA }}$ is the mass of the weight group, $\mathrm{HR}$ is the hourly labour rate in $£ / h r$ (assumed as $£ 0.05016 \mathrm{k} / \mathrm{hr}$ for UK shipyards) and $\mathrm{LR}_{\text {grpA }}$ is the required labour in manhours/te for that group (UCL 2010b). Detailed cost data for all weight groups and specific items are presented in the Appendices A13.1 for all three frigate designs. It should be noted that where cost data for specific items was inaccessible, data for similar 
equipment was used. In addition, if the data obtained was not in 2008 levels, it was converted using RPI annual inflation data provided by (ONS 2012). After calculating the cost of each weight group, the cost margins listed in Table A57 (see Appendix 13.1) were applied. The UPCs of the three frigate variants were calculated as approximately $£ 294 \mathrm{M}, £ 300 \mathrm{M}$ and $£ 308 \mathrm{M}$ respectively (with detailed results presented in Appendix 13.1). Figure 6.52 below illustrates the proportion of each weight group on the UPC of the three frigate design studies.

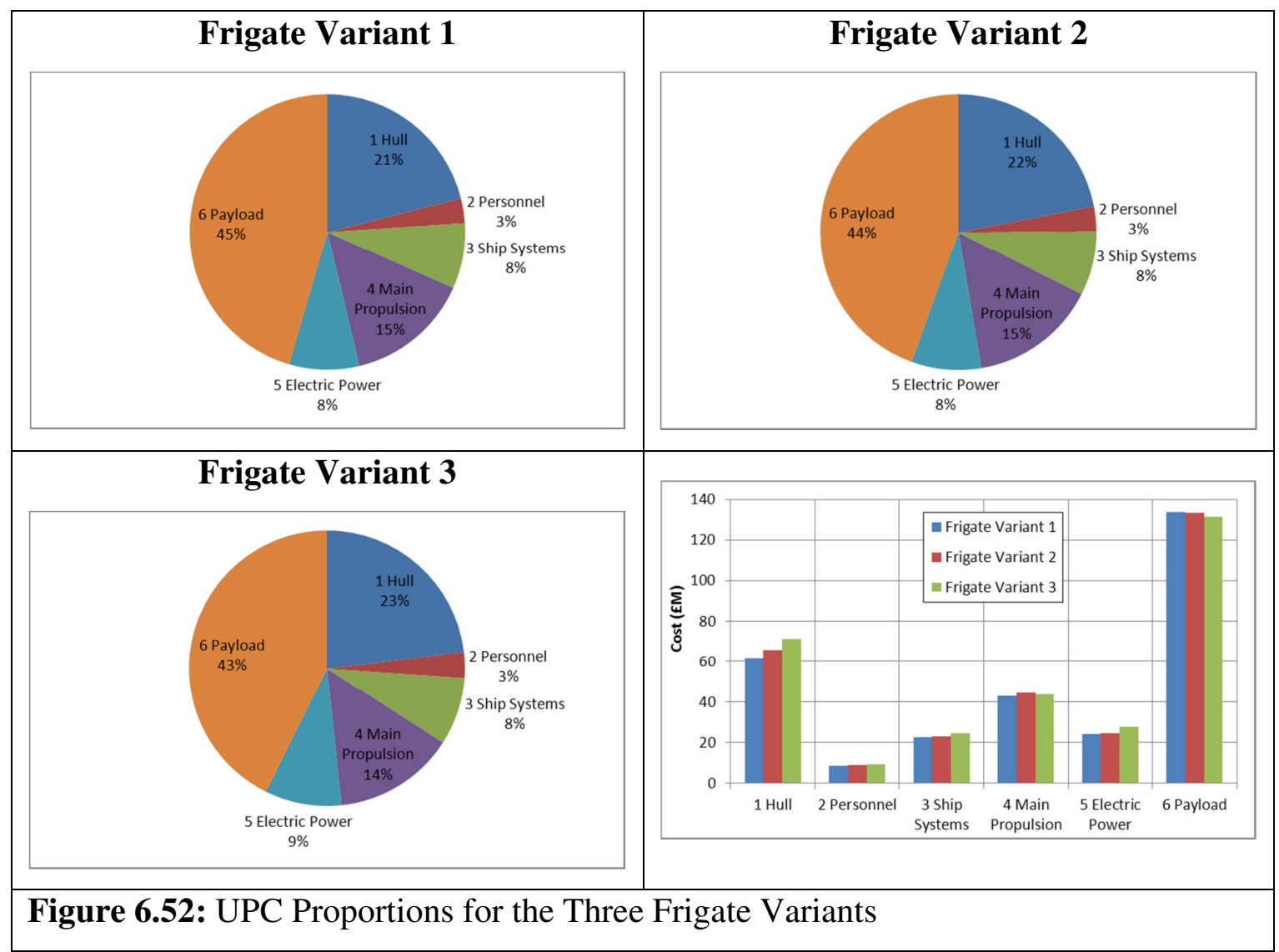

From the data and results presented above, the general observation is that UPC is not greatly affected (at least, to a much lesser extent than survivability performance) by ship configuration.

\subsubsection{Through Life Cost}

A number of TLC related costs were calculated for the three frigate designs.

Annual fuel consumption was calculated by assuming an annual operational period of 200 days and the simplified operational profile illustrated in Figure A107 (see Appendix 13.2), similar to that used by Dirksen (1996). The total annual fuel 
consumption for the three frigates was calculated as approximately 6,290te, 6,590te and 6,500te respectively. MDO prices were forecasted by plotting historical data from (MOT 2013) (and using $£$ to $\$$ exchange rates from (X-Rates 2012; BGFRS 2013)) and fitting an exponential trendline (see Figure A108 in Appendix 13.2). The resulting exponential relationship used to forecast MDO prices was:

$$
\text { MDO Price (Year), in } £ / \text { te }=4 \times 10^{-88} \times \mathrm{e}^{0.1032 \times \text { Year }}
$$

Although the equation above contains vast uncertainties, it is believed that it is more than sufficient for the comparative study being undertaken. Further research on future fuel prices is beyond the scope of this project. Moreover, a discount rate, r, of $6 \%$ was assumed (Dirksen 1996), where the discount factor is defined by Dirksen (1996) as "the increase in value of money, above inflation, over time, i.e. if the cost of a system or equipment is fixed it will effectively cost less if it is paid for later than sooner due to the fact that the cash can be invested and earn interest in the interim". Therefore, the present value, PV, of the future costs, F, (at year n after 2008, assumed year of start of construction) was calculated through the following relationship (Dirksen 1996; UCL 2010b):

$$
\mathrm{PV}=\mathrm{F} \div(1+\mathrm{r})^{\mathrm{n}}
$$

Detailed tables of forecasted fuel costs for the three frigate variants predicted using Equations 8 and 9 are presented in Appendix 13.2.

Crew costs were estimated by assuming the simplified salaries presented Table A61 of Appendix 13.2. Given the complement of each ship design (Table A2, Table A3 and Table A4 in Appendix 9.1) annual crew costs for the three frigate variants were estimated at $£ 3.18 \mathrm{M}, £ 3.23 \mathrm{M}$ and $£ 3.49 \mathrm{M}$ respectively. Rather than using a relationship similar to Equation 8, an annual inflation of $4 \%$ was assumed for crew costs. The present value was then calculated using Equation 9, with through life crew costs for the three frigates presented in Appendix 13.2.

The costs of consumables were forecasted using the data in Table A62 of Appendix 13.2. As with crew costs, the total annual consumable costs were calculated for each frigate design given the frigate complement $(£ 0.53 \mathrm{M}, £ 0.54 \mathrm{M}$ and $£ 0.58 \mathrm{M}$ respectively), inflated annually by $4 \%$ and discounted using Equation 9. Detailed results are presented in the Appendix 13.2. 
In order to calculate the annual maintenance costs, the data presented in Table A63 of Appendix 13.2 was utilised. This then led to an annual maintenance cost of $£ 4.40 \mathrm{M}, £ 4.40 \mathrm{M}$ and $£ 4.42 \mathrm{M}$ for each frigate variant respectively. After inflating and discounting the annual maintenance costs in a similar manner as described above, the through life maintenance costs presented in Appendix 13.2 were obtained.

The final TLC element is related to the costs associated with refits. It was assumed that major refits occurred every eight years with minor refits between major ones (UCL 2010b). Table A64 of Appendix 13.2 lists assumed cost data for refits. The hourly labour rate used was the same as in the UPC estimation section, i.e. $£ 0.05016 \mathrm{k} / \mathrm{hr}$ for UK shipyards (UCL 2010b). After applying the above relationships, the costs of major refits were estimated at $£ 52.12 \mathrm{M}, £ 53.02 \mathrm{M}$ and $£ 54.26 \mathrm{M}$ and minor refits at $£ 18.71 \mathrm{M}, £ 19.01 \mathrm{M}$ and $£ 19.43 \mathrm{M}$ for the three frigate designs respectively. By assuming that the vessel construction will take place over a period of 3 years with trials and commissioning completed in the 4th year (Dirksen 1996), the dates of planned refits were estimated and the associated costs were inflated and discounted accordingly, in the manner described above. Detailed forecasted refit associated costs are presented in Appendix 13.2.

The total TLCs for the three frigates were than calculated by assuming a service life of 30 years. Annual cost expenditure for the three frigate designs is presented in Figure 6.53 (and in Appendix 13.2). Although UPC is not part of TLC, UPC values (of the $4^{\text {th }}$ vessel in a class of twelve, as calculated above) are included in Figure 6.53. It was assumed that $30 \%$ of the total UPC is paid in each of the first 3 years with the remaining $10 \%$ paid by the end of the 4 th year (Dirksen 1996). 


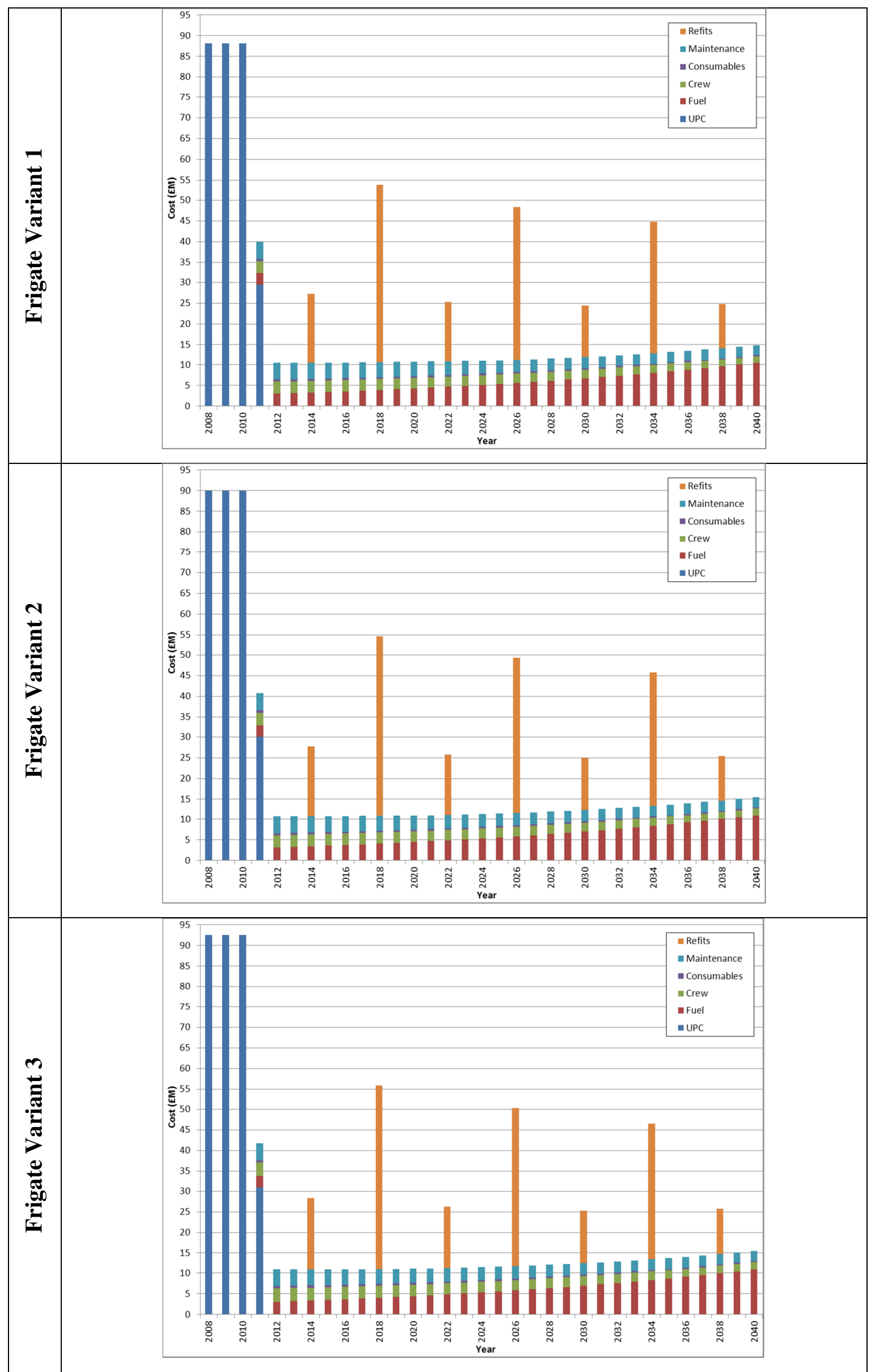

Figure 6.53: Annual Costs for the Three Frigate Variants 
TLC can also be separated into maintenance costs (consumables, maintenance and refits costs) and cost of ownership (fuel and crew costs) and compared to UPC, as illustrated in Figure 6.54.

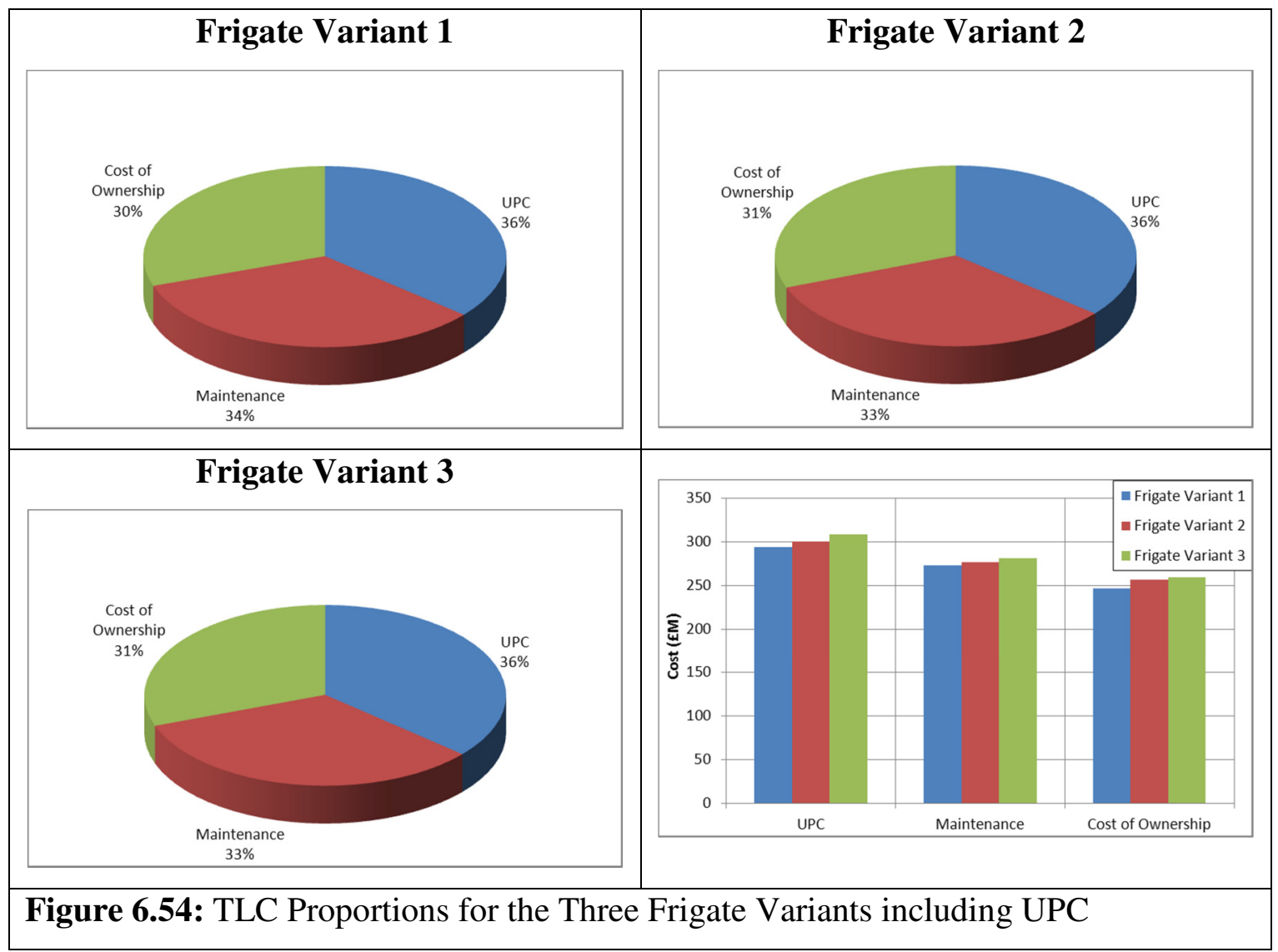

From the above data and analysis it follows that the TLC for the three frigate variants is equal to $£ 519.44 \mathrm{M}, £ 532.28 \mathrm{M}$ and $£ 540.45 \mathrm{M}$ respectively. Once again, the general observation is that ship cost (in this case TLC) is not as sensitive as survivability performance to variations in ship configuration.

\subsubsection{Whole Life Cost}

The final and most meaningful cost to be estimated is the whole life cost. This is calculated by combining the UPC, TLC, FOC costs and ship disposal costs of a class. First, it was assumed that the frigate class consisted of twelve ships in total and the UPC previously calculated was representative of the fourth vessel (UCL 2010b). Due to the shipyard learning curve, it is recognised that longer production runs may lead to reduced UPCs due to reasons such as increases of economies of scale brought about by increasing the amount of materials and number of equipment items purchased and 
improvements in production planning and processes in the shipyard as they become familiar with the design (UCL 2010b). The following relationship was used to estimate the UPC of each of the twelve vessels (UCL 2010b):

$$
\mathrm{UPC}_{\text {shipn }}=\mathrm{UPC}_{\text {ship } 4} \times 1.16 \times \operatorname{ship}_{\mathrm{n}}^{-0.105}
$$

Furthermore, the TLC of all vessels was assumed constant, i.e. no learning curve was assumed. FOC costs, or design and construction services, were assumed to be equal to $20 \%$ of the UPC of the $4^{\text {th }}$ vessel for a naval ship (UCL 2010b). This is a one-off cost including elements such as office setup, design, drawings, recruitment and administrative costs (UCL 2010b). Moreover, by assuming that all ships will be recycled within the UK when decommissioned (33 years after construction begins, Figure 6.53), a disposal cost of $£ 0.68 \mathrm{M}$ per ship was assumed (UCL 2010b). This value was inflated by applying the assumed annual $4 \%$ inflation and discounted annually by $6 \%$ as above, in order to get the present value of the disposal cost at the year of decommissioning. Finally, it was assumed that all twelve ships of the class (of each of the three frigate variants) were constructed and disposed of in the same years. This is clearly unlikely; however, it was assumed during this comparative study for simplification purposes and does not alter the essential WLC comparison. Figure 6.55 illustrates the relative proportions of all WLC elements mentioned above (detailed results are presented in Appendix 13.3). 


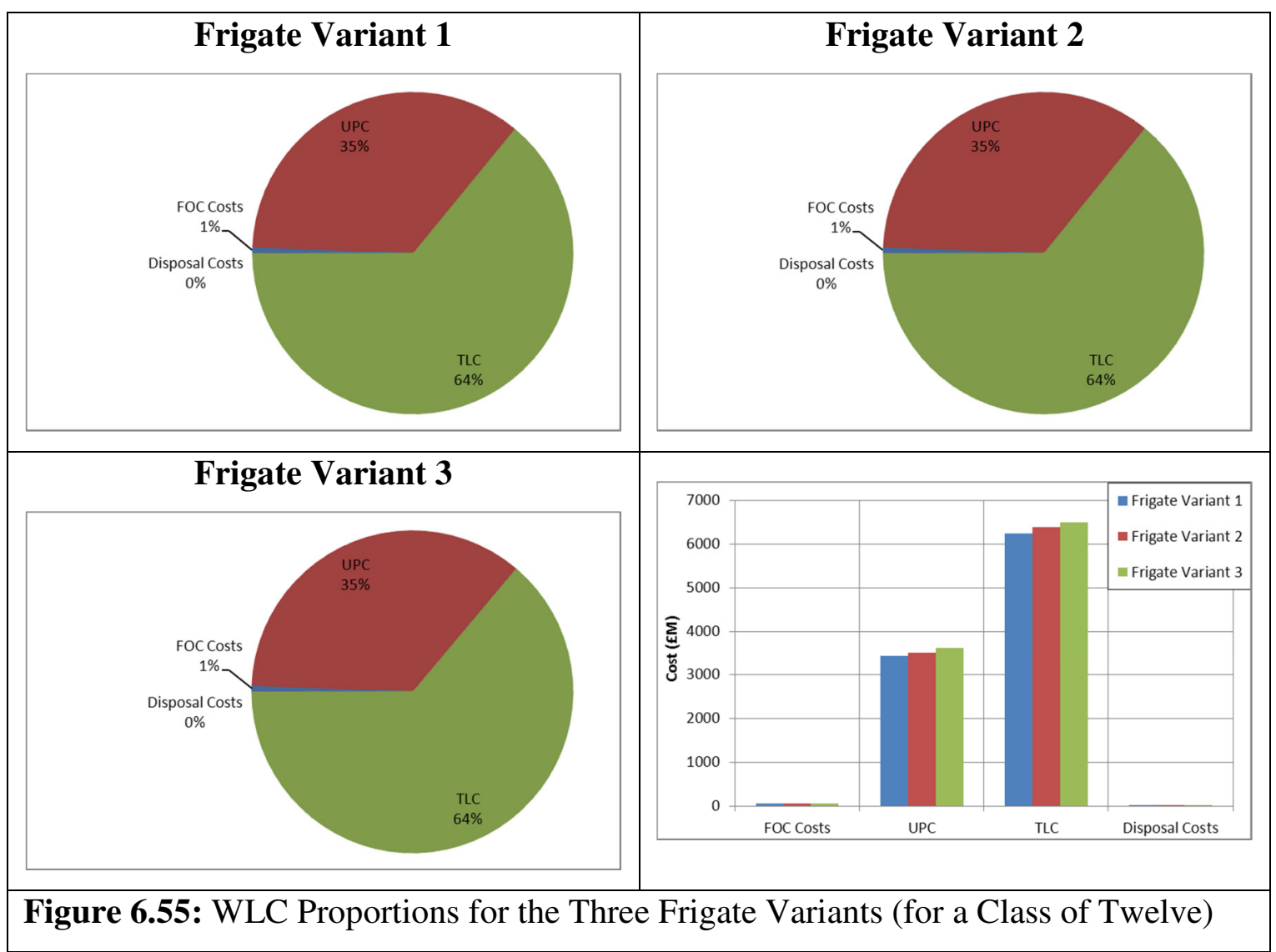

From above, the WLCs were estimated at $£ 9,740 \mathrm{M}, £ 9,970 \mathrm{M}$ and $£ 10,160 \mathrm{M}$ for each frigate variant class respectively. From the above results it is observed that WLC is not greatly affected by ship configuration. This was expected since WLC is almost entirely dependent on UPC and TLC, for which the same observations were made. This is additional confirmation that ship configuration has a greater influence on survivability as opposed to ship costs. 


\section{Chapter 7: Discussion}

The first five sections of this chapter discuss the analysis of the results reported in Chapter 6. The results were produced by applying the developed survivability assessment method (Chapter 4) to the ship design studies summarised in Chapter 5, as well as through the costing study undertaken. The final section of this chapter links the developed method to the research background (see Chapters 2 and 3). This is done to address whether the research aim (Chapter 1) has been met, to assess the extent to which that aim has been achieved and also to identify areas requiring further work.

\subsection{Susceptibility Assessment Results Analysis}

\subsubsection{Frigate Variants (Including Baseline)}

The susceptibility results produced for the three frigate variants are essentially the same since all ship designs had similar RCSs and identical defensive systems to counter the same postulated threat. The minor differences in the susceptibility results are solely caused by the small variations in RCS (and therefore, P(l)). These differences are minor since similar RCS reduction techniques were used during the design phase of the frigate variants; namely, the application of a $7^{\circ}$ slope to all superstructure sides and certain equipment and the placing of as much likely microgeometry as possible behind bulwarks. The small differences in the RCS values observed between the three frigate variants were caused by the variations in hull, superstructure and upperdeck arrangement between the three designs. Further analysis to identify the specific causes of these variations was not conducted for a number of reasons. For example, the variations are too small (less than 15\%) to justify any further work; a large amount of work has already been done in RCS reduction techniques and validated through full scale tests (see Section 3.3.2), therefore, being beyond the scope of this research; and most importantly, the RCS prediction software used, SPECTRE could not be accessed daily, since it was operated by the sponsor of this research (Dstl) rather than UCL itself. However, certain variations in susceptibility relevant features reported in Section 6.2 were investigated. These included alterations to microgeometry $\left(7^{\circ}\right.$ rotation of the RAS high points in Frigate Variant 1) and shaping (application of a $7^{\circ}$ flare on specific sections of the box structure and side hulls of Frigate Variant 3). The resultant improvement in the $\mathrm{P}(1)$ values for these designs (60\% and $75 \%$ respectively) seemed to 
match indications from adopting currently accepted RCS reduction techniques (Appendix 4.1) and justifies the efforts made in signatures reduction of current naval ships. In addition, it can be concluded that both hull and superstructure shaping and microgeometry management should be employed as extensively as possible.

It was also demonstrated that susceptibility results were to a large degree sensitive to the targeting pattern of the defensive missiles. By assuming that each pair of defensive missiles launched by the targeted ship could only home in on one attacking ASM, the frigate designs, each launching four defensive missiles, could only target two of the four attacking ASMs. If, however, each defensive missile was able to target a separate attacking ASM, all four ASMs could be targeted improving the $\mathrm{P}(\mathrm{h})$ by almost $40 \%$. This highlights the importance of selecting an appropriate defensive missile targeting pattern, which largely depends on the characteristics of the incoming threat, the prediction of which is extremely challenging.

\subsubsection{Corvette, Baseline Frigate and Destroyer}

When carrying out the comparative study between the Corvette, baseline frigate and Destroyer designs, larger variations in the results were observed. As expected, the Corvette design had the smallest RCS (and therefore, $\mathrm{P}(1)$ ) out of all combatant designs, due to its smaller size. However, the difference between the RCS of the Corvette design and the three frigate variants was relatively small (between $2 \%$ and $15 \%$ ). Conversely, the Destroyer design had the largest RCS by a significant margin, therefore, effectively rendering the use of a decoy system against the ASM threat of no use. The large RCS increase is not only attributed to its larger size, but also to the different equipment selected (e.g.: the two spherical, therefore, poor in terms of RCS, MFR Spectars and the larger helicopter) and the increased microgeometry (e.g.: the four additional $12.7 \mathrm{~mm}$ machine guns and two CIWSs) summarised in Table 5.4 of Section 5.2. Again, however, further analysis to identify detailed RCS characteristics was not conducted mainly since the RCS prediction software, SPECTRE, could not be accessed daily, although it would be of interest to detect the specific features (and their effect) that cause the approximately quadrupling of the Destroyers RCS, compared to the remaining combatants.

The second large variation between the Corvette and baseline frigate designs and the Destroyer design was related to the $\mathrm{P}(\mathrm{h})$. The first two designs, having identical AAW system, gave equal results. However, the much more capable AAW system of the 
Destroyer design led to a substantial improvement decreasing the $\mathrm{P}(\mathrm{h})$ by almost $90 \%$. This is the largest effect that a single survivability feature has presented out of all features investigated in the current project and more than eliminates the effect of the larger Destroyer RCS. Therefore, the Destroyer design is approximately two times less susceptible than the Corvette and baseline frigate, which present similar susceptibility characteristics to each other.

The Destroyers $\mathrm{P}(\mathrm{h})$ characteristics are further improved by approximately $30 \%$ if each defensive missile is allowed to independently target an attacking missile (rather than in pairs). The Destroyer, capable of firing eleven defensive missiles against the attacking ASMs, was initially assumed to target the first attacking missile with four defensive missiles, the second attacking missile with three defensive missiles and the remaining two attacking missiles with two defensive missiles each. By altering this pattern to three, three, three and two defensive missiles, the above improvement was observed. Finally, a further improvement was observed with the utilisation of longer range missiles, also having a larger maximum speed. These missiles, however, were limited by the ship design's radar horizon, therefore, limiting the $\mathrm{P}(\mathrm{h})$ improvement to approximately $8 \%$ (due to the additional defensive missile fired, totalling twelve). If, however, the limiting factor was not the radar horizon, the improvement to $\mathrm{P}(\mathrm{h})$ would be approximately $90 \%$ (due to the capability of firing eighteen defensive missiles), although this scenario is questionable given the sea-skimming nature of the examined threat.

\subsubsection{AOR Variants}

The most prominent variation regarding the susceptibility assessment of the two AOR variants is the much larger RCS of the first variant (approximately 3.7 times the RCS of the second variant) although both designs are similar in size and identical susceptibility reduction techniques were used for both. This large difference was caused by the two large RCS peaks towards the stern of AOR Variant 1 (not occurring in the second variant), at an azimuth of approximately $160^{\circ}$ and $200^{\circ}$, as shown in Figure 6.9 of Section 6.1.3. In order to identify the causes of these large peaks the designs were resent to Dstl and rerun in SPECTRE. Although the further analysis was not conclusive, it was observed that the forward RAS rig of AOR Variant 1 gave an unexpectedly high RCS when viewed from the above azimuth angles; it was suggested that the two peaks were caused by a distributed corner reflector (i.e. two or three facets that form a corner, 
but do not share a common edge) which typically causes RCS peaks of the magnitude and width observed (Patient 2013). Therefore, corrective design action could possibly eliminate this occurrence. However, the overall effect of the large RCS difference between the two variants on the $\mathrm{P}(\mathrm{l})$ was not proportional. In a similar manner to the Destroyer design, both AOR variants had an RCS close to or more than the $4000 \mathrm{~m}^{2}$ limit (i.e. twice that of the assumed chaff RCS) above which, in accordance with Equation 4, a constant $\mathrm{P}(\mathrm{l})$ equal to unity, was employed. Therefore, the larger RCS of AOR Variant 1 resulted to a worsening of $\mathrm{P}(1)$ by some $30 \%$. It follows that signature management loses its importance with ships of increasing signatures, unless decoy systems of much greater effectiveness can be utilised.

It is of interest that the AOR design variants (which included identical defensive systems and, therefore, $\mathrm{P}(\mathrm{h})$ values) gave $\mathrm{P}(\mathrm{h}) \mathrm{s}$ similar to the frigate variants and Corvette design, although the AOR designs had less capable AAW systems. In fact, the auxiliary ship designs were capable of firing only three defensive missiles against the four attacking ASMs, one less than the frigate variants and Corvette design. The similarity between the two $\mathrm{P}(\mathrm{h})$ values is observed solely due to the defensive missile targeting pattern adopted. The Corvette and frigate designs and the AOR designs were assumed to only target two of the attacking ASMs with their defensive missiles, as explained in Section 6.1. However, by allowing each defensive missile to independently target an attacking ASM, the Corvette and frigate variants would be capable of targeting all four attacking missiles, with the impressive $40 \%$ increase in effectiveness. Conversely, the AOR designs, launching only three defensive missiles could only target a maximum of three of the total four attacking ASMs. Although the $\mathrm{P}(\mathrm{h})$ value for the AOR designs was slightly improved by approximately $8 \%$, the $40 \%$ improvement in the combatants could not be matched.

When comparing the auxiliary ship designs with the combatant designs it is easily observed that the AORs are (at least three times) more susceptible to a seaskimming ASM threat mainly due to their greater signatures than the combatants.

\subsubsection{General Observations on Susceptibility Assessment}

One of the main differences between susceptibility assessment and vulnerability and recoverability assessment is that for the former, given the large variety of threats currently existent, a single method is not sufficient. A large number of tools are required in order to assess the susceptibility of a design to as many likely threats as possible. In 
contrast, a single tool to assess vulnerability, such as QinetiQ's SURVIVE software which can model the different current weapon mechanisms, and to assess recoverability should be adequate. However, in order to demonstrate the developed survivability assessment method, a specific scenario was chosen, that of four sea-skinning ASM against a ship design. A number of unclassified techniques were combined in order to simulate this scenario. Certain relationships, data and assumptions employed in this part of the method (summarised in Section 4.1 and 6.1), are considered to be oversimplistic. In addition to the assumptions mentioned in the above sections, it is useful to catalogue a number of points regarding the susceptibility assessment method. These were revealed from discussions with susceptibility experts at Dstl:-

- The ASM detection range is affected by the type of sensor and threat;

- The range of detection of a sea-skimming ASM is not equal to the maximum range of the defensive missile (which is usually relevant for larger targets at high altitudes, such as aircrafts);

- The speed profile and, therefore, manoeuvrability of the defensive missile varies with time and flight altitude;

- The defensive missile performance depends on the flight angle relative to the ASM (see Figure A15 and Figure A16 in Appendix 4.1);

- Jammers are not very useful as attacking missiles can home onto them; however, it is possible to launch the jammer away from ship via rocket/parachute;

- Different soft-kill techniques exist for different targets;

- The relationship between ship and decoy RCS is not linear (as assumed in Figure 4.2 and Equations 3 and 4 of Section 4.1). It is possible to manoeuver the ship in order to present the lowest RCS possible; so manoeuvrability (including speed and acceleration) of the ship plays an important role in susceptibility;

- Decoys are usually of similar and/or larger RCS than that the ship;

- Susceptibility is personnel dependent (as analysed in the next paragraph). For example an auxiliary ship would have less specialized people to deal with threat situations and deploy defensive measures;

- Chaff effectiveness depends on ASM sensor frequency.

It is therefore evident that not only a large variety of threats currently exists, but each of these involves a large number of variables, highlighting the challenges in susceptibility assessment. Numerous such assessment methods already exist; however, since they largely rely on classified weapon data, they are not able to be readily accessed. Thus the method outlined in Section 4.1 was selected and judged sufficient for the purposes of 
this research (since it was beyond the scope of this research to develop a new and improved susceptibility assessment method; rather, it was primarily about an integrated survivability approach at preliminary ship design).

Furthermore, it is evident that the susceptibility assessment method applied examines the susceptibility of the ship design, without, however, investigating situational awareness, which forms an important part of susceptibility performance. One of the outcomes of the above statement was the assumption of $\mathrm{P}(\mathrm{di})$ equalling 1 for all ship design studies. The probability of a ship being detected and identified largely depends on situational awareness and operational factors (rather than the ship design itself). It could be argued that the ship with lower signature would be more difficult to detect, therefore, it is possible that the susceptibility trend shown on the normalised susceptibility graphs (Figure 6.3, Figure 6.7 and Figure 6.11 in Section 6.1) would increase.

Situational awareness can be split into two sub-categories:-

- Ship situational awareness, being affected by such factors as sensor type, height and wavelength;

- Human situational awareness, depending upon the ship command's response to the situation, other human factors, manning, experience, length of watch, doctrine and training (including simulations and exercises used for training), scenario, aims and rules of engagement (e.g.: the target has to be identified before engaging it, so usually no action is carried out until hostile act occurs, such as with Fast Inshore Attack Crafts which may not pose an obvious threat).

It could be argued that the susceptibility method adopted accounts for ship situational awareness. However, this is not the case with human situational awareness. This is particularly important with asymmetric threats and it may be argued that such threats are not captured by the ASM attack scenario. Asymmetric threats usually occur in littoral (as opposed to blue) waters where the cluttered picture and the reduced reaction times, as well as the minimum range of certain sensors, leading to a greater reliance on lookouts, increase the importance of human situational awareness. (It might also be argued that in such threat situation, developing vulnerability reduction, such as armour plating, and recoverability enhancement features is more effective than, for example, improving sensor technology). Nevertheless, human performance, including human situational awareness, is difficult to quantify. However, it is important to note that the developed survivability assessment method does not assess human performance related aspects (which are assumed to be constant) but the ship design itself and how the choice 
of configuration has affected survivability. However, it could be argued that improving the ship design in regard to other aspects of human factors might improve the susceptibility aspect of survivability (Andrews et al 2008).

At this point it is useful to attempt and define the scale on the y-axis of the normalised susceptibility charts (Figure 6.3, Figure 6.7 and Figure 6.11 in Section 6.1). In the case of susceptibility assessment this is relatively easy, with zero being defined as the ship being assessed not being hit by a specified threat (i.e. one or more of the following probabilities: $\mathrm{P}(\mathrm{di}), \mathrm{P}(\mathrm{l})$ and $\mathrm{P}(\mathrm{h})$, have a value equal to zero). Unity can then be defined as the susceptibility performance of a baseline design. The definition of unity could be changed straightforwardly to: the ship being assessed is definitely hit by a specified threat (i.e. $\mathrm{P}(\mathrm{di}), \mathrm{P}(\mathrm{l})$ and $\mathrm{P}(\mathrm{h})$ are all equal to one), which would signify the worst possible case for susceptibility.

A general conclusion on susceptibility can be made that the large sensitivities (to relatively small changes) observed in the susceptibility part of the survivability assessment method, combined with the many simplistic assumptions made and the large variety of current threats which have to be accounted for, justify the considerable investigation in this topic. This research then justifies work carried out in this first survivability constituent and thus the need for an accurate susceptibility assessment method. Nevertheless, through the combination and use of simplified and unclassified susceptibility assessment tools, the design studies examined produced considerable (and traceable) results. This shows that such susceptibility assessment investigations are of significant worth during the preliminary ship design stages in order to not only select the most promising out of competing alternative designs, but also to tune the selected design in terms of susceptibility performance.

\subsection{Vulnerability Assessment Results Analysis}

In this section a summary of the analysis of the main results is presented, followed by some general observations resulting from the application of the vulnerability assessment approach to the seven ship design studies; a full results analysis is included in Appendix 14. This is done since the vulnerability assessment tool, SURVIVE Lite, was operated at UCL (unlike the RCS prediction tool, SPECTRE), allowing a large number of simulations and analysis; in addition, numerous ship systems were modelled (in contrast to a single system, i.e. the whole ship, modelled in susceptibility assessment). The above two reasons led to a vast amount of data and 
related analysis, most of which confirm already acknowledged vulnerability reduction design drivers.

\subsubsection{Frigate Variants (Including Baseline)}

Out of the six major systems modelled in the frigate design studies (for which reference should be made to the system architecture and tree diagrams included in Appendix 7 and detailed vulnerability results in the tables and charts of Section 6.3 and 6.4 and Appendix 11), Frigate Variant 1 was least vulnerable in two (forward SAM system and helicopter system), Frigate Variant 2 in one (naval gun system) and the third, trimaran variant, performed best in the remaining three (move system, ASM system and aft SAM system).

The design feature that had the largest impact on the vulnerability performance of the three frigate designs was almost certainly the degree of duplication and separation of identical sub-systems. For example, the move system of Frigate Variant 3 was invulnerable to the ASM threat investigated due to the split forward and aft propulsor configuration. The move system of the remaining two designs was vulnerable when the (two) HTS motors and/or (two) propeller shaft were affected. Although these items were duplicated, they were not reasonably separated as they were located in the same WT section. In addition, the power sub-system (containing all of the engines in parallel) was not vulnerable in any attack case investigated for any of the three frigate variants. This highlights the advantages of adopting an IFEP system with large distances between the power generation units. The benefits of redundancy and separation are also clear when considering that most ship systems modelled were most vulnerable when unduplicated (i.e. in-series) items were hit. For example, in all three frigate variants the naval gun system was vulnerable when items such as the magazine, gun power room, gunbay \& guntrunk and $155 \mathrm{~mm}$ gun were hit; the ASM system was vulnerable when the ASM launchers, LPCR (Local Power and Control Room) and Operations Room were hit; the aft and forward SAM systems were vulnerable when the VLSs and LCRs were hit. Furthermore, in two of the three frigate design studies (Frigate Variants 2 and 3 ) the helicopter system is the most vulnerable of the system modelled largely because it contains the most in-series (unduplicated equipment)

A vulnerability reduction advantage of trimaran configurations is the fact that the side hulls shield certain items which are located at the amidships portion of the hull. For example, items such as the Operations Room and the helo weapons lift were found 
to be less vulnerable in the trimaran frigate. This in turn had an impact on the vulnerability of ship systems which included these items in series, such as the ASM system and helicopter system. In addition, the effect on vulnerability of improving the system layout was perceived. For example, the large beam of the trimaran ship allowed for a wide weatherdeck. This in turn made possible the arrangement of the ASM launchers in a port-starboard configuration (as opposed to the forward-aft configuration in the first two variants) which dramatically reduced their vulnerability (assuming only broadside attack).

It was observed that equipment located deep in the hull was less vulnerable to the given, abovewater, threat (i.e. the sea-skimming ASM). This was particularly noticeable in the trimaran variant which had the deepest draught. The reduced vulnerability of certain items (such as the forward magazine and sonar instrument room, and the aft (helo) magazine and AVCAT tank) had an impact on the overall vulnerability of the naval gun system and helicopter system of that design. However, these effects are probably adverse when considering underwater attacks. Nevertheless, trimarans are also more vulnerable at the narrow forward and aft extremities of the hull. This was apparent in ship systems which included items at the extremities, such as the naval gun system (magazine, gun power room, gunbay \& guntrunk and 155mm gun) and the aft SAM system (VLSs and LCR).

After carrying out the sensitivity tests on the lengthwise probability hit distribution, (i.e. changing it from a normal to a linear distribution), it was observed that assessment of system vulnerability is highly dependent on this assumption. Specifically, ship systems with the majority of their items at the extremities of the hull became significantly more vulnerable, while the opposite was true for systems with the bulk of their related items located close to amidships. As is clear in Appendix 14, in some cases the vulnerability of a system was altered by more than $50 \%$ after changing the lengthwise probability hit distribution.

After normalising the system vulnerabilities and applying the weightings proposed by 1st Lt. Fonseca, the least overall vulnerable ship was found to be the trimaran variant followed by the baseline and the second variant. The trimaran variant presented an approximately $60 \%$ decrease in overall vulnerability compared to the baseline design. After carrying out the sensitivity tests on the applied major system weighting scheme, by using the weighting schemes proposed by the RN officers at Dstl for the three scenarios summarised in Section 6.4.1, the main conclusion was that ship vulnerability is more sensitive to the overall ship design features than to the weighting 
scheme or the operational scenario. The relatively small operational scenario related overall vulnerability variations are explained in Appendix 14. It is important to note that Frigate Variant 3 presented an improvement compared to the baseline frigate ranging from $28 \%$ to $42 \%$ (when considering all scenarios and the corresponding weighting schemed proposed by the RN staff) as opposed to the approximately $60 \%$ improvement observed with 1st Lt. Fonseca's weighting scheme. This is attributed to the much higher weightings given to the Fight major systems by the RN officers (compared to the weightings proposed by $1^{\text {st }}$ Lt. Fonseca). Therefore, the significance of the effect of the invulnerable move system of the trimaran variant was reduced.

\subsubsection{Corvette, Baseline Frigate and Destroyer}

Out of the six major systems modelled in the ship size and level of combat system and ship performance capability varied combatants (for which reference should be made to the system architecture and tree diagrams included in Appendix 7 and detailed vulnerability results in the tables and charts of Section 6.3 and 6.4 and Appendix 11), the Corvette design was least vulnerable in two (move system and aft SAM system) and most vulnerable in the remaining, Frigate Variant 1 was not the least vulnerable in any and the Destroyer design performed best in the remaining four (naval gun system, ASM system, forward SAM system and helicopter system).

In this case there were two major design features that influenced the vulnerability of the ship design studies; the degree of duplication and separation of similar sub-systems, and the overall ship size. The effects of the former were similar to those reported in the frigate variant comparative study. The move system of the Corvette design was invulnerable to the ASM threat investigated due to the split forward and aft propulsor configuration. In addition, the move systems of the baseline frigate and Destroyer were vulnerable when the duplicated but non-separated HTS motors and/or propeller shaft were hit. The power sub-system was not vulnerable in any attack case due to the widely separated IFEP system selected. The naval gun system was most vulnerable in all three designs when in-series, unduplicated, items, such as the magazine, gun power room/LCR, gunbay \& guntrunk, and gun were affected. The vulnerability of the Destroyers naval gun system was significantly reduced due to the presence of an additional sensor separated by a considerable distance (the Destroyer naval gun sensors included separated forward and aft MFRs and a GPEOD which was located at the forward mast together with the forward MFR; conversely, the Corvette 
and frigate designs included only a surveillance radar and GPEOD, both located on the forward mast). Similarly, the ASM system was most vulnerable when unduplicated items in series, such as the Operations Room, LPCR and launchers, were affected; as were the forward and aft SAM systems when the VLSs and LCRs were affected. However, the Destroyer design adopted a different, more capable (forward and aft) SAM system, with a different system tree diagram. For it, the LCRs are not in series but in parallel to the Operations Room, significantly reducing the vulnerability of the SAM control sub-system. However, the SAM systems of the Corvette and baseline frigate could operate with six sensors in total (forward and aft navigation radar, forward and aft IRST, surveillance radar and ESM/ECM module), while the Destroyer's SAM system could only operates with the two MFRs. This had a slight adverse effect on the Destroyer (forward and aft) SAM system vulnerability. Finally, in all three designs, the helicopter system was also most vulnerable when items such as the AVCAT tank, magazine, weapon lift, sonobuoy store, helicopter and flight deck, Operations Room and sonar instrument room, all of which are in series, were hit.

Increases in ship size led to a decreased system vulnerability in two regards. First, the larger volumetric size of the WT sections of larger ship designs can more easily contain, but also diffuse, the blast and fragmentation damage caused by the exploding ASM warhead. To this could be attributed the improved vulnerability performance of the Destroyer designs move system (compared to the identical such system in the baseline frigate) as well as improvements in the naval gun system, aft SAM system and helicopter system. The second advantage of larger designs arises from the fact that the number of WT sections in a design is proportional to the ship's length. Moreover, the footprint of identical/similar ship systems (in terms of WT sections containing items belonging to that ship system) was found to be almost unaffected by ship size. These two reasons lead to an increase in the proportion of ship's length which if hit affects a ship system (i.e. an increase in the vulnerability footprint of the system in terms of percentage of the overall length). Therefore, given that the ship is hit (i.e. susceptibility $=100 \%$ ), a system in a smaller ship would have a larger probability of being affected by the constant hit than a similar system in a larger ship. The above was observed to affect the vulnerability of systems such as the naval gun system, ASM system, forward SAM system and helicopter system, leading to improved performance in the Destroyer and decreased performance in the Corvette design.

Although in the comparative study between the size varied combatants sensitivity studies regarding the lengthwise probability hit distribution were not 
conducted, it should be noted that the ASM system is the most vulnerable of the six ship systems modelled in all three types of combatants. This is due to the concentration of the vulnerable equipment items amidships (therefore, most likely to be hit given the normal distribution). The effects of this assumption are thus once again profound, with more examples detailed in Appendix 14. A brief sensitivity test was, however, carried out on the Destroyer design to examine the effect of changing the elevation and azimuth angles of the attacking missile impact vector. In all system cases, vulnerability result fluctuations of over $50 \%$ were observed, indicating that hit location and weapon trajectory have a significant impact on system vulnerability.

After normalising the system vulnerabilities and applying the weightings proposed by 1 st Lt. Fonseca, the least overall vulnerable ship design was the larger Destroyer (approximately 30\% less than the baseline frigate). Despite the fact that the smaller Corvette design was the worst performing in four of the six major systems, it was overall less vulnerable than the baseline frigate, performing almost identically to the Destroyer design. This was a direct result of the considerably higher weighting applied to the move system (compared to the five Fight systems), which was invulnerable in the Corvette, as analysed above. If a weighting of 2 , rather than 9 , was used for the move system (i.e. in agreement with the weightings proposed by 1 st Lt. Fonseca for the Fight systems, Table 4.1 in Section 4.2), the Destroyer design would continue to be approximately $30 \%$ less vulnerable than the baseline frigate, but the Corvette design would now be the most vulnerable ship design, by approximately $20 \%$ compared to Frigate Variant 1. This contradicts the conclusions made in Section 7.2.1 regarding the relative insensitivity of the frigate variants overall vulnerability to the weighting scheme used.

\subsubsection{AOR Variants}

Out of the seven major systems/capabilities modelled in the two AOR variants (for which reference should be made to the system architecture and tree diagrams included in Appendix 7 and detailed vulnerability results in the tables and charts of Section 6.3 and Appendix 11), the first variant was least vulnerable in one (move system) and the second variant performed best in five (all RAS related capabilities and aviation support). The CIWS of both designs was assessed to be invulnerable.

It was once again observed that adopting a philosophy of duplication and separation has a great effect on vulnerability performance. As before, the move system 
of AOR Variant 1 is invulnerable owing to the vastly separated MMRs and propulsor units (realised by the adoption of an IFEP system). A forward propulsor unit was not included in the second variant, resulting in a vulnerable move system when the in-series and unduplicated pod drive room was affected. Furthermore, AOR Variant 2 presented the opportunity to duplicate the ship handling sub-system included in all RAS capability system tree diagrams. In AOR Variant 1 only the bridge can be used for ship handling; in the second variant, the presence of a single aft superstructure block led to the RAS control space (RASco) having an unobstructed view towards the ship's bow, therefore, being able to duplicate (and separate) the functions of the bridge. This in turn led to the invulnerability of the RAS liquid capabilities and significant reduction in the vulnerability of the RAS solid capabilities in AOR Variant 2. In addition, the split forward and aft nature of the AVCAT/dieso cargo tanks (and pumps), contrasting the grouping of dry stores and ordnance cargo spaces (and lifts) adjacently, had a significant impact on the vulnerability of the RAS capabilities in both ship designs. Finally the CIWS was invulnerable in both designs due to the duplication of the SeaRAM launchers, each including an independent sensors and control room.

The auxiliary ship design comparative study also highlighted the vulnerability reduction effects of protecting critical items by less critical spaces. This was realised through the almost two times more vulnerable aviation support system of AOR Variant 1. The absence of a forward superstructure block in AOR Variant 2 led a significant increase in the size of the (single) aft superstructure, which contained most items related to the aviation support system in both designs. The larger superstructure size (through the inclusion of, for example, accommodation spaces) resulted to the shielding of critical items belonging to this system.

Furthermore, the decreased vulnerability to abovewater threats to items located deep in the hull was again observed. More specifically, stores below No 6 Deck (i.e. ordnance stores) were invulnerable to the ASM threat simulated. It is also interesting to note that the auxiliary ship designs output considerably smaller system vulnerability values than the combatants, often not exceeding $2 \%$. This is possibly due to their significantly larger size (although different systems with different architectures and tree diagrams were modelled in the two ship types).

As in the previous comparative studies, the assumed normal lengthwise probability hit distribution has affected the vulnerability of the modeled systems, with systems located at the extremities (e.g. the move system) presenting considerably lower vulnerability values than amidships systems (e.g. RAS capabilities). After normalising 
the system vulnerabilities and applying the weightings proposed by Lt. Cdr. (rtd.) Day, AOR Variant 2 proved to be approximately $40 \%$ less vulnerable, predominantly due to the entirely invulnerable ability to RAS AVCAT and ability to RAS dieso, both of which were given maximum weighting.

\subsubsection{General Observations on Vulnerability Assessment}

In contrast to the susceptibility assessment method applied (with the exception of the RCS prediction tool), the vulnerability assessment method developed was based on the current established and accepted UK MOD vulnerability assessment software for concept stage ship designs, SURVIVE Lite (see Section 3.3.3). Also, in contrast to the susceptibility assessment method, SURVIVE Lite is able to simulate the damage effects of a large number of different weapons, with different characteristics (as summarised in Section 3.3.3); although the very specific case of a particular sea-skinning ASM was only examined in this research. Amongst the advantages given by the developed method is that the results can be easily backtracked in order to examine what caused each ship design to have its vulnerability characteristics and apply corrective actions.

However, a number of simplistic assumptions were made during the application of the method. These are in addition to the ones summarised in Section 4.2 and 6.3. For example:-

- The system tree diagrams developed (Appendix 7) imply that all weapon systems operate identically with any of the sensors in their sensor sub-system (although, clearly, the performance of certain weapon systems will be degraded depending on the available sensors);

- All engines fitted in the ship are assumed to be able to individually operate the ship in a fully capable manner (although clearly the loss of a more powerful engine will affect the ship in a different manner to the loss of a less powerful engine, and the loss of any engine could result to certain capability loss regarding not only propulsion but also operation of combat systems);

- Cargo stores in the AOR designs were modelled in parallel although in reality the loss of cargo spaces would lead to a loss in cargo capacity and, therefore, capability).

Such assumptions could be corrected in SURVIVE Lite by the inclusion of further system tree diagrams, each implying a different level of capability. This would, 
however, dramatically increase results processing and analysis time which is probably counterproductive in the concept phase of ship design.

There are also the issues regarding the use of appropriate weighting schemes which rely on human judgement rather than simulation. Certain of the design studies investigated were highly sensitive to the (subjective) weighting scheme used; although patterns in the weighting schemes proposed by different naval officers were observed, large results variations were still observed. Furthermore, certain of the officers interviewed made comments which are related to the operational scenario. For example, the duplicated (SAM, in the combatants) systems should each be given different weightings and that weighting schemes (and the selected systems to be modelled) are highly dependent on the ship's role as well as the operational scenario. This highlights the connection of ship overall vulnerability to human factors, such as how the operational situation is perceived by the ship's crew, which is largely defined by situational awareness. Developing system weighting schemes is an area where further research is necessary and the application of methods such as the Delphi Method mentioned in Section 3.3.4 might prove beneficial in using a single weighting scheme for each operational scenario investigated.

As with the susceptibility method developed, it is useful to attempt and define the scale of a design's vulnerability on the y-axis of normalised vulnerability charts (Figure 6.25, Figure 6.27 and Figure 6.29 in Section 6.3). This is relatively easy in vulnerability assessment, with zero being defined on the basis that all of the systems modeled in the ship being assessed not being vulnerable to the threat (ASM in this instance) given a hit at any location of the ship. The value of unity is defined as the vulnerability performance of a baseline design. The definition of unity could easily be changed to: all of the modeled systems in the ship being assessed are $100 \%$ vulnerable to the threat (ASM in this instance) given a hit at any location of the ship, (i.e. worst possible case). As already mentioned, in the case of the AOR variants, normalisations have been conducted against the worst performing ship design rather than an arbitrary baseline. This means that the score of unity is defined as the vulnerability performance of the worst performing design. This was done since both AOR designs included invulnerable (i.e. $0 \%$ vulnerable) systems. Thus, dividing (during the normalisation process) by zero is avoided since this would lead to impossible values. All other studies were normalized with respect to the corresponding baseline frigate values, which did not include any $0 \%$ vulnerable systems. This, however, was fortuitous possibly indicating that normalisation should always be made with respect to the worst 
performing of the designs being compared. It could be argued that this would also give a more meaningful definition to the value of unity on the y-axis of the normalised vulnerability charts and simplify presentation and comparison of results between alternative designs. For consistency, the AOR studies' total susceptibility and recoverability results were also normalised with respect to the worst performing design. In an alternative approach, experts could develop a set of minimum acceptable system vulnerability standards for each ship type and for a given threat (possibly based on the vulnerability performance of current ships), with new alternative designs being assessed against these standards. This latter approach would be analogous to traditional damage stability assessment.

A number of conclusions can be deduced from the vulnerability assessment results. Trimarans generally provide reduced vulnerability due to the protection provided by the side hulls, although they are more vulnerable at the narrow forward and aft ends; equipment located deep in the hull is less vulnerable to abovewater attack; increasing size tends to reduce vulnerability, duplication of identical systems greatly reduces vulnerability, provided that the redundant systems are reasonably separated; improved system architecture/layout (provided, for example, through the large box structure and associated DC deck and weatherdeck wide beam of a trimaran) decreases vulnerability and the protection of certain high value items by secondary compartments affects system vulnerability. However, system vulnerability results are largely dependent, not only on the trajectory of the attacking threat (a missile in all cases in this research), which can easily be simulated in SURVIVE Lite, but also on the (susceptibility related) assumed lengthwise hit probability distribution. The latter depends on a large variety of factors, such as ship and threat characteristics, environmental conditions, ship motions and manoeuvring and variations in weapon performance as outlined in Section 3.3.2. This can be seen to inhibit the development of a realistic prediction tool. It is relevant that although there have been efforts to link susceptibility and vulnerability assessment methods in order to calculate likely hit locations (Turner et al 2006; Martin 2007) (see Section 3.3.2), the official MOD maritime mission simulation programme (MISSION) does not utilise them. Rather, it simply assumes a normal lengthwise hit probability distribution. As mentioned in Section 7.1.4, further research is necessary in the area of susceptibility assessment, also because it has such an effect on the next survivability constituent, vulnerability. 


\subsubsection{Frigate Variants (Including Baseline)}

\section{Main Results}

Out of the eight PM groups (immediate DCFF or Float PMs (Category 1) shown in Table 4.3, major system recovery or recovery support PMs (Category 2) listed in Table 4.4 and the six individual major system, one Move and five Fight systems, recovery PMs (Category 3) summarised in Table 4.5; see Section 4.3) Frigate Variant 3 performed best in seven (float category, recovery support category and move, naval gun, ASM, aft SAM and forward SAM system recovery), while Frigate Variant 1 performed best in the helicopter system recovery category. These results can clearly be seen in the PM matrices presented in Section 6.5.1.

In the float category, the first two frigate variants performed almost identically, while the trimaran variant performed approximately $30 \%$ better. Some of the most noteworthy results include the fact that the third variant performed significantly worst in PM 1.1 and 1.2, due to the fact that, being a larger ship design than its counterparts, the FRPs had to travel longer distances and operate more WT doors in order to reach the affected section of the ship. In PM 1.4 Frigate Variant 2 performed best by a large margin due to the two passing decks presenting a greater number of alternative routes to the DC crew, followed by Frigate Variant 3, which included double, side passageways in the box structure (with single centreline passageways aft and forward) and finally the baseline (Variant 1), with its single centreline passageway presenting no alternative routes. Although in PM 1.3 the baseline frigate performed marginally better, due to the less number of decks in the hull, easing access, this was partially counterbalanced by the fact that the baseline had a full length superstructure, in contrast to the other two designs. Frigate Variant 3 performed much better in PMs 1.6 and 1.7 due to the fact that the firepumps in all variants were located deep in the hull. (As mentioned in Section 7.2.1, the deeper draught of the trimaran offered more protection from abovewater threats to items located at the lower decks, such as firepumps, therefore, minimising the probability that these items will be affected after an attack and increasing post hit availability). The aft FRP section base in Frigate Variants 2 and 3 is located one WT section aft than the corresponding item in the baseline frigate, therefore, the assumed normal lengthwise probability hit distribution, minimising the probability of that FRP 
section base being affected after a hit, leads to the significant improvement in PM 1.16. However, this also means that this FRP section base is less efficiently positioned (closer to the ships extremities) therefore, partially contributing to the decreased performance in PMs 1.1 and 1.2 for the last two frigate variants.

In PM 1.10 the baseline frigate performs significantly better than the other two variants, purely due to the fact that, conversely to Frigate Variants 2 and 3, the two remaining NBCD stores of the baseline are positioned one at each extremity of the ship, therefore, minimising the probability that they are affected after a given hit. In all remaining PMs, the two monohull variants performed almost identically, due to their similar layout, whereas the trimaran variant performed better, in some cases presenting more than $80 \%$ improved performance. This is attributed to the fact that, as analysed in Section 7.2.1, the vulnerability reduction features of the trimaran led to less items being affected by a given threat resulting in an increased availability of DCFF required items (such as ATU and ventilation, NBCD stores, power generation unit, SCC, Operations Room and FRPP, which were all quantified through their respective PMs) which are needed after the assumed, unvarying, attack. The general conclusion is that the two monohulls perform almost identically; although the trimaran variant performs worse in most access related PMs due to its larger size, this is more than counterbalanced by the increased availability of items necessary for DCFF after a given attack, due to the trimaran's increased inherent vulnerability reduction features. The relative difference between the performances of the three frigate designs also depends upon the weighting scheme employed.

In the recovery support PM group the two monohull frigates performed almost identically in all PMs, whereas the trimaran variant displayed an overall improved performance by approximately 40\%. Only in (low-weighted) PM 2.3 did the trimaran perform worse than the first two frigate variants, due to its naval stores being located closer to amidships (as opposed to the far aft positioned naval stores in Frigate Variants 2 and 3), therefore, increasing the probability that these stores are affected and unavailable after a hit. In all other PMs (related to workshops, spare gear stores, SCC and Operations Room) the trimaran variant performed up to approximately $60 \%$ better, despite the very similar arrangement of the above compartments in all three variants. This is attributed to the increased post attack availability of these items due to the inherent trimaran vulnerability reduction features, as analysed in Section 7.2.1.

In the move system recovery PM group, once again the almost identical system layout in the two monohulls leads to an almost identical performance in all PMs. 
Conversely, Frigate Variant 3 illustrated an improved performance by more than $60 \%$. Approximately half of this improvement is attributed to the fact that, by adopting a split forward and aft propulsion system, the high-weighted PM 3.1.1 outputted a value of zero as a result of the move system being invulnerable to any single ASM attack (therefore, requiring zero man-hours for the move system to be functioning post-attack). The remainder of the $60 \%$ improvement is attributed to the improved performance in all other PMs of this group. This was a direct result of a given constant threat, affecting fewer items in the less vulnerable trimaran; therefore, fewer items need to be accessed and recovered post-hit.

When considering the naval gun system recovery PM group, a near identical performance is observed in the two monohulls, again due to the almost identical system layout; Frigate Variant 3 displays an almost 30\% improvement. The improvement is less than that of the move system recovery PM group due to the fact that high-weighted PM 3.1.2 outputted a similar value in all frigate variants. This was expected since the naval gun system layout (regarding the in-series and therefore most vulnerable items, such as the magazine, gunbay \& guntrunk, gun power room and $155 \mathrm{~mm}$ gun) in the trimaran frigate was similar to that of the monohull variants, leading to similar overall vulnerability values. However, the increased protection and, therefore, post-hit availability, of duplicated system items, such as the engines contained in the power subsystem, led overall to less items being affected post-hit, as clearly shown by PM 3.2.2. Therefore, overall fewer items needed accessing and repair works, reflected on the improved PM values of the remainder PMs in the trimaran for this PM group.

In the ASM system recovery PM group, the baseline frigate is the worst performing, with the second variant outputting approximately $13 \%$ better results and the third variant resulting to a more than $30 \%$ improvement. Most in-series, i.e. more vulnerable, equipment items of this system, such as the launchers and LPCR, are located amidships for Frigate Variants 1 and 3, and at the aft end in the second variant. The assumed normal lengthwise hit distribution (meaning that the in-series equipment in Frigate Variant 2 have a lesser chance of being hit) leads to the second variant having a larger availability of such items post-hit, reflected on the almost $30 \%$ improvement in PM 3.1.3 (in contrast to the approximately 10\% improvement displayed in the trimaran variant, solely due to the improved system layout and inherent trimaran vulnerability reduction features). Once again, however, the least vulnerable trimaran leads to overall fewer items being affected and in need of access and repair works, therefore, showing improvements between $25 \%$ and $50 \%$ in all remaining PMs of this group. Conversely, 
the second variant produces very similar results to the baseline in the remaining PMs. This can be interpreted as the advantages presented being purely related to the location of the most vulnerable system items, rather than improvements in the ship's overall configuration which leads to greater post-hit availability of equipment.

A similar conclusion may be ascertained when comparing the PM values for the aft SAM system recovery PM group. The in-series most vulnerable equipment, such as the VLS launchers and LCR, are located one WT section closer to the aft extremity of the ship in Frigate Variant 2 compared to the baseline. This leads to PM 3.1.4 having a $30 \%$ improved performance than the baseline, while all other PMs output near identical results; this limits the overall improvement to approximately $10 \%$. On the contrary, the trimaran variant outputs an almost $70 \%$ improved performance. Approximately half of this improvement is attributed to the almost 90\% improvement in PM 3.1.4, which was mainly due to the positioning of the above vulnerable equipment closer to the aft extremity of the ship design, therefore decreasing the hit probability and increasing the post-hit availability of these items. The remaining improvement is attributed to the previously mentioned overall trimaran vulnerability reduction enhancements, captured by the improved performance in all other PMs, between approximately $40 \%$ and $75 \%$.

When considering the forward SAM system recovery PM group, the similar system layout between the two monohull frigates has led to almost identical performance in all PMs of this group. Once again, the trimaran outperforms the two monohulls in all PMs, leading to an overall improvement by approximately $50 \%$ (although the system layout is comparable to that of the two monohull frigates). This is again attributed to the fact that the inherent vulnerability reduction features of the trimaran lead to overall less items in need of access and repair, given a constant threat.

Finally, in the helicopter system recovery PM group, the baseline is the best performing, with Frigate Variant 3 producing a 12\% deterioration and Frigate Variant 2 almost $70 \%$. The above results are clearly related to the aft positioning of the majority of the baseline's helicopter facilities (such as the in-series AVCAT tank, magazine and weapon lift, sonobuoy store, helicopter and flight deck), compared to the amidships positioned helicopter facilities in the other two variants. The assumed normal lengthwise probability hit distribution leads to a smaller probability of these items being affected by a constant threat, therefore, increasing the post-hit availability and decreasing the number of man-hours required to put the system back to action in the baseline frigate. This is clearly shown in PM 3.1.6 where the second and third variants output approximately $130 \%$ and $60 \%$ worse results respectively. This worsened 
helicopter system layout, as well as the similar workshops and stores layout between the first two frigate variants, has led to all other PMs outputting worse values for Frigate Variant 2 compared to the baseline. However, the improved trimaran configuration previously mentioned has led to overall less system items being affected (when including duplicated system items, such as the engines contained in the power subsystem), leading to an improved PM 3.2.6 performance for Frigate Variant 3 as well as for some of the access PMs (PMs 3.4.6 and 3.5.6).

After normalising the PM group data and applying the weightings proposed by 1st Lt. Fonseca, as expected, the most recoverable ship was found to be the trimaran variant. It performed approximately $40 \%$ better than the baseline due to the overall improved performance in seven out of the eight PM groups. The recoverability characteristics of the second variant were virtually identical to the baseline, the approximately $5 \%$ worsening being attributed almost entirely to the less recoverable helicopter system.

\section{Sensitivity Study Results}

A sensitivity study to the lengthwise probability hit distribution was carried out by assuming a linear distribution across the length of the ship designs. The effect of this change to overall recoverability was not considerable, with the trimaran variant still outputting approximately $40 \%$ improved results. However, the effect of this change to certain PM groups was profound. The largest effect occurred to the helicopter system recovery PM group of the second variant, which now performed identically to that of the baseline. This led the second variant to output the second best recoverability performance, outperforming the baseline by a negligible $1 \%$.

Sensitivity studies were also carried out on the applied weighting scheme by using the weighting schemes proposed by the RN officers at Dstl for the three scenarios summarised in Section 6.4.1. As in the similar sensitivity study on the vulnerability assessment method, the main conclusion was that ship recoverability is more sensitive to the ship design than to the weighting scheme or the operational scenario. In fact, the output was much less sensitive to the weighting scheme than was the vulnerability output, possibly due to the increased number of variables (i.e. PMs) relevant to the recoverability assessment method. In all cases examined, the second frigate variant performed worse than the baseline by approximately $7 \%$ to $16 \%$ and the trimaran variant performed better by approximately $35 \%$ and $43 \%$. The decreased performance 
of Frigate Variant 2 in scenario 1 was once again displayed (however, to a lesser extent than in the vulnerability assessment section). This was a result of the comparatively large weighting given to the helicopter system by all $\mathrm{RN}$ officers for that scenario, due to the presence of a submarine threat (not present in the other two scenarios), highlighting once again the poor performance of the amidships helicopter system of that design variant.

The final sensitivity test carried out involved the decoupling of the recoverability assessment method from the vulnerability assessment SURVIVE Lite software. As explained in Section 6.6.3, this was done by assuming that an ASM hit at any WT section of the ship would affect all equipment and compartments modelled, and repopulating the PM matrices. This procedure led to some remarkable results. Certain patterns were observed and are briefly summarised. In the float category (Category 1), PMs 1.1-1.4 output identical values as before, since they are not related to any items being affected by the threat. All other float category, as well as recovery support (Category 2), PMs output identical, or near identical values (any negligible differences are attributed to slight differences in system layouts, such as the underwater exhaust of the forward auxiliary engine of the trimaran frigate design eliminating the abovewater vulnerability of an uptake). This was due to the (near) identical equipment and systems included in the three frigate designs, therefore, cancelling the advantages previously observed in the trimaran variant. For the same reasons, PMs 3.1 and 3.2 for all individual major system recovery PM groups output identical or near identical values, again cancelling the advantages previously observed, principally in the trimaran variant. The only major differences are observed when investigating the access related Category 3 PMs. When considering Frigate Variant 2, the fact that the aft spare gear stores and workshops are positioned one WT section aft compared to the baseline have led to all related PMs performing slightly worse. The far aft positioning of the ASM system launchers and LPCR (as opposed to the baselines amidships) has worsened the access PMs of this system from the forward spare gear stores and workshops. However, this aft system layout improved the performance of PM 3.8.3, due to the assumed normal lengthwise probability hit distribution. The adverse occurred in the respective PM of the helicopter system due to the amidships location of the majority of the helicopter facilities (as opposed to the baselines aft). However, access from the forward workshops and spare gear stores greatly improved, but access from the aft located naval stores worsened. All other access related Category 3 PMs output similar values due to the similarities presented in the two ship designs. When considering Frigate Variant 3 , the 
amidships-forward located naval store (as opposed to the aft naval store in the baseline) improved the performance of the related PMs of the forward positioned naval gun and forward SAM systems and amidships posited ASM systems; conversely, the equivalent PMs for the aft positioned aft SAM system and amidships positioned helicopter system worsened. Furthermore, all Category 3 PMs related to all spare gear stores and workshops performed worse for all major systems (excluding the move system which will be analysed later). This was clearly due to all the above stores and workshops being positioned less efficiently (i.e. closer to the ships extremities) in the trimaran, therefore, worsening access.

The split nature of the move system produced the most noteworthy results. In the baseline frigate (as well as the second variant) the naval stores, aft spare gear store and aft workshops were all located close to the aft end of the ship, where the propulsion machinery was located. In the trimaran variant with the split forward-aft steering and propulsion sub-system this is no longer the case, therefore, greatly worsening the results of PMs 3.3.1-3.5.1. However, the access PMs related to the forward spare gear stores and workshops were slightly improved due to the location of certain machinery in proximity. The overall conclusion is that, although a duplicated and separated system presents vulnerability related advantages, it inhibits recoverability (of the total system), due to the increased complexities relevant to accessing the dispersed system items. The above analysis led the trimaran to output an approximately 50\% worse performing move system recovery PM group, which is almost entirely responsible for the trimaran frigate now being the least recoverable design, outputting results approximately $20 \%$ worse than the baseline. The two monohull designs output almost identical recoverability performance due to the near identical equipment, systems and ship configuration. It is clear that the above procedure gives a good description of the efficiency of the location of the various stores and workshops required for repair works relative to the equipment distribution of the modelled ship systems. This was also captured by the recoverability assessment method as performed previously; however, the efficiency of the location of these compartments was often overpowered by factors such as the number of system items requiring repair (and, therefore, access) after a constant threat. By assuming that an ASM hit at any WT section of the ship would affect all equipment and compartments modelled, in effect, the constant threat assumption is no longer valid. This is because for the same amount of damage on different ship designs, a different threat is required. This, therefore, creates a major inconsistency in the above method and questions its validity. However, the fact that this 
inconsistency can only be overcome when involving vulnerability assessment software in a recoverability assessment method raises scepticism about the boundary between vulnerability and recoverability and if they should be separately considered.

\subsubsection{Corvette, Baseline Frigate and Destroyer}

Out of the eight PM groups (immediate DCFF or Float PMs (Category 1) shown in Table 4.3, major system recovery or recovery support PMs (Category 2) listed in Table 4.4 and the six individual major system, one Move and five Fight systems, recovery PMs (Category 3) summarised in Table 4.5; see Section 4.3) the Destroyer design performed best in six (float category and naval gun, ASM, aft SAM, fwd SAM and helicopter system recovery) while the baseline frigate performed best in the remaining two (recovery support category and move system recovery). These results can clearly be seen in the PM matrices presented in Section 6.5.2.

In the float category, the Destroyer design performed marginally better (by 4\%) than the baseline frigate, while the Corvette performed approximately $13 \%$ worse. Some of the most noteworthy results include that in PMs 1.1-1.3, the Corvette performed best, followed by the frigate and finally the Destroyer design. This is clearly due to the smaller ship designs involving reduced distances travelled and WT doors operated by DC crews, as well as generally less decks in each WT section. The Destroyer with its double side passageways outperformed the other two combatants in PM 1.4, while the Corvette with, the single and narrower than the baseline frigate centreline passageway, presented the worst result. It is interesting to note that when regarding equipment and compartments split in accordance to zoning, such as ATU and Ventilation (PM 1.5), firepumps (PM 1.6) and power generation units (PM 1.11), the Destroyer, with the greater number of (and therefore, smaller in size) zones, outputs the worst results. This signifies that the zones of this ship design are smaller than the ideal, therefore, the probability that one of the above items is affected after the corresponding zone is hit, would increase (i.e., a smaller zone is more vulnerable in the same way that a smaller ship is). It is also interesting that in PM 1.11 the Corvette outputs the most promising results, possibly due to the same number of engines as in the other two designs (i.e. five) spread over only two zones, therefore, significantly increasing availability. However, when considering the firepump and power system as a whole (PMs 1.7 and 1.12), the smaller Corvette performs worst due to possessing the most vulnerable such systems, decreasing post-hit availability. Conversely, when considering items not 
connected to the zoning arrangements, such as the SCC (PM 1.13), the Bridge (PM1.14) and the Operations Room (PM 1.15), the Destroyer design significantly outperforms the other two combatants due to the least vulnerable, larger Destroyer, leading to an increased availability of such items after the assumed, unvarying, attack.

Due to the different relative weightings of the NBCD stores (mentioned in Section 6.5.2) it is difficult to make a comparison of the related PMs. However, as a whole, the NBCD store related PMs of the Corvette design outperform the almost identically performed PMs of the other two combatants. This is attributed to the location of the stores, with the Corvette including only two such stores, both positioned close to the extremities (as opposed to the other two ship designs which also include amidships positioned NBCD stores). Therefore, given the normal lengthwise probability hit distribution, the probability that the stores are affected by a constant hit is decreased and post-hit availability increased. For a similar reason, the Destroyer design outperforms the other two combatants in the FRP related PMs, 1.16 and 1.17. Similarly to Section 7.3.1, it is observed that the Destroyer performs worse in most access related PMs, due to its larger size, but this is counterbalanced by the increased availability of items necessary for DCFF after a given attack, due to the increased inherent vulnerability reduction of the larger design.

In the recovery support PM group, both the Corvette and Destroyer designs output more than $30 \%$ worse results than the baseline frigate. The Corvette performs worse in every PM of that group (with the exception of PM 2.4) unsurprisingly since the increased vulnerability of the smaller ship leads to decreased post-hit availability of stores, workshops and command and control centres. PM 2.4 performs better due to the fact that the Corvette's aft spare gear store is only affected when the WT section in which it is located is hit (whereas the corresponding store in the frigate design is also affected when one adjacent WT section is hit), therefore, increasing post-hit availability. This occurs possibly since the Corvette's store is located at the middle of a WT section while the frigates store is located adjacent to a WT bulkhead. When considering the Destroyer design, as expected due to its larger, less vulnerable, size, it performs correspondingly or better in all PMs of this group, apart from PMs 2.1 and 2.4. The reason being that the larger aft workshops and aft spare gear stores of the Destroyer span the entire length of a WT section and are therefore affected by a hit in that and any of the two adjacent WT zones (as opposed to the two WT zone vulnerability footprint of the equivalent frigate compartments). Therefore, the probability that these compartments are affected by a constant threat increases in the Destroyer design, 
decreasing post-hit availability. This has almost caused a threefold decrease in the performance of PMs 2.1 and 2.4, overpowering the better performance of the remaining Destroyer PMs and contributing to the overall decrease of this PM group.

When considering the move system recovery PM group, once again the baseline frigate was the best performing, with the Corvette and Destroyer designs outputting approximately $4 \%$ and $27 \%$ worse results. The split, therefore, invulnerable to any single ASM attack forward and aft propulsion system of the Corvette lead to a value of zero for the high-weighted PM 3.1.1 (therefore, requiring zero man-hours for the move system to be functioning post-attack). However, all other PMs of this group outputted worse values since generally more system items (including duplicated equipment) were affected in the Corvette and were, therefore, in need of access and repair. Interestingly, the Destroyer was the worst performing in this PM group, largely because of its aft spare gear stores and workshops being located further from the propulsion machinery than in the frigate design, worsening the values outputted for the access related PMs 3.4.1 and 3.5.1.

Regarding the remaining (Fight) system recovery PM groups (naval gun, ASM, aft SAM, fwd SAM and helicopter system) the conclusions made are consistent. The Destroyer performs better by between $1 \%$ and $24 \%$ and the Corvette worse by between $10 \%$ and $71 \%$, when compared to the baseline frigate. (The margin by which each PM group differs from that of the baseline frigate depends on factors such as the number of items in need of repair, their location with respect to the stores and workshops and the relative weighting of each PM). The similar conclusions are a result of the similar system hierarchies and layouts in all three combatant designs and the general tendency to increase vulnerability and, therefore, the number of items requiring repair, with decreasing ship size. Regarding the Destroyer design, the only results worth further commenting on is the over $20 \%$ decrease in performance of PMs 3.4.3, 3.5.3, 3.4.6 and 3.5.6 (see Table 6.16 in Section 6.5.2) compared to the baseline frigate. This occurs due to the larger distance between the aft spare gears stores and aft workshops and the vulnerable, in-series, items of the ASM and helicopter systems (such as the ASM launchers and LPCR, and the AVCAT tank, magazine and weapon lift, sonobuoy store, helicopter, flight deck, Operations Room and sonar instrument room). Note that, additional to the above stores and workshops related PMs output worse values in the Destroyer than in the baseline frigate, however, by a negligible margin and so are not commented upon. The above is further justification on the increased access related complexity relevant in larger ship designs. However, these effects are balanced and 
sometimes overpowered by the fact that a larger, less vulnerable ship would require fewer items needing access and repair after a constant threat. For this reason, all access PMs of the system recovery (Category 3) PM groups of the Corvette perform worse than the two other combatant designs, although the distances involved are smaller. Regarding the Corvette design, the only result worth further commenting on is the over 20\% increased performance of PM 3.1.4 (see Table 6.16 in Section 6.5.2) compared to the baseline frigate (this being the only PM in the Category 3 PM groups where the Corvette performs better than the frigate). This is attributed to the in-series, i.e. more vulnerable, equipment items of the Corvette aft SAM system, such as the VLS launchers and LCR, being located closer towards the designs aft end. The assumed lengthwise hit distribution (meaning that the in-series equipment in the Corvette have a lesser chance of being hit) leads to the Corvette having a larger availability of such items post-hit. However, the worse performance of the Corvette in all other PMs of this group is indicative that the above advantages are purely related to the location of the most vulnerable system items, rather than improvements in the ship's overall configuration which would lead to greater post-hit availability of equipment.

From the above analysis one would expect the Destroyer design to output the best recoverability performance results, since it performed best in six out of eight PM groups. However, this is not the case since after normalising the PM group data and applying the weightings proposed by 1st Lt Fonseca, the Destroyer was found to be approximately $6 \%$ less recoverable than Frigate Variant 1 . As evident from Table 4.7 in Section 4.3.4, two of the PM groups (the Float PM group and move system recovery PM group) involved incomparably higher weightings (10 and 9 respectively) than all other PM groups (having a weighting of 1 or 2). In the first of these PM groups both the frigate and Destroyer designs outputted comparable results; in the latter group, however, the baseline frigate significantly outperformed the Destroyer, primarily due to the access PMs related to the aft spare gear stores and workshops, as summarised above. (The PM groups where the Destroyer outperformed the baseline frigate involved relatively small weightings). Therefore, it is a combination of access complexities encountered in larger ship designs during repair tasks and the weighting scheme selected that led to the above result. It could be deduced that there is an optimum combatant size where the damage caused by a threat (therefore, the amount of items requiring repair) combined with access implications would lead to the most recoverable ship. However this design point would be extremely difficult if not impossible to find since it would depend on aspects such as the weighting scheme employed and the threat considered. As expected the 
Corvette design was the least recoverable design (21\% worse performing than the baseline frigate) primarily due to the fact that a constant threat resulted in much greater damage in this smaller and more vulnerable ship, therefore, more items required accessing and repair works.

\subsubsection{AOR Variants}

Out of the nine PM groups (immediate DCFF or Float PMs (Category 1) shown in Table 4.3, major system recovery or recovery support PMs (Category 2) listed in Table 4.4 and the seven individual major system, one Move and six Fight systems, recovery PMs (Category 3) summarised in Table 4.5; see Section 4.3) AOR Variant 1 performed best in four (float category, recovery support category and ability to RAS ordnance and CIWS recovery) while AOR Variant 2 performed best in the remaining five (move, ability to RAS AVCAT and dieso, ability to RAS dry stores and aviation support system recovery). These results can be clearly seen in the PM matrices presented in Section 6.5.3.

In the float category AOR Variant 1 performed considerably better, by approximately $30 \%$ compared to its counterpart. Some of the most noteworthy results include that in PMs 1.1 and 1.2 AOR Variant 2 performed better. Although the aft FRP section bases in both ship designs were collocated (leading to similar values for PMs 1.8 and 1.16) the absence of systems and compartments other than cargo in the forward section of AOR Variant 2 led to the forward FRP section base being located at the forward end of the (single) aft superstructure block, therefore, closer to amidships. (In AOR Variant 1 the forward FRP section base was located at the far forward end since the philosophy was to split the two section bases between the two superstructure blocks containing the living and working spaces). The more efficient, closer to amidships, location of this section base led to smaller distances travelled and less WT doors operated by the FRPs; it also, however, led to much worse performing PMs 1.9 and 1.17 due to the increased vulnerability and decreased post-hit availability associated with items located close to amidships due to the assumed normal lengthwise hit probability distribution. The position relative to amidships of the ATU and ventilation compartments also led to AOR Variant 2 outperforming the first variant in PM 1.5. However, in all other PM of this group, either both auxiliary ship designs performed similarly or AOR Variant 1 performed better (in some cases more than 90\%). The reasoning is similar to that given for PMs 1.9 and 1.17, i.e., due to the absence of 
forward superstructure in the second variant, the single aft superstructure block had to be enlarged and extended towards amidships in order to fit all required systems and spaces. This led to equipment necessary for DC, such as firepumps (PMs 1.6 and 1.7), power generation units (PMs 1.11 and 1.12), SCC (PM 1.13), bridge (PM 1.14) and Operations Room (PM 1.15) being located closer to amidships, therefore, the probability of them being hit by the constant threat was increased, decreasing their posthit availability. Therefore, if it were to be assumed that the normal distribution used to describe the lengthwise hit probability is realistic, the above analysis demonstrated that concentration of systems at the extremities of the ship design (as was done in AOR Variant 1) decreases their vulnerability and increases their availability during recovery and repair efforts.

In the recovery support PM group AOR Variant 1 performed approximately $14 \%$ better than the second variant, the main reason being the improved performance of the SCC and Operations Room related PMs (2.6 and 2.7) summarised above. However, a comparison between the stores and workshops related PMs is difficult due to the different numbers of these compartments in the two variants (mentioned in Section 6.5.3).

When considering the move system recovery PM group, the second variant performed best by approximately $9 \%$. The split and therefore, invulnerable to any single ASM attack, forward and aft propulsion system of the first variant leads to a value of zero for the high-weighted PM 3.1.1 (requiring zero man-hours for the move system to be functioning post-attack). However, the total man-hours needed to restore all (including duplicated) affected items of the move system is comparable in both designs due to the identical hull and vulnerability characteristics (in fact PM 3.2.1 is slightly worse for AOR Variant 1 due to additional propulsion equipment, such as the forward motor and pumpjet, not included in AOR Variant 2). This led to AOR Variant 2 significantly outperforming the first variants in all access related PMs of this group. In the second variant, all power and propulsion machinery were concentrated at the aft end of the ship, as were the unduplicated naval and spare gear stores and workshops, therefore, providing easy access. In the second variant, not only was the propulsion system split in a forward and aft configuration, but so were the stores and workshops. This overcomplicated the access arrangements and more than overpowered the effects of the invulnerable move system. Once again, the importance of access to damaged equipment is highlighted, but should be treated with caution due to its high dependence 
on the weighting scheme used. In addition, the increased difficulty related to the recovery of duplicated and separated systems was again emphasized.

In the two RAS liquid capability recovery PM groups, AOR Variant 2 performs $16 \%$ and $19 \%$ better, respectively. This is because the duplicated ship handling subsystem of AOR Variant 2 (consisting of the bridge and RASco) has resulted to an invulnerable capability given a single ASM attack, therefore, requiring zero man-hours for post-attack capability restoration (PMs 3.1.2 and 3.1.3). All other PMs of this group output similar values due to the similar system configurations.

The duplicated ship handling sub-system of AOR Variant 2 also had an impact in PMs 3.1.4 and 3.1.5 of the two RAS solid capability recovery PM groups, therefore, reducing the number of man-hours to restore the capabilities after attack (however, not to zero, since, as analysed in Section 7.2.3 these capabilities are not invulnerably to a ASM attack). Therefore, the total improvement of AOR variant 2 in the ability to RAS dry stores recovery PM group was limited to approximately 5\% and the two variants performed identically in the ability to RAS ordnance recovery PM group.

Considering the aviation support system recovery PM group, the second variant outperformed AOR Variant 1 by approximately 33\%. As summarised in Section 7.2.3 the vulnerable, in-series, items of this system, such as the flight deck, hangar, ship's own AVCAT tank and all aviation workshops, are protected by additional structure in AOR Variant 2, therefore, leading to an improved performance in PM 3.1.6. Furthermore, similar to the move system in AOR Variant 2, the aviation support system was concentrated towards the aft end in both AOR designs. The concentration of stores and workshops required for repair works also towards the aft end of the second auxiliary variant (as opposed to the split forward and aft such compartments in AOR Variant 1) led to a considerable improvement in the access related PMs, further augmenting the performance of AOR Variant 2 in this group.

Finally, in the CIWS recovery PM group, AOR Variant 1 performed better to a level of more than $70 \%$ than its counterpart. Both systems were invulnerable to a single attack leading to zero values for PMs 3.1.7. However, the forward SeaRAM sub-system of the second variant was more vulnerable to fragmentation from the RAS posts and crane since it was not shielded by any structure, unlike in AOR Variant 1. This led to a larger total system vulnerability (including duplicated items), meaning that more system items were affected and needed repair in the second variant. Therefore, PM 3.2.7 of AOR Variant 2 performed worse by almost $100 \%$. The fact that more items needed repair implied that more items required accessing from the stores and workshops, 
therefore, dramatically decreasing the performance of all access related PMs of this group in AOR Variant 2. This was further amplified since all such stores and workshops were located at the ship's aft end and the most vulnerable CIWS sub-system was located at the forward end. Furthermore, access to damaged CIWS related items form the aft stores and workshops of AOR Variant 1 was unobstructed.

After normalising the PM group data and applying the weightings proposed by Lt. Cdr. (rtd.) Day it was found that the overall recoverability characteristics of the two AOR Variants were almost identical (less than $1 \%$ difference), although, as analysed above, certain remarkable differences were identified in the individual PM groups. However, this could be expected since there was an approximate balance in the number of PM groups (and their associated weightings) in which each variant outperformed its counterpart.

\subsubsection{General Observations on Recoverability Assessment}

Unlike the susceptibility and vulnerability assessment methods applied, it was necessary to develop a new recoverability assessment method. It was found that the recoverability assessment method developed could be relatively straightforwardly applied to different ship types encountering different threat scenarios. However, as mentioned in Sections 4.3 recoverability assessment is the most demanding and least researched area of survivability assessment and requires many assumptions, especially when the design is just at the concept definition level. The developed method involved various simplifying assumptions, in particular disregarding variations in human performance (although, theoretically, this could be addressed by varying the man-hour repair times employed, see Table 4.10 in Section 4.3.5). But also for fire (or flooding) spread (since it is simply assumed that only and all items in the hit WT section will be affected by fire) and the effects of excessive heel and trim (for example, from fire fighting). Despite this, the approach adopted can be used in the concept phase to investigate the efficiency of the arrangement of DC related items, major ship systems and stores and workshops required for repair works. This can be done in terms of magnitude of resulting damage and access to damaged items/WT sections. Therefore, the efficiency of the layout and system architecture can be improved in a concept stage ship design. As with the vulnerability assessment method, the recoverability assessment method facilitates results backtracking and provides ample insight into the effectiveness 
of the recoverability characteristics of each ship design, facilitating the subsequent adoption of corrective design actions.

It is, therefore, evident that the developed method examines recoverability of the ship design; however, this is done without regard to situational awareness, which forms an important part of recoverability (as well as susceptibility) performance. In Section 7.1.4 it was noted that human performance, including human situational awareness, is difficult to quantify. Moreover, the developed survivability assessment method does not assess human performance related aspects (which are assumed to be constant given that all ships compared are assumed to have the same crew once a selection between the designs was made and brought forward); rather, it assesses the ship design itself and how the choice of configuration has affected survivability. Therefore, the proposed recoverability assessment method is consistent with the other two components of the survivability assessment method. It is interesting to note that when asked to propose alternative weighting schemes, certain naval officers recommended the addition of PMs regarding unquantifiable entities such as training, situational awareness, availability of skills as well as prioritising between duplicated systems (such as FRPPs and NBCD stores) which also depend on human and operational factors due to decisions probably made by the CO. All these comments serve to highlight the link between human performance and naval ship recoverability.

Similarly to the vulnerability assessment method, there are issues regarding the use of appropriate weighting schemes which rely on human input (and are, therefore, subjective) rather than some numeric simulation. The impact of the applied weighting scheme on certain results was again highlighted, although very broad patterns were observed in the weighting schemes proposed by different naval officers. In Section 6.6.1, various remarks made by the interviewed naval officers were mentioned. It was noticed that there were disagreements regarding, for example, the contents and significance of NBCD, naval and spare gear stores which affected the weightings given by each officer. Dissimilarities were also perceived regarding the perceptions of the relative importance of the various compartments (represented by their corresponding PMs) on DCFF and the repair routines followed. In addition different naval officers proposed the addition of various quantifiable PMs (but possibly not available in preliminary ship design), for example, those relating to items such as fixed firefighting systems, detection equipment (which would affect ship situational awareness), communications, sickbay, smoke curtains and the Weapon Section Base. All this signifies that each officer draws on his own experiences, knowledge and specialisation. 
This means that the development of appropriate weighting schemes, as well as suitable $\mathrm{PMs}$, is an area where further research is required.

An interesting fact regarding the recoverability assessment of the two AOR variants is that the liquid and solid cargo tanks were assumed to belong to the fluid tanks and light engineering repair categories respectively (Table 4.10 in Section 4.3.5). This, however, gives an indication of the damage caused to the compartments themselves rather than the cargo and could be interpreted as the cargo tanks being empty. It would, therefore, be appropriate to investigate the recoverability characteristics of an AOR design with no cargo, as well as when loaded. This could be done by assuming that all cargo belongs to the last equipment repair category, i.e. humans and stores, assumed irreparable if affected by a hit.

Again the scale on the $y$-axis of the normalised recoverability charts (Figure 6.39, Figure 6.40 and Figure 6.41 in Section 6.5) needs careful definition (see Sections 7.1.4 and 7.2.4 for susceptibility and vulnerability axis definition respectively). Zero is defined as the damages caused by the threat (ASM in this instance) being instantly recoverable despite a hit at any location of the ship being assessed. This would mean that all PMs would output a value of zero, i.e. either the number of man-hours to repair damaged items would be zero (instantly reparable), or no items would be damaged (corresponding to a vulnerability of zero, in which case recoverability assessment would not follow). However, a value of zero in some cases (PMs 1.1-1.4) is impossible since it would imply that the ship design would have a length of zero with, therefore, no WT doors and decks, and infinite access routes. Unity is defined as the recoverability performance of a baseline design (or the worst performing design in the case of the AOR designs; see Section 7.2.4). In contrast to susceptibility and vulnerability assessment, the definition of unity could not be changed to something similar to: the ship being assessed and its systems are totally unrecoverable (100\% irreparable) to the threat, as this would imply infinite values in the PMs. For example, PMs quantified by man-hours to repair a specific equipment/system would require infinite values for irreparability. It becomes evident that defining the scale in recoverability assessment becomes increasingly difficult. Modifications to the PM recoverability assessment method would be required to achieve a more meaningful scale, indicating that this is an area for further research. These issues could be addressed partially through the development of a set of minimum acceptable recoverability standards for each ship type and for a given threat (possibly based on the recoverability performance of current ships), with all future alternative designs being assessed against these standards 
(analogous, for example, to damage stability assessment), in the same manner as outlined in Sections 7.2.4 for vulnerability assessment.

Various conclusions were made during the application of the recoverability assessment method on the seven ship design studies:-

- The impact of the number of damaged, unavailable, items which require repair and the access arrangements to the damaged areas, on recoverability performance was identified (including the effect of ship size on these two factors);

- The contrast between the improved vulnerability but degraded (total system) recoverability characteristics of duplicated and separated systems due to arising access complexities was observed. (Total system recoverability increases in importance when follow-on attacks are expected. This is another factor that could influence the weighting scheme since PM 3.1 just focuses on the recoverability performance of the system because of any redundancy while PM 3.2 focuses on the recoverability performance of the total system. The significance of the latter would increase if follow-on attacks are expected);

- The fact that there is an optimum ship size and number of zones per ship.

In addition, the impact of factors such as the applied weighting scheme and the susceptibility related assumed lengthwise hit probability distribution, which has been acknowledged as an aspect requiring further work due to its strong influence on all survivability constituents, were identified. Finally, the difficulty in distinguishing the boundary between vulnerability and recoverability was mentioned and the belief that they should be considered separately was questioned.

\subsection{Total Survivability Assessment Results Analysis}

\subsubsection{Total Survivability Star (Triangle) Plots}

The preferred method of presenting total survivability results is through the adoption of star plots in order to separate the results of the three constituents rather than providing a single (questionable) overall measure. Thus a smaller star (triangle) area for one design would indicate a more survivable ship (for a given weighting schemes and assumptions). The results illustrated by the star plots in Section 6.7 are those already discussed under the three survivability elements so further detailed constituent analysis is not required; however, some general survivability observations need to be made. 
In both sets of comparative studies of the frigate and AOR variants, the susceptibility results of the ship designs compared were similar. This was not the case with the size varied combatants comparative study, where the Destroyer significantly outperformed the remaining two combatant designs (due to the much more capable AAW system of the Destroyer design, resulting in the largest effect that a single survivability feature has presented out of all features investigated in the current project, see Section 7.1.2).

The choice of different hull configuration (such as in the frigate variants) was seen to have a much greater impact on vulnerability and recoverability than simply changing the ship size (such as in the combatants); although, the results were also affected by the choice of the applied weighting scheme, as well as, possibly, the specific hull configuration selected (hull configurations other than monohulls and a trimaran were not investigated). In addition, the AOR variant comparative study indicated that significant vulnerability reduction enhancements may be achieved by altering the internal and external configuration of a single hull configuration, despite identical dimensions, requirements and performance characteristics.

Finally the least vulnerable ship design is not necessarily the most recoverable one. This arose from recoverability performance not only relying on the magnitude of the damage to the ship systems. It also relies on factors such as the access arrangements, the distribution of DCFF and repair works related items, the system hierarchies and architectures (and the weighting scheme used). In addition, the recoverability assessment method investigated total system recoverability, whereas the vulnerability assessment method is biased in favour of redundant systems. Therefore, some cases (such as the split propulsion system of Frigate Variant 3 and the Corvette design and the duplicated ship handling sub-system of AOR Variant 2) can be seen to augment the difference in vulnerability performance of different ship designs. However, when the magnitude of the damage caused by the same threat significantly differed between the designs being compared, this was found to usually dominate the remaining recoverability related factors. This was observed in the frigate variant case where the considerably less vulnerable trimaran outperformed its two counterparts in recoverability, despite the access deficiencies due to its larger size.

An advantage of presenting the results in this star plot form is the avoidance of multiplying the three survivability constituents together, which side steps the issue as to whether the three survivability elements are truly statistically independent (see Sections 3.3.53 and 4.4). Furthermore, with a star plot results presentation, further spokes such as 
cost, displacement and power can be added; although it can only depict characteristics that are currently amenable to quantification (Vasudevan and Rusling 2007). The main disadvantage with this presentation is that the scales in the three axes (susceptibility, vulnerability and difficulty of recoverability) are not directly comparable (since only the definition of the origin, i.e. zero, is constant in all axes, as mentioned in Sections 7.1.4, 7.2.4 and 7.3.4). For example, if a design has a susceptibility of 0.7 and a vulnerability of 0.6 , it does not necessarily mean that the design is less vulnerable than it is susceptible, only that the improvement in vulnerability between that design and the baseline design it is being compared to is larger than the improvement in susceptibility. Since it is entirely a competitive approach, it is necessary to comprehend the survivability performance of the baseline in order to interpret the survivability performance of the designs being compared to the given baseline. This raises issues regarding how the diagram should be interpreted, with regards to the significance of the enclosed area. An obvious solution would be to equalise the definition of unity in all three scales (similar to the definition of zero, i.e. to set one as being equal to $100 \%$ susceptible, $100 \%$ vulnerable and $0 \%$ recoverable); however, as discussed in Sections 7.1.4, 7.2.4 and 7.3.4, this is possible for susceptibility and vulnerability performance, but not currently for recoverability performance. A partial solution would be to develop standards for each survivability constituent (for each ship type and threat), and set unity as being equal to the minimum acceptable relevant standard.

\subsubsection{Total Survivability Bar Charts}

The above disadvantage would be avoided if the individual survivability results were combined in the manner described in Section 4.4 and output the results illustrated in Figure 6.51 of Section 6.7. After following this procedure for the three frigate variants, it can be said that the results depicted in the total survivability star plot (Figure 6.48 in Section 6.7) were confirmed; i.e., overall, Frigate Variant 3 is the most survivable design, followed by the baseline and lastly the second variant. In addition since this procedure outputs a single survivability value it may be concluded that a better relative comparison can be made between the total survivability performances of the ship designs being compared. However, the main limitation of this method is that it is based on PM matrices which are specifically designed to investigate recoverability performance rather than total survivability. Therefore, the validity of the results and the relative survivability performance magnitude of each ship design is questionable. For 
this reason it was decided to apply this method to a limited number of ship design studies. Other limitations include the issues with regards to defining the y-axis (see Section 7.3.4) and the questionable statistical independence of the three constituents, required for a single numerical value.

It can be concluded that currently, the use of total survivability star plots is the most sensible means of presenting results. However, it is clear that visualisation of results and comparison methods are an area for further development.

\section{$\underline{7.5 \text { Ship Costing Results Analysis }}$}

\subsubsection{Unit Procurement Cost}

Certain assumptions, such as the material and equipment costs, labour costs, purchasing overhead factors, shipyard hourly charge-out rates and the parametric relationships used were based on historical (monohull) data and therefore are dubious in accounting for new technologies and innovative design features. It can be argued that when using specific item costs (as was done for engines, electric motors and payload items) more accurate cost estimation should be achieved; however, the lack of data in many cases led to estimations based on similar equipment and, therefore, further uncertainties.

One of the major uncertainties of the UPC cost estimation was the design contingency margin adopted. All UPC analysis margins were taken from references such as Dirksen (1996) and UCL (2010a). However, there was no indication as to what was an appropriate contingency margin for an innovative hull configuration, such as a trimaran, beyond a general comment regarding the use of larger margins for Advanced Marine Vehicles (AMVs). It was, therefore, simply decided to increase this margin by $1 \%$ between each frigate variant designs (see Table A57 in Appendix 13.1). From the above comments, the very similar UPCs were to be expected. Thus, Frigate Variant 3 is less than 5\% more expensive and Frigate Variant 2 approximately $2 \%$ more expensive than the baseline.

In all individual weight groups (with the exception of the main propulsion and payload groups) the trimaran gave larger costs, followed by the second variant and then the baseline. This was also expected since the parametric relationships scaled with weight and the ship designs descended in displacement in the same order. The main propulsion group for the second variant is slightly more expensive due to the larger 
generating capacity of that design (see Appendix 9.4) (compared to the identical power generation units used in Frigate Variants 1 and 3). The payload group follows a converse pattern due to all three ship designs incorporating a constant payload (meaning payload equipment costs were identical and installation costs similar) and a displacement scaling factor applied to all weight groups (which has an effect of decreasing relative costs for heavier ship designs). This raises issues as to if the displacement scaling factor should be applied to all or just to specific weight groups (such as the hull group in which, due to economies of scale, the purchase of larger steel quantities should result to cost benefits from cost per weight reductions). In all three frigate design cases studied, the payload group accounted for almost half of the UPC, followed by the hull and main propulsion groups, being the only groups responsible for more than $10 \%$ of the UPC. This suggests that cost cutting studies should focus on these areas. However, the effect this would have on survivability performance is unclear.

\subsubsection{Through Life Cost}

When estimating TLC, the fact that costs have to be forecasted can only increase the uncertainties of the resultant values. The projected discount rate, inflation and fuel inflation are likely to be sources of significant error. However, since in the current case different ship designs are compared, more emphasis is given to the relative, rather than the absolute, cost values, thus, decreasing the importance of the accuracy of the above values. However, elements such as the forecasted operational profile could mislead the costing outputs due to the different speed-power characteristics of the three frigates. Such errors would be enhanced by the fact that the assumed inflation of fuel is larger than the assumed discount rate, whereas the opposite is true for the assumed inflation of all other TLC elements.

Projected costs on maintenance and refits are derived though parametric relationships which are also based on historical (monohull) data and, therefore, often cannot account for new technologies and innovative design features. The fact that these parametric relationships scale with the already dubious UPC (and individual weight group cost) prediction increases uncertainty. As do the facts that all ships are assumed to be constructed in the same timeframe and all ships are assumed to undergo the same number of major and minor refits at identical intervals, regardless of the novelty level of the design. It is, therefore, not surprising that the TLC estimations of all frigates are 
very similar (Frigate Variant 3 TLC costs are approximately 4\% and Frigate Variant 2 approximately $2 \%$ higher than the baseline).

In all individual TLC elements (with the exception of the anticipated fuel costs) the trimaran outputted the larger costs, followed by the second variant and the baseline. Again, this was not surprising since crew and consumable costs are assumed to scale with complement (which in turn scaled with displacement) and maintenance and refit costs scaled with UPC (which also scaled with displacement). Larger fuel costs were projected for the second frigate variant due to the less efficient hull design (see Section 5.1.2). The largest contributors to TLC were in all cases, and by a large margin, the projected fuel and refits costs. Together they were responsible for approximately $66 \%$ of the total, therefore, suggesting that cost cutting research should focus on these costing elements. However, once again the effect of this on survivability is not obvious.

\subsubsection{Whole Life Cost}

The WLCs for the three frigate variants were estimated by adding the UPC, TLC, FOC costs and disposal costs for an assumed class of twelve frigates. A number of simplifying assumptions, such as a shipyard learning curve which would gradually decrease UPC, the scaling of FOC costs with UPC, the costs associated with scrapping a ship within the UK and the clearly unrealistic assumption of construction and disposal of all twelve ships of each class in the same years, were applied. It was found that the FOC and disposal costs were negligible compared to the UPC and TLC, therefore, leading to very similar WLCs for the three variants (the difference between the baseline design and the other two frigate designs was an increase of approximately $2 \%$ and $4 \%$ respectively). It is interesting to note that the discounted TLC accounts for approximately $64 \%$ of the WLC in all three frigate cases, with UPC accounting for approximately 35\%. However, cost cutting is usually biased towards UPC, not only because the effects of UPC are much more immediate and, therefore, of interest to final decision making authorities, but also since the elements of the TLC have to be forecasted through a much larger timeframe, spanning through decades, therefore, dramatically increasing the uncertainties embraced. Again, however, the parametric costing model employed cannot account for the effects of this on survivability enhancement features. 


\subsubsection{General Observations on Ship Costing and Survivability Assessment}

The major conclusion from the ship costing study applied to the three frigate variants is that the differences in ship related costs are negligible. This observation magnifies in importance if it is used to assess the merits of the survivability characteristics of the three frigate designs. It would seem that survivability performance is more sensitive to the ship (hull, internal and external) configuration than is ship cost. The costing method utilised was a parametric method, since such methods are generally more applicable to preliminary stage ship designs. As the parametric relationships used for all frigate designs were identical, the similarity between the results is not surprising. In addition, a vast number of simplifying assumptions and uncertainties were involved in the costing models, questioning the validity of the results.

However, since in western nations naval ship design has been characterised as 'cash limited' during recent decades (Brown 1986a), ship costing is one of the principal factors governing the inclusion of survivability (and other) features on ship designs. Clearly, the parametric costing model used is not capable of satisfactorily linking survivability performance to ship cost and, therefore, assessing the value of survivability. Survivability features such as the application of flare/tumblehome, the reduction of microgeometry and duplication/separation of equipment (to name a few) cannot be captured by the parametric costing model. It is evident that a more detailed costing model is needed; however, this might only be possible during the later design stages. The perception of the cost of survivability as being very (possibly unrealistically) high (National Warship Survivability Committee 2008) might however, mean that many survivability enhancement features will have already been withdrawn before reaching the later design stages. The need for an improved costing method during preliminary ship design and for a better understanding of the cost of survivability is, therefore, clear.

\subsection{Research Review}

This section considers whether the proposed approach in Chapter 4 addresses the gaps identified in Chapters 2 and 3 and, therefore, considers if the research aims in Chapter 1 were met and suggests a way forward for this research. The Research Review is split into nine sub-sections. The first three give background information on the topic, therefore, justifying the need for a survivability assessment method linked to 
architecturally orientated preliminary ship design. The following three sub-sections summarise the capabilities of current survivability assessment techniques, identify where further work was required and describe how this was tackled. The seventh subsection includes a comparison between the proposed approach and MISSION, the current MOD programme attempting to integrate all survivability constituents, and leads to the eighth sub-section which discusses some of the perceived advantages of the proposed approach. The final sub-section gives a list of what are considered the main areas for further work in this research. Those areas were identified by comparing the work accomplished to the initial research aims as well as by detecting problematic areas throughout the course of the project.

\subsubsection{The Need for Survivability Quantification}

It is commonly accepted that naval ship design has shifted from the technically best to the affordable design (Brown 1986a). The causes of this are a combination of various factors such as the above-inflation increase in UPC and the declining defence budgets, which have also led to a gradual reduction in the number of ships of most western navies. Another commonly accepted fact is that the main operational difference between naval and commercial ships is that the former are designed to be deliberately placed in harm's way. Naval ships, therefore, have to survive much tougher conditions, requiring additional measures to the ship's inherent survivability features. Such requirements establish the main distinction between naval and commercial vessels, contributing to the increased complexity and costs associated to warships.

The increased knowledge concerning naval ship survivability acquired over recent decades has resulted in various documents and publications from major navies, including survivability related rules and management strategies to be used as guidance by the designer. Such documents usually contained feature-based guidance based on historical experience, rather than performance based specifications, due to the difficulty in obtaining survivability quantification methods and tools. Thus, survivability features, whose performance is difficult to quantify, become hard to justify, presenting an apparently attractive area for cost cutting. It has been argued that such attitudes could lead to unbalanced and ineffective ship designs (Brown 1993), especially when considering the increase in magnitude and variety of threats that warships currently face and the decreasing number of warships. 
It has been argued that the best way to deal with the problems created by the increasing warship costs, declining budgets and requirements of increased capability is through innovations in both individual ship designs and the design process. However, prescriptive rule-based design inhibits novel and innovative designs (for which no historical data exist and are seen to be risky) and design processes; in addition, safety rules are always made following an incident. (This is also true in commercial ship design, where Table 1 of (Cai et al 2012) lists numerous safety regulations set after major incidents). Consequently, the need for methods to quantify survivability (and other important ship performance characteristics), which would allow innovations (therefore, theoretically enable the achievement of more cost effective designs) and justify survivability features is clear.

\subsubsection{The Importance of Survivability Assessment in Preliminary Ship Design}

Ship design is an iterative process, often depicted in the form of a design spiral (Andrews et al 2012). The role of the naval architect is increasingly demanding given the multitude of variables existent within the process (especially in the highly complex warship design area), with which the naval architect has to arrive to a balanced solution. The challenges faced escalate when considering that prototypes are very rarely built, implying that the solution does not have the reassurance of prototype testing before FOC construction. One easily understands the need for the exploration and assessment of a number of alternative designs at concept stage before proceeding to the later design stages. Thus, it is important that survivability is quantified during these early design stages through an appropriate assessment method. The existence of a preliminary design stage survivability assessment and quantification method would enable appropriate survivability measures in the initial iterative design process and offer increased certainty and confidence to the designer as well as the customer that the requirement will be met (Andrews et al 2012).

It is widely recognised that the time and resources expended during the concept design stages are minimal when regarding the entire project, despite the most major decisions and trade off studies which will define the final product being taken at these stages. Moreover, the identification and solution of a problem during the early design stages is much easier and cheaper than applying delayed corrective actions. Finally, addition of novel design features (aiming, for example, to increase capability and/or reduce cost) is much simpler to investigate and effectively incorporate during the 
preliminary design stages. It is, therefore, easily apprehended that the early design stages are the most crucial stages which will largely define the final solution, further justifying the need for a survivability assessment method able to operate with ship designs of minimal design definition.

\subsubsection{Survivability Assessment and Architecturally Orientated Design}

Most warships are described as 'architecture limited' (Purvis 1974) (see Section 2.1.4), therefore, a fully integrated and configurationally orientated preliminary design process would ease the identification of design drivers and risk areas (therefore, better dealing with complexity and increasing confidence), enable the incorporation of innovative solutions through increasing the creativity and contribution of the designer and facilitate the rapid exploration of various design alternatives. Also, by providing a more detailed concept design configurational description it would enable the investigation and assessment of various configuration dependant areas, such as ship operations, personnel evolutions, structural continuity, topside design and ease of production during the early design stages. Furthermore, survivability is also largely dependent on the layout/configuration/architecture of the ship, justifying the combination of a concept stage survivability assessment method with architecturally orientated preliminary design approaches.

The rapid development in computer technology and CAD systems over the last years has aided the production of powerful software fully integrating ship design synthesis with architectural factors in the centre and from the early stages of the process. One such method is the UCL DBB approach to preliminary ship design in which the designer separates the ship's functions and sub-functions into discrete elements (DBBs) and positions them appropriately, putting architectural factors in the centre of the process. The DBB approach was implemented through the SURFCON module in Paramarine, therefore, facilitating the use of all the naval architectural analytical tools available in the commercially established Paramarine CAD software. Thus, a fully integrated preliminary design process architecturally centred and combined with traditional naval architectural numerical analysis techniques to achieve balance is available and the advantages of linking this method to a concept stage survivability assessment method clear. 


\subsubsection{Susceptibility Assessment}

Regarding susceptibility assessment methods, a variety of validated tools able to assess and quantify the susceptibility performance of a ship design (in related areas such as signatures and defensive system effectiveness, and against numerous threats) have been identified, and many utilised in actual warship design projects (see Section 3.3.2). These tools can be applied on ship designs during different design stages (including preliminary). Therefore, most susceptibility assessment techniques can easily be linked to preliminary design methods such as the DBB approach and therefore should be incorporated in the Naval Architecture design process. It should be noted that since the need that was identified above was that of a survivability assessment technique investigating ship architecture the effects of human performance were not considered. That is to say, alternative ship designs should be assessed rather than alternative crews, therefore, assuming crew performance as constant.

For these reasons, the development of a new susceptibility assessment technique was deemed unnecessary. Since most susceptibility assessment techniques rely on restricted information and are not easily accessible, it was decided to combine an unclassified tool (CSEE) with the outputs given by a signature prediction tool (which being classified, was not run at UCL). This was done at the expense of results accuracy; however, since the detailed analysis of susceptibility features was beyond the scope of this study, the above method was considered acceptable. The method adopted led to a straightforward and easily comprehensible quantification of susceptibility and linked susceptibility assessment to preliminary architectural ship design, characterised by limited detailed design definition. This seemed to work satisfactorily across the range of ships studied.

\section{$\underline{\text { 7.6.5 Vulnerability Assessment }}$}

The extensive research in vulnerability assessment over recent decades has led to a multitude of relevant tools produced by different nations, able to simulate various weapons and predict probabilities of preserving capability (see Section 3.3.3). The recognised importance of preliminary ship design has even led to the development of such tools specifically aimed to concept design stages. Through the gradual development of such tools, it can be claimed that software such as SURVIVE (the current UK MOD survivability assessment technique) have reached a mature stage. 
Such tools can assess various threat types and weapon effects using a single ship model; do so in three dimensions, with various levels of detail; use validated algorithms, including flexible methods for system definition; are able to simulate cumulative damage caused by multiple hits; and able to model novel hullforms. An issue that possibly requires supplementary addressing is the further validation of concept stage vulnerability assessment tools operating with low levels of design detail (such as SURVIVE Lite), especially when investigating the effects of applying different zoning philosophies, where the zones are largely defined by the routings and connections between different items which are vague at best. Nevertheless, vulnerability assessment is already considered part of the iterative ship design process mentioned above. Vulnerability assessment software has been used in actual projects in order to manage vulnerability throughout the design process (Martin 1998; Reese et al 1998; Thornton et al 2007; Schofield et al 2012).

For the above reasons, and since access to SURVIVE Lite was granted, the development of a new vulnerability assessment technique was also deemed unnecessary for this project. SURVIVE Lite was used to develop an approach which quantified vulnerability (the quantification of which, however, depended on a subjective weighting scheme) and linked vulnerability assessment to preliminary architectural ship design, characterised by limited detailed design definitions. In addition, the approach facilitated the backtracking of the results and identification of undesirable vulnerability features, which could then be easily altered given the preliminary nature of the ship designs.

\subsubsection{Recoverability Assessment}

In marked contrast to susceptibility and vulnerability assessment, it was not possible to identify a suitable recoverability assessment method which quantified recoverability and was able to be applied easily, simply and rapidly to preliminary design stage ship designs. This can be attributed to various reasons (see Section 3.3.4 and 4.3) which constitute recoverability assessment considerably more demanding to achieve. For example, the reliance on human factors (since recoverability "is mainly an operational aspect relying mainly on the sufficient training of the crew although it may still pose several requirements to the designer" (Boulougouris and Papanikolaou 2012)); the time dependence (i.e. dynamic nature) of recoverability (in order to model secondary damage such as fire and flood progression and crew actions); the inadequate data available; and the difficulty in incorporating crew readiness and skill levels. 
A number of recoverability assessment techniques exist (see Section 3.3.4), however, many, such as safety and risk analysis methods, are qualitative. Thus, they are subjective, relying on expert judgment, and furthermore, do not take specific ship architectural features into account and so are considered to be of limited use. They could possibly be useful after preliminary ship design, when deciding on what DC equipment to have on board and what procedures to follow. Other methods are focused on a particular aspect of recoverability (rather than total ship recoverability), such as fire and flood spread or DC crew evolutions/personnel movement. Such techniques often rely on simulations, with often lengthy computational times (only worsened by the substantial number of scenarios which could be modelled) and require a detailed ship design definition not applicable to preliminary ship design. The latter issue also applies when total recoverability has to be simulated. The increased dependence on operational and human factors for recoverability has led to the development of DC simulation tools. These tools capture both the performance of the crew in various DC scenarios and the effect of the ship architecture. However, they are also aimed at detailed, if not existing, ship designs.

Since the required capability identified was that of a survivability assessment tool aimed at preliminary ship design and specifically investigating the effect of the choice of ship configuration on survivability (by linking the method to an architecturally orientated preliminary ship design approach) and none of the existing recoverability assessment techniques addressed this, the development of a new recoverability assessment technique was clearly identified. Simulations might just be possible late in preliminary ship design (Casarosa 2011) but an early assessment of survivability was considered preferable (for the level of ship definition in the ship design studies explored in this research). Furthermore, assessment of recoverability requires temporal metrics such as the time taken to repair systems, which would be produced by simulation.

An alternative analytical method was developed in order to generate this data (and, therefore, decouple the method from chronological dependence). The new recoverability assessment approach developed a number of Performance Measures (PMs) and an appropriate weighting scheme in order to attempt to overcome the difficulties of recoverability modelling (such as lack of data, human performance and time dependence). The PMs are aimed at investigating the post-hit availability of the DC system, various major ship systems and the access to damaged items, thus capturing the effects of ship architecture. It can be said that, since recovery operations have a 
storyboard, in ship designs with a low level of detail, the best way to describe this is by tying each action into a spatial feature represented by a corresponding PM which in turn indicates the performance of the design. The level of detail in the ship designs does not allow the detailed investigation of human performance and actions (with which the storyboard would be described); in addition, as mentioned, the need that was identified was that of a survivability assessment technique investigating ship architecture rather than human performance.

However, since recoverability is more of an operational rather than a design aspect, the complete decoupling of human performance from recoverability performance is impossible. In the developed method it was incorporated through assuming man-hour data for the repair of the various affected items, which in turn defined the value of the PMs. Theoretically, human performance could be assessed by altering this data; however, the main objective of the developed method is to investigate ship architecture, i.e. to assess the survivability performance of alternative ship designs rather than alternative crews, therefore, assuming crew performance as constant.

It could be argued that the developed method has some similarities and could be considered an evolution (since factors such as major system recovery and access were incorporated) of the suggestion of MOD (2001) that, given the immature stage of recoverability assessment, an interim solution would be to incorporate recoverability by modelling the vulnerability of the whole DC system, without, however, considering progressive damage.

The proposed approach links recoverability assessment to preliminary architectural ship design, characterised by very low detail design definitions. In addition, the approach facilitates the backtracking of the results and identification of undesirable features, which could then be easily altered given the preliminary nature of the ship designs. Finally, the method partially succeeds in quantifying recoverability (the quantification of which, however, depends on a subjective weighting scheme). The major problem in this regard is the difficulty in defining the recoverability performance scale (due to the absence of a minimum recoverability value, see Section 7.3.4), an area requiring further research.

\subsubsection{Comparison between MISSION and Proposed Approach}

At this point, it would be useful to undertake a brief comparison between the developed survivability assessment method and MISSION, the current MOD 
programme attempting to integrate all survivability constituents. The main top-level differences between the two methods can be summarised as:-

- "MISSION is an event simulation tool designed to simulate a maritime mission" (White and Allwood 2011; Parry 2012; Parry and White 2012); inputs include tasks and movement of blue and red forces and, therefore, it can be considered as an operational analysis simulation tool;

The proposed approach is a design optimization tool, designed to compare ship variants (or even ship types) with regards to their survivability performance; it is closely linked to CASD software and is designed to incorporate survivability in the iterative preliminary ship design process (in a similar manner to, for example, powering and stability analysis);

- MISSION simulates “at a task-force level" (White and Allwood 2011; Parry 2012; Parry and White 2012);

The proposed approach looks at single ship designs;

- MISSION gives a variety of outputs, the most important being the Probability of Mission Success;

The proposed approach outputs survivability performance in terms of the three survivability constituents;

- In MISSION, capabilities (and repair tasks) are prioritized, and crew and casualties are modelled and used for recoverability modelling;

The proposed approach uses scenario dependant weighted PMs for recoverability assessment;

- MISSION, being a Monte-Carlo model, involves the repetition of simulations in order to generate statistical results;

The proposed approach, being a deterministic method/algorithm, outputs the same results given constant inputs; therefore, each ship design is investigated once;

- "MISSION uses a time step simulation method" (White and Allwood 2011; Parry 2012; Parry and White 2012);

Proposed approach uses an analytical method.

Some top-level similarities between MISSION and the proposed approach include:-

- Both methods are only defensive, i.e. blue forces are unable to target red forces but only the weapons attacking the blue forces;

- Both methods disregard environmental factors; 
- Both methods use SURVIVE (Lite in the latter case) for vulnerability assessment, using a near identical philosophy for system/capability definition;

- Both methods use external models (which can be replaced/substituted) to input susceptibility and vulnerability (and in the case of the proposed survivability assessment approach, recoverability) data.

In addition to the above, there are various lower-level differences and similarities between the two approaches. It should also be recognised that both approaches are currently in their development phase. The general conclusion is that MISSION, being a maritime operational analysis simulation tool, is generally more complex and requires much more input detail and computational time. The proposed approach solely investigates the survivability characteristics of competing ship designs in an attempt to incorporate survivability analysis in the iterative, architecturally orientated, preliminary design process. Moreover, the proposed approach, being constituted by three external methods to assess the three survivability constituents is able to output the relative recoverability (and susceptibility and vulnerability) performance of a ship design being assessed. MISSION is not able to do that for recoverability performance since it does not output a specific recoverability related value (it does, however, for susceptibility and vulnerability since it uses external models for their assessment). This also makes it easier to suggest improvements to the ship design when using the proposed approach, as negative results (e.g. worst performing PMs) can be traced back.

\subsubsection{Advantages of the Proposed Approach}

The alternative proposals as to how to combine the results from the three survivability constituents in the proposed approach led to the decision to regard them separately and present them together in the form of star plots. This process is advantageous in that it gives a clear indication of the survivability performance of competing designs and avoids the uncertainties involved in the combination of the three constituents (see Section 7.4.1). It is believed that the proposed approach would support the move from prescriptive, feature-based to performance-based design for survivability by providing a supplementary tool for designers to assess survivability and ensure the attainment of the required performance from the commencement of the design phase. However, the issue regarding the meaningful quantification of recoverability performance must first be addressed, through for example, the development by experts 
of recoverability (and susceptibility and vulnerability) standards for each ship type and for a given threat (possibly based on the recoverability performance of current ships), with these standards defining the recoverability performance scale (see Section 7.3.4). The proposed approach would thus support the establishment of survivability statements and detailed requirements/goals during the requirement formulation stage, rather than viewing them as further constraints to an already complicated process.

Furthermore, the survivability assessment approach proposed would assist in achieving a balance between the three (equally investigated) survivability components and other design features, with consideration to their associated weight, volume and cost penalties, therefore, justifying the required survivability enhancement measures despite reducing budgets.

\subsubsection{Future Work}

A number of issues must be addressed before the proposed survivability assessment approach is considered mature:-

- The issue regarding the meaningful quantification of the recoverability performance scale (Figure 6.39, Figure 6.40 and Figure 6.41 in Section 6.5) has been flagged up (see Section 7.3.4). This might be addressed through the development of recoverability standards which would define a scale, or through alterations to the recoverability assessment method;

- Further investigation is required regarding the use of appropriate weighting schemes for a given operational scenario (as well as appropriate equipment repair times). The Delphi Method (see Section 3.3.4) is seen as a promising technique for converging to a single weighting scheme per scenario and easing the concerns regarding the subjectivity of the weighting schemes (and repair times) and, therefore, vulnerability and recoverability results. In addition, it could be used to suggest alternative major ship systems and PMs that should be included in the vulnerability and recoverability assessment sub-methods respectively;

- A major issue requiring further research is the modelling of secondary damage through the progression of fire and flooding, as well as the difficulties associated with excessive post-damage heel and trim in the recoverability assessment part of the method. In the proposed approach demonstrated it was simply assumed that fire (the effects of which are often worse than the effects from the weapon 
detonation) would only affect all those items within the hit WT section (flooding was not considered since only abovewater threats were modelled and excessive heel and trim were neglected). In addition, fire effects were not accounted for in the PMs relating to the immediate actions and effects after attack (i.e. Category 1 PMs), but only in the PMs relating to recovery operations after secondary effects have been dealt with (i.e. Category 2 and 3 PMs). This was assumed in order to avoid the use of complex simulation tools. The use of such tools would not only require a much higher level of design definition but would also disable the decoupling of the recoverability assessment method from time dependence, introducing further requirements, such as the modelling of crew evolutions. This would then result in a highly complicated simulation tool, similar to ones already existing (see Section 3.3.4), which are inappropriate for use in preliminary ship design. Of relevance is Cootes' (2010) remark that "whilst it is imperative to implement survivability analysis at the early stages of design, the level of detail needs to be kept minimal early on". Furthermore, the very detailed and accurate input data required by advanced fire modelling tools, as well as the amount of factors influencing compartment fires and required assumptions outlined in (Institution of Structural Engineers 2007), could limit the realism of fire analysis even at detailed level. Therefore, a certain level of damage (hit WT section) was simply assumed. However, this is an area regarding further investigation;

- The proposed approach could be further developed in order to include the effects of multiple and varying threats (as opposed to the current demonstration just using radar homing sea-skinning ASMs) as well as protection offered by other ships in the task group (as opposed to assuming that each ship design only uses its own defensive systems). These developments could be addressed relatively easily given the plethora of susceptibility assessment techniques capable of the above (they could also be used to improve on the assumption of $\mathrm{P}(\mathrm{di})=1$ for all ship designs) and the ability of SURVIVE Lite to model various threat types and cumulative damage;

- In order to fully regard the developed method as a constituent of the iterative architecturally orientated DBB approach to preliminary ship design, it should be computer coded, therefore, saving remodelling and method application time.

- In Section 7.4.1 it was mentioned that the proposed survivability assessment approach demonstrated that the choice of hull configuration had a great impact 
on vulnerability and recoverability performance. This observation requires further examination through the application of the proposed approach on additional hull configurations (other than the monohull and trimaran configurations investigated in this research);

- The need for an improved costing method during preliminary ship design which would be able to capture the cost of survivability enhancement features, as well as the importance of a better understanding of the cost of survivability was identified in Section 7.5.4.

A final problematic issue identified during this research (and summarised in Section 7.3.1), which is not directly related to the survivability assessment method developed but to the subject of naval ship survivability as a whole, is the correct identification of the boundary between vulnerability and recoverability, or even whether they should be separately considered. There is an inconsistency in the definitions of survivability given by various authors (see Appendix 3), where some consider the constituents of survivability as being susceptibility and vulnerability (which included recoverability) while others regarding recoverability as a separate topic. In addition, most authors do not seem to recognise that the susceptibility related lengthwise probability hit distribution of a ship (i.e. the location of the hit, an additional topic which requires further definition) directly affects the ship's vulnerability (and, therefore, recoverability) performance, while not playing any role in the ship's total susceptibility performance. The proposed survivability assessment approach did incorporate this relatively straightforward feature; however, the issues regarding the assessment of vulnerability and recoverability remain and need to be resolved. 


\section{Chapter 8: Conclusions and Future Work}

The discussion in Chapter 7 details a large amount of issues arising from the application of the proposed survivability assessment approach outlined in Chapter 4 to the ship design studies described in Chapter 5 in order to output the results presented in Chapter 6. Several central matters emerged throughout this research, which are summarised in this concluding chapter. In general, the main research aim defined in Chapter 1 was met; however, a substantial amount of further work is required in order to classify the topic as mature. The major areas for further work are also summarised in this chapter.

The main aim was defined as: to propose an integrated survivability assessment approach and demonstrate it on a range of ship types and hullform configurations during the preliminary stages of ship design. It was rapidly identified that the linking of such a method to an architecturally orientated preliminary ship design approach, such as the DBB approach, would be advantageous for various reasons. Chiefly, attempting to assess human performance (upon which aspects of survivability, primarily susceptibility and recoverability, are dependant) is difficult, possibly unachievable in the concept design stages, with limited ship definition. Therefore, it was decided that the developed approach should investigate the effect that configurational choice had on survivability performance (ship configuration, i.e. design, as opposed to human, factors, being the other main driver of survivability). The advantages of the DBB approach were quickly realised during the development of the seven ship design studies on which the proposed survivability assessment approach was demonstrated. For example, the ability to quickly produce and compare alternative ship designs, locate related spaces efficiently, identify drivers and risk areas, encourage novel configurations, account for zoning in the early design stages and understand the implications of innovative technologies such as IFEP, were clear. It was concluded that a new ship design must be described architecturally, in terms of its functional breakdown, and also (traditionally) in terms of its weight breakdown, in order for one to be able to justify the selected design. Without an architectural description of a ship design, an adequate survivability analysis would not be possible.

Various susceptibility and vulnerability assessment techniques which are applicable to preliminary ship design already exist. These tools have been utilised in actual warship design projects in recent decades, contributing to the relative maturity of susceptibility and vulnerability assessment. It was, therefore, judged unnecessary to 
develop new such tools. Due to the sensitivity relevant in the area susceptibility (assessment) it was decided to simulate a simplified ASM attack using an unclassified existing method with unclassified but representative data, in combination with a signature prediction tool operated by the sponsor of this research. Although the method and data used included extensive simplifications, various important features were highlighted after its application to the ship design studies. For example, the effect of signatures through varying ship size, managing microgeometry and shaping were confirmed as was the decreasing importance of signature management with increasing ship size. The consequences of varying defensive missile targeting pattern and defensive missile types were also observed. In fact, it was concluded that the largest effect that a single survivability feature presented (out of all features investigated in the current project) was the use of a more capable defensive AAW system (in the destroyer design), justifying the extensive investments made in susceptibility assessment techniques and reduction features. The method adopted led to a straightforward and easily comprehensible quantification of susceptibility and linked susceptibility assessment to preliminary architectural ship design, characterised by limited detailed design definition. This seemed to work satisfactorily across the range of ships studied.

For vulnerability assessment, QinetiQ's SURVIVE Lite, being currently the principal concept stage vulnerability assessment code of the UK MOD, was utilised. A number of major ship systems were modelled and combined with a weighting scheme in order to assess the effects of the above ASM attack scenario. This approach also led to valuable data by giving indications of performance enhancements when adopting practices such as duplication and separation of identical systems, adoption of IFEP, increasing ship size, improving system layout (facilitated in trimaran configurations) and shielding critical equipment by other, less critical compartments. In addition, the considerable reduction in vulnerability of trimaran configurations through protection provided by the side hulls was observed. The dependence of vulnerability on issues such as the assumed lengthwise probability hit distribution, the attack trajectory and the subjective weighting scheme used, was also observed and those issues require further definition. Nevertheless, the developed approach quantified vulnerability and linked vulnerability assessment to preliminary architectural ship design, characterised by limited detailed design definitions. In addition, the approach allowed the backtracking of the results and identification of undesirable vulnerability features, which could then be easily altered given the preliminary nature of the ship designs. 
A recoverability assessment tool aimed specifically at preliminary ship designs and specifically investigating how ship configuration would affect overall naval ship recoverability does not seem to exist. It was, therefore, decided that a new recoverability assessment method should be developed to investigate such issues at early stage design. Since simulations are not appropriate during the early concept design stages and recoverability is strongly time dependent, the development of a number of Performance Measures (PMs) together with an appropriate (but subjective) weighting scheme was selected in order to decouple the method form chronological dependence. The PMs investigate the post-hit availability of the DC system, various major ship systems and the access to damaged items, thus capturing the effects of ship architecture. In addition, as mentioned in Section 7.6.6, since recovery operations have a storyboard, in ship designs with a low level of detail the best way to describe this is by tying each action into a spatial feature. Spatial features are represented by a corresponding PM which in turn indicates the performance of the design. The level of detail in the ship designs did not allow the detailed investigation of human performance and actions (with which the storyboard would be described). However, the strong operational (as opposed to design) aspect of recoverability did not allow the complete decoupling of human performance from recoverability performance. Specifically, human performance was incorporated through assuming man-hour data for the repair of the various affected items, which in turn defined the value of the PMs. The application of the proposed approach led to significant observations, such as the dominant effect of post-attack equipment availability (and, therefore, the efficiency of the ship layout as well as the assumed lengthwise probability hit distribution) on recoverability performance and the decreased access efficiency of larger ships, as well as the relative advantages of different access philosophies. Moreover, it was identified that there is an optimum ship size (since posthit equipment availability increases due to decreasing vulnerability, but access efficiency decreases, with ship size) for recoverability performance, as well as an optimum number of zones per ship (although zones are largely defined by the routings and connections between different items which are unclear during the preliminary ship design stages, therefore, requiring further work). The proposed approach links recoverability assessment to preliminary architectural ship design, characterised by very low detail design definitions. In addition, the approach facilitates the backtracking of the results and identification of undesirable features, which could then be easily altered given the preliminary nature of the ship designs. However, the method partially succeeds in quantifying recoverability, the major problem in this regard being the 
difficulty in defining the recoverability performance scale (mentioned in Section 7.3.4); an area requiring further research.

From above, it is clear that the proposed design optimization approach is able to identify the principal survivability drivers of various ship types and hullform configurations. In addition, it is able to investigate the effect of factors such as ship configuration, hull configuration and ship size on survivability performance. Moreover, by assessing all three survivability constituents equally, a balance between survivability features may confidently be attempted. The fact that the proposed survivability assessment approach is linked to an architecturally orientated preliminary design approach allows the investigation of alternative designs, as well as specific survivability features in the earliest design stages. During these stages, ship designs are amenable to modifications, which would be expensive or even unfeasible in the later design stages; it is noteworthy that the resources spent during the early ship design stages are minimal compared to those spent during the entire project, although the decisions made during these design stages largely determine the final solution. Finally, the quantification of survivability would justify survivability enhancement features, therefore, discouraging cost cutting in this area. Moreover, through the quantification of survivability, the proposed approach encourages a more meaningful performance-based (rather than prescriptive, feature-based) requirements specification for a ship design project, with survivability analysis commencing at the early design stages, making survivability assessment an integral part of the design process.

A brief costing study was undertaken in an attempt to use ship cost as a measure against which survivability measures can be assessed. However, the parametric costing method used (applicable to preliminary stage ship designs) was unable to evaluate the value of survivability. Survivability features, such as the application of flare/tumblehome, the reduction of microgeometry and duplication/separation of equipment, were not captured. More detailed costing models are required for such analyses; however, they might not be applicable to ship designs of limited design definition, potentially leading to the omission of survivability features. The need for a better understanding of the cost of survivability, especially during preliminary design, is clearly identified.

Other main areas requiring further work (which have been detailed in Section 7.6.9, therefore, are briefly mentioned here) are:-

- The meaningful quantification of the recoverability performance scale; 
- The use of appropriate weighting schemes and equipment repair times as well as the modelling of supplementary major ship systems and PMs in the vulnerability and recoverability assessment methods;

- The modelling of secondary damage and excessive post-damage heel and trim in the recoverability assessment method;

- The inclusion of multiple and varying threats and protection offered by other ships in the task group in the proposed survivability assessment approach;

- The computer coding of the proposed survivability assessment approach;

- The application of the proposed approach on additional hull configurations;

- The correct identification of the boundary between vulnerability and recoverability, as well as a better definition of the lengthwise probability hit distribution of a ship.

Despite the considerable amount of further work required, it is considered that this research has contributed to advancing knowledge of what is possible in the early stages of the design process of "the most complex, diverse and highly integrated of any engineering systems" produced today (Graham 1982). 


\section{$\underline{\text { References }}$}

Adams, T.P. (1988) Guidance Rationale for Future Area Defence Missile Systems. Journal of Naval Engineering, 31 (2).

Afanasieff, L. and Mabry, J.P. (1994) The Design of the FF-21 Multi-Mission Frigate. Naval Engineers Journal, 106 (3).

American Superconductor. (2011) HTS Ship Propulsion Motors [WWW]. Available from: http://www.amsc.com/products/motorsgenerators/shipPropulsion.html [Accessed: 11/01/2011].

Andersen, P and Guldhammer, H.E. (1986) A Computer-Oriented Power Prediction Procedure. In: Proceedings of the International Conference on Computer Aided Design, Manufacture and Operation in the Marine and Offshore Industries, Washington, DC, USA.

Andrews, D.J. (1981) Creative Ship Design. RINA Transactions, Vol. 123.

Andrews, D.J. (1984) Synthesis in Ship Design. (PhD), University of London.

Andrews, D.J. (1986) An Integrated Approach to Ship Synthesis. RINA Transactions, Vol. 128.

Andrews, D.J. (1987) Explorations in the Nature of Frigate Preliminary Design. In: Proceedings of RINA Warship 1987: Anti-submarine Warfare, London, UK.

Andrews, D.J. (1992) The Management of Warship Design - The MOD Warship Project Manager's Perspective. RINA Transactions, Vol. 134.

Andrews, D.J. (1993) Preliminary Warship Design. RINA Transactions, Vol. 135.

Andrews, D.J. (1998) A Comprehensive Methodology for the Design of Ships (and Other Complex Systems). In: Proceedings of The Royal Society, London, UK, 1998.

Andrews, D.J. (2003) A Creative Approach to Ship Architecture. International Journal of Maritime Engineering, 145 (3).

Andrews, D.J. (2004) Chapter 46: Multi-Hulled Vessels. In: Lamb, T. (ed.) Ship Design and Construction: Volume II. Jersey City, NJ, USA: SNAME.

Andrews, D.J. (2006) Simulation and the Design Building Block Approach in the Design of Ships and Other Complex Systems. In: Proceedings of The Royal Society, London, UK, 2006.

Andrews, D.J. (2011) Art and Science in the Design of Physically Large and Complex Systems. In: Proceedings of The Royal Society, London, UK, 2011.

Andrews, D.J. (2012) Is Marine Design Now a Mature Discipline. In: Proceedings of 
IMDC 2012, Glasgow, UK.

Andrews, D.J., Atlar, M., Drake, K., Gee, N., Levander, K., Sen, P. and Snaith, G.R. (1997) IMDC State of the Art Report on Design Methodology. In: Proceedings of IMDC 97, Newcastle, UK.

Andrews D.J. and Brown D.K. (1982) Cheap Warships are not Simple. In: Proceedings of the SNAME Symposium on Ship Costs and Energy, New York, NY, USA, 1982. Andrews, D.J. Casarosa, L. Pawling, R.G., Gelea, E., Deere, S. and Lawrence, P. (2008) Integrating Personnel Movement Simulation into Preliminary Ship Design. International Journal of Maritime Engineering, 150 (A1).

Andrews, D.J., Cudmore, A.C., Humble, P. and Wilson, D. (1996) SUBCON - A New Approach to Submarine Concept Design. In: Proceedings of RINA Warship 1996: Naval Submarines 5, London, UK.

Andrews, D.J. and Dicks, C.A. (1997) The Building Block Design Methodology Applied to Advanced Naval Ship Design. In: Proceedings of IMDC 97, Newcastle, UK.

Andrews, D.J., Duchateau, E., Gillespe, J., Hopman, H., Pawling, R. and Singer, D. (2012a) State of the Art Report: Design for X - Design for Layout. In: Proceedings of IMDC 2012, Glasgow, UK.

Andrews, D.J., Greig, A.R. and Pawling R.G. (2004) The Implications of an All Electric Ship Approach on the Configuration of a Warship. In: Proceedings of IMarEST INEC 2004: Marine Technology in Transition, Amsterdam, NL.

Andrews, D.J. and Hall, H.J. (1995) The Trimaran Frigate - Recent Research and Potential for the Next Generation. In: Proceeding of IMDEX 95, RNC Greenwich, $U K$.

Andrews, D.J., Keane, R.G., Lamb, T., Sen, P. and Vassalos, D. (2006) IMDC 2006 State of the Art Report: Design Methodology. In: Proceedings of IMDC 2006, Ann Arbor, MI, USA.

Andrews, D.J., Papanikolaou, A., Erichen, S. and Vasudevan, S. (2009) IMDC 2009 State of the Art Report on Design Methodology. In: Proceedings of IMDC 2009, Trondheim, NOR.

Andrews, D.J. and Pawling, R.G. (2003) SURFCON - A $21^{\text {st }}$ Century Ship Design Tool. In: Proceedings of IMDC 03, Athens, GR.

Andrews, D.J. and Pawling, R.G. (2006a) Innovative Ship Design for High Speed Adaptable Littoral Warfare. In: Proceedings of RINA Warship 2006: Future Surface Ships, London, UK. 
Andrews, D.J. and Pawling, R.G. (2006b) The Applications of Computer Aided Graphics to Preliminary Ship Design. In: Proceedings of IMDC 2006, Ann Arbor, MI, USA.

Andrews, D.J. and Pawling, R.G. (2007) Concept Studies for a Joint Support Ship. In: Proceedings of RINA Warship 2007: The Affordable Warship, Bath, UK.

Andrews, D.J. and Pawling, R.G. (2008) A Case Study in Preliminary Ship Design. International Journal of Maritime Engineering, 150 (A3).

Andrews, D.J. and Pawling, R.G. (2009) The Impact of Simulation on Preliminary Ship Design. In: Proceedings of IMDC 2009, Trondheim, NOR.

Andrews, D.J., Percival, V. and Pawling, R. (2012b) Just How Valid is the Ship Design Spiral Given the Existence of Cliffs and Plateaux? In: Proceedings of IMDC 2012, Glasgow, UK.

Andrews, D.J. and Zhang, J.W. (1995) Considerations in the Design of a Trimaran Frigate. In: Proceedings of the International Symposium: High Speed Vessels for Transport and Defence, London, UK, 1995.

Archer, B. (1979) Design as a Discipline - Whatever became of Design Methodology? Design Studies, 1 (1).

Army Technology. (2011) Vertical Launch Mica [WWW]. Available from: http://www.army-technology.com/projects/vlmica/ [Accessed: 06/02/2012].

Ashe, G., Bosman, T., Cheng, F., Dow, R., Ferraris, S., Ferreiro, L., Kaeding, P., Kaneko, H., McGeorge, D., Norwood, M., Park, J. (2006) Naval Ship Design. In: Proceedings of the $16^{\text {th }}$ International Ship and Offshore Structures Congress, Southampton, UK, 2006.

Azzi, C., Pennycott, A., Mermiris, G. and Vassalos, D. (2011) Evacuation Simulation of Shipboard Fire Scenarios. In: Proceedings of the Fire and Evacuation Modelling Technical Conference 2011, Baltimore, MD, USA.

Bain, G. (2006) Warship Recoverability Modelling. In: Proceedings of the European Survivability Workshop, Toulouse, FR, 2006.

Ball, R.E. and Calvano, C.N. (1994) Establishing the Fundamentals of a Surface Ship Survivability Design Discipline. Naval Engineers Journal, 106 (1).

Barnett, R.W. (1998) Surface Ship Survivability: Risk Management and Network Centric Warfare. Newport, RI, USA: Naval War College, Center for Naval Warfare Studies, Strategic Research Department.

Barton, M.A. (2009) From Incident to Sinking - What is the Typical Time? A Look at the Data from WWII. Journal of Naval Engineering, 45 (1). 
Bastisch, C. (2002) Operator Involvement in Battle Damage Control Systems -

Requirements and Limitations. In: Proceedings of IMarEST INEC 2002: The

Marine Engineer in the Electronic Age, Glasgow, UK.

Bebbington, J and Groves, A. (2006) Internal Blast Performance of Maritime

Composite Sandwich Panels. In: Proceedings of the European Survivability

Workshop, Toulouse, FR, 2006.

Beedall, R. (2011a) PAAMS - Principal Anti Air Missile System [WWW]. Available from: http://navy-matters.beedall.com/paams.htm [Accessed: 05/04/2013].

Begg, A.G., Paton, W. and Whiteford, J.S. (1990) The Resilient Warship. In:

Proceedings of RINA Warship 1990: The Future for Surface Warships, London, $U K$.

Belcher, M. (2008) Survivability Primer: An Introduction to Naval Combat

Survivability. Halifax, NS, CA: Canadian Department of National Defence.

BMT Defence Services. (2012) Aegir: A Family of Naval Task Force Support Ship

Designs. [Brochure]. Bath, UK: BMT Defence Services.

Board of Governors of the Federal Reserve System. (2013) U.S. / U.K. Foreign

Exchange Rate [WWW]. Available from: http://research.stlouisfed.org/fred2/ data/EXUSUK.txt [Accessed: 05/04/2013].

Bodegraven, K.S., Logtmeijer, R.A., Stanley, J., de Wildt, F.W.J., Janssen, J.A.A.J.,

Smit, C.S., Annakin, B. and Doherty, G. (2012) Reducing the Effect of Damage

on Operational Effectiveness with DINCS Technology. In: Proceedings of IMarEST INEC 2012: Engineering Naval Capability, Edinburgh, UK.

Bole, M. (2007) Introducing Damage Structural Assessment to Onboard Decision

Support Tools. In: Proceedings of COMPIT 2007, Cortona, IT.

Boughton, J. (2012) Personal communication, Dstl Portsdown West, August 2012.

Boulougouris, E.K. and Papanikolaou, A.D. (2004) Optimisation of the Survivability of

Naval Ships by Genetic Algorithms. In: Proceedings of COMPIT 2004,

Siguenza, ES.

Boulougouris, E. and Papanikolaou, A. (2012) Risk-Based Design of Naval

Combatants. In: Proceedings of IMDC 2012, Glasgow, UK.

Bradbeer, N. and Andrews, D.J. (2012a), Affordability, Ship Impact and Shock

Response Implications of Simpler Warship Structural Styles. In: Proceedings of RINA Warship 2012: The Affordable Warship, Bath, UK.

Bradbeer, N. and Andrews, D.J. (2012b) Shock Response Implications of Lower-Cost

Warship Structural Styles. In: Proceedings of IMDC 2012, Glasgow, UK. 
Braid, P., Dunn-Rogers, J. and Webster, K. (2009) Empow!erment-Completing the Kill Chain. [Poster]. Portsdown West, UK: Dstl.

Bricknell, D.J., Skarda, R. and Vedlog, P.E. (2006) A Family of Underway Replenishment Ships. In: Proceedings of IMarEST WMTC 2006, London, UK. Bricknell, D.J. and Vedlog, P.E. (2007) Capturing the Commercial Cost Base in Delivering Naval Auxiliaries. In: Proceedings of RINA Warship 2007: The Affordable Warship, Bath, UK.

Brown, D.K. (1986a) Defining a Warship. Naval Engineers Journal, 98 (2).

Brown, D.K. (1986b) Design to Survive. From: MOD Mid-Career Update Course. Department of Mechanical Engineering, University College London, on $27^{\text {th }}$ May 1986. MOD reference: D/SSC/DCNA/50/36.

Brown, D.K. (1987) The Architecture of Frigates. In: Proceedings of RINA Warship 1987: Anti-submarine Warfare, London, UK.

Brown, D.K. (1989) Safety and Survivability. In: Proceedings of the $13^{\text {th }}$ WEGMTAdvanced Vehicles, Delft, NL, 1989.

Brown, D.K. (1990) The Battleworthy Frigate. North East Coast Institution of Engineers and Shipbuilders Transactions.

Brown, D.K. (1991) The Future British Surface Fleet-Options For Medium-Sized Navies. London, UK: Conway Maritime Press Ltd.

Brown, D.K. (1993) Naval Architecture. Naval Engineers Journal, 105 (1).

Brown, D.K. (1997) In Harms Way: Warship Vulnerability 1939-98, Experience and Statistics. Portsmouth, UK: Naval Historical Branch. NHB Study 39/97.

Brown, D.K., and Andrews, D.J. (1980) The Design of Cheap Warships. In: Proceedings of the International Naval Technology Expo 80, Rotterdam, NL. Brown, D.K. and Brown J.D. (1986) Vulnerability - The Lessons of World War II. DERA. MOD reference: D/SSC/DCNA/109/1.

Brown, D.K., and Tupper, E.C. (1989) The Naval Architecture of Surface Warships. RINA Transactions, Vol. 131.

Bulitko, V.V. and Wilkins, D.C. (1999) Automated Instructor Assistant for Ship Damage Control. In: Proceedings of the $11^{\text {th }}$ Conference on Innovative Applications of Artificial Intelligence, Orlando, FL, USA, 1999.

Cai, W., Konovessis, D. and Vassalos, D. (2012) A Methodology of Marine Accident Investigation for Risk-Based Design. In: Proceedings of IMDC 2012, Glasgow, $U K$.

Carreyette, J. (1977) Preliminary Ship Cost Estimation. RINA Transactions, Vol. 119. 
Carter III, E.W. (1988) Combatant Vulnerability: So What's New. United States Naval Institute Proceedings / Naval Review.

Casarosa, L. (2011) The Integration of Human Factors, Operability and Personnel Movement Simulation into the Preliminary Design of Ships Utilizing the Design Building Block Approach. (PhD), Department of Mechanical Engineering, University College London.

Cerminara, J. and Kotacka, R.O. (1990) Ship Service Electrical Systems - Designing for Survivability. Naval Engineers Journal, 102 (5).

Chalmers, D.W. (1993a) Chapter 6: Structural Synthesis. In: Chalmers, D.W. Ministry of Defence - Design of Ships' Structures. London, UK: HMSO.

Chalmers, D.W. (1993b) Chapter 15: Structural Vulnerability. In: Chalmers, D.W. Ministry of Defence - Design of Ships' Structures. London, UK: HMSO.

Cherming Countermeasures Ltd. (2006) Product Data Sheet No.12 - Issue 4: Cartridge Dual Chaff/IR 130mm Seduction CHIMERA [Brochure]. Salisbury, UK: Cherming Countermeasures Ltd.

Cherming Countermeasures Ltd. (2011) Cartridge Countermeasure Chaff 130mm Distraction CCM216 Mk1 Type 1 [Brochure]. Salisbury, UK: Cherming Countermeasures Ltd.

Chislett, H.W.J. (1972) Replenishment at Sea. RINA Transactions, Vol. 114.

Chow, W.K. (2002) Proposed Fire Safety Ranking System EB-FSRS for Existing HighRise Nonresidential Buildings in Hong Kong. Journal of Architectural Engineering, 8 (4).

Cimino, D. and Filipopoulos, C. (2001) Section 5.0: Weight and CG Margins. In: SAWE and SNAME (eds.) Weight Estimating and Margin Manual for Marine Vehicles. Los Angeles, CA, USA: SAWE.

Clements, D.J. and Kneebone, G.P. (1985) Integrated Damage Surveillance and Control in Surface Warships. In: Proceedings of IMarE Conference on Ship Fires in the 1980s, London, UK, 1985.

Collins, L.E. and Ward, P.A. (2012) The Affordable Modular Frigate - A Design Study. In: Proceedings of RINA Warship 2012: The Affordable Warship, Bath, UK.

Conklin, A. (1988) We Gave $110 \%$ and Saved the Stark. United States Naval Institute Proceedings, 114 (12).

Cooper, S.L., Berger, D.P.G. and McDonald, T.P. (2007) Concepts for a Fleet Tanker: An Exploration into Options and Pricing. In: Proceedings of the RINA International Conference: Military Support Ships, London, UK, 2007. 
Coote, W. (2010) The Effect of Architectural Choices on Warship Vulnerability. (MSc), Department of Mechanical Engineering, University College London.

Covich, P. (1988) Survivability and Ship Design Requirements. From: USN NAVSEA $55 \mathrm{X}$, on $2^{\text {nd }}$ September 1988

Crees, M.R. (2009) Lessons from the Loss of HMS SHEFFIELD. (MSc), Department of Mechanical Engineering, University College London.

Crow, A.C.D. (2001) Influences in Surface Naval Ship Design. In: Proceedings of RINA Warship 2001, London, UK.

Day, T. (2012) Personal communication, Dstl Portsdown West, July 2012.

Deere, S.J. (2012) Ship Design with the Human Factor: Evacuation and Normal Operations Modelling in the Ship Design Process. (PhD), School of Computing and Mathematical Sciences, University of Greenwich.

Deere, S.J., Galea, E.R. and Lawrence P.J. (2009) A Systematic Methodology to Assess the Impact of Human Factors in Ship Design. Applied Mathematical Modelling, $33(2)$.

Dicker, R.J.L. (1986) Survivability and the Modular Frigate - New Developments from Blohm \& Voss. International Defense Review, 19 (7).

Dicks, C.A. (2000) Preliminary Design of Conventional and Unconventional Surface Ships Using a Building Block Approach. (PhD), Department of Mechanical Engineering, University College London.

Dirksen, G.C. (1996) Consideration of Warship Cost. (MSc), Department of Mechanical Engineering, University College London.

Doerry, N. (2007) Designing Electrical Power Systems for Survivability and Quality of Service. Naval Engineers Journal. 19 (2).

Donnelly, K. (1985) Aesthetics in Warship Design. Naval Architect, (June).

Edwards, M.W. and Carr, D. (1998) Men and Machines: The Survivability Balance. In: Proceedings of IMarEST INEC 1998: Surviving the War, London, UK.

Ellison, P. and Escott, H. (2012) Current Developments in the Systems Engineering of the IPMS for the Royal Navy's Queen Elizabeth Class Carriers. In: Proceedings of IMarEST INEC 2012: Engineering Naval Capability, Edinburgh, UK.

Evans, J.H. (1959) Basic Design Concepts. Naval Engineers Journal, 71 (4).

Ferreiro, L.D. and Stonehouse, M.H. (1994) A Comparative Study of US and UK Frigate Design. RINA Transactions, Vol. 136.

Fireproof. (2013) FIREPROOF: Probabilistic Framework for Onboard Fire Safety 
[WWW]. Available from: http://www.fireproof-project.eu [Accessed: 25/03/2013].

Flensburger Schiffbau-Gesellschaft mbH \& Co. KG. (2012) Combat Support Ship / $E G V$ [WWW]. Available from: http://www.fsg-ship.de/101-1-EGV.html [Accessed: 06/02/2012].

Fonseca, P. (2011) Personal communication, Department of Mechanical Engineering, University College London, September 2011.

Foxwell, D. (1990a) Stealth: The Essence of Modern Frigate Design. International Defense Review, 23 (9).

Foxwell, D. (1990b) Soviet Navy Reduces Warship IR Signatures. International Defense Review, 23 (11).

Foxwell, D. (1991a) Signature Reduction: Smart Materials for Active Control. International Defense Review, 24 (11).

Foxwell, D. (1991b) Submarine Defensive Warfare: US to Refit Enhanced Torpedo Defenses. International Defense Review, 24 (11).

Friedman, N. (1991) Stealth in Naval Warfare. Naval Forces, 12 (4).

Friedman, N. (2006) The Naval Institute Guide to World Naval Weapons Systems. $5^{\text {th }}$ ed. Annapolis, MD, USA: US Naval Institute Press.

Fuller, M. (ed.) (2008) Jane's Naval Weapon Systems. $49^{\text {th }}$ ed. Coulsdon, UK: Jane's Information Group.

Fung, S.C. and Leibman, L. (1995) Revised Speed-Dependent Powering Predictions for High-Speed Transom Stern Hull Forms. In: Proceedings of FAST '95: Third International Conference on Fast Sea Transportation, Lubeck-Travemunde, DE. Gale, P.A. (2003) Chapter 5: The Ship Design Process. In: Lamb, T. (ed.) Ship Design and Construction: Volume I. Jersey City, NJ, USA: SNAME.

Gallin, C. and Lemenkuhler, H.J. (1989) Progress on Noise and Space Reduction of Power Transmission for Naval Ships. In: Proceedings of the International Conference on New Developments in Warship Propulsion, London, UK, 1989. Garre, L., Perassi, A., Raffetti, A. and Rizzuto, E. (2010) An Application of Bayesian Networks for the Optimisation of a Bridge Layout. Journal of Engineering for the Maritime Environment, 224 (M).

Garzke, W.H. and Kerr, G. (1985) A New Warship Design Strategy - A Perspective. SNAME Transactions, Vol. 93.

Gates, P.J. (2005) Surface Warships: An Introduction to Design Principles. Revised Edition. London, UK: Brassey's Defence Publishers. 
Gates, P.J. and Rusling, S.C. (1982) The Impact of Weapons Electronics on Surface Warship Design. RINA Transactions, Vol. 124.

GE Marine. (2012) LM2500 Marine Gas Turbine [Brochure]. Evendale, OH, USA: GE Aviation.

Geertsma, R.D. (1999) MSc Naval Architecture Ship Design Exercise 1999: High Survivability Frigate. (MSc), Department of Mechanical Engineering, University College London.

Geertsma, R.D., Badon Ghijben, N.A. and Middeldrop, E.J. (2012) Advanced PMS Functionality in the Royal Netherlands Navy: Automation to Support the Internal Battle. In: Proceedings of IMarEST INEC 2012: Engineering Naval Capability, Edinburgh, UK.

Gerdemann, G., Kuramochi, N.F., Mooshage, O., Puppe, D. and Rudius, M. (2012) VORGES - A Comprehensive Approach for the Planning of Future Naval Vessels. In: Proceedings of RINA Warship 2012: The Affordable Warship, Bath, $U K$.

Gillespie, S.R. (2004) Exploration of Warship Configuration Against Fire Effects. (MSc), Department of Mechanical Engineering, University College London. Glass, J.W. (1988) Damage Control: Adopting an Unwanted Stepchild. United States Naval Institute Proceedings, 114 (12).

Goossens, I.L. (1992) What's Driving the Hydrodynamic Ship Design? In: Hydrodynamics: Computations, Model Tests, and Reality / Proceedings of MARIN Workshops on Advanced Vessels, Station Keeping, Propulsor-Hull Interaction, and Nautical Simulators, Wageningen, NL, 1992.

Gougoulidis, G. (2012) The Affordable Hovercraft: A Flexible Modularised Design. In: Proceedings of RINA Warship 2012: The Affordable Warship, Bath, UK.

Graham, C. quoted in Gates, P.J. and Rusling, S.C. (1982) The Impact of Weapons Electronics on Surface Warship Design. RINA Transactions, Vol. 124.

Grois, E., Hsu, W.H., Voloshin, M. and Wilkins, D.C. (1998) Bayesian Network Models for Generation of Crisis Management Training Scenarios. In: Proceedings of the $10^{\text {th }}$ Conference on Innovative Applications of Artificial Intelligence, Madison, WI, USA, 1998.

Harmathy, T.Z. (1982) The Delphi Method - A Complement to Research. Fire and Materials, 6 (2).

Harms-Ringdahl, L. (2003) Assessing Safety Functions - Results from a Case Study at an Industrial Workplace. Safety Science 2003, 41 (8). 
Harney, R.C. (2010) Broadening the Trade Space in Designing for Warship

Survivability. Naval Engineers Journal, 122 (1).

Harold, T. and Hooper, E. and Qiu, X.Q. (2012) Supportability of a Warship Control

System: Learning Hard Lessons about Requirements Engineering. In:

Proceedings of IMarEST INEC 2012: Engineering Naval Capability,

Edinburgh, $U K$.

Havron, P.R., Howard, S.M. and Brinklow, I. (2012) Whole Life Cost Considerations

for the Procurement of an RFA Tanker. In: Proceedings of RINA Warship 2012:

The Affordable Warship, Bath, UK.

Heather, R.G. (1990) Preliminary Design Considerations for Fast Warships. In:

Proceedings of RINA Warship 1990: The Future for Surface Warships, London, $U K$.

Herman, S. and Loeser, C.T. (1992) Damage Control - The Last Line of Shipboard

Defense. Naval Engineers Journal. 104 (1).

Heywood, M. and Lear, T. (2006) PREVENT - A Tool to Reduce Vulnerability Early

in the Design. In: Proceedings of RINA Warship 2006: Future Surface

Warships, London, UK.

Holmberg, T. and Kotiranta, R. (2012) Bringing Efficiency in the Naval Ship Design

Process With COTS Software. In: Proceedings of RINA Warship 2012: The Affordable Warship, Bath, UK.

Holtrop, J. (1984) A Statistical Re-analysis of Resistance and Propulsion Data.

International Shipbuilding Progress, Vol. 31.

Hood, K. (2012) Personal communication, Dstl Portsdown West, July 2012.

Hudson, B., Shepherd, D. and Ferris, J. (1996) Warship Design: What's So Different? A Canadian Experience. In: Proceedings of IMarEST INEC 1996: Warship Design: What Is So Different?, Den Helder, NL.

IAI Ltd. (2009) Surveillance \& Threat Alert Radar - STAR [Brochure]. Lod, IL: IAI Ltd.

Institution of Structural Engineers. (2007) Guide to the advanced fire safety engineering of structures. London, UK: Institution of Structural Engineers.

International Maritime Organisation. (1993) Resolution A.749(18): Code on Intact

Stability for All Types of Ships Covered by IMO Instruments.

International Maritime Organisation. (2004) International Convention for the Safety of Life at Sea (SOLAS).

International Maritime Organisation. (2011) Passenger Ships [WWW]. Available from: 
http://www.imo.org/OurWork/Safety/Regulations/Pages/PassengerShips.aspx

[Accessed: 07/02/2012].

Jasionowski, A. (2012) The Art of Naval Architecture: Science or Craft? In:

Proceedings of IMDC 2012, Glasgow, UK.

Jasionowski, A. Vassalos, D. and Scott, A. (2007) Ship Vulnerability to Flooding. In:

Proceedings of $3^{\text {rd }}$ International Maritime Conference on Design for Safety,

Berkley, CA, USA.

Jones, J.C. (1970) Design Methods: Seeds of Human Futures. New York and

Chichester, NY, USA: John Wiley \& Sons.

Jones, A. and Kimber, A. (2012) Balancing Survivability, Operability and Cost for a

Corvette Design. In: Proceedings of RINA Warship 2012: The Affordable Warship, Bath, UK.

Kadinopoulos, B. (2012) Personal communication, Dstl Portsdown West, August 2012.

Kang, H.J., Choi, J., Lee, J.K., Yang, Y. and Lee, D. (2012) A Conceptual Design of

Ship Safety Assessment Model for Ship Systems' Safety Assessment. In:

Proceedings of IMDC 2012, Glasgow, UK.

Kiely, D.G. (1988) Brassey's Sea Power: Naval Vessels, Weapon Systems and

Technology Series, Volume 5: Naval Electronic Warfare. London, UK:

Brassey's Defence Publishers.

Knight, S. (2012a) Challenges in Designing Affordable Warships. In: Proceedings of

IMarEST INEC 2012: Engineering Naval Capability, Edinburgh, UK.

Knight, S.D.T. (2012b) Some Challenges in Designing Affordable Warships. In:

Proceedings of RINA Warship 2012: The Affordable Warship, Bath, UK.

Koheeallee, M. (2012) Personal communication, Dstl Portsdown West, August 2012.

Korotkin, I.M. (1960) Battle Damage to Surface Ships During WWII. Leningrad, USSR:

Sudpromgiz.

Le Garsmeur, P. and d'Anselme, G. (1998) Simulating the Spread of Fire - The

LUCIFER Software. In: Proceedings of IMarEST INEC 1998: Surviving the

War, London, UK.

Lee, D., Lee, J. and Lee, K.H. (2002) A Decision-Support System to Improve Damage

Survivability of Submarine. In: Proceedings of IEA/AIE 2002: Developments in

Applied Artificial Intelligence, Cairns, QLD, AU.

Ling, N. (1985) Merchantmen in the Gulf Front Line. In: Moore, J.E. (ed.) Jane's Naval

Review. $4^{\text {th }}$ ed. London, UK: Jane's Publishing Ltd.

Linklater, N. (2012) The Implications of an All-Electric Ship Approach on Frigate 
Configuration. (MSc), Department of Mechanical Engineering, University College London.

Liwang, H., Ringsberg, J. W. and Norsell, M. (2012) Probabilistic Risk Assessment for Integrating Survivability and Safety Measures on Naval Ships. International Journal of Maritime Engineering, 154 (A1).

Lloyd, A.R.J.M., and Hanson, P.J. (1985) The Operational Effectiveness of the Shipborne Naval Helicopter. In: Proceedings of the RINA International Symposium on The Air Threat at Sea, London, UK, 1985.

Lok, J.J. (1993) Swedish Navy Studies Stealth Fleet Tactics. Jane's Defence Weekly, 20 (10).

Longworth, B. (1983) Sea Wolf and Beyond - The Future of UK Point Defence Missiles. Jane's Defence Weekly, 3 (4).

Manley, D. (2012) The Falklands Conflict 30 Years On - Lessons on Ship Design for the Modern Era. Presented to the DE\&S 2012 Marine Engineering Conference (MEC), held at Abbey Wood on May 3rd 2012.

Mant, J. (2012) Personal communication, Dstl Portsdown West, July 2012.

Martin, A.A. (1998) The Place of Survivability in the Design of Future Surface Warships. In: Proceeding of RINA Warship 1998: Surface Warships - The Next Generation, London, UK.

Martin, A.A. (2001) MSc Naval Architecture Ship Design Exercise 2000-2001:

Battleworthy Royal Fleet Auxiliary. (MSc), Department of Mechanical

Engineering, University College London.

Martin, A.A. (2007) Survivability and the Affordable Warship. In: Proceedings of RINA Warship 2007: The Affordable Warship, Bath, UK.

Mayfield, T.F. and Morrissey, M.S. (1998) The Potential Use of Virtual Reality in Warship Survivability. In: Proceedings of IMarEST INEC 1998: Surviving the War, London, UK.

MBDA Missile Systems. (2012) ASTER Anti-Missile Missile [Brochure]. Paris, FR: MBDA Missile Systems.

McDonald, T. (2010) Combat Systems Effectiveness Exercise. From: MSc Naval Architecture Course, Ship Design module. Department of Mechanical Engineering, University College London.

Mermiris, G., Grandison, A., Themelis, N. and Pawling, R. (2012) Fire Risk Modelling. In: Proceedings of IMDC 2012, Glasgow, UK.

Ministry of Defence, UK. (1988) BR 4007: Guide to Ship Firefighting. $2^{\text {nd }}$ Edition. 
London, UK: HMSO.

Ministry of Defence, UK. (2000) Defence Standard 02-109 (NES 109) - Stability

Standards for Surface Ships: Part 1 Conventional Ships. Bristol, UK: Sea

Technology Group, Defence Procurement Agency. Issue 4 (February).

Ministry of Defence, UK. (2001) The Development of Vulnerability Requirements for

Warships and Auxiliaries. Bristol, UK: Sea Technology Group, Defence

Procurement Agency. Issue 1, (December).

Ministry of Defence, UK. (2010) Defence Standard 02-163 - Classification for Weight

Groups for Surface Ships. Glasgow, UK: Defence Equipment and Support, UK

Defence Standardization. Issue 2 (March).

Ministry of Transport, NZ. (2013) Transport-Related Price Indices : Prices [WWW].

Available from: http://www.transport.govt.nz/ourwork/TMIF/Pages/TI008.aspx

[Accessed: 05/04/2013].

Moss, N.J. (1990) Noise Performance on SWATH Vessels: An Experiment on a Small

SWATH. In: Proceedings of the Undersea Defence Technology Conference,

London, UK, 1990.

Munoz, J.A. and Forrest, C.J.M. (2002) Advantages of Software Integration from Initial

Design Through to Production Design. In: Proceedings of ICCAS 2002, Malmo, $S E$.

National Warship Survivability Committee. (2008) Procuring Survivability. Portsdown

West, UK: Dstl.

NATO. (2003a) Allied Naval Engineering Publication on Ship Combat Survivability

(ANEP 43). $2^{\text {nd }}$ Edition. NATO Classified Document.

NATO. (2003b) Cost Structure and Life Cycle Costs for Military Systems [WWW].

Available from: http://www.dtic.mil/cgi-bin/GetTRDoc?AD=ADA418708

[Accessed: 15/03/2013].

NATO. (2007) Methods and Models for Life Cycle Costing [WWW]. Available from:

http://www.dtic.mil/cgi-bin/GetTRDoc?AD=ADA515584 [Accessed:

15/03/2013].

NATO. (2009) Code of Practice for Life Cycle Costing [WWW]. Available from:

http://ftp.rta.nato.int/public//PubFullText/RTO/TR/RTO-TR-SAS-069///\$TR-

SAS-069-ALL.pdf [Accessed: 21/11/2012].

NATO. (2011) ANEP - 77 (Edition 3): Naval Ship Code [WWW]. Available from:

http://navalshipcode.org/ [Accessed: 08/08/2012].

Naval War College. (2002) Littoral Combat Ship: Concept of Operations Development 
SITREP [WWW]. Available from: http://www.nps.navy.mil/orfacpag/resumem

Pages/LCS\%20CONOPS\%20brief\%2011-15pt1.ppt [Accessed: 11/05/2012].

O’Brien, T. (2012) Personal communication, Dstl Portsdown West, August 2012.

Office for National Statistics. (2012) Consumer Price Indices, October 2012 [WWW].

Available from: http://www.ons.gov.uk/ons/rel/cpi/consumer-price-indices/ october-2012/index.htmlt [Accessed: 05/04/2013].

Pakenham, W.T.T. (1989) Brassey's Sea Power: Naval Vessels, Weapon Systems and

Technology Series, Volume 8: Naval Command and Control. London, UK:

Brassey's Defence Publishers.

Papanikolaou, A. and Boulougouris, E. (1998) Design Aspects of Survivability of

Surface Naval and Merchant Ships. In: Proceedings of the International

Conference on Naval Technology, Piraeus, GR, 1998.

Parry, S.J. (2012) MISSION (v597) User Guide. UK MOD Classified Document.

Parry, S.J. and White, K. (2012) MISSION (v597) Model Description. UK MOD

Classified Document.

Patient, D. (2013) Subject title: Duplicate Predictions [E-mail]. DEPATIENT@mail. dstl.gov.uk [Sent: 19/03/2013].

Patil, S.V. and Kar, A.R. (2012) An Alternative Way of Escape Route for Fire Safety and Evacuation. In: Proceedings of IMDC 2012, Glasgow, UK.

Pegg, N., Roberts, P. and Macadam, T. (2012) Improving the Integration of Ship Model Data in Simulation-Based Design and Management. In: Proceedings of IMDC 2012, Glasgow, UK.

Percival, V. (2010) An Exploration of Design Drivers within Ship Synthesis. (MSc), Department of Mechanical Engineering, University College London.

Petersen, L. (2006) Survivability in Naval Ship Classification. In: Proceedings of RINA Warship 2006: Future Surface Ships, London, UK.

Phillips, R.T.R. (1998) Opening Address. In: Proceedings of IMarEST INEC 1998:

Surviving the War, London, UK.

Piperakis, A.S., Andrews, D.J. and Pawling, R. (2012) An Integrated Approach to Naval Ship Survivability in Preliminary Ship Design. In: Proceedings of RINA Warship 2012: The Affordable Warship, Bath, UK.

Preston, A. (1990) Cost-effective Ship Destruction. Defence, 21 (10).

Project Management Office JSS. (2005a) JSS Ship Concept. Department of National

Defence, Canada.

Project Management Office JSS. (2005b) JSS SRD Narrative. Department of National 


\section{Defence, Canada}

Pugh, R. (2006) A Rapid Maritime Concept Vulnerability Analysis Tool. In:

Proceedings of the European Survivability Workshop, Toulouse, FR, 2006.

Purvis, M.K. (1974) Post War RN Frigate and Guided Missile Destroyer Design 19441969. RINA Transactions, Vol. 116.

QinetiQ Group PLC. (2005a) Assessing Vulnerability to Attack [Brochure].

Farnborough, UK: QinetiQ Group PLC.

QinetiQ Group PLC. (2005b) Building and Infrastructure Protection. [Brochure].

Farnborough, UK: QinetiQ Group PLC.

QinetiQ Group PLC. (2011a) Applicability of Powering Methods. In: Online Help

Pages for Paramarine V7.0.

QinetiQ Group PLC. (2011b) Equipment and Systems. In: SURVIVE Lite User Guide.

QinetiQ Group PLC. (2012) QinetiQ GRC [WWW]. Available from: http://www2. qinetiq.com/home_grc.html [Accessed: 02/02/2012].

Randles, I. (2009) Exploration of Frigate Layout on Vulnerability of Ship's Systems. (MSc), Department of Mechanical Engineering, University College London.

Rattenbury, N. (2004) Selection and Use of Standards for Naval Ships. In: Proceedings of IMarEST INEC 2004: Marine Technology in Transition, Amsterdam, NL.

Rawson, K.J. (1973) Towards Economic Warship Acquisition and Ownership. RINA Transactions, Vol. 115.

Rawson, K.J. and Tupper, E.C. (2001) Basic Ship Theory. $5^{\text {th }}$ ed. Oxford, UK: Butterworth-Heinemann.

Reese, R.M., Calvano, C.N. and Hopkins, T.M. (1998) Operationally Orientated Vulnerability Requirements in the Ship Design Process. Naval Engineers Journal, 110 (1).

Ren, H., Li, C., Feng, Q. and Li, H. (2008) Calculation Method of the Residual Capability of Damaged Warships. In: Proceedings of the ASME $27^{\text {th }}$ International Conference on Offshore Mechanics and Arctic Engineering, Estoril, PT.

Richards, M.G., Ross, A.M., Hastings, D.E. and Rhodes, D.H. (2008) Empirical Validation of Design Principles for Survivable System Architecture. In: Proceedings of the $2^{\text {nd }}$ IEEE Systems Conference, Montreal, QC., CA., 2008.

Robb, M., Horstmann, P., Manley, D. and Tanner, B. (2010) Affordable Survivability for the Modern Surface Combatant. In: Proceedings of IMarEST INEC 2010: The Affordable Future Fleet, Portsmouth, UK. 
Rogers, P.D. (1988) Fixing Battle Damage. United States Naval Institute Proceedings, $114(6)$.

Rolls-Royce PLC. (2002) Fact Sheet: MT30 Marine Gas Turbine [Brochure]. Bristol, UK: Rolls-Royce PLC.

Rolls-Royce PLC. (2008) Fuelling-At-Sea Systems [Brochure]. Newcastle Upon Tyne, UK: Rolls-Royce PLC.

Ross, R., Meijer, C.G. and van de Mheen, R.J. (2012) Degaussing by Normal and Superconductive Windings. In: Proceedings of IMarEST INEC 2012: Engineering Naval Capability, Edinburgh, UK.

Rudius, M. (2012) Approaches to Cost Risk Analysis in Naval Shipbuilding. In: Proceedings of RINA Warship 2012: The Affordable Warship, Bath, UK.

Said, M.O. (1995) Theory and Practice of Total Ship Survivability for Ship Design. Naval Engineers Journal. 107 (4).

Sajdak, J.A.W. and Karni, Z.H. (2006) Determination of a Measure of Total Integrated System Survivability. In: Proceedings of RINA Warship 2006: Future Surface Ships, London, UK.

Sarmento, N. and Duarte, J. (2005) SINGRAR - Sistema INtegrado para a Gestão de Prioridades de Reparação e Afectação de Recursos (Integrated System for Repair Priority Management and Resource Assignment). From: Portuguese Navy General Staff - CIS Division, Lisbon, PT.

Saunders, S. (ed.) (2008) Jane's Fighting Ships 2008-2009. $111^{\text {th }}$ ed. Coulsdon, UK: Jane's Information Group.

Schofield, J. (2006) Bringing a Naval Philosophy to the Survivability Assessment of Buildings and Infrastructures. In: Proceedings of the European Survivability Workshop, Toulouse, FR, 2006.

Schofield, J.S., Horstmann, P.O., Robb, M.D. and Lewis, D.H (2012) The Influence of Survivability in the Design of a Capable, Affordable Surface Combatant. In: Proceedings of RINA Warship 2012: The Affordable Warship, Bath, UK.

Schottel Group. (2010a) SPJ PUMP JET: Operating Principle [WWW]. Available from: http://www.schottel.de/marine-propulsion/spj-pump-jet/operatingprinciple/ [Accessed: 03/04/2013].

Schottel Group. (2010b) SPJ PUMP JET: Technical Data [WWW]. Available from: http://www.schottel.de/marine-propulsion/spj-pump-jet/technical-data/ [Accessed: 03/04/2013].

Scott, R. (2010) Extending Reach: JSS seeks to rewrite the theory of lift. Jane's Navy 
International, 115 (5).

Scrase, T. (1991) Signature Management - The Material Facts. Maritime Defence, (September).

Sharp, R.D. (2011) Subject title: Fire vulnerability modelling in SURVIVE Lite; Attached document title: Fire and Smoke [E-mail]. RDSHARP@ qinetiq.com [Sent: 09/08/2011].

Surko, W.S. (1994) An Assessment of Current Warship Damage Stability Criteria. Naval Engineers Journal, 106 (3).

Sutcliffe, J. (2012) Personal communication, Dstl Portsdown West, July 2012.

Thales Group. (2012) Integrated Mast [WWW]. Available from: http://www. thalesgroup.com/integratedmast/ [Accessed: 06/02/2012].

Thomas, P.H. (1967). The Spread of Fire in Buildings: A Statistical Approach. Fire Research Note \#694. Borehamwood, UK: Fire Research Station.

Thornton, J.S. (2011) Personal communication, Dstl Portsdown West, August 2011. Thornton, J.S., Coulthard, R. and Hardy, J.N. (2006) Modelling Maritime Survivability. In: Proceedings of RINA Warship 2006: Future Surface Ships, London, UK.

Thornton, J.S., Courts, M.D. and Robb, M. (2007) Making Warship Survivability Affordable. In: Proceedings of RINA Warship 2007: The Affordable Warship, Bath, UK.

Thornton, J.S. and Day, T. (2012) Personal communication, Dstl Portsdown West, July 2012.

Thornton, J.S., White, K. and Parry, S.J. (2011) Personal communication, Dstl Portsdown West, August 2011.

Thornton, J.S., White, K. and Parry, S.J. (2012) Personal communication, Dstl Portsdown West, July 2012.

Tozer, V.B. (1993) Aspects of Vulnerability. Dunfermline, UK: Vulnerability Assessment Section, DRA.

Truver, S.C. (2001) Fire! Fire! Fire! All Hands Man Your Battle Stations! United States Naval Institute Proceedings, 127 (1).

TTS Marine ASA. (2010) Product Specification: TTS Cargo Cranes [Brochure]. Bergen, NO: TTS Marine ASA.

Turner, S.D., Horstmann, P. and Bain, G. (2006) Warship Survivability. In: Proceedings of RINA Warship 2006: Future Surface Ships, London, UK. UCL. (2001) Topside and Sonar Layout Considerations for Warships. From: MSc Naval Architecture Course, Ship Design module. Department of Mechanical 
Engineering, University College London.

UCL. (2006a) Naval Architecture Warship Design Exercise - Procedure. From:

Undergraduate Naval Architecture Course, Ship Design module. Department of Mechanical Engineering, University College London.

UCL. (2006b) $3^{\text {rd }}$ Year Frigate Design Exercise: Design Data. From: Undergraduate Naval Architecture Course, Ship Design module. Department of Mechanical Engineering, University College London.

UCL. (2010a) Ship Design Procedure. From: MSc Naval Architecture Course, Ship Design module. Department of Mechanical Engineering, University College London.

UCL. (2010b) Ship Design Data Book. From: MSc Naval Architecture Course, Ship Design module. Department of Mechanical Engineering, University College London.

Usher, P.J. and Dorey, A.L. (1982) A Family of Warships. RINA Transactions, Vol. 124.

van Oers, B., van Ingens, G. and, Stapersma, D. (2012) An Integrated Approach for the Design of Resilient Ship Services Systems. In: Proceedings of IMarEST INEC 2012: Engineering Naval Capability, Edinburgh, UK.

Vasilakos, J., Devries, R. and Tompkins, K.T. (2000) Total Ship Open Systems Architecture. Naval Engineers Journal, 112 (4).

Vassalos, D. (2012) State of the Art Report: Design for Safety: Risk-Based Design LifeCycle Risk Management. In: Proceedings of IMDC 2012, Glasgow, UK. Vasudevan, S. and Rusling, S.C. (2007) A Ship Design Tool Using Genetic Algorithms. In: Proceedings of the International Conference on Computer Applications in Shipbuilding, Portsmouth, UK, 2007.

Vestdavit AS. (2012) Vestdavit - The world-Leading Provider of Efficient and Innovative Boat-Handling Systems [WWW]. Available form: http://www. vestdavit.no/index.php [Accessed: 28/02/2012].

Walton W.D. and Thomas P.H. (1995) Estimating Temperatures in Compartment Fires. In: P.J. DiNenno, P.J., Beyler, C.L., Custer, R.L.P. and Walton, W.D. (eds.) SFPE Handbook of Fire Protection Engineering. $2^{\text {nd }}$ ed. Quincy, MA, USA: National Fire Protection Association.

Wartsila Corporation. (2010) 2010 Ship Power Product Catalogue [Brochure].

Helsinki, FI: Wartsila Corporation.

Watson, D.G.M. (1998) Elsevier Ocean Engineering Book Series, Volume 1: Practical 
Ship Design. Oxford, UK: Elsevier Science Ltd.

Watson, D.G.M. and Gilfillan, A.W. (1976) Some Ship Design Methods. RINA

Transactions, Vol. 118.

Wettern, D. (1991) Warship "Survivability" - Soviet Thinking. Warship Technology, 14.

White, K. and Allwood, M. (2011) MISSION Validation Log Book. UK MOD Classified Document.

X-Rates. (2012) Historic Lookup [WWW]. Available form: http://www.X-rates.com/ historical/?from=USD\&amount=1.00\&date=2013-04-05 [Accessed: 05/04/2013]. 